\title{
Enantioselective Synthesis of Multifunctionalized 4H-Pyrans via Formal [4 + 2] Annulation Process by Bifunctional Phosphonium Salt Catalysis
}

Jia-Hong Wu, ${ }^{\dagger}$ Jianke Pan,${ }^{\dagger}$ Juan Du, ${ }^{\dagger}$ Xiaoxia Wang, ${ }^{\dagger}$ Xuemei Wang, ${ }^{\dagger}$ Chunhui Jiang, ${ }^{\dagger} \S$ Tianli Wang ${ }^{\dagger} \uparrow$

${ }^{\dagger}$ Key Laboratory of Green Chemistry \& Technology of Ministry of Education, College of Chemistry, Sichuan University, Chengdu 610064, P. R. China.

$\S$ School of Environmental and Chemical Engineering, Jiangsu University of Science and Technology, 2 Mengxi Road, Zhenjiang 212003 P. R. China.

E-mail: wangtl@ scu.edu.cn

1. General Information 2

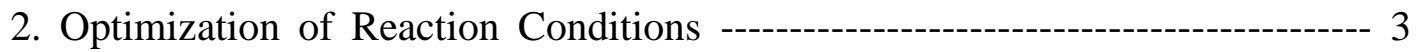

3. Preparation of Catalysts ----------------------------------------------------------------- 5

4. Preparation of 3-Alkenyl-oxindoles and Allenoates ----------------------------- 9

5. Representative Procedure for [4 + 2] Annulation Reaction--------------------- 17

6. Determination of Absolute Configuration of the Products --------------------82

7. Gram-scale Synthesis and Transformations ------------------------------------83

8. Preliminary Mechanistic Studies and Proposed Reaction Cycle ----------------86

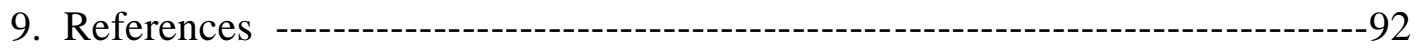

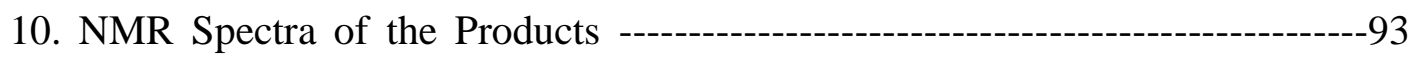




\section{General Information}

All the starting materials were obtained from commercial sources and used without further purification unless otherwise stated. ${ }^{1} \mathrm{H}$ and ${ }^{13} \mathrm{C}$ NMR spectra were recorded at ambient temperature in $\mathrm{CDCl}_{3}$ on a Brüker Advance 400 spectrometer. Chemical shifts $(\delta)$ were given in parts per million $(\mathrm{ppm})$, and the residual solvent peak was used as an internal reference $\left(\mathrm{CDCl}_{3}: \delta 7.26 \mathrm{ppm}{ }^{1} \mathrm{H} ; \delta 77.16 \mathrm{ppm}{ }^{13} \mathrm{C}\right)$. Multiplicity was indicated as follows: $\mathrm{s}$ (singlet), $\mathrm{d}$ (doublet), $\mathrm{t}$ (triplet), q (quartet), m (multiplet), dd (doublet of doublet), br s (broad singlet). Coupling constants $(J)$ were reported in Hertz (Hz). High resolution mass spectra were obtained on a Thermo LTQ mass spectrometer. For thin layer chromatography (TLC), Merck pre-coated TLC plates (Merck 60 F254) were used, and compounds were visualized with a UV light at 254 nm. Further visualization was achieved by staining with iodine, or ceric ammonium molybdate followed by heating on a hot plate. Flash chromatographic separations were performed on Merck 60 (0.040-0.063 mm) mesh silica gel. Enantiomeric excess was determined by HPLC analysis using chiral column described below in detail. Optical rotations were measured with polarimeter.

All phosphonium salt catalysts $\mathbf{P}_{\mathbf{1}}-\mathbf{P}_{\mathbf{1 0}}$ used in this study were prepared via a P-alkylation reaction of our previously reported organophosphines according to the known procedures. ${ }^{[1]}$ The unknown catalysts $\mathbf{P}_{\mathbf{1 0}}$ was fully characterized. All 3-alkenyl-oxindoles $\mathbf{1}$ and $\mathbf{5}$ were synthesized following the methods reported in the literature, ${ }^{[2]}$ and allenoates 2 were synthesized according to the known procedures. ${ }^{[3]}$ The unknown compound 1f, 1h-i, 1o, 1r-s, 1u-y, 5b-c, 5i, 2d-e was fully characterized. The absolute configurations of multisubstituted 4H-pyran products were assigned by X-ray crystallographic analysis of the single crystal of 3l (Figure $\mathrm{S} 1)$. 


\section{Optimization of Reaction Conditions}
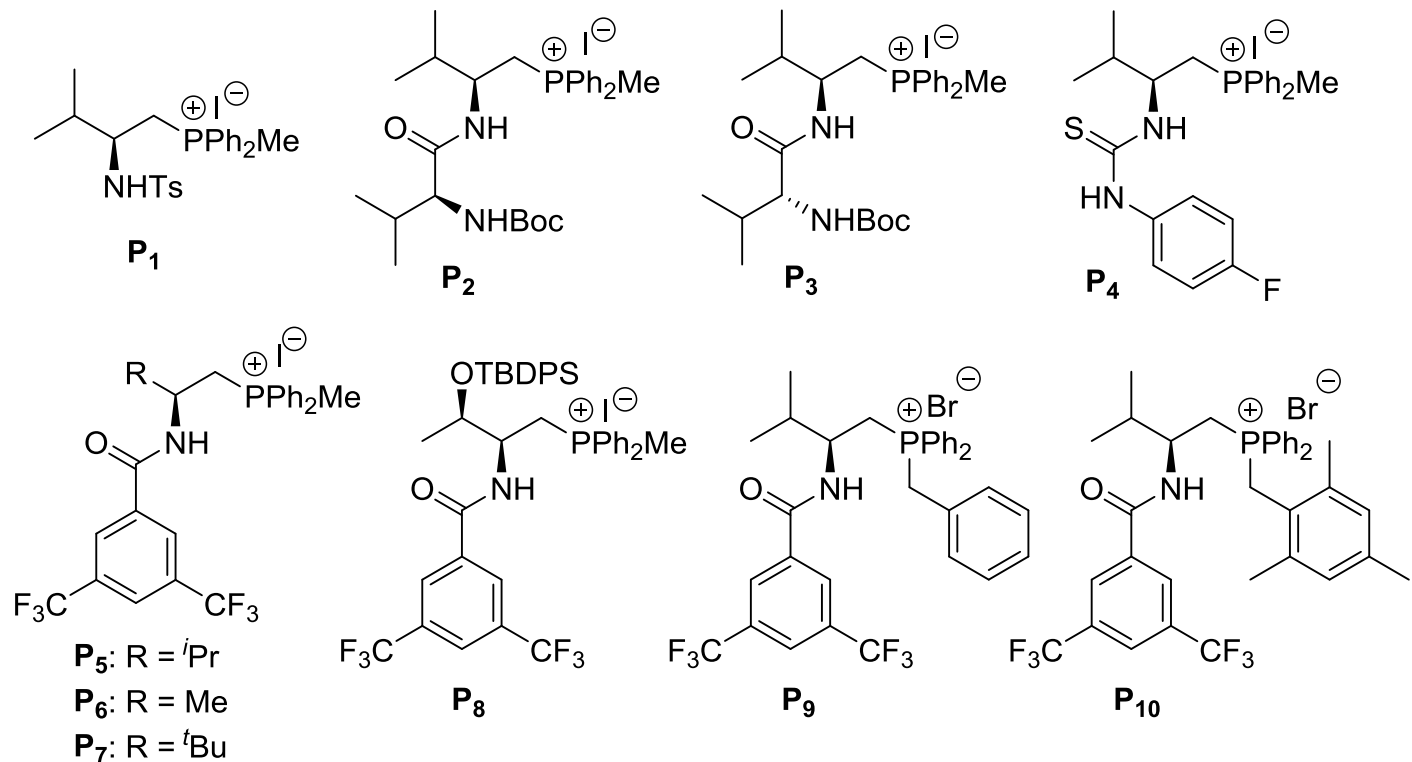

Scheme S1: Chiral phosphonium salt catalysts used in this study.

$($ TBDPS $=$ tert-butyldiphenylsilyl, Ts $=4$-toluenesulfonyl $)$

A. Optimization of reaction conditions for the asymmetric [4+2] annulations of 3-alkenyl-oxindoles and allenoates

Table S1: Asymmetric [4+2] annulations of 3-alkenyl-oxindoles 1a and allenoates 2a catalyzed by different chiral phosphonium salts in toluene. ${ }^{[\mathrm{a}]}$

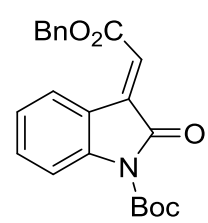

1a

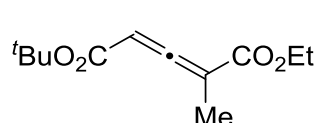

2a

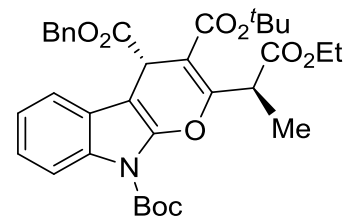

3a

\begin{tabular}{lllll}
\hline entry & cat. & $t[\mathrm{~h}]$ & yield $(\%)^{b}$ & $e e(\%)^{c}$ \\
\hline 1 & $\mathbf{P}_{\mathbf{1}}$ & 8 & 76 & 15 \\
2 & $\mathbf{P}_{\mathbf{2}}$ & 8 & 91 & 5
\end{tabular}




\begin{tabular}{lllll}
3 & $\mathbf{P}_{\mathbf{3}}$ & 8 & 90 & 3 \\
4 & $\mathbf{P}_{\mathbf{4}}$ & 8 & 92 & 7 \\
5 & $\mathbf{P}_{\mathbf{5}}$ & 8 & 97 & 50 \\
6 & $\mathbf{P}_{\mathbf{6}}$ & 8 & 93 & 25 \\
7 & $\mathbf{P}_{\mathbf{7}}$ & 8 & 96 & 14 \\
8 & $\mathbf{P}_{\mathbf{8}}$ & 8 & 95 & 45 \\
9 & $\mathbf{P}_{\mathbf{9}}$ & 8 & 98 & 73 \\
$\mathbf{1 0}$ & $\mathbf{P}_{\mathbf{1 0}}$ & $\mathbf{8}$ & $\mathbf{9 8}$ & $\mathbf{8 1}$ \\
\hline${ }^{a}$ Reaction conditions: 1a $(0.1 \mathrm{mmol}), \mathbf{2 a}\left(0.12\right.$ mmol), catalyst $(0.02 \mathrm{mmol}), \mathrm{Cs}_{2} \mathrm{CO}_{3}$ \\
$(0.2$ mmol), toluene $(2.0 \mathrm{~mL})$ at room temperature for 8 h. All dr values were \\
determined by HPLC analysis of crude product. ${ }^{b}$ Isolated yields. ${ }^{c}$ Determined by \\
HPLC on a chiral stationary phase.
\end{tabular}

Table S2: Asymmetric [4+2] annulations of 3-alkenyl-oxindoles 1a and allenoates 2a catalyzed by $\mathbf{P}_{10}$ in toluene: screening of the bases. ${ }^{[a]}$

7

\begin{tabular}{lllll}
\hline entry & base (x equiv.) & $t[\mathrm{~h}]$ & yield $(\%)^{b}$ & $e e(\%)^{c}$ \\
\hline 1 & $\mathrm{PhONa}_{2}(2.0)$ & 8 & 67 & 17 \\
2 & $\mathrm{~K}_{2} \mathrm{CO}_{3}(2.0)$ & 8 & 89 & 83 \\
3 & $\mathrm{Na}_{2} \mathrm{CO}_{3}(2.0)$ & 8 & 54 & 62 \\
4 & $\mathrm{KHCO}_{3}(2.0)$ & 8 & 65 & 74 \\
5 & $\mathrm{Cs}_{2} \mathrm{CO}_{3}(2.0)$ & 8 & 98 & 81 \\
6 & $\mathrm{Na}_{3} \mathrm{PO}_{4}(2.0)$ & 8 & 80 & 68 \\
7 & $\mathrm{~K}_{3} \mathrm{PO}_{4}(2.0)$ & $\mathbf{8}$ & $\mathbf{9 8}$ & $\mathbf{9 1}$ \\
8 & $\mathrm{~K}_{3} \mathrm{PO}_{4} \cdot 3 \mathrm{H}_{2} \mathrm{O}(2.0)$ & 8 & 96 & 89 \\
9 & $\mathrm{~K}_{3} \mathrm{PO}_{4} \cdot 7 \mathrm{H}_{2} \mathrm{O}(2.0)$ & 8 & 95 & 85 \\
10 & $\mathrm{~K}_{3} \mathrm{PO}_{4}(0.5)$ & 8 & 92 & 86 \\
11 & $\mathrm{~K}_{3} \mathrm{PO}_{4}(1.0)$ & 8 & 95 & 89 \\
12 & $\mathrm{~K}_{3} \mathrm{PO}_{4}(4.0)$ & 8 & 98 & 87 \\
13 & $\mathrm{~K}_{3} \mathrm{PO}_{4}(6.0)$ & 8 & 97 & 83 \\
\hline
\end{tabular}

${ }^{a}$ Reaction conditions: 1a $(0.1 \mathrm{mmol}), \mathbf{2 a}(0.12 \mathrm{mmol}), \mathbf{P}_{\mathbf{1 0}}(0.02 \mathrm{mmol})$, toluene $(2.0$ $\mathrm{mL}$ ) at room temperature for $8 \mathrm{~h}$. All dr values were determined by HPLC analysis of crude product. ${ }^{b}$ Isolated yields. ${ }^{c}$ Determined by HPLC on a chiral stationary phase. 
Table S3: Asymmetric [4+2] annulations of 3-alkenyl-oxindole 1a and allenoate 2a catalyzed by $\mathbf{P}_{\mathbf{1 0}}$ : screening solvents, catalyst loading. ${ }^{[\mathrm{a}}$

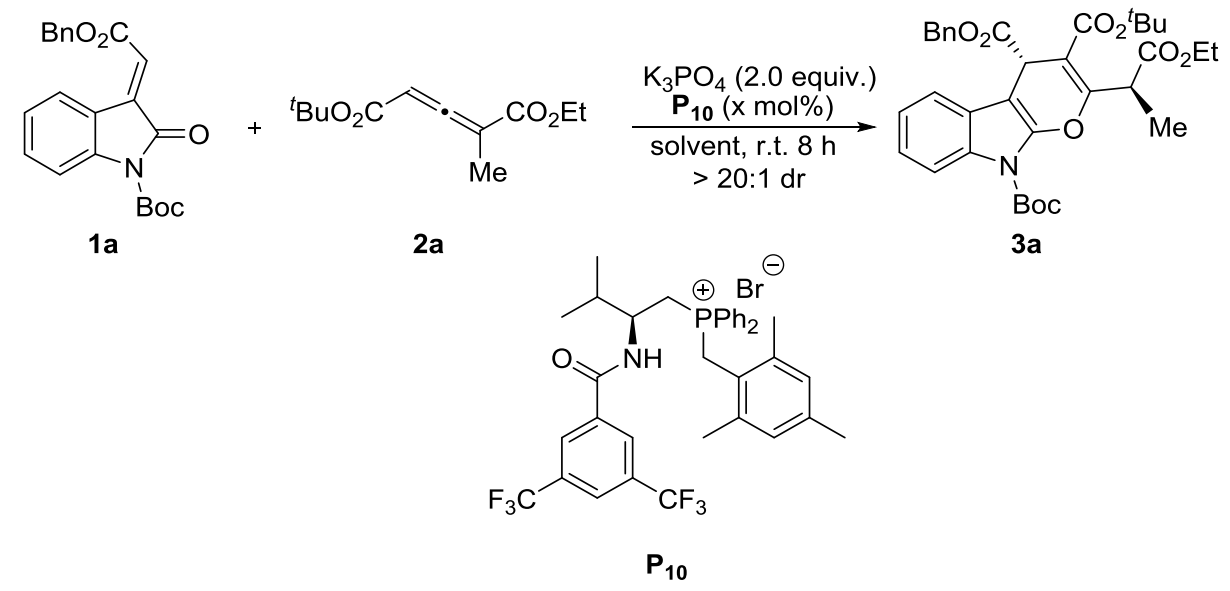

\begin{tabular}{llllll}
\hline entry & solvent & cat. $(\mathrm{mol} \%)$ & $t[\mathrm{~h}]$ & yield $(\%)^{b}$ & $e e(\%)^{c}$ \\
\hline 1 & toluene & 20 & 8 & 98 & 91 \\
2 & chlorobenzene & 20 & 8 & 98 & 85 \\
3 & $\mathrm{CHCl}_{3}$ & 20 & 8 & 89 & 62 \\
4 & $\mathrm{CH}_{2} \mathrm{Cl}_{2}$ & 20 & 8 & 61 & 70 \\
5 & hexane & 20 & 8 & 89 & 78 \\
6 & ethyl acetate & 20 & 8 & 98 & 71 \\
$\mathbf{7}$ & Et $2 \mathrm{O}$ & 20 & 8 & 95 & 79 \\
8 & xylene & 20 & 8 & 98 & 93 \\
10 & $o$-xylene & 20 & 8 & 98 & 90 \\
11 & $m$-xylene & 20 & 8 & 97 & 88 \\
12 & $p$-xylene & 20 & 8 & 97 & 91 \\
$\mathbf{1 3}$ & xylene & $\mathbf{1 0}$ & $\mathbf{8}$ & $\mathbf{9 8}$ & $\mathbf{9 3}$ \\
14 & xylene & 5 & 8 & 96 & 82 \\
\hline
\end{tabular}

${ }^{a}$ Reaction conditions: 1a $(0.1 \mathrm{mmol}), 2 \mathrm{a}(0.12 \mathrm{mmol})$, solvent $(2.0 \mathrm{~mL})$ at room temperature for $8 \mathrm{~h}$. All $\mathrm{dr}$ values were determined by HPLC analysis of crude product. ${ }^{b}$ Isolated yields. ${ }^{c}$ Determined by HPLC on a chiral stationary phase.

\section{Preparation of Catalysts}

\section{A. General procedures for preparation of phosphonium salts}



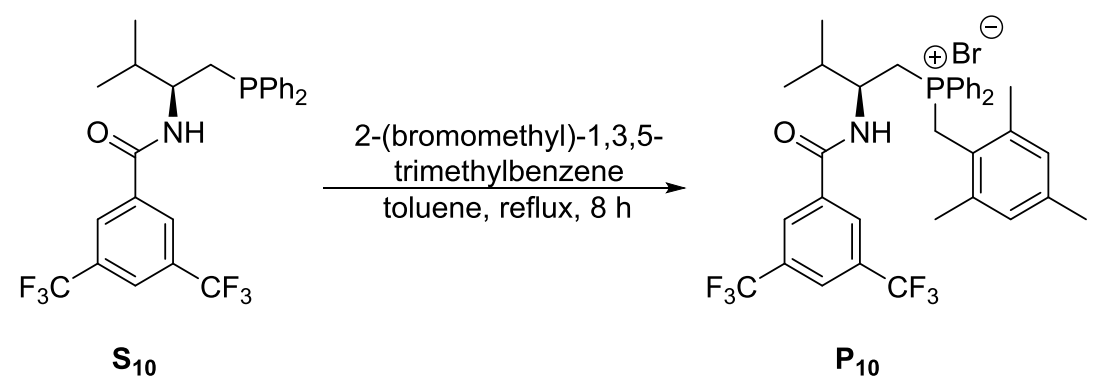

The 2-(bromomethyl)-1,3,5-trimethylbenzene (26 mg, $0.12 \mathrm{mmol})$ and $\mathbf{S}_{\mathbf{1 0}}(51 \mathrm{mg}$, $0.1 \mathrm{mmol})$ dissolved in toluene $(2 \mathrm{~mL})$, then the mixture was refluxed in oil bath for $8 \mathrm{~h}$. The resulting solution was cooled to room temperature and the solvent was removed under reduced pressure. The residue was purified by flash column chromatography on silica gel (petroleum ether /ethyl acetate $=1: 1$ to methanol) to afford the the desired chiral phosphonium salt $\mathbf{P}_{\mathbf{1 0}}(69 \mathrm{mg}, 95 \%$ yield) as a white solid.

\section{$\underline{(S)-(2-(3,5-b i s(t r i f l u o r o m e t h y l) b e n z a m i d o)-3-m e t h y l b u t y l) d i p h e n y l(2,4,6-t r i m e t h ~}$}

\section{ylbenzyl)phosphonium $\left(\mathbf{P}_{10}\right)$}

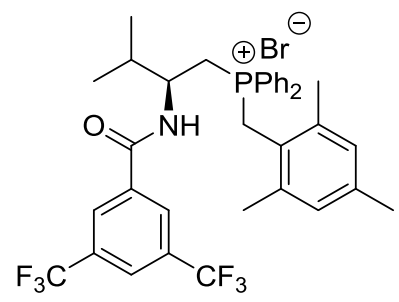

A white solid (95\%); ${ }^{1} \mathrm{H}$ NMR $\left(400 \mathrm{MHz}, \mathrm{CDCl}_{3}\right) \delta 9.36(\mathrm{~d}, J=8.7 \mathrm{~Hz}, 1 \mathrm{H}), 8.39(\mathrm{~s}$, 2H), $7.81(\mathrm{~s}, 1 \mathrm{H}), 7.70(\mathrm{dd}, J=12.0,7.7 \mathrm{~Hz}, 2 \mathrm{H}), 7.65-7.59(\mathrm{~m}, 2 \mathrm{H}), 7.58(\mathrm{t}, J=7.9$ $\mathrm{Hz}, 1 \mathrm{H}), 7.45(\mathrm{td}, J=7.5,3.1 \mathrm{~Hz}, 2 \mathrm{H}), 7.31-7.22(\mathrm{~m}, 3 \mathrm{H}), 6.59(\mathrm{~s}, 2 \mathrm{H}), 5.05-4.83$ $(\mathrm{m}, 1 \mathrm{H}), 4.51-4.41(\mathrm{~m}, 1 \mathrm{H}), 4.38(\mathrm{dd}, J=14.2,4.3 \mathrm{~Hz}, 2 \mathrm{H}), 3.03-2.94(\mathrm{~m}, 1 \mathrm{H})$, $2.69-2.61(\mathrm{~m}, 1 \mathrm{H}), 2.23(\mathrm{dd}, J=12.8,6.3 \mathrm{~Hz}, 1 \mathrm{H}), 2.08(\mathrm{~s}, 3 \mathrm{H}), 1.79(\mathrm{~s}, 6 \mathrm{H}), 0.97(\mathrm{~s}$, $6 \mathrm{H}) ;{ }^{13} \mathrm{C}$ NMR $\left(100 \mathrm{MHz}, \mathrm{CDCl}_{3}\right) \delta 163.8(\mathrm{~s}), 138.3(\mathrm{~d}, J=4.2 \mathrm{~Hz}), 137.6(\mathrm{~d}, J=5.4$ Hz), $134.8(\mathrm{~d}, J=2.8 \mathrm{~Hz}), 134.7(\mathrm{~s}), 134.3(\mathrm{~d}, J=2.8 \mathrm{~Hz}), 134.0(\mathrm{~d}, J=9.5 \mathrm{~Hz})$, $133.7(\mathrm{~d}, J=8.7 \mathrm{~Hz}), 131.2(\mathrm{q}, J=33.8 \mathrm{~Hz}), 129.8(\mathrm{~d}, J=6.7 \mathrm{~Hz}), 129.7(\mathrm{~d}, J=1.2$ $\mathrm{Hz}), 129.4(\mathrm{~d}, J=12.3 \mathrm{~Hz}), 128.7(\mathrm{~d}, J=2.5 \mathrm{~Hz}), 124.6(\mathrm{q}, J=2.7 \mathrm{~Hz}), 123.1(\mathrm{q}, J=$ 
$273.1 \mathrm{~Hz}), 122.1$ (d, $J=9.1 \mathrm{~Hz}), 117.5$ (d, $J=80.8 \mathrm{~Hz}), 116.3(\mathrm{~d}, J=81.6 \mathrm{~Hz}), 50.3$ $(\mathrm{d}, J=5.9 \mathrm{~Hz}), 34.7(\mathrm{~d}, J=12.8 \mathrm{~Hz}), 26.6(\mathrm{~d}, J=46.3 \mathrm{~Hz}), 25.2(\mathrm{~d}, J=47.8 \mathrm{~Hz})$, $21.1(\mathrm{~d}, J=1.1 \mathrm{~Hz}), 20.7,19.3,18.9 ;{ }^{31} \mathrm{P} \mathrm{NMR}\left(162 \mathrm{MHz}, \mathrm{CDCl}_{3}\right) \delta 21.7$. (ESI) $m / z$ calcd for $\mathrm{C}_{36} \mathrm{H}_{37} \mathrm{~F}_{6} \mathrm{NOP}[\mathrm{M}-\mathrm{Br}]^{+}=644.2511$, found $=644.2545$.

\section{$\underline{(S)-(2-(3,5-b i s(t r i f l u o r o m e t h y l) b e n z a m i d o)-3-m e t h y l b u t y l) d i p h e n y l(2,4,6-t r i m e t h ~}$}

\section{ylbenzyl)phosphonium ( $\mathbf{P}_{\mathbf{1 0 - 2}}$}

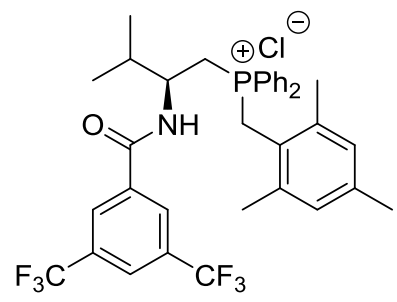

A white solid (91\%); ${ }^{1} \mathrm{H}$ NMR (400 MHz, $\left.\mathrm{CDCl}_{3}\right) \delta 10.18(\mathrm{~d}, J=8.5 \mathrm{~Hz}, 1 \mathrm{H}), 8.56(\mathrm{~s}$, 2H), $7.85(\mathrm{~s}, 1 \mathrm{H}), 7.73-7.54(\mathrm{~m}, 5 \mathrm{H}), 7.47-7.42(\mathrm{~m}, 2 \mathrm{H}), 7.37-7.27(\mathrm{~m}, 3 \mathrm{H}), 6.64$ (s, 2H), $5.16(\mathrm{dd}, J=27.0,11.7 \mathrm{~Hz}, 1 \mathrm{H}), 4.58-4.40(\mathrm{~m}, 1 \mathrm{H}), 4.37$ (dd, $J=14.3,4.9$ $\mathrm{Hz}, 2 \mathrm{H}), 3.42(\mathrm{q}, J=7.0 \mathrm{~Hz}, 1 \mathrm{H}), 2.88-2.74(\mathrm{~m}, 1 \mathrm{H}), 2.24(\mathrm{dd}, J=13.0,6.5 \mathrm{~Hz}, 1 \mathrm{H})$, $2.13(\mathrm{~d}, J=2.5 \mathrm{~Hz}, 3 \mathrm{H}), 1.81(\mathrm{~s}, 6 \mathrm{H}), 1.01(\mathrm{~d}, J=6.7 \mathrm{~Hz}, 6 \mathrm{H}) .{ }^{13} \mathrm{C} \mathrm{NMR}(100 \mathrm{MHz}$, $\left.\mathrm{CDCl}_{3}\right) \delta 163.8(\mathrm{~s}), 138.4(\mathrm{~d}, J=4.2 \mathrm{~Hz}), 137.6(\mathrm{~d}, J=5.4 \mathrm{~Hz}), 134.8(\mathrm{~s}), 134.7(\mathrm{~d}, J$ $=3.0 \mathrm{~Hz}), 134.3(\mathrm{~d}, J=3.0 \mathrm{~Hz}), 133.9(\mathrm{~d}, J=9.3 \mathrm{~Hz}), 133.7$ (d, $J=8.7 \mathrm{~Hz}), 131.3(\mathrm{q}$, $J=33.7 \mathrm{~Hz}), 129.8(\mathrm{~s}), 129.77(\mathrm{~d}, J=15.1 \mathrm{~Hz}), 129.4(\mathrm{~d}, J=12.3 \mathrm{~Hz}), 128.8(\mathrm{~d}, J=$ $2.4 \mathrm{~Hz}), 124.7-124.6(\mathrm{~m}), 123.1(\mathrm{q}, J=273.1 \mathrm{~Hz}), 122.1(\mathrm{~d}, J=9.2 \mathrm{~Hz}), 117.2(\mathrm{t}, J$ $=81.5 \mathrm{~Hz}), 50.2(\mathrm{~d}, J=5.9 \mathrm{~Hz}), 34.7(\mathrm{~d}, J=13.0 \mathrm{~Hz}), 26.4(\mathrm{~d}, J=46.1 \mathrm{~Hz}), 24.8(\mathrm{~d}$, $J=47.8 \mathrm{~Hz}), 21.1(\mathrm{~d}, J=1.4 \mathrm{~Hz}), 20.7(\mathrm{~d}, J=1.2 \mathrm{~Hz}), 18.9(\mathrm{~d}, J=10.5 \mathrm{~Hz}) ;{ }^{31} \mathrm{P}$ NMR $\left(162 \mathrm{MHz}, \mathrm{CDCl}_{3}\right) \delta 21.3 ;{ }^{19} \mathrm{~F} \mathrm{NMR}\left(376 \mathrm{MHz}, \mathrm{CDCl}_{3}\right) \delta-62.5$ (s). (ESI) $\mathrm{m} / \mathrm{z}$ calcd for $\mathrm{C}_{36} \mathrm{H}_{37} \mathrm{~F}_{6} \mathrm{NOP}[\mathrm{M}-\mathrm{Cl}]^{+}=644.2511$, found $=644.2537$.

\section{B. Preparation of phosphonium salts $\mathbf{P}_{10-1}$}




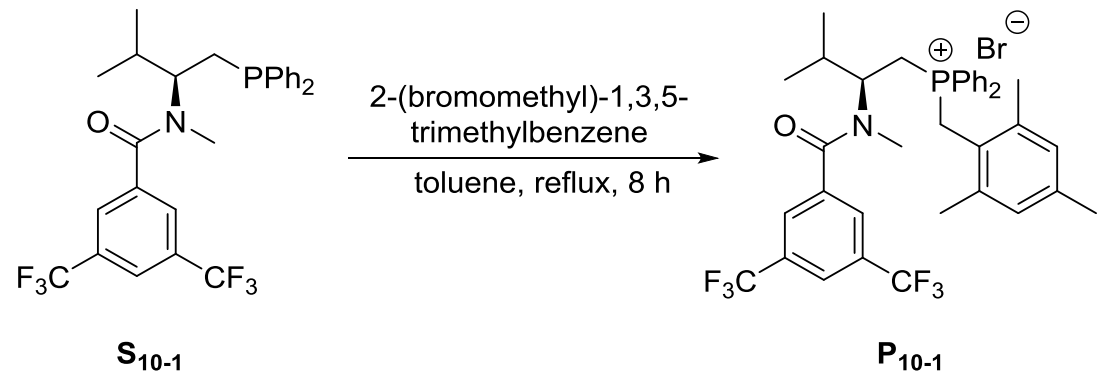

The 2-(bromomethyl)-1,3,5-trimethylbenzene (26 $\mathrm{mg}, 0.12 \mathrm{mmol}$ ) and $\mathbf{S}_{\mathbf{1 0 - 1}}$ (53 $\mathrm{mg}, 0.1 \mathrm{mmol})$ dissolved in toluene $(2 \mathrm{~mL})$, then the mixture was refluxed in oil bath for $8 \mathrm{~h}$. The resulting solution was cooled to room temperature and the solvent was removed under reduced pressure. The residue was purified by flash column chromatography on silica gel (petroleum ether /ethyl acetate $=1: 1$ to methanol) to afford the the desired chiral phosphonium salt $\mathbf{P}_{\mathbf{1 0 - 1}}(67 \mathrm{mg}, 91 \%$ yield $)$ as a white solid.

\section{$\underline{(S)-(3-m e t h y l-2-(N-m e t h y l-3,5-b i s(t r i f l u o r o m e t h y l) b e n z a m i d o) b u t y l) d i p h e n y l(2,4 ~}$}

\section{6-trimethylbenzyl)phosphonium $\left(\mathbf{P}_{10-1}\right)$}<smiles>Cc1cc(C)c(C[Pb]([O-])(Br)C[C@@H](C(C)C)N(C)C(=O)c2cc(C(F)(F)F)cc(C(F)(F)F)c2)c(C)c1</smiles>

A white solid (91\%); ${ }^{1} \mathrm{H}$ NMR $\left(400 \mathrm{MHz}, \mathrm{CDCl}_{3}\right) \delta 8.03(\mathrm{dd}, J=12.4,7.6 \mathrm{~Hz}, 2 \mathrm{H})$, $7.72(\mathrm{~s}, 1 \mathrm{H}), 7.66(\mathrm{dd}, J=10.2,8.3 \mathrm{~Hz}, 3 \mathrm{H}), 7.56(\mathrm{dd}, J=8.0,6.3 \mathrm{~Hz}, 1 \mathrm{H}), 7.52-$ $7.41(\mathrm{~m}, 4 \mathrm{H}), 7.07(\mathrm{~s}, 2 \mathrm{H}), 6.43(\mathrm{~s}, 2 \mathrm{H}), 5.05-4.92(\mathrm{~m}, 1 \mathrm{H}), 4.82-4.66(\mathrm{~m}, 2 \mathrm{H}), 4.59$ $(\mathrm{t}, J=15.0 \mathrm{~Hz}, 1 \mathrm{H}), 3.79-3.58(\mathrm{~m}, 1 \mathrm{H}), 2.88(\mathrm{~s}, 3 \mathrm{H}), 2.24-2.11(\mathrm{~m}, 1 \mathrm{H}), 2.01(\mathrm{~s}$, $3 \mathrm{H}), 1.62(\mathrm{~s}, 6 \mathrm{H}), 1.16(\mathrm{~d}, J=6.5 \mathrm{~Hz}, 3 \mathrm{H}), 0.81(\mathrm{~d}, J=6.6 \mathrm{~Hz}, 3 \mathrm{H}) ;{ }^{13} \mathrm{C}$ NMR $(100$ $\left.\mathrm{MHz}, \mathrm{CDCl}_{3}\right) \delta 168.6,137.9(\mathrm{~d}, J=5.4 \mathrm{~Hz}), 137.6(\mathrm{~d}, J=4.3 \mathrm{~Hz}), 137.2,134.8(\mathrm{~d}, J$ $=2.7 \mathrm{~Hz}), 134.5(\mathrm{~d}, J=2.8 \mathrm{~Hz}), 134.4(\mathrm{~d}, J=10.0 \mathrm{~Hz}), 134.1(\mathrm{~d}, J=8.4 \mathrm{~Hz}), 131.4$ $(\mathrm{q}, J=33.8 \mathrm{~Hz}), 129.5(\mathrm{~d}, J=6.0 \mathrm{~Hz}), 129.4,129.4(\mathrm{~d}, J=1.9 \mathrm{~Hz}), 127.1(\mathrm{~d}, J=2.2$ Hz), $123.4(\mathrm{q}, J=3.2 \mathrm{~Hz}), 122.7(\mathrm{~d}, J=9.3 \mathrm{~Hz}), 122.7(\mathrm{q}, J=273.0 \mathrm{~Hz}), 118.1(\mathrm{~d}, J$ $=80.1 \mathrm{~Hz}), 115.9(\mathrm{~d}, J=81.5 \mathrm{~Hz}), 54.9,33.5,31.5(\mathrm{~d}, J=13.0 \mathrm{~Hz}), 29.6(\mathrm{~d}, J=45.9$ $\mathrm{Hz}), 24.2(\mathrm{~d}, J=46.7 \mathrm{~Hz}), 21.1(\mathrm{~d}, J=1.4 \mathrm{~Hz}), 20.7,20.6(\mathrm{~d}, J=1.1 \mathrm{~Hz}), 19.1 ;{ }^{31} \mathrm{P}$ 
NMR (162 MHz, $\left.\mathrm{CDCl}_{3}\right) \delta$ 22.6. (ESI) $\mathrm{m} / z$ calcd for $\mathrm{C}_{37} \mathrm{H}_{39} \mathrm{~F}_{6} \mathrm{NOP}[\mathrm{M}-\mathrm{Br}]^{+}=$ 658.2668 , found $=658.2645$.

\section{Preparation of 3-Alkenyl-oxindoles and Allenoates}

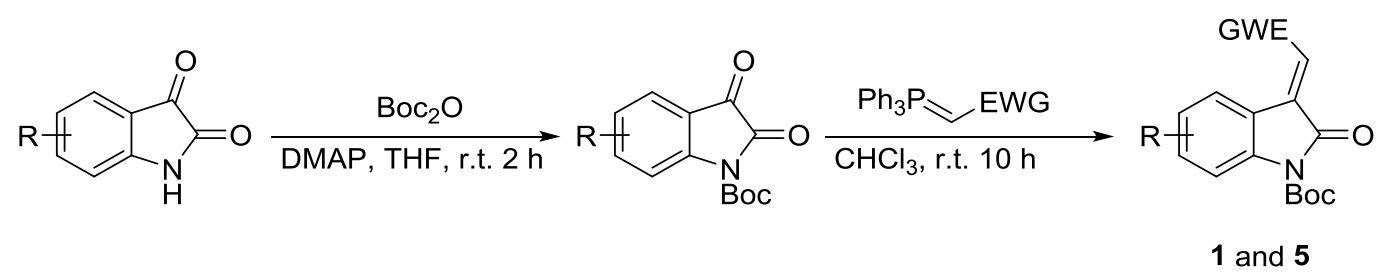

All 3-alkenyl-oxindoles $\mathbf{1}$ and $\mathbf{5}$ were synthesized following the methods reported in the literature (51-93\% yields), ${ }^{[2]}$ and allenoates 2 were synthesized according to the known procedures. ${ }^{[3]}$ The unknown compound 1f, 1h-i, 1o, 1r-s, 1u-y, 5b-c, 5i, 2d-e was fully characterized.

tert-butyl (E)-3-(2-((9H-fluoren-9-yl)methoxy)-2-oxoethylidene)-2-oxoindoline-1carboxylate (1f)

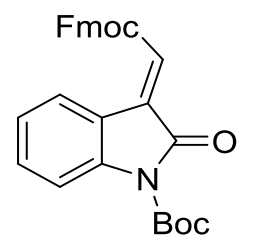

A yellow solid (81\%); ${ }^{1} \mathrm{H}$ NMR (400 MHz, $\left.\mathrm{CDCl}_{3}\right) \delta 8.82(\mathrm{~d}, J=7.7 \mathrm{~Hz}, 1 \mathrm{H}), 7.93(\mathrm{~d}$, $J=8.2 \mathrm{~Hz}, 1 \mathrm{H}), 7.69(\mathrm{~d}, J=7.5 \mathrm{~Hz}, 2 \mathrm{H}), 7.61(\mathrm{~d}, J=7.5 \mathrm{~Hz}, 2 \mathrm{H}), 7.50-7.40(\mathrm{~m}$, $3 \mathrm{H}), 7.31(\mathrm{td}, J=7.5,0.9 \mathrm{~Hz}, 2 \mathrm{H}), 7.29-7.18(\mathrm{ddd}, J=15.5,8.1,0.7 \mathrm{~Hz}, 1 \mathrm{H}), 6.96$ $(\mathrm{d}, J=16.1 \mathrm{~Hz}, 2 \mathrm{H}), 1.64(\mathrm{~s}, 9 \mathrm{H}) ;{ }^{13} \mathrm{C} \mathrm{NMR}\left(100 \mathrm{MHz}, \mathrm{CDCl}_{3}\right) \delta 166.2,165.6,148.8$, $142.1,141.5,141.2,138.8,137.2,133.1,129.8,128.7,128.0,126.1,125.4,124.7$, 122.4, 120.2, 116.8, 115.0, 84.9, 76.1, 28.1. HRMS (ESI) $\mathrm{m} / z$ calcd for $\mathrm{C}_{29} \mathrm{H}_{25} \mathrm{NO}_{5}$ $[\mathrm{M}+\mathrm{Na}]^{+}=490.1630$, found $=490.1627$. (E)-3-(2-(benzyloxy)-2-oxoethylidene)-4-chloro-2-oxoindoline-1carboxylate (1h) 


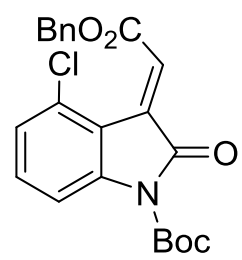

A yellow solid (76\%); ${ }^{1} \mathrm{H} \mathrm{NMR}\left(400 \mathrm{MHz}, \mathrm{CDCl}_{3}\right) \delta 7.84(\mathrm{~d}, J=8.2 \mathrm{~Hz}, 1 \mathrm{H}), 7.61(\mathrm{~s}$, 1H), $7.46(\mathrm{dd}, J=7.8,1.4 \mathrm{~Hz}, 2 \mathrm{H}), 7.40-7.33(\mathrm{~m}, 3 \mathrm{H}), 7.30(\mathrm{t}, J=8.2 \mathrm{~Hz}, 1 \mathrm{H}), 7.14$ $(\mathrm{d}, J=8.2 \mathrm{~Hz}, 1 \mathrm{H}), 5.38(\mathrm{~s}, 2 \mathrm{H}), 1.64(\mathrm{~s}, 9 \mathrm{H}) ;{ }^{13} \mathrm{C} \mathrm{NMR}\left(100 \mathrm{MHz}, \mathrm{CDCl}_{3}\right) \delta 166.1$, $162.4,148.7,141.7,135.2,131.4,130.5,129.5,128.9,128.6,128.5,128.2,125.9$, 117.8, 113.8, 85.1, 67.8, 28.1. HRMS (ESI) $\mathrm{m} / z$ calcd for $\mathrm{C}_{22} \mathrm{H}_{20} \mathrm{ClNO}_{5}[\mathrm{M}+\mathrm{Na}]^{+}=$ 436.0928, found $=436.0922$.

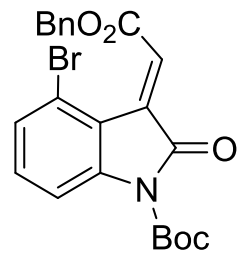

A yellow solid (69\%); ${ }^{1} \mathrm{H}$ NMR $\left(400 \mathrm{MHz}, \mathrm{CDCl}_{3}\right) \delta 7.90(\mathrm{dd}, J=8.2,0.7 \mathrm{~Hz}, 1 \mathrm{H})$, $7.83(\mathrm{~s}, 1 \mathrm{H}), 7.46(\mathrm{dd}, J=7.8,1.5 \mathrm{~Hz}, 2 \mathrm{H}), 7.42-7.31(\mathrm{~m}, 4 \mathrm{H}), 7.21(\mathrm{t}, J=8.2 \mathrm{~Hz}$, 1H), $5.38(\mathrm{~s}, 2 \mathrm{H}), 1.64(\mathrm{~s}, 9 \mathrm{H}) ;{ }^{13} \mathrm{C} \mathrm{NMR}\left(100 \mathrm{MHz}, \mathrm{CDCl}_{3}\right) \delta$ 166.1, 162.5, 148.6, $142.1,135.2,131.5,130.1,129.2,128.9,128.6,128.5,127.7,119.1,118.6,114.3$, 85.1, 67.8, 28.1. HRMS (ESI) $\mathrm{m} / z$ calcd for $\mathrm{C}_{22} \mathrm{H}_{20} \mathrm{BrNO}_{5}[\mathrm{M}+\mathrm{Na}]^{+}=480.0423$, found $=480.0430$. 


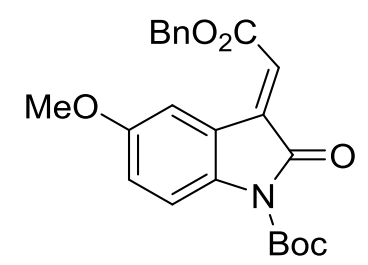

A red solid (88\%); ${ }^{1} \mathrm{H}$ NMR $\left(400 \mathrm{MHz}, \mathrm{CDCl}_{3}\right) \delta 8.35(\mathrm{~d}, J=2.8 \mathrm{~Hz}, 1 \mathrm{H}), 7.81(\mathrm{~d}, J$ $=9.0 \mathrm{~Hz}, 1 \mathrm{H}), 7.45-7.32(\mathrm{~m}, 5 \mathrm{H}), 6.99(\mathrm{dd}, J=9.0,2.8 \mathrm{~Hz}, 1 \mathrm{H}), 6.96(\mathrm{~s}, 1 \mathrm{H}), 5.31$ (s, 2H), 3.82 (s, 3H), $1.64(\mathrm{~s}, 9 \mathrm{H}) ;{ }^{13} \mathrm{C} \mathrm{NMR}\left(100 \mathrm{MHz}, \mathrm{CDCl}_{3}\right) \delta 165.8,165.2,156.6$, $148.8,137.3,135.8,135.3,128.7,128.5,128.3,122.7,120.9,119.5,115.9,112.8$, 84.6, 67.1, 55.7, 28.1. HRMS (ESI) $m / z$ calcd for $\mathrm{C}_{23} \mathrm{H}_{23} \mathrm{NO}_{6}[\mathrm{M}+\mathrm{Na}]^{+}=432.1423$, found $=432.1421$.

tert-butyl (E)-3-(2-(benzyloxy)-2-oxoethylidene)-6-methoxy-2-oxoindoline-1carboxylate (1r)<smiles>COc1ccc2c(c1)N(C(=O)OCc1ccccc1)C(=O)/C2=C/C=O</smiles>

A yellow solid (84\%); ${ }^{1} \mathrm{H} \mathrm{NMR}\left(400 \mathrm{MHz}, \mathrm{CDCl}_{3}\right) \delta 8.68(\mathrm{~d}, J=8.8 \mathrm{~Hz}, 1 \mathrm{H}), 7.52$ (d, $J=2.4 \mathrm{~Hz}, 1 \mathrm{H}), 7.46-7.31(\mathrm{~m}, 5 \mathrm{H}), 6.79(\mathrm{~s}, 1 \mathrm{H}), 6.68(\mathrm{dd}, J=8.8,2.5 \mathrm{~Hz}, 1 \mathrm{H}), 5.29$ (s, 2H), $3.88(\mathrm{~s}, 3 \mathrm{H}), 1.65$ (s, 9H); ${ }^{13} \mathrm{C}$ NMR (100 MHz, $\left.\mathrm{CDCl}_{3}\right) \delta 166.4,165.7,163.5$, 148.8, 144.0, 136.6, 135.5, 130.4, 128.7, 128.4 (d, $J=13.9$ Hz), 118.8, 113.3, 110.3, 101.4, 84.8, 66.8, 55.7, 28.1. HRMS (ESI) $\mathrm{m} / \mathrm{z}$ calcd for $\mathrm{C}_{23} \mathrm{H}_{23} \mathrm{NO}_{6}[\mathrm{M}+\mathrm{Na}]^{+}=$ 432.1423, found $=432.1421$. 


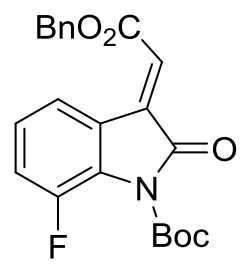

A yellow solid (56\%); ${ }^{1} \mathrm{H}$ NMR (400 MHz, $\left.\mathrm{CDCl}_{3}\right) \delta 8.46(\mathrm{dd}, J=7.6,1.0 \mathrm{~Hz}, 1 \mathrm{H})$, $7.44-7.33(\mathrm{~m}, 5 \mathrm{H}), 7.23-7.10(\mathrm{~m}, 2 \mathrm{H}), 7.02(\mathrm{~s}, 1 \mathrm{H}), 5.31(\mathrm{~s}, 2 \mathrm{H}), 1.61(\mathrm{~s}, 9 \mathrm{H}) ;{ }^{13} \mathrm{C}$ NMR (100 MHz, $\left.\mathrm{CDCl}_{3}\right) \delta 165.37,164.80,148.55(\mathrm{~d}, J=250.4 \mathrm{~Hz}), 147.1,136.4(\mathrm{~d}$, $J=3.2 \mathrm{~Hz}), 135.1,128.7,128.6,128.4,125.5(\mathrm{~d}, J=6.8 \mathrm{~Hz}), 124.5(\mathrm{~d}, J=3.5 \mathrm{~Hz})$, $124.4,122.9(\mathrm{~d}, J=2.4 \mathrm{~Hz}), 121.0,120.8,85.4,67.3,27.7 ;{ }^{19} \mathrm{~F}$ NMR $(376 \mathrm{MHz}$, $\left.\mathrm{CDCl}_{3}\right) \delta$-120.0. HRMS (ESI) $\mathrm{m} / z$ calcd for $\mathrm{C}_{22} \mathrm{H}_{20} \mathrm{FNO}_{5}[\mathrm{M}+\mathrm{Na}]^{+}=420.1223$, found $=420.1227$. (E)-3-(2-(benzyloxy)-2-oxoethylidene)-7-bromo-2-oxoindoline-1$\underline{\text { carboxylate (1u) }}$<smiles>CC(C)(C)OC(=O)N1C(=O)/C(=C/C(=O)OCc2ccccc2)c2cccc(Br)c21</smiles>

A yellow solid (73\%); ${ }^{1} \mathrm{H}$ NMR $\left(400 \mathrm{MHz}, \mathrm{CDCl}_{3}\right) \delta 8.62(\mathrm{dd}, J=7.8,0.8 \mathrm{~Hz}, 1 \mathrm{H})$, $7.57(\mathrm{~d}, J=8.1 \mathrm{~Hz}, 1 \mathrm{H}), 7.44-7.33(\mathrm{~m}, 5 \mathrm{H}), 7.04$ (t, $J=8.0 \mathrm{~Hz}, 1 \mathrm{H}), 6.97(\mathrm{~s}, 1 \mathrm{H})$, $5.31(\mathrm{~s}, 2 \mathrm{H}), 1.64(\mathrm{~s}, 9 \mathrm{H}) ;{ }^{13} \mathrm{C} \mathrm{NMR}\left(100 \mathrm{MHz}, \mathrm{CDCl}_{3}\right) \delta$ 166.2, 164.8, 147.6, 140.9, 137.3, 136.7, 135.1, 128.7, 128.6, 128.4, 127.6, 125.7, 124.2, 123.3, 106.8, 85.9, 67.2, 27.7. HRMS (ESI) $\mathrm{m} / z$ calcd for $\mathrm{C}_{22} \mathrm{H}_{20} \mathrm{BrNO}_{5}[\mathrm{M}+\mathrm{Na}]^{+}=480.0423$, found $=$ 480.0430 . 


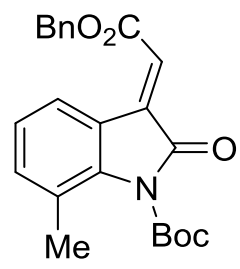

A yellow solid (71\%); ${ }^{1} \mathrm{H}$ NMR $\left(400 \mathrm{MHz}, \mathrm{CDCl}_{3}\right) \delta 8.49(\mathrm{~d}, J=7.7 \mathrm{~Hz}, 1 \mathrm{H}), 7.45-$ 7.31 (m, 5H), 7.23 (d, $J=7.7 \mathrm{~Hz}, 1 \mathrm{H}), 7.09$ (t, $J=7.8 \mathrm{~Hz}, 1 \mathrm{H}), 6.92$ (s, 1H), 5.30 (s, 2H), $2.23(\mathrm{~s}, 3 \mathrm{H}), 1.63(\mathrm{~s}, 9 \mathrm{H}) ;{ }^{13} \mathrm{C} \mathrm{NMR}\left(100 \mathrm{MHz}, \mathrm{CDCl}_{3}\right) \delta$ 166.9, 165.1, 148.9, $140.7,137.5,135.9,135.3,128.7,128.5,128.4,126.2,124.6,123.7,122.4,121.3$, 85.2, 67.0, 27.8, 19.5. HRMS (ESI) $\mathrm{m} / z$ calcd for $\mathrm{C}_{23} \mathrm{H}_{23} \mathrm{NO}_{5}[\mathrm{M}+\mathrm{Na}]^{+}=416.1474$, found $=416.1477$.

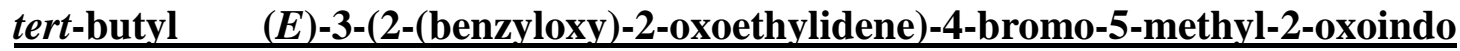
line-1-carboxylate (1w)<smiles>C/C=C1/C(=O)N(C(C)(C)C)c2ccc(C)c(Br)c21</smiles>

A yellow solid (61\%); ${ }^{1} \mathrm{H}$ NMR (400 MHz, $\left.\mathrm{CDCl}_{3}\right) \delta 7.93(\mathrm{~s}, 1 \mathrm{H}), 7.79(\mathrm{~d}, J=8.3 \mathrm{~Hz}$, 1H), $7.46(\mathrm{dd}, J=7.8,1.4 \mathrm{~Hz}, 2 \mathrm{H}), 7.41-7.32(\mathrm{~m}, 3 \mathrm{H}), 7.24(\mathrm{~d}, J=8.4 \mathrm{~Hz}, 1 \mathrm{H}), 5.38$ (s, 2H), 2.40 (s, 3H), 1.63 (s, 9H); ${ }^{13} \mathrm{C}$ NMR (100 MHz, $\left.\mathrm{CDCl}_{3}\right) \delta$ 166.4, 162.7, 148.7, $140.0,135.3,134.8,132.4,130.6,128.9,128.6,128.5,127.7,121.2,119.4,114.0$, 84.9, 67.8, 28.1, 22.5. HRMS (ESI) $\mathrm{m} / z$ calcd for $\mathrm{C}_{23} \mathrm{H}_{22} \mathrm{BrNO}_{5}[\mathrm{M}+\mathrm{Na}]^{+}=494.0579$, found $=494.0571$.

tert-butyl (E)-3-(2-(benzyloxy)-2-oxoethylidene)-5,7-dimethyl-2-oxoindoline-1carboxylate (1x) 


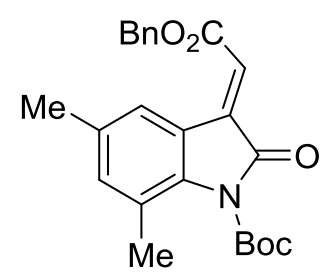

A yellow solid (49\%); ${ }^{1} \mathrm{H}$ NMR (400 MHz, $\left.\mathrm{CDCl}_{3}\right) \delta 8.20(\mathrm{~s}, 1 \mathrm{H}), 7.42-7.22(\mathrm{~m}$, 5H), $6.97(\mathrm{~s}, 1 \mathrm{H}), 6.82(\mathrm{~s}, 1 \mathrm{H}), 5.23(\mathrm{~s}, 2 \mathrm{H}), 2.21(\mathrm{~s}, 3 \mathrm{H}), 2.11(\mathrm{~s}, 3 \mathrm{H}), 1.55(\mathrm{~s}, 7 \mathrm{H})$; ${ }^{13} \mathrm{C}$ NMR $\left(100 \mathrm{MHz}, \mathrm{CDCl}_{3}\right) \delta 167.1,165.2,148.9,138.6,137.6,136.6,135.4,134.2$, $128.7,128.5,128.4,126.5,123.5,121.9,121.3,84.9,67.0,27.8,21.0,19.5 ;$ HRMS (ESI) $\mathrm{m} / z$ calcd for $\mathrm{C}_{24} \mathrm{H}_{25} \mathrm{NO}_{5}[\mathrm{M}+\mathrm{Na}]^{+}=430.1630$, found $=430.1626$.

tert-butyl (E)-3-(2-(benzyloxy)-2-oxoethylidene)-5,6-difluoro-2-oxoindoline-1carboxylate $(1 \mathrm{y})$<smiles>CC(C)(C)OC(=O)N1C(=O)/C(=C/C(=O)OCc2ccccc2)c2cc(F)c(F)cc21</smiles>

A yellow solid (52\%); ${ }^{1} \mathrm{H} \mathrm{NMR}\left(400 \mathrm{MHz}, \mathrm{CDCl}_{3}\right) \delta 8.68(\mathrm{dd}, J=10.7,8.5 \mathrm{~Hz}, 1 \mathrm{H})$, $7.85(\mathrm{dd}, J=11.4,7.1 \mathrm{~Hz}, 1 \mathrm{H}), 7.47-7.33(\mathrm{~m}, 5 \mathrm{H}), 6.94(\mathrm{~s}, 1 \mathrm{H}), 5.31$ (s, 2H), 1.64 (s, $9 \mathrm{H}) ;{ }^{13} \mathrm{C}$ NMR $\left(100 \mathrm{MHz}, \mathrm{CDCl}_{3}\right) \delta 164.9,164.8,152.7(\mathrm{dd}, J=255.5,13.9 \mathrm{~Hz})$, $148.5147 .2(\mathrm{dd}, J=245.4,13.3 \mathrm{~Hz}), 138.9(\mathrm{dd}, J=10.4,2.6 \mathrm{~Hz}), 135.7(\mathrm{t}, J=2.7$ $\mathrm{Hz}), 135.1,128.8,128.7,128.5,123.3(\mathrm{~d}, J=3.7 \mathrm{~Hz}), 117.7(\mathrm{~d}, J=22.1 \mathrm{~Hz}), 116.0$ $(\mathrm{dd}, J=8.1,4.1 \mathrm{~Hz}), 105.5(\mathrm{~d}, J=25.4 \mathrm{~Hz}), 85.5,67.4,28.0 ;{ }^{19} \mathrm{~F}$ NMR $(376 \mathrm{MHz}$, $\left.\mathrm{CDCl}_{3}\right) \delta-126.6(\mathrm{~d}, J=20.9 \mathrm{~Hz}),-141.3(\mathrm{~d}, J=20.9 \mathrm{~Hz})$. HRMS (ESI) $\mathrm{m} / \mathrm{z}$ calcd for $\mathrm{C}_{22} \mathrm{H}_{19} \mathrm{~F}_{2} \mathrm{NO}_{5}[\mathrm{M}+\mathrm{Na}]^{+}=438.1129$, found $=438.1134$. 


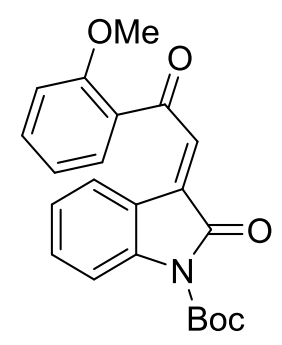

A yellow solid (90\%); ${ }^{1} \mathrm{H}$ NMR (400 MHz, $\left.\mathrm{CDCl}_{3}\right) \delta 8.45(\mathrm{~d}, J=7.8 \mathrm{~Hz}, 1 \mathrm{H}), 7.90$ (d, $J=8.2 \mathrm{~Hz}, 1 \mathrm{H}), 7.85(\mathrm{dd}, J=7.8,1.8 \mathrm{~Hz}, 1 \mathrm{H}), 7.83(\mathrm{~s}, 1 \mathrm{H}), 7.57-7.51(\mathrm{~m}, 1 \mathrm{H}), 7.45$ - $7.38(\mathrm{~m}, 1 \mathrm{H}), 7.15$ (t, $J=7.7 \mathrm{~Hz}, 1 \mathrm{H}), 7.07$ (t, $J=7.5 \mathrm{~Hz}, 1 \mathrm{H}), 7.00$ (d, $J=8.4 \mathrm{~Hz}$, 1H), 3.91 (s, 3H), 1.67 (s, 9H); ${ }^{13} \mathrm{C}$ NMR (100 MHz, $\left.\mathrm{CDCl}_{3}\right) \delta 191.7,166.9,159.4$, $149.0,141.5,134.9,132.3,132.2,132.2,131.0,128.2,126.8,124.5,121.0,120.7$, 114.9, 111.9, 84.6, 55.9, 28.1. HRMS (ESI) $\mathrm{m} / z$ calcd for $\mathrm{C}_{22} \mathrm{H}_{21} \mathrm{NO}_{5}[\mathrm{M}+\mathrm{Na}]^{+}=$ 402.1317, found $=402.1315$.

tert-butyl (E)-3-(2-(3-fluorophenyl)-2-oxoethylidene)-2-oxoindoline-1-carboxylate $\underline{(5 c)}$

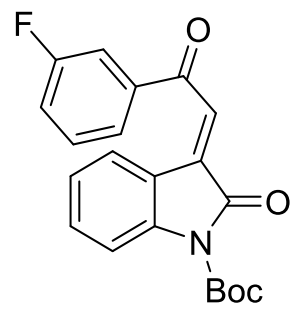

A yellow solid (56\%); ${ }^{1} \mathrm{H}$ NMR (400 MHz, $\left.\mathrm{CDCl}_{3}\right) \delta 8.40(\mathrm{~d}, J=7.6 \mathrm{~Hz}, 1 \mathrm{H}), 7.93(\mathrm{~d}$, $J=8.2 \mathrm{~Hz}, 1 \mathrm{H}), 7.86(\mathrm{dd}, J=7.8,0.5 \mathrm{~Hz}, 1 \mathrm{H}), 7.82(\mathrm{~s}, 1 \mathrm{H}), 7.77(\mathrm{~d}, J=9.3 \mathrm{~Hz}, 1 \mathrm{H})$, $7.51(\mathrm{td}, J=8.0,5.5 \mathrm{~Hz}, 1 \mathrm{H}), 7.45(\mathrm{t}, J=7.9 \mathrm{~Hz}, 1 \mathrm{H}), 7.33(\mathrm{td}, J=8.2,2.5 \mathrm{~Hz}, 1 \mathrm{H})$, $7.16(\mathrm{t}, J=7.7 \mathrm{~Hz}, 1 \mathrm{H}), 1.67(\mathrm{~s}, 9 \mathrm{H}) ;{ }^{13} \mathrm{C} \mathrm{NMR}\left(100 \mathrm{MHz}, \mathrm{CDCl}_{3}\right) \delta$ 189.6, 166.1, $163.0(\mathrm{~d}, J=249.0 \mathrm{~Hz}), 148.8,142.0,139.5(\mathrm{~d}, J=6.3 \mathrm{~Hz}), 135.7,133.2,130.6(\mathrm{~d}, J$ = 7.6 Hz), 127.3, 126.1, 124.6, $124.6(\mathrm{~d}, J=3.0 \mathrm{~Hz}), 121.0(\mathrm{~d}, J=21.6 \mathrm{~Hz}), 120.2$, $115.3(\mathrm{~d}, J=22.5 \mathrm{~Hz}), 115.1,84.8,28.1 ;{ }^{19} \mathrm{~F} \mathrm{NMR}\left(376 \mathrm{MHz}, \mathrm{CDCl}_{3}\right) \delta-111.0$. HRMS (ESI) $m / z$ calcd for $\mathrm{C}_{21} \mathrm{H}_{18} \mathrm{FNO}_{4}[\mathrm{M}+\mathrm{Na}]^{+}=390.1118$, found $=390.1124$. 


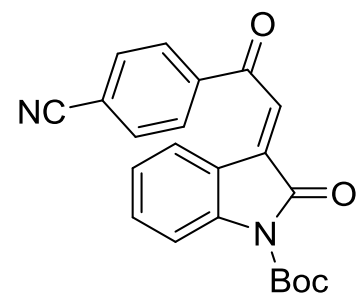

A yellow solid (47\%); ${ }^{1} \mathrm{H} \mathrm{NMR}\left(400 \mathrm{MHz}, \mathrm{CDCl}_{3}\right) \delta 8.50(\mathrm{dd}, J=7.9,1.2 \mathrm{~Hz}, 1 \mathrm{H})$, $8.22-8.15(\mathrm{~m}, 2 \mathrm{H}), 7.95(\mathrm{~d}, J=8.0 \mathrm{~Hz}, 1 \mathrm{H}), 7.87-7.81(\mathrm{~m}, 3 \mathrm{H}), 7.53-7.42(\mathrm{~m}$, $1 \mathrm{H}), 7.26(\mathrm{~s}, 1 \mathrm{H}), 7.19(\mathrm{td}, J=7.8,1.0 \mathrm{~Hz}, 1 \mathrm{H}), 1.67(\mathrm{~s}, 9 \mathrm{H}) ;{ }^{13} \mathrm{C}$ NMR $(100 \mathrm{MHz}$, $\left.\mathrm{CDCl}_{3}\right) \delta 189.3,166.0,148.7,142.4,140.6,136.8,133.8,132.8,129.1,127.6,124.8$, 120.1, 117.8, 117.0, 115.2, 85.0, 28.1. HRMS (ESI) $\mathrm{m} / z$ calcd for $\mathrm{C}_{22} \mathrm{H}_{18} \mathrm{~N}_{2} \mathrm{O}_{4}$ $[\mathrm{M}+\mathrm{Na}]^{+}=397.1164$, found $=397.1159$.

\section{5-(tert-butyl) 1-ethyl 2-butylpenta-2,3-dienedioate (2d)}<smiles>CCCC(=C=CC(=O)OCC)COC(C)(C)C</smiles>

A colorless oil (51\%); ${ }^{1} \mathrm{H} \mathrm{NMR}\left(400 \mathrm{MHz}, \mathrm{CDCl}_{3}\right) \delta 5.81(\mathrm{t}, J=2.8 \mathrm{~Hz}, 1 \mathrm{H}), 4.19$ (q, $J=7.1 \mathrm{~Hz}, 2 \mathrm{H}), 2.41-2.20(\mathrm{~m}, 2 \mathrm{H}), 1.50-1.39(\mathrm{~m}, 11 \mathrm{H}), 1.39-1.31(\mathrm{~m}, 2 \mathrm{H}), 1.25$ $(\mathrm{t}, J=7.1 \mathrm{~Hz}, 3 \mathrm{H}), 0.88(\mathrm{t}, J=7.2 \mathrm{~Hz}, 3 \mathrm{H}) ;{ }^{13} \mathrm{C} \mathrm{NMR}\left(100 \mathrm{MHz}, \mathrm{CDCl}_{3}\right) \delta 217.9$, $165.5,163.5,104.6,93.3,81.5,61.3,29.8,28.0,27.9,22.0,14.2,13.8$. HRMS (ESI) $m / z$ calcd for $\mathrm{C}_{15} \mathrm{H}_{24} \mathrm{O}_{4}[\mathrm{M}+\mathrm{Na}]^{+}=291.1572$, found $=291.1579$.

\section{5-(tert-butyl) 1-ethyl 2-benzylpenta-2,3-dienedioate (2e)}<smiles>CCOC(=O)C=C=C(COC(C)(C)C)Cc1ccccc1</smiles> 
A colorless oil (74\%); ${ }^{1} \mathrm{H} \mathrm{NMR}\left(400 \mathrm{MHz}, \mathrm{CDCl}_{3}\right) \delta 7.29-7.25(\mathrm{~m}, 4 \mathrm{H}), 7.24-7.18$

$(\mathrm{m}, 1 \mathrm{H}), 5.78(\mathrm{t}, J=2.5 \mathrm{~Hz}, 1 \mathrm{H}), 4.20$ (q, $J=7.2 \mathrm{~Hz}, 2 \mathrm{H}), 3.65(\mathrm{ddd}, J=37.2,15.2$, $2.5 \mathrm{~Hz}, 2 \mathrm{H}), 1.47(\mathrm{~s}, 9 \mathrm{H}), 1.25(\mathrm{t}, J=7.1 \mathrm{~Hz}, 3 \mathrm{H}) ;{ }^{13} \mathrm{C} \mathrm{NMR}\left(100 \mathrm{MHz}, \mathrm{CDCl}_{3}\right) \delta$ 218.5, 165.0, 163.1, 137.8, 128.9, 128.3, 126.7, 104.3, 93.7, 81.7, 61.6, 34.9, 28.1, 14.2. HRMS (ESI) $m / z$ calcd for $\mathrm{C}_{18} \mathrm{H}_{22} \mathrm{O}_{4}[\mathrm{M}+\mathrm{Na}]^{+}=325.1416$, found $=325.1411$.

\section{Representative Procedure for $[4+2]$ Annulation Reaction}

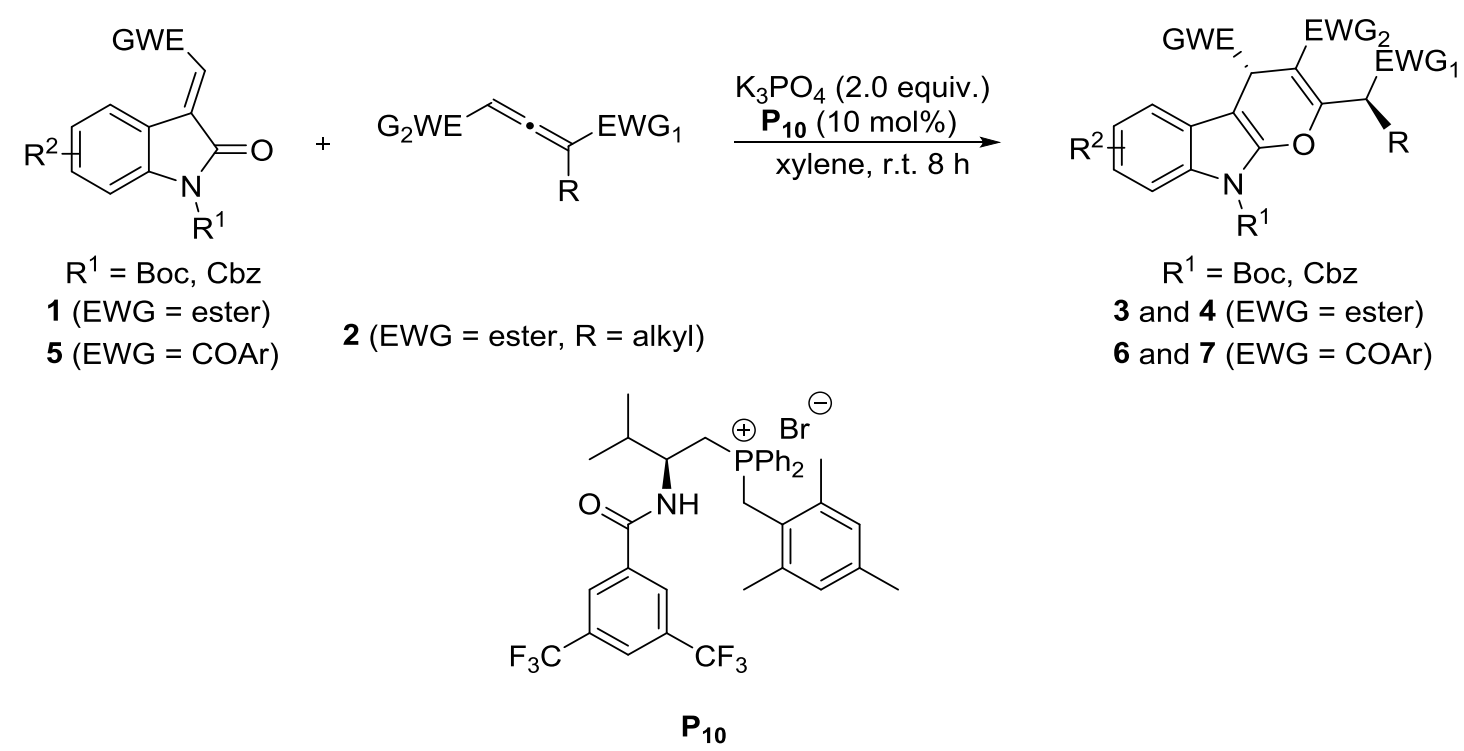

To a flame round bottle flask with a magnetic stirring bar were added the 3-alkenyl-oxindole 1a $(0.2 \mathrm{mmol})$, allenoates 2 (0.24 mmol), phosphonium salt $\mathbf{P}_{10}$ (14.5 mg, $0.02 \mathrm{mmol})$ and $\mathrm{K}_{3} \mathrm{PO}_{4}(85 \mathrm{mg}, 0.4 \mathrm{mmol}$ ), followed by the addition of xylene $(4.0 \mathrm{~mL})$. The reaction mixture was stirred at room temperature for $8 \mathrm{~h}$. The solvent was removed under reduced pressure, and the residue was purified by column chromatography on silica gel (petroleum ether/ethyl acetate $=15: 1)$ to afford 3a $(119$ mg, $98 \%$ yield) as a white solid.

4-benzyl 3,9-di-tert-butyl $(S)$-2-((S)-1-ethoxy-1-oxopropan-2-yl)pyrano[2,3-b] indole-3,4,9(4H)-tricarboxylate $(3 \mathrm{a})$ 


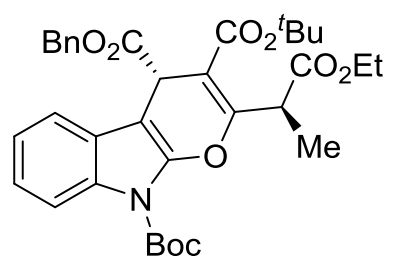

A white solid (119mg, 98\%); $[\alpha]^{25}{ }_{\mathrm{D}}=-30.0\left(c 0.80, \mathrm{CHCl}_{3}\right) ;{ }^{1} \mathrm{H}$ NMR $(400 \mathrm{MHz}$, $\left.\mathrm{CDCl}_{3}\right) \delta 8.13(\mathrm{~d}, J=8.2 \mathrm{~Hz}, 1 \mathrm{H}), 7.57(\mathrm{~d}, J=7.1 \mathrm{~Hz}, 1 \mathrm{H}), 7.36-7.28(\mathrm{~m}, 5 \mathrm{H}), 7.25$ $(\mathrm{td}, J=7.4,1.4 \mathrm{~Hz} 1 \mathrm{H}), 7.17(\mathrm{td}, J=7.6,1.0 \mathrm{~Hz}, 1 \mathrm{H}), 5.17-5.06(\mathrm{~m}, 3 \mathrm{H}), 4.92(\mathrm{~s}, 1 \mathrm{H})$, $4.26-4.00(\mathrm{~m}, 2 \mathrm{H}), 1.64(\mathrm{~s}, 9 \mathrm{H}), 1.58(\mathrm{~d}, J=7.2 \mathrm{~Hz}, 3 \mathrm{H}), 1.44(\mathrm{~s}, 9 \mathrm{H}), 1.19$ (t, $J=$ $7.1 \mathrm{~Hz}, 3 \mathrm{H}) ;{ }^{13} \mathrm{C} \mathrm{NMR}\left(100 \mathrm{MHz}, \mathrm{CDCl}_{3}\right) \delta 171.4,171.3,165.1,161.1,148.6,142.4$, $135.4,131.9,128.5,128.4,128.3,125.3,123.6,123.3,118.9,114.9,104.9,104.9$, 90.8, 84.6, 82.1, 67.2, 61.0, 41.2, 40.2, 28.2, 28.0, 14.2. HRMS (ESI) $\mathrm{m} / z$ calcd for $\mathrm{C}_{34} \mathrm{H}_{39} \mathrm{NO}_{9}[\mathrm{M}+\mathrm{Na}]^{+}=628.2523$, found $=628.2501$; The ee value was $93 \%, \mathrm{t}_{\mathrm{R}}$ (minor) $=11.8 \mathrm{~min}, \mathrm{t}_{\mathrm{R}}$ (major) $=15.5 \mathrm{~min}($ Chiralcel IE, $\lambda=254 \mathrm{~nm}, 2 \% i$-PrOH$/$ hexanes, flow rate $=1.0 \mathrm{~mL} / \mathrm{min})$.

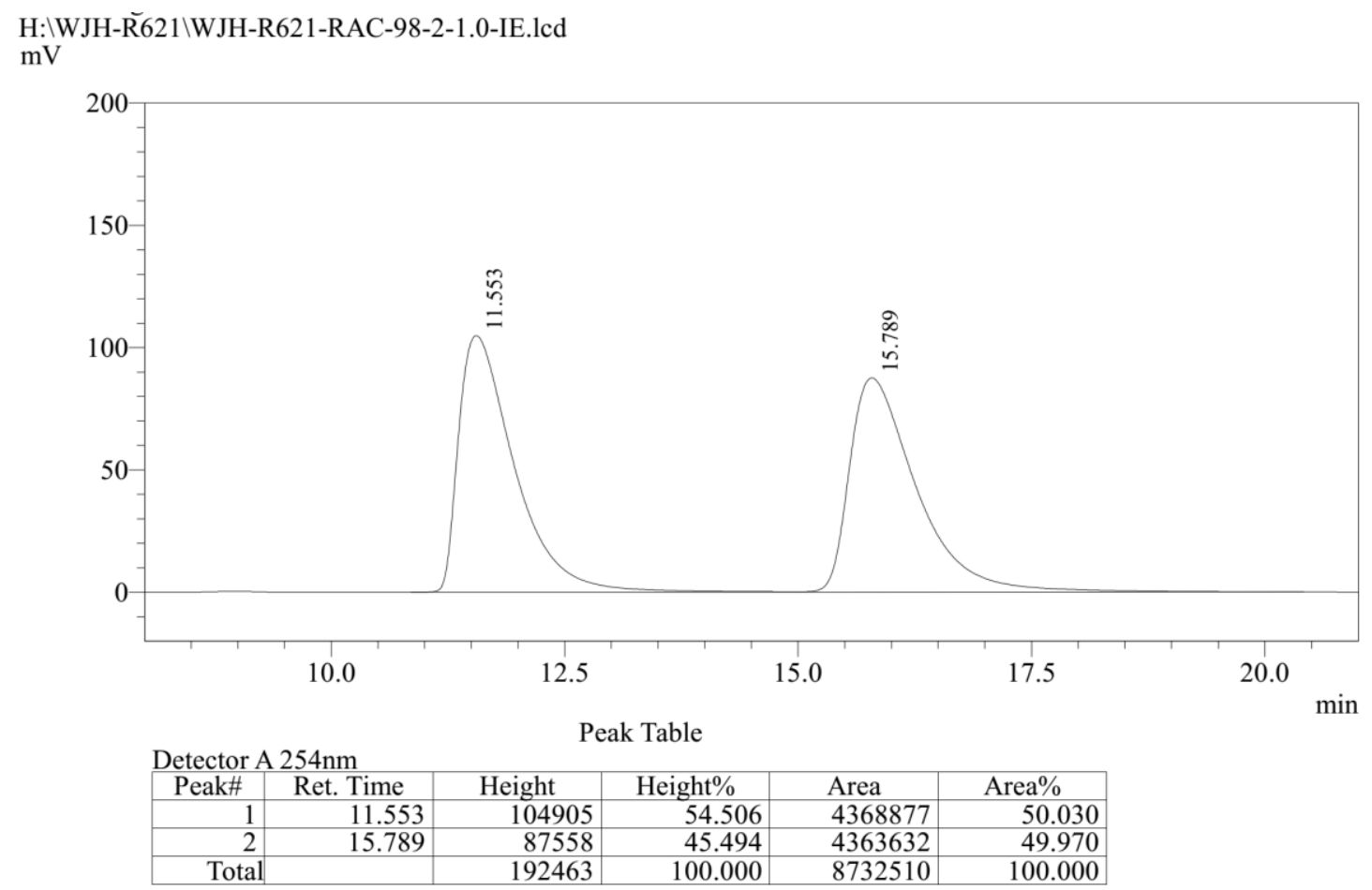


H:IWJH-Ř621 \WJH-R621-CHI-98-2-1.0-IE-25.1.lcd $\mathrm{mV}$

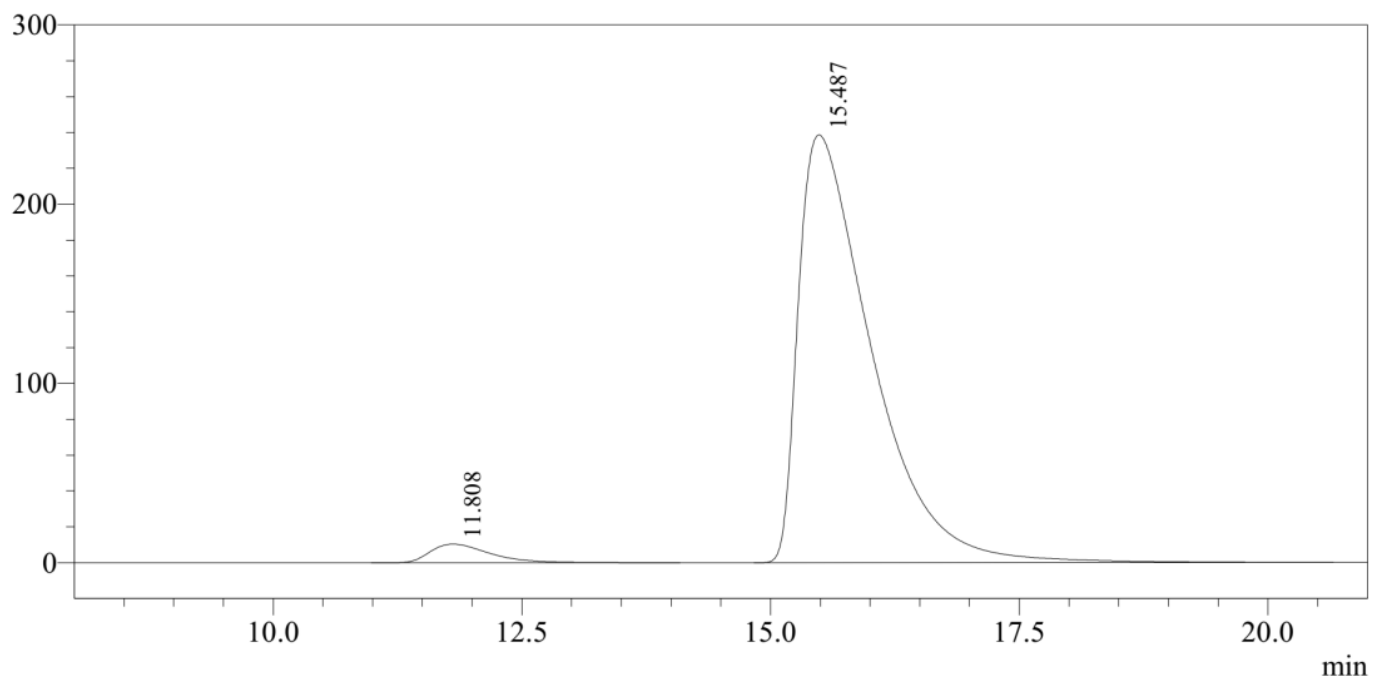

\begin{tabular}{|c|c|c|c|c|c|}
\hline \multicolumn{6}{|c|}{ Detector A 254nm } \\
\hline Peak\# & Ret. Time & Height & Height $\%$ & Area & Area $\%$ \\
\hline 1 & 11.808 & 10441 & 4.191 & 441684 & 3.473 \\
\hline 2 & 15.487 & 238691 & 95.809 & 12277764 & 96.527 \\
\hline Total & & 249133 & 100.000 & 12719447 & 100.000 \\
\hline
\end{tabular}

\section{3,9-di-tert-butyl 4-methyl $(S)$-2-( $(S)$-1-ethoxy-1-oxopropan-2-yl)pyrano[2,3-b]} indole-3,4,9(4H)-tricarboxylate $(3 \mathrm{~b})$

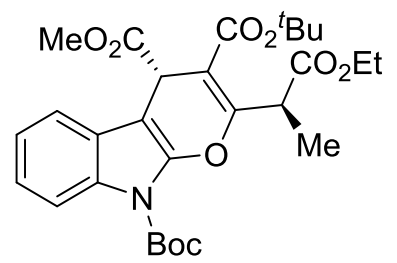

A white solid (104mg, 98\%); $[\alpha]^{25}{ }_{\mathrm{D}}=-41.8\left(c\right.$ 1.0, $\left.\mathrm{CHCl}_{3}\right) ;{ }^{1} \mathrm{H}$ NMR (400 MHz, $\left.\mathrm{CDCl}_{3}\right) \delta 8.22-8.09(\mathrm{~m}, 1 \mathrm{H}), 7.73-7.57(\mathrm{~m}, 1 \mathrm{H}), 7.33-7.21(\mathrm{~m}, 2 \mathrm{H}), 5.07(\mathrm{q}, J=$ $7.2 \mathrm{~Hz}, 1 \mathrm{H}), 4.89(\mathrm{~s}, 1 \mathrm{H}), 4.27-4.04(\mathrm{~m}, 2 \mathrm{H}), 3.71(\mathrm{~s}, 3 \mathrm{H}), 1.64(\mathrm{~s}, 9 \mathrm{H}), 1.59(\mathrm{~d}, J=$ $7.2 \mathrm{~Hz}, 3 \mathrm{H}), 1.51(\mathrm{~s}, 9 \mathrm{H}), 1.20(\mathrm{t}, J=7.1 \mathrm{~Hz}, 3 \mathrm{H}) ;{ }^{13} \mathrm{C} \mathrm{NMR}\left(100 \mathrm{MHz}, \mathrm{CDCl}_{3}\right) \delta$ $172.0,171.3,165.1160 .9,148.6,142.3,131.9,125.3,123.6,123.4,118.6,114.9$, 105.0, 90.8, 84.5, 82.0, 61.0, 52.2, 41.2, 40.0, 28.2, 28.0, 14.2, 14.2. HRMS (ESI) $\mathrm{m} / \mathrm{z}$ calcd for $\mathrm{C}_{28} \mathrm{H}_{35} \mathrm{NO}_{9}[\mathrm{M}+\mathrm{Na}]^{+}=552.2210$, found $=552.2200$; The ee value was $99 \%$, $\mathrm{t}_{\mathrm{R}}($ minor $)=9.4 \mathrm{~min}, \mathrm{t}_{\mathrm{R}}($ major $)=12.4 \min ($ Chiralcel $\mathrm{IE}, \lambda=254 \mathrm{~nm}, 2 \%$ $i$-PrOH/hexanes, flow rate $=1.0 \mathrm{~mL} / \mathrm{min}$ ). 
$\mathrm{mV}$

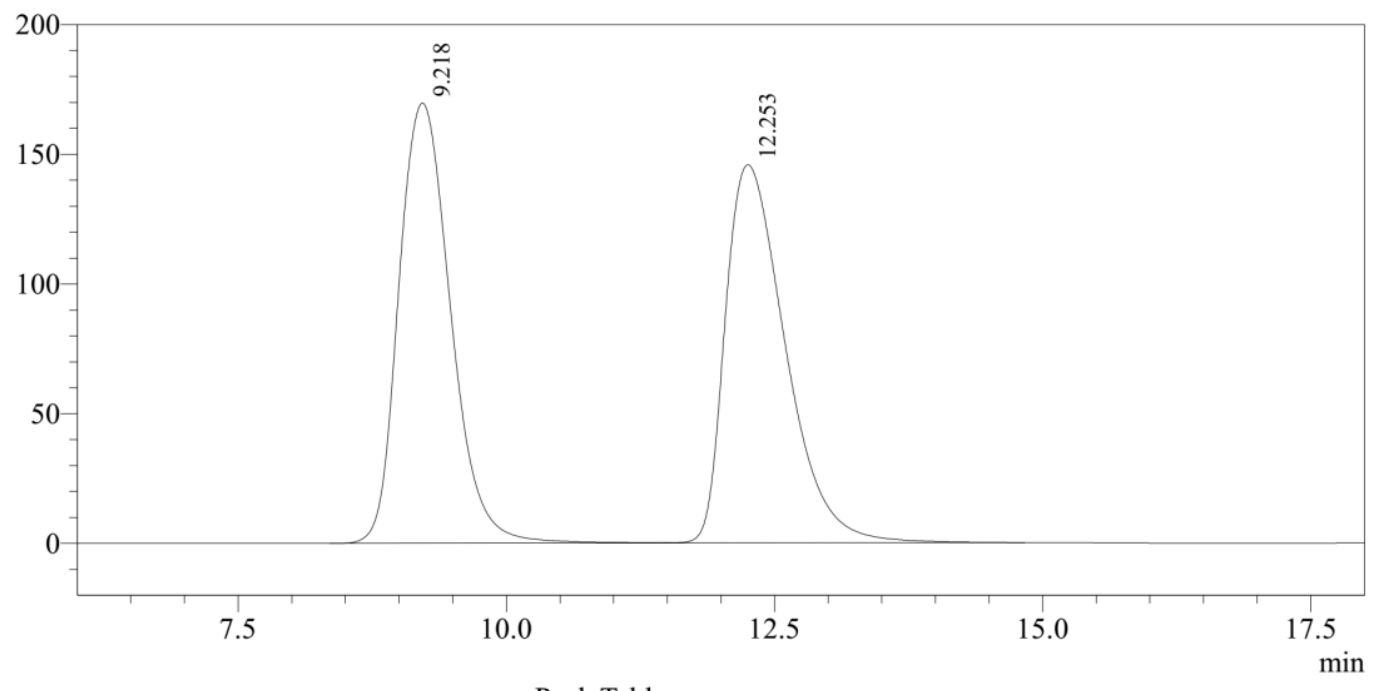

Peak Table

Detector A $254 \mathrm{~nm}$
\begin{tabular}{|r|r|r|r|r|r|}
\hline Peak\# & Ret. Time & \multicolumn{1}{|c|}{ Height } & Height $\%$ & \multicolumn{1}{|c|}{ Area } & \multicolumn{1}{|c|}{ Area $\%$} \\
\hline 1 & 9.218 & 169760 & 53.797 & 5704390 & 50.084 \\
\hline 2 & 12.253 & 145799 & 46.203 & 5685164 & 49.916 \\
\hline Total & & 315558 & 100.000 & 11389554 & 100.000 \\
\hline
\end{tabular}

H:IWJH-Ř708।WJH-R623-2CHRI-98-2-1.0-IE-25-3.6-106.lcd $\mathrm{mV}$

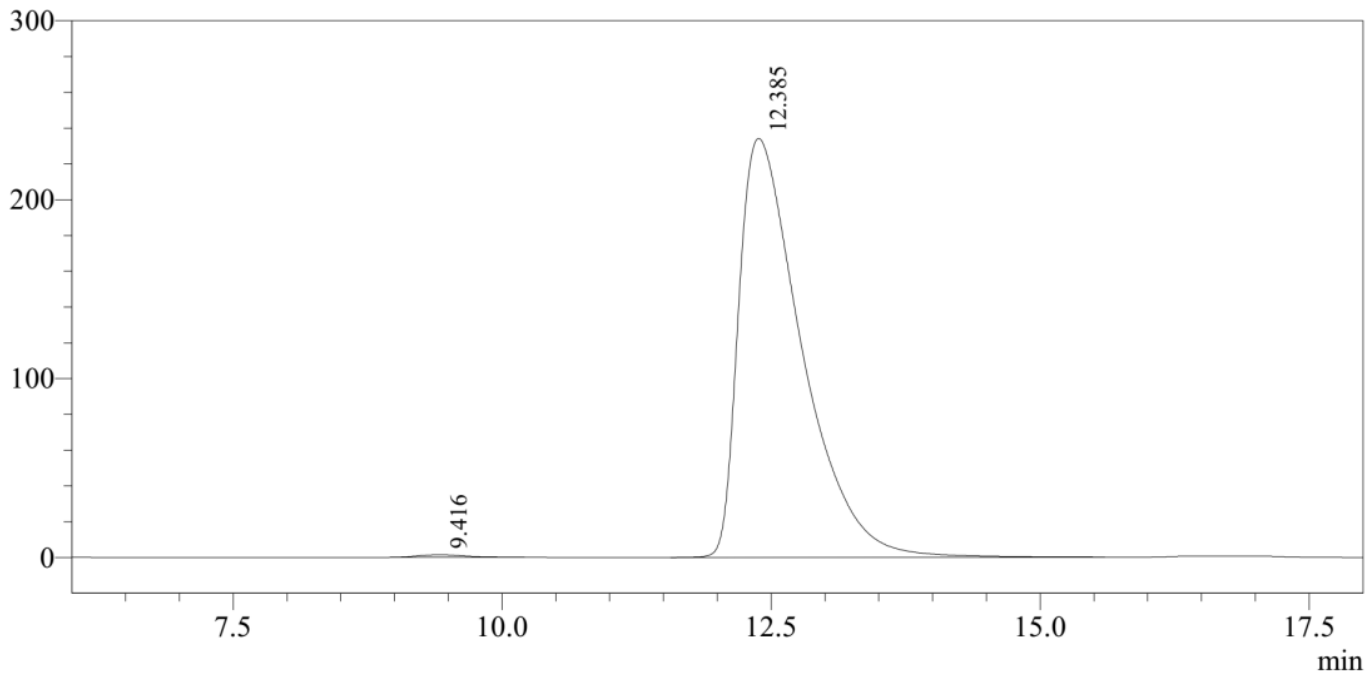

Peak Table

Detector A $254 \mathrm{~nm}$
\begin{tabular}{|r|r|r|r|r|r|}
\hline Peak\# & Ret. Time & Height & Height $\%$ & \multicolumn{1}{|c|}{ Area } & \multicolumn{1}{|c|}{ Area $\%$} \\
\hline 1 & 9.416 & 1485 & 0.630 & 45786 & 0.478 \\
\hline 2 & 12.385 & 234157 & 99.370 & 9539794 & 99.522 \\
\hline Total & & 235641 & 100.000 & 9585580 & 100.000 \\
\hline
\end{tabular}

\section{3,9-di-tert-butyl 4-ethyl $(S)$-2-((S)-1-ethoxy-1-oxopropan-2-yl)pyrano[2,3-b]} indole-3,4,9(4H)-tricarboxylate $(3 \mathrm{c})$ 


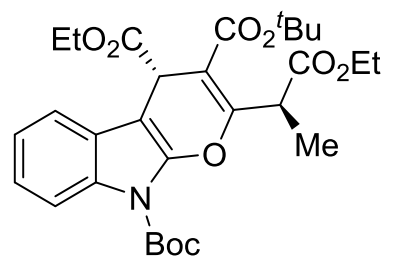

A white solid (104mg, 96\%); $[\alpha]^{25}=-42.9\left(\right.$ c 1.0, $\left.\mathrm{CHCl}_{3}\right) ;{ }^{1} \mathrm{H}$ NMR $(400 \mathrm{MHz}$, $\left.\mathrm{CDCl}_{3}\right) \delta 8.25-7.96(\mathrm{~m}, 1 \mathrm{H}), 7.79-7.50(\mathrm{~m}, 1 \mathrm{H}), 7.37-7.13(\mathrm{~m}, 3 \mathrm{H}), 5.10(\mathrm{q}, J=$ $7.2 \mathrm{~Hz}, 1 \mathrm{H}), 4.88(\mathrm{~s}, 1 \mathrm{H}), 4.43-3.91(\mathrm{~m}, 4 \mathrm{H}), 1.65$ (s, 9H), 1.60 (d, J = 7.2 Hz, 3H), $1.52(\mathrm{~s}, 9 \mathrm{H}), 1.28(\mathrm{t}, J=7.1 \mathrm{~Hz}, 3 \mathrm{H}), 1.21(\mathrm{t}, J=7.1 \mathrm{~Hz}, 3 \mathrm{H}) ;{ }^{13} \mathrm{C} \mathrm{NMR}(100 \mathrm{MHz}$, $\left.\mathrm{CDCl}_{3}\right) \delta 171.7,171.4,165.2,161.0,148.6,142.4,132.0,125.5,123.6,123.3,118.8$, 115.0, 105.1, 91.0, 84.6, 82.0, 61.4, 61.0, 41.3, 40.1, 28.2, 28.1, 14.3. HRMS (ESI) $m / z$ calcd for $\mathrm{C}_{29} \mathrm{H}_{37} \mathrm{NO}_{9}[\mathrm{M}+\mathrm{Na}]^{+}=566.2366$, found $=566.2356$; The ee value was $93 \%, \mathrm{t}_{\mathrm{R}}($ minor $)=8.9 \mathrm{~min}, \mathrm{t}_{\mathrm{R}}($ major $)=11.3 \mathrm{~min}($ Chiralcel $\mathrm{IE}, \lambda=254 \mathrm{~nm}, 2 \% i-\mathrm{PrOH}$ /hexanes, flow rate $=1.0 \mathrm{~mL} / \mathrm{min}$ ).

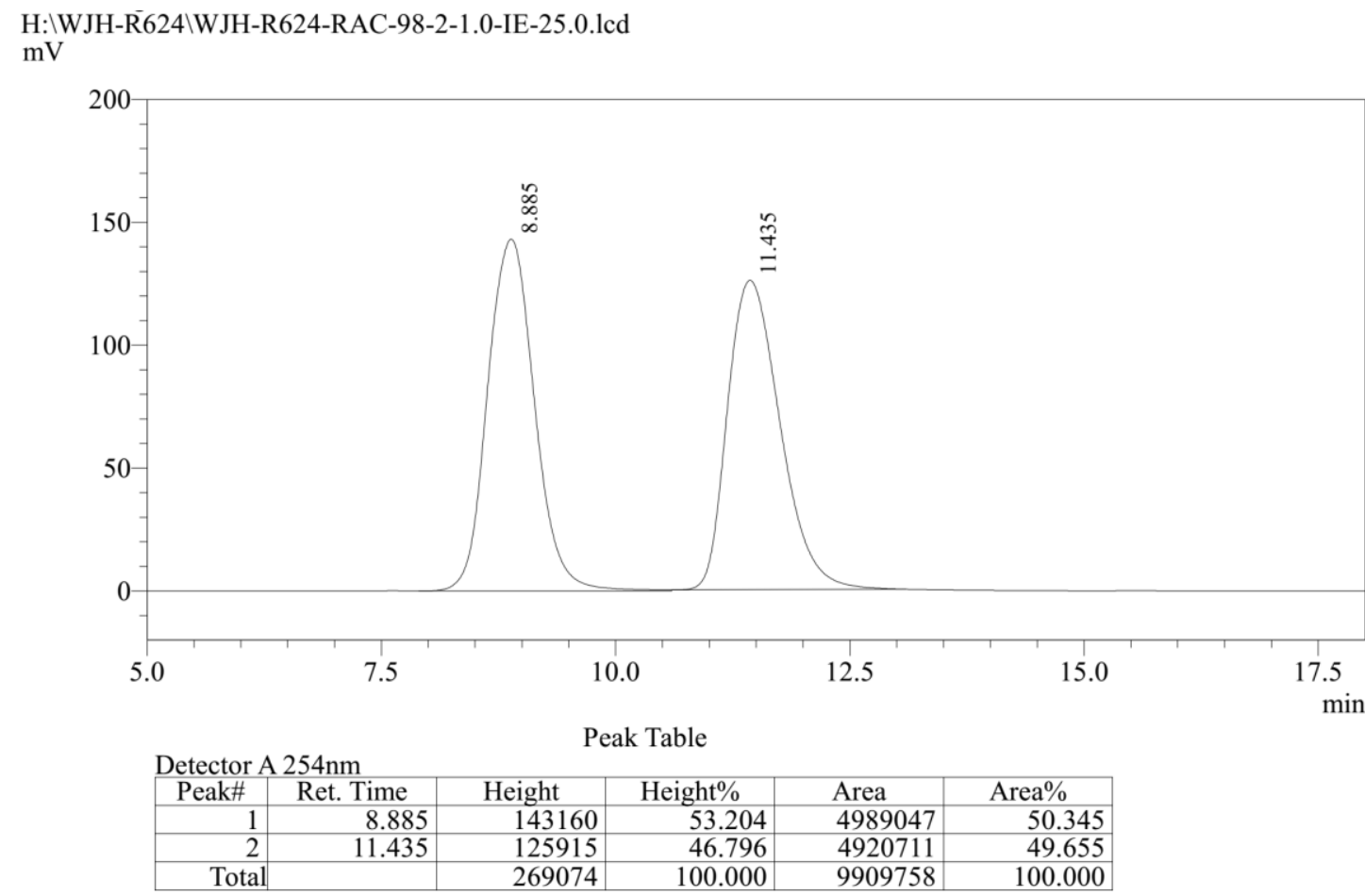


H:IWJH-Ř624|WJH-R624-CHI-98-2-1.0-IE-25-4.4.lcd $\mathrm{mV}$

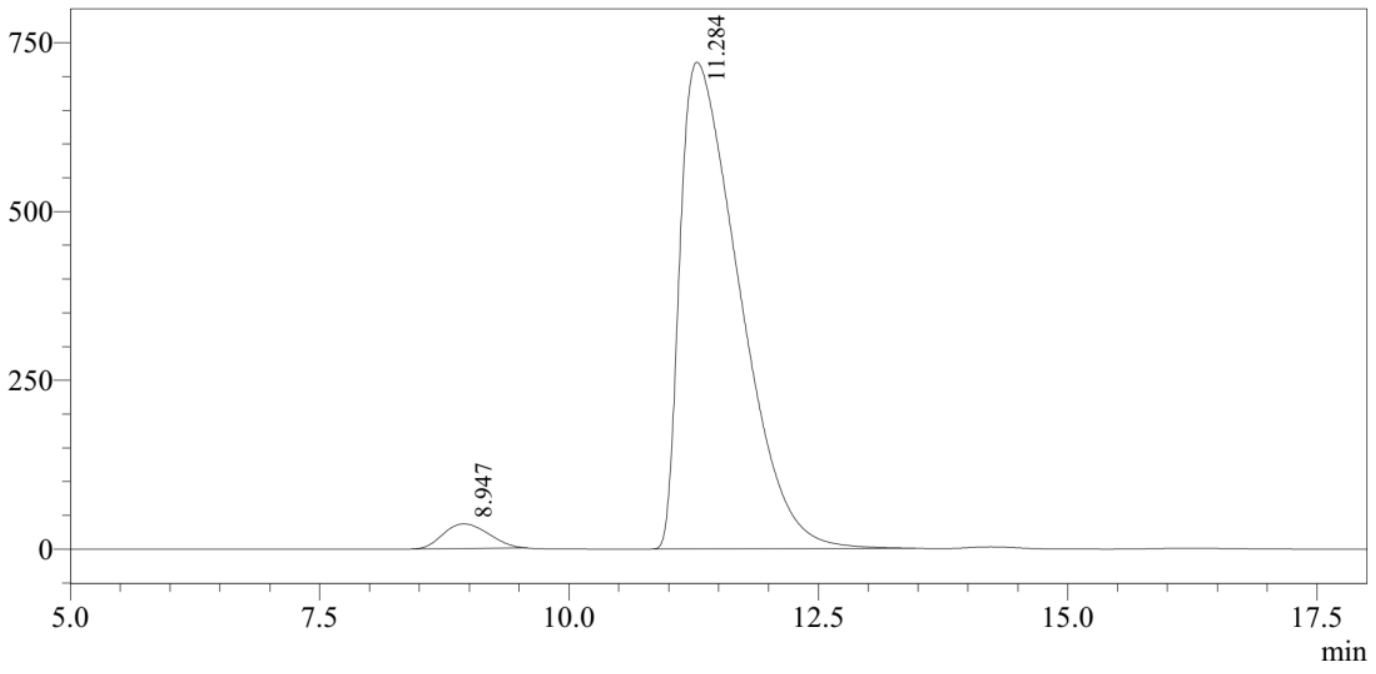

Peak Table

\begin{tabular}{|c|c|c|c|c|c|}
\hline \multicolumn{6}{|c|}{ etector A $254 \mathrm{~nm}$} \\
\hline & Ret. Time & Height & Height $\%$ & Area & Area $\%$ \\
\hline 1 & 8.947 & 36650 & 4.840 & 1164144 & 3.771 \\
\hline 2 & 11.284 & 720659 & 95.160 & 29702870 & 96.229 \\
\hline Total & & 757309 & 100.000 & 30867014 & 100.000 \\
\hline
\end{tabular}

\section{3,9-di-tert-butyl 4-isopropyl $(S)$-2-((S)-1-ethoxy-1-oxopropan-2-yl)pyrano[2,3-b]} indole-3,4,9(4H)-tricarboxylate $(3 \mathrm{~d})$

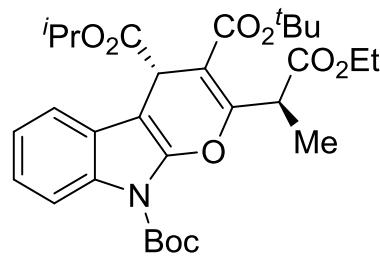

A white solid $(107 \mathrm{mg}, 96 \%) ;[\alpha]^{25}=-60.6\left(c 1.0, \mathrm{CHCl}_{3}\right) ;{ }^{1} \mathrm{H}$ NMR (400 MHz, $\left.\mathrm{CDCl}_{3}\right) \delta 8.20-8.07(\mathrm{~m}, 1 \mathrm{H}), 7.75-7.65(\mathrm{~m}, 1 \mathrm{H}), 7.32-7.18(\mathrm{~m}, 2 \mathrm{H}), 5.10(\mathrm{q}, J=$ $7.2 \mathrm{~Hz}, 1 \mathrm{H}), 5.02-4.88$ (m, 1H), 4.85 (s, 1H), $4.26-4.05$ (m, 2H), 1.64 (s, 9H), 1.59 $(\mathrm{d}, J=7.2 \mathrm{~Hz}, 3 \mathrm{H}), 1.52(\mathrm{~s}, 9 \mathrm{H}), 1.29(\mathrm{~d}, J=6.2 \mathrm{~Hz}, 3 \mathrm{H}), 1.23-1.13(\mathrm{~m}, 6 \mathrm{H}) ;{ }^{13} \mathrm{C}$ NMR $\left(100 \mathrm{MHz}, \mathrm{CDCl}_{3}\right) \delta 171.4,171.2,165.2,160.9,148.6,142.4,132.0,125.4$, $123.5,123.1,119.0,115.9,105.1,91.1,84.5,81.9,69.2,60.9,41.2,40.2,28.2,28.1$, 21.9, 21.7, 14.2. HRMS (ESI) $\mathrm{m} / z$ calcd for $\mathrm{C}_{30} \mathrm{H}_{39} \mathrm{NO}_{9}[\mathrm{M}+\mathrm{Na}]^{+}=580.2523$, found $=$ 580.2516 ; The ee value was $93 \%, \mathrm{t}_{\mathrm{R}}($ minor $)=8.2 \mathrm{~min}, \mathrm{t}_{\mathrm{R}}($ major $)=10.0 \mathrm{~min}$ (Chiralcel IE, $\lambda=254 \mathrm{~nm}, 2 \% i$-PrOH$/$ hexanes, flow rate $=1.0 \mathrm{~mL} / \mathrm{min}$ ). 
H:IWJH-Ř625\WJH-R625-RAC-98-2-1.0-IE-25.0.lcd $\mathrm{mV}$

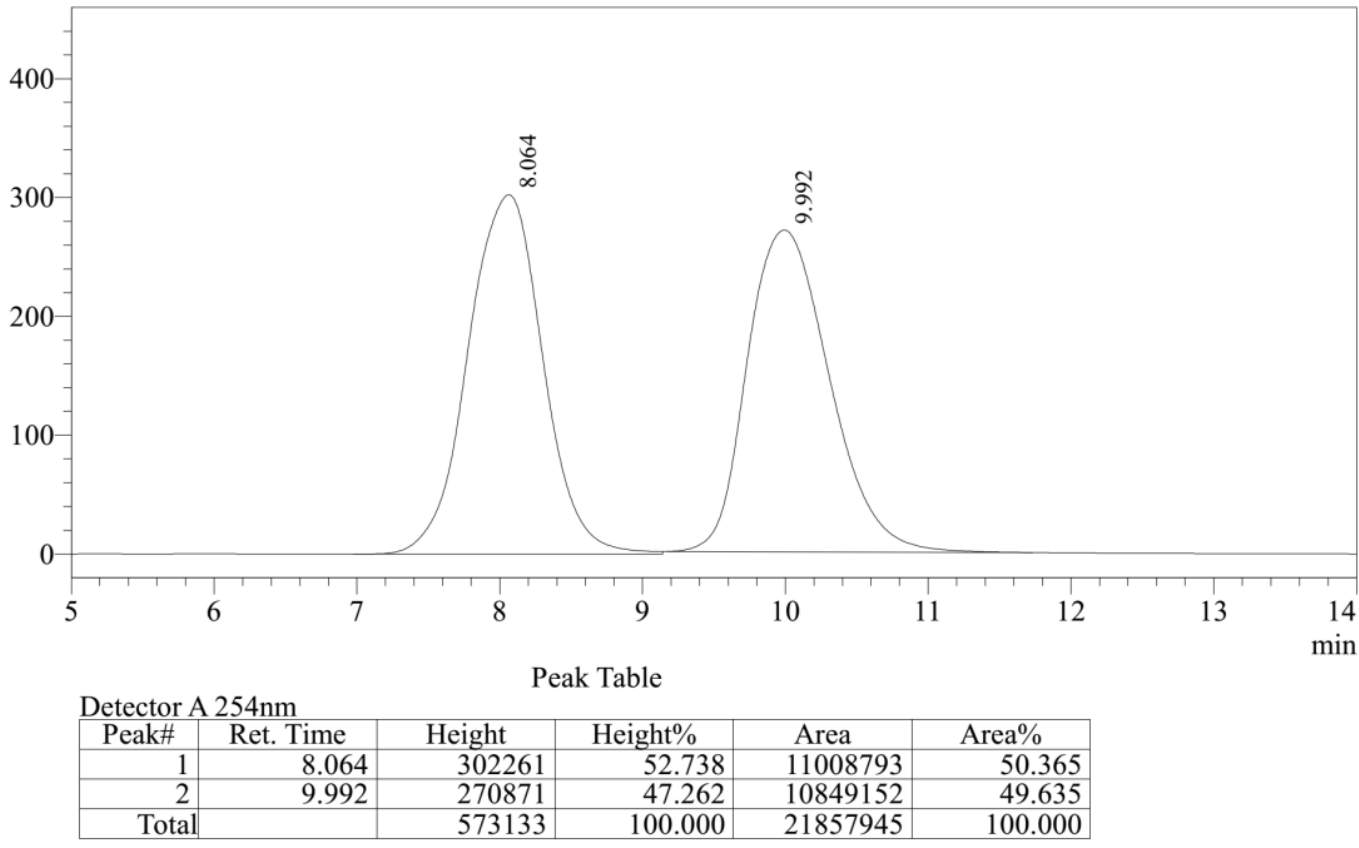

H:IWJH-R625\WJH-R625-CHI-98-2-1.0-IE-25-4.4.lcd $\mathrm{mV}$

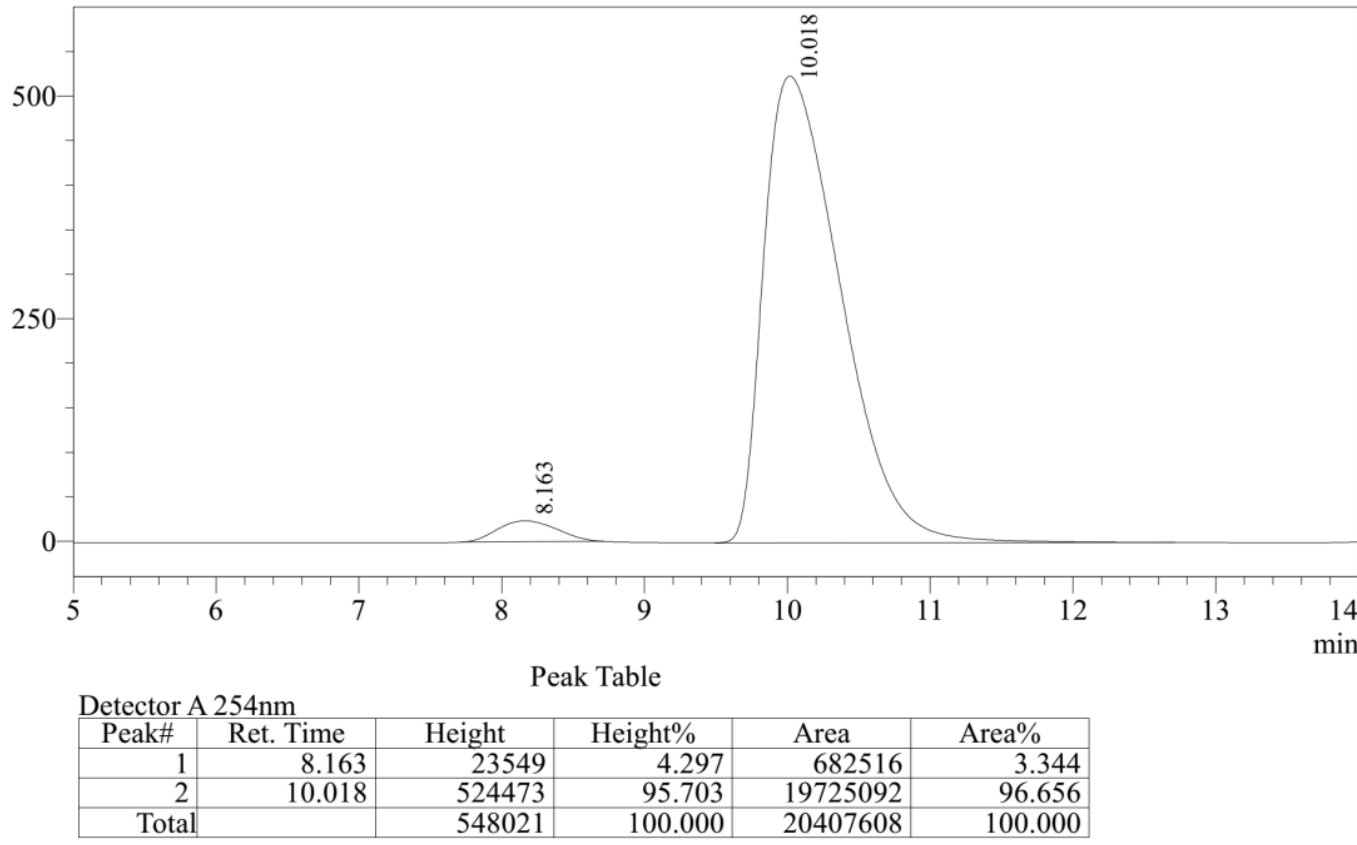




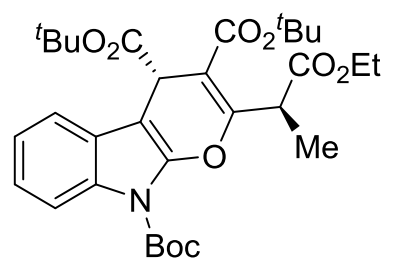

A white solid $(103 \mathrm{mg}, 90 \%)$; $[\alpha]^{25} \mathrm{D}=-53.1\left(c 1.0, \mathrm{CHCl}_{3}\right) ;{ }^{1} \mathrm{H}$ NMR $(400 \mathrm{MHz}$, $\left.\mathrm{CDCl}_{3}\right) \delta 8.19-8.08(\mathrm{~m}, 1 \mathrm{H}), 7.73-7.63(\mathrm{~m}, 1 \mathrm{H}), 7.29-7.18(\mathrm{~m}, 1 \mathrm{H}), 5.09(\mathrm{q}, J=$ $7.2 \mathrm{~Hz}, 1 \mathrm{H}), 4.78(\mathrm{~s}, 1 \mathrm{H}), 4.28-4.04(\mathrm{~m}, 2 \mathrm{H}), 1.63(\mathrm{~s}, 9 \mathrm{H}), 1.58(\mathrm{~d}, J=7.2 \mathrm{~Hz}, 3 \mathrm{H})$, $1.52(\mathrm{~s}, 9 \mathrm{H}), 1.42(\mathrm{~s}, 9 \mathrm{H}), 1.18(\mathrm{t}, J=7.1 \mathrm{~Hz}, 3 \mathrm{H}) ;{ }^{13} \mathrm{C} \mathrm{NMR}\left(100 \mathrm{MHz}, \mathrm{CDCl}_{3}\right) \delta$ 171.4, 170.9, 165.3, 160.8, 148.7, 142.3, 132.0, 125.5, 123.4, 123.0, 119.0, 114.9, 105.4, 91.4, 84.5, 81.8, 81.7, 60., 41.2, 40.9, 28.2, 28.1, 28.0, 14.2. HRMS (ESI) $\mathrm{m} / \mathrm{z}$ calcd for $\mathrm{C}_{31} \mathrm{H}_{41} \mathrm{NO}_{9}[\mathrm{M}+\mathrm{Na}]^{+}=594.2679$, found $=594.2671$; The ee value was 93\%, $t_{R}($ minor $)=7.3 \min , t_{R}$ (major) $=9.4 \min ($ Chiralcel $\mathrm{IE}, \lambda=254 \mathrm{~nm}, 2 \%$ $i$-PrOH/hexanes, flow rate $=1.0 \mathrm{~mL} / \mathrm{min}$ ).

H:IWJH-Ř626।WJH-R626-RAC-98-2-1.0-IE-25.0.lcd $\mathrm{mV}$

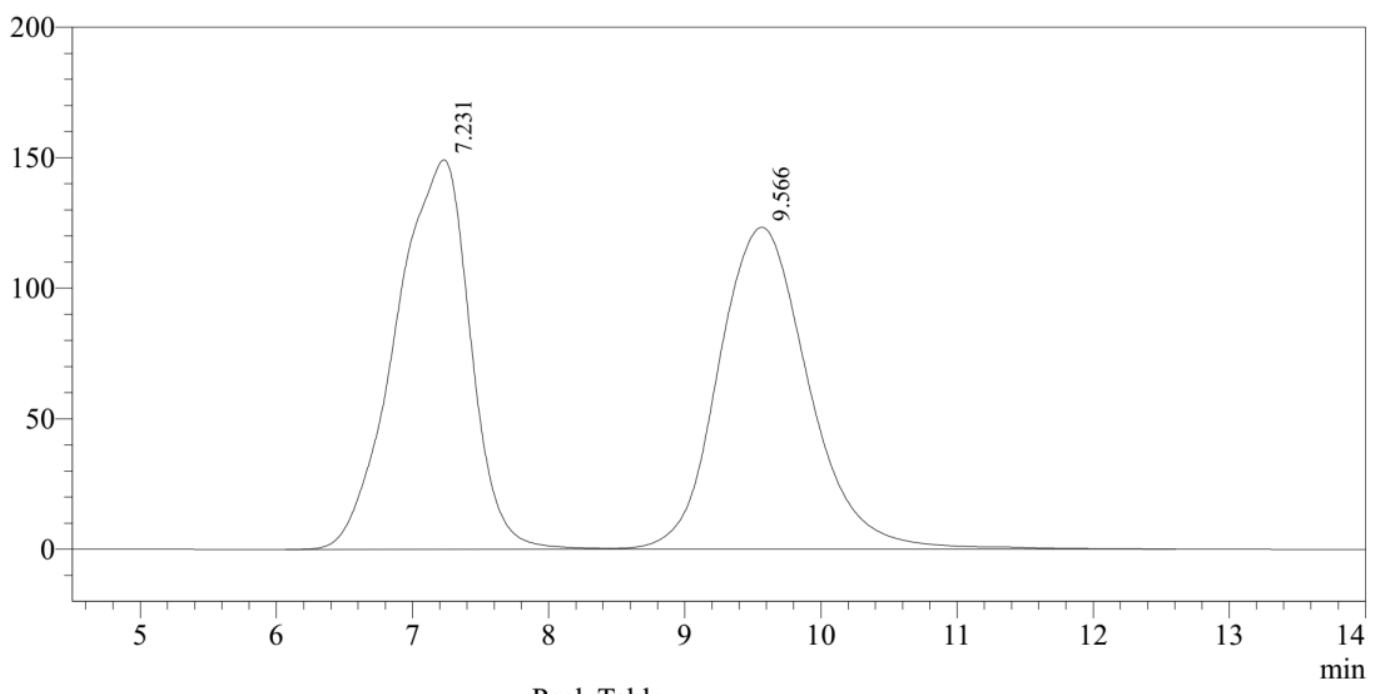

Peak Table

\begin{tabular}{|c|c|c|c|c|c|}
\hline \multicolumn{6}{|c|}{ Detector A $254 \mathrm{~nm}$} \\
\hline Peak\# & Ret. Time & Height & Height $\%$ & Area & Area\% \\
\hline 1 & 7.231 & 149285 & 54.752 & 5669734 & 49.960 \\
\hline 2 & 9.566 & 123370 & 45.248 & 5678845 & 50.040 \\
\hline Total & & 272655 & 100.000 & 11348580 & 100.000 \\
\hline
\end{tabular}




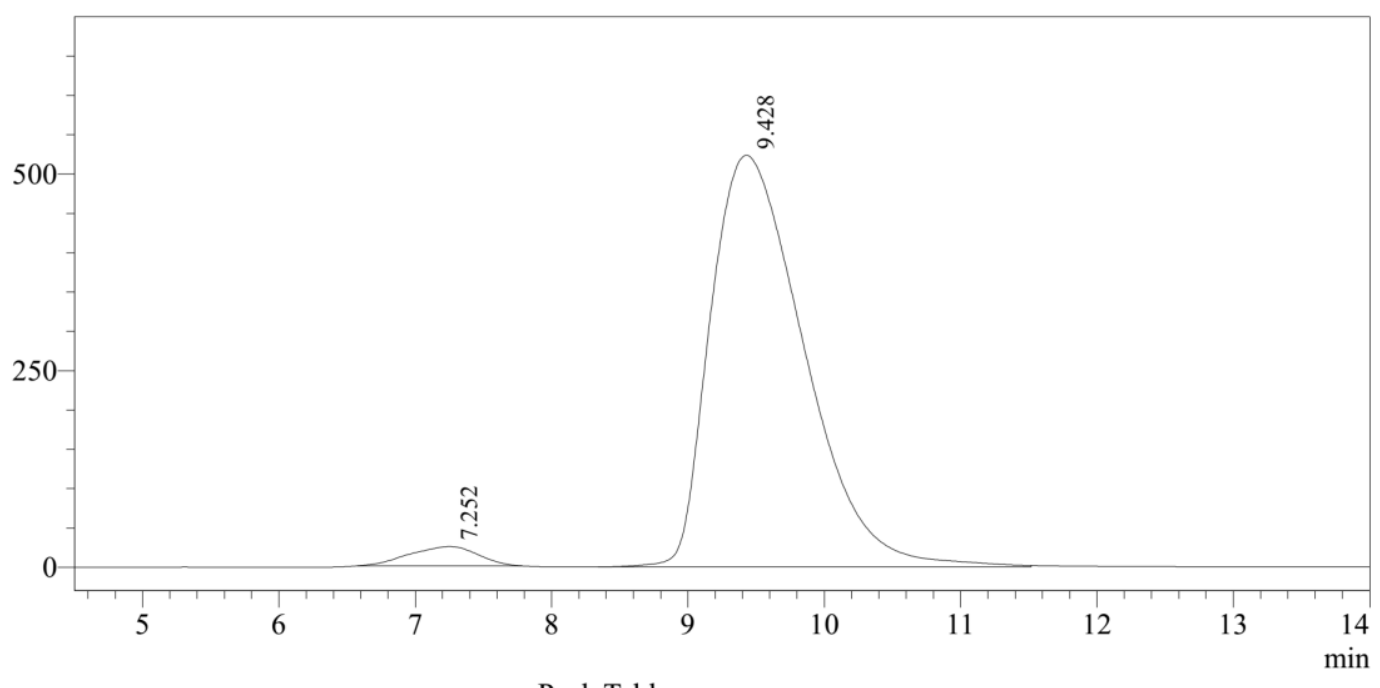

\begin{tabular}{|c|c|c|c|c|c|}
\hline \multirow{2}{*}{\multicolumn{6}{|c|}{ Detector A $254 \mathrm{~nm}$}} \\
\hline & & & & & \\
\hline Peak\# & Ret. Time & Height & Height $\%$ & Area & Area $\%$ \\
\hline 1 & 7.25 & 24750 & 4.515 & 859354 & 3.321 \\
\hline 2 & 9.428 & 523387 & 95.485 & 25018085 & 96.679 \\
\hline Tota & & 548137 & 100.000 & 25877439 & 100.000 \\
\hline
\end{tabular}

\section{3,9-di-tert-butyl 4-(9H-fluoren-9-yl) $(S)$-2-( $(S)$-1-ethoxy-1-oxopropan-2-yl)pyrano}

\section{[2,3-b]indole-3,4,9(4H)-tricarboxylate $(3 f)$}

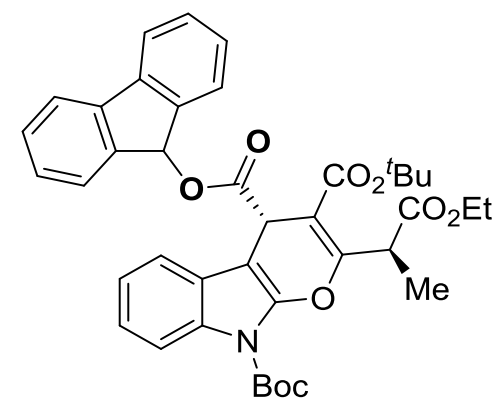

A white solid (125mg, 92\%); $[\alpha]^{25}{ }_{\mathrm{D}}=-29.6\left(c\right.$ 1.0, $\left.\mathrm{CHCl}_{3}\right) ;{ }^{1} \mathrm{H}$ NMR (400 MHz, $\left.\mathrm{CDCl}_{3}\right) \delta 8.15(\mathrm{~d}, J=8.3 \mathrm{~Hz}, 1 \mathrm{H}), 7.65(\mathrm{~d}, J=7.5 \mathrm{~Hz}, 2 \mathrm{H}), 7.56(\mathrm{dd}, J=7.4,3.8 \mathrm{~Hz}$, 2H), $7.42-7.35(\mathrm{~m}, 2 \mathrm{H}), 7.29-7.10(\mathrm{~m}, 6 \mathrm{H}), 6.68(\mathrm{~s}, 1 \mathrm{H}), 5.10(\mathrm{q}, J=7.2 \mathrm{~Hz}, 1 \mathrm{H})$, 4.99 (s, 1H), $4.26-4.04(\mathrm{~m}, 2 \mathrm{H}), 1.65$ (s, 9H), 1.57 (d, J= $7.2 \mathrm{~Hz}, 3 \mathrm{H}), 1.45$ (s, 9H), $1.18(\mathrm{t}, J=7.1 \mathrm{~Hz}, 3 \mathrm{H}) ;{ }^{13} \mathrm{C} \mathrm{NMR}\left(100 . \mathrm{MHz}, \mathrm{CDCl}_{3}\right) \delta 172.5,171.3,165.2,161.3$, $148.6,142.6,141.9,141.4-140.8$ (m), 132.0, 129.5, 129.47, 127.8, 127.6, 126.7, $126.3,125.1,123.7,123.1,119.9,119.3,114.9,105.0,91.0,84.6,82.3,76.4,61.0$, $41.4,40.2,28.2,28.0,14.2,14.2$. HRMS (ESI) $\mathrm{m} / z$ calcd for $\mathrm{C}_{40} \mathrm{H}_{41} \mathrm{NO}_{9}[\mathrm{M}+\mathrm{Na}]^{+}=$ 
702.2679 , found $=702.2683$; The ee value was $86 \%, t_{R}($ minor $)=9.6$ min, $t_{R}($ major $)=$ $11.6 \mathrm{~min}$ (Chiralcel IE, $\lambda=254 \mathrm{~nm}, 3 \% i-\mathrm{PrOH} / \mathrm{h}$ exanes, flow rate $=1.0 \mathrm{~mL} / \mathrm{min}$ ).

H:IWJH-R677\WJH-R677-RAC-97-3-1.0-IE-30-3.3-106.lcd $\mathrm{mV}$

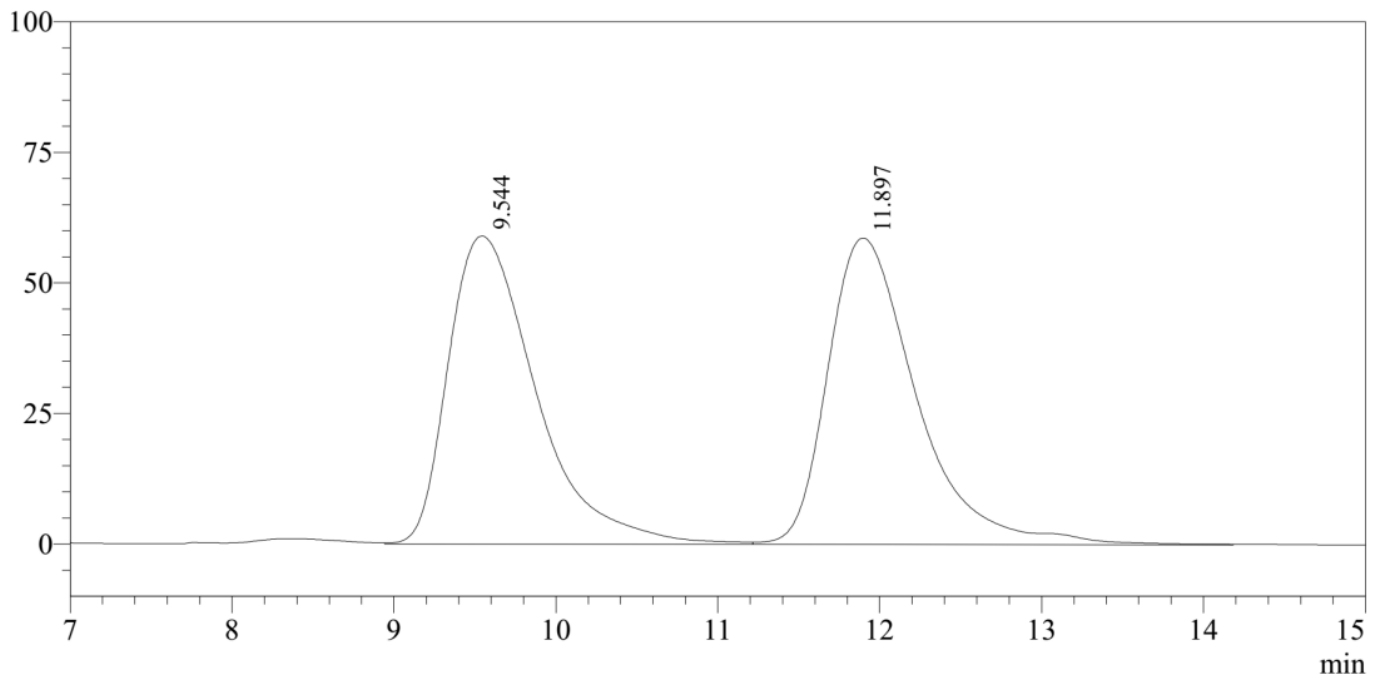

Peak Table

\begin{tabular}{|c|c|c|c|c|c|}
\hline \multicolumn{6}{|c|}{ Detector A $254 \mathrm{~nm}$} \\
\hline Peak\# & Ret. Time & Height & Height $\%$ & Area & Area\% \\
\hline 1 & 9.544 & 58999 & 50.130 & 2225951 & 49.796 \\
\hline 2 & 11.897 & 58692 & 49.870 & 2244168 & 50.204 \\
\hline Total & & 117691 & 100.000 & 4470120 & 100.000 \\
\hline
\end{tabular}

H:IWJH-R6̄677\WJH-R677-CHRI2-97-3-1.0-IE-30-3.3-106.lcd $\mathrm{mV}$

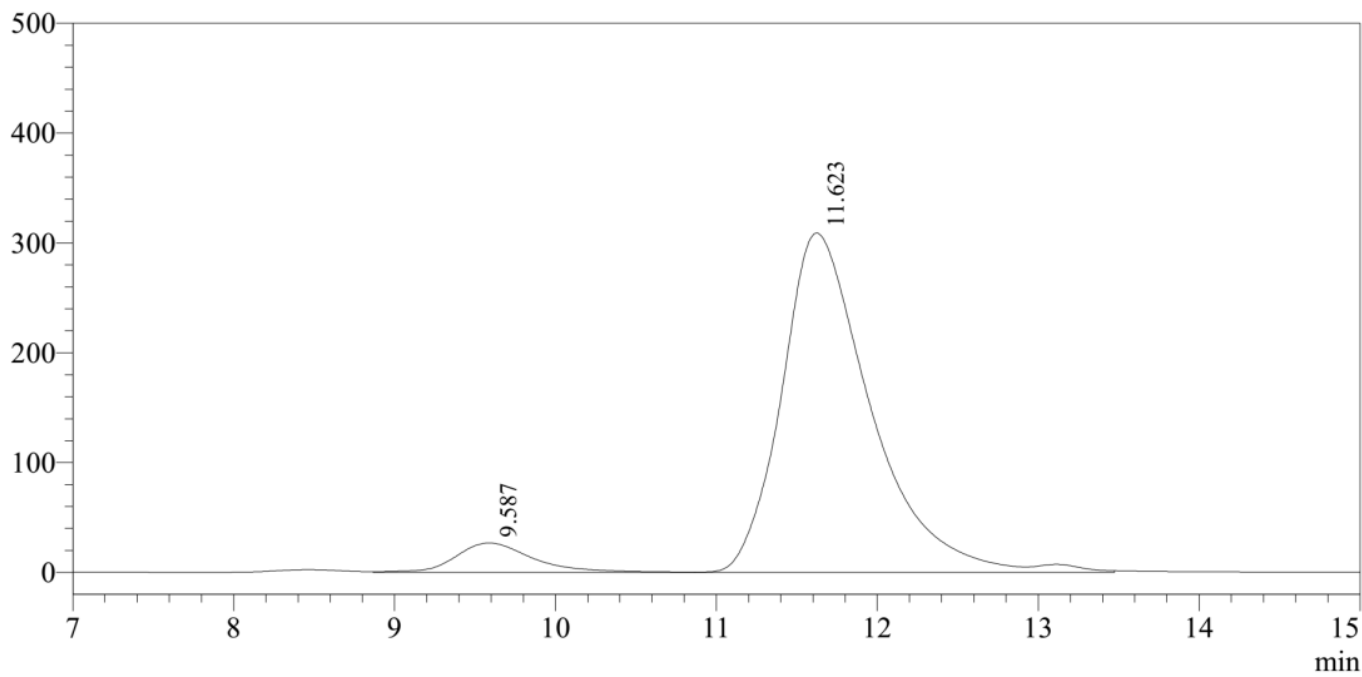

Peak Table

\begin{tabular}{|c|c|c|c|c|c|}
\hline Peak\# & Ret. Time & Height & Height $\%$ & Area & Area $\%$ \\
\hline 1 & 9.587 & 26772 & 7.976 & 900934 & 7.008 \\
\hline 2 & 11.623 & 308903 & 92.024 & 11954085 & 92.992 \\
\hline Total & & 335675 & 100.000 & 12855019 & 100.000 \\
\hline
\end{tabular}

\section{4,9-dibenzyl 3-(tert-butyl) $\quad(S)$-2-((S)-1-ethoxy-1-oxopropan-2-yl)pyrano[2,3-b]}




\section{indole- $3,4,9(4 H)$-tricarboxylate $(3 \mathrm{~g})$}

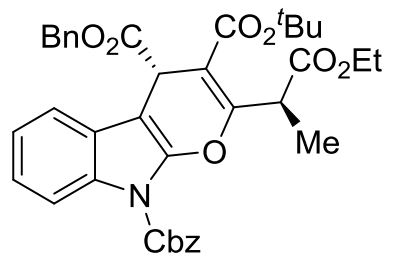

A white solid (113mg, 88\%); $[\alpha]^{25}{ }_{\mathrm{D}}=-5.6\left(c 0.8, \mathrm{CHCl}_{3}\right) ;{ }^{1} \mathrm{H} \mathrm{NMR}\left(400 \mathrm{MHz}, \mathrm{CDCl}_{3}\right)$ $\delta 8.14(\mathrm{~d}, J=8.2 \mathrm{~Hz}, 1 \mathrm{H}), 7.59-7.52(\mathrm{~m}, 1 \mathrm{H}), 7.52-7.47(\mathrm{~m}, 2 \mathrm{H}), 7.41-7.33(\mathrm{~m}$, 3H), $7.33-7.27(\mathrm{~m}, 5 \mathrm{H}), 7.27-7.21(\mathrm{~m}, 1 \mathrm{H}), 7.20-7.14(\mathrm{~m}, 1 \mathrm{H}), 5.52-5.32(\mathrm{~m}$, 2H), $5.10(\mathrm{dd}, J=36.0,12.2 \mathrm{~Hz}, 2 \mathrm{H}), 4.95$ (q, $J=7.2 \mathrm{~Hz}, 1 \mathrm{H}), 4.89$ (s, 1H), $4.16-$ $4.03(\mathrm{~m}, 2 \mathrm{H}), 1.42(\mathrm{~s}, 9 \mathrm{H}), 1.39(\mathrm{~d}, J=7.2 \mathrm{~Hz}, 3 \mathrm{H}), 1.16(\mathrm{t}, J=7.1 \mathrm{~Hz}, 3 \mathrm{H}) ;{ }^{13} \mathrm{C}$ NMR $\left(100 \mathrm{MHz}, \mathrm{CDCl}_{3}\right) \delta 171.3,171.1,164.8,160.6,149.8,142.2,135.2,134.6$, 131.6, 129.0, 129.0, 128.7, 128.6, 128.4, 128.3, 128.2, 128.1, 125.4, 123.8, 123.6, 119.0, 114.9, 104.8, 91.3, 82.0, 68.9, 67.1, 60.9, 41.4, 40.1, 27.8, 14.1, 13.8. HRMS (ESI) $m / z$ calcd for $\mathrm{C}_{37} \mathrm{H}_{37} \mathrm{NO}_{9}[\mathrm{M}+\mathrm{Na}]^{+}=662.2366$, found $=662.2370$; The ee value was $84 \%, \mathrm{t}_{\mathrm{R}}($ minor $)=37.8 \mathrm{~min}, \mathrm{t}_{\mathrm{R}}($ major $)=41.0 \min ($ Chiralcel IE, $\lambda=254 \mathrm{~nm}, 2 \%$ $i-\mathrm{PrOH} / \mathrm{h}$ exanes, flow rate $=1.0 \mathrm{~mL} / \mathrm{min})$.

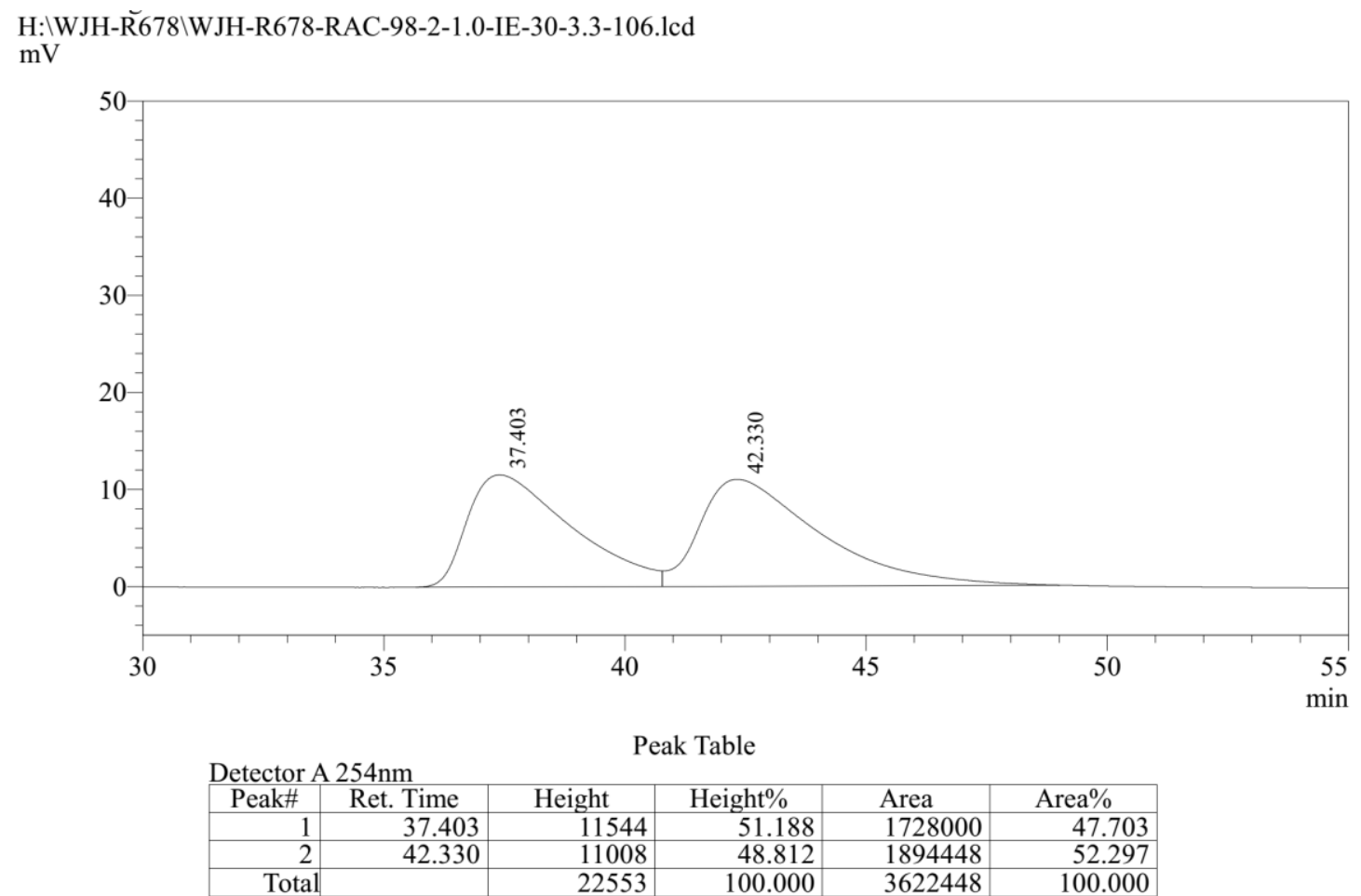


H:IWJH-R6-678।WJH-R678-CHRI-98-2-1.0-IE-30-3.3-106.lcd $\mathrm{mV}$

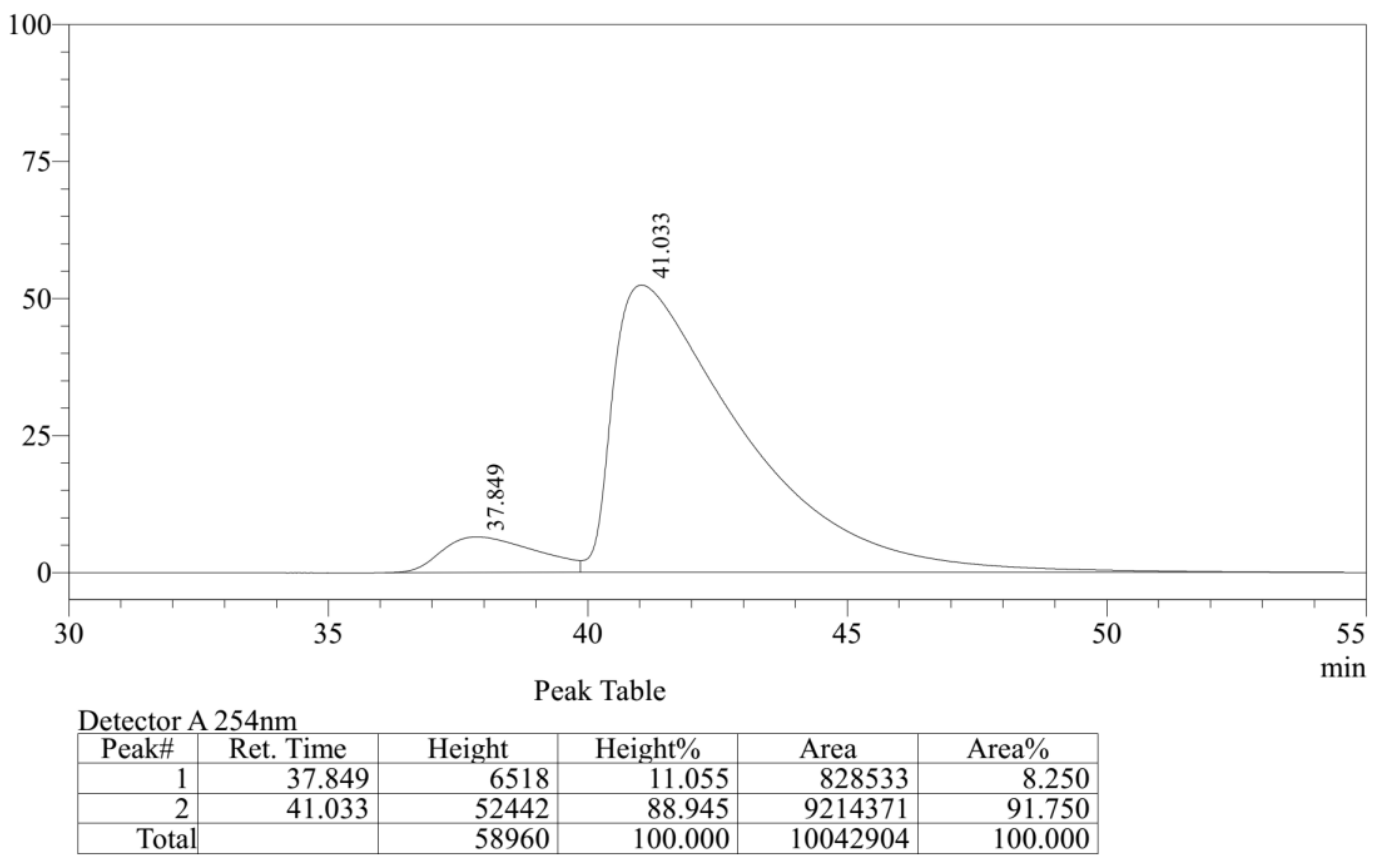

4-benzyl 3,9-di-tert-butyl $(S)$-5-chloro-2-( $(S)$-1-ethoxy-1-oxopropan-2-yl)pyrano [2,3-b]indole-3,4,9(4H)-tricarboxylate $(3 \mathrm{~h})$<smiles>CCOC(=O)C1=C(C(=O)OCC)C(C(=O)OCc2ccccc2)c2c(n(C(=O)OCc3ccccc3)c3cccc(Br)c23)O1</smiles>

A white solid $(123 \mathrm{mg}, 96 \%) ;[\alpha]^{25}=-41.1\left(c\right.$ 1.0, $\left.\mathrm{CHCl}_{3}\right) ;{ }^{1} \mathrm{H}$ NMR (400 MHz, $\left.\mathrm{CDCl}_{3}\right) \delta 8.17-8.06(\mathrm{~m}, 1 \mathrm{H}), 7.37-7.25(\mathrm{~m}, 5 \mathrm{H}), 7.23-7.13(\mathrm{~m}, 2 \mathrm{H}), 5.50(\mathrm{~s}, 1 \mathrm{H})$, $5.16(\mathrm{q}, J=12.4 \mathrm{~Hz}, 2 \mathrm{H}), 4.77(\mathrm{q}, J=7.2 \mathrm{~Hz}, 1 \mathrm{H}), 4.24-4.07(\mathrm{~m}, 2 \mathrm{H}), 1.66(\mathrm{~s}, 9 \mathrm{H})$, $1.55(\mathrm{~d}, J=7.2 \mathrm{~Hz}, 3 \mathrm{H}), 1.47(\mathrm{~s}, 9 \mathrm{H}), 1.18(\mathrm{t}, J=7.1 \mathrm{~Hz}, 3 \mathrm{H}) ;{ }^{13} \mathrm{C}$ NMR $(100 \mathrm{MHz}$, $\left.\mathrm{CDCl}_{3}\right) \delta 171.5,171.1,165.3,158.8,148.3,143.8,135.6,133.2,128.4,128.1(\mathrm{~d}, J=$ $3.9 \mathrm{~Hz}), 124.8,124.2,123.9,123.2,113.7,106.8,91.2,85.1,82.2,67.1,61.0,41.1$, 39.7, 28.10, 27.9, 14.2, 14.0. HRMS (ESI) $m / z$ calcd for $\mathrm{C}_{34} \mathrm{H}_{38} \mathrm{ClNO}_{9}[\mathrm{M}+\mathrm{Na}]^{+}=$ 662.2133, found $=662.2114 ;$ The ee value was $92 \%, t_{R}($ major $)=15.1 \mathrm{~min}, \mathrm{t}_{\mathrm{R}}($ minor $)$ $=18.2 \mathrm{~min}($ Chiralcel IG, $\lambda=254 \mathrm{~nm}, 2 \% i-\mathrm{PrOH} / \mathrm{h}$ exanes, flow rate $=0.50 \mathrm{~mL})$ 
H:\WJH-R̄640 \WJH-R640-RAC-98-2-0.5-IG-30-1.4-106.lcd $\mathrm{mV}$

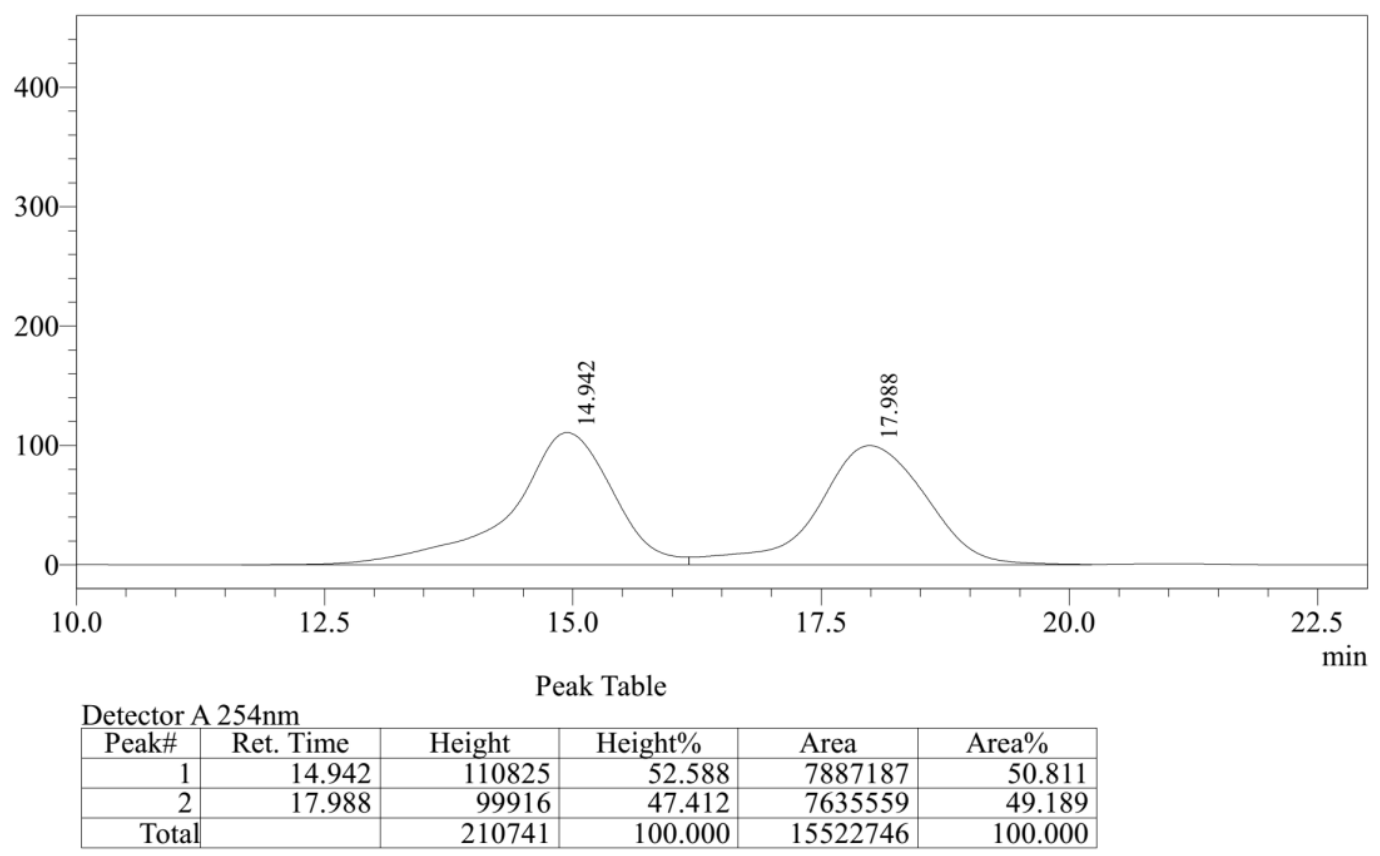

H:IWJH-R640|WJH-R640-CHRI-98-2-0.5-IG-30-1.4-106.lcd $\mathrm{mV}$

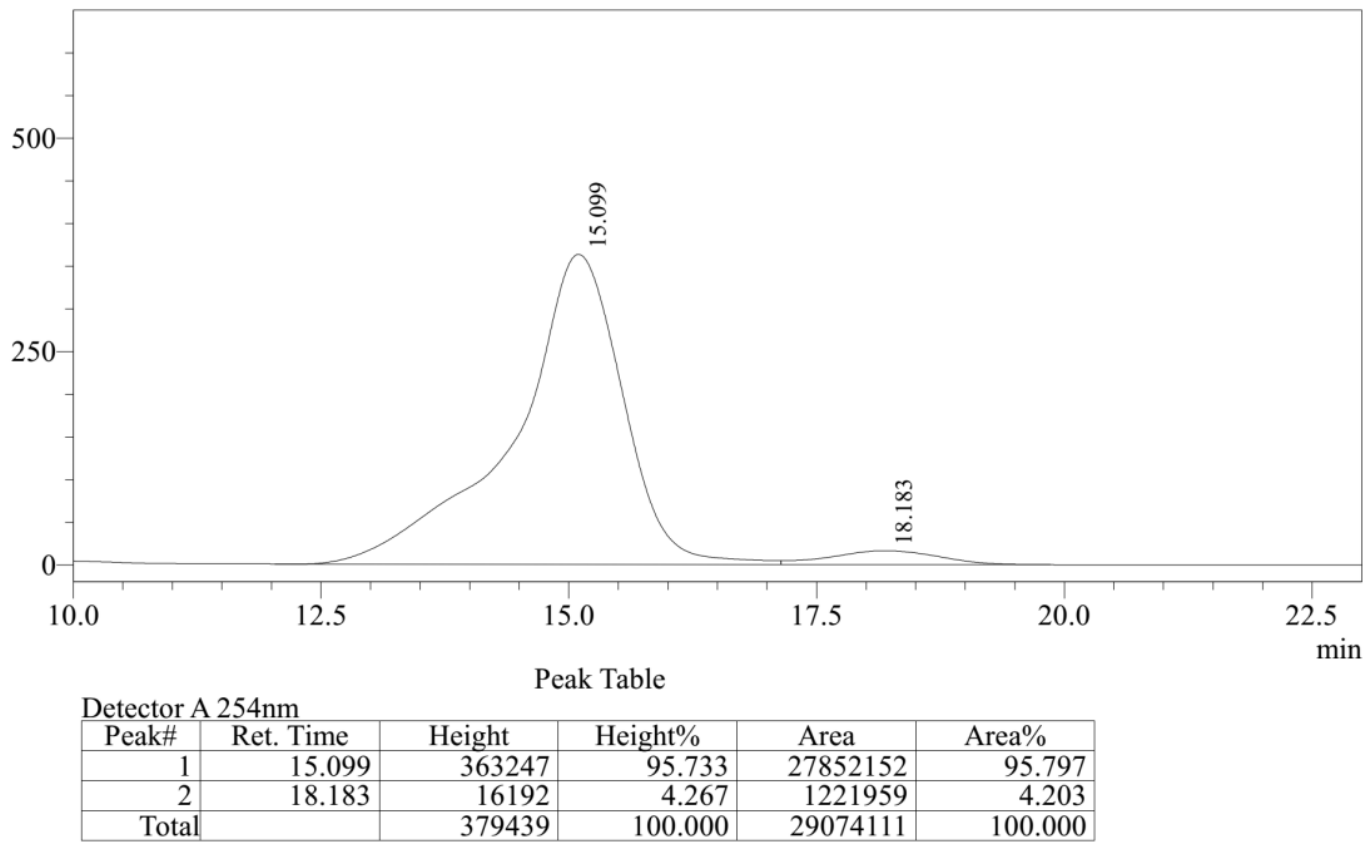

4-benzyl 3,9-di-tert-butyl $(S)$-5-bromo-2-((S)-1-ethoxy-1-oxopropan-2-yl)pyrano [2,3-b]indole-3,4,9(4H)-tricarboxylate $(3 \mathrm{i})$ 


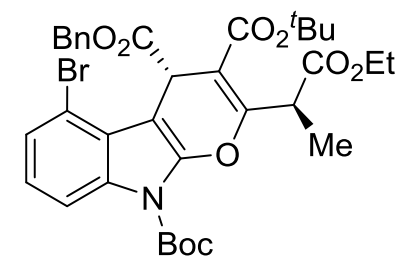

A white solid $(124 \mathrm{mg}, 91 \%) ;[\alpha]^{25}=-40.1\left(c 1.0, \mathrm{CHCl}_{3}\right) ;{ }^{1} \mathrm{H}$ NMR $(400 \mathrm{MHz}$, $\left.\mathrm{CDCl}_{3}\right) \delta 8.15(\mathrm{~d}, J=8.3 \mathrm{~Hz}, 1 \mathrm{H}), 7.35(\mathrm{~d}, J=7.8 \mathrm{~Hz}, 1 \mathrm{H}), 7.33-7.24(\mathrm{~m}, 6 \mathrm{H}), 7.08$ $(\mathrm{t}, J=8.1 \mathrm{~Hz}, 1 \mathrm{H}), 5.61(\mathrm{~s}, 1 \mathrm{H}), 5.14(\mathrm{q}, J=12.4 \mathrm{~Hz}, 2 \mathrm{H}), 4.71(\mathrm{q}, J=7.1 \mathrm{~Hz}, 1 \mathrm{H})$, $4.21-4.03(\mathrm{~m}, 2 \mathrm{H}), 1.64$ (s, 9H), 1.51 (d, $J=7.2 \mathrm{~Hz}, 3 \mathrm{H}), 1.45$ (s, 9H), $1.15(\mathrm{t}, J=$ $7.1 \mathrm{~Hz}, 1 \mathrm{H}) ;{ }^{13} \mathrm{C}$ NMR $\left(100 \mathrm{MHz}, \mathrm{CDCl}_{3}\right) \delta 171.4,171.0,158.6,128.4,128.2,128.1$, 127.5, 124.3, 114.2, 112.5, 85.1, 82.2, 67.2, 61.0, 41.1, 39.4, 28.1, 27.9, 14.2, 13.9. HRMS (ESI) $\mathrm{m} / z$ calcd for $\mathrm{C}_{34} \mathrm{H}_{38} \mathrm{BrNO}_{9}[\mathrm{M}+\mathrm{Na}]^{+}=706.1628$, found $=706.1611$; The ee value was $91 \%, t_{R}($ major $)=11.1 \mathrm{~min}, \mathrm{t}_{\mathrm{R}}($ minor $)=13.5 \mathrm{~min}($ Chiralcel IE, $\lambda=254$ $\mathrm{nm}, 2 \% i-\mathrm{PrOH} / \mathrm{hexanes}$, flow rate $=1.0 \mathrm{~mL} / \mathrm{min}$ ).

H:IWJH-R641 \WJH-R641-RAC-98-2-1.0-30-3.4-106.lcd $\mathrm{mV}$

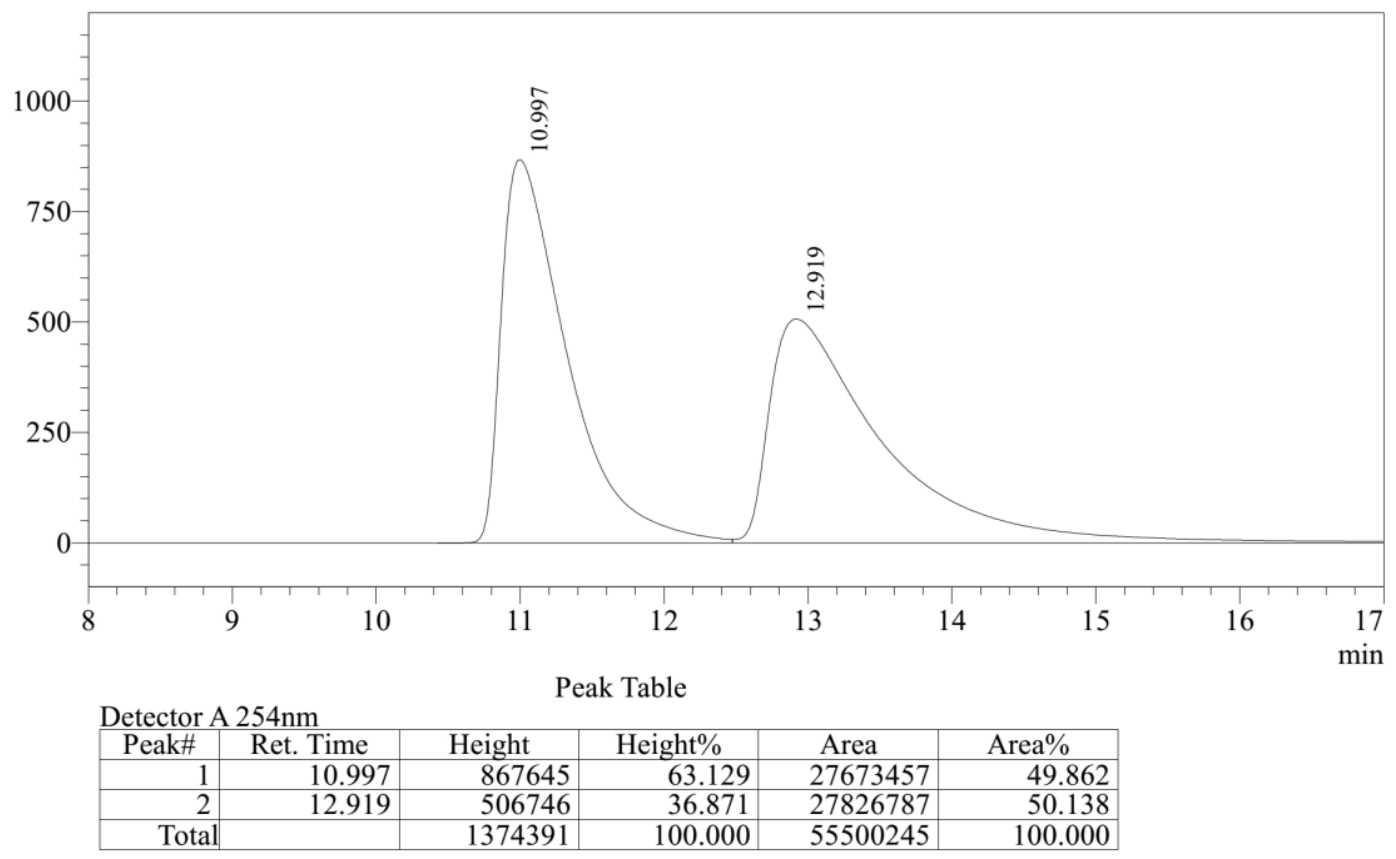


H:IWJH-Ř641 \WJH-R641-CHRI-98-2-1.0-30-3.4-106.lcd $\mathrm{mV}$

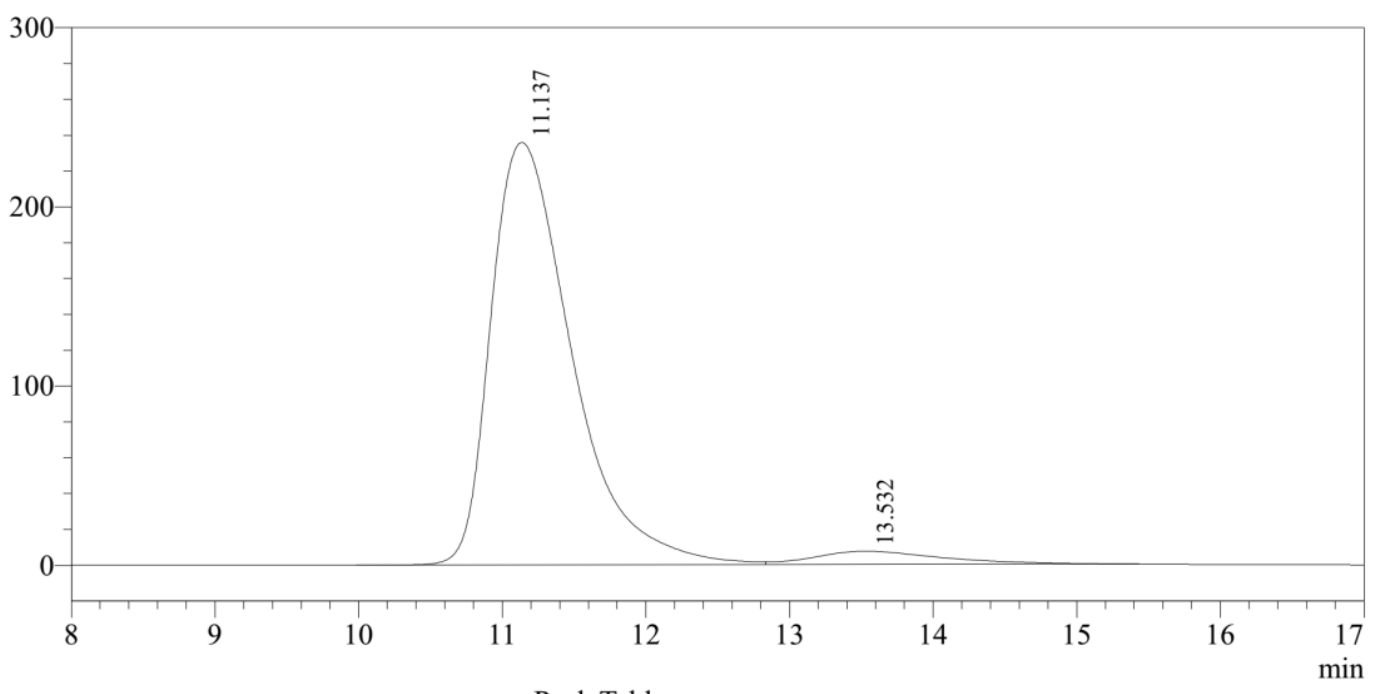

Peak Table
\begin{tabular}{|r|r|r|r|r|r|} 
Detector A 254nm \\
\hline Peak\# & Ret. Time & \multicolumn{1}{|c|}{ Height } & \multicolumn{1}{|c|}{ Height $\%$} & \multicolumn{1}{c|}{ Area } & \multicolumn{1}{|c|}{ Area $\%$} \\
\hline 1 & 11.137 & 235668 & 96.985 & 9245615 & 95.276 \\
\hline 2 & 13.532 & 7325 & 3.015 & 458416 & 4.724 \\
\hline Total & & 242994 & 100.000 & 9704031 & 100.000 \\
\hline
\end{tabular}

4-benzyl 3,9-di-tert-butyl $(S)$-2-( $(S)$-1-ethoxy-1-oxopropan-2-yl)-6-fluoropyrano [2,3-b]indole-3,4,9(4H)-tricarboxylate $(3 \mathrm{j})$<smiles>CCOC(=O)C1=C(C(C)C(=O)OCC)Oc2c(c3cc(F)ccc3n2C(=O)OCc2ccccc2)C1C(=O)OCc1ccccc1</smiles>

A white solid (116mg, 93\%); $[\alpha]^{25}{ }_{\mathrm{D}}=-40.1\left(c\right.$ 1.0, $\left.\mathrm{CHCl}_{3}\right) ;{ }^{1} \mathrm{H}$ NMR $(400 \mathrm{MHz}$, $\left.\mathrm{CDCl}_{3}\right) \delta 8.08(\mathrm{dd}, J=9.1,4.6 \mathrm{~Hz}, 1 \mathrm{H}), 7.36-7.30(\mathrm{~m}, 5 \mathrm{H}), 7.25(\mathrm{dd}, J=8.8,2.5 \mathrm{~Hz}$ 1H), $6.94(\mathrm{td}, J=9.1,2.7 \mathrm{~Hz}, 1 \mathrm{H}), 5.19-5.02(\mathrm{~m}, 3 \mathrm{H}), 4.86(\mathrm{~s}, 1 \mathrm{H}), 4.25-4.07(\mathrm{~m}$, $2 \mathrm{H}), 1.63(\mathrm{~s}, 9 \mathrm{H}), 1.57(\mathrm{~d}, J=7.2 \mathrm{~Hz}, 3 \mathrm{H}), 1.44(\mathrm{~s}, 9 \mathrm{H}), 1.20(\mathrm{t}, J=7.1 \mathrm{~Hz}, 3 \mathrm{H}) ;{ }^{13} \mathrm{C}$ NMR (100 MHz, $\left.\mathrm{CDCl}_{3}\right) \delta 171.2,171.1,164.9,161.0,159.5(\mathrm{~d}, J=239.7 \mathrm{~Hz}), 148.4$, 143.3, 135.1, 128.6, 128.6, 128.5, 128.1 (d, $J=1.1 \mathrm{~Hz}), 126.3(\mathrm{~d}, J=10.5 \mathrm{~Hz}), 116.1$ $(\mathrm{d}, J=9.0 \mathrm{~Hz}), 110.9(\mathrm{~d}, J=24.6 \mathrm{~Hz}), 105.0(\mathrm{~d}, J=25.3 \mathrm{~Hz}), 90.8(\mathrm{~d}, J=3.8 \mathrm{~Hz})$, 84.8, 82.2, 67.4, 61.0, 41.1, 40.0, 28.1, 27.9, 14.2, 14.2; ${ }^{19} \mathrm{~F}$ NMR (376 MHz, $\left.\mathrm{CDCl}_{3}\right)$ $\delta$-119.1. HRMS (ESI) $\mathrm{m} / z$ calcd for $\mathrm{C}_{34} \mathrm{H}_{38} \mathrm{FNO}_{9}[\mathrm{M}+\mathrm{Na}]^{+}=646.2428$, found $=$ 646.2422; The ee value was $90 \%, \mathrm{t}_{\mathrm{R}}($ major $)=9.0 \mathrm{~min}, \mathrm{t}_{\mathrm{R}}($ minor $)=11.4 \mathrm{~min}($ Chiralcel IE, $\lambda=254 \mathrm{~nm}, 2 \% i-\mathrm{PrOH} / \mathrm{hexanes}$, flow rate $=1.0 \mathrm{~mL} / \mathrm{min})$. 
H:IWJH-R̄643।WJH-R643-RAC-98-2-1.0-IE-30-3.3.lcd $\mathrm{mV}$

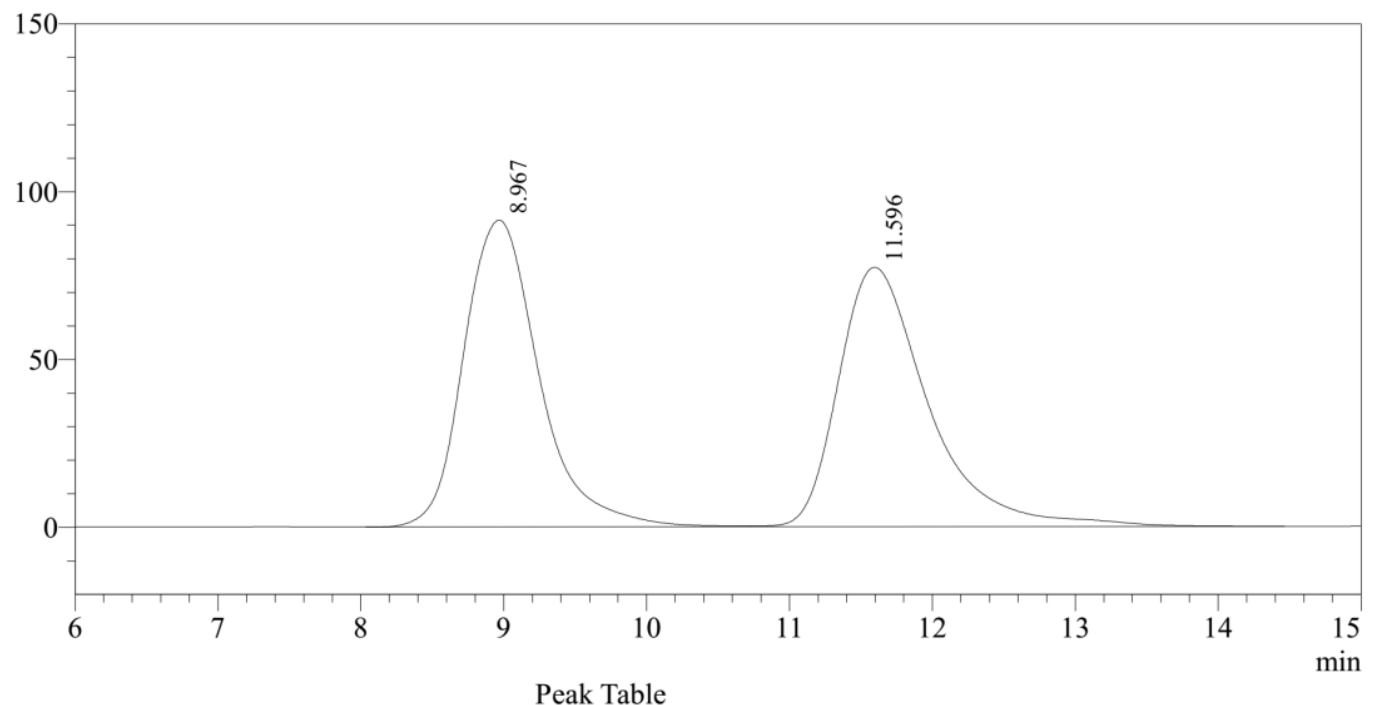

\begin{tabular}{|c|c|c|c|c|c|}
\hline \multicolumn{6}{|c|}{ Detector A $254 \mathrm{~nm}$} \\
\hline Peak\# & Ret. Time & Height & Height $\%$ & Area & Area $\%$ \\
\hline 1 & 8.967 & 91450 & 54.192 & 3357304 & 49.805 \\
\hline 2 & 11.596 & 77301 & 45.808 & 3383551 & 50.195 \\
\hline Total & & 168751 & 100.000 & 6740856 & 100.000 \\
\hline
\end{tabular}

H:IWJH-Ř643 \WJH-R643-CHRI-98-2-1.0-IE-30-3.3.lcd $\mathrm{mV}$

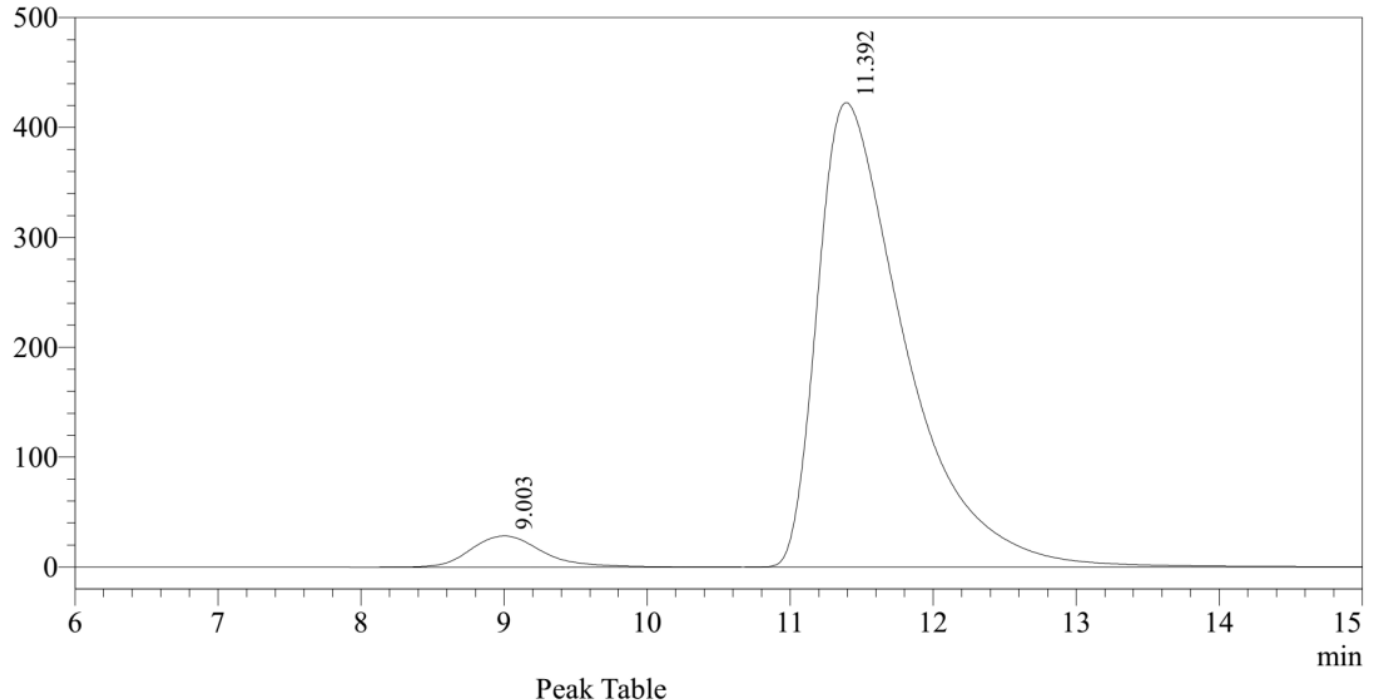

Detector A $254 \mathrm{~nm}$
\begin{tabular}{|r|r|r|r|r|r|}
\hline Peak\# & Ret. Time & Height & Height $\%$ & Area & Area $\%$ \\
\hline 1 & 9.003 & 28357 & 6.288 & 992927 & 5.221 \\
\hline 2 & 11.392 & 422613 & 93.712 & 18025549 & 94.779 \\
\hline Total & & 450970 & 100.000 & 19018475 & 100.000 \\
\hline
\end{tabular}

4-benzyl 3,9-di-tert-butyl (S)-6-chloro-2-((S)-1-ethoxy-1-oxopropan-2-yl)pyrano [2,3-b]indole-3,4,9(4H)-tricarboxylate $(3 \mathrm{k})$ 


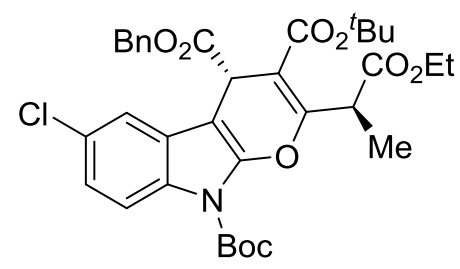

A white solid (118mg, 92\%); $[\alpha]^{25}{ }_{\mathrm{D}}=-45.7\left(c\right.$ 0.5, $\left.\mathrm{CHCl}_{3}\right) ;{ }^{1} \mathrm{H}$ NMR $(400 \mathrm{MHz}$, $\left.\mathrm{CDCl}_{3}\right) \delta 8.06(\mathrm{~d}, J=8.8 \mathrm{~Hz}, 1 \mathrm{H}), 7.57(\mathrm{~d}, J=2.1 \mathrm{~Hz}, 1 \mathrm{H}), 7.38-7.31(\mathrm{~m}, 5 \mathrm{H}), 7.19$ $(\mathrm{dd}, J=8.8,2.2 \mathrm{~Hz}, 1 \mathrm{H}), 5.20-5.03(\mathrm{~m}, 3 \mathrm{H}), 4.86(\mathrm{~s}, 1 \mathrm{H}), 4.25-4.08(\mathrm{~m}, 2 \mathrm{H}), 1.63$ (s, 9H), $1.57(\mathrm{~d}, J=7.2 \mathrm{~Hz}, 3 \mathrm{H}), 1.45(\mathrm{~s}, 9 \mathrm{H}), 1.20(\mathrm{t}, J=7.1 \mathrm{~Hz}, 3 \mathrm{H}) ;{ }^{13} \mathrm{C}$ NMR (100 $\left.\mathrm{MHz}, \mathrm{CDCl}_{3}\right) \delta 171.2,164.8,161.0,148.3,143.2,135.0,130.2,129.0,128.7,128.6$, $128.49,126.5,123.7,118.6,116.1,105.0,90.4,85.1,82.2,67.6,61.0,41.1,39.9,28.2$, 28.0, 14.2, 14.2. HRMS (ESI) $m / z$ calcd for $\mathrm{C}_{34} \mathrm{H}_{38} \mathrm{ClNO}_{9}[\mathrm{M}+\mathrm{Na}]^{+}=662.2133$, found $=662.2114 ;$ The ee value was $87 \%, \mathrm{t}_{\mathrm{R}}($ minor $)=9.1 \mathrm{~min}, \mathrm{t}_{\mathrm{R}}$ (major) $=11.4 \mathrm{~min}$ (Chiralcel IE, $\lambda=254 \mathrm{~nm}, 2 \% i$-PrOH$/$ hexanes, flow rate $=1.0 \mathrm{~mL} / \mathrm{min}$ ).

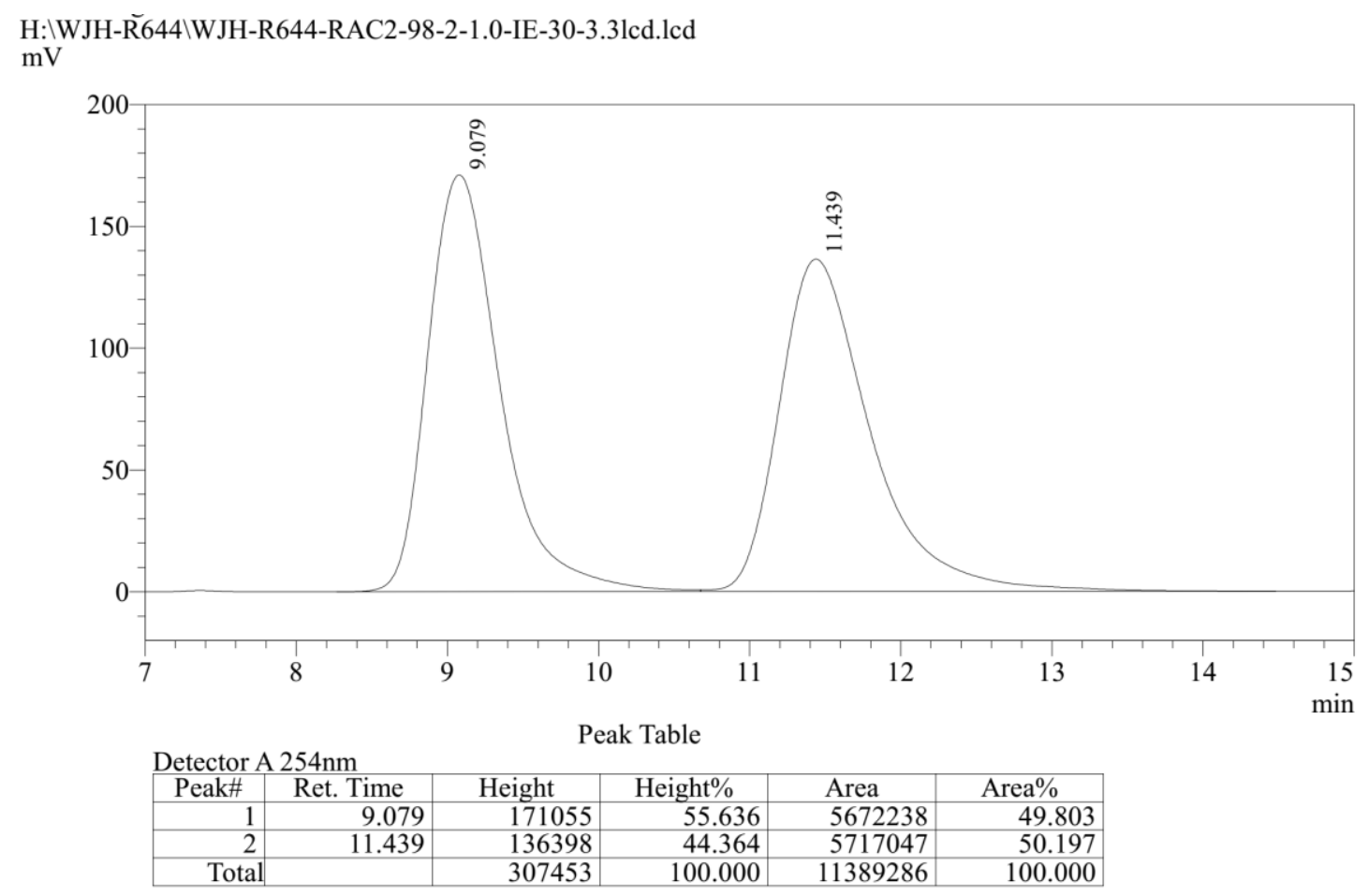


H:IWJH-Ř644|WJH-R644-CHRI-98-2-1.0-IE-30-3.3.lcd $\mathrm{mV}$

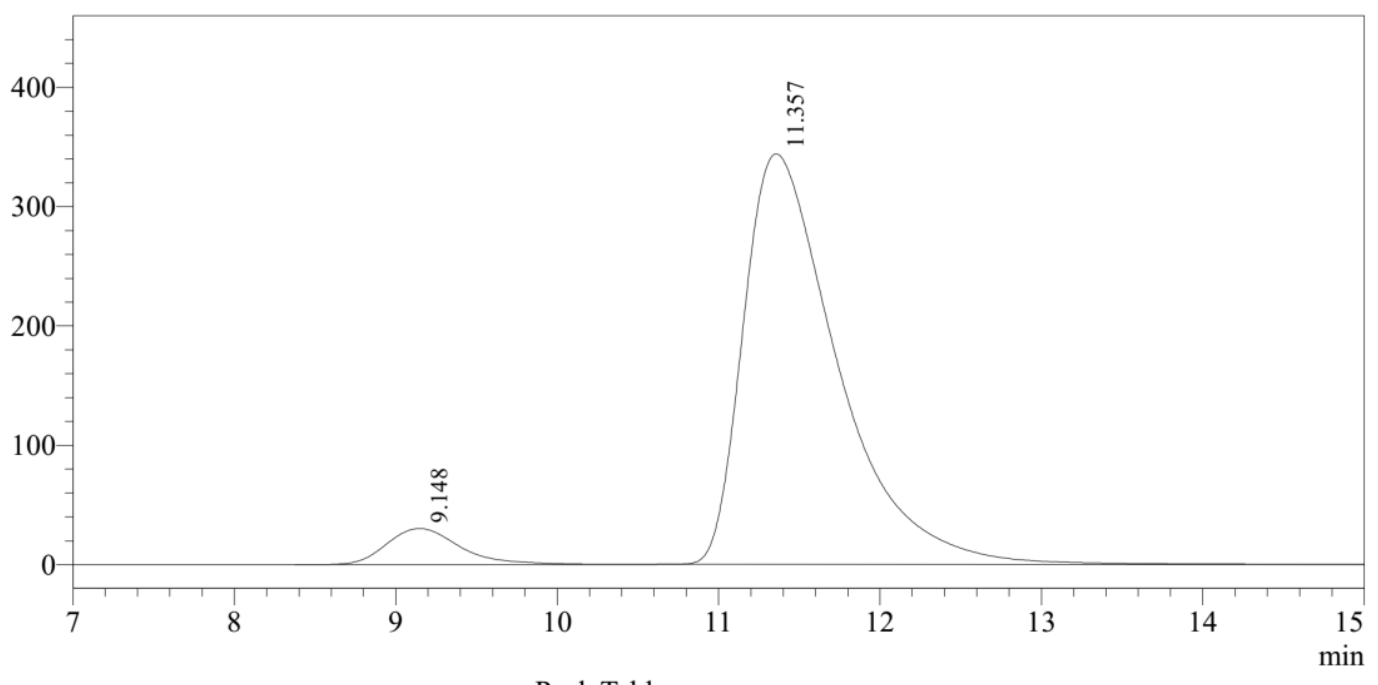

Peak Table

Detector A $254 \mathrm{~nm}$
\begin{tabular}{|r|r|r|r|r|r|}
\hline Peak\# & Ret. Time & Height & Height $\%$ & Area & Area $\%$ \\
\hline 1 & 9.148 & 30245 & 8.079 & 966322 & 6.432 \\
\hline 2 & 11.357 & 344112 & 91.921 & 14057398 & 93.568 \\
\hline Total & & 374357 & 100.000 & 15023720 & 100.000 \\
\hline
\end{tabular}

\section{4-benzyl 3,9-di-tert-butyl $(S)$-6-bromo-2-( $(S)$-1-ethoxy-1-oxopropan-2-yl)pyrano} [2,3-b]indole-3,4,9(4H)-tricarboxylate $(31)$<smiles>CCOC(=O)C1=C(C(=O)OCC)C(C(=O)OCc2ccccc2)c2c(n(C(=O)OC(C)(C)C)c3ccc(Br)cc23)O1</smiles>

A white solid $(123 \mathrm{mg}, 90 \%) ;[\alpha]^{25}=-23.9\left(c\right.$ 1.0, $\left.\mathrm{CHCl}_{3}\right) ;{ }^{1} \mathrm{H}$ NMR (400 MHz, $\left.\mathrm{CDCl}_{3}\right) \delta 8.01(\mathrm{~d}, J=8.8 \mathrm{~Hz}, 1 \mathrm{H}), 7.73(\mathrm{~d}, J=1.9 \mathrm{~Hz}, 1 \mathrm{H}), 7.39-7.31(\mathrm{~m}, 6 \mathrm{H}), 5.20$ $-5.02(\mathrm{~m}, 3 \mathrm{H}), 4.86(\mathrm{~s}, 1 \mathrm{H}), 4.24-4.07(\mathrm{~m}, 2 \mathrm{H}), 1.63(\mathrm{~s}, 9 \mathrm{H}), 1.57(\mathrm{~d}, J=7.2 \mathrm{~Hz}$, $3 \mathrm{H}), 1.45(\mathrm{~s}, 9 \mathrm{H}), 1.20(\mathrm{t}, J=7.1 \mathrm{~Hz}, 3 \mathrm{H}) ;{ }^{13} \mathrm{C} \mathrm{NMR}\left(100 \mathrm{MHz}, \mathrm{CDCl}_{3}\right) \delta 171.2$, $164.8,161.0,148.3,143.0,135.0,130.6,128.7,128.7,128.5,127.0,126.4,121.5$, $116.7,116.5,105.0,90.2,85.1,82.2,67.6,61.0,41.1,39.9,28.1,28.0,14.2,14.2$. HRMS (ESI) $m / z$ calcd for $\mathrm{C}_{34} \mathrm{H}_{38} \mathrm{BrNO}_{9}[\mathrm{M}+\mathrm{Na}]^{+}=706.1628$, found $=706.1605$; The ee value was $92 \%, \mathrm{t}_{\mathrm{R}}($ minor $)=9.7 \mathrm{~min}, \mathrm{t}_{\mathrm{R}}($ major $)=11.8 \min ($ Chiralcel $\mathrm{IE}, \lambda=254$ $\mathrm{nm}, 2 \% i-\mathrm{PrOH} / \mathrm{hexanes}$, flow rate $=1.0 \mathrm{~mL} / \mathrm{min})$. 
H:IWJH-R645 \WJH-R645-RAC-98-2-1.0-IE-30-3.3.lcd $\mathrm{mV}$

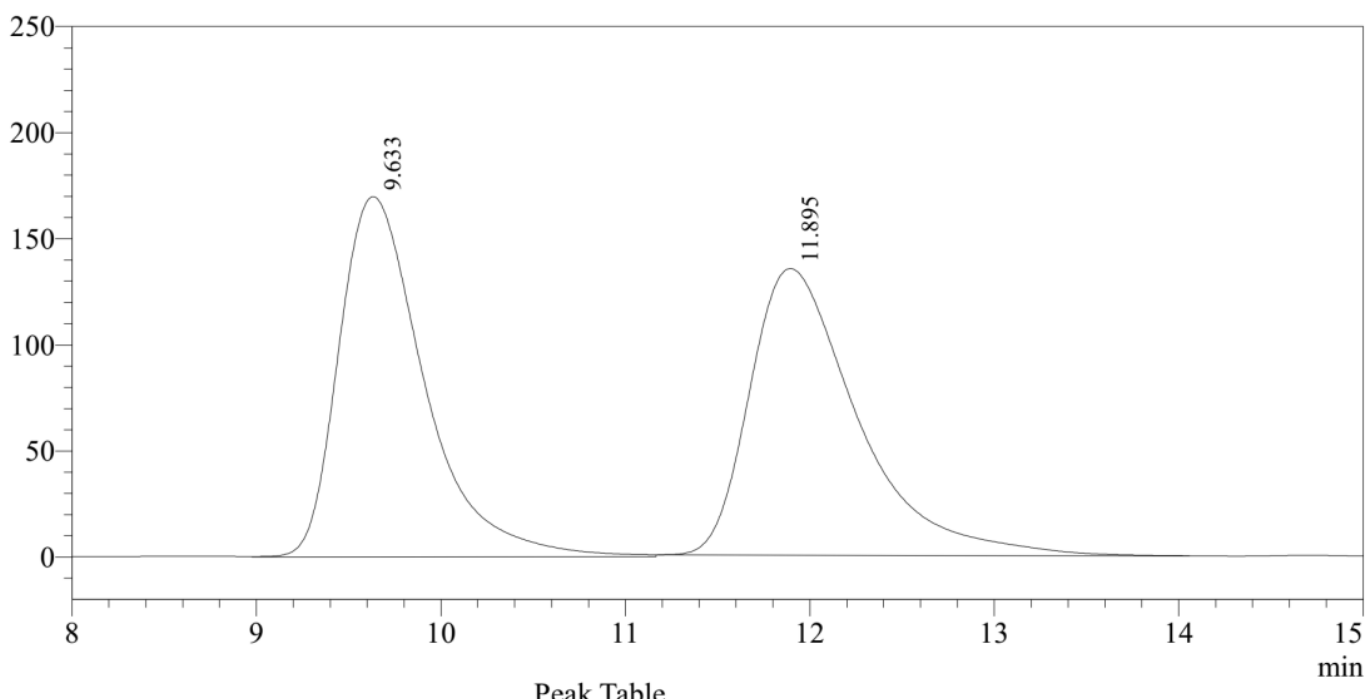

\begin{tabular}{|c|c|c|c|c|c|}
\hline etector & $\mathrm{nm}$ & & & & \\
\hline Peak\# & Ret. Time & Height & Height $\%$ & Area & Area\% \\
\hline 1 & 9.633 & 169787 & 55.707 & 5480744 & 49.947 \\
\hline 2 & 11.895 & 134997 & 44.293 & 5492267 & 50.053 \\
\hline Tota & & 304784 & 100.000 & 10973011 & 100.000 \\
\hline
\end{tabular}

H:IWJH-Ř645 \WJH-R645-CHRI-98-2-1.0-IE-30-3.3.lcd $\mathrm{mV}$

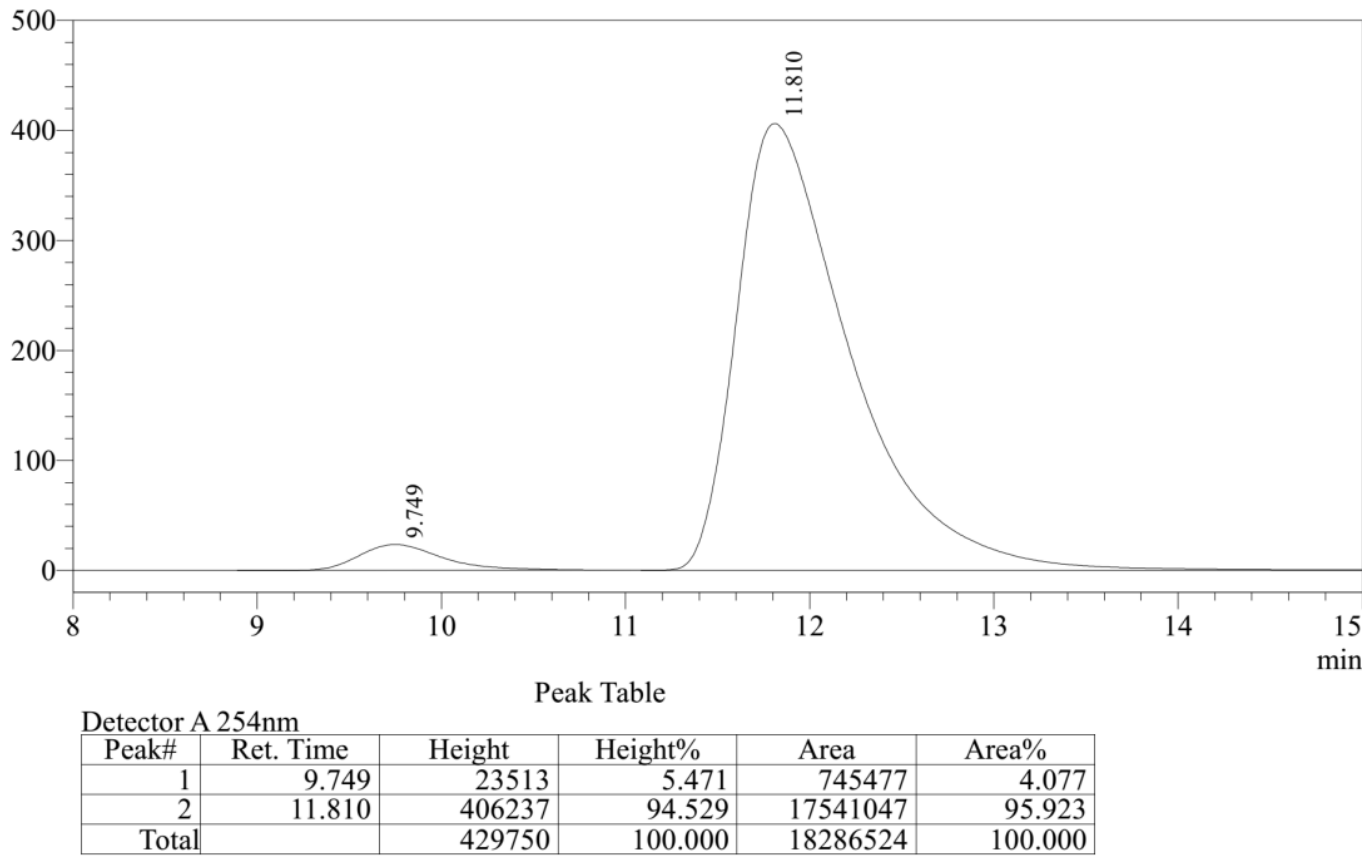

4-benzyl 3,9-di-tert-butyl $(S)$-2-( $(S)$-1-ethoxy-1-oxopropan-2-yl)-6-nitropyrano [2,3-b]indole-3,4,9(4H)-tricarboxylate $(3 \mathrm{~m})$ 


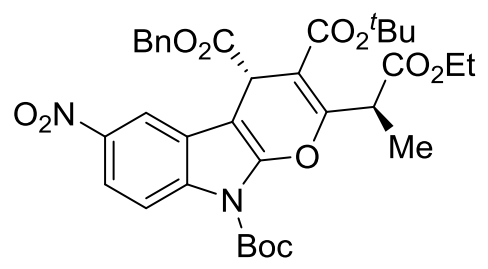

A white solid (91mg, 70\%); $[\alpha]^{25}=-18.4\left(c 0.5, \mathrm{CHCl}_{3}\right) ;{ }^{1} \mathrm{H}$ NMR $\left(400 \mathrm{MHz}, \mathrm{CDCl}_{3}\right)$ $\delta 8.48(\mathrm{~d}, J=2.3 \mathrm{~Hz}, 1 \mathrm{H}), 8.27(\mathrm{~d}, J=9.1 \mathrm{~Hz}, 1 \mathrm{H}), 8.14(\mathrm{dd}, J=9.1,2.3 \mathrm{~Hz}, 1 \mathrm{H})$, $7.43-7.29(\mathrm{~m}, 5 \mathrm{H}), 5.32-5.05(\mathrm{~m}, 3 \mathrm{H}), 4.97(\mathrm{~s}, 1 \mathrm{H}), 4.28-4.11(\mathrm{~m}, 2 \mathrm{H}), 1.68(\mathrm{~s}$, 9H), $1.62(\mathrm{~d}, J=2.2 \mathrm{~Hz}, 3 \mathrm{H}), 1.48(\mathrm{~s}, 9 \mathrm{H}), 1.23(\mathrm{t}, J=7.0 \mathrm{~Hz}, 3 \mathrm{H}) ;{ }^{13} \mathrm{C}$ NMR $(100$ $\left.\mathrm{MHz}, \mathrm{CDCl}_{3}\right) \delta 171.1,171.0,164.5,160.9,147.9,144.4,144.0,135.2,134.8,128.9$, $128.6,128.6,128.5,125.4,118.9,115.2,114.9,105.3,91.3,86.1,82.5,67.7,61.1$, 41.0, 39.7, 29.7, $28.0(\mathrm{~d}, J=13.9 \mathrm{~Hz}), 14.2,14.2$. HRMS (ESI) $\mathrm{m} / z$ calcd for $\mathrm{C}_{34} \mathrm{H}_{38} \mathrm{~N}_{2} \mathrm{O}_{11}[\mathrm{M}+\mathrm{Na}]^{+}=673.2373$, found $=673.2362$; The ee value was $85 \%, \mathrm{t}_{\mathrm{R}}$ (minor) $=30.6 \mathrm{~min}, \mathrm{t}_{\mathrm{R}}($ major $)=34.5 \mathrm{~min}($ Chiralcel IE, $\lambda=254 \mathrm{~nm}, 2 \% i-\mathrm{PrOH} / \mathrm{hexanes}$, flow rate $=1.0 \mathrm{~mL} / \mathrm{min})$.

H:IWJH-Ř647\WJH-R647-RAC-98-2-1.0-IE-30-3.3-106.lcd $\mathrm{mV}$

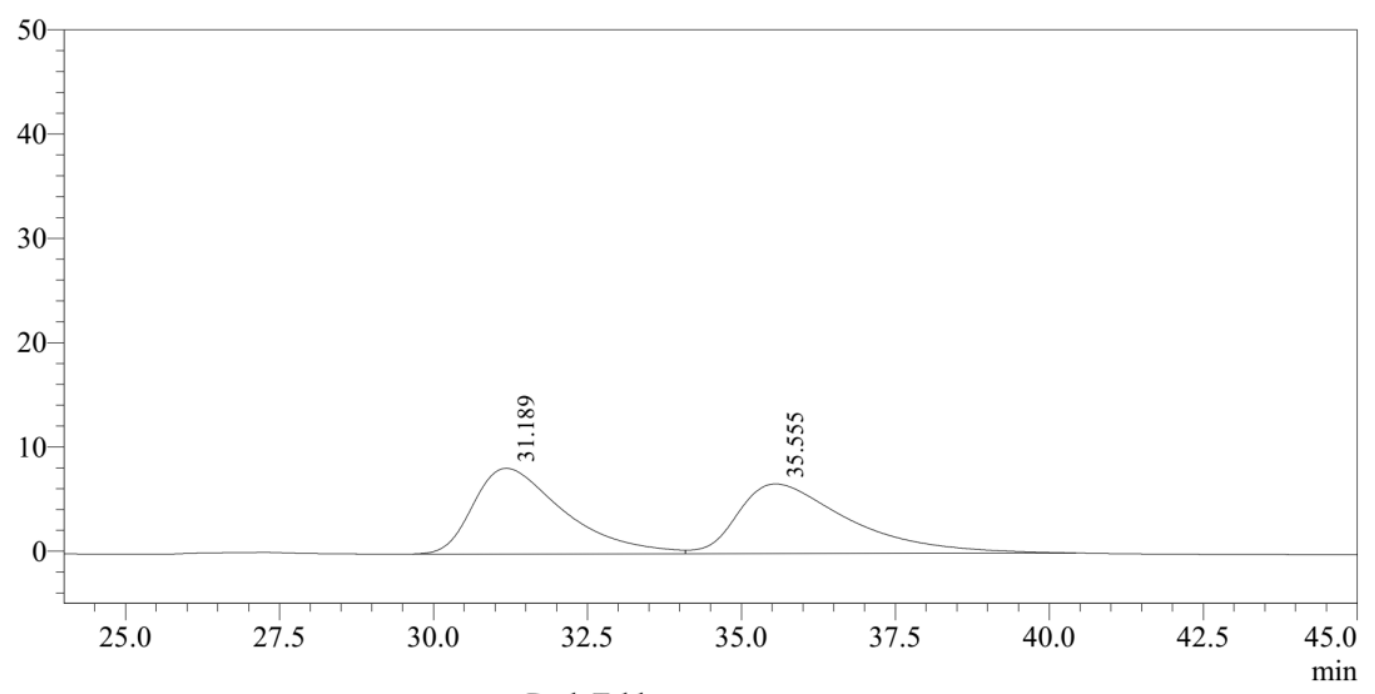

Peak Table

Detector A $254 \mathrm{~nm}$
\begin{tabular}{|r|r|r|r|r|r|}
\hline Peak\# & Ret. Time & Height & Height $\%$ & \multicolumn{1}{|c|}{ Area } & \multicolumn{1}{|c|}{ Area $\%$} \\
\hline 1 & 31.189 & 8201 & 55.167 & 836947 & 50.060 \\
\hline 2 & 35.555 & 6664 & 44.833 & 834933 & 49.940 \\
\hline Total & & 14865 & 100.000 & 1671879 & 100.000 \\
\hline
\end{tabular}


H:IWJH-Ř647\WJH-R647-CHRI-98-2-1.0-IE-30-3.4-106.lcd $\mathrm{mV}$

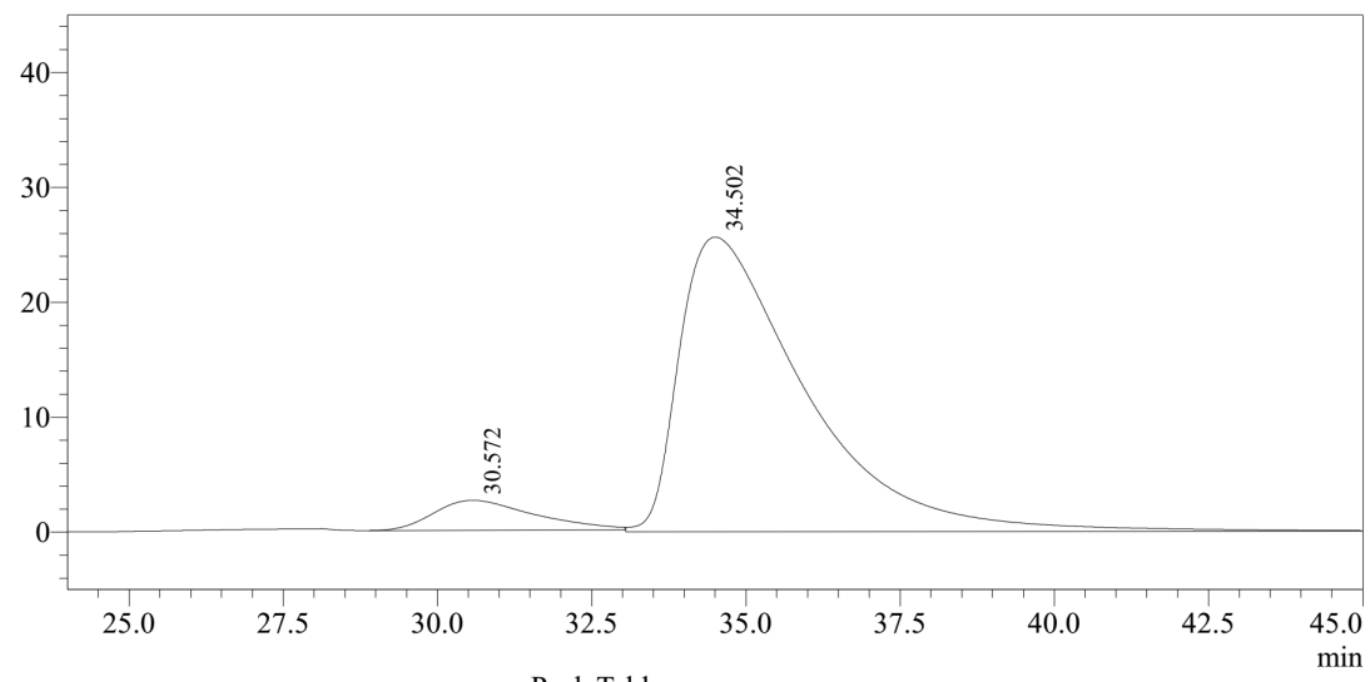

\begin{tabular}{|c|c|c|c|c|c|}
\hline \multicolumn{6}{|c|}{ Detector A $254 \mathrm{~nm}$} \\
\hline Peak\# & Ret. Time & Height & Height $\%$ & Area & Area\% \\
\hline 1 & 30.572 & 2608 & 9.232 & 296202 & 7.466 \\
\hline 2 & 34.502 & 25645 & 90.768 & 3670943 & 92.534 \\
\hline Tota & & 28253 & 100.000 & 3967145 & 100.000 \\
\hline
\end{tabular}

\section{4-benzyl 3,9-di-tert-butyl $(S)$-2-((S)-1-ethoxy-1-oxopropan-2-yl)-6-methylpyrano} [2,3-b]indole-3,4,9(4H)-tricarboxylate $(3 \mathrm{n})$

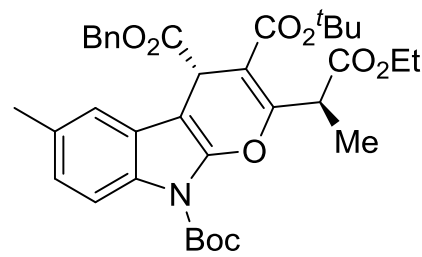

A white solid (110mg, 89\%); $[\alpha]^{25}{ }_{\mathrm{D}}=-31.4\left(c 0.80, \mathrm{CHCl}_{3}\right) ;{ }^{1} \mathrm{H}$ NMR (400 MHz, $\left.\mathrm{CDCl}_{3}\right) \delta 7.99(\mathrm{~d}, J=8.5 \mathrm{~Hz}, 1 \mathrm{H}), 7.36-7.31(\mathrm{~m}, 6 \mathrm{H}), 7.05(\mathrm{dd}, J=8.5,1.3 \mathrm{~Hz}, 1 \mathrm{H})$, $5.21-4.98(\mathrm{~m}, 3 \mathrm{H}), 4.89(\mathrm{~s}, 1 \mathrm{H}), 4.26-4.06(\mathrm{~m}, 2 \mathrm{H}), 2.32(\mathrm{~s}, 3 \mathrm{H}), 1.63(\mathrm{~s}, 9 \mathrm{H}), 1.58$ $(\mathrm{d}, J=7.2 \mathrm{~Hz}, 3 \mathrm{H}), 1.46(\mathrm{~s}, 9 \mathrm{H}), 1.19(\mathrm{t}, J=7.1 \mathrm{~Hz}, 3 \mathrm{H}) ;{ }^{13} \mathrm{C}$ NMR $(100 \mathrm{MHz}$, $\left.\mathrm{CDCl}_{3}\right) \delta 171.5,171.3,165.1,161.2,148.6,142.4,135.3,132.8,130.0,128.5,128.5$, $125.4,124.8,118.9,114.6,104.9,90.6,84.4,82.0,67.3,61.0,53.4,41.2,40.2,28.2$, 28.0, 21.3, 14.2. HRMS (ESI) $\mathrm{m} / z$ calcd for $\mathrm{C}_{35} \mathrm{H}_{41} \mathrm{NO}_{9}[\mathrm{M}+\mathrm{Na}]^{+}=642.2679$, found $=$ 642.2674; The ee value was $91 \%, \mathrm{t}_{\mathrm{R}}($ minor $)=11.6 \mathrm{~min}, \mathrm{t}_{\mathrm{R}}($ major $)=15.8 \mathrm{~min}$ (Chiralcel IE, $\lambda=254 \mathrm{~nm}, 2 \% i$-PrOH$/$ hexanes, flow rate $=1.0 \mathrm{~mL} / \mathrm{min}$ ). 


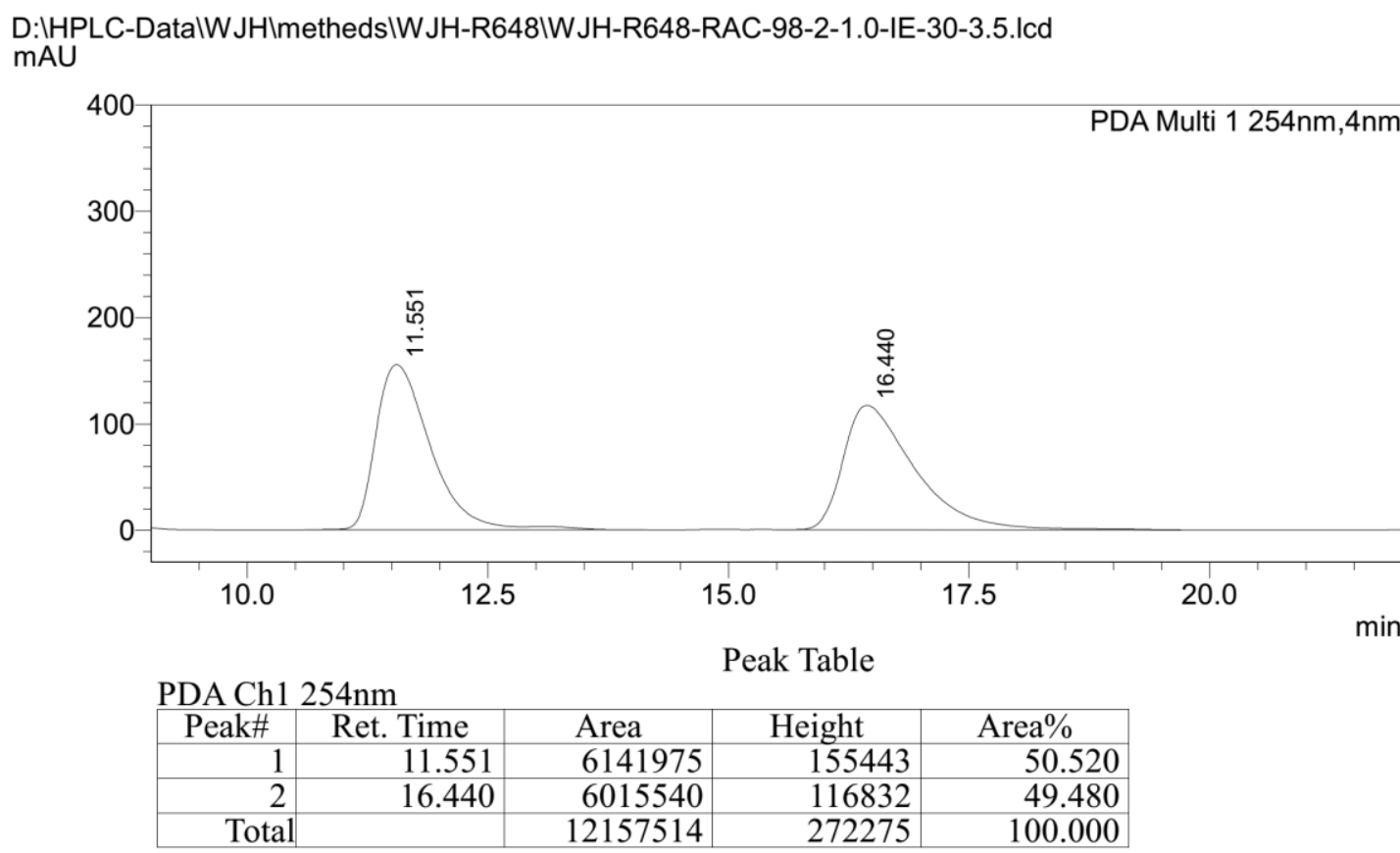

H:IWJH-Ř648|WJH-R648-CHRI-98-2-1.0-IE-30-3.3.lcd $\mathrm{mV}$

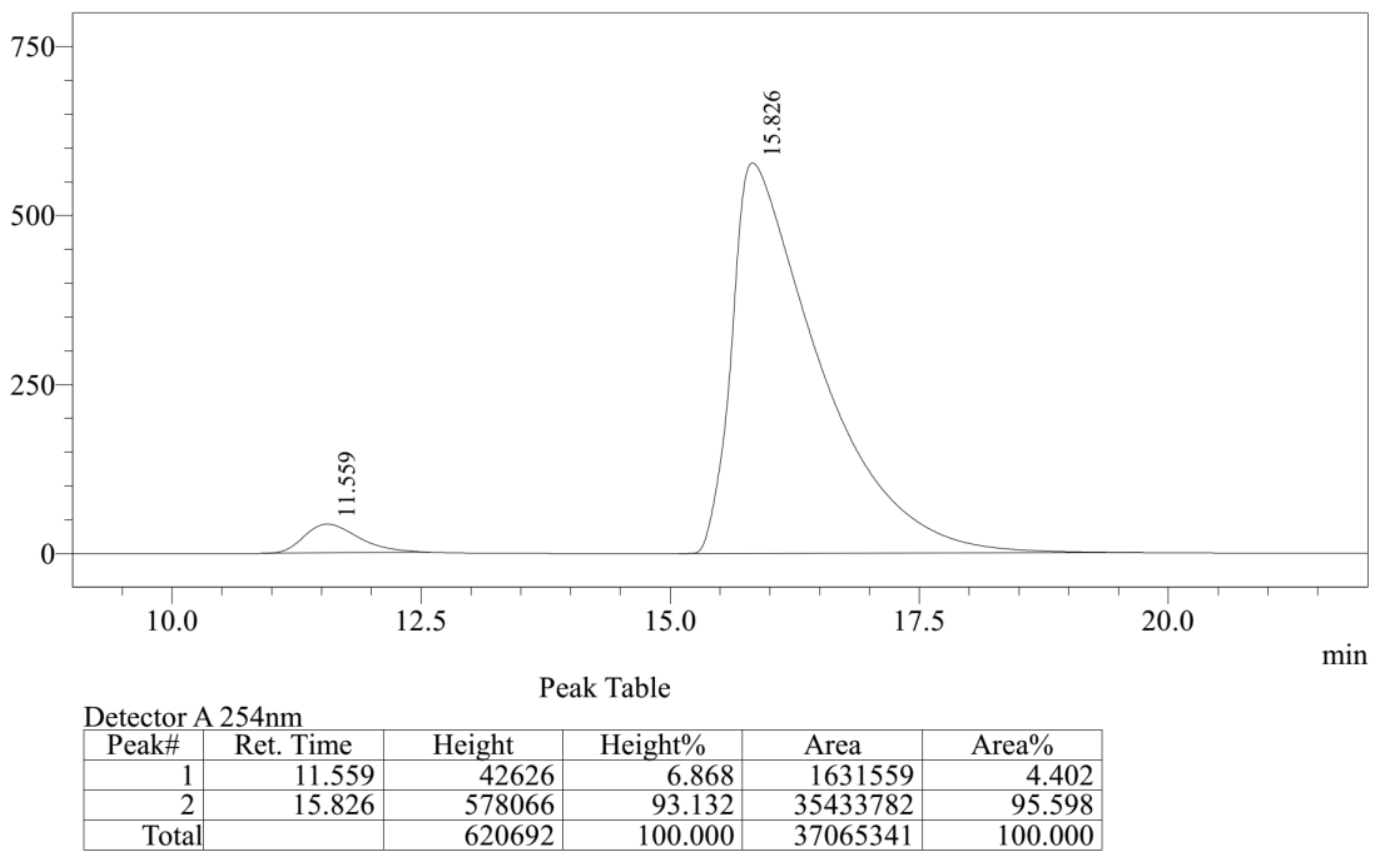

4-benzyl 3,9-di-tert-butyl $(S)$-2-((S)-1-ethoxy-1-oxopropan-2-yl)-6-methoxypy rano[2,3-b]indole-3,4,9(4H)-tricarboxylate $(30)$ 


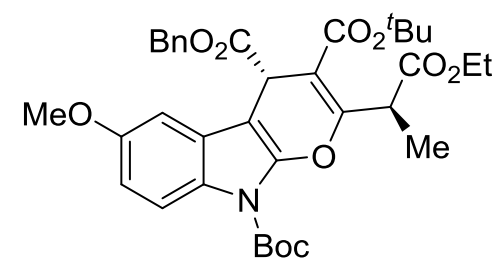

A white solid (122mg, 96\%); $[\alpha]^{25}{ }_{\mathrm{D}}=-9.3\left(c\right.$ 1.5, $\left.\mathrm{CHCl}_{3}\right) ;{ }^{1} \mathrm{H} \mathrm{NMR}\left(400 \mathrm{MHz}, \mathrm{CDCl}_{3}\right)$ $\delta 8.01(\mathrm{~d}, J=9.0 \mathrm{~Hz}, 1 \mathrm{H}), 7.37-7.28(\mathrm{~m}, 5 \mathrm{H}), 7.03(\mathrm{~d}, J=2.6 \mathrm{~Hz}, 1 \mathrm{H}), 6.82(\mathrm{dd}, J=$ 9.0, $2.6 \mathrm{~Hz}, 1 \mathrm{H}), 5.26-5.01(\mathrm{~m}, 3 \mathrm{H}), 4.89(\mathrm{~s}, 1 \mathrm{H}), 4.27-4.05(\mathrm{~m}, 2 \mathrm{H}), 3.61(\mathrm{~s}, 3 \mathrm{H})$, $1.63(\mathrm{~s}, 9 \mathrm{H}), 1.58(\mathrm{~d}, J=7.2 \mathrm{~Hz}, 3 \mathrm{H}), 1.46(\mathrm{~s}, 9 \mathrm{H}), 1.20(\mathrm{t}, J=7.1 \mathrm{~Hz}, 3 \mathrm{H}),{ }^{13} \mathrm{C} \mathrm{NMR}$ $\left(100 \mathrm{MHz}, \mathrm{CDCl}_{3}\right) \delta 171.4,171.3,165.1,161.2,156.2,148.5,142.7,135.4,128.5$, 128.3, 126.2, 126.1, 115.8, 112.0, 104.8, 101.8, 100.0, 90.9, 84.4, 82.0, 67.2, 61.0, 55.4, 41.2, 40.1, 28.2, 28.0, 14.2. HRMS (ESI) $m / z$ calcd for $\mathrm{C}_{35} \mathrm{H}_{41} \mathrm{NO}_{10}[\mathrm{M}+\mathrm{Na}]^{+}=$ 658.2628 , found $=658.2612$; The ee value was $93 \%, t_{R}($ minor $)=18.2 \mathrm{~min}, \mathrm{t}_{\mathrm{R}}$ (major) $=24.5 \mathrm{~min}$ (Chiralcel IE, $\lambda=254 \mathrm{~nm}, 2 \% i$-PrOH$/$ hexanes, flow rate $=1.0 \mathrm{~mL} / \mathrm{min})$.

H:\WJH-Ř649 \WJH-R649-RAC2-98-2-1.0-IE-30-3.31cd.lcd $\mathrm{mV}$

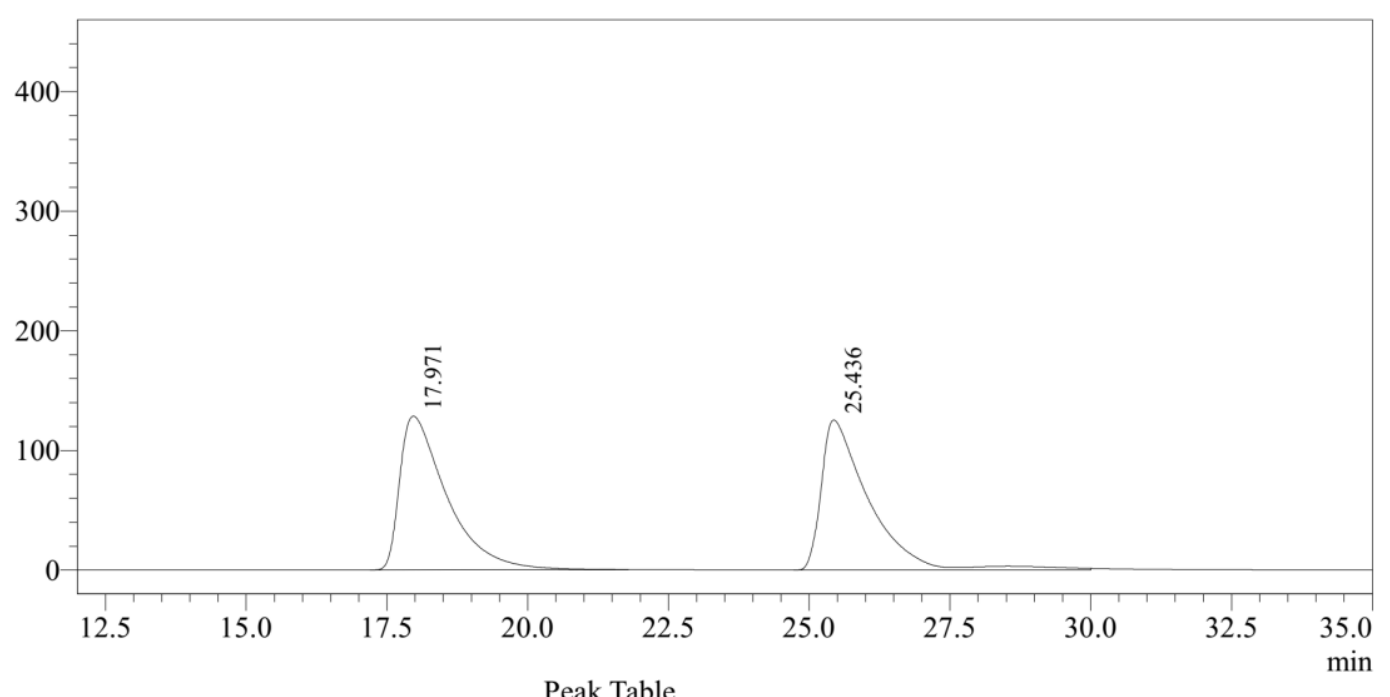

Detector A 254nm
\begin{tabular}{|r|r|r|r|r|r|}
\hline Peak\# & Ret. Time & \multicolumn{1}{|c|}{ Height } & \multicolumn{1}{|c|}{ Height $\%$} & \multicolumn{1}{c|}{ Area } & Area $\%$ \\
\hline 1 & 17.971 & 128537 & 50.618 & 7472464 & 50.154 \\
\hline 2 & 25.436 & 125396 & 49.382 & 7426520 & 49.846 \\
\hline Total & & 253933 & 100.000 & 14898983 & 100.000 \\
\hline
\end{tabular}


H:IWJH-Ř649 |WJH-R649-CHRI-98-2-1.0-IE-30-3.3.lcd

$\mathrm{mV}$

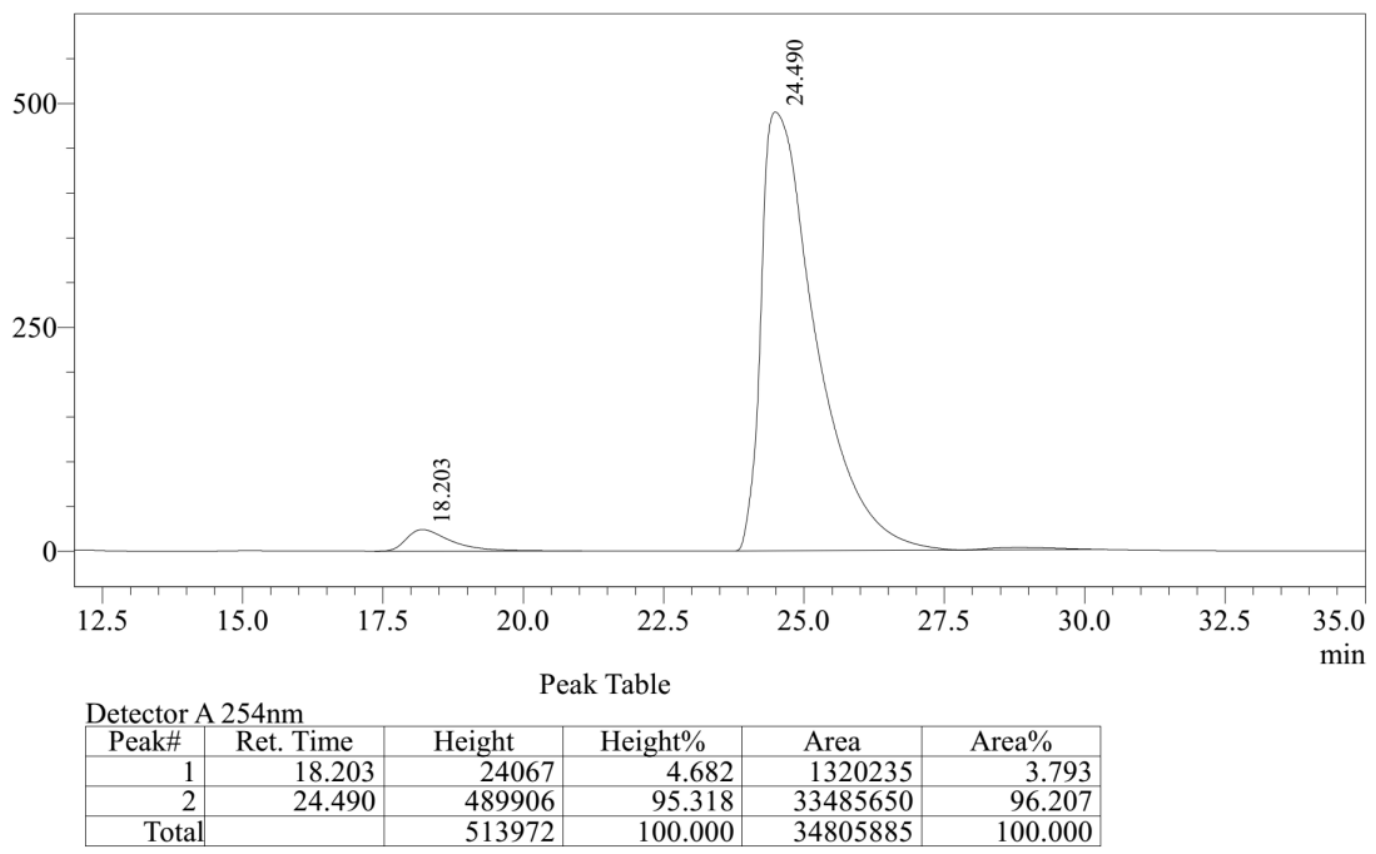

\section{4-benzyl 3,9-di-tert-butyl (S)-7-chloro-2-((S)-1-ethoxy-1-oxopropan-2-yl)pyrano} [2,3-b]indole-3,4,9(4H)-tricarboxylate $(3 p)$<smiles>CCOC(=O)C1=C(C(=O)OCC)C(C(=O)OCc2ccccc2)c2c(n(C(=O)OC(C)(C)C)c3cc(Cl)ccc23)O1</smiles>

A white solid (116mg, 91\%); $[\alpha]_{\mathrm{D}}^{25}=-41.4\left(c\right.$ 0.80, $\left.\mathrm{CHCl}_{3}\right) ;{ }^{1} \mathrm{H}$ NMR $(400 \mathrm{MHz}$, $\left.\mathrm{CDCl}_{3}\right) \delta 8.19(\mathrm{~d}, J=1.9 \mathrm{~Hz}, 1 \mathrm{H}), 7.45(\mathrm{~d}, J=8.4 \mathrm{~Hz}, 1 \mathrm{H}), 7.35-7.27(\mathrm{~m}, 5 \mathrm{H}), 7.12$ $(\mathrm{dd}, J=8.4,1.9 \mathrm{~Hz}, 1 \mathrm{H}), 5.21-4.99(\mathrm{~m}, 3 \mathrm{H}), 4.89(\mathrm{~s}, 1 \mathrm{H}), 4.27-4.06(\mathrm{~m}, 2 \mathrm{H}), 1.64$ $(\mathrm{s}, 9 \mathrm{H}), 1.57(\mathrm{~d}, J=7.2 \mathrm{~Hz}, 3 \mathrm{H}), 1.44(\mathrm{~s}, 9 \mathrm{H}), 1.20(\mathrm{t}, J=7.1 \mathrm{~Hz}, 3 \mathrm{H}) ;{ }^{13} \mathrm{C}$ NMR $(100$ $\left.\mathrm{MHz}, \mathrm{CDCl}_{3}\right) \delta 171.2,171.1,164.9,161.0,148.3,142.7,135.2,132.2,129.3,128.5$, $128.5,128.4,123.7,119.6,115.3,105.0,90.7,85.2,82.2,67.3,61.0,41.1,40.0,28.1$, 28.0, 14.2, 14.2. HRMS (ESI) $\mathrm{m} / z$ calcd for $\mathrm{C}_{34} \mathrm{H}_{38} \mathrm{ClNO}_{9}[\mathrm{M}+\mathrm{Na}]^{+}=662.2133$, found $=662.2101 ;$ The ee value was $90 \%, t_{R}($ major $)=7.9 \min , t_{R}($ minor $)=8.9 \min$ (Chiralcel ID, $\lambda=254 \mathrm{~nm}, 5 \% i$-PrOH/hexanes, flow rate $=0.7 \mathrm{~mL} / \mathrm{min}$ ). 
H: \WJH-Ř650 \WJH-R650-RAC-95-5-0.7-ID-30-2.0-106.lcd $\mathrm{mV}$

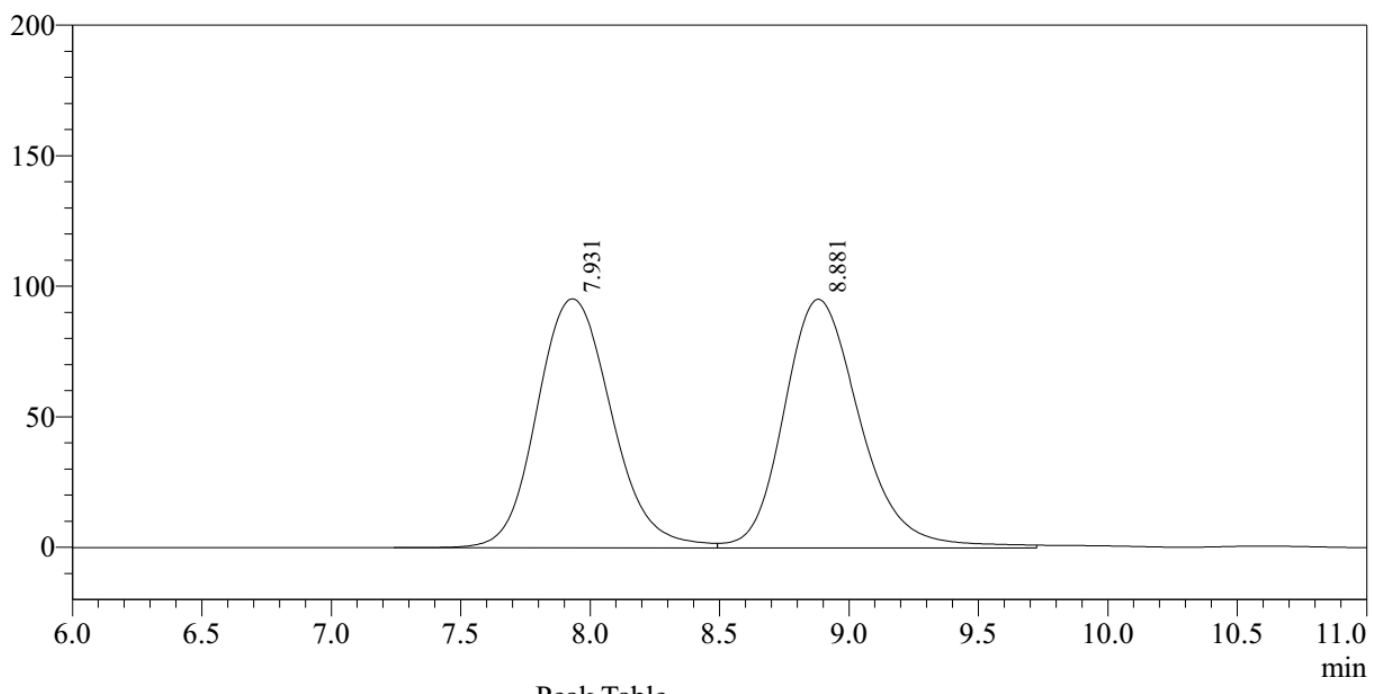

Peak Table

Detector A $254 \mathrm{~nm}$
\begin{tabular}{|r|r|r|r|r|r|}
\hline Peak\# & Ret. Time & Height & Height $\%$ & Area & \multicolumn{1}{c|}{ Area $\%$} \\
\hline 1 & 7.931 & 95369 & 50.044 & 1916727 & 49.593 \\
\hline 2 & 8.881 & 95201 & 49.956 & 1948166 & 50.407 \\
\hline Total & & 190570 & 100.000 & 3864893 & 100.000 \\
\hline
\end{tabular}

H:\WJH-R650 \WJH-R650-CHRI-95-5-0.7-ID-30-2.0-106.lcd $\mathrm{mV}$

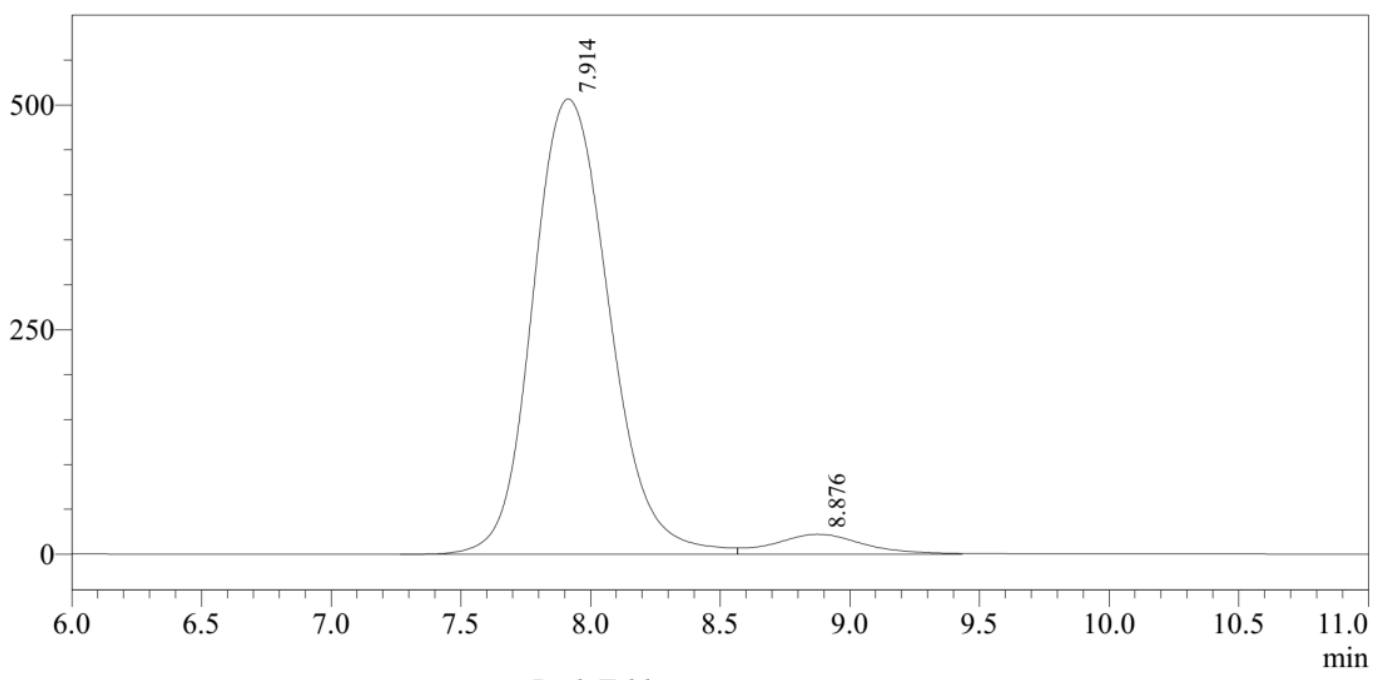

Peak Table

Detector A $254 \mathrm{~nm}$
\begin{tabular}{|r|r|r|r|r|r|}
\hline Peak\# & Ret. Time & \multicolumn{1}{|c|}{ Height } & Height $\%$ & \multicolumn{1}{c|}{ Area } & \multicolumn{1}{c|}{ Area $\%$} \\
\hline 1 & 7.914 & 506733 & 95.812 & 10437316 & 95.176 \\
\hline 2 & 8.876 & 22150 & 4.188 & 529007 & 4.824 \\
\hline Total & & 528883 & 100.000 & 10966323 & 100.000 \\
\hline
\end{tabular}

\section{4-benzyl 3,9-di-tert-butyl $(S)$-7-bromo-2-( $(S)$-1-ethoxy-1-oxopropan-2-yl)pyrano} $[2,3$-b]indole-3,4,9(4H)-tricarboxylate $(3 q)$ 


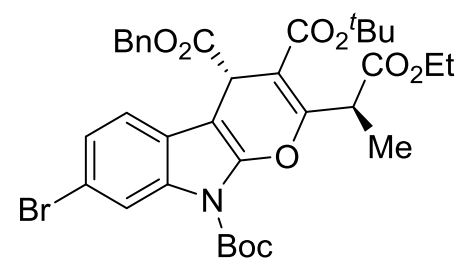

A white solid $(127 \mathrm{mg}, 93 \%) ;[\alpha]^{25}{ }_{\mathrm{D}}=-40.5\left(c 0.80, \mathrm{CHCl}_{3}\right) ;{ }^{1} \mathrm{H}$ NMR $(400 \mathrm{MHz}$, $\left.\mathrm{CDCl}_{3}\right) \delta 8.37(\mathrm{~d}, J=1.7 \mathrm{~Hz}, 1 \mathrm{H}), 7.43(\mathrm{~d}, J=8.4 \mathrm{~Hz}, 1 \mathrm{H}), 7.36-7.27(\mathrm{~m}, 5 \mathrm{H}), 7.24$ $(\mathrm{d}, J=8.4 \mathrm{~Hz}, 1 \mathrm{H}), 5.23-5.06(\mathrm{~m}, 3 \mathrm{H}), 4.91(\mathrm{~s}, 1 \mathrm{H}), 4.28-4.09(\mathrm{~m}, 2 \mathrm{H}), 1.66(\mathrm{~s}$, 9H), $1.59(\mathrm{~d}, J=7.2 \mathrm{~Hz}, 3 \mathrm{H}), 1.46(\mathrm{~s}, 9 \mathrm{H}), 1.22(\mathrm{t}, J=7.1 \mathrm{~Hz}, 3 \mathrm{H}) ;{ }^{13} \mathrm{C} \mathrm{NMR}(100$ $\left.\mathrm{MHz}, \mathrm{CDCl}_{3}\right) \delta 171.2,171.1,164.9,161.0,148.3,135.2,132.5,128.5,128.5,128.5$, 126.5, 124.1, 120.0, 118.1, 116.9, 105.0, 90.7, 85.2, 82.2, 67.3, 61.0, 41.1, 40.0, 28.1, 28.0, 14.2, 14.2. HRMS (ESI) $m / z$ calcd for $\mathrm{C}_{34} \mathrm{H}_{38} \mathrm{BrNO}_{9}[\mathrm{M}+\mathrm{Na}]^{+}=706.1628$, found $=706.1601$; The ee value was $95 \%, t_{R}($ minor $)=15.4 \mathrm{~min}, t_{R}($ major $)=17.2$ min (Chiralcel IE, $\lambda=254 \mathrm{~nm}, 2 \% i$-PrOH/hexanes, flow rate $=0.7 \mathrm{~mL} / \mathrm{min}$ )

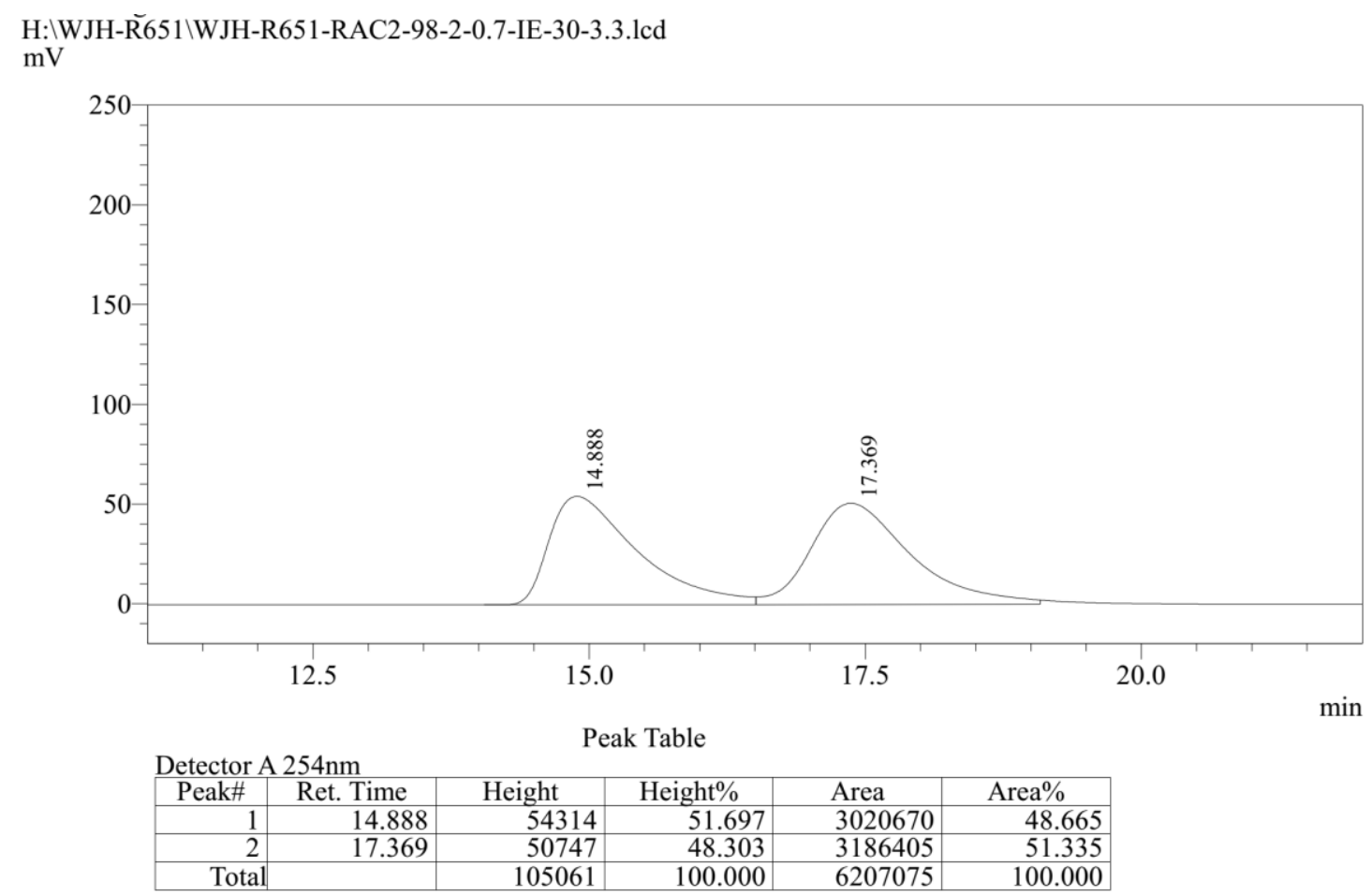


H:IWJH-Ř651 \WJH-R651-CHRI2-98-2-0.7-IE-30-2.3-106.lcd $\mathrm{mV}$

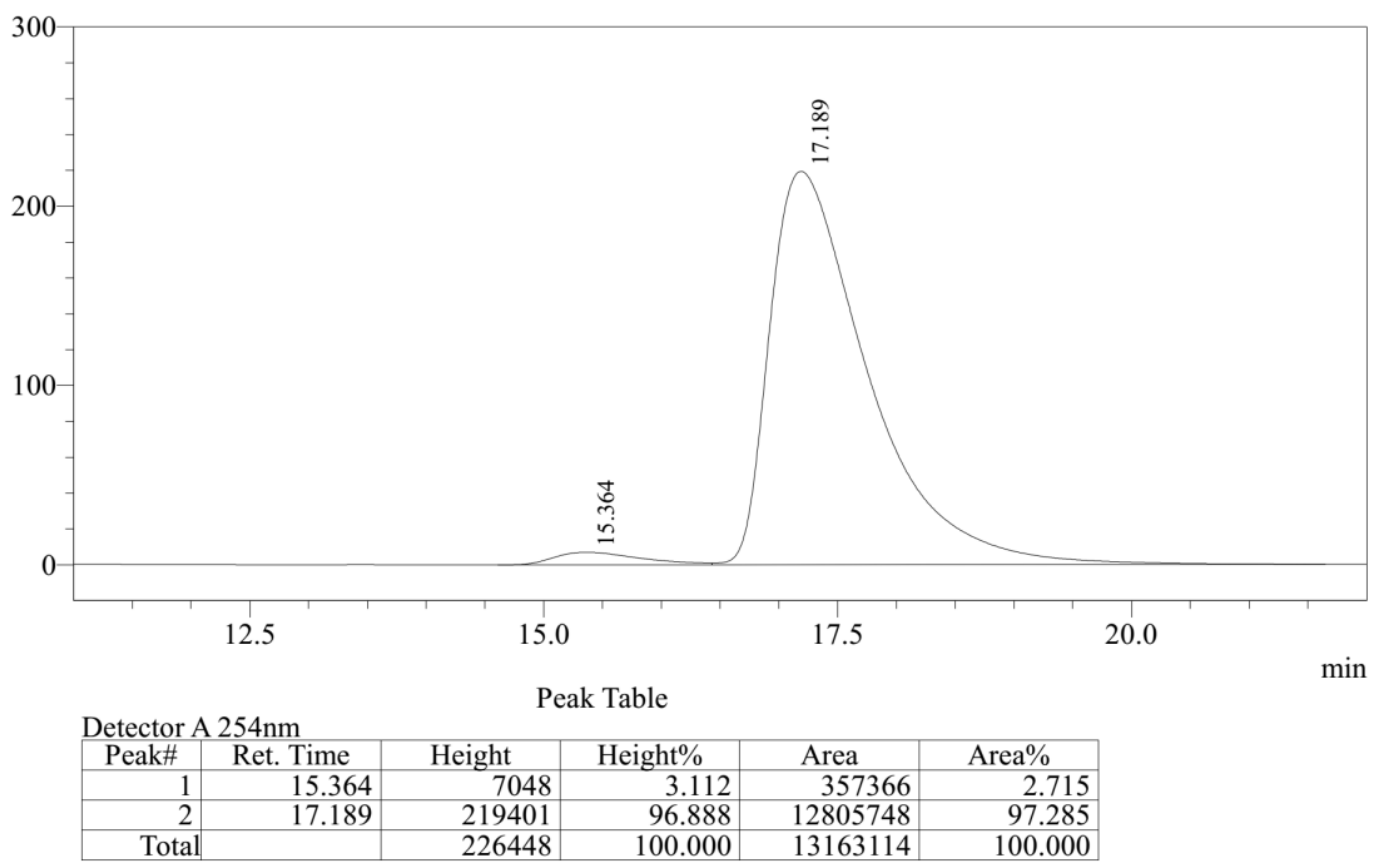

\section{4-benzyl 3,9-di-tert-butyl $\quad(S)$-2-( $(S)$-1-ethoxy-1-oxopropan-2-yl)-7-methoxypy} rano[2,3-b]indole-3,4,9(4H)-tricarboxylate $(3 r)$<smiles>CCOC(=O)C1=C(C(C)C(C)C)Oc2c(c3ccc(OC)cc3n2C(=O)OCc2ccccc2)C1C(=O)OCc1ccccc1</smiles>

A white solid (121mg, 95\%); $[\alpha]^{25}=-36.5\left(c\right.$ 1.0, $\left.\mathrm{CHCl}_{3}\right) ;{ }^{1} \mathrm{H}$ NMR $(400 \mathrm{MHz}$, $\left.\mathrm{CDCl}_{3}\right) \delta 7.77(\mathrm{~d}, J=2.3 \mathrm{~Hz}, 1 \mathrm{H}), 7.43(\mathrm{~d}, J=8.6 \mathrm{~Hz}, 1 \mathrm{H}), 7.35-7.30(\mathrm{~m}, 5 \mathrm{H}), 6.79$ $(\mathrm{dd}, J=8.6,2.4 \mathrm{~Hz}, 1 \mathrm{H}), 5.21-4.99(\mathrm{~m}, 3 \mathrm{H}), 4.89(\mathrm{~s}, 1 \mathrm{H}), 4.24-4.08$ (m, 2H), 3.86 (s, 3H), $1.64(\mathrm{~s}, 9 \mathrm{H}), 1.57(\mathrm{~d}, J=7.2 \mathrm{~Hz}, 3 \mathrm{H}), 1.43(\mathrm{~s}, 9 \mathrm{H}), 1.20(\mathrm{t}, J=7.1 \mathrm{~Hz}, 3 \mathrm{H})$; ${ }^{13} \mathrm{C}$ NMR $\left(100 \mathrm{MHz}, \mathrm{CDCl}_{3}\right) \delta 171.4,171.4,165.1,161.0,157.2,148.8,141.3,135.4$, $132.9,128.5,128.5,128.3,119.4,118.8,111.9,104.6,99.8,90.7,84.5,81.9,67.2$, 60.9, 55.7, 41.2, 40.2, 28.2, 28.0, 14.2. HRMS (ESI) $\mathrm{m} / \mathrm{z}$ calcd for $\mathrm{C}_{35} \mathrm{H}_{41} \mathrm{BrNO}_{10}$ $[\mathrm{M}+\mathrm{Na}]^{+}=658.2628$, found $=658.2626$; The ee value was $90 \%, \mathrm{t}_{\mathrm{R}}($ major $)=11.3 \mathrm{~min}$, $\mathrm{t}_{\mathrm{R}}($ minor $)=12.9 \mathrm{~min}($ Chiralcel ID, $\lambda=254 \mathrm{~nm}, 5 \% i-\mathrm{PrOH} /$ hexanes, flow rate $=0.7$ $\mathrm{mL} / \mathrm{min}$ ). 
H:IWJH-R̄652।WJH-R652-RAC-95-5-0.7-ID-30-2.0-107.lcd

$\mathrm{mV}$

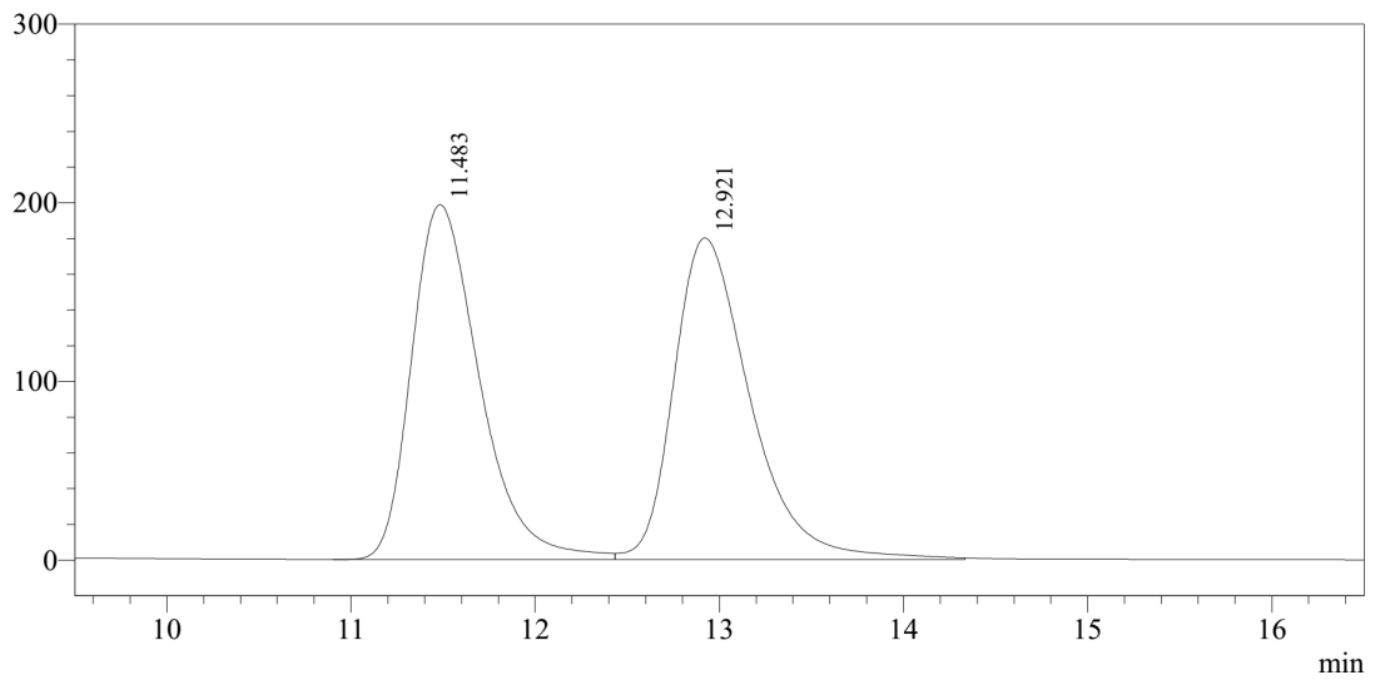

Peak Table

\begin{tabular}{|c|c|c|c|c|c|}
\hline etector $A$ & $254 \mathrm{~nm}$ & & & & \\
\hline Peak\# & Ret. Time & Height & Height $\%$ & Area & Area $\%$ \\
\hline 1 & 11.483 & 198746 & 52.474 & 5108171 & 49.566 \\
\hline 2 & 12.921 & 180008 & 47.526 & 5197614 & 50.434 \\
\hline Total & & 378755 & 100.000 & 10305784 & 100.000 \\
\hline
\end{tabular}

H:IWJH-Ř652 \WJH-R652-CHRI-95-5-0.7-ID-30-2.0-107.lcd $\mathrm{mV}$

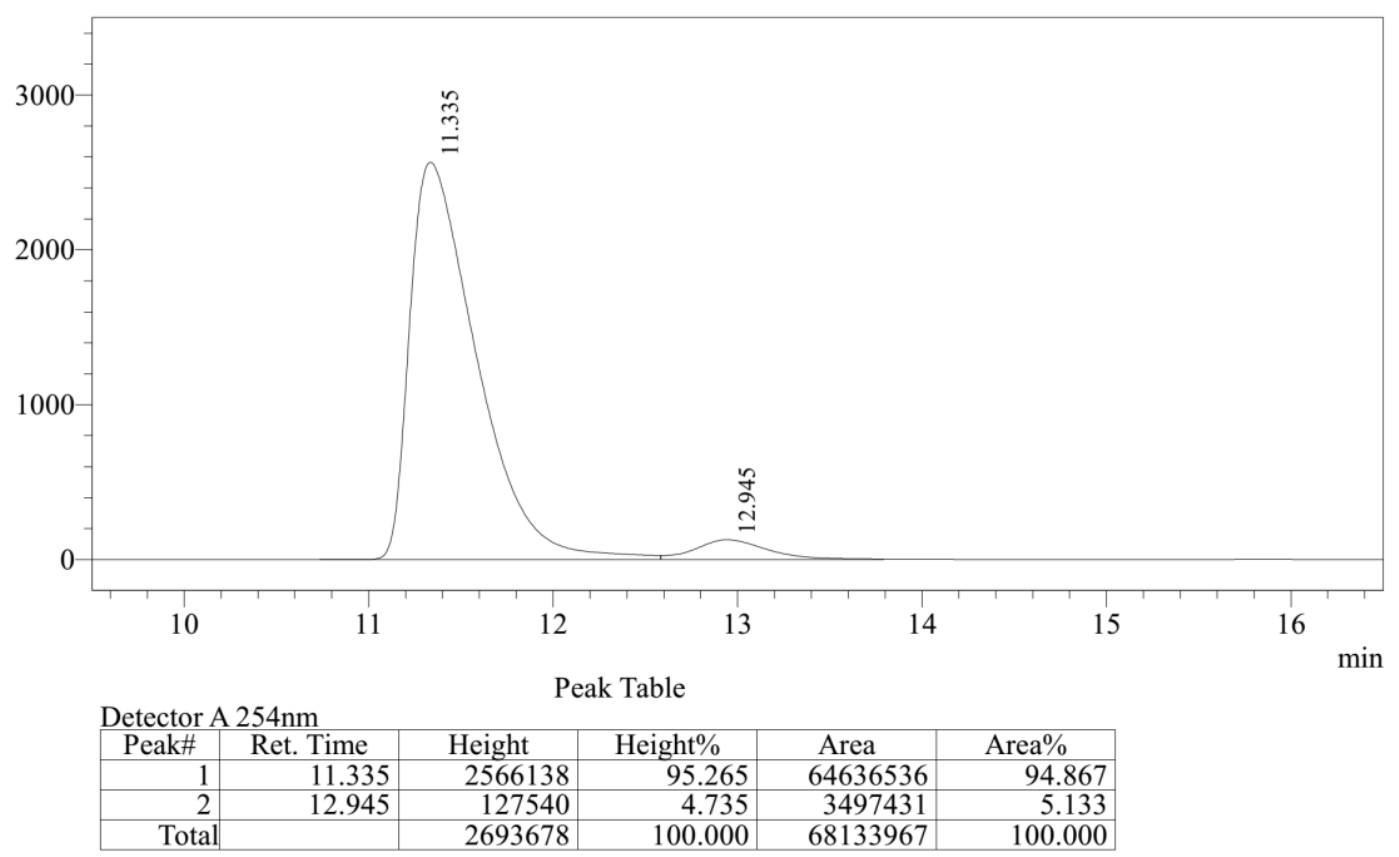

4-benzyl 3,9-di-tert-butyl $(S)$-2-((S)-1-ethoxy-1-oxopropan-2-yl)-8-fluoropyrano [2,3-b]indole-3,4,9(4H)-tricarboxylate $(3 \mathrm{~s})$ 


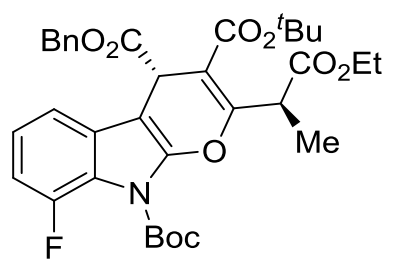

A white solid (90mg, 72\%); $[\alpha]^{25}{ }_{\mathrm{D}}=-21.8\left(c\right.$ 0.50, $\left.\mathrm{CHCl}_{3}\right) ;{ }^{1} \mathrm{H}$ NMR $(400 \mathrm{MHz}$, $\left.\mathrm{CDCl}_{3}\right) \delta 7.36-7.27(\mathrm{~m}, 6 \mathrm{H}), 7.09(\mathrm{td}, J=8.0,4.3 \mathrm{~Hz}, 1 \mathrm{H}), 6.95(\mathrm{ddd}, J=12.3,8.1$, $0.9 \mathrm{~Hz}, 1 \mathrm{H}), 5.21-5.02(\mathrm{~m}, 3 \mathrm{H}), 4.89(\mathrm{~s}, 1 \mathrm{H}), 4.21-4.10(\mathrm{~m}, 2 \mathrm{H}), 1.62(\mathrm{~s}, 9 \mathrm{H}), 1.57$ $(\mathrm{d}, J=7.4 \mathrm{~Hz}, 3 \mathrm{H}), 1.43(\mathrm{~s}, 9 \mathrm{H}), 1.20(\mathrm{t}, J=7.1 \mathrm{~Hz}, 3 \mathrm{H}) ;{ }^{13} \mathrm{C} \mathrm{NMR}(100 \mathrm{MHz}$, $\left.\mathrm{CDCl}_{3}\right) \delta 171.2,171.1,164.9,160.8,149.7(\mathrm{~d}, J=253.5 \mathrm{~Hz}), 147.0,143.3,135.3$, $128.9(\mathrm{~d}, J=3.5 \mathrm{~Hz}), 128.5,128.5,128.3(\mathrm{~d}, J=6.0 \mathrm{~Hz}), 124.0(\mathrm{~d}, J=7.4 \mathrm{~Hz}), 118.4$ $(\mathrm{d}, J=9.6 \mathrm{~Hz}), 114.8(\mathrm{~d}, J=3.7 \mathrm{~Hz}), 110.8(\mathrm{~d}, J=21.0 \mathrm{~Hz}), 105.23,90.3$ (d, $J=2.1$ $\mathrm{Hz}), 85.1,82.2,67.2,61.1,41.3,40.3,28.0,27.8,14.2 .{ }^{19} \mathrm{~F} \mathrm{NMR}\left(376 \mathrm{MHz}, \mathrm{CDCl}_{3}\right) \delta$ -118.4. HRMS (ESI) $\mathrm{m} / z$ calcd for $\mathrm{C}_{34} \mathrm{H}_{38} \mathrm{BrNO}_{9}[\mathrm{M}+\mathrm{Na}]^{+}=646.2428$, found $=$ 646.2422; The ee value was $93 \%, \mathrm{t}_{\mathrm{R}}$ (minor) $=16.1 \mathrm{~min}, \mathrm{t}_{\mathrm{R}}$ (major) $=20.5 \mathrm{~min}$ (Chiralcel IE, $\lambda=254 \mathrm{~nm}, 2 \% i-\mathrm{PrOH} / \mathrm{h}$ exanes, flow rate $=1.0 \mathrm{~mL} / \mathrm{min}$ ).

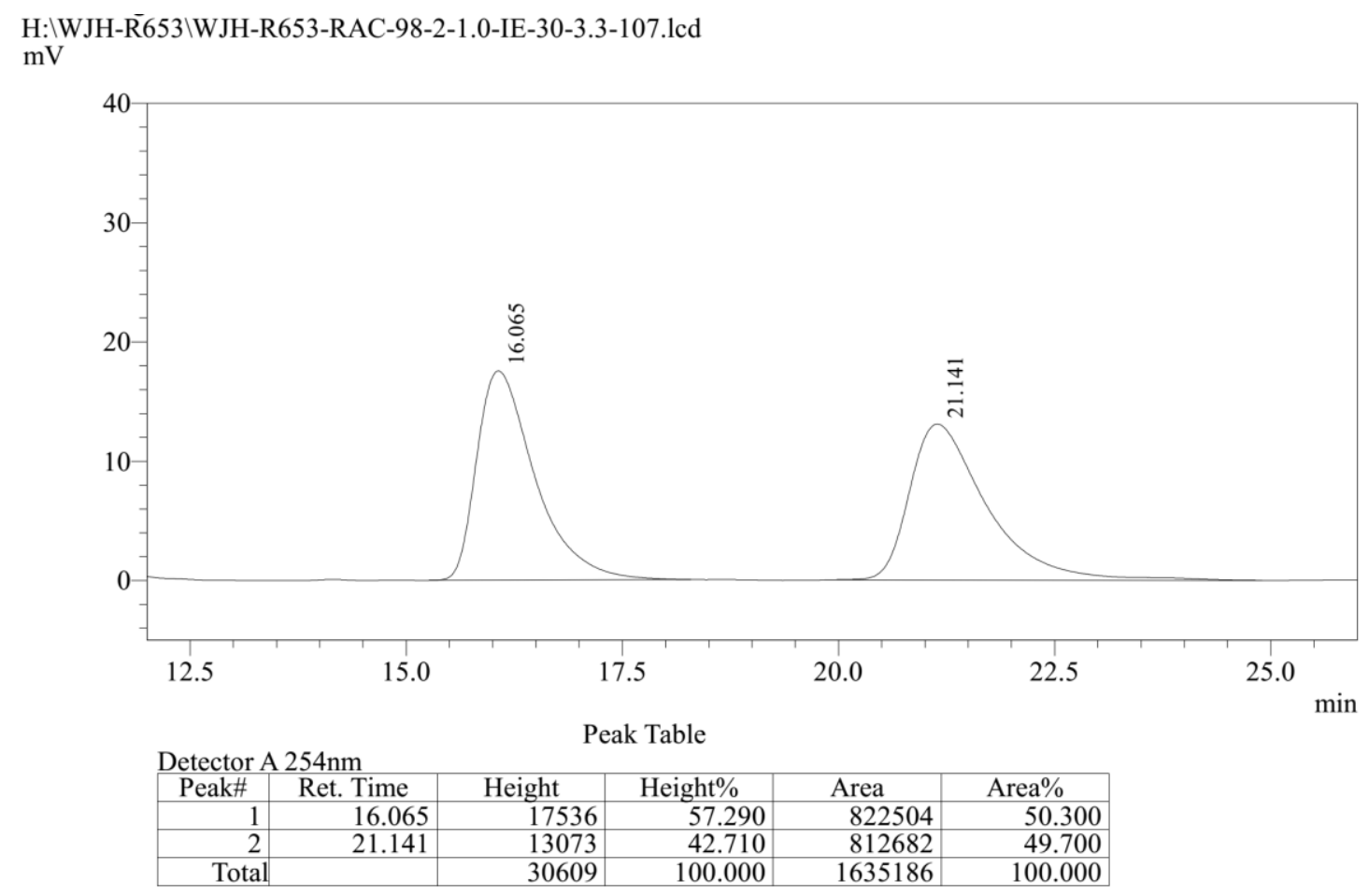


H:|WJH-Ř653।WJH-R653-CHRI-98-2-1.0-IE-30-3.3-106lcd.lcd $\mathrm{mV}$

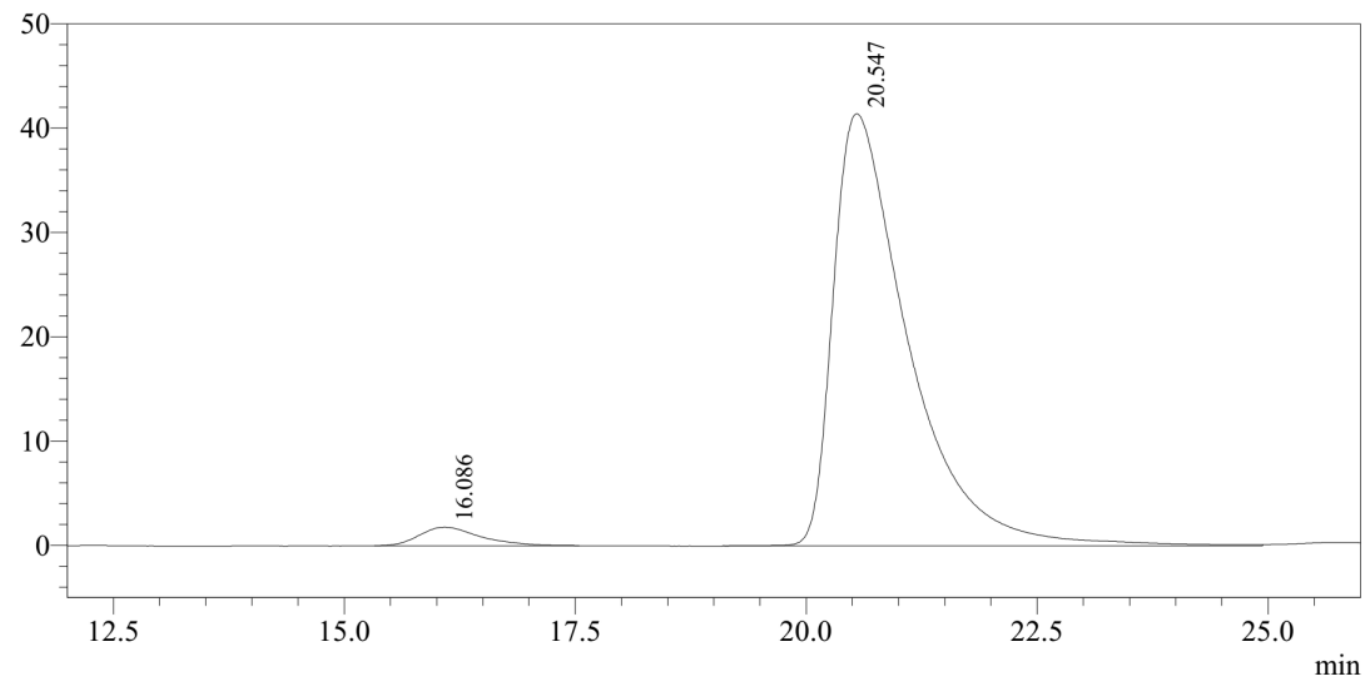

Peak Table

\begin{tabular}{|c|c|c|c|c|c|}
\hline ctor & $254 \mathrm{~nm}$ & & & & \\
\hline Peak\# & Ret. Time & Height & Height $\%$ & Area & Area $\%$ \\
\hline 1 & 16.086 & 1771 & 4.099 & 80421 & 3.325 \\
\hline 2 & 20.547 & 41427 & 95.901 & 2337915 & 96.675 \\
\hline Total & & 43198 & 100.000 & 2418336 & 100.000 \\
\hline
\end{tabular}

\section{4-benzyl 3,9-di-tert-butyl (S)-8-chloro-2-((S)-1-ethoxy-1-oxopropan-2-yl)pyrano}

\section{[2,3-b]indole-3,4,9(4H)-tricarboxylate $(3 \mathrm{t})$}

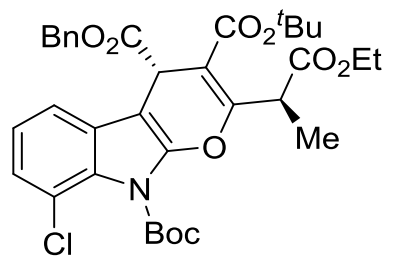

A white solid (116mg, 91\%); $[\alpha]^{25}=-14.8\left(c\right.$ 1.0, $\left.\mathrm{CHCl}_{3}\right) ;{ }^{1} \mathrm{H}$ NMR (400 MHz, $\left.\mathrm{CDCl}_{3}\right) \delta 7.46(\mathrm{dd}, J=7.8,1.0 \mathrm{~Hz}, 1 \mathrm{H}), 7.33-7.30(\mathrm{~m}, 5 \mathrm{H}), 7.20(\mathrm{dd}, J=7.9,1.0 \mathrm{~Hz}$, 1H), $7.06(\mathrm{t}, J=7.9 \mathrm{~Hz}, 1 \mathrm{H}), 5.20-4.99(\mathrm{~m}, 3 \mathrm{H}), 4.90(\mathrm{~s}, 1 \mathrm{H}), 4.22-4.09(\mathrm{~m}, 2 \mathrm{H})$, $1.64(\mathrm{~s}, 9 \mathrm{H}), 1.56(\mathrm{~d}, J=7.2 \mathrm{~Hz}, 3 \mathrm{H}), 1.43(\mathrm{~s}, 9 \mathrm{H}), 1.20(\mathrm{t}, J=7.1 \mathrm{~Hz}, 3 \mathrm{H}) ;{ }^{13} \mathrm{C} \mathrm{NMR}$ $\left(100 \mathrm{MHz}, \mathrm{CDCl}_{3}\right) \delta 171.2,171.0,164.9,160.5,146.9,143.5,135.3,128.5,128.5$, $128.4,128.1,124.7,123.8,119.4,117.7,105.5,88.8,85.5,82.2,67.2,61.1,41.1,40.3$, 27.9, 27.6, 14.2, 14.1. HRMS (ESI) $\mathrm{m} / z$ calcd for $\mathrm{C}_{34} \mathrm{H}_{38} \mathrm{ClNO}_{9}[\mathrm{M}+\mathrm{Na}]^{+}=662.2133$, found $=662.2121 ;$ The ee value was $96 \%, t_{R}($ minor $)=12.6$ min, $t_{R}($ major $)=15.7 \min$ (Chiralcel IE, $\lambda=254 \mathrm{~nm}, 2 \% i$-PrOH$/$ hexanes, flow rate $=1.0 \mathrm{~mL} / \mathrm{min}$ ). 
H:IWJH-R654|WJH-R654-RAC-98-2-1.0-IE-30-3.3.lcd $\mathrm{mV}$

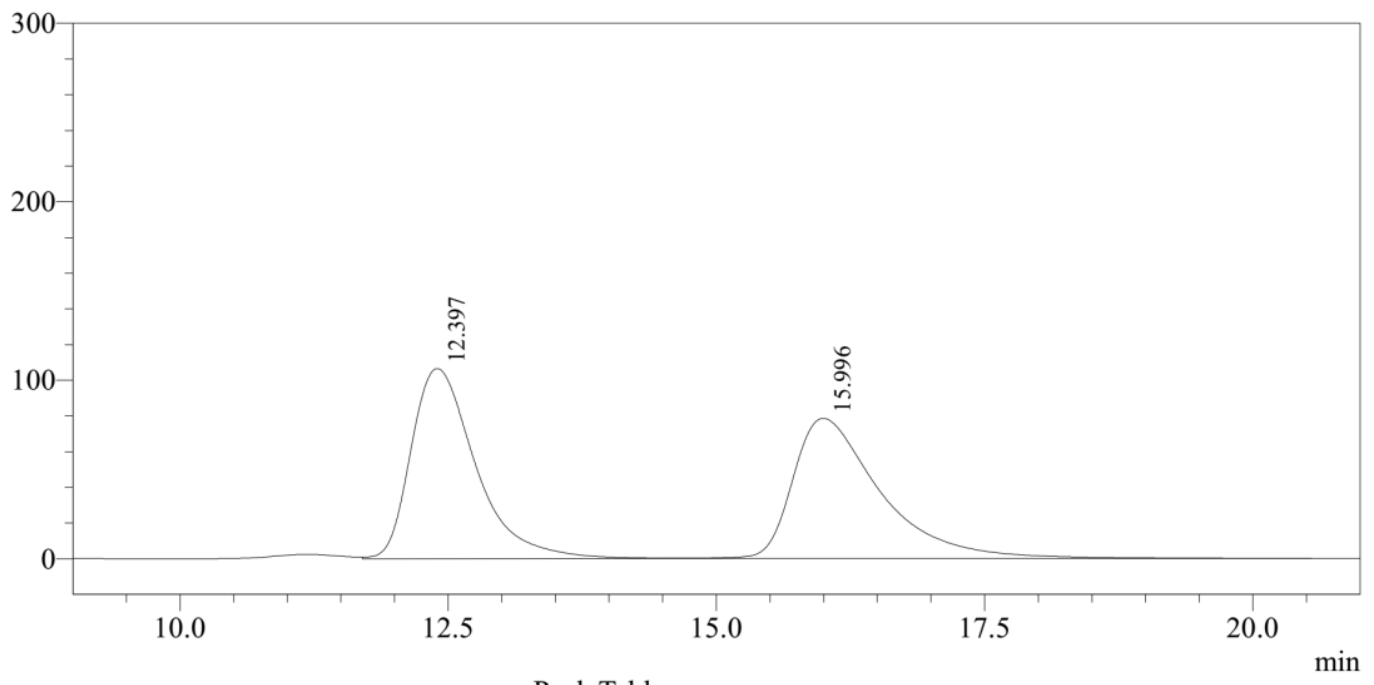

\begin{tabular}{|c|c|c|c|c|c|}
\hline \multirow{2}{*}{\multicolumn{6}{|c|}{ Detector A $254 \mathrm{~nm}$}} \\
\hline & & & & & \\
\hline 1 & 12.397 & 106508 & 57.540 & 4474686 & 49.909 \\
\hline 2 & 15.996 & 78595 & 42.460 & 4491070 & 50.091 \\
\hline Total & & 185103 & 100.000 & 8965755 & 100.000 \\
\hline
\end{tabular}

H:IWJH-R̄654|WJH-R654-CHRI2-98-2-1.0-IE-30-3.3.lcd $\mathrm{mV}$

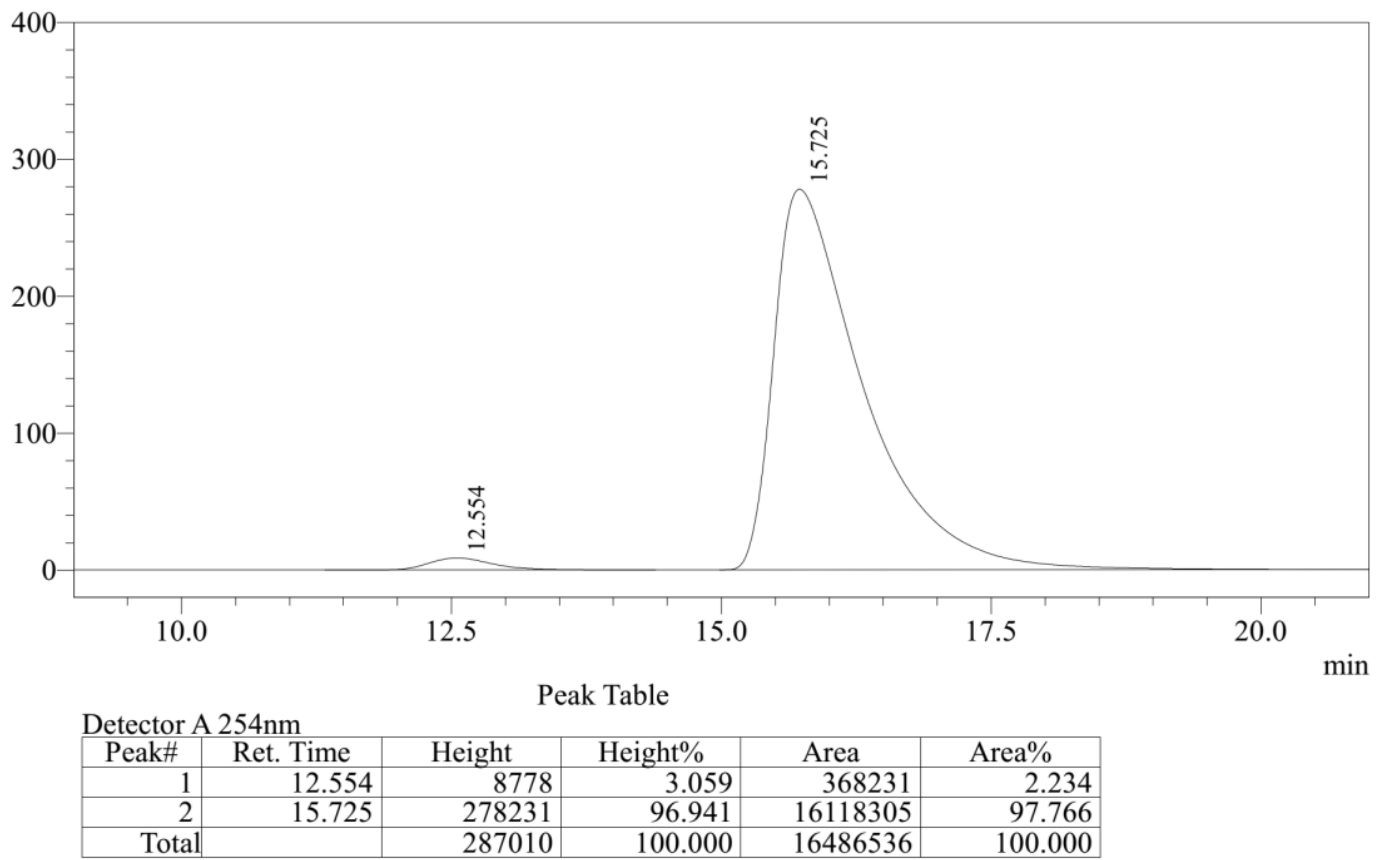

4-benzyl 3,9-di-tert-butyl (S)-8-bromo-2-( $(S)$-1-ethoxy-1-oxopropan-2-yl)pyrano [2,3-b]indole-3,4,9(4H)-tricarboxylate $(3 \mathrm{u})$ 


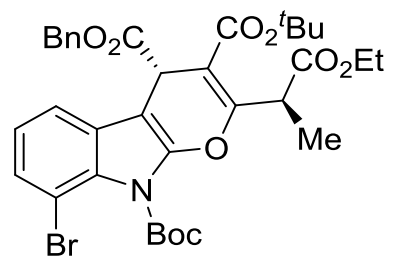

A white solid (126mg, 92\%); $[\alpha]^{25}{ }_{\mathrm{D}}=-17.1\left(c 1.0, \mathrm{CHCl}_{3}\right) ;{ }^{1} \mathrm{H}$ NMR $(400 \mathrm{MHz}$, $\left.\mathrm{CDCl}_{3}\right) \delta 7.51(\mathrm{dd}, J=7.8,0.9 \mathrm{~Hz}, 1 \mathrm{H}), 7.39(\mathrm{dd}, J=7.9,0.9 \mathrm{~Hz}, 1 \mathrm{H}), 7.34-7.27(\mathrm{~m}$, $5 \mathrm{H}), 7.00(\mathrm{t}, J=7.9 \mathrm{~Hz}, 1 \mathrm{H}), 5.20-4.97(\mathrm{~m}, 3 \mathrm{H}), 4.90(\mathrm{~s}, 1 \mathrm{H}), 4.20-4.08(\mathrm{~m}, 2 \mathrm{H})$, 1.65 (s, 9H), 1.56 (d, $J=7.2 \mathrm{~Hz}, 3 \mathrm{H}), 1.43$ (s, 9H), $1.20(\mathrm{t}, J=7.1 \mathrm{~Hz}, 3 \mathrm{H}) ;{ }^{13} \mathrm{C}$ NMR $\left(100 \mathrm{MHz}, \mathrm{CDCl}_{3}\right) \delta 171.2,171.0,164.9,160.4,146.8,143.5,135.3,129.8,128.7$, $128.5,128.4,128.3,128.0,124.2,118.3,107.0,105.5,88.7,85.5,82.2,67.2,61.1$, 41.0, 40.2, 27.9, 27.6, 14.2, 14.1. HRMS (ESI) $\mathrm{m} / z$ calcd for $\mathrm{C}_{34} \mathrm{H}_{38} \mathrm{BrNO}_{9}[\mathrm{M}+\mathrm{Na}]^{+}=$ 706.1628, found $=706.1604 ;$ The ee value was $94 \%, t_{R}(\operatorname{minor})=12.9$ min, $t_{R}($ major $)$ $=15.7 \mathrm{~min}$ (Chiralcel IE, $\lambda=254 \mathrm{~nm}, 2 \% i-\mathrm{PrOH} / \mathrm{hexanes}$, flow rate $=1.0 \mathrm{~mL} / \mathrm{min})$.

H:\WJH-Ř655 $\mathrm{mWJH}-\mathrm{R} 655-R A C 2-98-2-1.0-I E-30-3.3 . l c d$
mV

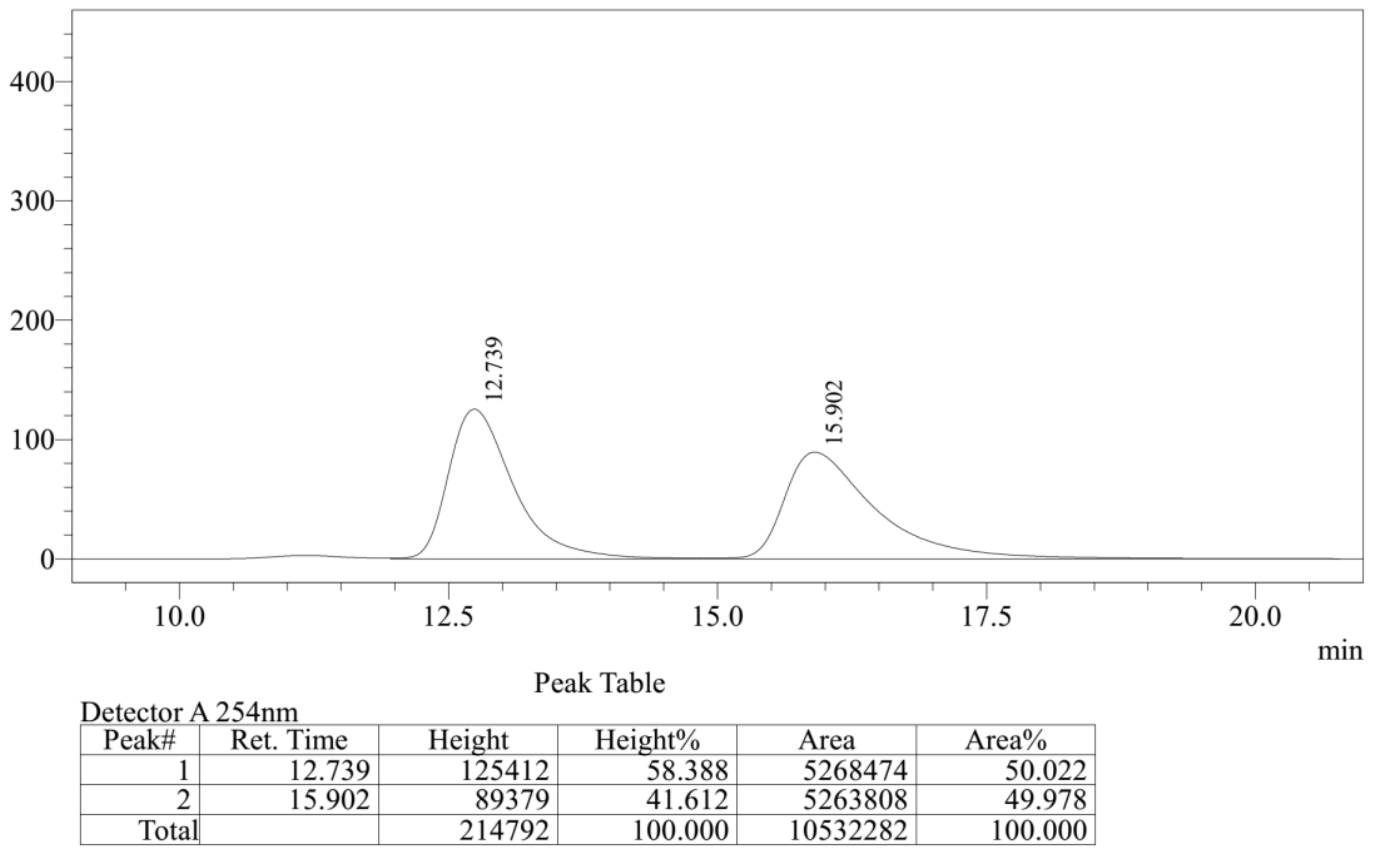


H:IWJH-Ř655 \WJH-R655-CHRI2-98-2-1.0-IE-30-3.3.lcd $\mathrm{mV}$

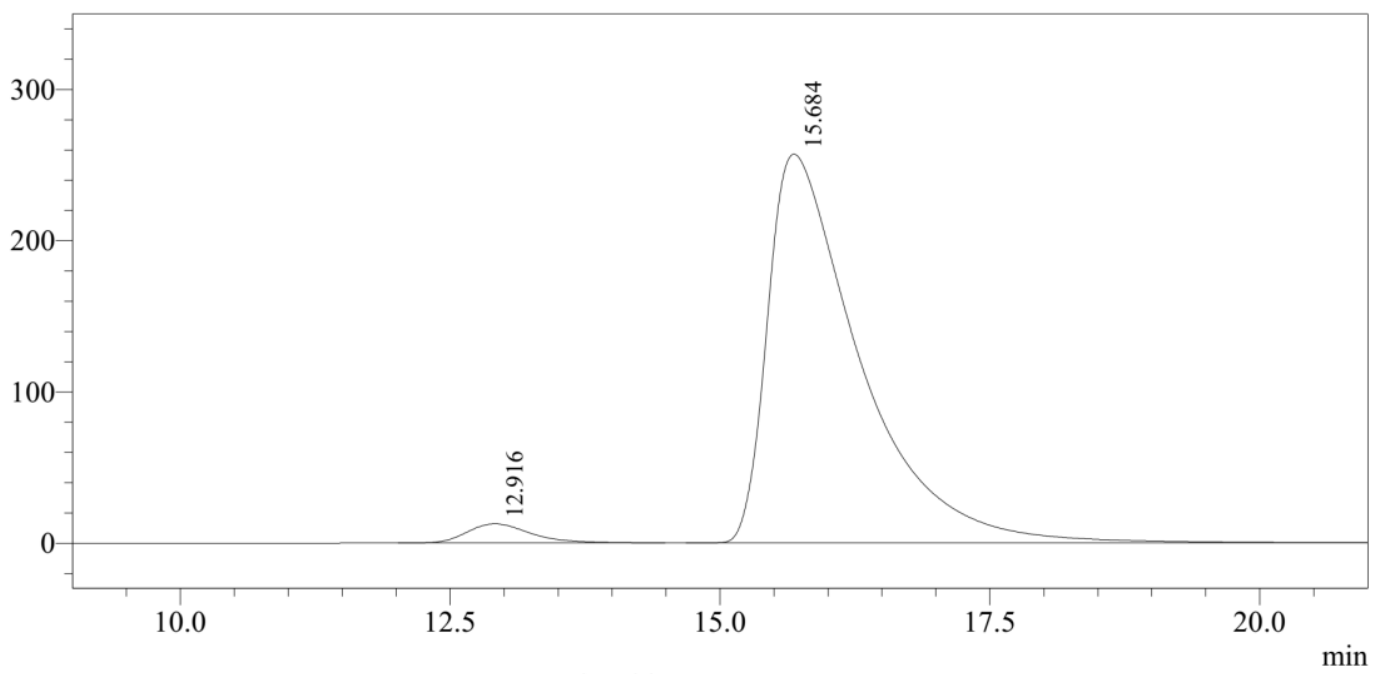

Peak Table
\begin{tabular}{|r|r|r|r|r|r|} 
Detector A 254nm \\
\hline Peak\# & Ret. Time & \multicolumn{1}{c|}{ Height } & Height $\%$ & \multicolumn{1}{c|}{ Area } & \multicolumn{1}{c|}{ Area $\%$} \\
\hline 1 & 12.916 & 12632 & 4.682 & 520247 & 3.280 \\
\hline 2 & 15.684 & 257188 & 95.318 & 15339797 & 96.720 \\
\hline Total & & 269820 & 100.000 & 15860045 & 100.000 \\
\hline
\end{tabular}

\section{4-benzyl 3,9-di-tert-butyl $(S)$-2-((S)-1-ethoxy-1-oxopropan-2-yl)-8-methylpyrano} [2,3-b]indole-3,4,9(4H)-tricarboxylate $(3 \mathrm{v})$

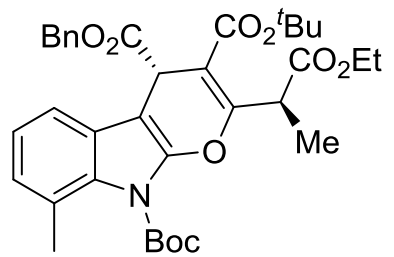

A white solid (123mg, 99\%); $[\alpha]^{25}=-10.0\left(c\right.$ 1.5, $\left.\mathrm{CHCl}_{3}\right) ;{ }^{1} \mathrm{H}$ NMR (400 MHz, $\left.\mathrm{CDCl}_{3}\right) \delta 7.42(\mathrm{~d}, J=7.6 \mathrm{~Hz}, 1 \mathrm{H}), 7.35-7.27(\mathrm{~m}, 5 \mathrm{H}), 7.08(\mathrm{t}, J=7.6 \mathrm{~Hz}, 1 \mathrm{H}), 7.01$ $(\mathrm{d}, J=7.4 \mathrm{~Hz}, 1 \mathrm{H}), 5.22-4.95(\mathrm{~m}, 3 \mathrm{H}), 4.90(\mathrm{~s}, 1 \mathrm{H}), 4.18-4.08(\mathrm{~m}, 2 \mathrm{H}), 2.39(\mathrm{~s}$, $3 \mathrm{H}), 1.62(\mathrm{~s}, 9 \mathrm{H}), 1.56(\mathrm{~d}, J=7.2 \mathrm{~Hz}, 3 \mathrm{H}), 1.42(\mathrm{~s}, 9 \mathrm{H}), 1.18(\mathrm{t}, J=7.1 \mathrm{~Hz}, 3 \mathrm{H}) ;{ }^{13} \mathrm{C}$ NMR $\left(100 \mathrm{MHz}, \mathrm{CDCl}_{3}\right) \delta 171.4,165.2,160.7,148.3,142.7,135.5,130.7,128.5$, $128.5,128.5,128.3,126.5,126.5,124.3,123.4,116.8,105.4,89.3,84.7,82.1,67.2$, 61.1, 41.1, 40.4, 28.0, 27.8, 20.5, 14.2, 14.2. HRMS (ESI) $\mathrm{m} / z$ calcd for $\mathrm{C}_{35} \mathrm{H}_{41} \mathrm{NO}_{9}$ $[\mathrm{M}+\mathrm{Na}]^{+}=642.2679$, found $=642.2670$; The ee value was $91 \%, \mathrm{t}_{\mathrm{R}}($ minor $)=10.7 \mathrm{~min}$, $\mathrm{t}_{\mathrm{R}}($ major $)=14.3 \mathrm{~min}($ Chiralcel IE, $\lambda=254 \mathrm{~nm}, 2 \% i-\mathrm{PrOH} / \mathrm{hexanes}$, flow rate $=1.0$ $\mathrm{mL} / \mathrm{min})$. 
G:IWJH-R657IWJH-R657-RAC-98-2-1.0-IE-30-3.5.Icd mAU

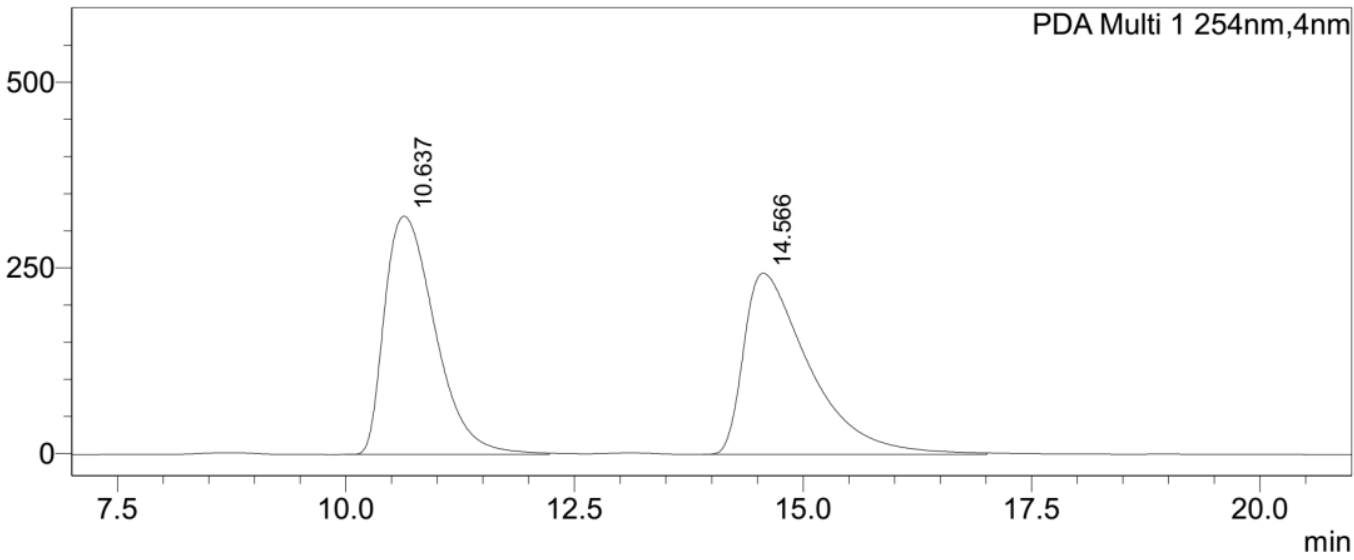

Peak Table

PDA Ch1 $254 \mathrm{~nm}$
\begin{tabular}{|r|r|r|r|r|}
\hline Peak\# & Ret. Time & Area & Height & \multicolumn{1}{|c|}{ Area $\%$} \\
\hline 1 & 10.637 & 12345853 & 320667 & 50.340 \\
\hline 2 & 14.566 & 12178957 & 243744 & 49.660 \\
\hline Total & & 24524810 & 564411 & 100.000 \\
\hline
\end{tabular}

H:IWJH-Ř657 \WJH-R657-CHRI-98-2-1.0-IE-30-3.3.lcd $\mathrm{mV}$

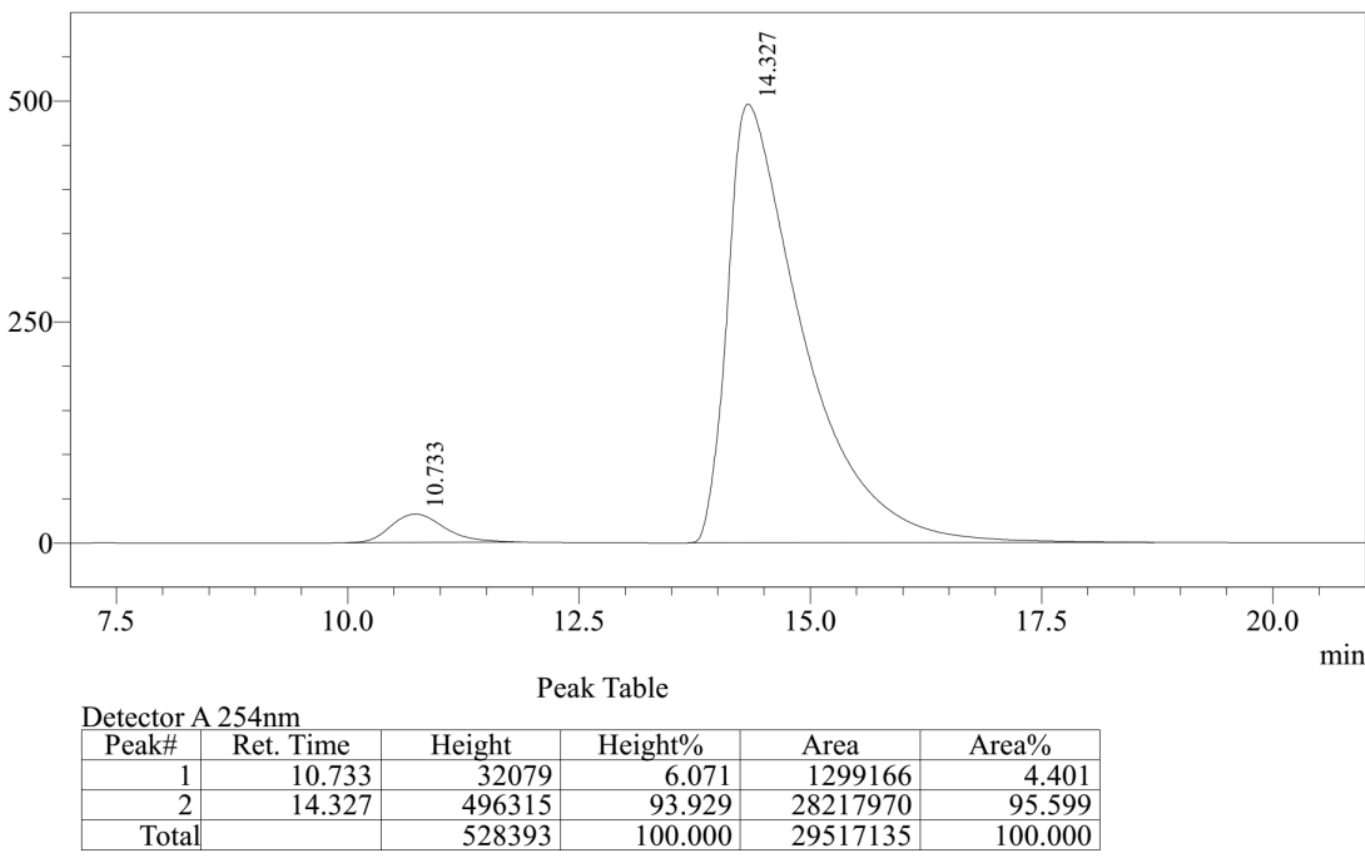

4-benzyl 3,9-di-tert-butyl $(S)$-5-bromo-2-((S)-1-ethoxy-1-oxopropan-2-yl)-6methylpyrano[2,3-b]indole-3,4,9(4H)-tricarboxylate $(3 \mathrm{w})$ 


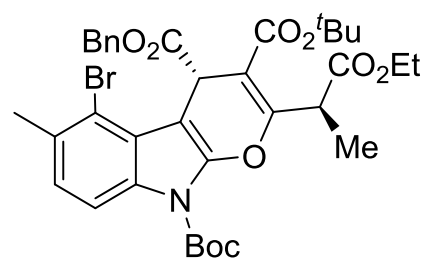

A white solid (128mg, 92\%); $[\alpha]^{25}{ }_{\mathrm{D}}=-18.1\left(c 1.0, \mathrm{CHCl}_{3}\right) ;{ }^{1} \mathrm{H}$ NMR $(400 \mathrm{MHz}$, $\left.\mathrm{CDCl}_{3}\right) \delta 8.03(\mathrm{~d}, J=8.4 \mathrm{~Hz}, 1 \mathrm{H}), 7.31-7.24(\mathrm{~m}, 5 \mathrm{H}), 7.09(\mathrm{~d}, J=8.5 \mathrm{~Hz}, 1 \mathrm{H}), 5.70$ (s, 1H), $5.15(\mathrm{q}, J=12.4 \mathrm{~Hz}, 2 \mathrm{H}), 4.68(\mathrm{q}, J=7.1 \mathrm{~Hz}, 1 \mathrm{H}), 4.21-4.04(\mathrm{~m}, 2 \mathrm{H}), 2.43$ (s, 3H), 1.63 (s, 9H), 1.50 (d, $J=7.2 \mathrm{~Hz}, 3 \mathrm{H}), 1.45$ (s, 9H), 1.15 (t, $J=7.1 \mathrm{~Hz}, 3 \mathrm{H}$ ); ${ }^{13} \mathrm{C}$ NMR $\left(100 \mathrm{MHz}, \mathrm{CDCl}_{3}\right) \delta 171.5,171.0,165.5,158.3,148.3,144.0,135.6,133.0$, 131.3, 128.3, 128.1, 128.1, 125.6, 125.1, 114.8, 113.9, 106.8, 92.0, 84.8, 82.1, 67.1, 61.0, 41.1, 39.7, 28.1, 27.9, 22.5, 14.2, 13.9. HRMS (ESI) $\mathrm{m} / 2$ calcd for $\mathrm{C}_{35} \mathrm{H}_{40} \mathrm{BrNO}_{9}$ $[\mathrm{M}+\mathrm{Na}]^{+}=720.1784$, found $=720.1772$; The ee value was $85 \%, \mathrm{t}_{\mathrm{R}}($ major $)=11.7 \mathrm{~min}$, $\mathrm{t}_{\mathrm{R}}(\operatorname{minor})=14.1 \mathrm{~min}($ Chiralcel IG, $\lambda=254 \mathrm{~nm}, 2 \% i$-PrOH/hexanes, flow rate $=0.7$ $\mathrm{mL} / \mathrm{min})$.

H:IWJH-Ř680 \WJH-R680-RAC-98-2-0.7-IG-30-2.1-106.lcd $\mathrm{mV}$

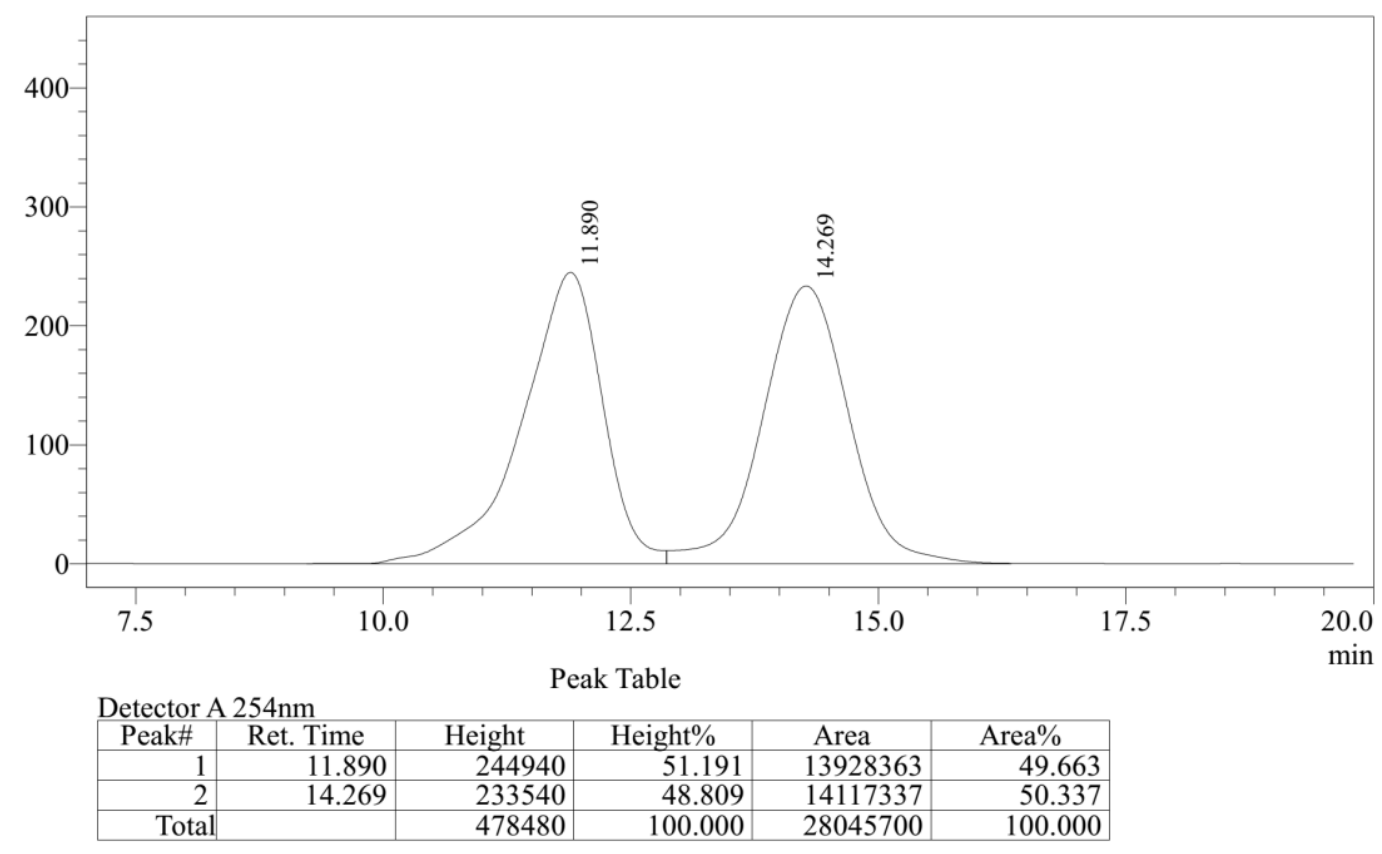


H:IWJH-Ř680 \WJH-R680-CHRI-98-2-0.7-IG-30-2.1-106.1cd $\mathrm{mV}$

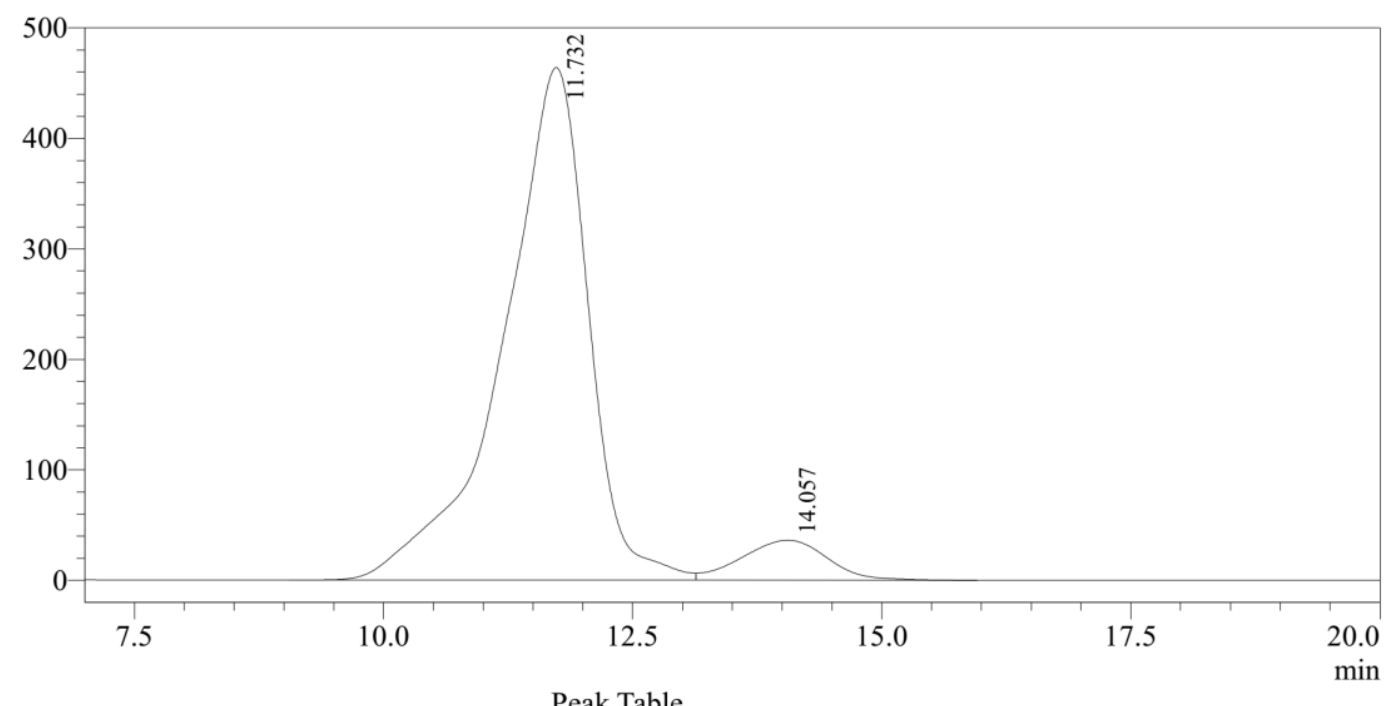

\begin{tabular}{|c|c|c|c|c|c|}
\hline \multirow{2}{*}{\multicolumn{6}{|c|}{ Detector A 254nm }} \\
\hline & & & & & \\
\hline Pea & Time & Height & Height $\%$ & Area & Area\% \\
\hline 1 & 11.732 & 463885 & 92.758 & 27513073 & 92.667 \\
\hline 2 & 14.057 & & 7.242 & 2177144 & 7.333 \\
\hline Total & & 500100 & 100.000 & 29690216 & 100.000 \\
\hline
\end{tabular}

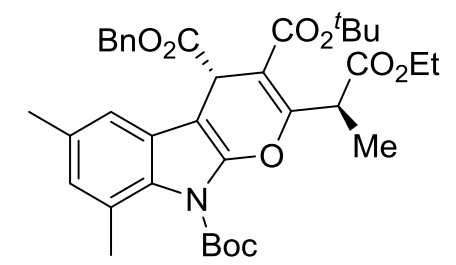

A colorless oil $(117 \mathrm{mg}, 92 \%) ;[\alpha]^{25}{ }_{\mathrm{D}}=-31.9\left(c 0.5, \mathrm{CHCl}_{3}\right) ;{ }^{1} \mathrm{H}$ NMR $(400 \mathrm{MHz}$, $\left.\mathrm{CDCl}_{3}\right) \delta 7.44-7.30(\mathrm{~m}, 5 \mathrm{H}), 7.22(\mathrm{~s}, 1 \mathrm{H}), 6.85(\mathrm{~s}, 1 \mathrm{H}), 5.16(\mathrm{dd}, J=13.2,6.1 \mathrm{~Hz}$, 2H), $5.02(\mathrm{~d}, J=12.2 \mathrm{~Hz}, 1 \mathrm{H}), 4.88(\mathrm{~s}, 1 \mathrm{H}), 4.15(\mathrm{q}, J=7.1 \mathrm{~Hz}, 2 \mathrm{H}), 2.37$ (s, 3H), 2.29 (s, 3H), 1.63 (s, 9H), 1.57 (d, J= $7.2 \mathrm{~Hz}, 3 \mathrm{H}), 1.46(\mathrm{~s}, 9 \mathrm{H}), 1.19$ (t, J = 7.1 Hz, $3 \mathrm{H}) ;{ }^{13} \mathrm{C} \mathrm{NMR}\left(100 \mathrm{MHz}, \mathrm{CDCl}_{3}\right) \delta 171.4,171.3,160.8,148.3,142.7,135.4,132.8$, 128.8, 128.5, 128.5, 127.7, 126.7, 123.9, 116.8, 105.2, 89.1, 84.4, 82.0, 67.2, 61.0, 41.0, 40.3, 27.97, 27.7, 21.1, 20.4, 14.2. HRMS (ESI) $\mathrm{m} / z$ calcd for $\mathrm{C}_{36} \mathrm{H}_{43} \mathrm{NO}_{9}$ $[\mathrm{M}+\mathrm{Na}]^{+}=656.2836$, found $=656.2833$; The ee value was $88 \%, \mathrm{t}_{\mathrm{R}}($ minor $)=10.5 \mathrm{~min}$, $\mathrm{t}_{\mathrm{R}}($ major $)=14.9 \mathrm{~min}($ Chiralcel IE, $\lambda=254 \mathrm{~nm}, 2 \% i-\mathrm{PrOH} / \mathrm{hexanes}$, flow rate $=1.0$ $\mathrm{mL} / \mathrm{min})$. 
G:IWJH-R658IWJH-R658-RAC-98-2-1.0-IE-30.Icd mAU

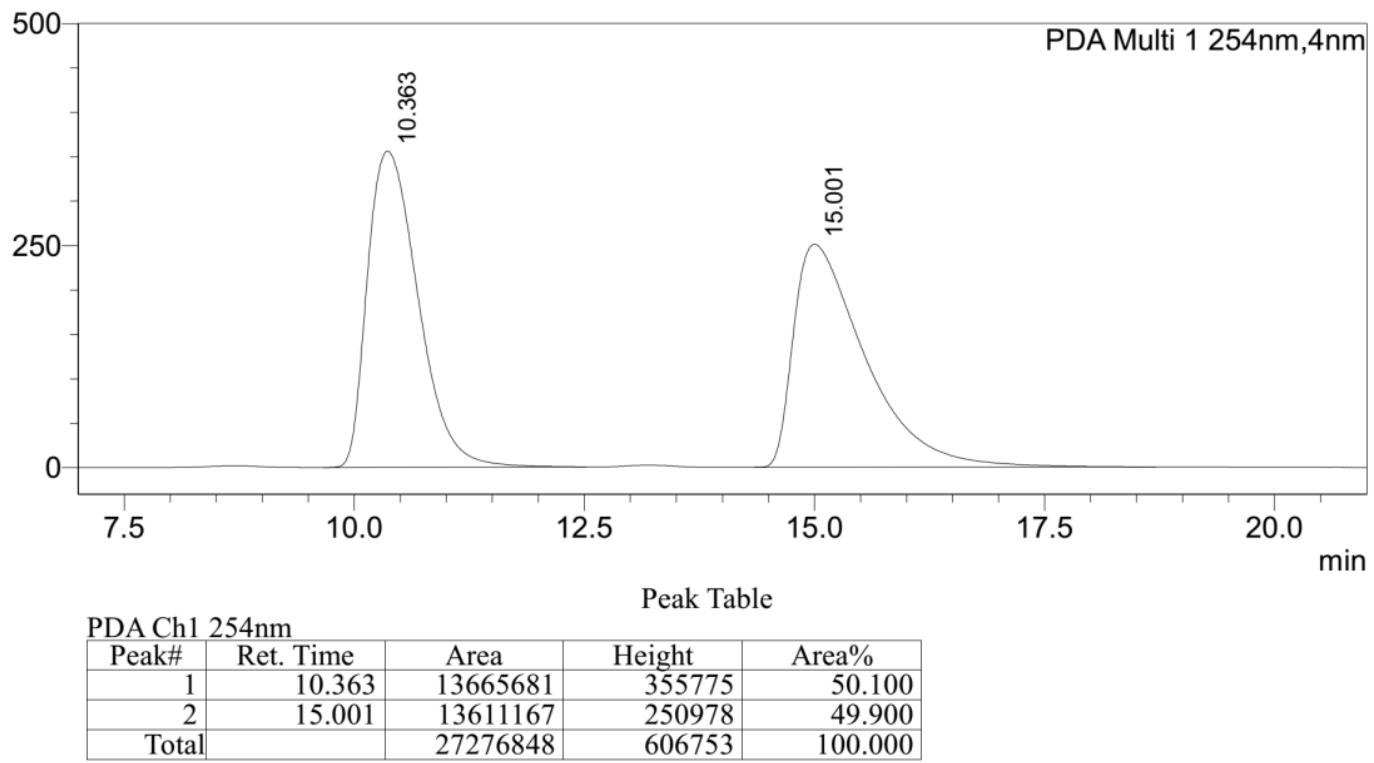

H:IWJH-R̄658|WJH-R658-CHRI-98-2-1.0-IE-30-3.3.lcd $\mathrm{mV}$

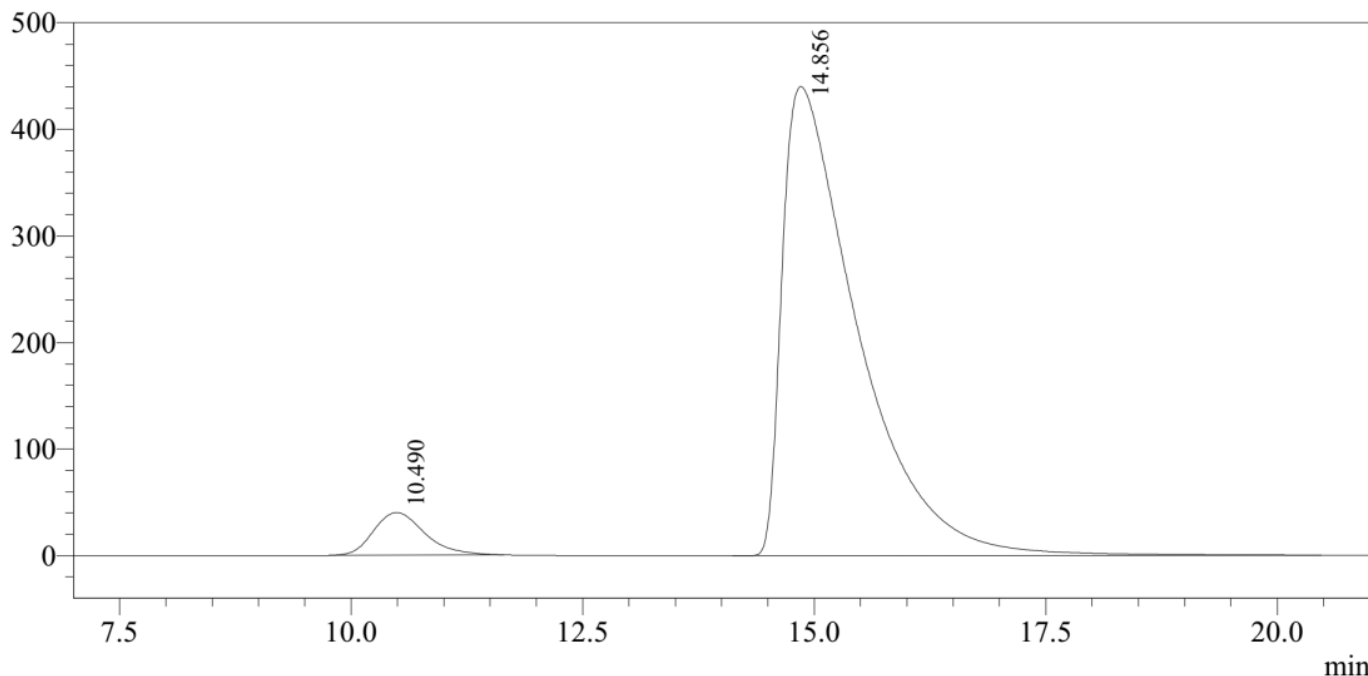

Peak Table

Detector A $254 \mathrm{~nm}$
\begin{tabular}{|r|r|r|r|r|r|}
\hline Peak\# & Ret. Time & Height & Height $\%$ & \multicolumn{1}{c|}{ Area } & \multicolumn{1}{c|}{ Area $\%$} \\
\hline 1 & 10.490 & 39909 & 8.316 & 1539472 & 5.809 \\
\hline 2 & 14.856 & 440010 & 91.684 & 24962951 & 94.191 \\
\hline Total & & 479919 & 100.000 & 26502423 & 100.000 \\
\hline
\end{tabular}




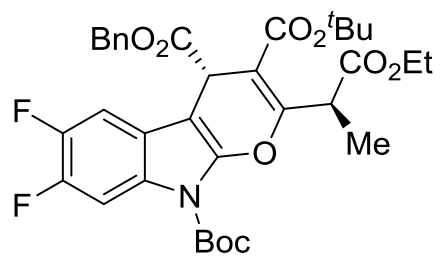

A yellow oil $(109 \mathrm{mg}, 85 \%) ;[\alpha]^{25}=-18.1\left(c 1.0, \mathrm{CHCl}_{3}\right) ;{ }^{1} \mathrm{H} \mathrm{NMR}\left(400 \mathrm{MHz}, \mathrm{CDCl}_{3}\right)$ $\delta 8.02(\mathrm{dd}, J=11.7,7.1 \mathrm{~Hz}, 1 \mathrm{H}), 7.36-7.29(\mathrm{~m}, 6 \mathrm{H}), 5.22-5.01(\mathrm{~m}, 3 \mathrm{H}), 4.84(\mathrm{~s}$, 1H), $4.24-4.08(\mathrm{~m}, 2 \mathrm{H}), 1.63(\mathrm{~s}, 9 \mathrm{H}), 1.56(\mathrm{~d}, J=7.2 \mathrm{~Hz}, 3 \mathrm{H}), 1.44(\mathrm{~s}, 9 \mathrm{H}), 1.20$ (t, $J=7.1 \mathrm{~Hz}, 3 \mathrm{H}) ;{ }^{13} \mathrm{C}$ NMR $\left(100 \mathrm{MHz}, \mathrm{CDCl}_{3}\right) \delta 171.2,171.0,164.8,160.8,148.2$, $147.9(\mathrm{dd}, J=242.0,6.6 \mathrm{~Hz}), 147.8(\mathrm{dd}, J=242.0,6.3 \mathrm{~Hz}), 142.9(\mathrm{~d}, J=3.4 \mathrm{~Hz})$, 135.0, 128.6, 128.6, 128.6, $127.0(\mathrm{dd}, J=10.7,1.5 \mathrm{~Hz}), 121.0(\mathrm{dd}, J=8.7,2.2 \mathrm{~Hz})$, $106.5(\mathrm{~d}, J=20.7 \mathrm{~Hz}), 104.9,104.7(\mathrm{~d}, J=25.0 \mathrm{~Hz}), 90.4(\mathrm{dd}, J=3.0,1.4 \mathrm{~Hz}), 85.3$, 82.3, 67.5, 61.0, 41.1, 40.0, 28.1, 27.9, 14.2, 14.2; ${ }^{19} \mathrm{~F}$ NMR $\left(376 \mathrm{MHz}, \mathrm{CDCl}_{3}\right) \delta$ $-141.5(\mathrm{~d}, J=21.3 \mathrm{~Hz}),-142.7(\mathrm{~d}, J=21.2 \mathrm{~Hz})$. HRMS $(\mathrm{ESI}) \mathrm{m} / z$ calcd for $\mathrm{C}_{35} \mathrm{H}_{40} \mathrm{BrNO}_{9}[\mathrm{M}+\mathrm{Na}]^{+}=664.2334$, found $=664.2337 ;$ The ee value was $90 \%, \mathrm{t}_{\mathrm{R}}$ $($ minor $)=12.5 \mathrm{~min}, \mathrm{t}_{\mathrm{R}}($ major $)=13.9 \mathrm{~min}($ Chiralcel $\mathrm{IE}, \lambda=254 \mathrm{~nm}, 2 \%$ $i$-PrOH/hexanes, flow rate $=0.7 \mathrm{~mL} / \mathrm{min})$.

H:IWJH-R682\WJH-R682-RAC-98-2-0.7-IE-30-2.3-106.lcd $\mathrm{mV}$

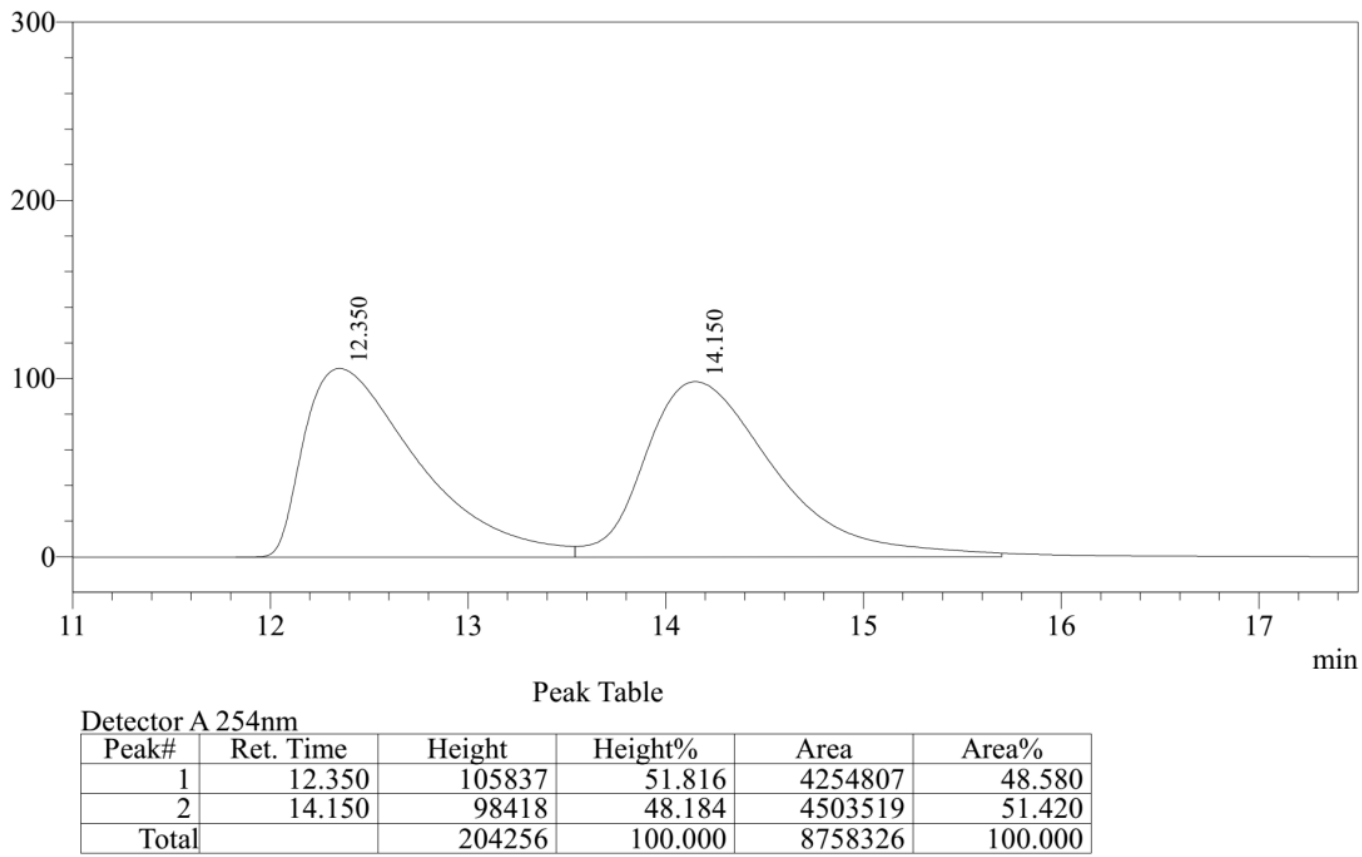




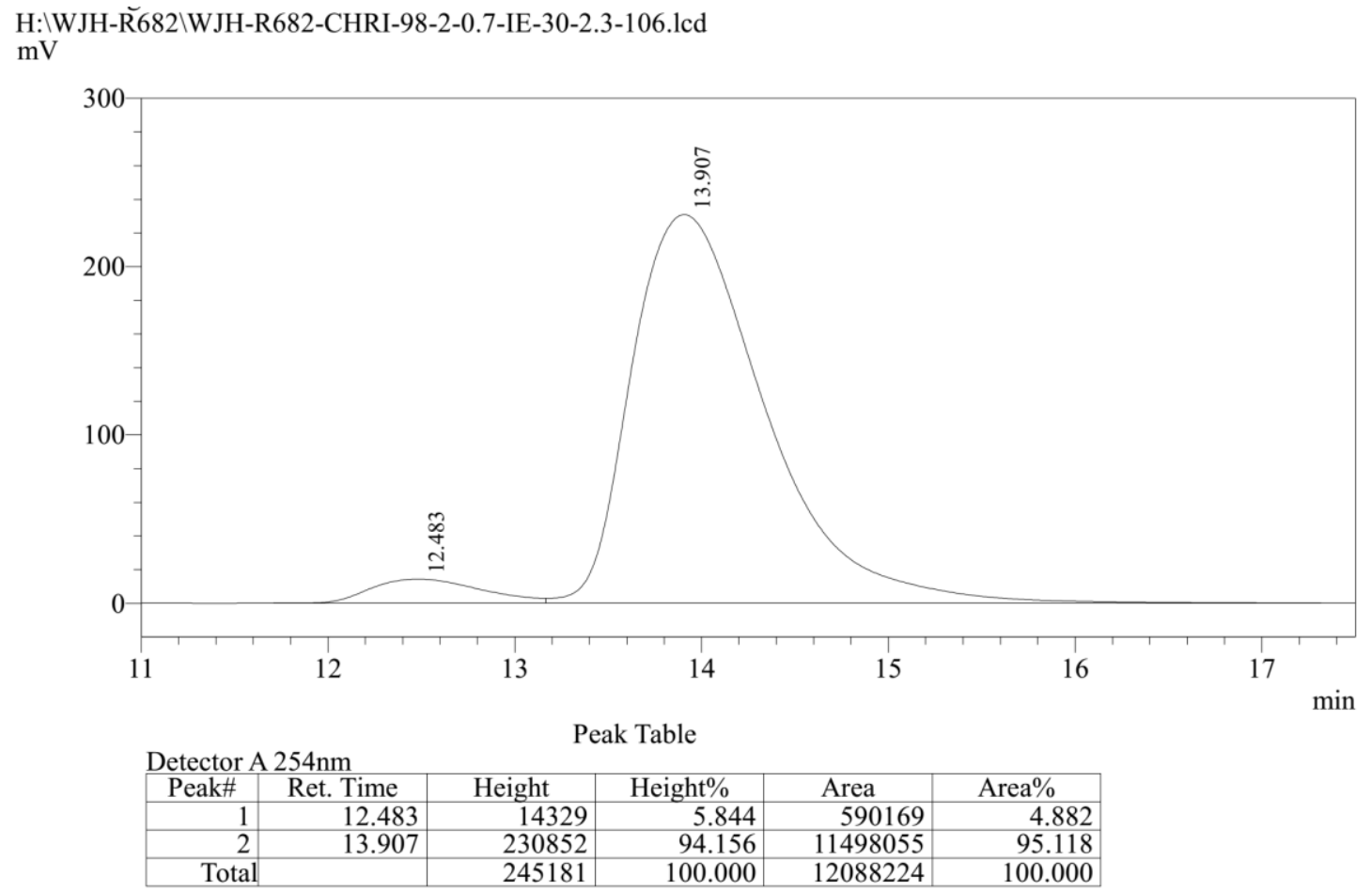

4-benzyl 9-(tert-butyl) 3-methyl $(S)$-2-((S)-1-ethoxy-1-oxopropan-2-yl)pyrano [2,3-b]indole-3,4,9(4H)-tricarboxylate $(4 \mathrm{a})$

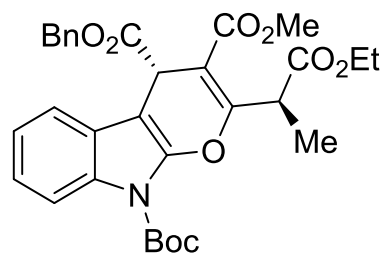

A colorless oil $(110 \mathrm{mg}, 98 \%) ;[\alpha]^{25}{ }_{\mathrm{D}}=-34.6\left(c\right.$ 1.0, $\left.\mathrm{CHCl}_{3}\right) ;{ }^{1} \mathrm{H}$ NMR $(400 \mathrm{MHz}$, $\left.\mathrm{CDCl}_{3}\right) \delta 8.15(\mathrm{~d}, J=8.2 \mathrm{~Hz}, 1 \mathrm{H}), 7.61-7.58(\mathrm{~m}, 1 \mathrm{H}), 7.37-7.28(\mathrm{~m}, 5 \mathrm{H}), 7.27-$ $7.24(\mathrm{~m}, 1 \mathrm{H}), 7.20(\mathrm{td}, J=7.5,1.0 \mathrm{~Hz}, 1 \mathrm{H}), 5.20-5.06(\mathrm{~m}, 3 \mathrm{H}), 4.97(\mathrm{~s}, 1 \mathrm{H}), 4.26-$ $4.06(\mathrm{~m}, 2 \mathrm{H}), 3.62(\mathrm{~s}, 3 \mathrm{H}), 1.65(\mathrm{~s}, 9 \mathrm{H}), 1.59$ (d, $J=7.2 \mathrm{~Hz}, 3 \mathrm{H}), 1.19(\mathrm{t}, J=7.1 \mathrm{~Hz}$, $3 \mathrm{H}) ;{ }^{13} \mathrm{C} \mathrm{NMR}\left(100 \mathrm{MHz}, \mathrm{CDCl}_{3}\right) \delta 171.2,171.1,166.4,162.2,148.5,142.3,135.6$, 132.0, 128.5, 128.4, 128.3, 125.1, 123.8, 123.4, 118.9, 115.0, 103.6, 90.8, 84.7, 67.1, 61.1, 52.0, 41.4, 39.7, 28.2, 14.2. HRMS (ESI) $m / z$ calcd for $\mathrm{C}_{31} \mathrm{H}_{33} \mathrm{NO}_{9}[\mathrm{M}+\mathrm{Na}]^{+}=$ 586.2053 , found $=586.2053$; The ee value was $82 \%, \mathrm{t}_{\mathrm{R}}($ minor $)=20.6 \mathrm{~min}, \mathrm{t}_{\mathrm{R}}$ (major) $=22.4 \mathrm{~min}$ (Chiralcel IE, $\lambda=254 \mathrm{~nm}, 2 \% i-\mathrm{PrOH} / \mathrm{h}$ exanes, flow rate $=1.0 \mathrm{~mL} / \mathrm{min}$ ). 
H:IWJH-Ř721 \WJH-R721-RAC-98-2-1.0-IE-30-3.5.lcd

$\mathrm{mV}$

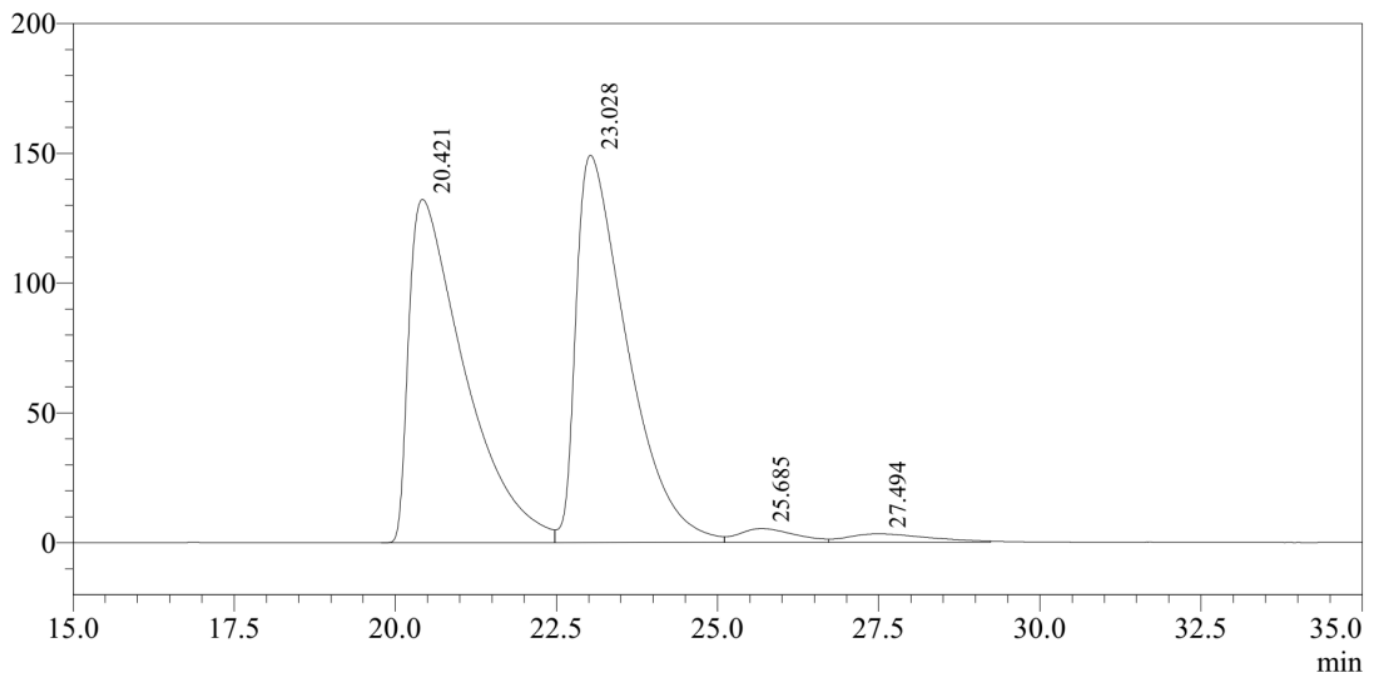

Peak Table

Detector A $254 n m$
\begin{tabular}{|r|r|r|r|r|r|}
\hline Peak\# & Ret. Time & \multicolumn{1}{|c|}{ Height } & Height $\%$ & \multicolumn{1}{c|}{ Area } & Area $\%$ \\
\hline 1 & 20.421 & 132272 & 45.578 & 7973423 & 47.243 \\
\hline 2 & 23.028 & 149216 & 51.417 & 8274456 & 49.026 \\
\hline 3 & 25.685 & 5396 & 1.859 & 332635 & 1.971 \\
\hline 4 & 27.494 & 3325 & 1.146 & 297101 & 1.760 \\
\hline Total & & 290209 & 100.000 & 16877615 & 100.000 \\
\hline
\end{tabular}

H:IWJH-R̄721 \WJH-R721-CHRI-98-2-1.0-IE-30-3.5.lcd $\mathrm{mV}$

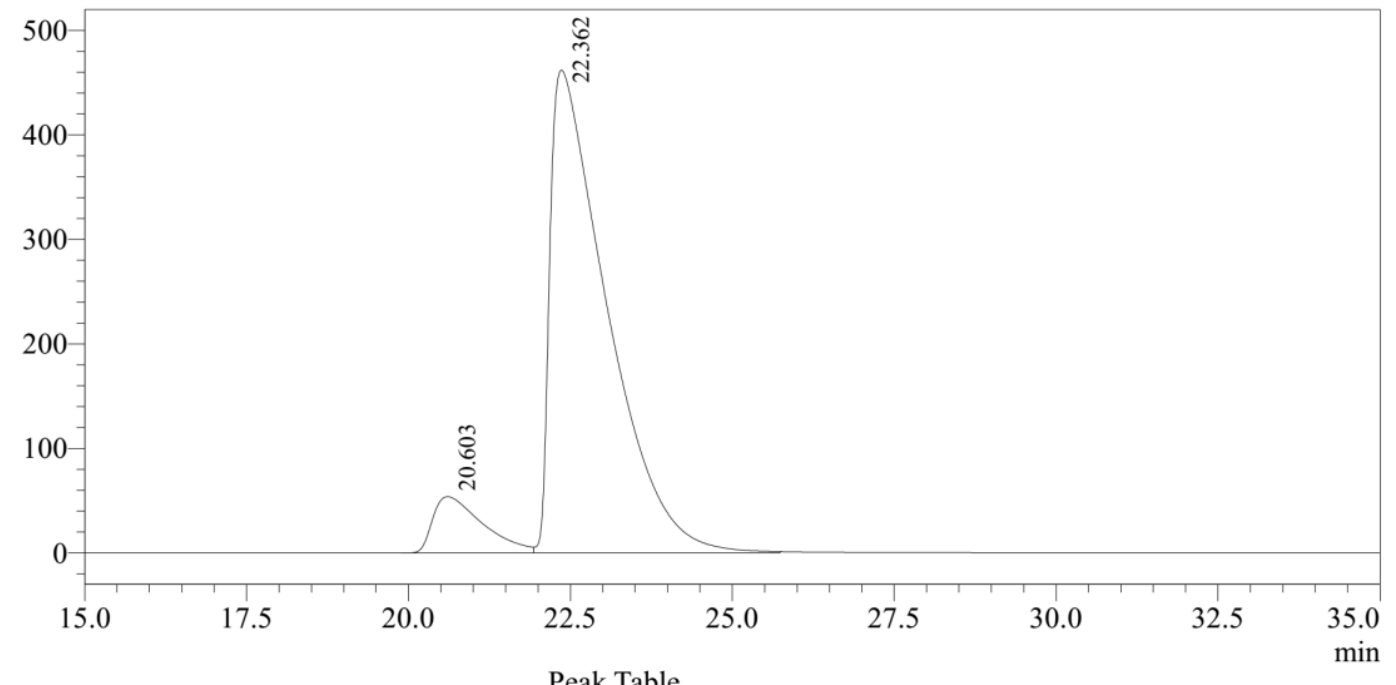

Detector A $254 \mathrm{~nm}$
\begin{tabular}{|r|r|r|r|r|r|}
\hline Peak\# & Ret. Time & Height & Height $\%$ & Area & \multicolumn{1}{|c|}{ Area $\%$} \\
\hline 1 & 20.603 & 53884 & 10.443 & 2888355 & 9.256 \\
\hline 2 & 22.362 & 462101 & 89.557 & 28316659 & 90.744 \\
\hline Total & & 515985 & 100.000 & 31205014 & 100.000 \\
\hline
\end{tabular}

4-benzyl 9-(tert-butyl) 3-ethyl $(S)$-2-((S)-1-ethoxy-1-oxopropan-2-yl)pyrano [2,3-b]indole-3,4,9(4H)-tricarboxylate $(4 \mathrm{~b})$ 


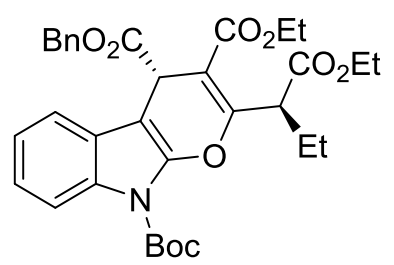

A colorless oil $(112 \mathrm{mg}, 97 \%) ;[\alpha]^{25}{ }_{\mathrm{D}}=-13.1\left(\right.$ c 1.0, $\left.\mathrm{CHCl}_{3}\right) ;{ }^{1} \mathrm{H}$ NMR $(400 \mathrm{MHz}$, $\left.\mathrm{CDCl}_{3}\right) \delta 8.16(\mathrm{~d}, J=8.2 \mathrm{~Hz}, 1 \mathrm{H}), 7.60(\mathrm{dd}, J=7.7,0.6 \mathrm{~Hz}, 1 \mathrm{H}), 7.38-7.30(\mathrm{~m}, 5 \mathrm{H})$, $7.28(\mathrm{td}, J=7.4,1.4 \mathrm{~Hz}, 1 \mathrm{H}), 7.21(\mathrm{td}, J=7.6,1.0 \mathrm{~Hz}, 1 \mathrm{H}), 5.26-5.04(\mathrm{~m}, 3 \mathrm{H}), 5.00$ (s, 1H), $4.27-4.08(\mathrm{~m}, 4 \mathrm{H}), 1.66(\mathrm{~s}, 9 \mathrm{H}), 1.61(\mathrm{~d}, J=7.2 \mathrm{~Hz}, 3 \mathrm{H}), 1.29-1.15(\mathrm{~m}$, $6 \mathrm{H}) ;{ }^{13} \mathrm{C} \mathrm{NMR}\left(100 \mathrm{MHz}, \mathrm{CDCl}_{3}\right) \delta 171.3,171.2,165.9,162.0,148.5,142.3,135.5$, 132.0, 128.5, 128.4, 128.3, 125.2, 123.7, 123.4, 118.9, 114.9, 103.7, 90.9, 84.6, 67.2, $61.1(\mathrm{~d}, J=11.7 \mathrm{~Hz}), 41.4,39.8,28.2,14.2,14.2,13.9$. HRMS (ESI) $\mathrm{m} / \mathrm{z}$ calcd for $\mathrm{C}_{32} \mathrm{H}_{35} \mathrm{NO}_{9}[\mathrm{M}+\mathrm{Na}]^{+}=600.2210$, found $=600.2210$; The ee value was $85 \%, \mathrm{t}_{\mathrm{R}}$ (minor) $=14.0 \mathrm{~min}, \mathrm{t}_{\mathrm{R}}($ major $)=16.7 \mathrm{~min}($ Chiralcel IE, $\lambda=254 \mathrm{~nm}, 3 \% i$-PrOH/hexanes, flow rate $=1.0 \mathrm{~mL} / \mathrm{min})$.

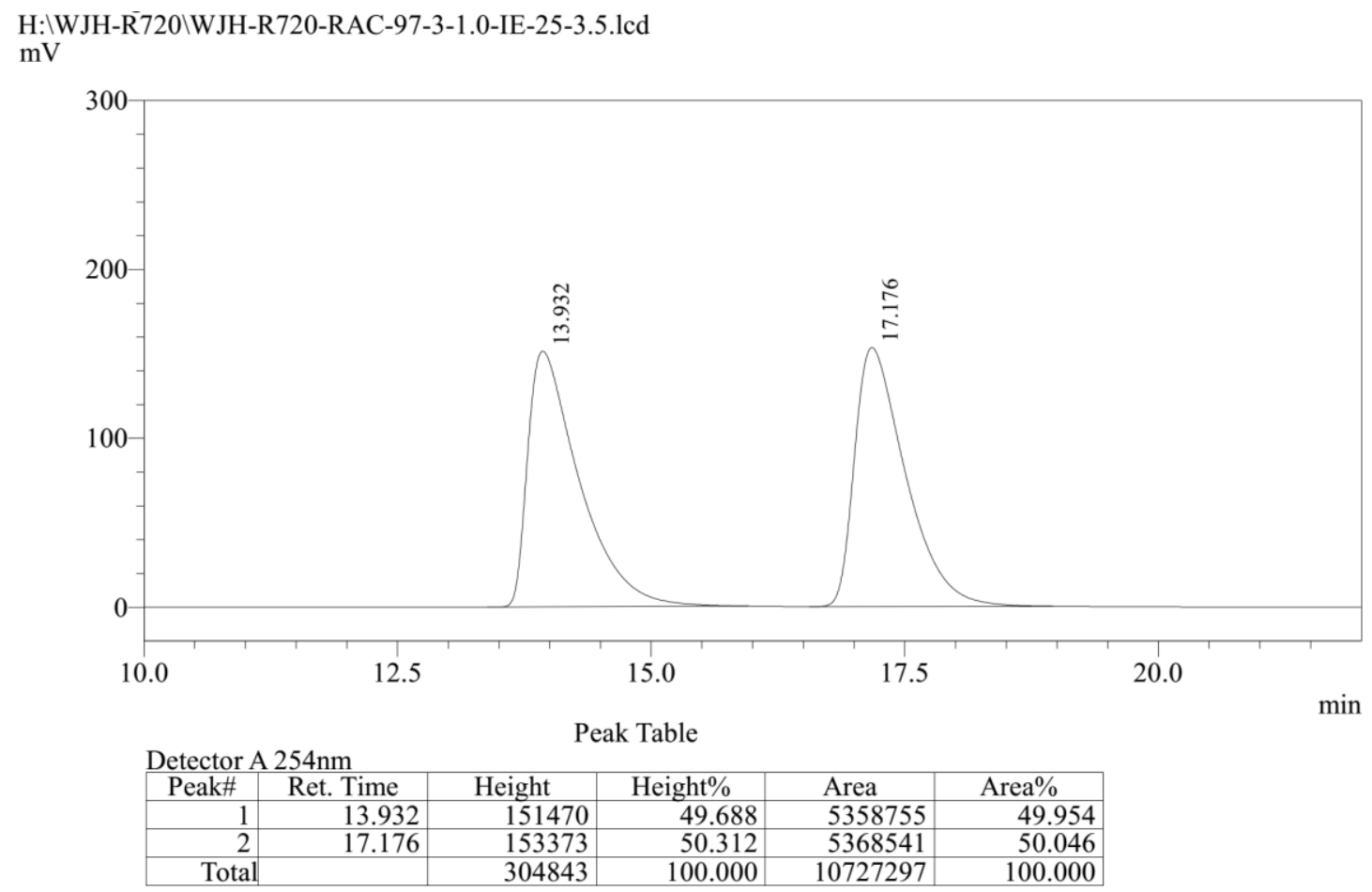




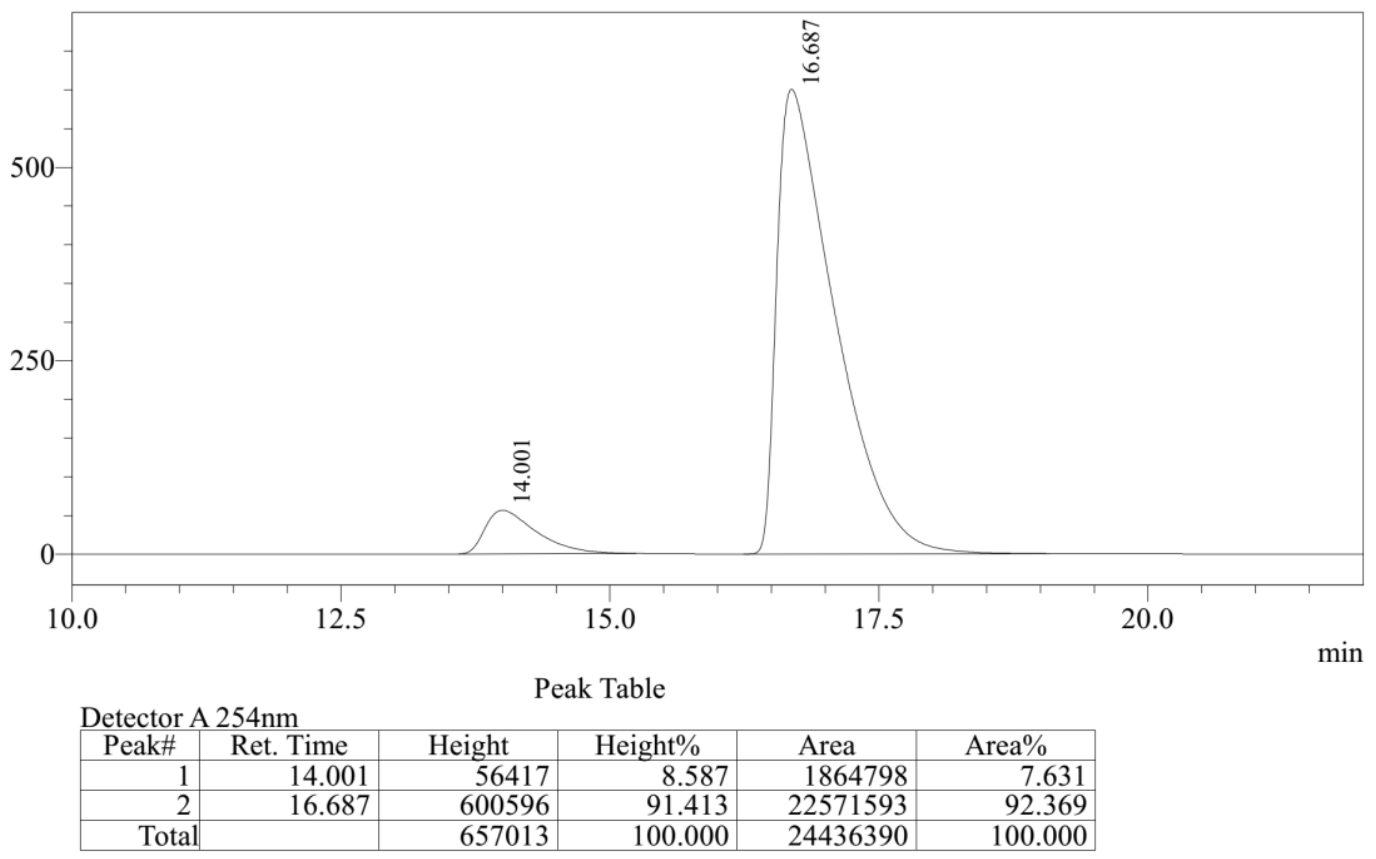

\section{4-benzyl 3,9-di-tert-butyl $\quad(S)$-2-((S)-1-(tert-butoxy)-1-oxopropan-2-yl)pyrano}

\section{[2,3-b]indole-3,4,9(4H)-tricarboxylate $(4 \mathrm{c})$}

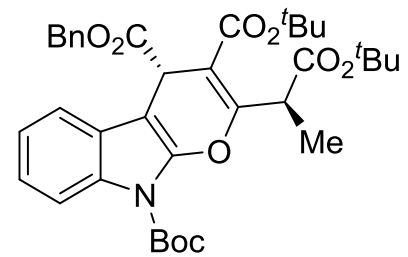

A colorless oil $(111 \mathrm{mg}, 88 \%) ;[\alpha]_{\mathrm{D}}^{25}=-20.4\left(c 1.0, \mathrm{CHCl}_{3}\right) ;{ }^{1} \mathrm{H}$ NMR $(400 \mathrm{MHz}$, $\left.\mathrm{CDCl}_{3}\right) \delta 8.15(\mathrm{~d}, J=8.2 \mathrm{~Hz}, 1 \mathrm{H}), 7.57(\mathrm{dd}, J=7.1,0.7 \mathrm{~Hz}, 1 \mathrm{H}), 7.35-7.28(\mathrm{~m}, 5 \mathrm{H})$, $7.28-7.21(\mathrm{~m}, 1 \mathrm{H}), 7.17(\mathrm{td}, J=7.6,1.1 \mathrm{~Hz}, 1 \mathrm{H}), 5.12(\mathrm{dd}, J=29.7,12.3 \mathrm{~Hz}, 2 \mathrm{H})$, $5.01(\mathrm{q}, J=7.2 \mathrm{~Hz}, 1 \mathrm{H}), 4.93(\mathrm{~s}, 1 \mathrm{H}), 1.65(\mathrm{~s}, 9 \mathrm{H}), 1.52$ (d, $J=7.2 \mathrm{~Hz}, 3 \mathrm{H}), 1.44$ (s, 9H), 1.39 (s, 9H); ${ }^{13} \mathrm{C}$ NMR (100 MHz, $\left.\mathrm{CDCl}_{3}\right) \delta 171.4,170.5,165.2,161.5,148.6$, $142.6,135.4,132.0,128.5,128.4,128.3,125.4,123.5,123.3,118.9,115.0,104.7$, 90.8, 84.6, 81.9, 80.9, 67.1, 42.1, 40.1, 28.2, 28.0, 28.0, 13.9. HRMS (ESI) $\mathrm{m} / z$ calcd for $\mathrm{C}_{36} \mathrm{H}_{43} \mathrm{NO}_{9}[\mathrm{M}+\mathrm{Na}]^{+}=656.2836$, found $=656.2871$; The ee value was $80 \%, \mathrm{t}_{\mathrm{R}}$ $($ minor $)=7.9 \mathrm{~min}, \mathrm{t}_{\mathrm{R}}($ major $)=9.9 \mathrm{~min}($ Chiralcel IE, $\lambda=254 \mathrm{~nm}, 2 \% i$-PrOH$/$ hexanes, flow rate $=1.0 \mathrm{~mL} / \mathrm{min})$. 
G:IWJH-R633IWJH-R632-RAC-98-2-1.0-IE-30.Icd mAU

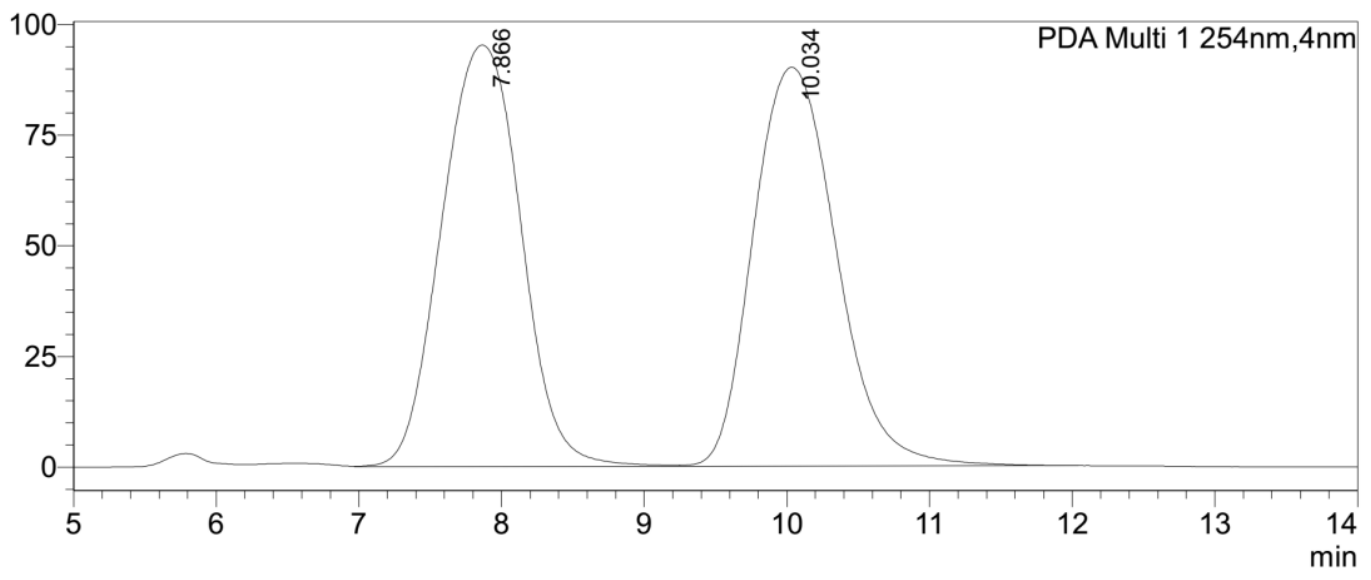

Peak Table

PDA Ch1 $254 \mathrm{~nm}$
\begin{tabular}{|r|r|r|r|r|}
\hline Peak\# & Ret. Time & Area & Height & \multicolumn{1}{|c|}{ Area $\%$} \\
\hline 1 & 7.866 & 3677773 & 95187 & 50.168 \\
\hline 2 & 10.034 & 3653193 & 90079 & 49.832 \\
\hline Total & & 7330966 & 185265 & 100.000 \\
\hline
\end{tabular}

G:IWJH-R633IWJH-R633-CHRI-1-98-2-1.0-IE-30.Icd mAU

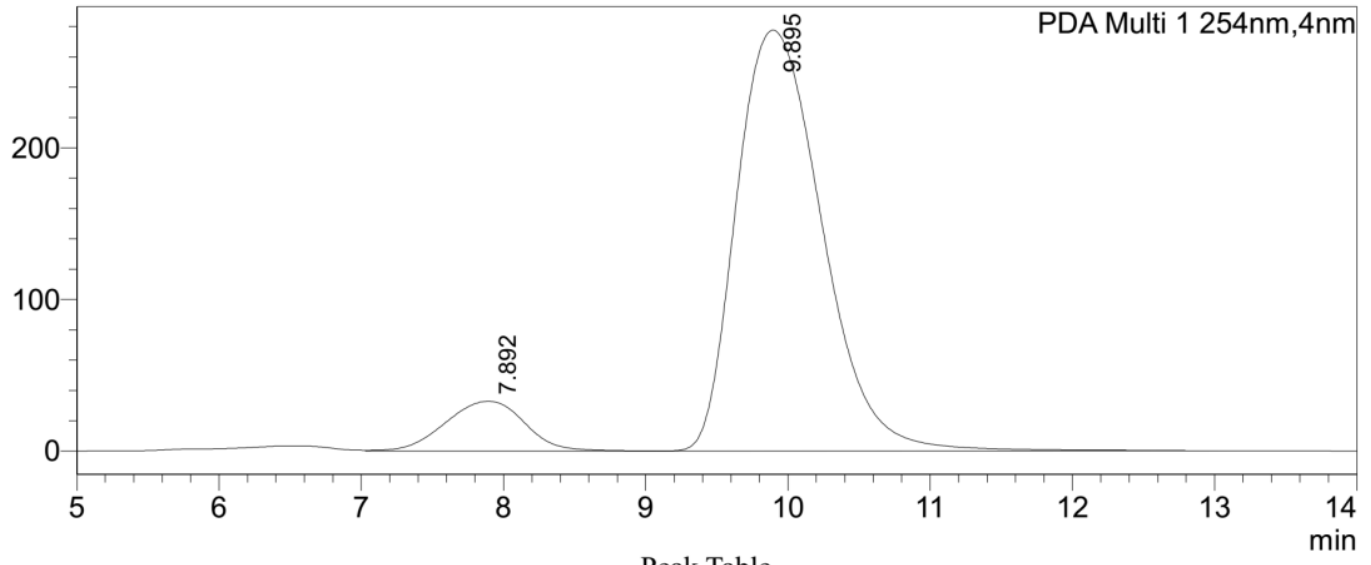

PDA Ch1 $254 \mathrm{~nm}$
\begin{tabular}{|r|r|r|r|r|}
\hline Peak\# & Ret. Time & \multicolumn{1}{|c|}{ Area } & Height & \multicolumn{1}{|c|}{ Area $\%$} \\
\hline 1 & 7.892 & 1281864 & 32827 & 9.934 \\
\hline 2 & 9.895 & 11622508 & 277476 & 90.066 \\
\hline Total & & 12904372 & 310303 & 100.000 \\
\hline
\end{tabular}

\section{4-benzyl 3,9-di-tert-butyl $\quad(S)$-2-( $(S)$-1-ethoxy-1-oxohexan-2-yl)pyrano[2,3-b]} indole-3,4,9 $(4 H)$-tricarboxylate $(4 \mathrm{~d})$ 


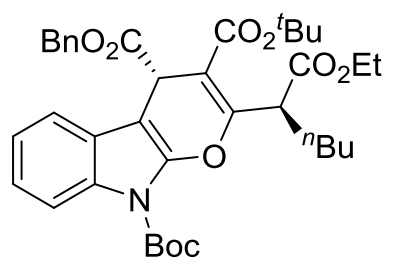

A colorless oil $(124 \mathrm{mg}, 96 \%) ;[\alpha]^{25}{ }_{\mathrm{D}}=-24.4\left(c\right.$ 1.0, $\left.\mathrm{CHCl}_{3}\right) ;{ }^{1} \mathrm{H}$ NMR $(400 \mathrm{MHz}$, $\left.\mathrm{CDCl}_{3}\right) \delta 8.15(\mathrm{~d}, J=8.2 \mathrm{~Hz}, 1 \mathrm{H}), 7.57(\mathrm{dd}, J=7.7,0.6 \mathrm{~Hz}, 1 \mathrm{H}), 7.34-7.29(\mathrm{~m}, 5 \mathrm{H})$, $7.27-7.22(\mathrm{~m}, 1 \mathrm{H}), 7.17(\mathrm{td}, J=7.6,1.1 \mathrm{~Hz}, 1 \mathrm{H}), 5.21-4.99(\mathrm{~m}, 3 \mathrm{H}), 4.90(\mathrm{~s}, 1 \mathrm{H})$, $4.24-4.05(\mathrm{~m}, 2 \mathrm{H}), 2.21-1.93(\mathrm{~m}, 2 \mathrm{H}), 1.64(\mathrm{~s}, 9 \mathrm{H}), 1.50-1.40(\mathrm{~m}, 11 \mathrm{H}), 1.39-$ $1.26(\mathrm{~m}, 2 \mathrm{H}), 1.19(\mathrm{t}, J=7.1 \mathrm{~Hz}, 3 \mathrm{H}), 0.88(\mathrm{t}, J=7.2 \mathrm{~Hz}, 3 \mathrm{H}) ;{ }^{13} \mathrm{C}$ NMR $(100 \mathrm{MHz}$, $\left.\mathrm{CDCl}_{3}\right) \delta 171.2,171.0,165.2,160.1,148.6,142.4,135.4,131.9,128.5,128.5,128.3$, 125.3, 123.6, 123.3, 118.8, 114.9, 106.3, 90.9, 84.5, 82.1, 67.2, 60.9, 46.0, 40.4, 28.8, 28.5, 28.2, 28.0, 22.5, 14.2, 13.8. HRMS (ESI) $\mathrm{m} / z$ calcd for $\mathrm{C}_{37} \mathrm{H}_{45} \mathrm{NO}_{9}[\mathrm{M}+\mathrm{Na}]^{+}=$ 670.2992 , found $=670.2993$; The ee value was $93 \%, t_{R}($ minor $)=11.0 \mathrm{~min}, \mathrm{t}_{\mathrm{R}}$ (major) $=13.5 \mathrm{~min}$ (Chiralcel IE, $\lambda=254 \mathrm{~nm}, 2 \% i$-PrOH$/$ hexanes, flow rate $=0.8 \mathrm{~mL} / \mathrm{min}$ ).

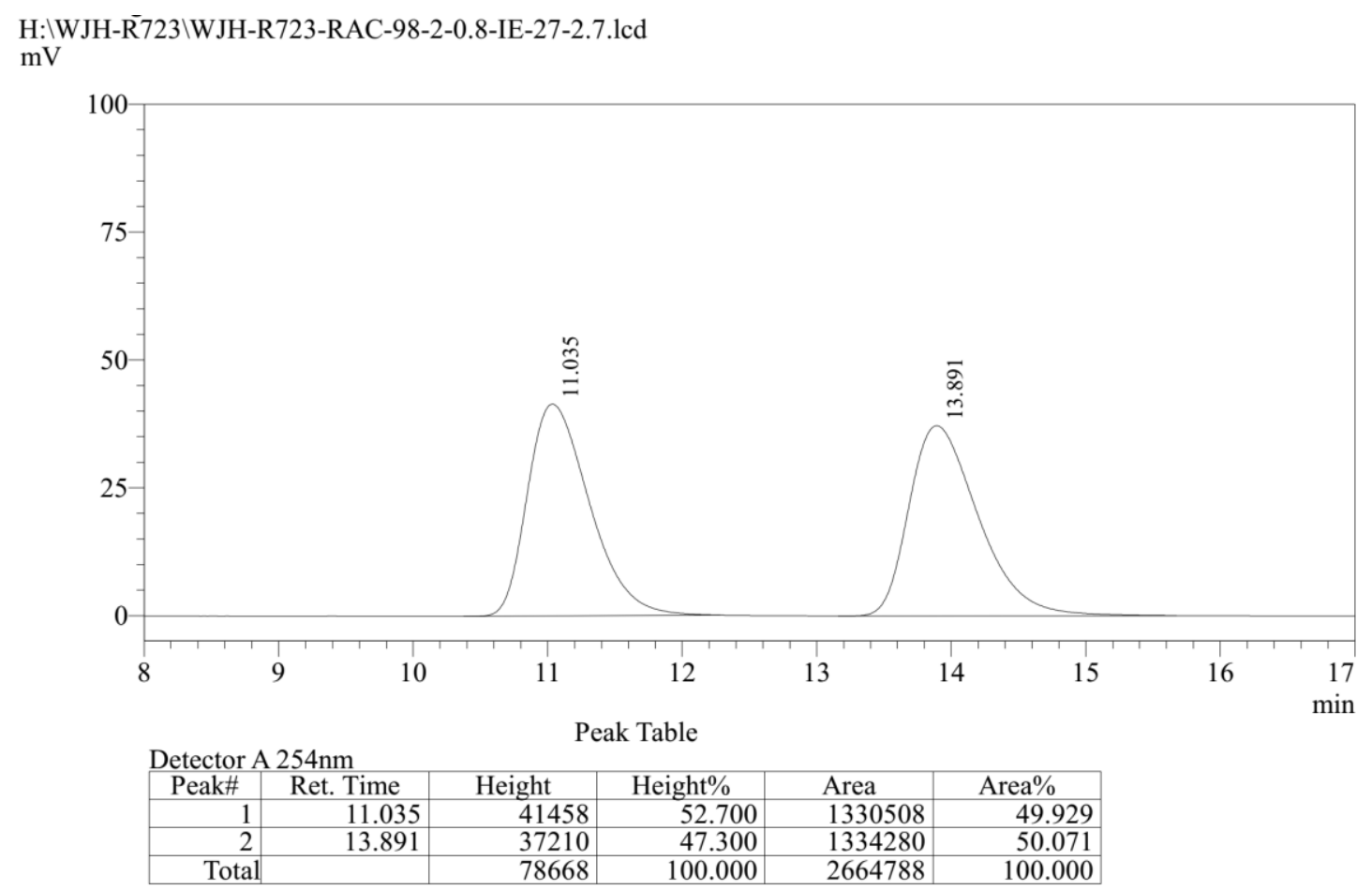




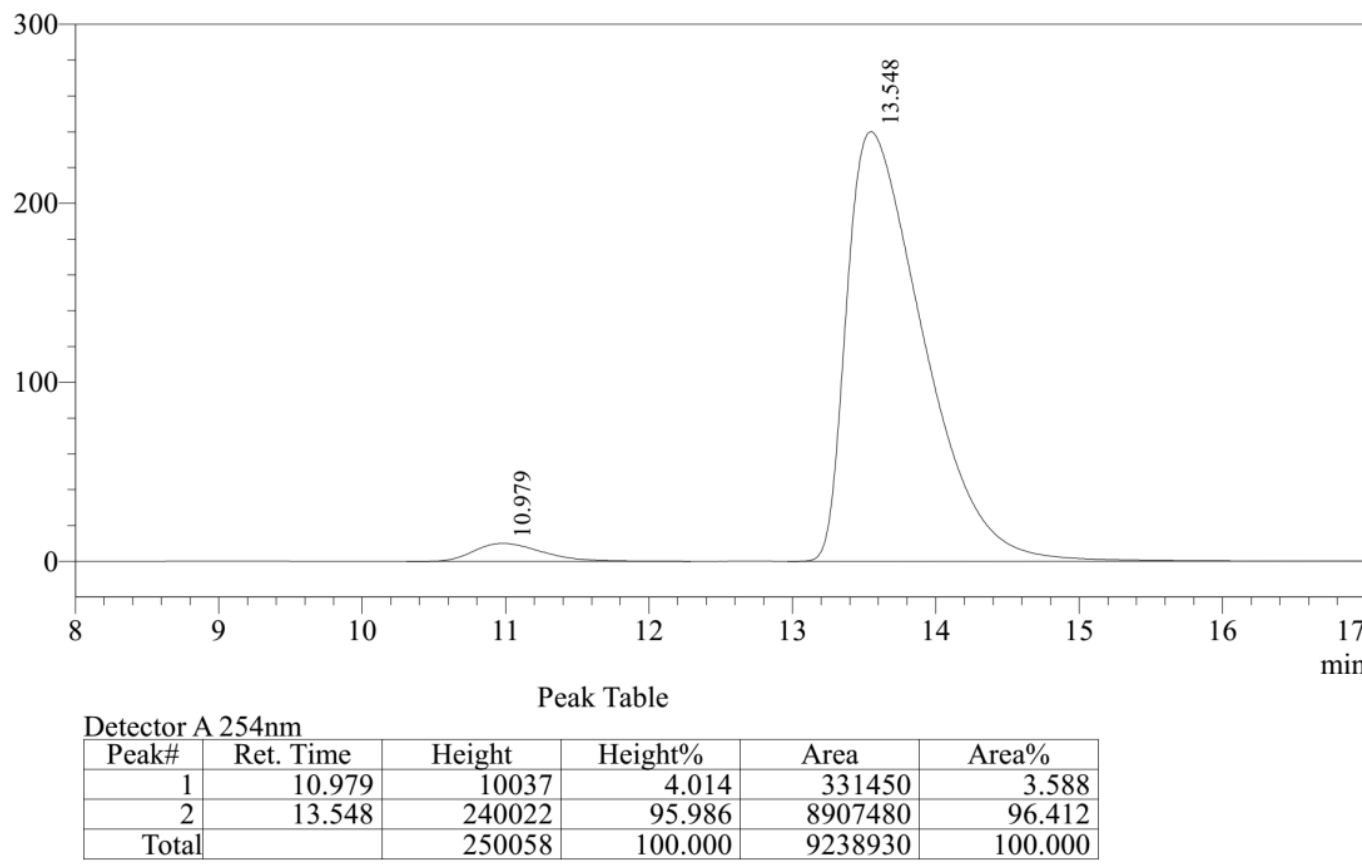

\section{4-benzyl 3,9-di-tert-butyl $(S)$-2-( $(S)$-1-ethoxy-1-oxo-3-phenylpropan-2-yl)pyrano}

\section{[2,3-b]indole-3,4,9(4H)-tricarboxylate $(4 \mathrm{e})$}

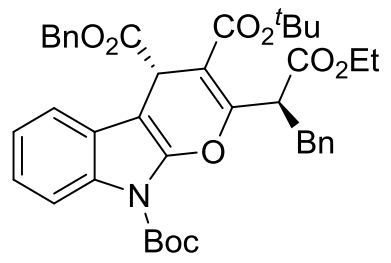

A colorless oil $(132 \mathrm{mg}, 97 \%) ;[\alpha]^{25}{ }_{\mathrm{D}}=-12.6\left(c 0.5, \mathrm{CHCl}_{3}\right) ;{ }^{1} \mathrm{H}$ NMR $(400 \mathrm{MHz}$, $\left.\mathrm{CDCl}_{3}\right) \delta 8.07(\mathrm{~d}, J=8.2 \mathrm{~Hz}, 1 \mathrm{H}), 7.48-7.44(\mathrm{~m}, 1 \mathrm{H}), 7.30-7.26(\mathrm{~m}, 2 \mathrm{H}), 7.23-$ $7.13(\mathrm{~m}, 9 \mathrm{H}), 7.08$ (dd, $J=11.1,4.0 \mathrm{~Hz}, 2 \mathrm{H}), 5.37$ (dd, $J=8.1,6.6 \mathrm{~Hz}, 1 \mathrm{H}), 4.96(\mathrm{dd}$, $J=45.5,12.2 \mathrm{~Hz}, 2 \mathrm{H}), 4.78(\mathrm{~s}, 1 \mathrm{H}), 4.17-3.99(\mathrm{~m}, 2 \mathrm{H}), 3.30(\mathrm{ddd}, J=22.0,13.9$, $7.3 \mathrm{~Hz}, 2 \mathrm{H}), 1.63(\mathrm{~s}, 9 \mathrm{H}), 1.27$ (s, 9H), 1.10 (t, $J=7.1 \mathrm{~Hz}, 3 \mathrm{H}) ;{ }^{13} \mathrm{C}$ NMR (100 MHz, $\left.\mathrm{CDCl}_{3}\right) \delta 170.9,170.3,164.8,159.3,148.6,142.3,138.7,135.5,131.9,129.3,128.4$, $128.2,128.2,126.3,125.2,123.6,123.3,118.9,114.9,106.2,91.0,84.7,82.0,67.0$, 61.1, 48.0, 40.3, 35.0, 28.3, 27.8, 14.2. HRMS (ESI) $\mathrm{m} / \mathrm{z}$ calcd for $\mathrm{C}_{40} \mathrm{H}_{43} \mathrm{NO}_{9}$ $[\mathrm{M}+\mathrm{Na}]^{+}=704.2836$, found $=704.2835$; The ee value was $90 \%, \mathrm{t}_{\mathrm{R}}($ minor $)=11.3 \mathrm{~min}$, 
$\mathrm{t}_{\mathrm{R}}($ major $)=12.2 \mathrm{~min}($ Chiralcel IE, $\lambda=254 \mathrm{~nm}, 3 \% i-\mathrm{PrOH} / \mathrm{h}$ exanes, flow rate $=0.8$ $\mathrm{mL} / \mathrm{min})$.

H:IWJH-R̄722 \WJH-R722-RAC-97-3-0.8-IE-26.1-2.7.lcd

$\mathrm{mV}$

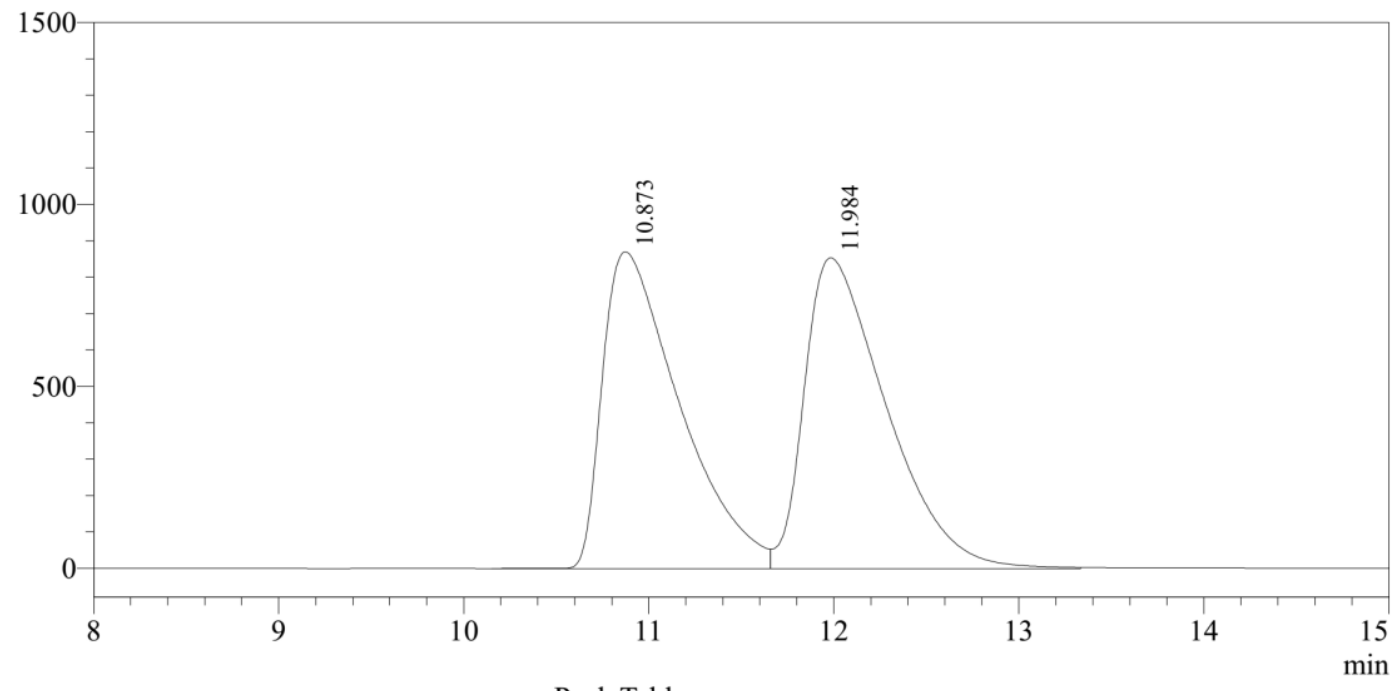

Peak Table

Detector A 254nm
\begin{tabular}{|r|r|r|r|r|r|}
\hline Peak\# & Ret. Time & \multicolumn{1}{|c|}{ Height } & Height $\%$ & Area & \multicolumn{1}{c|}{ Area $\%$} \\
\hline 1 & 10.873 & 870630 & 50.478 & 25222864 & 49.016 \\
\hline 2 & 11.984 & 854140 & 49.522 & 26235686 & 50.984 \\
\hline Total & & 1724769 & 100.000 & 51458550 & 100.000 \\
\hline
\end{tabular}

H:IWJH-R̄722\WJH-R722-CHRI-97-3-0.8-IE-26.1-2.7.lcd

$\mathrm{mV}$

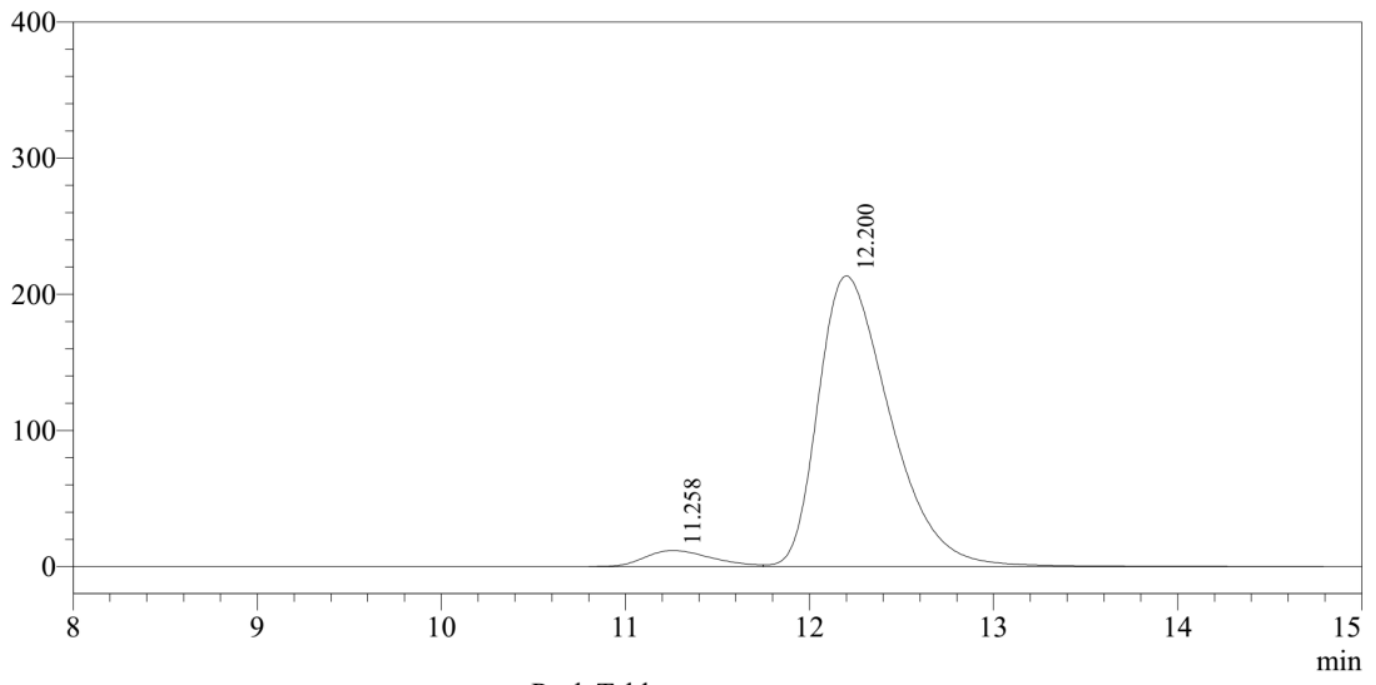

Peak Table

\begin{tabular}{l} 
Detector A 254nm \\
\hline Peak\#
\end{tabular}




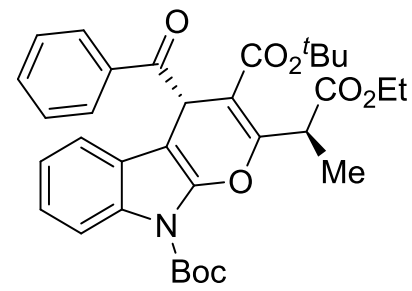

A white solid $(104 \mathrm{mg}, 90 \%) ;[\alpha]^{25}{ }_{\mathrm{D}}=+36.9\left(c 0.8, \mathrm{CHCl}_{3}\right) ;{ }^{1} \mathrm{H}$ NMR $(400 \mathrm{MHz}$, $\left.\mathrm{CDCl}_{3}\right) \delta 8.12-8.06(\mathrm{~m}, 3 \mathrm{H}), 7.61(\mathrm{t}, J=7.4 \mathrm{~Hz}, 1 \mathrm{H}), 7.50(\mathrm{t}, J=7.6 \mathrm{~Hz}, 2 \mathrm{H}), 7.15$ (ddd, $J=8.5,6.9,1.7 \mathrm{~Hz}, 1 \mathrm{H}), 7.04-6.94(\mathrm{~m}, 2 \mathrm{H}), 5.74(\mathrm{~s}, 1 \mathrm{H}), 5.07$ (q, $J=7.2 \mathrm{~Hz}$, 1H), $4.25-4.12(\mathrm{~m}, 2 \mathrm{H}), 1.66(\mathrm{~s}, 9 \mathrm{H}), 1.63(\mathrm{~d}, J=7.2 \mathrm{~Hz}, 3 \mathrm{H}), 1.33$ (s, 9H), 1.22 (t, $J=7.1 \mathrm{~Hz}, 3 \mathrm{H}) ;{ }^{13} \mathrm{C} \mathrm{NMR}\left(100 \mathrm{MHz}, \mathrm{CDCl}_{3}\right) \delta 199.1,171.4,165.1,161.3,148.6$, $142.5,136.9,133.2,131.9,129.0,128.6,125.1,123.4,123.2,118.4,114.9,106.6$, 92.0, 84.6, 82.5, 61.0, 42.6, 41.5, 28.2, 28.0, 14.2, 14.1. HRMS (ESI) $\mathrm{m} / z$ calcd for $\mathrm{C}_{33} \mathrm{H}_{37} \mathrm{NO}_{8}[\mathrm{M}+\mathrm{Na}]^{+}=598.2417$, found $=598.2411$; The ee value was 98\%, $\mathrm{t}_{\mathrm{R}}$ (minor) $=14.8 \mathrm{~min}, \mathrm{t}_{\mathrm{R}}($ major $)=17.8 \mathrm{~min}($ Chiralcel IE, $\lambda=254 \mathrm{~nm}, 3 \% i$-PrOH$/$ hexanes, flow rate $=1.0 \mathrm{~mL} / \mathrm{min})$.

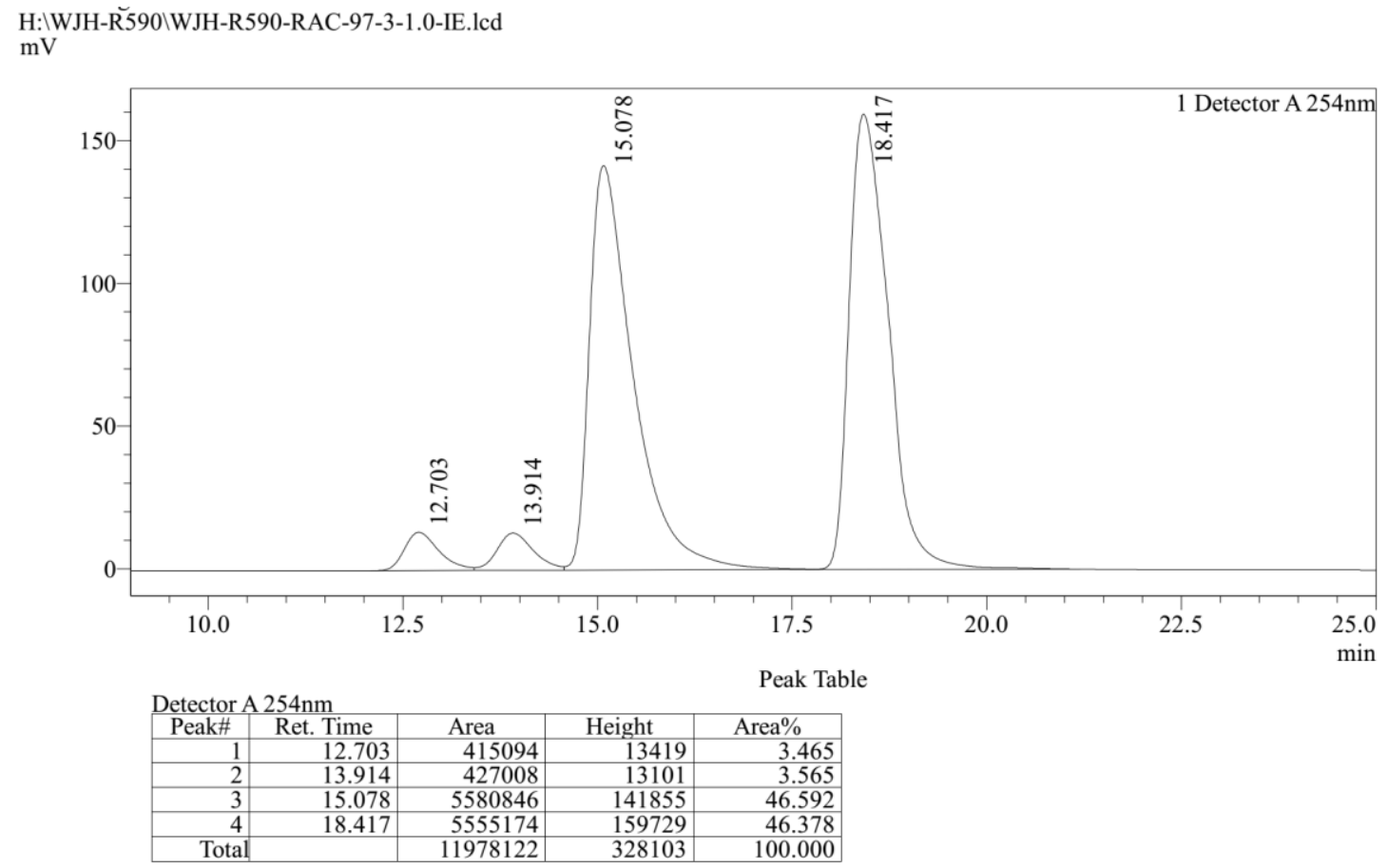


D:LLC-DatalWJH\WJH-R595\WJH-R595-14.lcd $\mathrm{mV}$

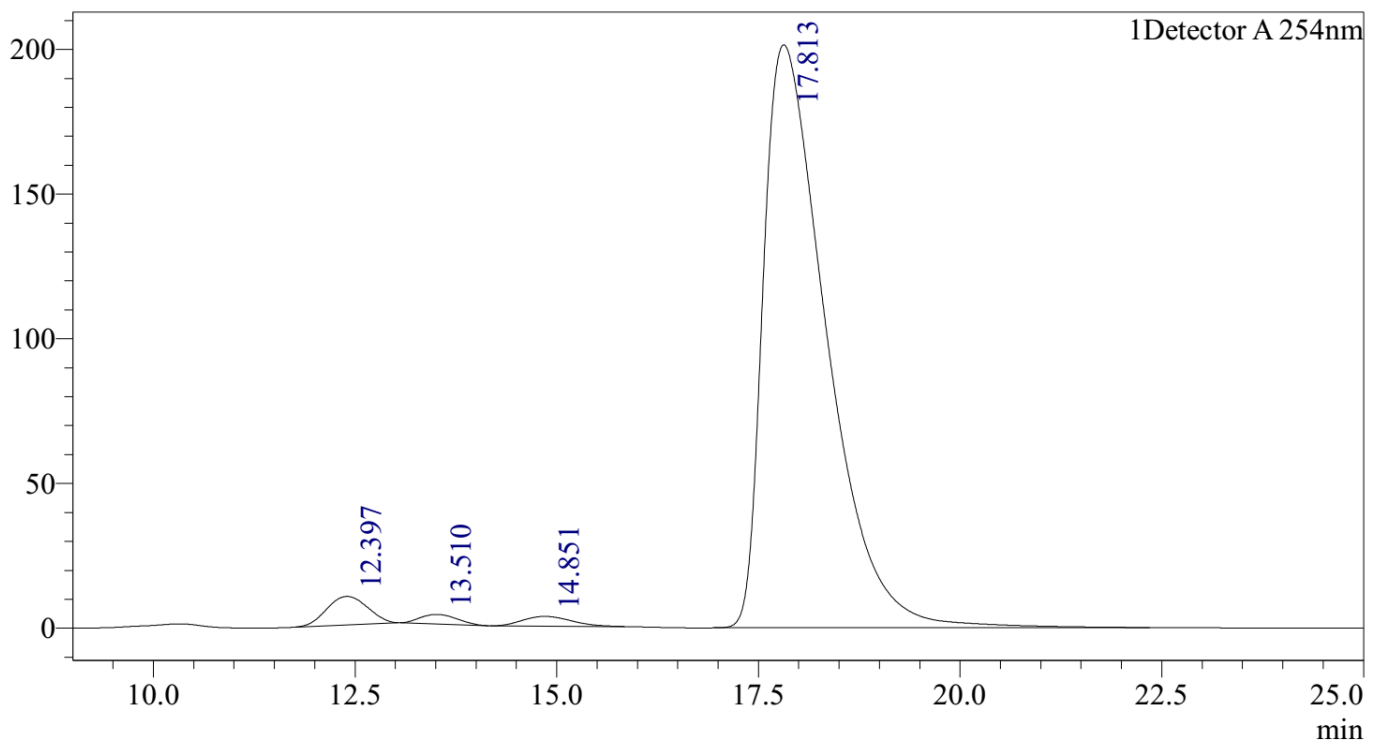

Detector A 254nm
\begin{tabular}{r|r|r|r|r|r|} 
Peak Table \\
\hline Peak\# & Ret. Time & Area & Height & Height $\%$ & Area $\%$ \\
\hline 1 & 12.397 & 346970 & 9868 & 4.526 & 3.005 \\
\hline 2 & 13.510 & 107233 & 3342 & 1.533 & 0.929 \\
\hline 3 & 14.851 & 140876 & 3349 & 1.536 & 1.220 \\
\hline 4 & 17.813 & 10952517 & 201477 & 92.406 & 94.847 \\
\hline Total & & 11547597 & 218035 & 100.000 & 100.000 \\
\hline
\end{tabular}

di-tert-butyl $(S)$-2-((S)-1-ethoxy-1-oxopropan-2-yl)-4-(2-methoxybenzoyl)pyrano [2,3-b]indole-3,9(4H)-dicarboxylate $(6 \mathrm{~b})$

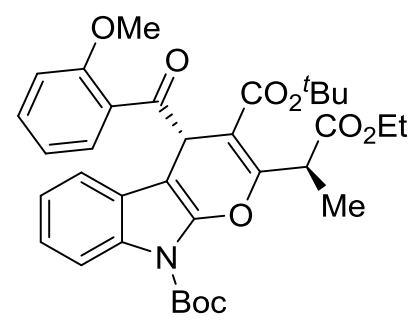

A white solid $(116 \mathrm{mg}, 96 \%) ;[\alpha]^{25}=+20.6\left(c 0.8, \mathrm{CHCl}_{3}\right) ;{ }^{1} \mathrm{H}$ NMR $(400 \mathrm{MHz}$, $\left.\mathrm{CDCl}_{3}\right) \delta 8.10(\mathrm{~d}, J=8.3 \mathrm{~Hz}, 1 \mathrm{H}), 7.61(\mathrm{dd}, J=7.6,1.7 \mathrm{~Hz}, 1 \mathrm{H}), 7.46(\mathrm{ddd}, J=9.0$, 7.5, $1.8 \mathrm{~Hz}, 1 \mathrm{H}), 7.15$ (ddd, $J=8.4,6.1,2.5 \mathrm{~Hz}, 1 \mathrm{H}), 7.05-7.01$ (m, 2H), $7.00-6.92$ (m, 2H), $5.80(\mathrm{~s}, 1 \mathrm{H}), 5.00$ (q, $J=7.2 \mathrm{~Hz}, 1 \mathrm{H}), 4.24-4.06(\mathrm{~m}, 2 \mathrm{H}), 3.77$ (s, 3H), 1.65 (s, 9H), $1.57(\mathrm{~d}, J=7.2 \mathrm{~Hz}, 3 \mathrm{H}), 1.39$ (s, 9H), $1.18(\mathrm{t}, J=7.1 \mathrm{~Hz}, 3 \mathrm{H}) ;{ }^{13} \mathrm{C}$ NMR (100 $\left.\mathrm{MHz}, \mathrm{CDCl}_{3}\right) \delta 200.2,171.5,165.4,160.9,157.8,148.7,142.7,133.3,131.9,130.9$, $128.2,125.5,123.1,123.1,120.5,118.4,114.7,111.4,106.3,92.3,84.4,82.0,60.9$, 
55.3, 46.0, 41.4, 28.2, 28.0, 14.2. HRMS (ESI) $m / z$ calcd for $\mathrm{C}_{34} \mathrm{H}_{39} \mathrm{NO}_{9}[\mathrm{M}+\mathrm{Na}]^{+}=$ 628.2523 , found $=628.2522 ;$ The ee value was $97 \%, \mathrm{t}_{\mathrm{R}}($ major $)=18.9 \mathrm{~min}, \mathrm{t}_{\mathrm{R}}($ minor $)$ $=28.9 \mathrm{~min}($ Chiralcel IG, $\lambda=254 \mathrm{~nm}, 3 \% i-\mathrm{PrOH} / \mathrm{hexanes}$, flow rate $=1.0 \mathrm{~mL} / \mathrm{min})$.

D: \LC-Dă̌a $\backslash W J H \backslash W J H-R 640-680 \backslash W J H-R 662-R A C-97-3-1.0-2.8-I G--106-A U T O 01.1 c d$ $\mathrm{mV}$

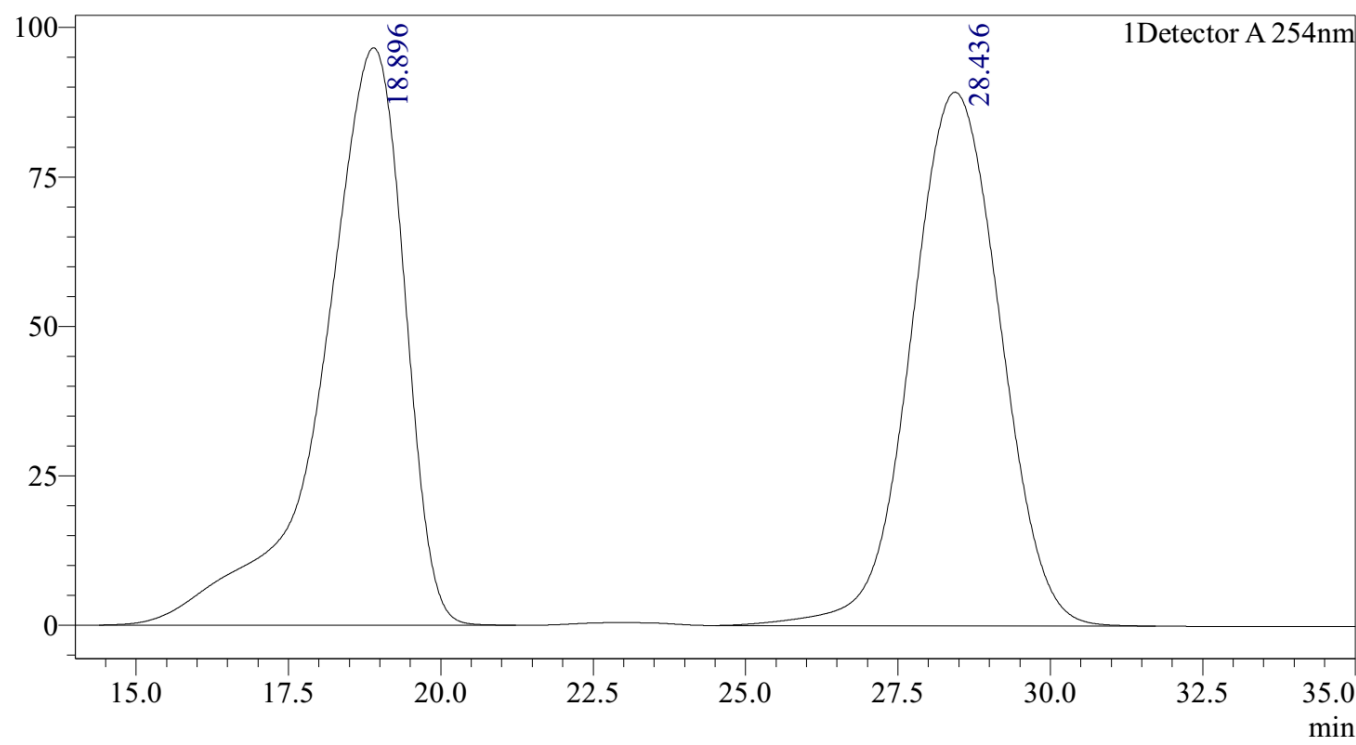

Peak Table

Detector A $254 \mathrm{~nm}$
\begin{tabular}{|r|r|r|r|r|r|}
\hline Peak\# & Ret. Time & \multicolumn{1}{|c|}{ Area } & Height & Height $\%$ & \multicolumn{1}{c|}{ Area $\%$} \\
\hline 1 & 18.896 & 9136237 & 96641 & 51.974 & 50.068 \\
\hline 2 & 28.436 & 9111269 & 89300 & 48.026 & 49.932 \\
\hline Total & & 18247506 & 185941 & 100.000 & 100.000 \\
\hline
\end{tabular}

H: IWJH-Ř662 \WJH-R662-CHRI-97-3-1.0-IG-30-2.9-106.1cd $\mathrm{mV}$

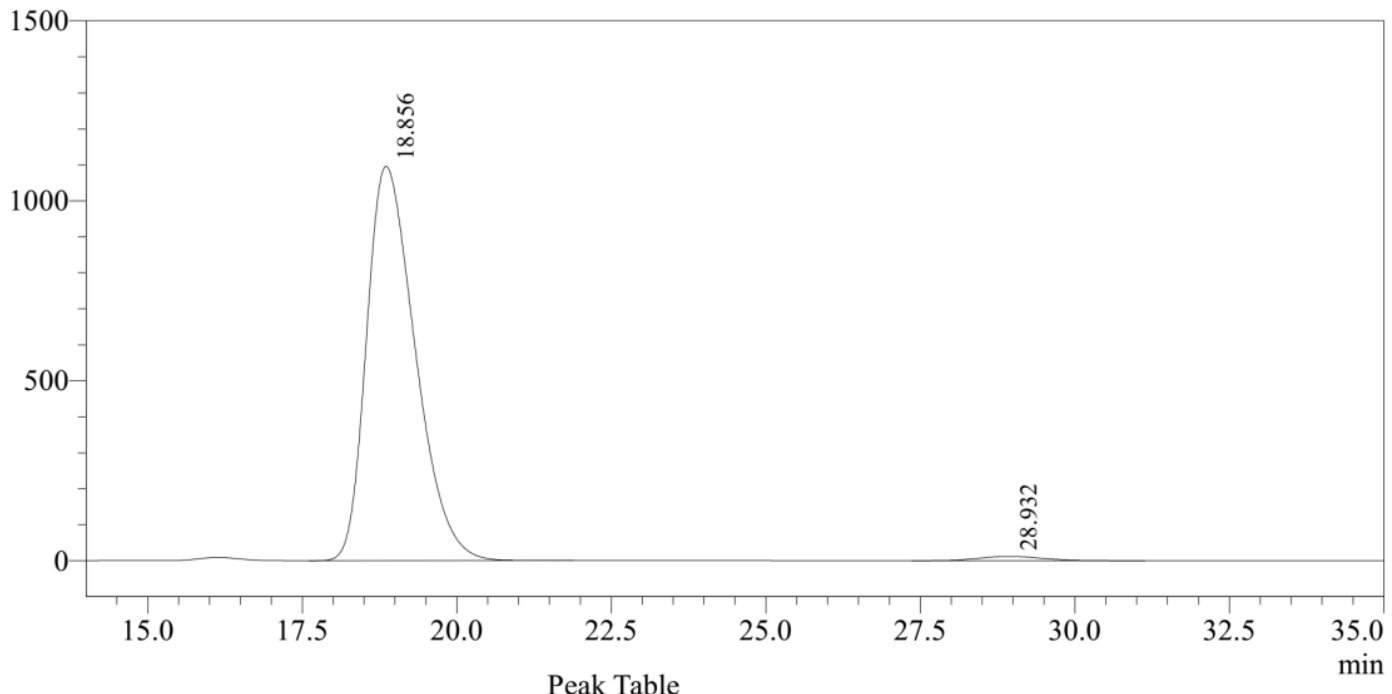

Detector A $254 \mathrm{~nm}$
\begin{tabular}{|r|r|r|r|r|r|}
\hline Peak\# & Ret. Time & \multicolumn{1}{|c|}{ Height } & Height $\%$ & \multicolumn{1}{c|}{ Area } & \multicolumn{1}{|c|}{ Area\% } \\
\hline 1 & 18.856 & 1095748 & 98.915 & 60011883 & 98.583 \\
\hline 2 & 28.932 & 12017 & 1.085 & 862830 & 1.417 \\
\hline Total & & 1107764 & 100.000 & 60874712 & 100.000 \\
\hline
\end{tabular}


di-tert-butyl $(S)$-2-((S)-1-ethoxy-1-oxopropan-2-yl)-4-(3-Sfluorobenzoyl)pyrano [2,3-b]indole-3,9(4H)-dicarboxylate $(6 \mathrm{c})$

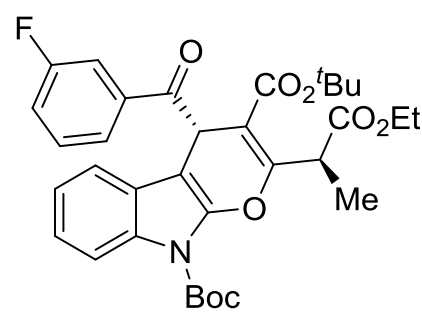

A white solid $(109 \mathrm{mg}, 92 \%) ;[\alpha]^{25}{ }_{\mathrm{D}}=+29.9\left(c 0.8, \mathrm{CHCl}_{3}\right) ;{ }^{1} \mathrm{H}$ NMR $(400 \mathrm{MHz}$, $\left.\mathrm{CDCl}_{3}\right) \delta 8.11(\mathrm{~d}, J=8.3 \mathrm{~Hz}, 1 \mathrm{H}), 7.90(\mathrm{~d}, J=7.8 \mathrm{~Hz}, 1 \mathrm{H}), 7.75-7.68(\mathrm{~m}, 1 \mathrm{H}), 7.49$ $(\mathrm{td}, J=8.0,5.6 \mathrm{~Hz}, 1 \mathrm{H}), 7.34-7.27(\mathrm{~m}, 1 \mathrm{H}), 7.17(\mathrm{ddd}, J=8.5,7.1,1.5 \mathrm{~Hz}, 1 \mathrm{H})$, $7.01(\mathrm{dtd}, J=8.6,7.9,0.9 \mathrm{~Hz}, 2 \mathrm{H}), 5.66(\mathrm{~s}, 1 \mathrm{H}), 5.04(\mathrm{q}, J=7.2 \mathrm{~Hz}, 1 \mathrm{H}), 4.22-4.11$ (m, 2H), $1.66(\mathrm{~s}, 9 \mathrm{H}), 1.62(\mathrm{~d}, J=7.2 \mathrm{~Hz}, 3 \mathrm{H}), 1.34$ (s, 9H), $1.22(\mathrm{t}, J=7.1 \mathrm{~Hz}, 3 \mathrm{H})$; ${ }^{13} \mathrm{C} \mathrm{NMR}\left(100 \mathrm{MHz}, \mathrm{CDCl}_{3}\right) \delta 197.7,171.3,165.0,162.6(\mathrm{~d}, J=248.2 \mathrm{~Hz}), 161.4$, 148.5, 142.5, 138.8 (d, $J=6.2 \mathrm{~Hz}), 131.9,130.3(\mathrm{~d}, J=7.6 \mathrm{~Hz}), 125.0,124.7$ (d, $J=$ $3.0 \mathrm{~Hz}), 123.5,123.3,120.2(\mathrm{~d}, J=21.3 \mathrm{~Hz}), 118.2,115.7$ (d, $J=22.5 \mathrm{~Hz}), 115.0$, 106.5, 91.7, 84.7, 82.8, 61.0, 43.0, 41.5, 28.2, 28.0, 14.2, 14.1; ${ }^{19} \mathrm{~F}$ NMR (376 MHz, $\left.\mathrm{CDCl}_{3}\right) \delta$-111.4. HRMS (ESI) $m / z$ calcd for $\mathrm{C}_{33} \mathrm{H}_{36} \mathrm{FNO}_{8}[\mathrm{M}+\mathrm{Na}]^{+}=616.2323$, found $=616.2326$; The ee value was $95 \%, \mathrm{t}_{\mathrm{R}}($ minor $)=9.2 \mathrm{~min}, \mathrm{t}_{\mathrm{R}}($ major $)=11.5 \mathrm{~min}$ (Chiralcel IE, $\lambda=254 \mathrm{~nm}, 3 \% i$-PrOH$/$ hexanes, flow rate $=1.0 \mathrm{~mL} / \mathrm{min}$ ). 
G:IWJH-R663IWJH-R663-RAC-97-3-1.0-IE-30-3.7-202.Icd $\mathrm{mAU}$

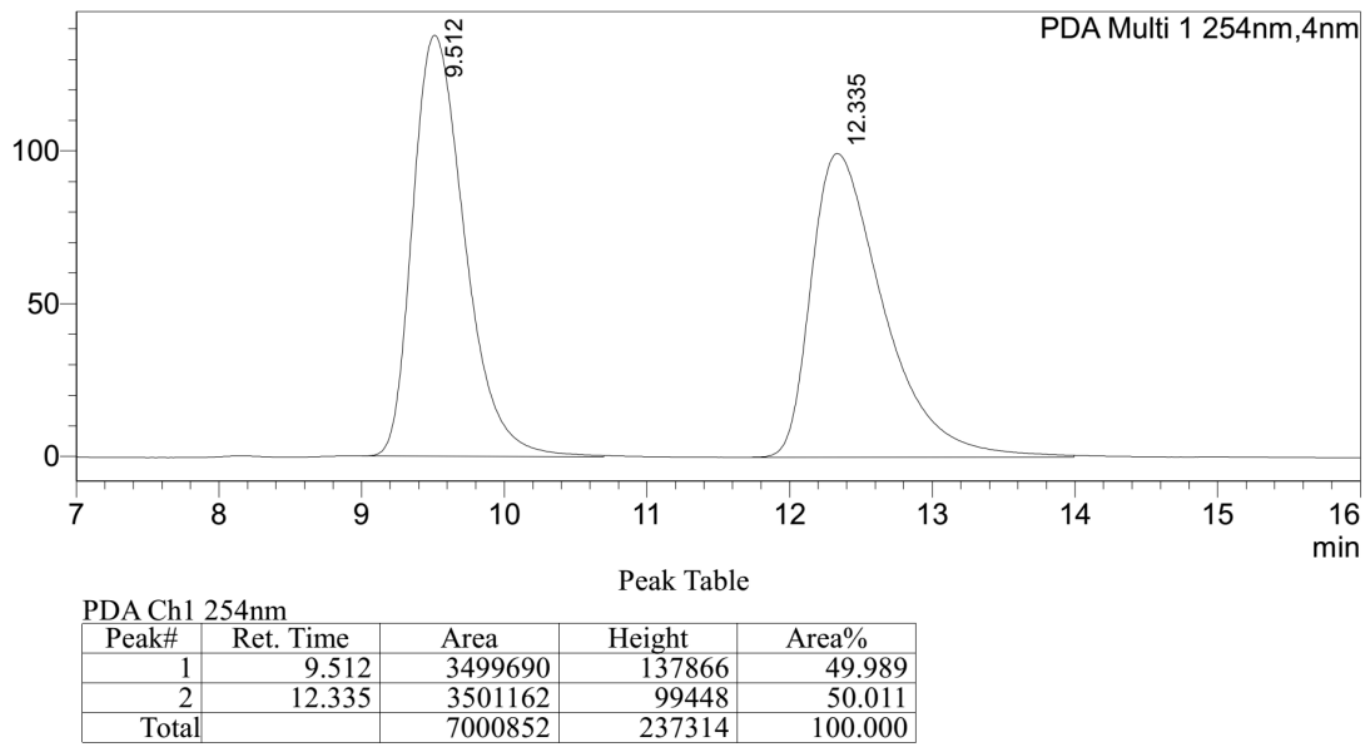

H:\WJH-Ř663 \WJH-R663-CHRI-97-3-1.0-IE-30-3.3-106.lcd $\mathrm{mV}$

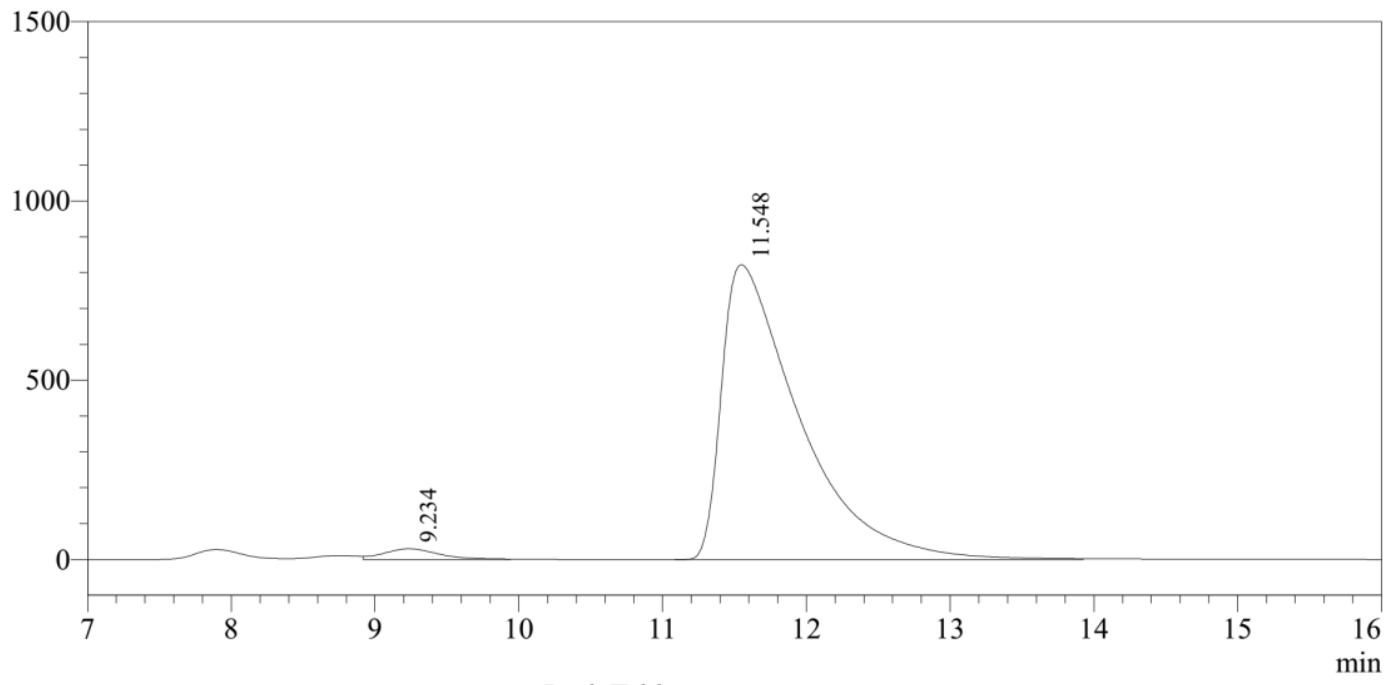

Peak Table

\begin{tabular}{|c|c|c|c|c|c|}
\hline Peak\# & Ret. Time & Height & Height $\%$ & Area & Area $\%$ \\
\hline r & 9.234 & 29472 & 3.461 & 794184 & 2.502 \\
\hline 2 & 11.548 & 821959 & 96.539 & 30948685 & 97.498 \\
\hline Total & & 851430 & 100.000 & 31742869 & 100.000 \\
\hline
\end{tabular}

di-tert-butyl $(S)$-4-(3-chlorobenzoyl)-2-((S)-1-ethoxy-1-oxopropan-2-yl)pyrano [2,3-b]indole-3,9(4H)-dicarboxylate $(6 \mathrm{~d})$ 


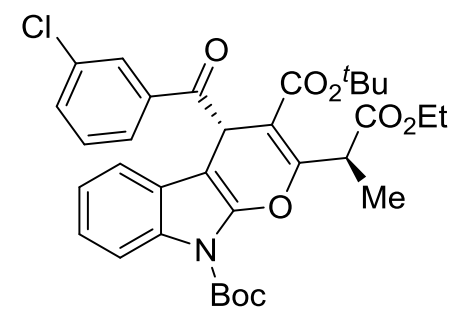

A white solid $(117 \mathrm{mg}, 96 \%)$; $[\alpha]^{25}{ }_{\mathrm{D}}=+12.3\left(\mathrm{c} 1.5, \mathrm{CHCl}_{3}\right) ;{ }^{1} \mathrm{H}$ NMR $(400 \mathrm{MHz}$, $\left.\mathrm{CDCl}_{3}\right) \delta 8.11(\mathrm{~d}, J=8.3 \mathrm{~Hz}, 1 \mathrm{H}), 8.01(\mathrm{t}, J=1.8 \mathrm{~Hz}, 1 \mathrm{H}), 7.97-7.93(\mathrm{~m}, 1 \mathrm{H}), 7.56$ $(\mathrm{ddd}, J=8.0,2.0,1.0 \mathrm{~Hz}, 1 \mathrm{H}), 7.44(\mathrm{t}, J=7.9 \mathrm{~Hz}, 1 \mathrm{H}), 7.18(\mathrm{ddd}, J=8.5,7.2,1.5 \mathrm{~Hz}$, 1H), $7.08-6.98(\mathrm{~m}, 2 \mathrm{H}), 5.63(\mathrm{~s}, 1 \mathrm{H}), 5.06(\mathrm{q}, J=7.2 \mathrm{~Hz}, 1 \mathrm{H}), 4.24-4.11(\mathrm{~m}, 2 \mathrm{H})$, $1.66(\mathrm{~s}, 9 \mathrm{H}), 1.62(\mathrm{~d}, J=7.2 \mathrm{~Hz}, 3 \mathrm{H}), 1.36(\mathrm{~s}, 9 \mathrm{H}), 1.22(\mathrm{t}, J=7.1 \mathrm{~Hz}, 3 \mathrm{H}) ;{ }^{13} \mathrm{C} \mathrm{NMR}$ $\left(100 \mathrm{MHz}, \mathrm{CDCl}_{3}\right) \delta 197.6,171.2,164.9,161.4,148.5,142.4,138.3,134.7,133.0$, $131.9,129.9,128.9,127.0,124.9,123.6,123.4,118.3,115.0,106.5,91.7,84.7,82.8$, 61.0, 43.2, 41.5, 28.2, 28.0, 14.2, 14.1. HRMS (ESI) $\mathrm{m} / z$ calcd for $\mathrm{C}_{33} \mathrm{H}_{36} \mathrm{ClNO}_{8}$ $[\mathrm{M}+\mathrm{Na}]^{+}=632.2027$, found $=632.2024$; The ee value was $96 \%, \mathrm{t}_{\mathrm{R}}($ minor $)=20.1 \mathrm{~min}$, $\mathrm{t}_{\mathrm{R}}($ major $)=23.9 \mathrm{~min}($ Chiralcel IE-ID, $\lambda=254 \mathrm{~nm}, 8 \% i$-PrOH/hexanes, flow rate $=$ $0.6 \mathrm{~mL} / \mathrm{min})$.

G:IWJH-R664IWJH-R664-RAC-92-8-0.6-IE-ID-30-4.0-202.Icd mAU

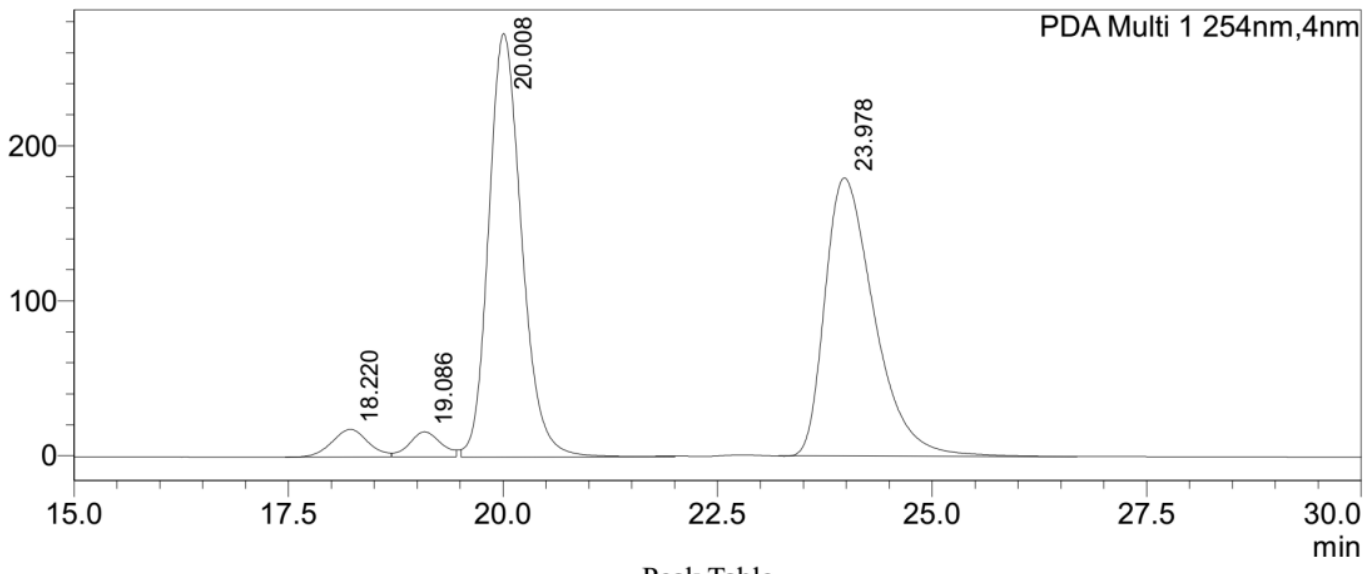

Peak Table

\begin{tabular}{|c|c|c|c|c|}
\hline \multicolumn{5}{|c|}{ DA Ch1 $254 \mathrm{~nm}$} \\
\hline Peak\# & Ret. Time & Area & Height & Area $\%$ \\
\hline 1 & 18.220 & 523995 & 17840 & 3.40 \\
\hline 2 & 19.086 & 415198 & 16198 & 2.694 \\
\hline 3 & & 731 & & 47.476 \\
\hline 4 & 23.978 & 7155599 & 179255 & 46.430 \\
\hline Total & & 15411551 & 486545 & 100.000 \\
\hline
\end{tabular}


H:|WJH-Ř664|WJH-R664-CHRI-92-8-0.6-IE-ID-30-3.6-106.lcd $\mathrm{mV}$

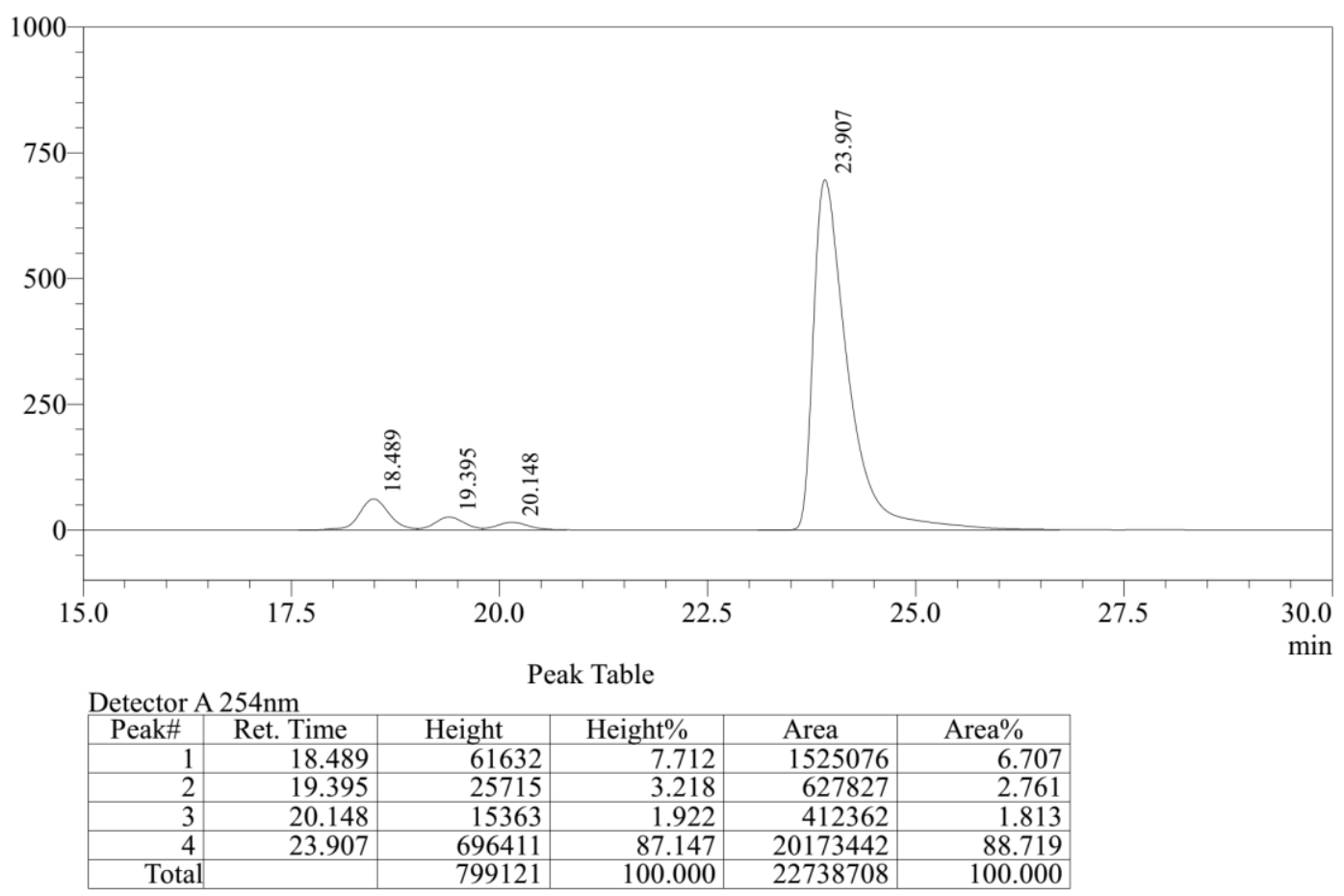

di-tert-butyl $(S)$-4-(3-bromobenzoyl)-2-((S)-1-ethoxy-1-oxopropan-2-yl)pyrano [2,3-b]indole-3,9(4H)-dicarboxylate $(6 \mathrm{e})$

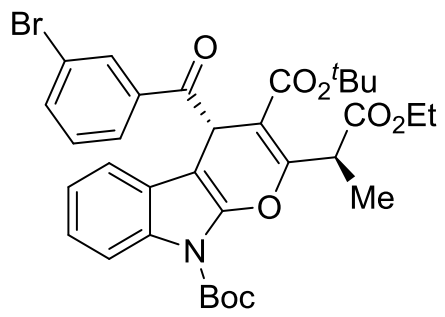

A white solid (122mg, 93\%); $[\alpha]^{25}{ }_{\mathrm{D}}=+35.3\left(c\right.$ 1.0, $\left.\mathrm{CHCl}_{3}\right) ;{ }^{1} \mathrm{H}$ NMR (400 MHz, $\left.\mathrm{CDCl}_{3}\right) \delta 8.17(\mathrm{t}, J=1.7 \mathrm{~Hz}, 1 \mathrm{H}), 8.11(\mathrm{~d}, J=8.3 \mathrm{~Hz}, 1 \mathrm{H}), 8.01-7.95(\mathrm{~m}, 1 \mathrm{H}), 7.71$ $(\mathrm{ddd}, J=8.0,1.8,0.9 \mathrm{~Hz}, 1 \mathrm{H}), 7.37$ (t, $J=7.9 \mathrm{~Hz}, 1 \mathrm{H}), 7.18(\mathrm{ddd}, J=8.4,7.1,1.5 \mathrm{~Hz}$, 1H), $7.04(\mathrm{dtd}, J=8.6,7.8,0.9 \mathrm{~Hz}, 2 \mathrm{H}), 5.62(\mathrm{~s}, 1 \mathrm{H}), 5.06(\mathrm{q}, J=7.2 \mathrm{~Hz}, 1 \mathrm{H}), 4.25-$ $4.11(\mathrm{~m}, 2 \mathrm{H}), 1.66(\mathrm{~s}, 9 \mathrm{H}), 1.62(\mathrm{~d}, J=7.2 \mathrm{~Hz}, 3 \mathrm{H}), 1.36(\mathrm{~s}, 9 \mathrm{H}), 1.22(\mathrm{t}, J=7.1 \mathrm{~Hz}$, $3 \mathrm{H}) ;{ }^{13} \mathrm{C} \mathrm{NMR}\left(100 \mathrm{MHz}, \mathrm{CDCl}_{3}\right) \delta 197.5,171.2,164.9,161.4,148.5,142.4,138.5$, $135.9,131.9,131.8,130.2,127.4,124.9,123.6,123.4,122.7,118.3,115.0,106.4$, 91.7, 84.7, 82.8, 61.0, 43.3, 41.5, 28.2, 28.0, 14.2, 14.2. HRMS (ESI) $\mathrm{m} / \mathrm{z}$ calcd for $\mathrm{C}_{33} \mathrm{H}_{36} \mathrm{BrNO}_{8}[\mathrm{M}+\mathrm{Na}]^{+}=676.1522$, found $=676.1516$; The ee value was $95 \%, \mathrm{t}_{\mathrm{R}}$ 
$($ minor $)=23.0 \mathrm{~min}, \mathrm{t}_{\mathrm{R}}($ major $)=28.9 \mathrm{~min}($ Chiralcel IE-ID, $\lambda=254 \mathrm{~nm}, 6 \%$ $i$ - $\mathrm{PrOH} / \mathrm{hexanes,}$ flow rate $=0.6 \mathrm{~mL} / \mathrm{min})$.

H:IWJH-R665IWJH-R665-RAC-94-6-0.6-IE-ID-30-3.8-202.Icd mAU

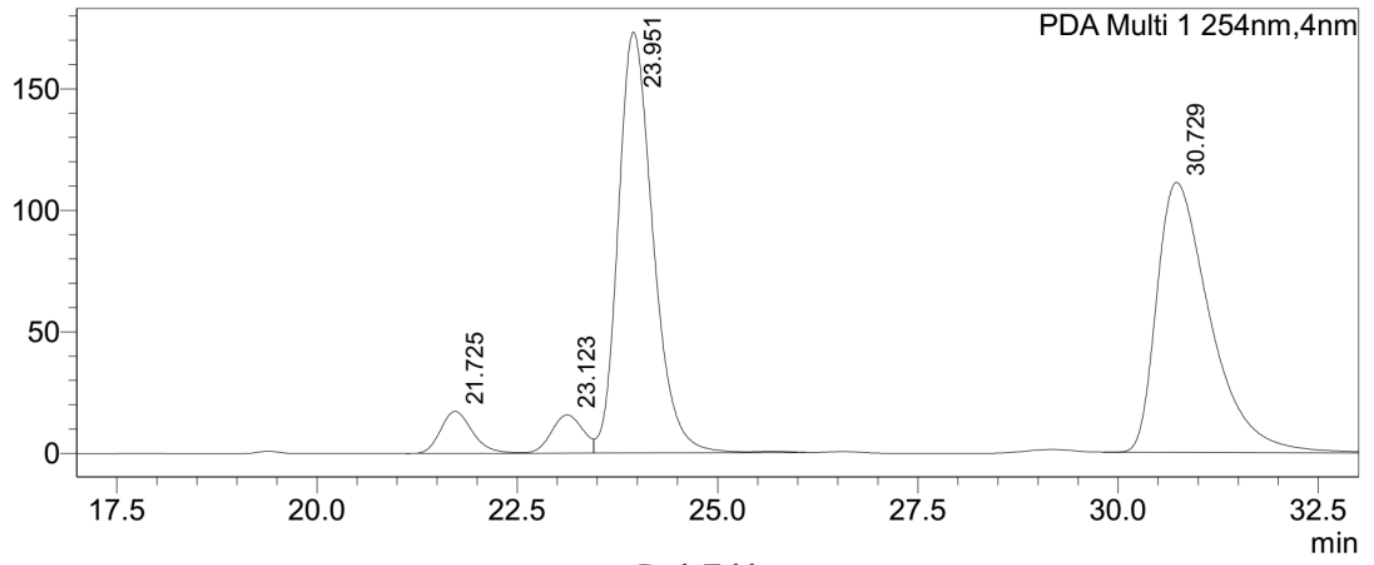

Peak Table

PDA Ch1 $254 \mathrm{~nm}$
\begin{tabular}{|r|r|r|r|r|}
\hline Peak\# & Ret. Time & \multicolumn{1}{|c|}{ Area } & Height & Area $\%$ \\
\hline 1 & 21.725 & 473893 & 17236 & 4.191 \\
\hline 2 & 23.123 & 429782 & 15727 & 3.801 \\
\hline 3 & 23.951 & 5243439 & 173255 & 46.372 \\
\hline 4 & 30.729 & 5160225 & 11118 & 45.636 \\
\hline Total & & 11307339 & 317335 & 100.000 \\
\hline
\end{tabular}

H:IWJH-Ř665 \WJH-R665-CHRI-94-6-0.6-IE-ID-30-3.5-106.lcd $\mathrm{mV}$

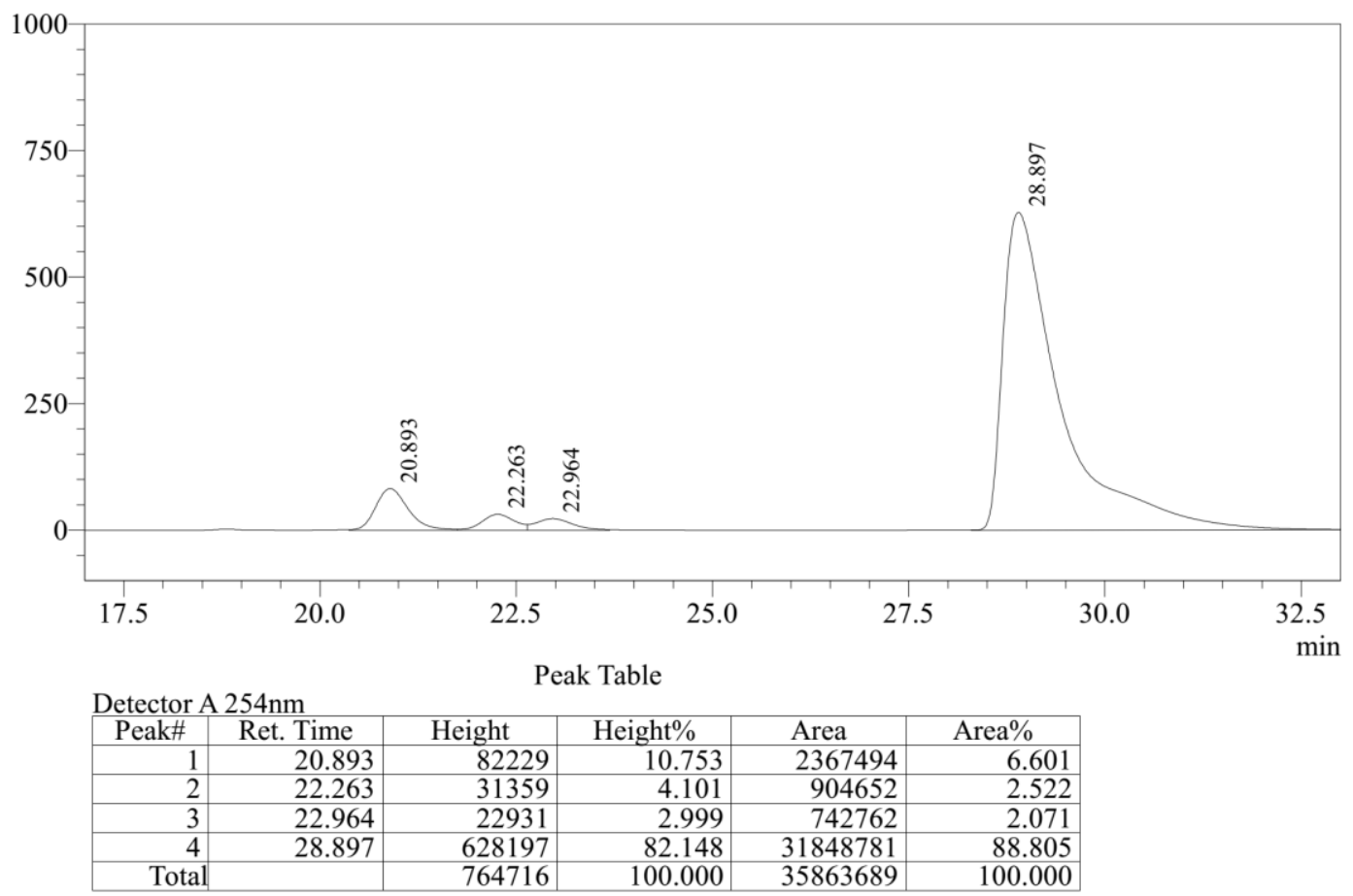


di-tert-butyl $(S)$-2-((S)-1-ethoxy-1-oxopropan-2-yl)-4-(3-methoxybenzoyl)pyrano [2,3-b]indole-3,9(4H)-dicarboxylate (6f)

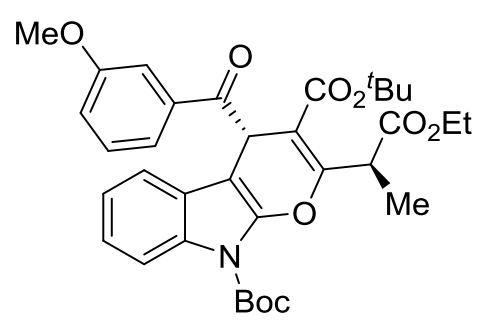

A white solid (114mg, 94\%); $[\alpha]^{25}{ }_{\mathrm{D}}=+27.9\left(\mathrm{c} 1.0, \mathrm{CHCl}_{3}\right) ;{ }^{1} \mathrm{H}$ NMR $(400 \mathrm{MHz}$, $\left.\mathrm{CDCl}_{3}\right) \delta 8.10(\mathrm{~d}, J=8.3 \mathrm{~Hz}, 1 \mathrm{H}), 7.77(\mathrm{~d}, J=7.7 \mathrm{~Hz}, 1 \mathrm{H}), 7.53(\mathrm{dd}, J=2.4,1.6 \mathrm{~Hz}$, 1H), $7.43(\mathrm{t}, J=8.0 \mathrm{~Hz}, 1 \mathrm{H}), 7.18-7.13(\mathrm{~m}, 2 \mathrm{H}), 7.01(\mathrm{~d}, J=3.9 \mathrm{~Hz}, 2 \mathrm{H}), 5.74(\mathrm{~s}$, 1H), 5.07 (q, J = 7.2 Hz, 1H), $4.28-4.09(\mathrm{~m}, 2 \mathrm{H}), 3.82(\mathrm{~s}, 3 \mathrm{H}), 1.65(\mathrm{~s}, 9 \mathrm{H}), 1.63(\mathrm{~d}$, $J=7.2 \mathrm{~Hz}, 3 \mathrm{H}), 1.34(\mathrm{~s}, 9 \mathrm{H}), 1.21(\mathrm{t}, J=7.1 \mathrm{~Hz}, 3 \mathrm{H}) ;{ }^{13} \mathrm{C} \mathrm{NMR}\left(100 \mathrm{MHz}, \mathrm{CDCl}_{3}\right) \delta$ $198.9,171.4,165.1,161.3,159.8,148.5,142.5,138.1,131.9,129.5,125.1,123.4$, $123.2,121.6,119.8,118.4,114.9,113.2,106.6,92.0,84.6,82.5,61.0,55.4,42.5,41.5$, 28.2, 28.0, 14.2, 14.2. HRMS (ESI) $m / z$ calcd for $\mathrm{C}_{34} \mathrm{H}_{39} \mathrm{NO}_{9}[\mathrm{M}+\mathrm{Na}]^{+}=628.2523$, found $=628.2520$; The ee value was $98 \%, \mathrm{t}_{\mathrm{R}}($ minor $)=34.3 \mathrm{~min}, \mathrm{t}_{\mathrm{R}}($ major $)=44.9 \mathrm{~min}$ (Chiralcel IE-ID, $\lambda=254 \mathrm{~nm}, 6 \% i$-PrOH/hexanes, flow rate $=0.6 \mathrm{~mL} / \mathrm{min}$ ). 
D: \LC-Dă̌a $\backslash W J H \backslash W J H-R 640-680 \backslash W J H-R 666-R A C-94-6-0.6-I E-I D-30-4.0-106-A U T O . l c d$ $\mathrm{mV}$

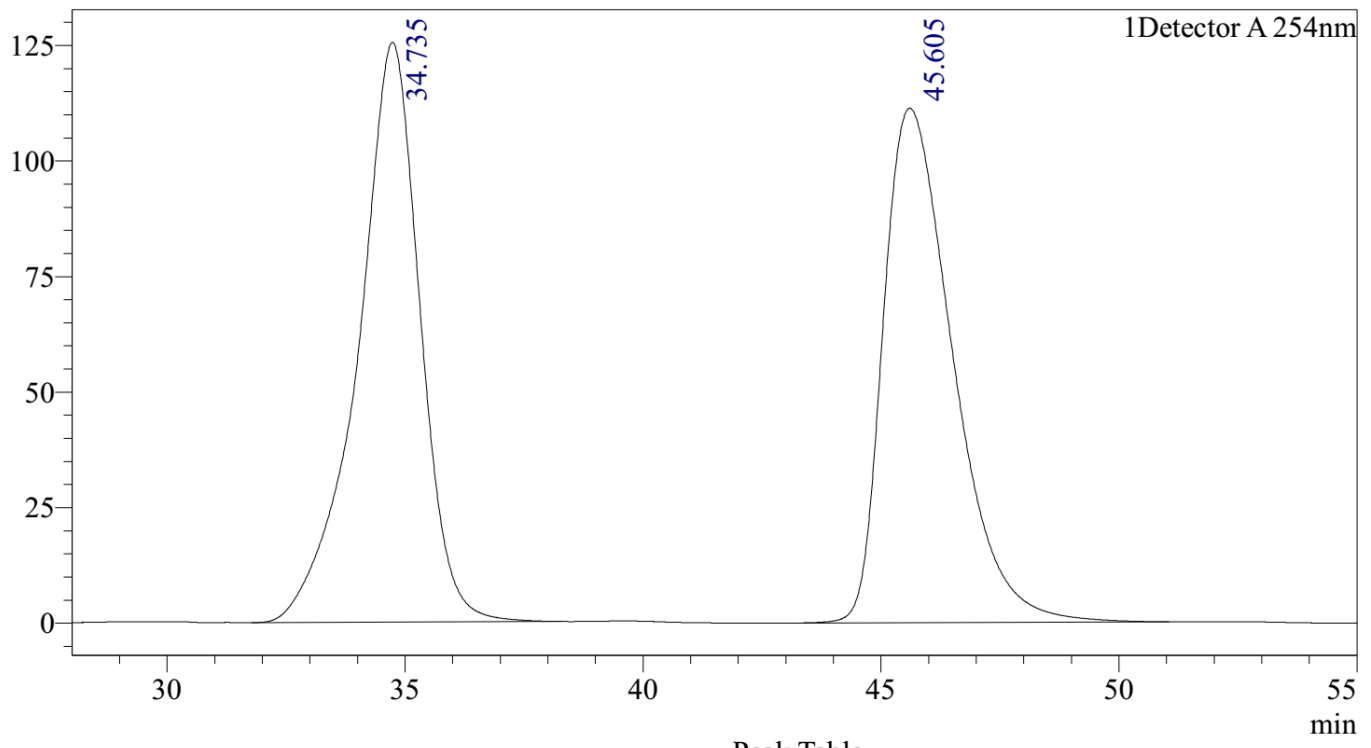

\begin{tabular}{|c|c|c|c|c|c|}
\hline & & & & & \\
\hline \multicolumn{6}{|c|}{ Detector A 254nm } \\
\hline & Ret. Time & & Height & Height $\%$ & Area\% \\
\hline 1 & 34.735 & 9820 & 125490 & 52.984 & 50.108 \\
\hline$\frac{1}{2}$ & 45.605 & 113 & & 47.016 & 49.892 \\
\hline Total & & 22650601 & 236843 & 100.000 & 100.000 \\
\hline
\end{tabular}

H:IWJH-Ř666 \JH-R666-CHRI-94-6-0.6-IE-ID-30-3.5-106.1cd $\mathrm{mV}$

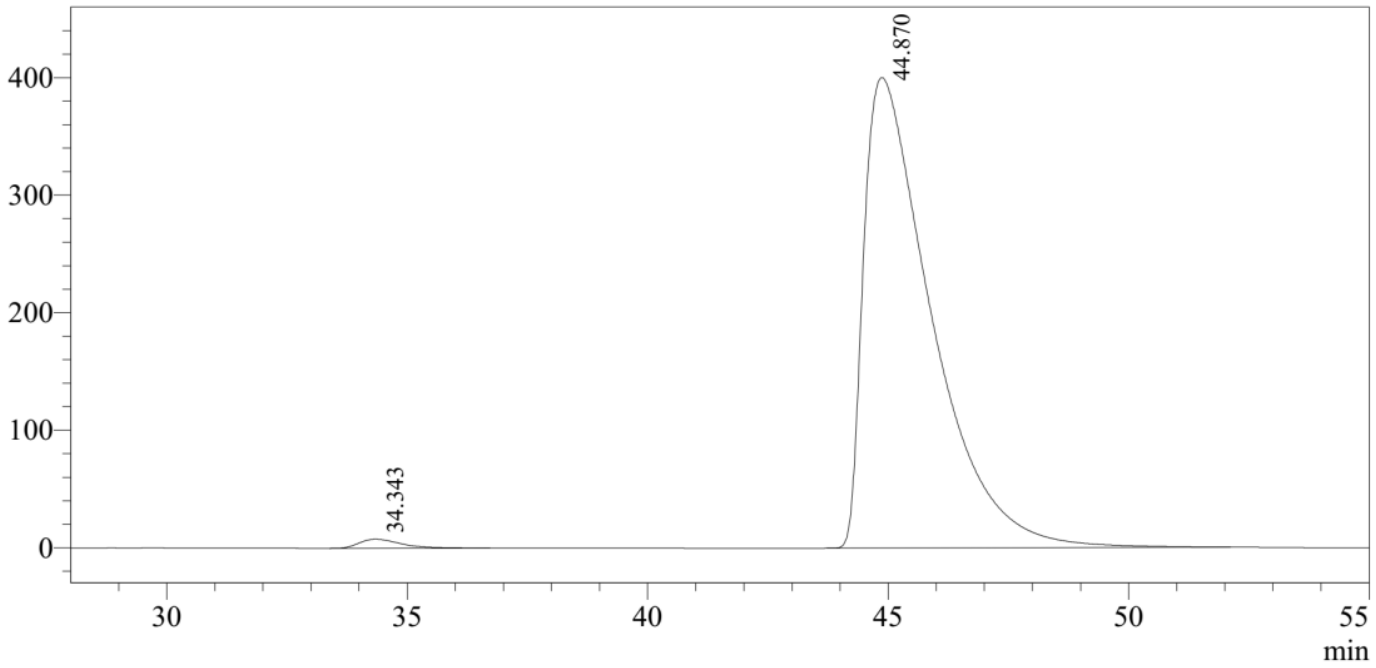

\begin{tabular}{|c|c|c|c|c|c|}
\hline \multicolumn{6}{|l|}{ Dete } \\
\hline Den & Ret. Time & Height & Height $\%$ & Area & Area $\%$ \\
\hline 1 & 34.343 & 7699 & 1.886 & 469461 & 1.180 \\
\hline 2 & 44.870 & 400427 & 98.114 & 39301056 & 98.820 \\
\hline Total & & 408126 & 100.000 & 39770517 & 100.000 \\
\hline
\end{tabular}

\section{di-tert-butyl $(S)$-2-((S)-1-ethoxy-1-oxopropan-2-yl)-4-(4-methylbenzoyl)pyrano} [2,3-b]indole-3,9(4H)-dicarboxylate $(6 \mathrm{~g})$ 


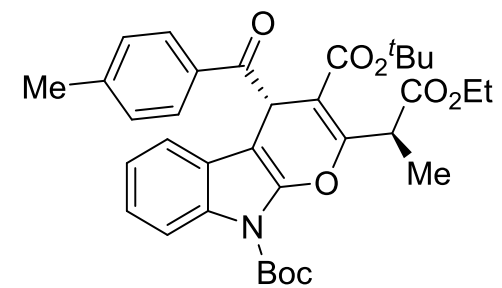

A white solid $(112 \mathrm{mg}, 95 \%) ;[\alpha]^{25}{ }_{\mathrm{D}}=+37.3\left(c 0.8, \mathrm{CHCl}_{3}\right) ;{ }^{1} \mathrm{H}$ NMR $(400 \mathrm{MHz}$, $\left.\mathrm{CDCl}_{3}\right) \delta 8.09(\mathrm{~d}, J=8.3 \mathrm{~Hz}, 1 \mathrm{H}), 8.01(\mathrm{~d}, J=8.3 \mathrm{~Hz}, 2 \mathrm{H}), 7.30(\mathrm{~d}, J=8.0 \mathrm{~Hz}, 2 \mathrm{H})$, $7.14(\mathrm{dt}, J=8.5,4.3 \mathrm{~Hz}, 1 \mathrm{H}), 7.00(\mathrm{~d}, J=4.0 \mathrm{~Hz}, 2 \mathrm{H}), 5.73(\mathrm{~s}, 1 \mathrm{H}), 5.07(\mathrm{q}, J=7.2$ Hz, 1H), $4.26-4.10$ (m, 2H), 2.44 (s, 3H), 1.65 (s, 9H), 1.63 (d, J= 7.2 Hz, 3H), 1.32 (s, 9H), $1.21(\mathrm{t}, J=7.1 \mathrm{~Hz}, 3 \mathrm{H}) ;{ }^{13} \mathrm{C} \mathrm{NMR}\left(100 \mathrm{MHz}, \mathrm{CDCl}_{3}\right) \delta 198.6,171.4,165.1$, 161.2 , 148.6, 144.1, 142.5, 134.2, 131.9, 129.3, 129.1, 125.2, 123.3, 123.2, 118.4, $114.9,106.7,92.2,84.5,82.5,61.0,42.2,41.4,28.2,28.0,21.7,14.2,14.1$. HRMS (ESI) $m / z$ calcd for $\mathrm{C}_{34} \mathrm{H}_{39} \mathrm{NO}_{8}[\mathrm{M}+\mathrm{Na}]^{+}=612.2573$, found $=612.2528$; The ee value was $95 \%, \mathrm{t}_{\mathrm{R}}($ minor $)=22.0 \mathrm{~min}, \mathrm{t}_{\mathrm{R}}($ major $)=25.4 \min ($ Chiralcel IE, $\lambda=254 \mathrm{~nm}, 3 \%$ $i$-PrOH/hexanes, flow rate $=1.0 \mathrm{~mL} / \mathrm{min})$.

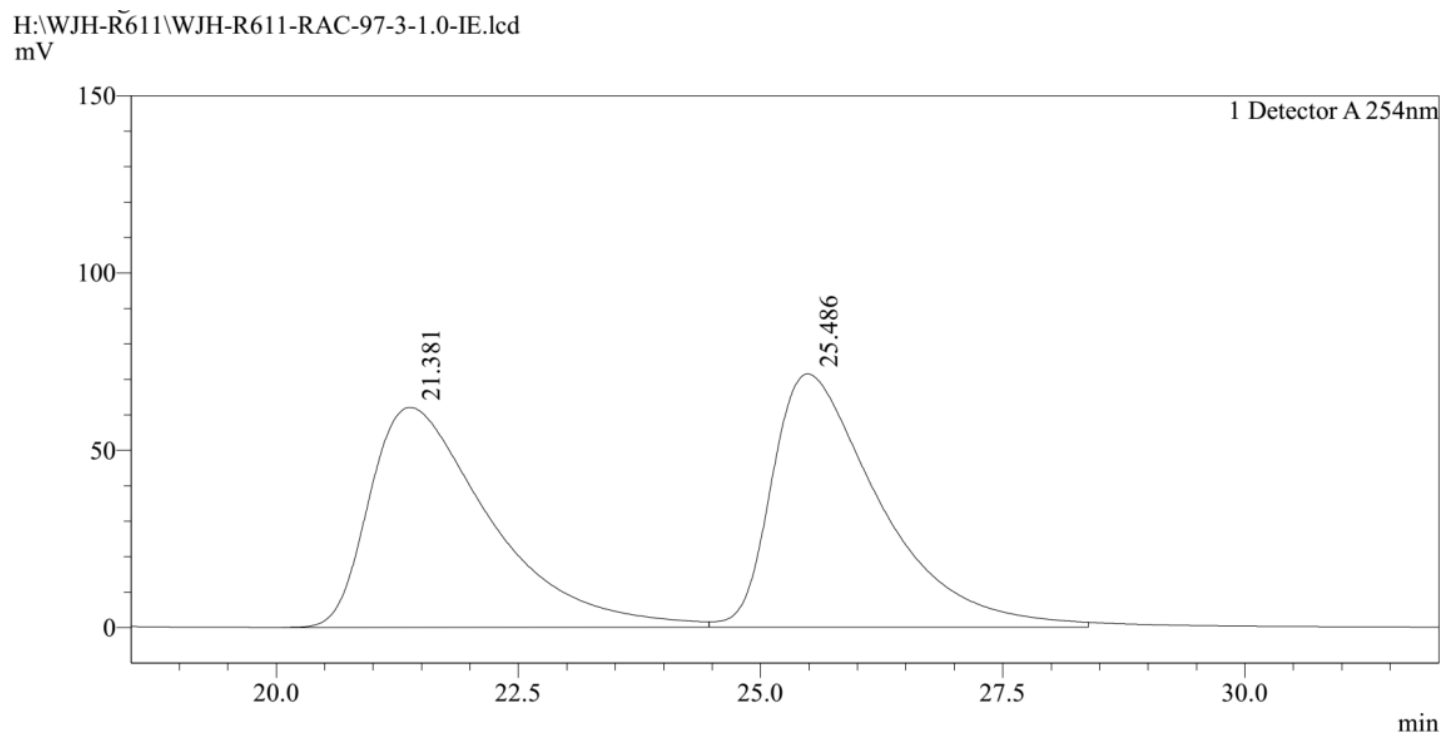

Peak Table

Detector A $254 \mathrm{~nm}$
\begin{tabular}{|r|r|r|r|r|}
\hline Peak\# & Ret. Time & \multicolumn{1}{|c|}{ Area } & Height & \multicolumn{1}{|c|}{ Area $\%$} \\
\hline 1 & 21.381 & 5401266 & 62065 & 49.213 \\
\hline 2 & 25.486 & 5574037 & 71442 & 50.787 \\
\hline Total & & 10975303 & 133507 & 100.000 \\
\hline
\end{tabular}


H:IWJH-Ř611 \WJH-R611-CHI-97-3-1.0-IE.lcd $\mathrm{mV}$

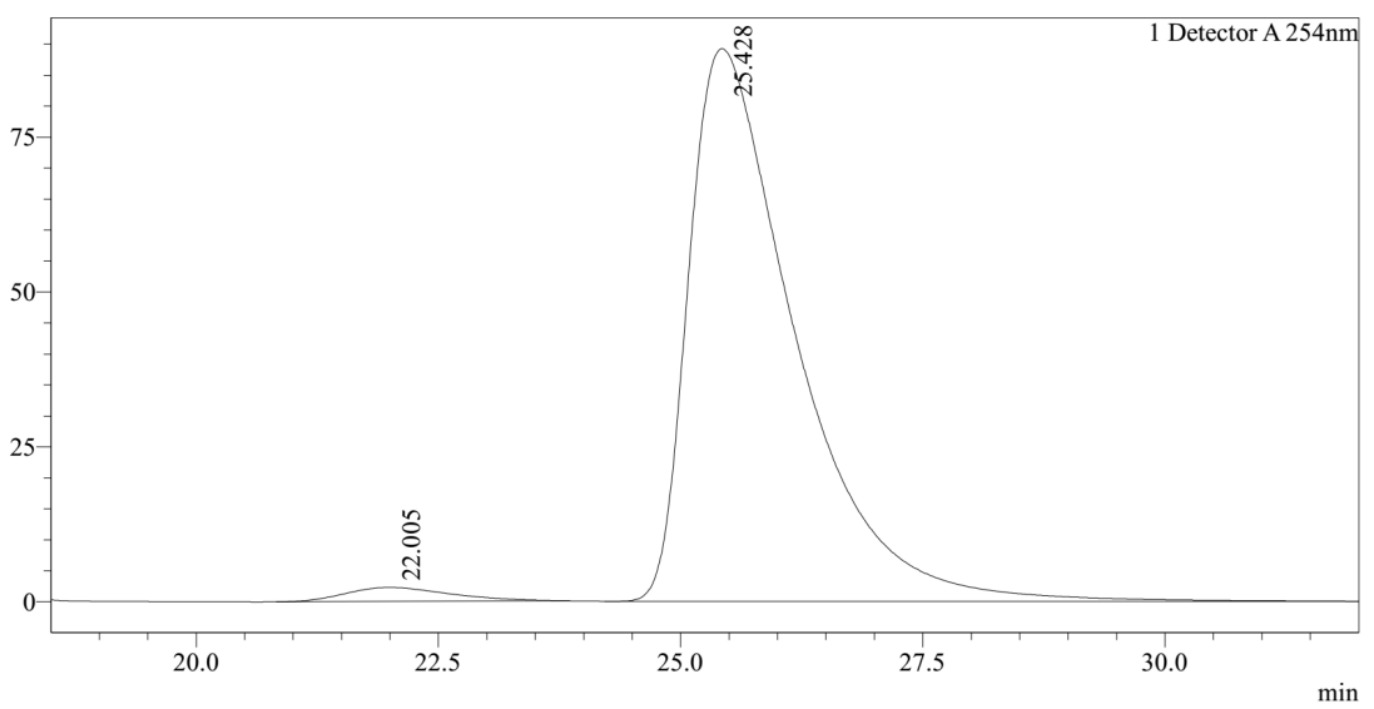

Peak Table

Detector A $254 \mathrm{~nm}$
\begin{tabular}{|r|r|r|r|r|}
\hline Peak\# & Ret. Time & Area & Height & \multicolumn{1}{|c|}{ Area $\%$} \\
\hline 1 & 22.005 & 170729 & 2261 & 2.434 \\
\hline 2 & 25.428 & 6844805 & 89209 & 97.566 \\
\hline Total & & 7015534 & 91470 & 100.000 \\
\hline
\end{tabular}

di-tert-butyl $(S)$-2-((S)-1-ethoxy-1-oxopropan-2-yl)-4-(4-methoxybenzoyl)pyrano [2,3-b]indole-3,9(4H)-dicarboxylate $(6 \mathrm{~h})$

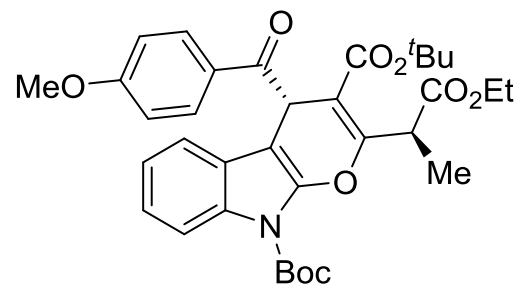

A white solid $(117 \mathrm{mg}, 97 \%) ;[\alpha]^{25}{ }_{\mathrm{D}}=+42.6\left(c 0.8, \mathrm{CHCl}_{3}\right) ;{ }^{1} \mathrm{H}$ NMR (400 MHz, $\left.\mathrm{CDCl}_{3}\right) \delta 8.15-8.07(\mathrm{~m}, 1 \mathrm{H}), 7.16-7.11(\mathrm{~m}, 1 \mathrm{H}), 7.03-6.95(\mathrm{~m}, 4 \mathrm{H}), 5.73(\mathrm{~s}, 1 \mathrm{H})$, $5.07(\mathrm{q}, J=7.2 \mathrm{~Hz}, 1 \mathrm{H}), 4.27-4.10(\mathrm{~m}, 2 \mathrm{H}), 3.90(\mathrm{~s}, 3 \mathrm{H}), 1.65$ (s, 9H), 1.63 (d, $J=$ $7.2 \mathrm{~Hz}, 3 \mathrm{H}), 1.32(\mathrm{~s}, 9 \mathrm{H}), 1.22(\mathrm{t}, J=7.1 \mathrm{~Hz}, 3 \mathrm{H}) ;{ }^{13} \mathrm{C} \mathrm{NMR}\left(100 \mathrm{MHz}, \mathrm{CDCl}_{3}\right) \delta$ 197.6, 171.5, 165.2, 163.6, 161.1, 148.6, 142.6, 131.9, 131.3, 129.6, 125.2, 123.3, 123.2, 118.4, 114.9, 113.8, 106.8, 92.3, 84.5, 82.4, 61.0, 55.5, 41.8, 41.4, 28.2, 28.0, 14.2, 14.2. HRMS (ESI) $\mathrm{m} / z$ calcd for $\mathrm{C}_{34} \mathrm{H}_{39} \mathrm{NO}_{9}[\mathrm{M}+\mathrm{Na}]^{+}=628.2523$, found $=$ 628.2523; The ee value was $96 \%, \mathrm{t}_{\mathrm{R}}($ minor $)=27.9 \mathrm{~min}, \mathrm{t}_{\mathrm{R}}$ (major) $=40.2 \mathrm{~min}$ (Chiralcel IG, $\lambda=254 \mathrm{~nm}, 3 \% i$-PrOH$/$ hexanes, flow rate $=1.0 \mathrm{~mL} / \mathrm{min}$ ). 
D: LLC-Dăa $\mid W J H \backslash W J H-R 640-680 \backslash W J H-R 674-R A C-97-3-I G-30-2.8-106-A U T O 01 . l c d$ $\mathrm{mV}$

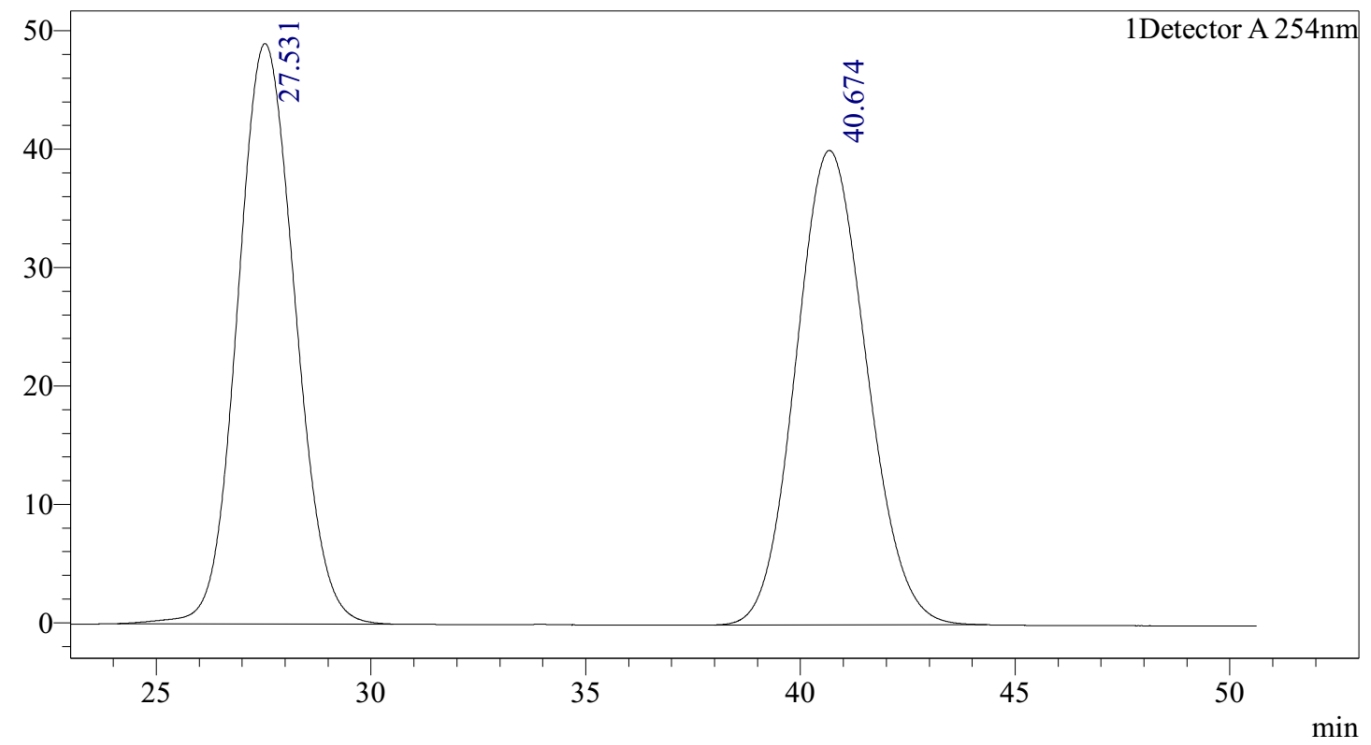

Peak Table

Detector A $254 \mathrm{~nm}$
\begin{tabular}{|r|r|r|r|r|r|}
\hline Peak\# & Ret. Time & Area & Height & Height $\%$ & \multicolumn{1}{|c|}{ Area\% } \\
\hline 1 & 27.531 & 4573218 & 49015 & 55.019 & 49.919 \\
\hline 2 & 40.674 & 4588003 & 40072 & 44.981 & 50.081 \\
\hline Total & & 9161221 & 89087 & 100.000 & 100.000 \\
\hline
\end{tabular}

H:|WJH-R̄674|WJH-R674-CHRI-97-3-1.0-IG-30-2.9-106.1cd $\mathrm{mV}$

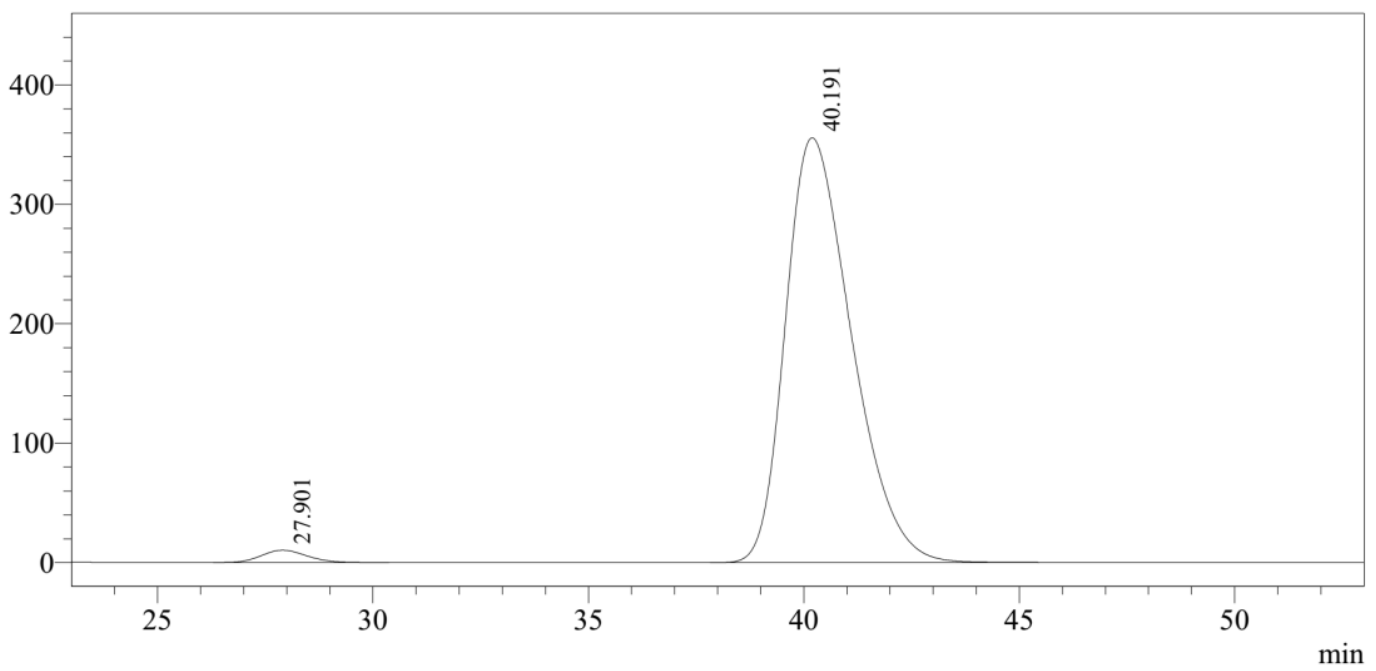

Peak Table

Detector A 254nm
\begin{tabular}{|r|r|r|r|r|r|}
\hline Peak\# & Ret. Time & Height & Height $\%$ & \multicolumn{1}{|c|}{ Area } & \multicolumn{1}{|c|}{ Area $\%$} \\
\hline 1 & 27.901 & 10389 & 2.836 & 772034 & 1.989 \\
\hline 2 & 40.191 & 355880 & 97.164 & 38038844 & 98.011 \\
\hline Total & & 366269 & 100.000 & 38810878 & 100.000 \\
\hline
\end{tabular}

di-tert-butyl $(S)$-4-(4-cyanobenzoyl)-2-( $(S)$-1-ethoxy-1-oxopropan-2-yl)pyrano [2,3-b]indole-3,9(4H)-dicarboxylate $(6 \mathrm{i})$ 


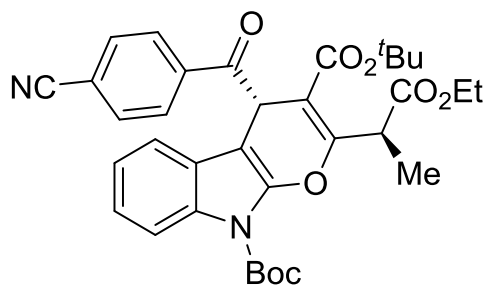

A white solid (90mg, 75\%); $[\alpha]^{25}=+20.8\left(c 1.0, \mathrm{CHCl}_{3}\right) ;{ }^{1} \mathrm{H}$ NMR $\left(400 \mathrm{MHz}, \mathrm{CDCl}_{3}\right)$ $\delta 8.15-8.08(\mathrm{~m}, 3 \mathrm{H}), 7.83-7.77(\mathrm{~m}, 2 \mathrm{H}), 7.19(\mathrm{ddd}, J=8.5,7.4,1.2 \mathrm{~Hz}, 1 \mathrm{H}), 7.07$ $-7.01(\mathrm{~m}, 1 \mathrm{H}), 6.90(\mathrm{~d}, J=7.5 \mathrm{~Hz}, 1 \mathrm{H}), 5.64(\mathrm{~s}, 1 \mathrm{H}), 4.98(\mathrm{q}, J=7.2 \mathrm{~Hz}, 1 \mathrm{H}), 4.27-$ $4.10(\mathrm{~m}, 2 \mathrm{H}), 1.66(\mathrm{~s}, 9 \mathrm{H}), 1.61(\mathrm{~d}, J=7.2 \mathrm{~Hz}, 3 \mathrm{H}), 1.37(\mathrm{~s}, 9 \mathrm{H}), 1.22(\mathrm{t}, J=7.1 \mathrm{~Hz}$, $3 \mathrm{H}) ;{ }^{13} \mathrm{C}$ NMR $\left(100 \mathrm{MHz}, \mathrm{CDCl}_{3}\right) \delta 171.1,148.4,142.5,140.2,132.4,131.9,129.2$, $124.8,123.7,123.4,118.0,116.3,115.2,106.4,91.4,84.9,83.0,61.1,43.5,41.7,28.2$, 28.0, 14.2, 14.1. HRMS (ESI) $\mathrm{m} / z$ calcd for $\mathrm{C}_{34} \mathrm{H}_{36} \mathrm{~N}_{2} \mathrm{O}_{8}[\mathrm{M}+\mathrm{Na}]^{+}=623.2369$, found $=$ 623.2366; The ee value was 93\%, $\mathrm{t}_{\mathrm{R}}($ minor $)=18.1 \mathrm{~min}, \mathrm{t}_{\mathrm{R}}($ major $)=22.9 \mathrm{~min}$ (Chiralcel IG, $\lambda=254 \mathrm{~nm}, 3 \% i$-PrOH/hexanes, flow rate $=1.0 \mathrm{~mL} / \mathrm{min}$ ).

D:|LC-Data $\mid W J H \backslash W J H-R 640-680 \backslash W J H-R 670-R A C-97-3-1.0-2.8-I G--106-A U T O 01.1 c d$ $\mathrm{mV}$

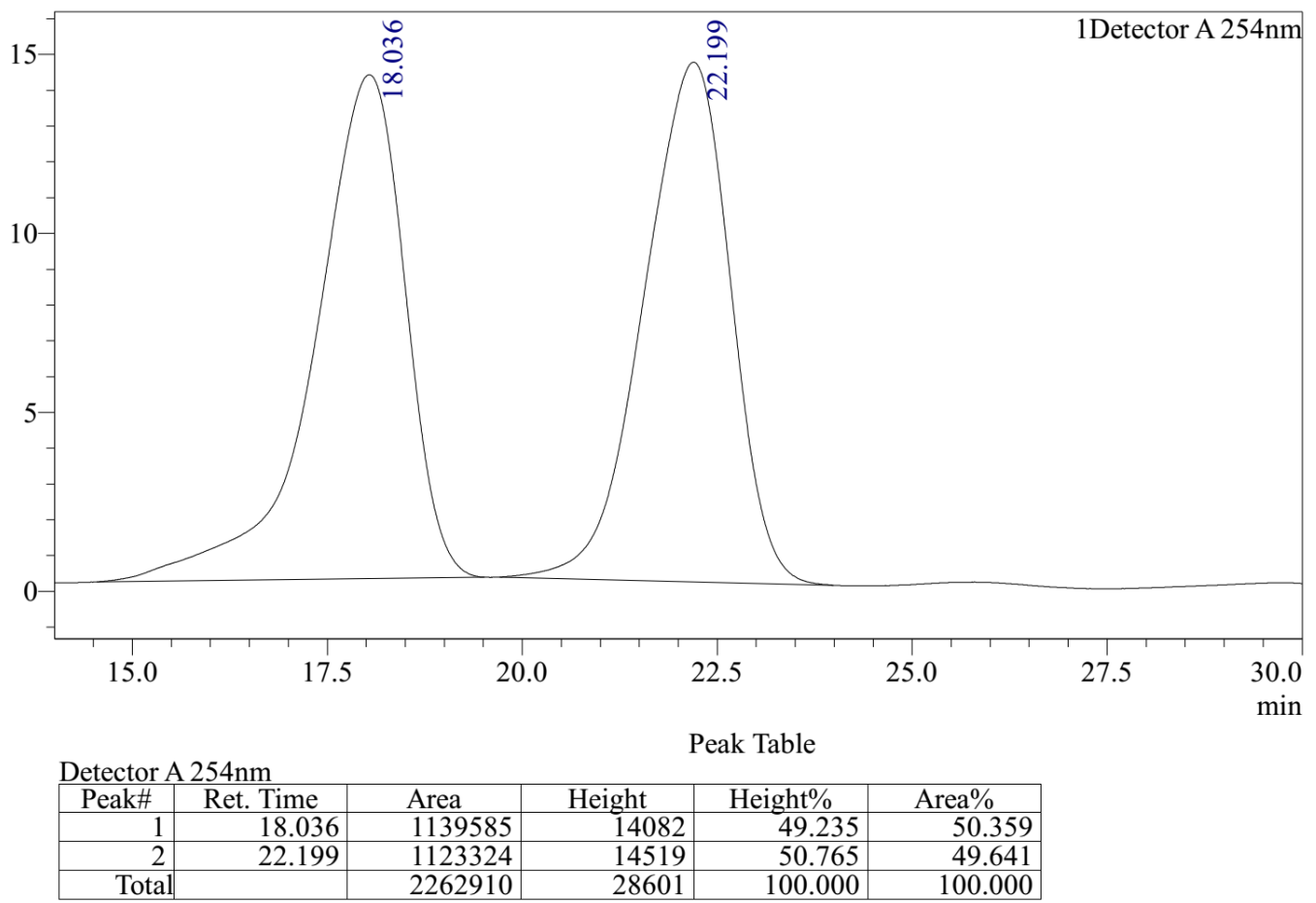




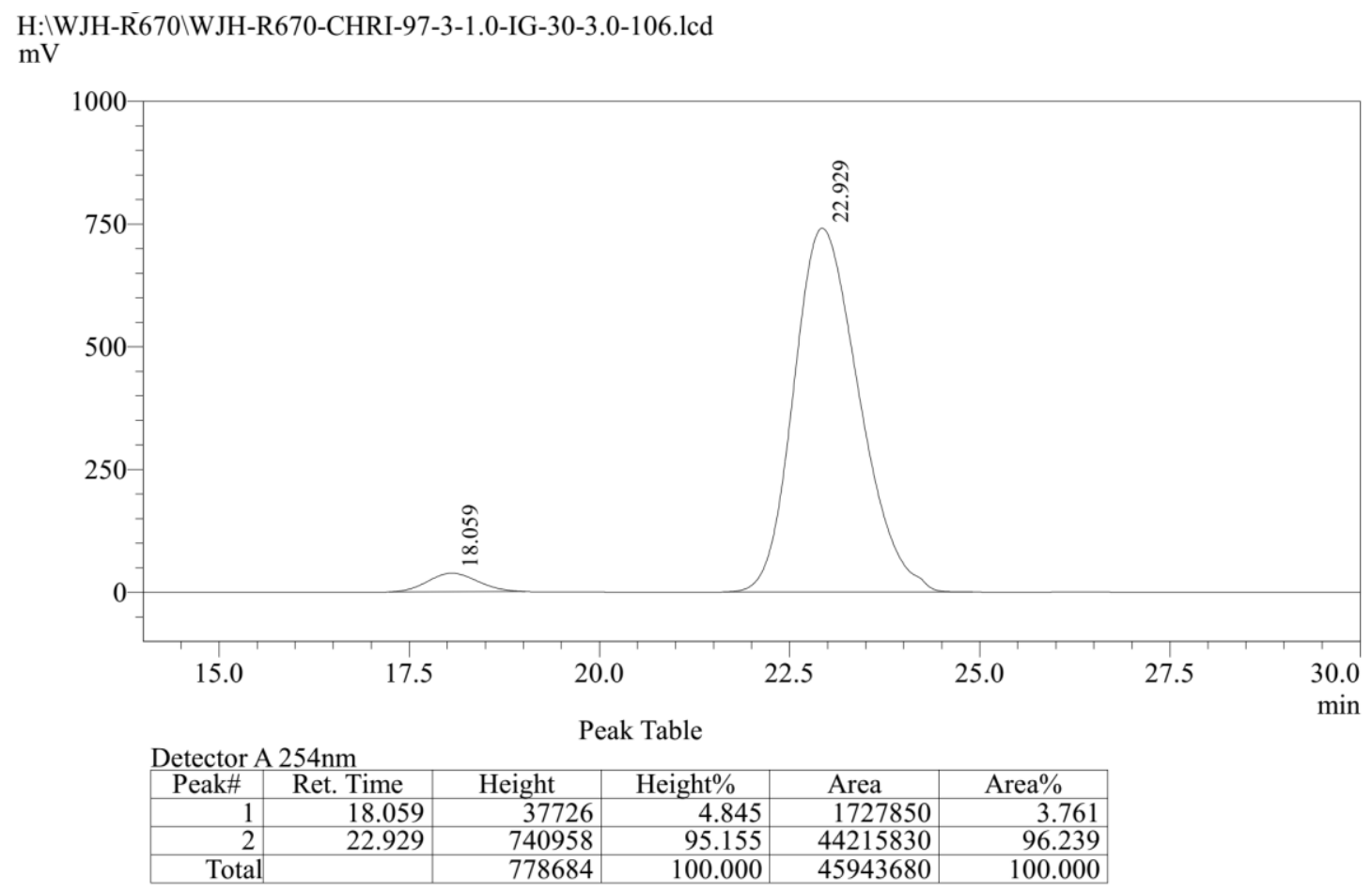

di-tert-butyl $(S)$-4-(2-naphthoyl)-2-((S)-1-ethoxy-1-oxopropan-2-yl)pyrano[2,3-b] indole-3,9(4H)-dicarboxylate $(6 \mathbf{j})$

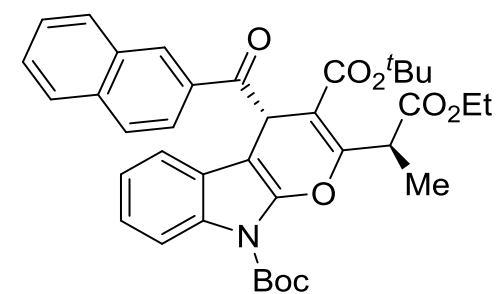

A white solid $(123 \mathrm{mg}, 98 \%)$; $[\alpha]^{25}{ }_{\mathrm{D}}=+66.1\left(\mathrm{c} 1.0, \mathrm{CHCl}_{3}\right) ;{ }^{1} \mathrm{H} \mathrm{NMR}(400 \mathrm{MHz}$, $\left.\mathrm{CDCl}_{3}\right) \delta 8.72(\mathrm{~s}, 1 \mathrm{H}), 8.12-8.07(\mathrm{~m}, 2 \mathrm{H}), 8.00-7.88(\mathrm{~m}, 3 \mathrm{H}), 7.64-7.55(\mathrm{~m}, 2 \mathrm{H})$, 7.13 (ddd, $J=8.4,7.3,1.3 \mathrm{~Hz}, 1 \mathrm{H}), 7.01(\mathrm{~d}, J=7.3 \mathrm{~Hz}, 1 \mathrm{H}), 6.96-6.89(\mathrm{~m}, 1 \mathrm{H})$, $5.89(\mathrm{~s}, 1 \mathrm{H}), 5.10(\mathrm{q}, J=7.1 \mathrm{~Hz}, 1 \mathrm{H}), 4.28-4.11(\mathrm{~m}, 2 \mathrm{H}), 1.68(\mathrm{~s}, 9 \mathrm{H}), 1.66(\mathrm{~d}, J=$ $7.2 \mathrm{~Hz}, 3 \mathrm{H}), 1.31(\mathrm{~s}, 9 \mathrm{H}), 1.22(\mathrm{t}, J=7.1 \mathrm{~Hz}, 3 \mathrm{H}) ;{ }^{13} \mathrm{C} \mathrm{NMR}\left(100 \mathrm{MHz}, \mathrm{CDCl}_{3}\right) \delta$ $198.9,171.4,165.1,161.3,148.6,142.5,135.7,134.0,132.3,131.9,130.5,129.6$, 128.6, 128.6, 127.8, 126.9, 125.1, 124.8, 123.3 (d, $J=11.0 \mathrm{~Hz}), 118.4,114.9,106.8$, 92.2, 84.6, 82.6, 61.0, $42.741 .5,28.2,28.0,14.2$. HRMS (ESI) $\mathrm{m} / z$ calcd for $\mathrm{C}_{37} \mathrm{H}_{39} \mathrm{NO}_{8}[\mathrm{M}+\mathrm{Na}]^{+}=648.2573$, found $=648.2565$; The ee value was $97 \%, \mathrm{t}_{\mathrm{R}}$ (minor) 
$=27.2 \mathrm{~min}, \mathrm{t}_{\mathrm{R}}$ (major) $=30.6 \mathrm{~min}($ Chiralcel IG, $\lambda=254 \mathrm{~nm}, 3 \% i$-PrOH$/$ hexanes, flow rate $=1.0 \mathrm{~mL} / \mathrm{min}$ ).

D: \LC-Dăă $\backslash W J H \backslash W J H-R 640-680 \backslash W J H-R 675-R A C-97-3-1.0-I G-30-2.8-106-A U T O 01.1 c d$ $\mathrm{mV}$

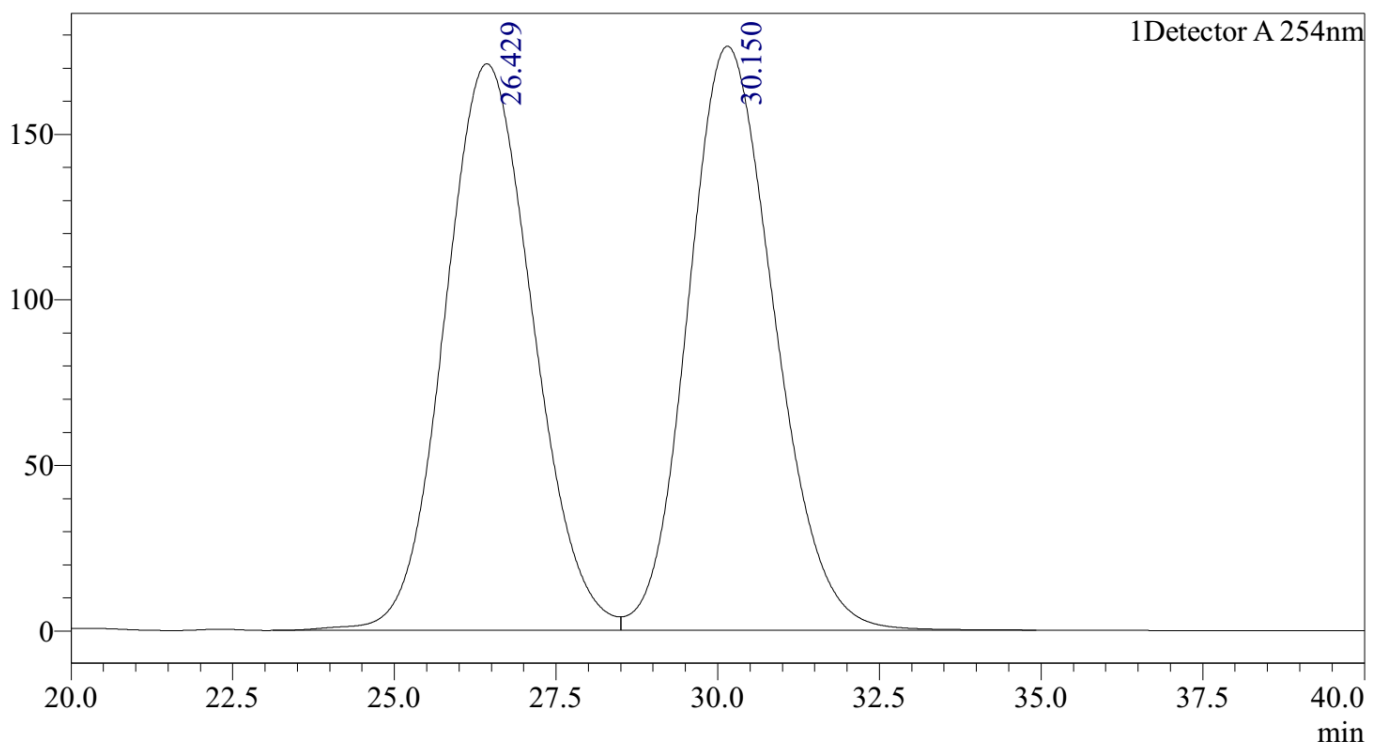

Peak Table

Detector A $254 \mathrm{~nm}$
\begin{tabular}{|r|r|r|r|r|r|}
\hline Peak\# & Ret. Time & \multicolumn{1}{|c|}{ Area } & Height & Height $\%$ & \multicolumn{1}{|c|}{ Area $\%$} \\
\hline 1 & 26.429 & 16236494 & 171110 & 49.236 & 49.792 \\
\hline 2 & 30.150 & 16372468 & 176420 & 50.764 & 50.208 \\
\hline Total & & 32608962 & 347531 & 100.000 & 100.000 \\
\hline
\end{tabular}

H:IWJH-Ř675।WJH-R675-CHRI-97-3-1.0-IG-30-2.9-106.lcd

$\mathrm{mV}$

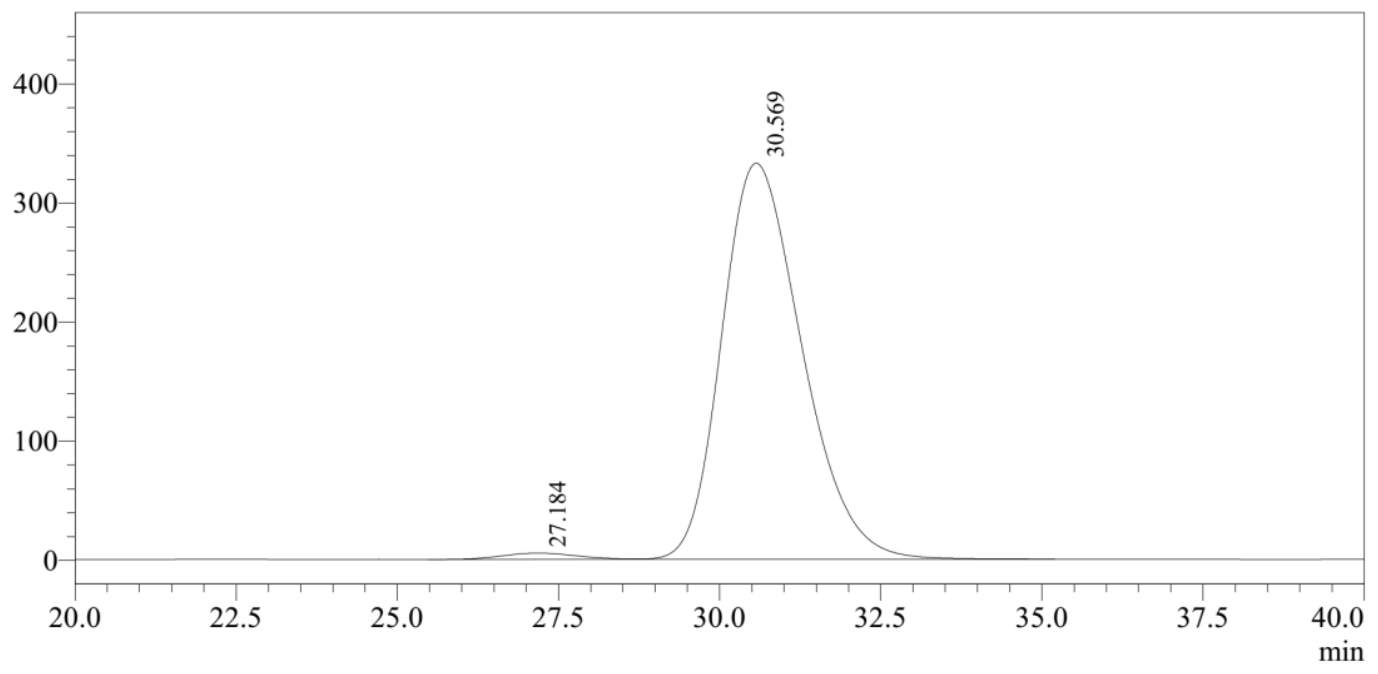

Peak Table

\begin{tabular}{|r|r|r|r|r|r|} 
Detector A $254 \mathrm{~nm}$ \\
\hline Peak\# & Ret. Time & Height & Height $\%$ & \multicolumn{1}{|c|}{ Area } & \multicolumn{1}{|c|}{ Area $\%$} \\
\hline 1 & 27.184 & 5343 & 1.581 & 434846 & 1.486 \\
\hline 2 & 30.569 & 332687 & 98.419 & 28830715 & 98.514 \\
\hline Total & & 338030 & 100.000 & 29265561 & 100.000 \\
\hline
\end{tabular}


9-benzyl 3-(tert-butyl) $\quad(S)$-4-benzoyl-2-( $(S)$-1-ethoxy-1-oxopropan-2-yl)pyrano

\section{[2,3-b]indole-3,9(4H)-dicarboxylate $(6 \mathrm{k})$}

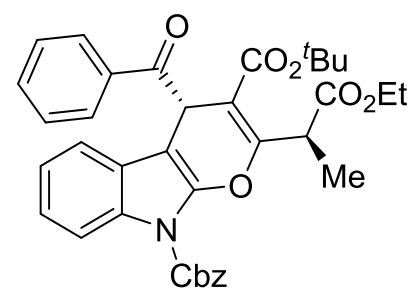

A white solid (113mg, 93\%); $[\alpha]^{25}{ }_{\mathrm{D}}=+43.6\left(c 0.8, \mathrm{CHCl}_{3}\right) ;{ }^{1} \mathrm{H}$ NMR $(400 \mathrm{MHz}$, $\left.\mathrm{CDCl}_{3}\right) \delta 8.14-8.03(\mathrm{~m}, 3 \mathrm{H}), 7.66-7.57(\mathrm{~m}, 1 \mathrm{H}), 7.53-7.48(\mathrm{~m}, 4 \mathrm{H}), 7.45-7.37$ (m, 3H), 7.15 (ddd, $J=8.5,7.3,1.3 \mathrm{~Hz}, 1 \mathrm{H}), 7.03-6.97(\mathrm{~m}, 1 \mathrm{H}), 6.92$ (d, $J=7.4 \mathrm{~Hz}$, 1H), $5.73(\mathrm{~s}, 1 \mathrm{H}), 5.52-5.36(\mathrm{~m}, 2 \mathrm{H}), 4.91(\mathrm{q}, J=7.2 \mathrm{~Hz}, 1 \mathrm{H}), 4.13(\mathrm{q}, J=7.1 \mathrm{~Hz}$, 2H), $1.45(\mathrm{~d}, J=7.2 \mathrm{~Hz}, 3 \mathrm{H}), 1.32(\mathrm{~s}, 9 \mathrm{H}), 1.19(\mathrm{t}, J=7.1 \mathrm{~Hz}, 3 \mathrm{H}) ;{ }^{13} \mathrm{C}$ NMR $(100$ $\left.\mathrm{MHz}, \mathrm{CDCl}_{3}\right) \delta 199.0$ 171.5, 165.0, 160.9, 149.9, 142.4, 136.8, 134.7, 133.2, 131.7, $129.1,128.9,128.8,128.7,128.6,125.3,123.6,123.6,118.5,115.0,106.6,92.6,82.5$, 69.0, 61.0, 42.5, 41.82, 27.9, 14.2, 13.9. HRMS (ESI) $\mathrm{m} / \mathrm{z}$ calcd for $\mathrm{C}_{36} \mathrm{H}_{35} \mathrm{NO}_{8}$ $[\mathrm{M}+\mathrm{Na}]^{+}=632.2260$, found $=632.2260$; The ee value was $88 \%, \mathrm{t}_{\mathrm{R}}($ minor $)=14.4 \mathrm{~min}$, $\mathrm{t}_{\mathrm{R}}($ major $)=16.3 \mathrm{~min}($ Chiralcel IC, $\lambda=254 \mathrm{~nm}, 4 \% i$-PrOH/hexanes, flow rate $=1.0$ $\mathrm{mL} / \mathrm{min})$. 
Chromatogram

H:IWJH-R614 \WJH-R614-4-RAC-96-4-1.0-IC.lcd

$\mathrm{mV}$

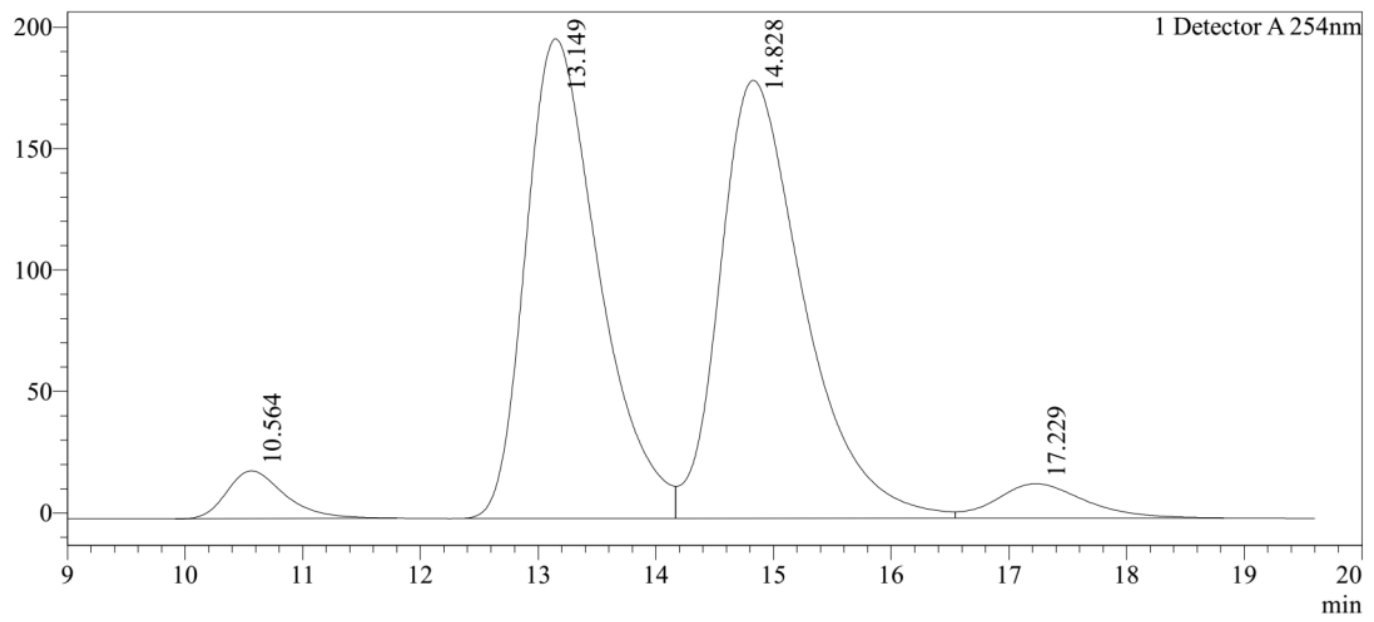

Peak Table

\begin{tabular}{|c|c|c|c|c|}
\hline \multicolumn{5}{|c|}{ Detector A $254 \mathrm{~nm}$} \\
\hline Peak\# & Ret. Time & Area & Height & Area $\%$ \\
\hline 1 & 10.564 & 672603 & 19686 & 3.560 \\
\hline 2 & 13.149 & 8540063 & 197531 & 45.203 \\
\hline 3 & 14.828 & 8940897 & 180334 & 47.325 \\
\hline 4 & 17.229 & 739148 & 14138 & 3.912 \\
\hline Total & & 18892710 & 411689 & 100.000 \\
\hline
\end{tabular}

Chromatogram

H:IWJH-R614 \WJH-R614-4-CHI-96-4-1.0-IC.lcd

$\mathrm{mV}$

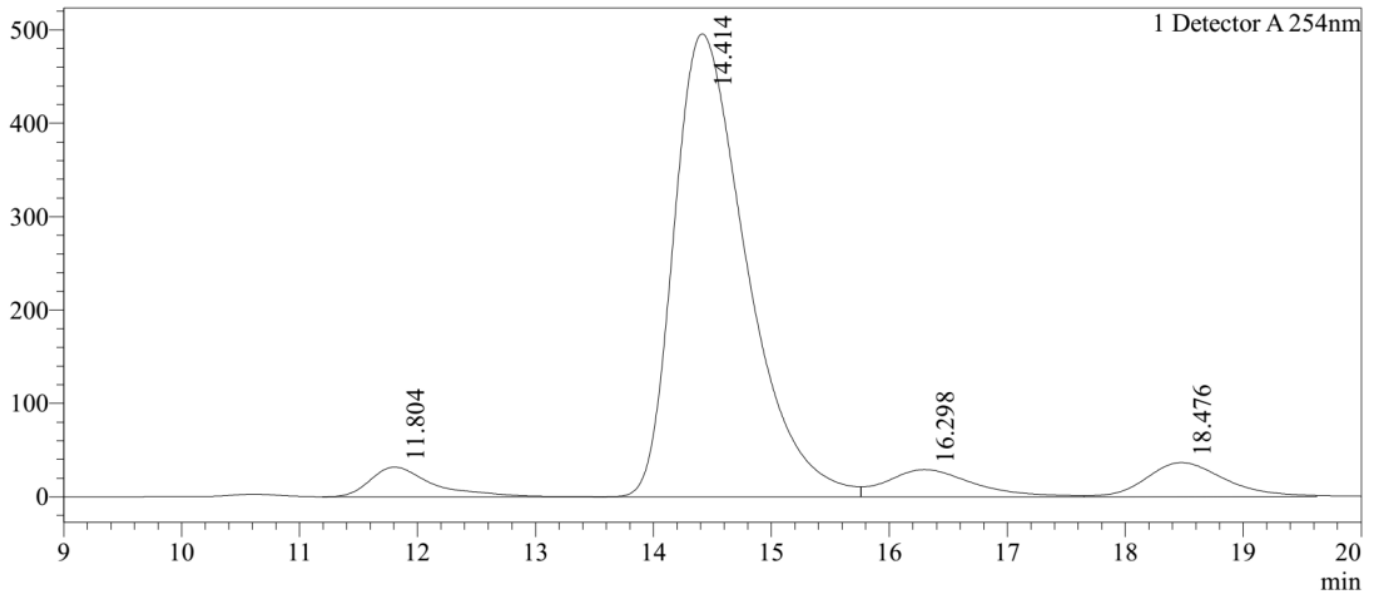

Peak Table

\begin{tabular}{|c|c|c|c|c|}
\hline & & & & \\
\hline Peak\# & Ret. Time & Area & Height & Area $\%$ \\
\hline 1 & 11.804 & 1185060 & 31867 & 4.651 \\
\hline 2 & 14.414 & 21132575 & 495578 & 82.933 \\
\hline 3 & 16.298 & 1475106 & 29048 & 5.789 \\
\hline 4 & 18.476 & 1688831 & 36510 & 6.628 \\
\hline Total & & 25481572 & 593003 & 100.000 \\
\hline
\end{tabular}




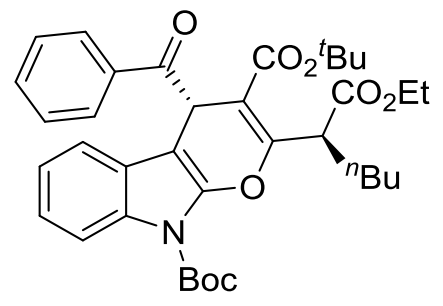

A colorless oil $(114 \mathrm{mg}, 92 \%) ;[\alpha]^{25}=+33.4\left(c 1.0, \mathrm{CHCl}_{3}\right) ;{ }^{1} \mathrm{H}$ NMR (400 MHz, $\left.\mathrm{CDCl}_{3}\right) \delta 8.10(\mathrm{~d}, J=7.5 \mathrm{~Hz}, 3 \mathrm{H}), 7.61(\mathrm{t}, J=7.4 \mathrm{~Hz}, 1 \mathrm{H}), 7.50(\mathrm{t}, J=7.6 \mathrm{~Hz}, 2 \mathrm{H})$, $7.19-7.10(\mathrm{~m}, 1 \mathrm{H}), 6.97(\mathrm{t}, J=7.5 \mathrm{~Hz}, 1 \mathrm{H}), 6.89(\mathrm{~d}, J=7.5 \mathrm{~Hz}, 1 \mathrm{H}), 5.76(\mathrm{~s}, 1 \mathrm{H})$, $5.09(\mathrm{dd}, J=9.6,5.5 \mathrm{~Hz}, 1 \mathrm{H}), 4.25-4.10(\mathrm{~m}, 2 \mathrm{H}), 2.20-2.01(\mathrm{~m}, 2 \mathrm{H}), 1.65(\mathrm{~s}, 9 \mathrm{H})$, $1.54-1.45(\mathrm{~m}, 2 \mathrm{H}), 1.46-1.36(\mathrm{~m}, 2 \mathrm{H}), 1.34(\mathrm{~s}, 9 \mathrm{H}), 1.21(\mathrm{t}, J=7.1 \mathrm{~Hz}, 3 \mathrm{H}), 0.94(\mathrm{t}$, $J=7.2 \mathrm{~Hz}, 3 \mathrm{H}) ;{ }^{13} \mathrm{C} \mathrm{NMR}\left(100 \mathrm{MHz}, \mathrm{CDCl}_{3}\right) \delta 199.1,171.1,165.3,160.4,142.7$, $137.0,133.2,131.9,129.0,128.6,125.1,123.3,123.1,118.2,115.0,107.9,92.0,84.5$, 82.5, 60.9, 46.3, 42.5, 29.0, 28.6, 28.2, 28.0, 22.6, 14.3, 13.9. HRMS (ESI) $\mathrm{m} / z$ calcd for $\mathrm{C}_{36} \mathrm{H}_{43} \mathrm{NO}_{8}[\mathrm{M}+\mathrm{Na}]^{+}=640.2886$, found $=640.2882$; The ee value was $>99.9 \%, \mathrm{t}_{\mathrm{R}}$ $($ minor $)=13.6 \mathrm{~min}, \mathrm{t}_{\mathrm{R}}$ (major) $=15.5 \mathrm{~min}($ Chiralcel $\mathrm{ID}, \lambda=254 \mathrm{~nm}, 2 \%$ $i$-PrOH/hexanes, flow rate $=0.5 \mathrm{~mL} / \mathrm{min})$.

D:IHPLC-DatalWJHIWJH-R827IWJH-R827-RAC-98-2-0.5-ID.Icd
mAU

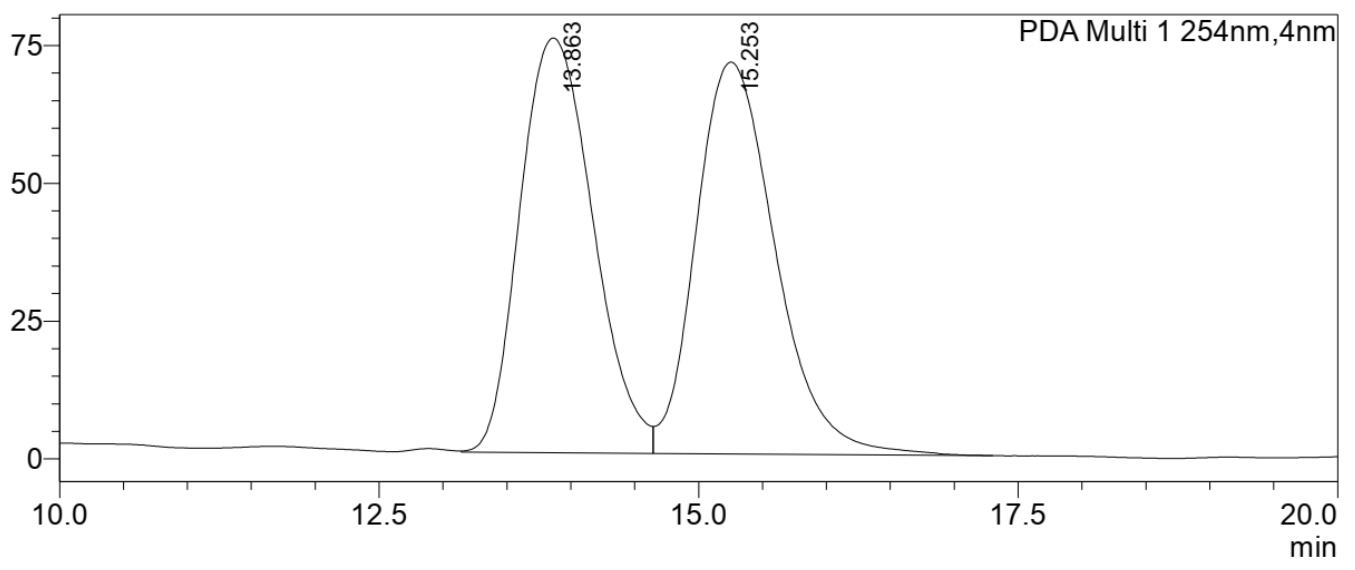

Peak Table

PDA Ch1 254nm

\begin{tabular}{|r|r|r|r|r|r|}
\hline Peak\# & Ret. Time & \multicolumn{1}{c|}{ Area } & \multicolumn{1}{c|}{ Height } & \multicolumn{1}{c|}{ Height $\%$} & \multicolumn{1}{c|}{ Area $\%$} \\
\hline 1 & 13.863 & 3002611 & 75217 & 51.410 & 49.467 \\
\hline 2 & 15.253 & 3067307 & 71090 & 48.590 & 50.533 \\
\hline Total & & 6069918 & 146308 & & 100.000 \\
\hline
\end{tabular}


D:IHPLC-DatalWJHIWJH-R827IWJH-R827-CHI-98-2-0.5-ID.Icd mAU

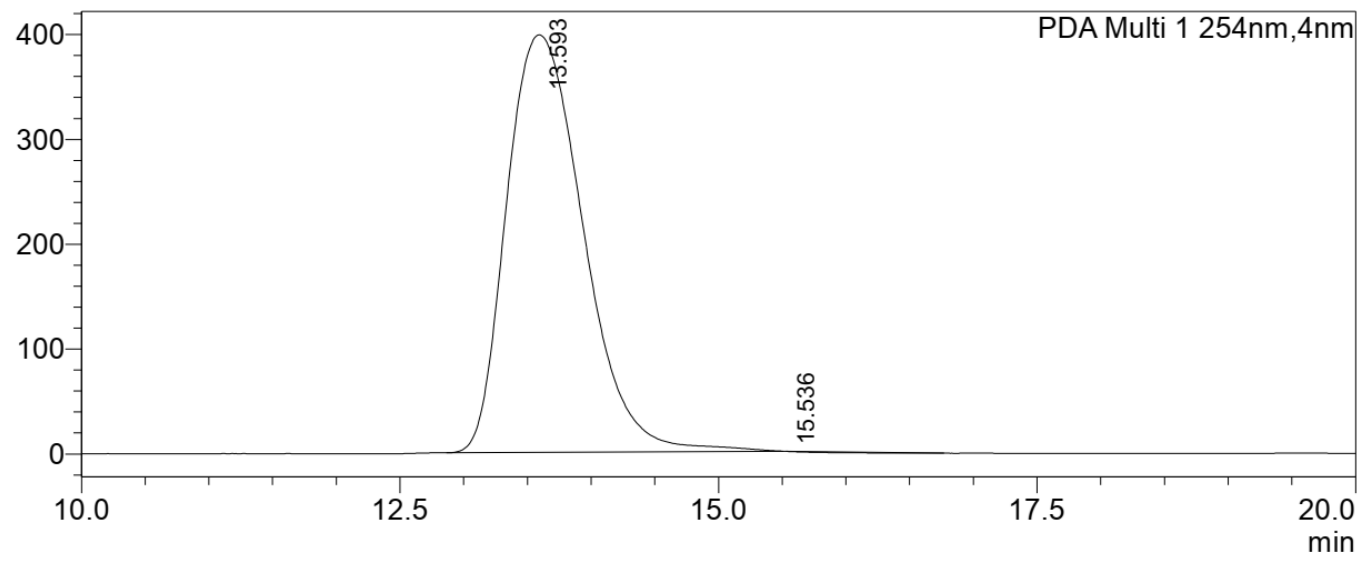

Peak Table

PDA Ch1 254nm

\begin{tabular}{|r|r|r|r|r|r|}
\hline Peak\# & Ret. Time & \multicolumn{1}{|c|}{ Area } & Height & \multicolumn{1}{|c|}{ Height $\%$} & \multicolumn{1}{|c|}{ Area\% } \\
\hline 1 & 13.593 & 16682666 & 398220 & 99.998 & 100.186 \\
\hline 2 & 15.536 & -31026 & 8 & 0.002 & -0.186 \\
\hline Total & & 16651640 & 398228 & 100.000 & 100.000 \\
\hline
\end{tabular}

\section{Determination of Absolute Configuration of the Products}

A The absolute configuration of the product $3 \mathbf{I}(S, S)$ was assigned by X-ray crystallographic analysis of a single crystal of $\mathbf{3 1}$ (Figure S3). The configurations of other products were assigned by analogy. CCDC 1947826 contains the supplementary crystallographic data of the adduct $\mathbf{3 l}$ for this paper. These data can be obtained free of charge from The Cambridge Crystallographic Data Centre via www.ccdc.cam.ac.uk/data_request/cif.

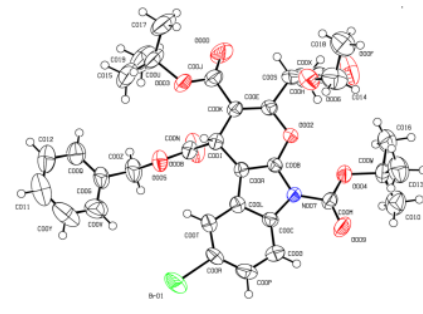

3I

(CCDC 1947826)

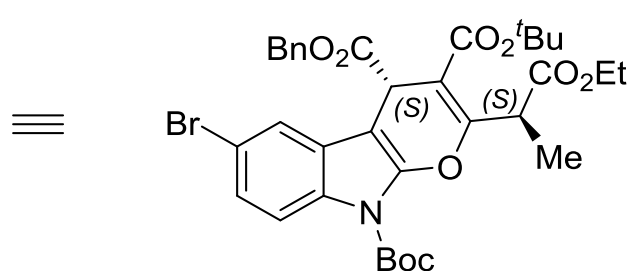

31

4-benzyl 3,9-di-tert-butyl (S)-6-bromo-

2-((S)-1-ethoxy-1-oxopropan-2yl)pyrano[2,3-b]indole-3,4,9(4H)tricarboxylate

Figure S1. X-ray structure of $\mathbf{3 1}$. 
Table 1 Crystal data and structure refinement for 31 .

\begin{tabular}{|c|c|}
\hline Identification code & 31 \\
\hline Empirical formula & $\mathrm{C}_{34} \mathrm{H}_{38} \mathrm{BrNO}_{9}$ \\
\hline Formula weight & 684.56 \\
\hline Temperature/K & $294.9(3)$ \\
\hline Crystal system & monoclinic \\
\hline Space group & $\mathrm{P}_{21}$ \\
\hline $\mathrm{a} / \AA$ & $9.6668(6)$ \\
\hline $\mathrm{b} / \AA$ & $14.4314(8)$ \\
\hline $\mathrm{c} / \AA$ & $12.7564(6)$ \\
\hline$\alpha /^{\circ}$ & 90 \\
\hline$\beta /{ }^{\circ}$ & $107.035(6)$ \\
\hline$\gamma /{ }^{\circ}$ & 90 \\
\hline Volume $/ \AA^{3}$ & $1701.51(16)$ \\
\hline $\mathrm{Z}$ & 2 \\
\hline pcalcg $/ \mathrm{cm}^{3}$ & 1.336 \\
\hline$\mu / \mathrm{mm}^{-1}$ & 2.091 \\
\hline $\mathrm{F}(000)$ & 712.0 \\
\hline Crystal size $/ \mathrm{mm}^{3}$ & $0.7 \times 0.5 \times 0.15$ \\
\hline Radiation & $\mathrm{CuK} \alpha(\lambda=1.54184)$ \\
\hline $2 \Theta$ range for data collection ${ }^{\circ}$ & 7.248 to 146.974 \\
\hline Index ranges & $-9 \leq \mathrm{h} \leq 12,-17 \leq \mathrm{k} \leq 14,-15 \leq 1 \leq 14$ \\
\hline Reflections collected & 12523 \\
\hline Independent reflections & $5387[$ Rint $=0.0390$, Rsigma $=0.0364]$ \\
\hline Data/restraints/parameters & $5387 / 1 / 414$ \\
\hline Goodness-of-fit on $\mathrm{F}^{2}$ & 1.038 \\
\hline Final $R$ indexes $[\mathrm{I}>=2 \sigma(\mathrm{I})]$ & $\mathrm{R} 1=0.0531, \mathrm{wR} 2=0.1427$ \\
\hline Final $\mathrm{R}$ indexes [all data] & $\mathrm{R} 1=0.0567, \mathrm{wR} 2=0.1492$ \\
\hline Largest diff. peak/hole / e $\AA^{-3}$ & $0.41 /-0.49$ \\
\hline Flack parameter & $-0.017(13)$ \\
\hline
\end{tabular}




\section{Gram-scale Synthesis and Transformations}

a) Gram-scale synthesis:

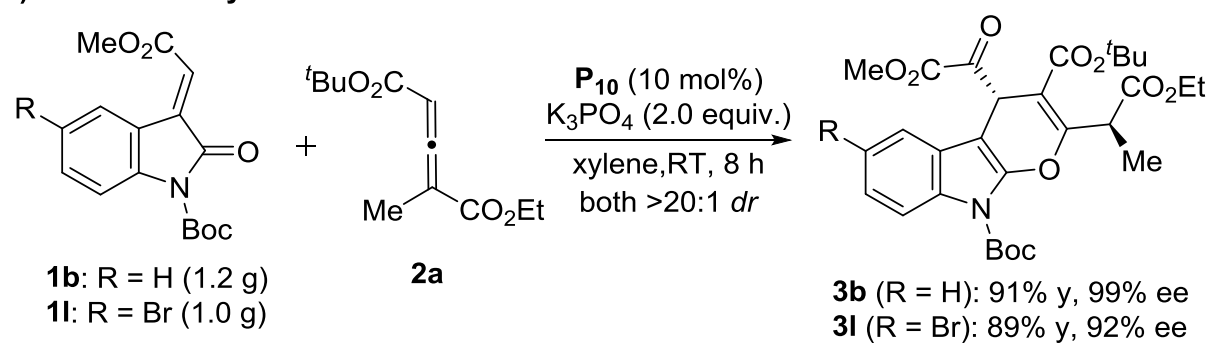

b) Synthetic manipulations of 31:

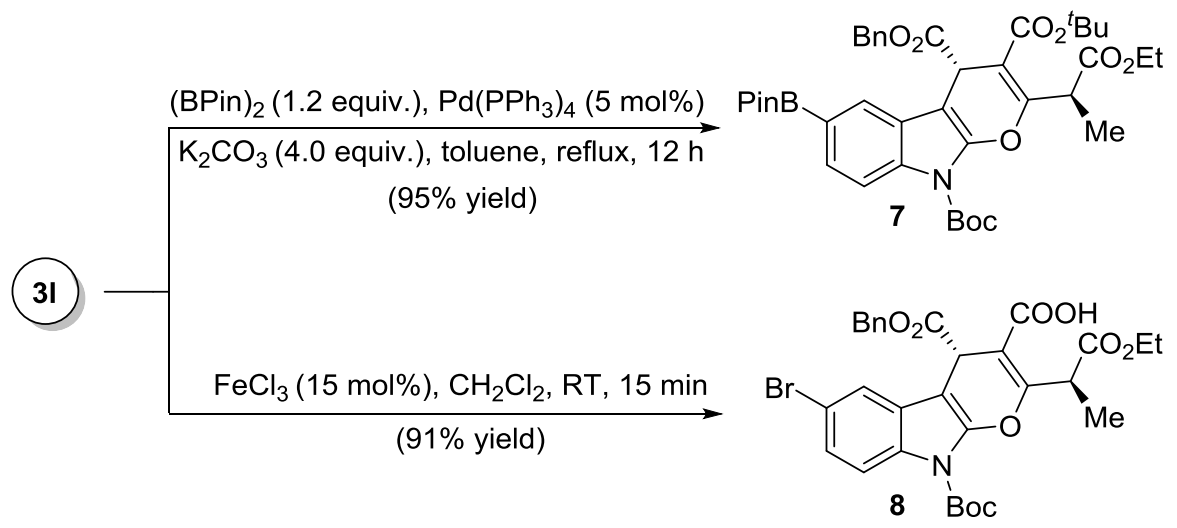

Figure S2. Gram-scale synthesis and transformations.

\section{A. Procedure for the gram scale synthesis of $3 \mathrm{~b}$}<smiles>COC(=O)/C=C1/C(=O)N(C(=O)OC(C)(C)C)c2ccccc21</smiles>

$1 \mathrm{~b}(1.21 \mathrm{~g})$

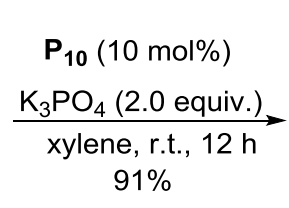

$2 \mathbf{a}$<smiles>CCOC(=O)C1=C(C(C)C)Oc2c(c3ccccc3n2C(=O)OC(C)(C)C)[C@@H]1C(=O)OC</smiles>

3b

$>20: 1 d r, 99 \%$ ee

To a flame-dried round bottle flask with a magnetic stirring bar were added the insatin derivatives $\mathbf{1 b}$ (1.21 g, $4 \mathrm{mmol})$, allenoates $\mathbf{2 a}$ (1.09 g, $4.8 \mathrm{mmol})$, phosphonium salt $\mathbf{P}_{10}(290 \mathrm{mg}, 0.4 \mathrm{mmol})$ and $\mathrm{K}_{3} \mathrm{PO}_{4}(1.7 \mathrm{~g}, 8 \mathrm{mmol})$, followed by the addition of xylene $(80 \mathrm{~mL})$. The reaction mixture was stirred at room temperature for $12 \mathrm{~h}$. Then, the reaction was added $\mathrm{H}_{2} \mathrm{O}(20 \mathrm{~mL})$, and the mixture was extracted with ethyl acetate (20 mL x 3), dried over $\mathrm{MgSO}_{4}$, and the solvent was removed under reduced pressure, and the residue was purified by column chromatography on silica gel (petroleum 
ether/ethyl acetate $=25: 1)$ to give $\mathbf{3 b}(1.93 \mathrm{~g}, 91 \%$ yield, $>20: 1 d r,>99 \%$ ee $)$ as a white solid.

\section{B. Procedure for the gram scale synthesis of 31}<smiles>CC(C)(C)OC(=O)N1C(=O)/C(=C/C(=O)OBr)c2cc(Br)ccc21</smiles>

1I (1.0g)<smiles>CCOC(=O)C=CC(=O)OCC</smiles>

2a
$\mathbf{P}_{\mathbf{1 0}}(10 \mathrm{~mol} \%)$

$\underset{\text { xylene, r.t., } 12 \mathrm{~h}}{\stackrel{\mathrm{K}_{3} \mathrm{PO}_{4}(2.0 \text { equiv. })}{\longrightarrow}}$ $91 \%$

$>20: 1 d r, 92 \%$ ee

To a flame-dried round bottle flask with a magnetic stirring bar were added the insatin derivatives 11 (1.0 g, $2.18 \mathrm{mmol})$, allenoates $2 \mathbf{a}$ (593 mg, $2.61 \mathrm{mmol})$, phosphonium salt $\mathbf{P}_{10}(158 \mathrm{mg}, 0.22 \mathrm{mmol})$ and $\mathrm{K}_{3} \mathrm{PO}_{4}(927 \mathrm{mg}, 4.36 \mathrm{mmol})$, followed by the addition of xylene $(80 \mathrm{~mL})$. The reaction mixture was stirred at room temperature for $12 \mathrm{~h}$. Then, the reaction was added $\mathrm{H}_{2} \mathrm{O}(20 \mathrm{~mL})$, and the mixture was extracted with ethyl acetate $\left(20 \mathrm{~mL}\right.$ x 3), dried over $\mathrm{MgSO}_{4}$, and the solvent was removed under reduced pressure, and the residue was purified by column chromatography on silica gel (petroleum ether/ethyl acetate $=25: 1)$ to give $31(1.33 \mathrm{~g}, 89 \%$ yield, $>20: 1 d r, 92 \%$ ee) as a white solid.

\section{Transformations of the product 31}

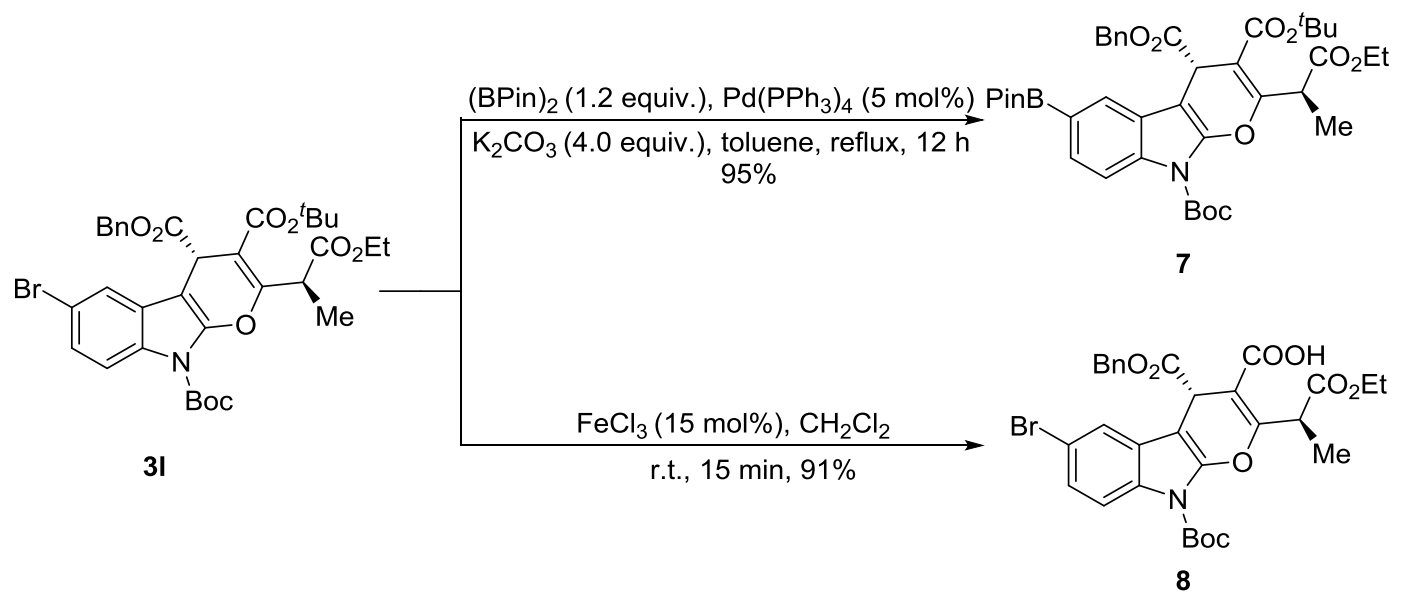




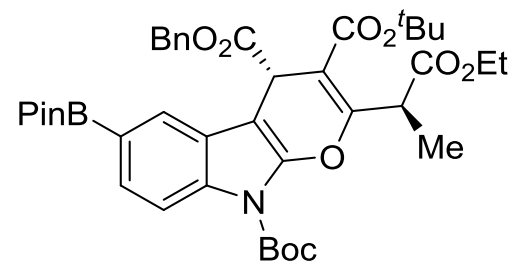

Under a nitrogen atmosphere, the product 31 (274 $\mathrm{mg}, 0.4 \mathrm{mmol})$, bis(pinacolato)diboron (122 mg, $0.48 \mathrm{mmol})$, tetrakis(triphenylphosphine)palladium (23 mg, 0.02) and potassium carbonate $(221 \mathrm{mg}, 1.6 \mathrm{mmol}$ ) were dissolved in toluene $(8 \mathrm{ml})$. the mixture was refluxed in oil bath for $12 \mathrm{~h}$. Then, the reaction mixture was cooled to room temperature, and was added $\mathrm{H}_{2} \mathrm{O}(20 \mathrm{~mL})$, and the mixture was extracted with ethyl acetate $\left(20 \mathrm{~mL}\right.$ x 3), dried over $\mathrm{MgSO}_{4}$, and the solvent was removed under reduced pressure, and the residue was purified by column chromatography on silica gel (petroleum ether/ethyl acetate $=10: 1)$ to give $7(230 \mathrm{mg}$, 95\% yield) as a white solid. ${ }^{1} \mathrm{H}$ NMR (400 MHz, $\left.\mathrm{CDCl}_{3}\right) \delta 8.16-8.12(\mathrm{~m}, 2 \mathrm{H}), 7.72$ $(\mathrm{dd}, J=8.5,1.0 \mathrm{~Hz}, 1 \mathrm{H}), 7.42-7.35(\mathrm{~m}, 2 \mathrm{H}), 7.33-7.27(\mathrm{~m}, 3 \mathrm{H}), 5.20-5.05(\mathrm{~m}$, 3H), $4.95(\mathrm{~s}, 1 \mathrm{H}), 4.25-4.08(\mathrm{~m}, 2 \mathrm{H}), 1.64(\mathrm{~s}, 9 \mathrm{H}), 1.57(\mathrm{~d}, J=7.2 \mathrm{~Hz}, 3 \mathrm{H}), 1.43$ (s, 9H), $1.38(\mathrm{~s}, 6 \mathrm{H}), 1.36(\mathrm{~s}, 6 \mathrm{H}), 1.19(\mathrm{t}, J=7.1 \mathrm{~Hz}, 3 \mathrm{H}) ;{ }^{13} \mathrm{C} \mathrm{NMR}\left(100 \mathrm{MHz}, \mathrm{CDCl}_{3}\right)$ $\delta 170.5,170.3,164.0,160.1,147.5,134.4,133.2,129.2,127.4,127.4,127.2,124.5$, $123.9,113.2,104.1,90.1,83.7,82.7,81.0,66.1,60.0,40.1,38.9,27.2,27.0,24.0$, 23.8, 13.2. HRMS (ESI) $\mathrm{m} / \mathrm{z}$ calcd for $\mathrm{C}_{40} \mathrm{H}_{50} \mathrm{BNO}_{11}[\mathrm{M}+\mathrm{Na}]^{+}=754.3375$, found $=$ 754.3382 .

(S)-4-((benzyloxy)carbonyl)-6-bromo-9-(tert-butoxycarbonyl)-2-((S)-1-ethoxy-1-0 xopropan-2-yl)-4,9-dihydropyrano[2,3-b]indole-3-carboxylic acid (8)<smiles>CCOC(C)C1=C(C(=O)O)[C@@H](C(=O)O)c2c(n(C(=O)O)c3ccc(Br)cc23)O1</smiles> 
The product 31 (274 mg, $0.4 \mathrm{mmol})$ dissolved in dry dichloromethane $(10 \mathrm{ml})$, and iron chloride $(9.7 \mathrm{mg}, 0.06 \mathrm{mmol})$ was added at room temperature. $10 \mathrm{~min}$ later, the mixture was added $\mathrm{H}_{2} \mathrm{O}(10 \mathrm{~mL})$ and extracted with ethyl acetate $(20 \mathrm{~mL}$ x 3), dried over $\mathrm{MgSO}_{4}$, and the solvent was removed under reduced pressure, and the residue was purified by column chromatography on silica gel (petroleum ether/ethyl acetate $=$ 3:1) to give 8 (229 mg, 91\% yield) as a white solid. ${ }^{1} \mathrm{H}$ NMR (400 $\left.\mathrm{MHz}, \mathrm{CDCl}_{3}\right) \delta$ $8.56(\mathrm{~s}, 1 \mathrm{H}), 7.52(\mathrm{~d}, J=1.9 \mathrm{~Hz}, 1 \mathrm{H}), 7.31-7.21(\mathrm{~m}, 5 \mathrm{H}), 7.11(\mathrm{dd}, J=8.5,1.9 \mathrm{~Hz}$, 1H), $6.95(\mathrm{~d}, J=8.5 \mathrm{~Hz}, 1 \mathrm{H}), 5.05-4.94(\mathrm{~m}, 3 \mathrm{H}), 4.38(\mathrm{~s}, 1 \mathrm{H}), 4.14(\mathrm{q}, J=7.1 \mathrm{~Hz}$, 2H), $1.44(\mathrm{~d}, J=7.2 \mathrm{~Hz}, 3 \mathrm{H}), 1.38(\mathrm{~s}, 9 \mathrm{H}), 1.19(\mathrm{t}, J=7.1 \mathrm{~Hz}, 3 \mathrm{H}) ;{ }^{13} \mathrm{C}$ NMR $(100$ $\left.\mathrm{MHz}, \mathrm{CDCl}_{3}\right) \delta 171.6,170.6,164.1,158.8,142.7,134.2,128.5,127.6,127.6,127.3$, 125.5, 123.1, 120.5, 112.6, 111.1, 104.7, 84.2, 80.9, 66.3, 60.4, 40.4, 39.0, 27.0, 13.2. HRMS (ESI) m/z calcd for $\mathrm{C}_{30} \mathrm{H}_{30} \mathrm{BrNO}_{9}[\mathrm{M}-\mathrm{H}]^{-}=626.1026$, found $=626.1023$.

\section{Preliminary Mechanistic Studies and Proposed Reaction Cycle}

\section{A) Deuterium-labeling experiment:}

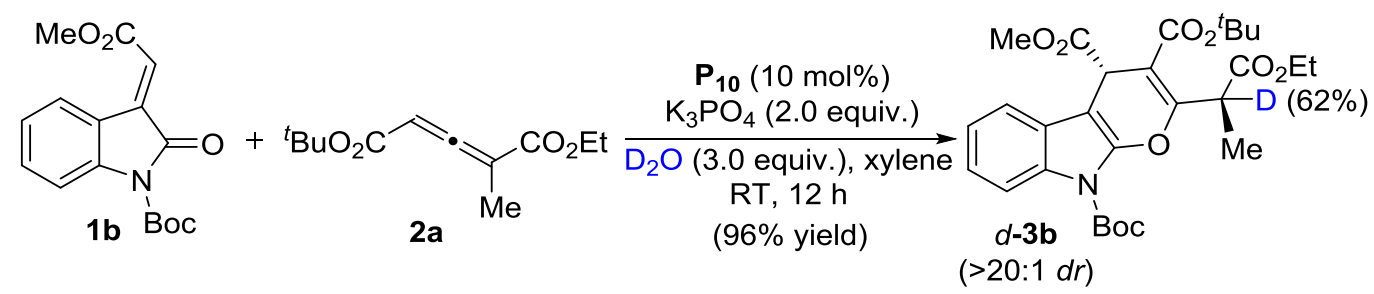

To a flame (dried in muffle furnace for $4 \mathrm{~h}$ ) round bottle flask with a magnetic stirring bar were added the insatin derivatives $\mathbf{1 b}(121 \mathrm{mg}, 0.4 \mathrm{mmol})$, allenoates $\mathbf{2 a}(109 \mathrm{mg}$, $0.48 \mathrm{mmol}), \mathbf{P}_{10}(29 \mathrm{mg}, 0.04 \mathrm{mmol})$ and $\mathrm{K}_{3} \mathrm{PO}_{4}(170 \mathrm{mg}, 0.8 \mathrm{mmol})$, followed by the addition of the mixture of xylene $(8 \mathrm{~mL})$ and deuterium oxide $(24 \mu \mathrm{L}, 1.2 \mathrm{mmol})$. The reaction mixture was stirred at room temperature for $12 \mathrm{~h}$. Then, the reaction was filtered and the solvent was removed under reduced pressure. The residue was purified by column chromatography on silica gel (petroleum ether/ethyl acetate = 25:1) to give $d$-3b (204 mg, 96\% yield) as a white solid. ${ }^{1} \mathrm{H}$ NMR (400 $\mathrm{MHz}, \mathrm{CDCl}_{3}$ ) $\delta 8.21-8.13(\mathrm{~m}, 1 \mathrm{H}), 7.68-7.61(\mathrm{~m}, 1 \mathrm{H}), 7.31-7.25(\mathrm{~m}, 2 \mathrm{H}), 5.10(\mathrm{q}, J=7.2 \mathrm{~Hz}$, $0.38 \mathrm{H}), 4.91(\mathrm{~s}, 1 \mathrm{H}), 4.28-4.10(\mathrm{~m}, 2 \mathrm{H}), 3.74(\mathrm{~s}, 3 \mathrm{H}), 1.66(\mathrm{~s}, 9 \mathrm{H}), 1.62(\mathrm{~d}, J=6.5$ 
$\mathrm{Hz}, 3 \mathrm{H}), 1.54$ (s, 9H), $1.22(\mathrm{t}, J=7.1 \mathrm{~Hz}, 3 \mathrm{H})$.

\section{B) Control experiments:}
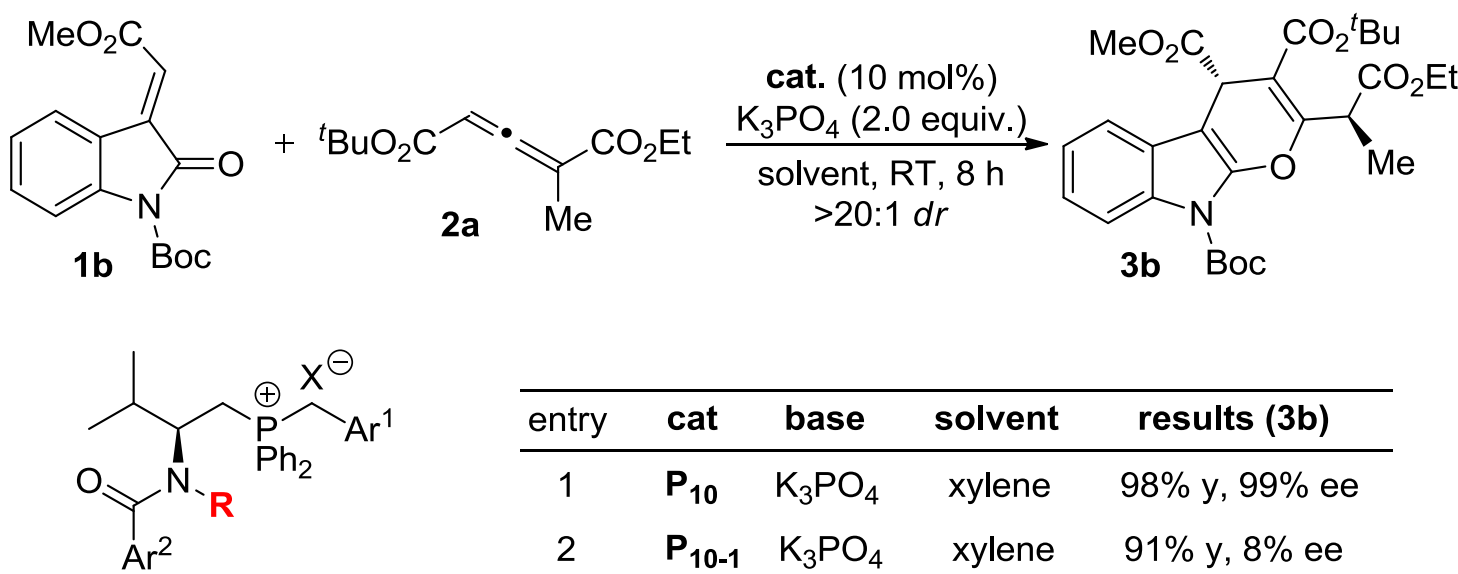

\begin{tabular}{cllll}
\hline entry & cat & base & solvent & \multicolumn{1}{c}{ results (3b) } \\
\hline 1 & $\mathbf{P}_{\mathbf{1 0}}$ & $\mathrm{K}_{3} \mathrm{PO}_{4}$ & xylene & $98 \% \mathrm{y}, 99 \%$ ee \\
2 & $\mathbf{P}_{10-1}$ & $\mathrm{~K}_{3} \mathrm{PO}_{4}$ & xylene & $91 \% \mathrm{y}, 8 \% \mathrm{ee}$ \\
3 & none & $\mathrm{K}_{3} \mathrm{PO}_{4}$ & xylene & $89 \% \mathrm{y}, 0 \% \mathrm{ee}$ \\
\hline 4 & $\mathbf{P}_{\mathbf{1 0}-\mathbf{2}}$ & $\mathrm{K}_{3} \mathrm{PO}_{4}$ & xylene & $93 \% \mathrm{y}, 89 \%$ ee \\
5 & $\mathbf{P}_{\mathbf{1 0}}$ & $\mathrm{K}_{3} \mathrm{PO}_{4}$ & $\mathrm{MeOH}$ & messy \\
6 & $\mathbf{P}_{\mathbf{1 0}}$ & none & xylene & no reaction \\
\hline
\end{tabular}

To a flame (dried in muffle furnace for $4 \mathrm{~h}$ ) round bottle flask with a magnetic stirring bar were added the insatin derivatives $\mathbf{1 b}(121 \mathrm{mg}, 0.4 \mathrm{mmol})$, allenoates $\mathbf{2 a}$ (109 $\mathrm{mg}$, $0.48 \mathrm{mmol}), \mathbf{P}_{10-1}(30 \mathrm{mg}, 0.04 \mathrm{mmol})$ and $\mathrm{K}_{3} \mathrm{PO}_{4}(170 \mathrm{mg}, 0.8 \mathrm{mmol})$, followed by the addition of the mixture of xylene $(8 \mathrm{~mL})$. The reaction mixture was stirred at room temperature for $8 \mathrm{~h}$. Then, the reaction was filtered and the solvent was removed under reduced pressure. The residue was purified by column chromatography on silica gel (petroleum ether/ethyl acetate $=25: 1)$ to give $\mathbf{3 b}(189 \mathrm{mg}, 91 \%$ yield $)$ as a white solid. The ee value was $8 \%, \mathrm{t}_{\mathrm{R}}($ minor $)=9.1 \mathrm{~min}, \mathrm{t}_{\mathrm{R}}($ major $)=12.0 \mathrm{~min}($ Chiralcel IE, $\lambda$ $=254 \mathrm{~nm}, 2 \% i-\mathrm{PrOH} /$ hexanes, flow rate $=1.0 \mathrm{~mL} / \mathrm{min})$. 
$\mathrm{mV}$

H: IWJH-Ř623 \WJH-R623-RAC-98-2-1.0-IE-25.0.1cd

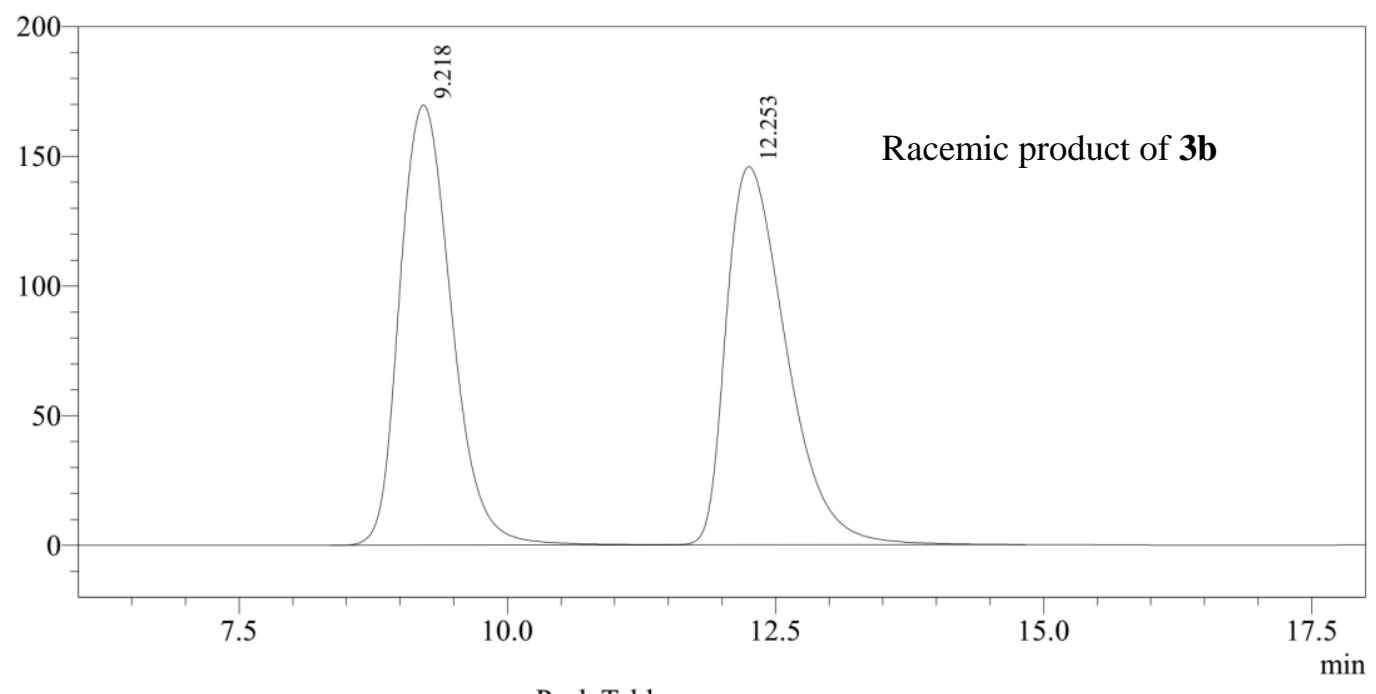

\begin{tabular}{|c|c|c|c|c|c|}
\hline \multirow{2}{*}{\multicolumn{6}{|c|}{ Detector A $254 \mathrm{~nm}$}} \\
\hline & & & & & \\
\hline Peak & Ret. Time & Height & Height $\%$ & Area & Area $\%$ \\
\hline 1 & 9.218 & 169760 & 53.797 & 5704390 & 50.084 \\
\hline 2 & 12.253 & 145799 & 46.203 & 5685164 & 49.916 \\
\hline Tota & & 315558 & 100.000 & 11389554 & 100.000 \\
\hline
\end{tabular}

Chromatogram D: LLC-DatalZLX YYSZZLX-Ph-race.lcd

H:IWJH-R 708।WJH-R623-2CHRI-98-2-1.0-IE-25-3.6-106.lcd $\mathrm{mV}$

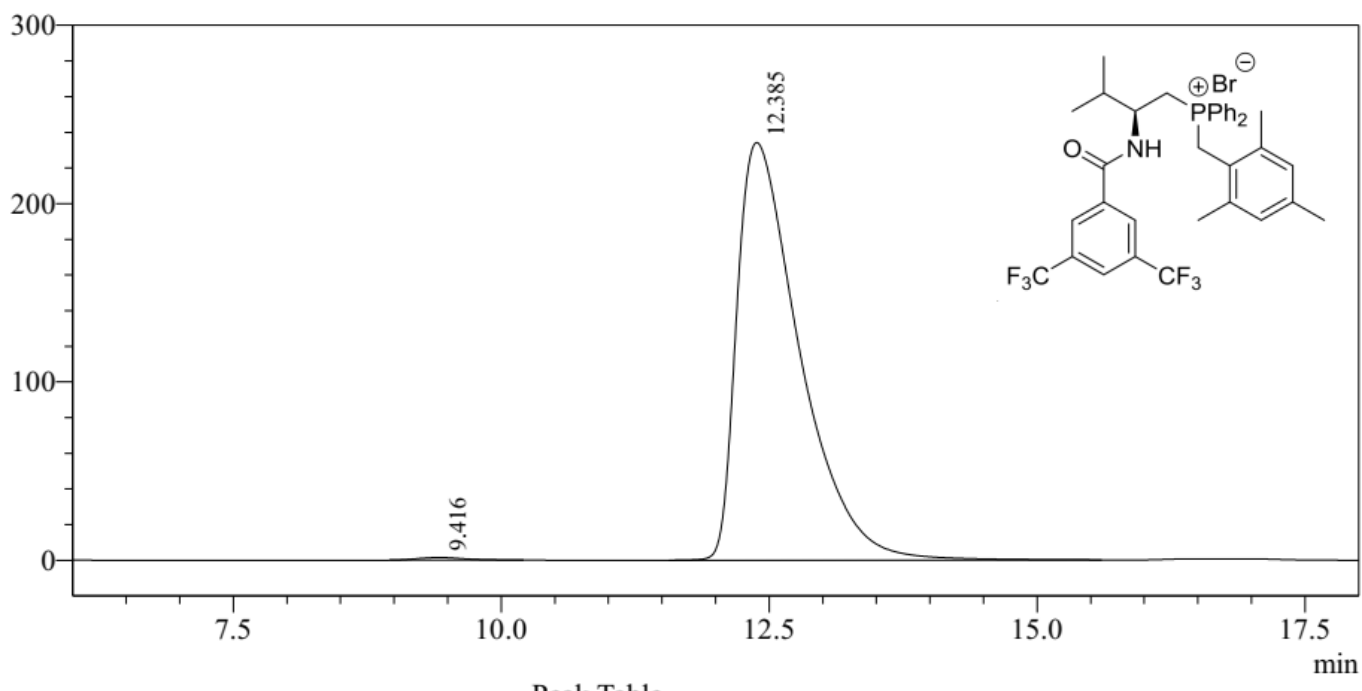

Detector A $254 \mathrm{~nm}$

Peak Table

\begin{tabular}{|r|r|r|r|r|r|}
\hline \multicolumn{1}{|c|}{ Peak\# } & Ret. Time & \multicolumn{1}{|c|}{ Height } & \multicolumn{1}{c|}{ Height $\%$} & Area & \multicolumn{1}{c|}{ Area $\%$} \\
\hline 1 & 9.416 & 1485 & 0.630 & 45786 & 0.478 \\
\hline 2 & 12.385 & 234157 & 99.370 & 9539794 & 99.522 \\
\hline Total & & 235641 & 100.000 & 9585580 & 100.000 \\
\hline
\end{tabular}


Chromatogram D: LCC-DatalZLX $\mid Y S Z Z L X-P h-r a c e . l c d$

D:LLC-DatalWJH $|W J H-R 710| W J H-R 710-C H R I-98-2-1.0-I E .1 c d$

$\mathrm{mV}$

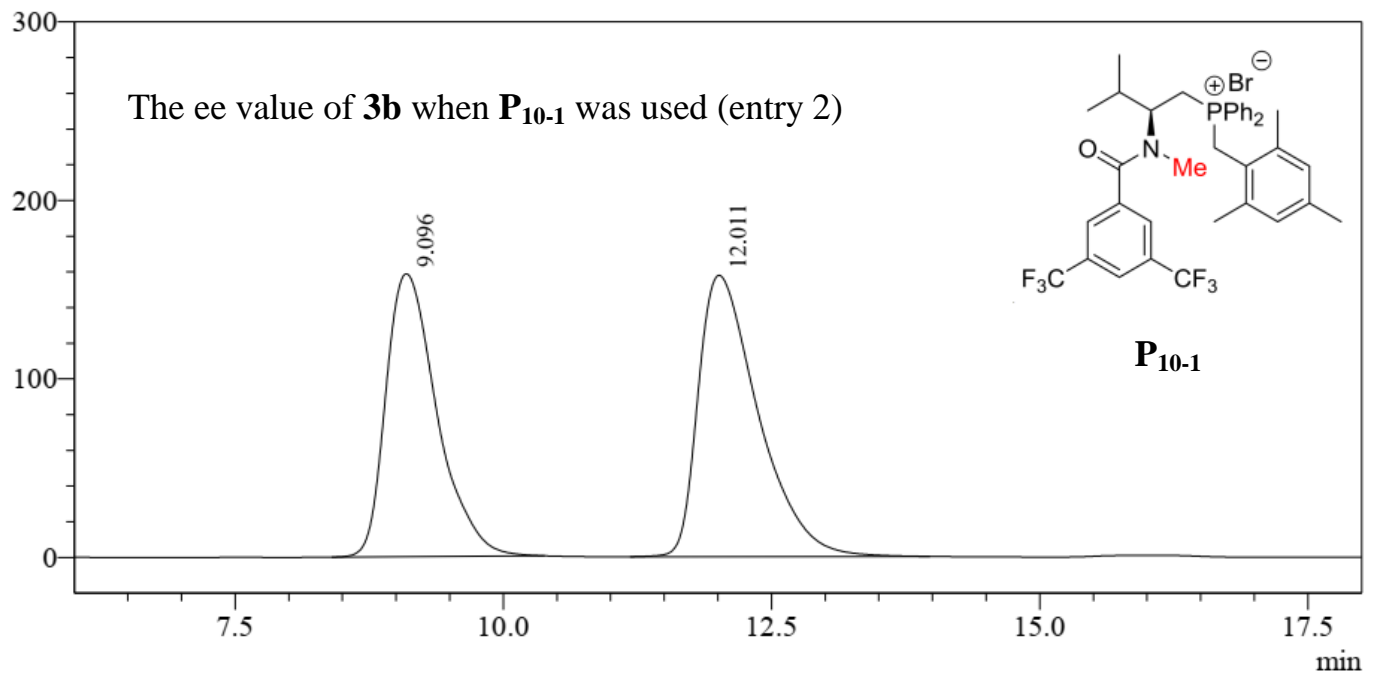

Peak Table

Detector A $254 \mathrm{~nm}$
\begin{tabular}{|r|r|r|r|r|r|}
\hline Peak\# & Ret. Time & Height & Height $\%$ & \multicolumn{1}{|c|}{ Area } & \multicolumn{1}{c|}{ Area $\%$} \\
\hline 1 & 9.096 & 158502 & 50.140 & 5251358 & 46.110 \\
\hline 2 & 12.011 & 157620 & 49.860 & 6137296 & 53.890 \\
\hline Total & & 316122 & 100.000 & 11388654 & 100.000 \\
\hline
\end{tabular}

Chromatogram D:LLC-DataZLLXYSLZLX-Ph-race.lcd

D:LLC-DatalWJH $\backslash W J H-R 708 \backslash W J H-R 708-C a t-C l-98-2-1.0-I E . l c d$

$\mathrm{mV}$

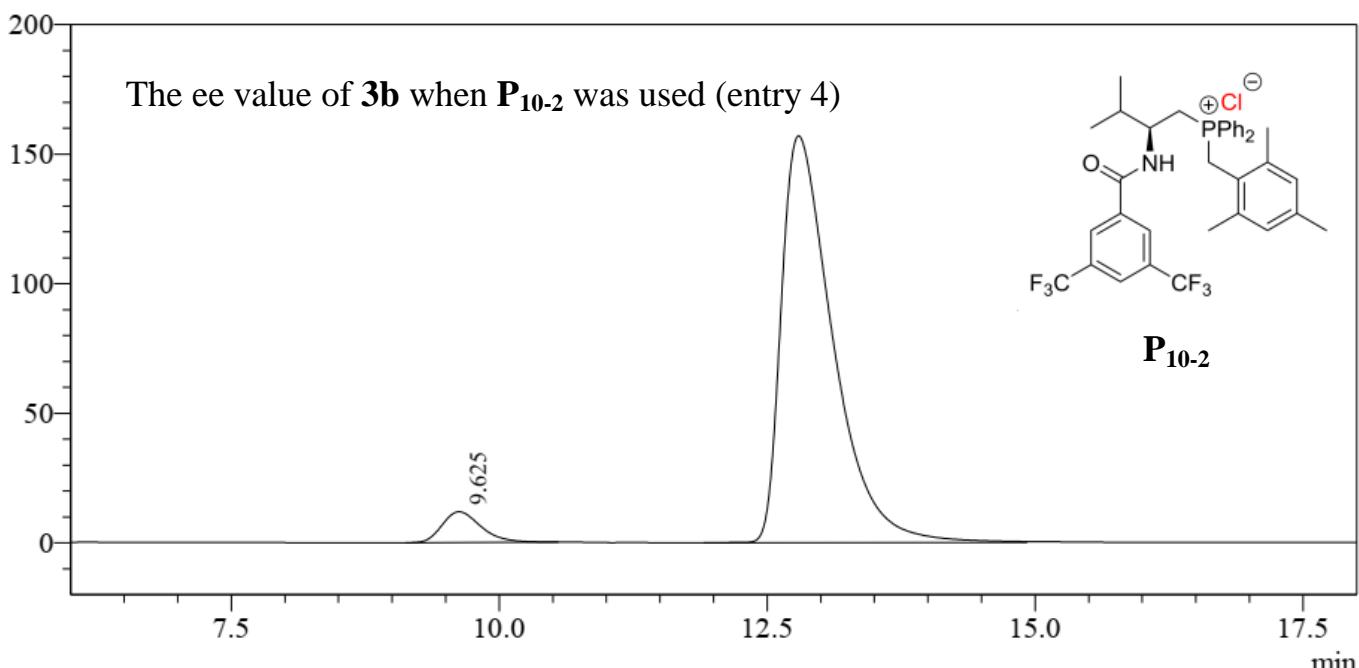

Peak Table

Detector A $254 \mathrm{~nm}$
\begin{tabular}{|r|r|r|r|r|r|}
\hline Peak\# & Ret. Time & Height & Height $\%$ & \multicolumn{1}{c|}{ Area } & \multicolumn{1}{|c|}{ Area $\%$} \\
\hline 1 & 9.625 & 11875 & 7.033 & 302175 & 5.425 \\
\hline 2 & 12.793 & 156979 & 92.967 & 5267655 & 94.575 \\
\hline Total & & 168854 & 100.000 & 5569829 & 100.000 \\
\hline
\end{tabular}


C) Proposed Reaction Cycle: 

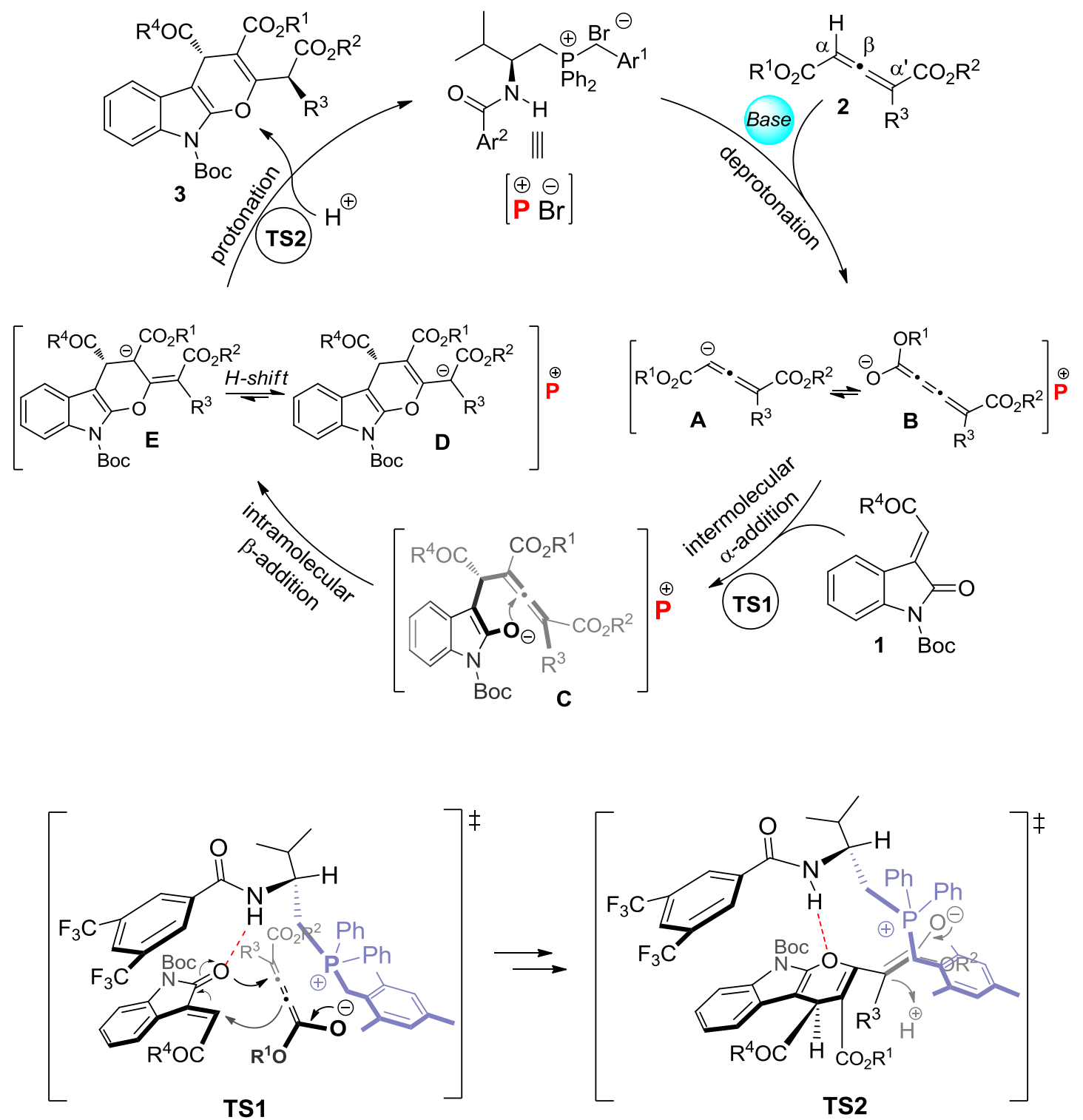

Figure S3. Proposed reaction cycle and TS models. 


\section{References}

[1] a) X. Han, Y. Wang, F. Zhong, Y. Lu, J. Am. Chem. Soc. 2011, 133, 1726; b) X. Han, F. Zhong, Y. Wang, Y. Lu, Angew. Chem. Int. Ed. 2012, 51, 767; c) F. Zhong, X. Han, Y. Wang, Y. Lu, Chem. Sci. 2012, 3, 1231; d) F. Zhong, X. Han, Y. Wang, Y. Lu, Angew. Chem. Int. Ed. 2011, 50, 7837; e) F. Zhong, J. Luo, G.-Y. Chen, X. Dou, Y. Lu, J. Am. Chem. Soc. 2012, 134, 10222; f) F. Zhong, X. Dou, X. Han, W. Yao, Q, Zhu, Y. Meng, Y. Lu, Angew. Chem. Int. Ed. 2013, 52, 943.

[2] a) B. Tan, G. Hernández-Torres, C. F. Barbas. III. J. Am. Chem. Soc. 2011, 133, 1235; b) B. Tan, N. R. Candeias, C. F. Barbas. III. J. Am. Chem. Soc. 2011, 133, 4672.

[3] a) T. Hashimoto, Y. Naganawa, K.Maruoka, J. Am. Chem. Soc. 2009, 131, 6614; b) T. Hashimoto, K. Sakata, F. Tamakuni, M. J. Dutton, K. Maruoka, Nat. Chem. 2013, 5, 240. 


\section{NMR Spectra of the Products}

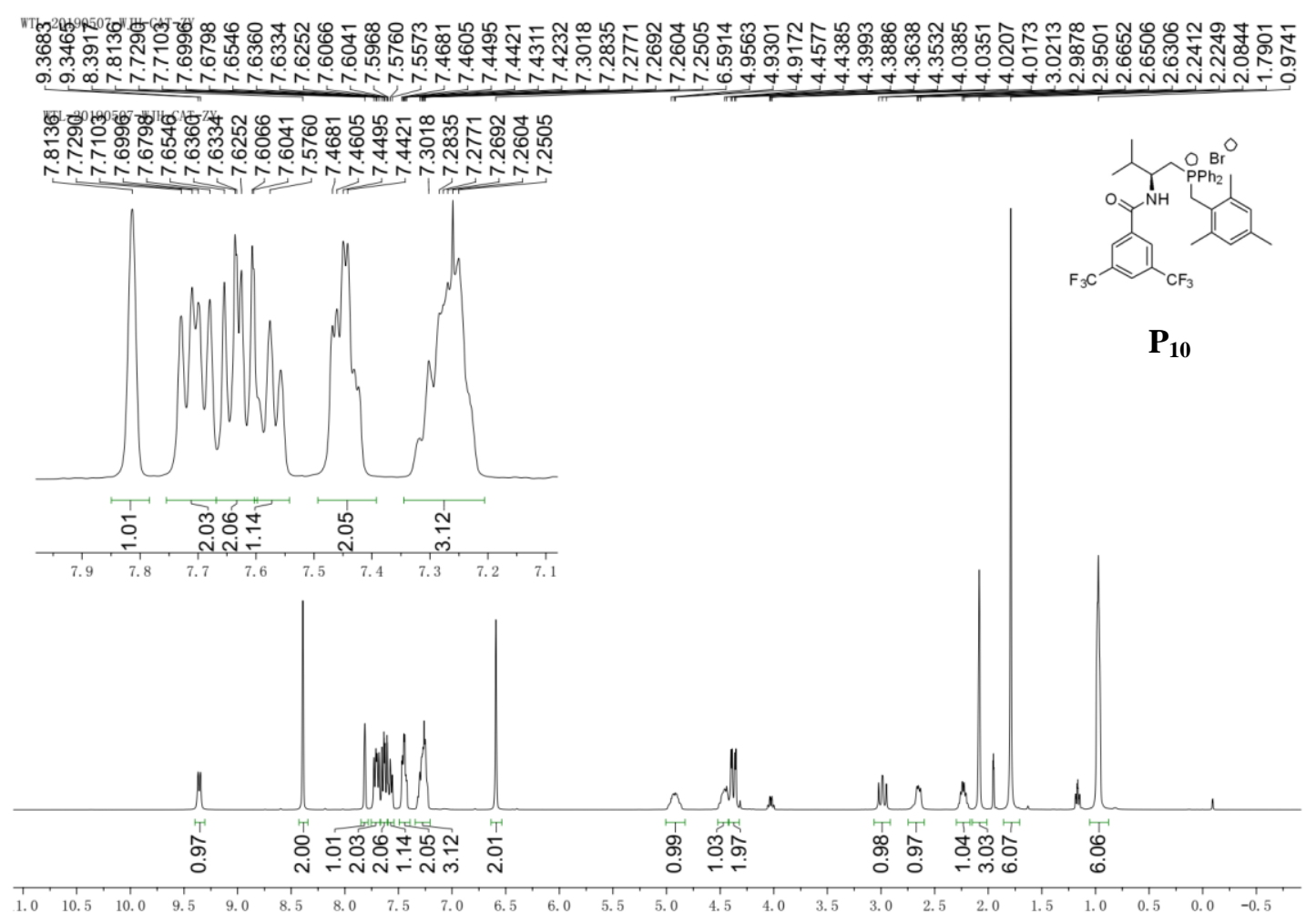

WTL-20191106-WWJH-ZY-2

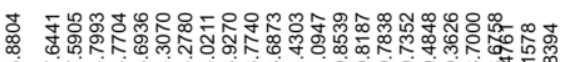

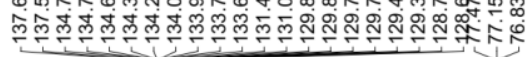

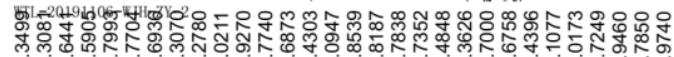

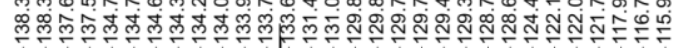

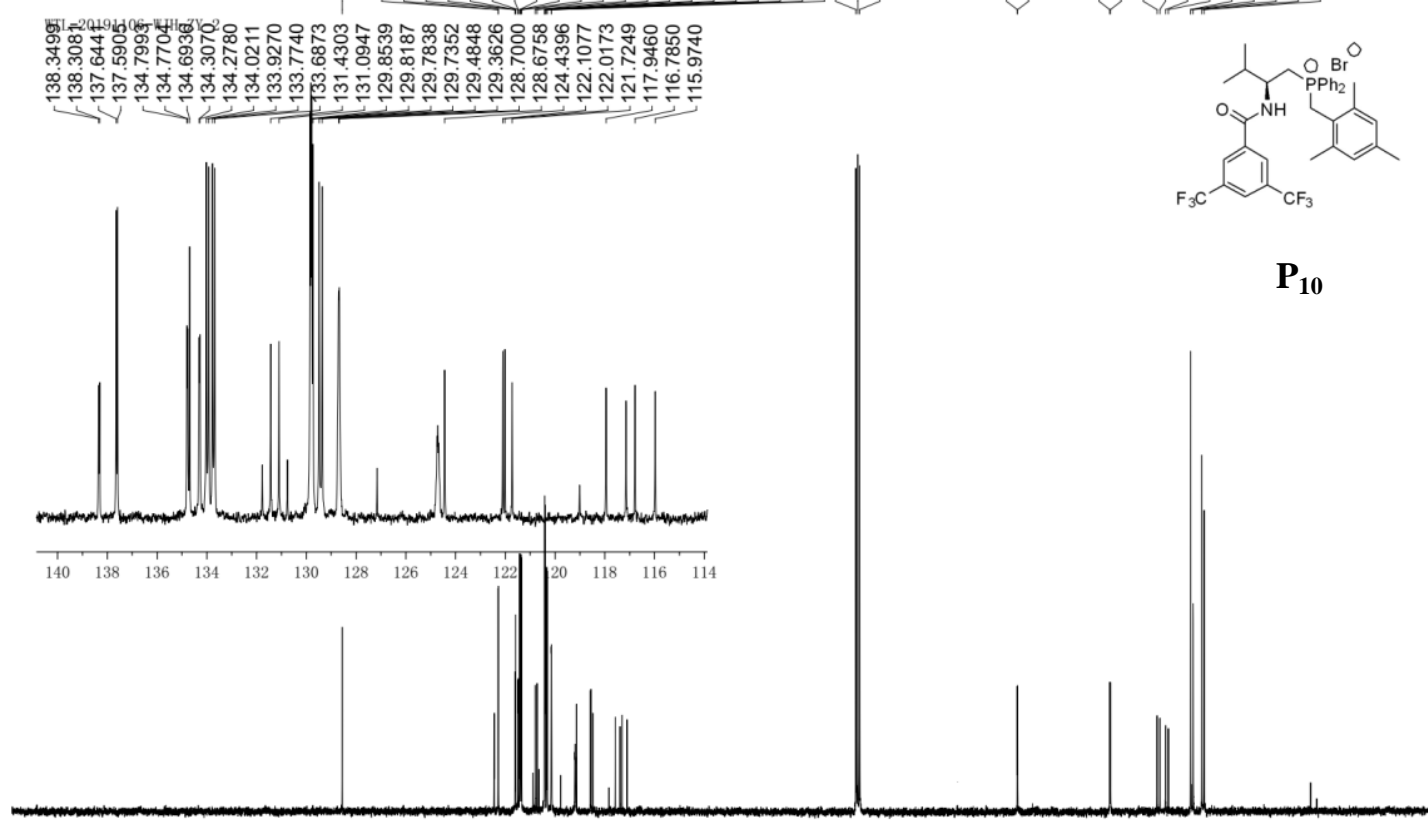

\begin{tabular}{lllllllllllll}
\hline 10 & 200 & 190 & 180 & 170 & 160 & 150 & 140 & 130 & 120 & 110 & 1 \\
\hline
\end{tabular}

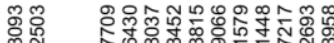

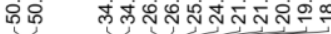


WTL-20190507-WJH-CAT-ZY

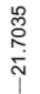

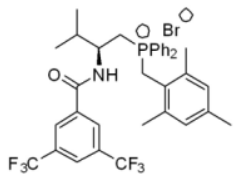

$\mathbf{P}_{10}$

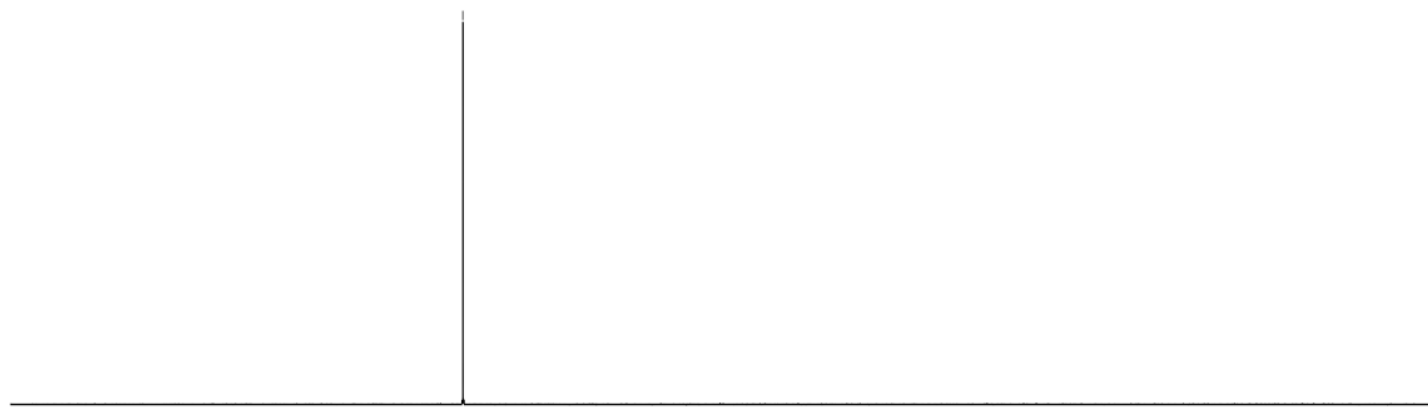

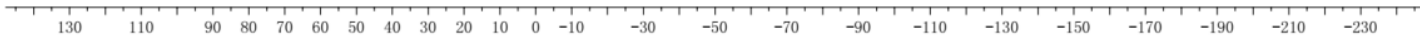

WTL-20191130-WJH-RP10@़

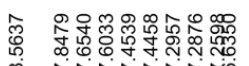

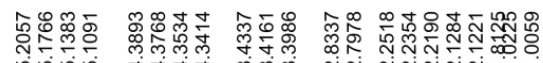

둥으

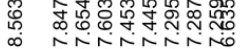

की

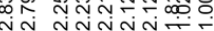

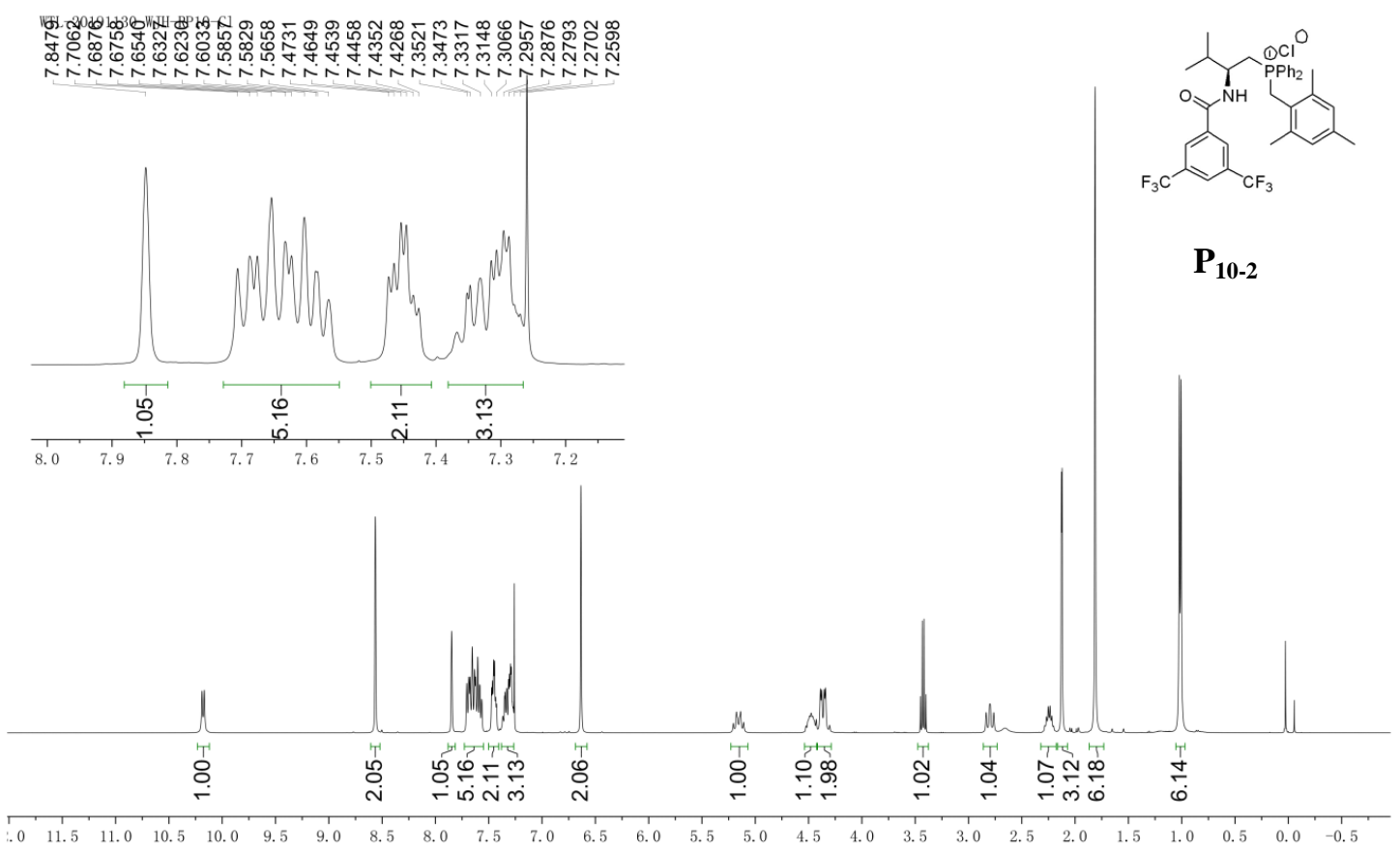



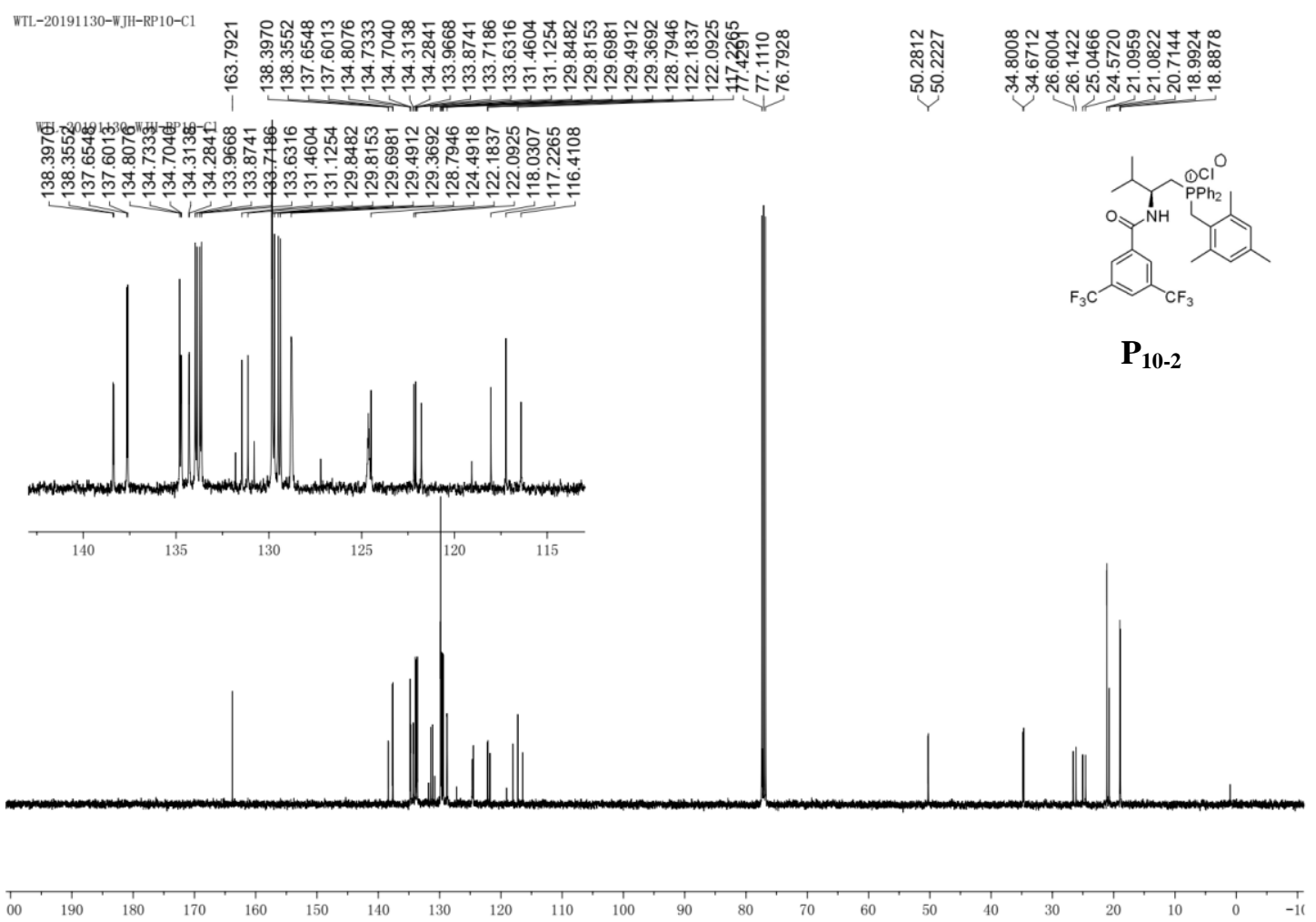

WTL-20191130-WJH-RP10-Cl

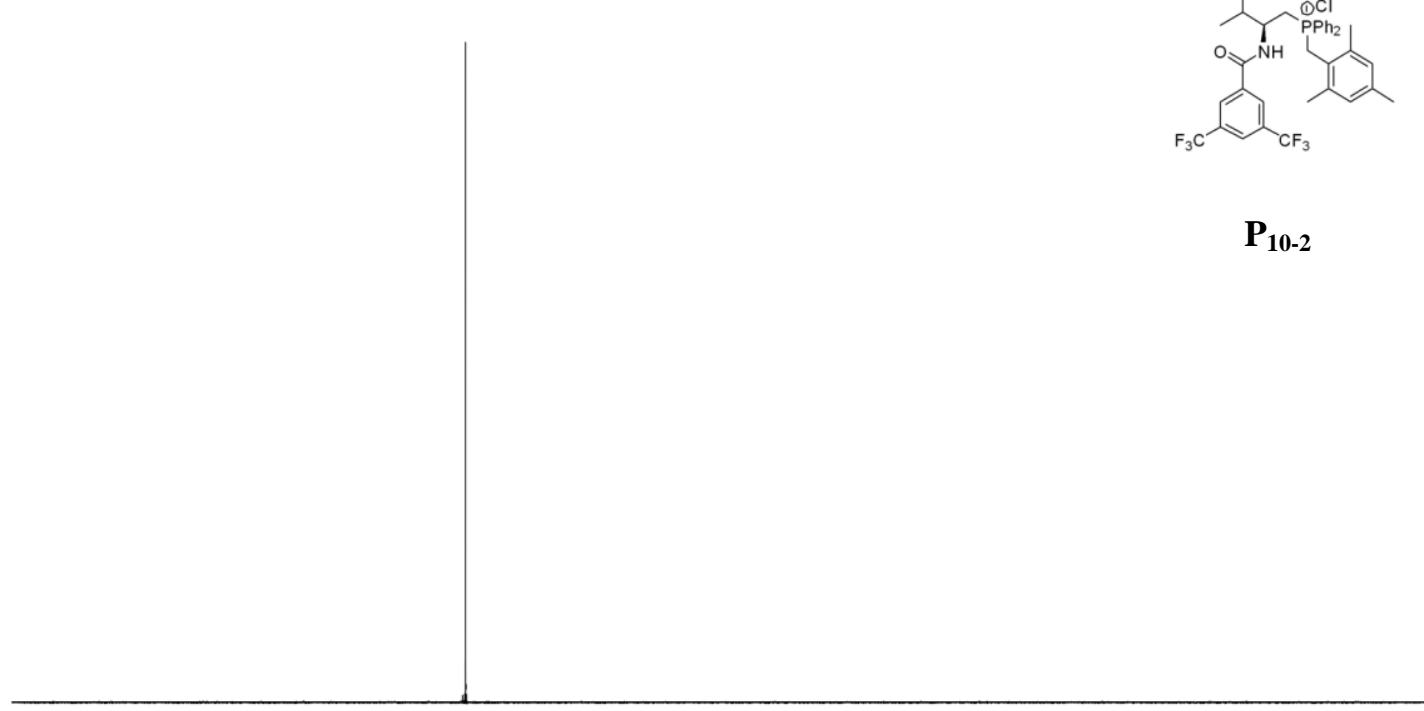

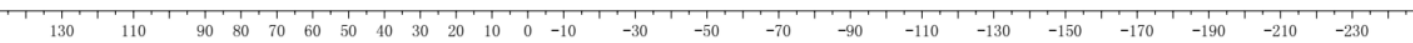




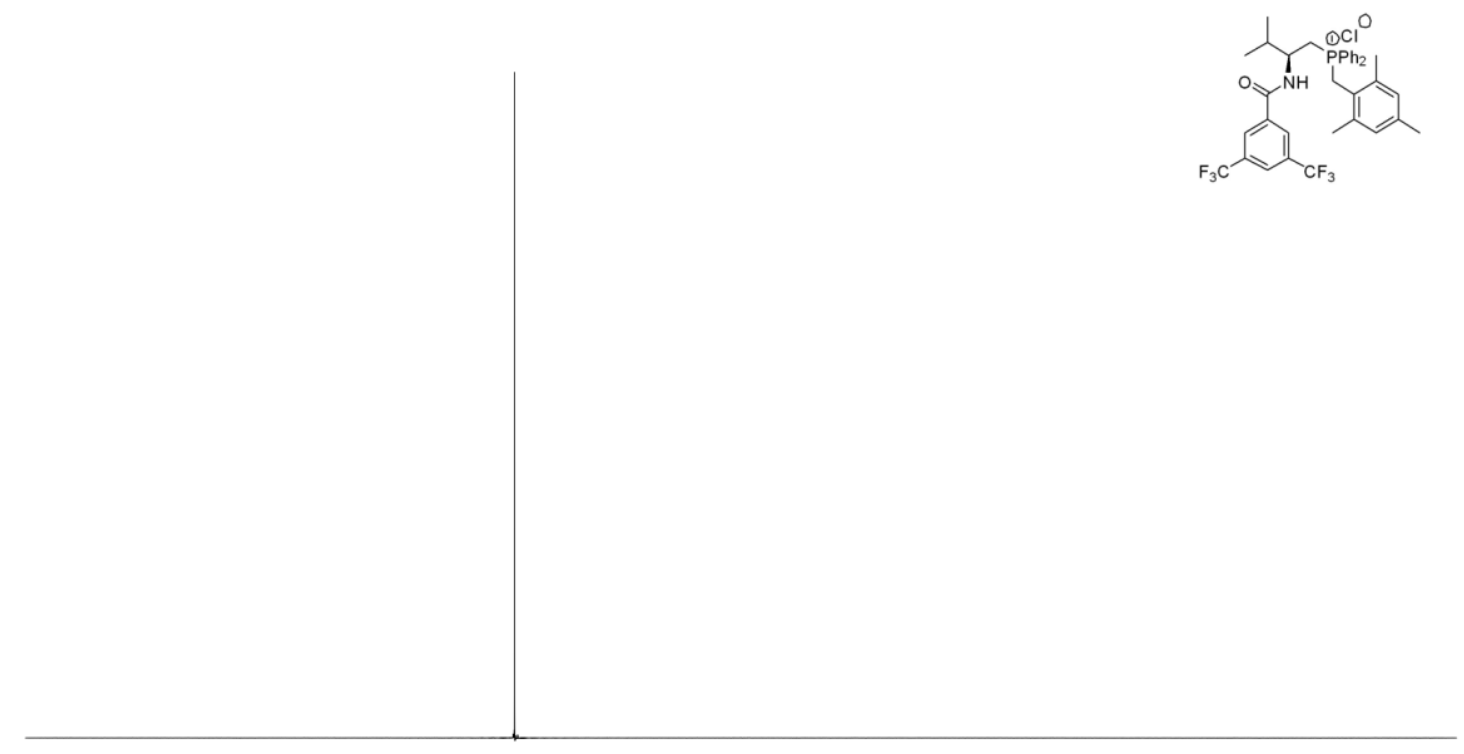

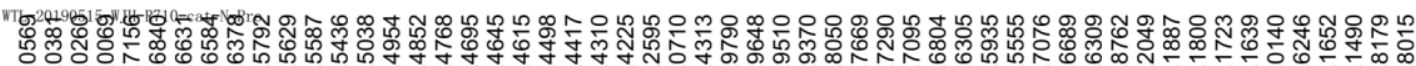

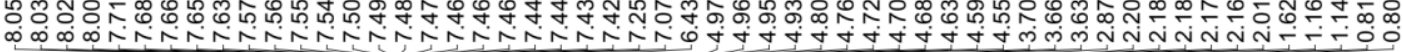

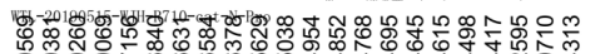

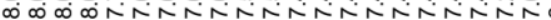
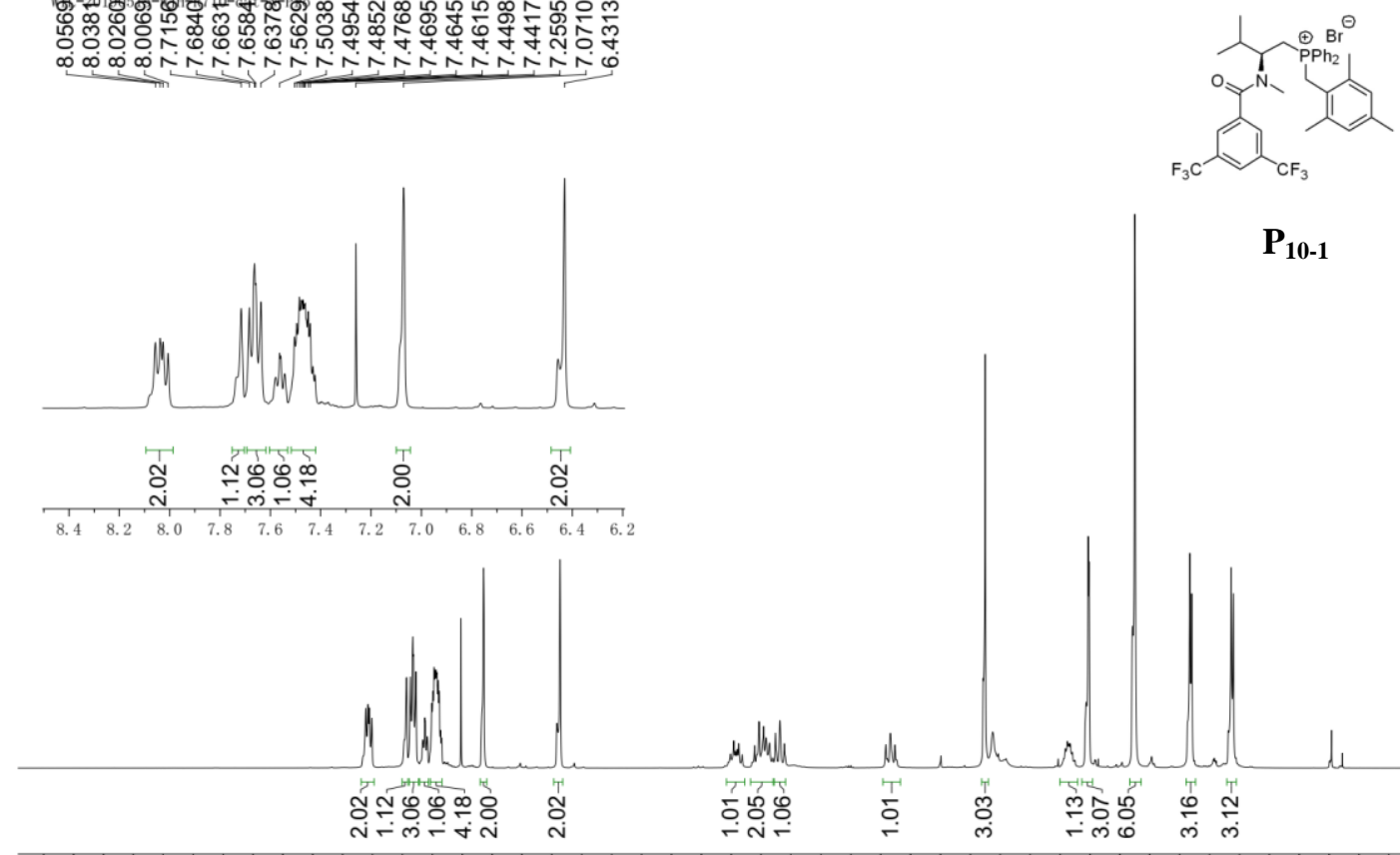

$\mathbf{P}_{10-1}$

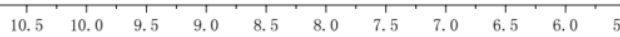

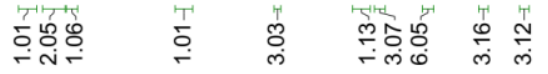



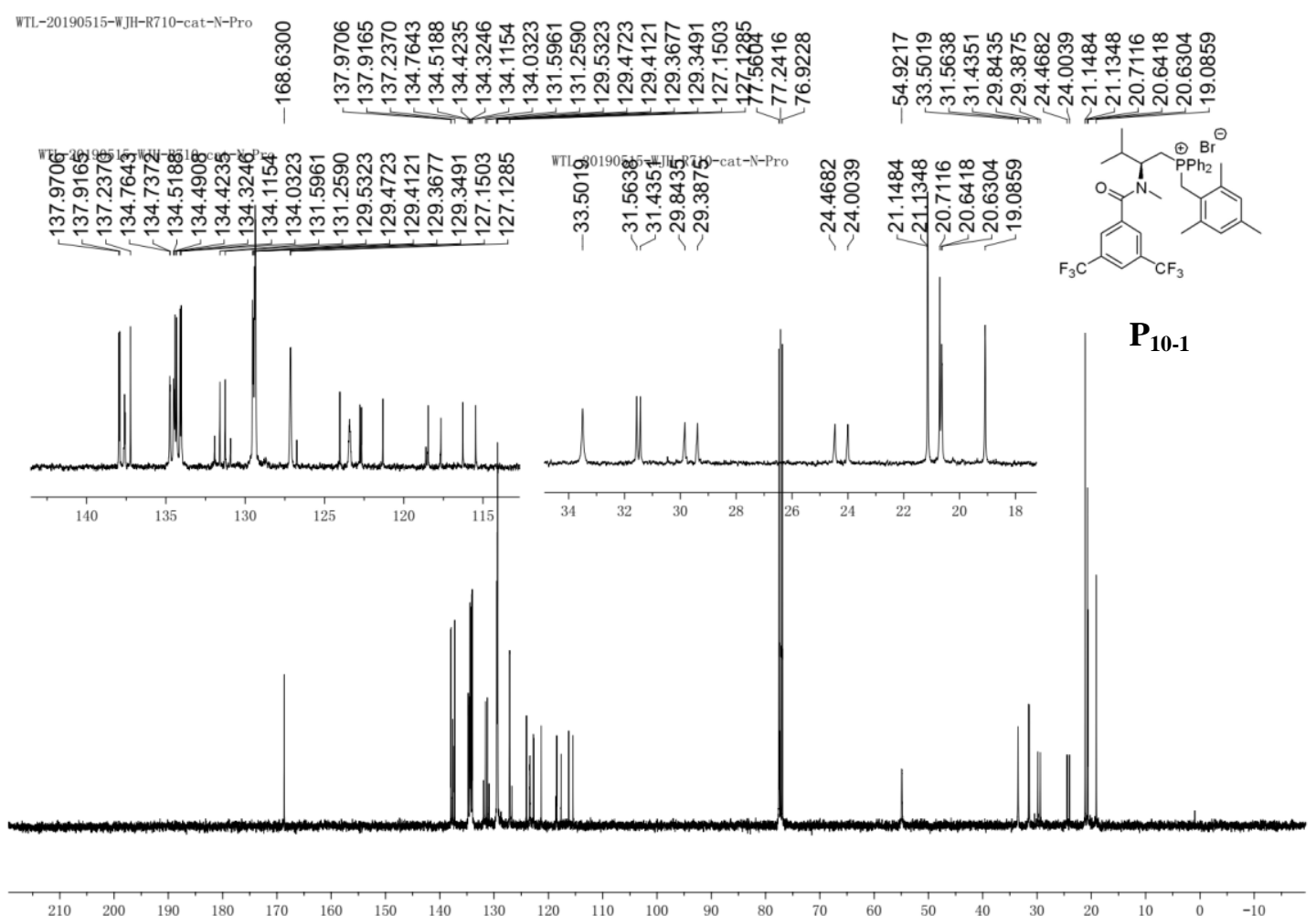

WTL-20190515-W JH-R710-cat-N-Pro

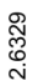

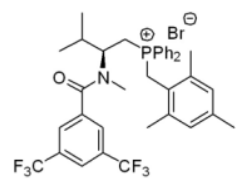

$\mathbf{P}_{10-1}$

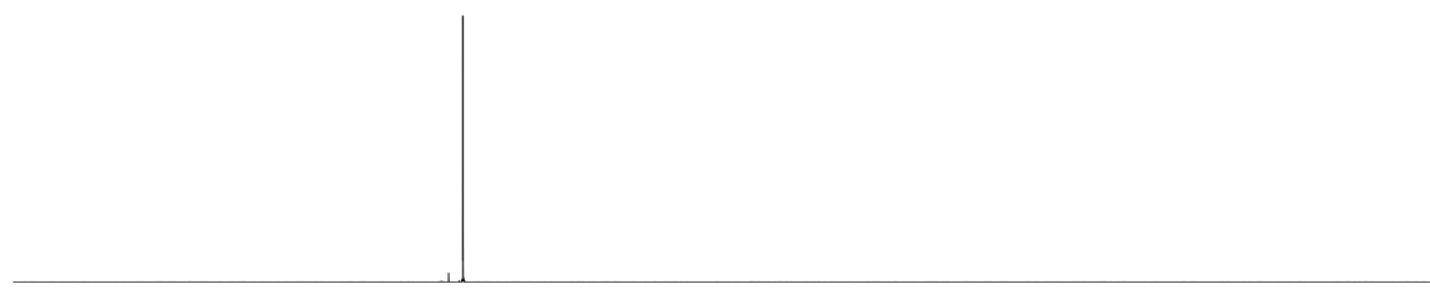

${ }_{130}^{1}$
110 


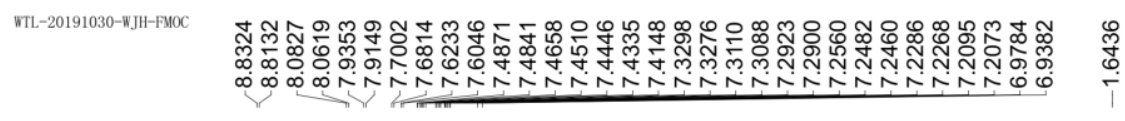

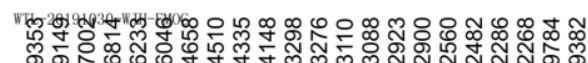

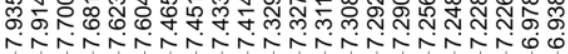

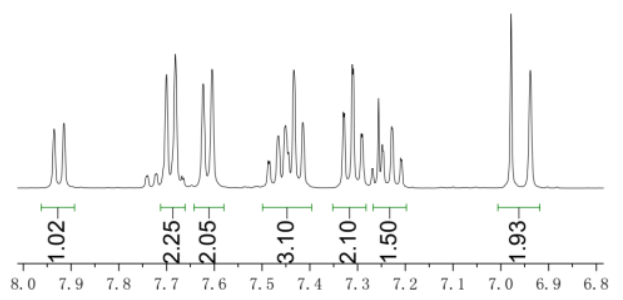

Boc

1f

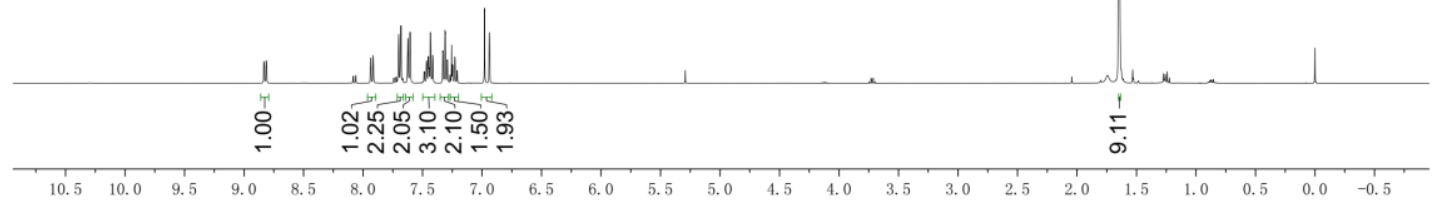

WTL-20191029-WJH-Fmoc

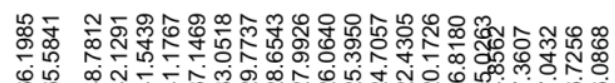

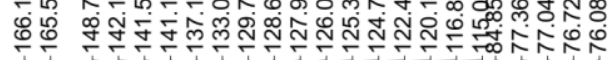

ผุํำ

办
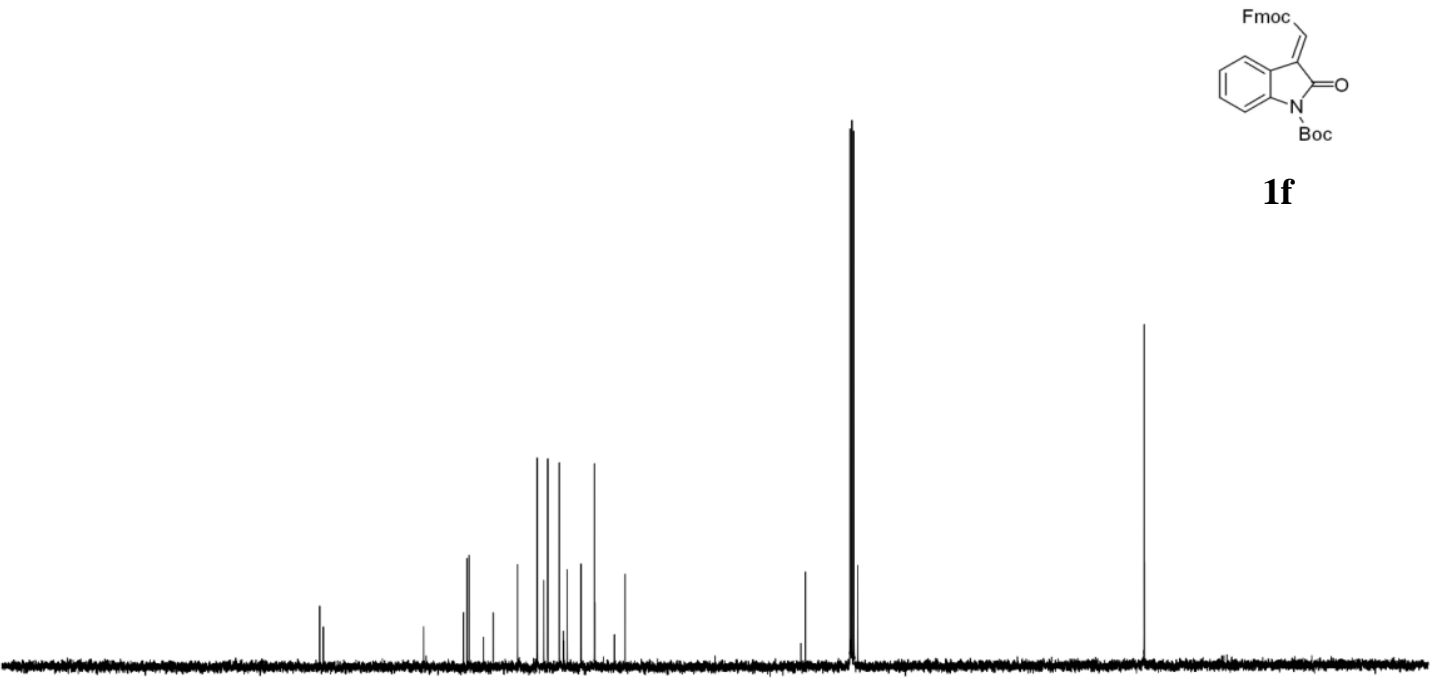

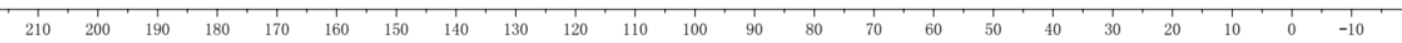



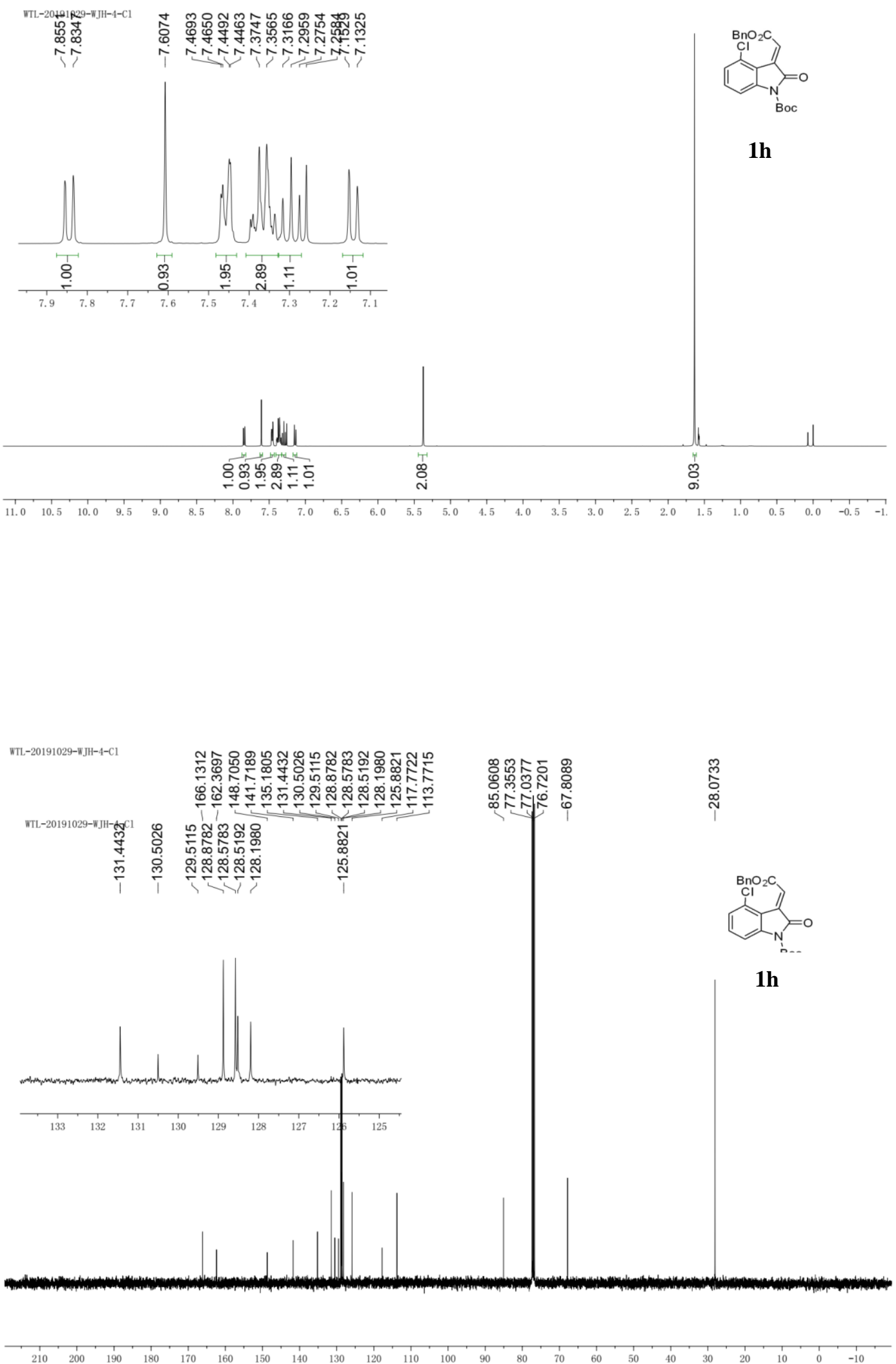


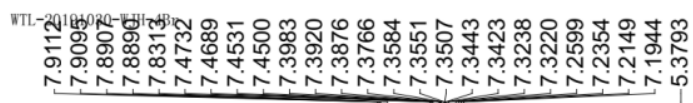

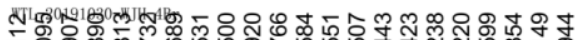

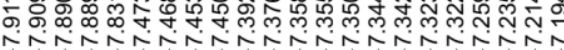
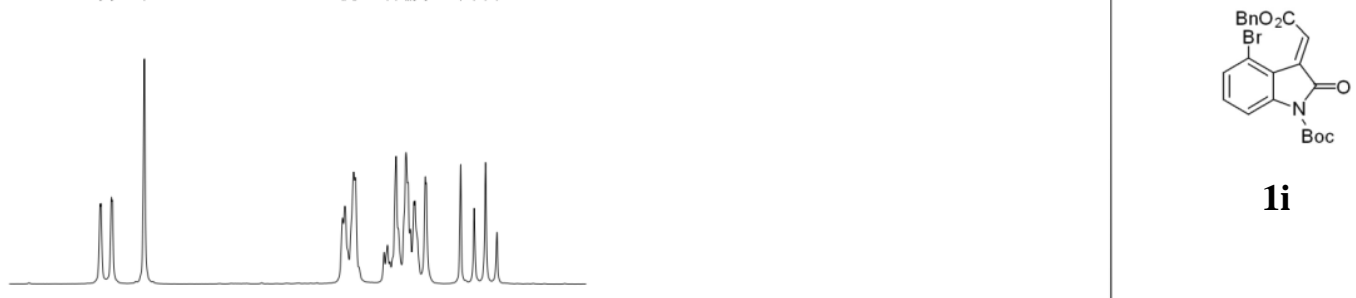

8 म

ले ले ธ

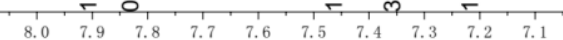

$1 \mathrm{i}$

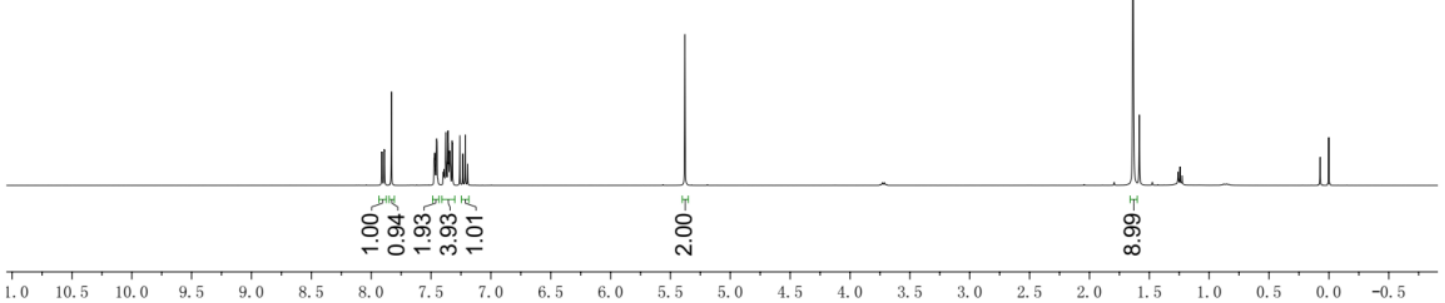

WTL-20191029-WJH-4-Br

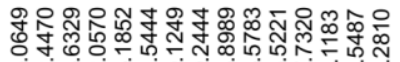

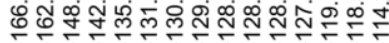

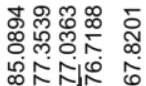

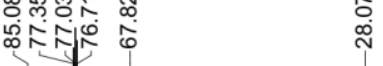

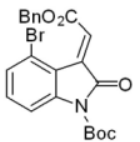

$1 \mathbf{i}$

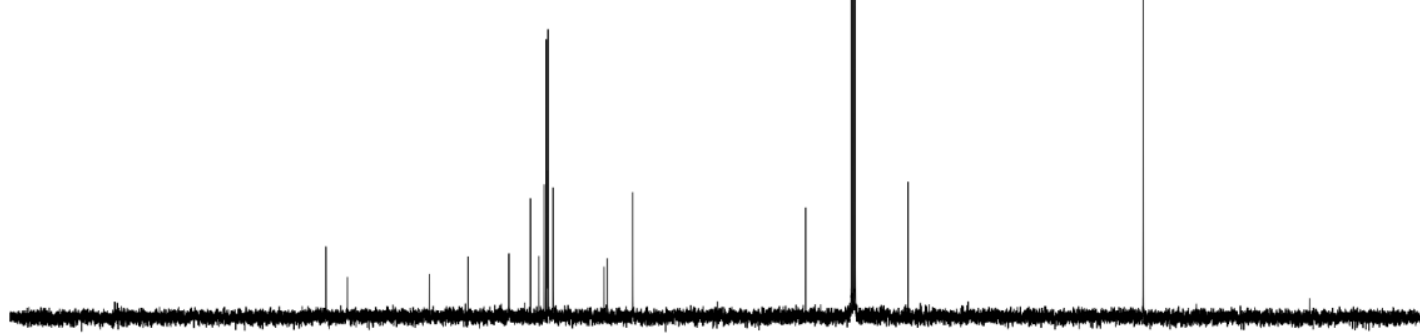

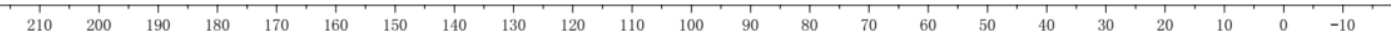



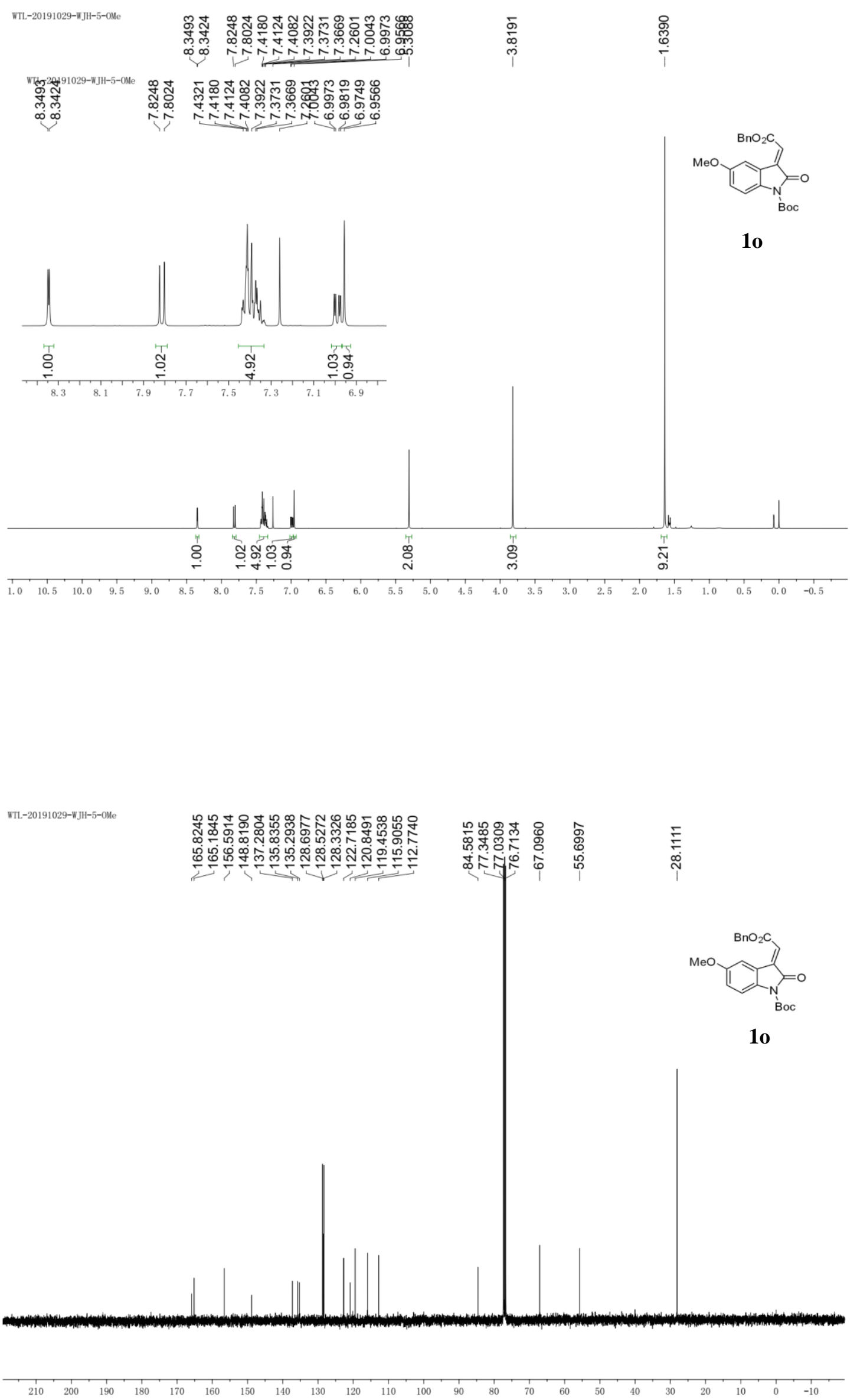

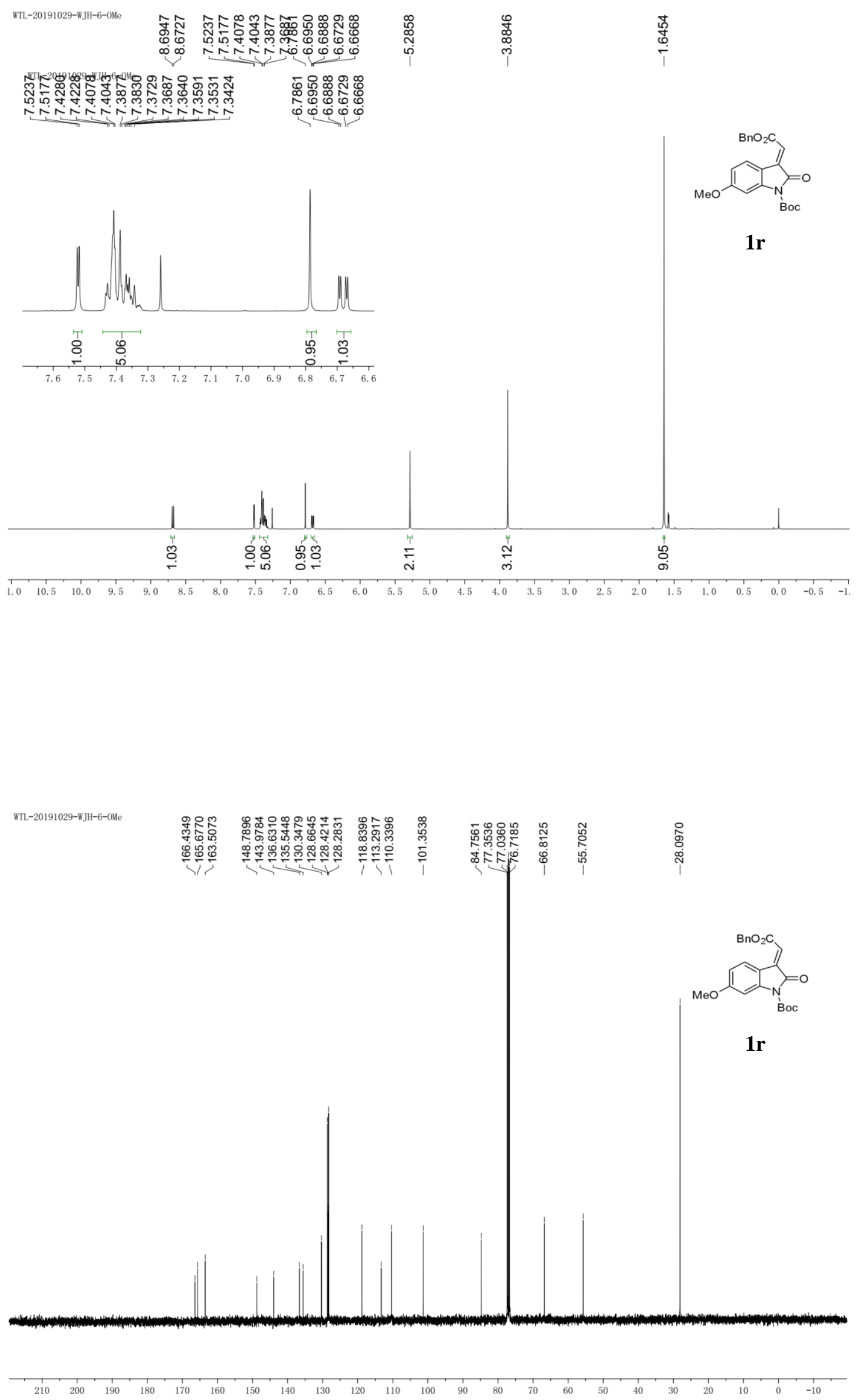

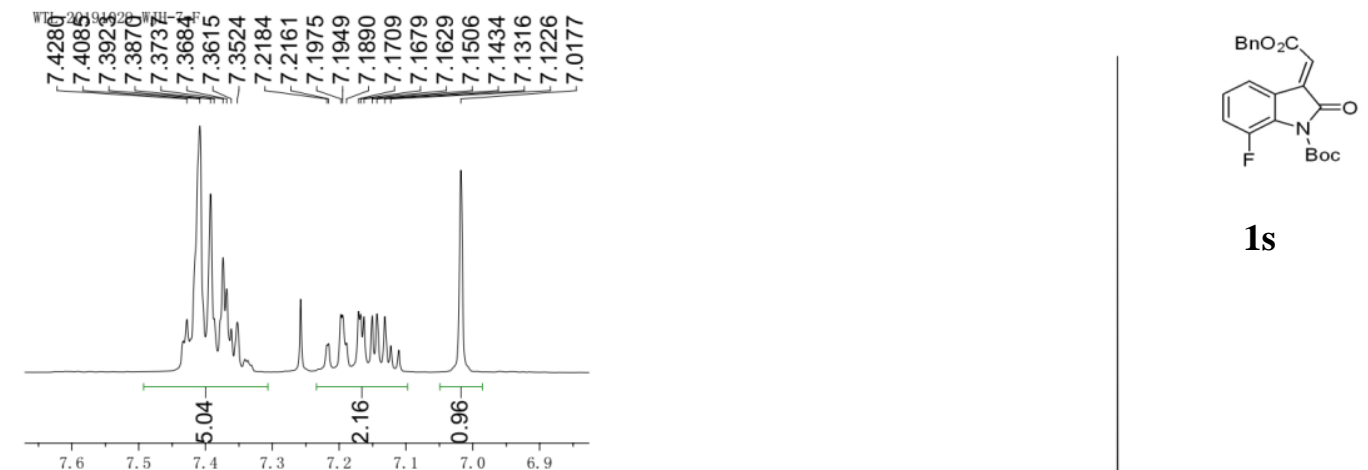

1s

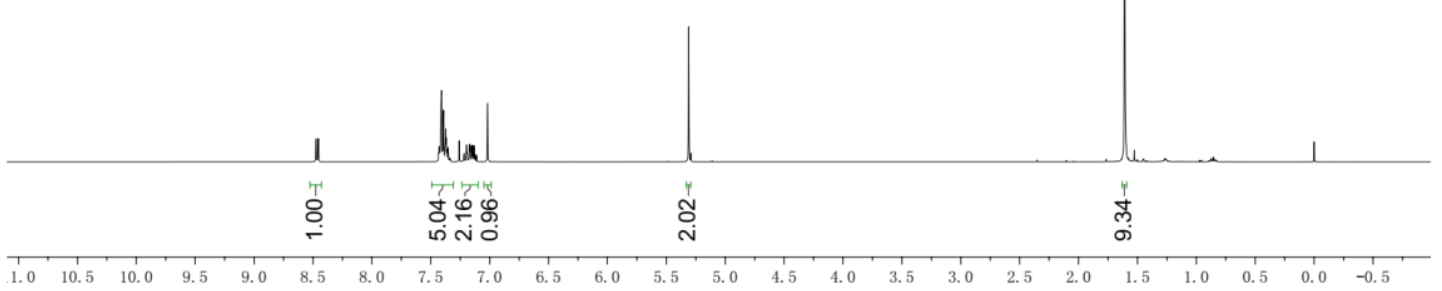

WTL-20191029-WJH-7-F

J
$\stackrel{0}{0}$
$\stackrel{\sim}{N}$

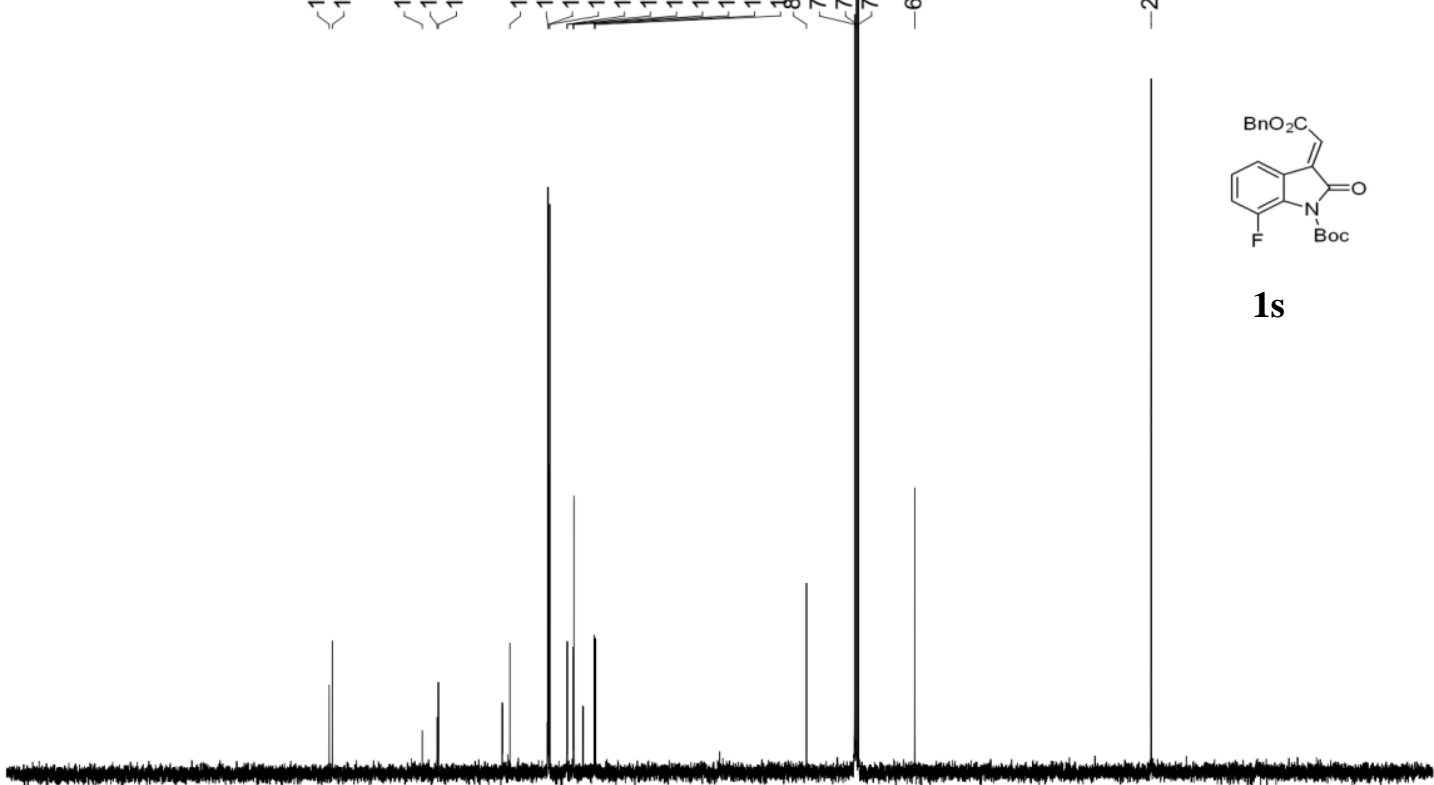



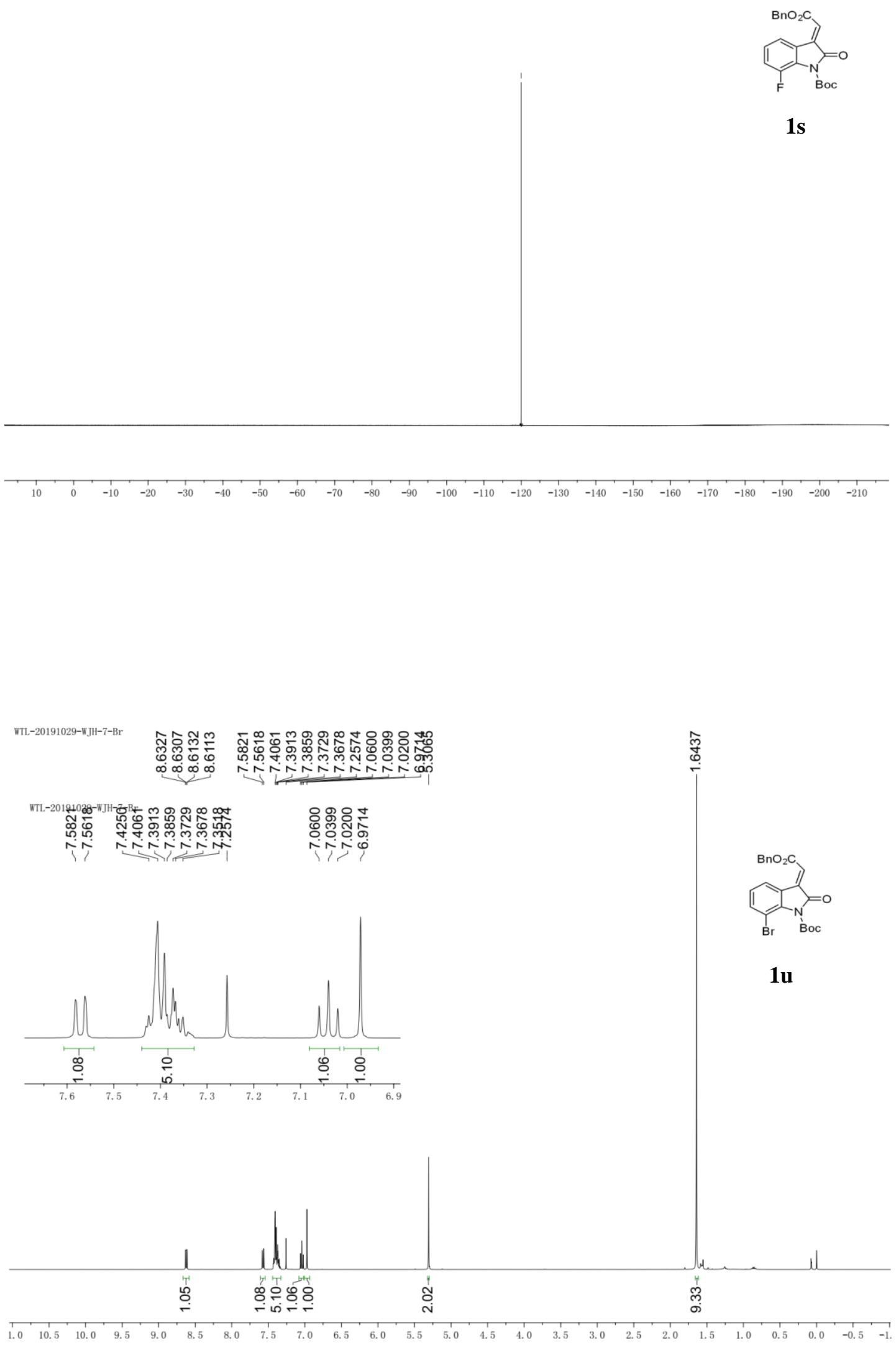


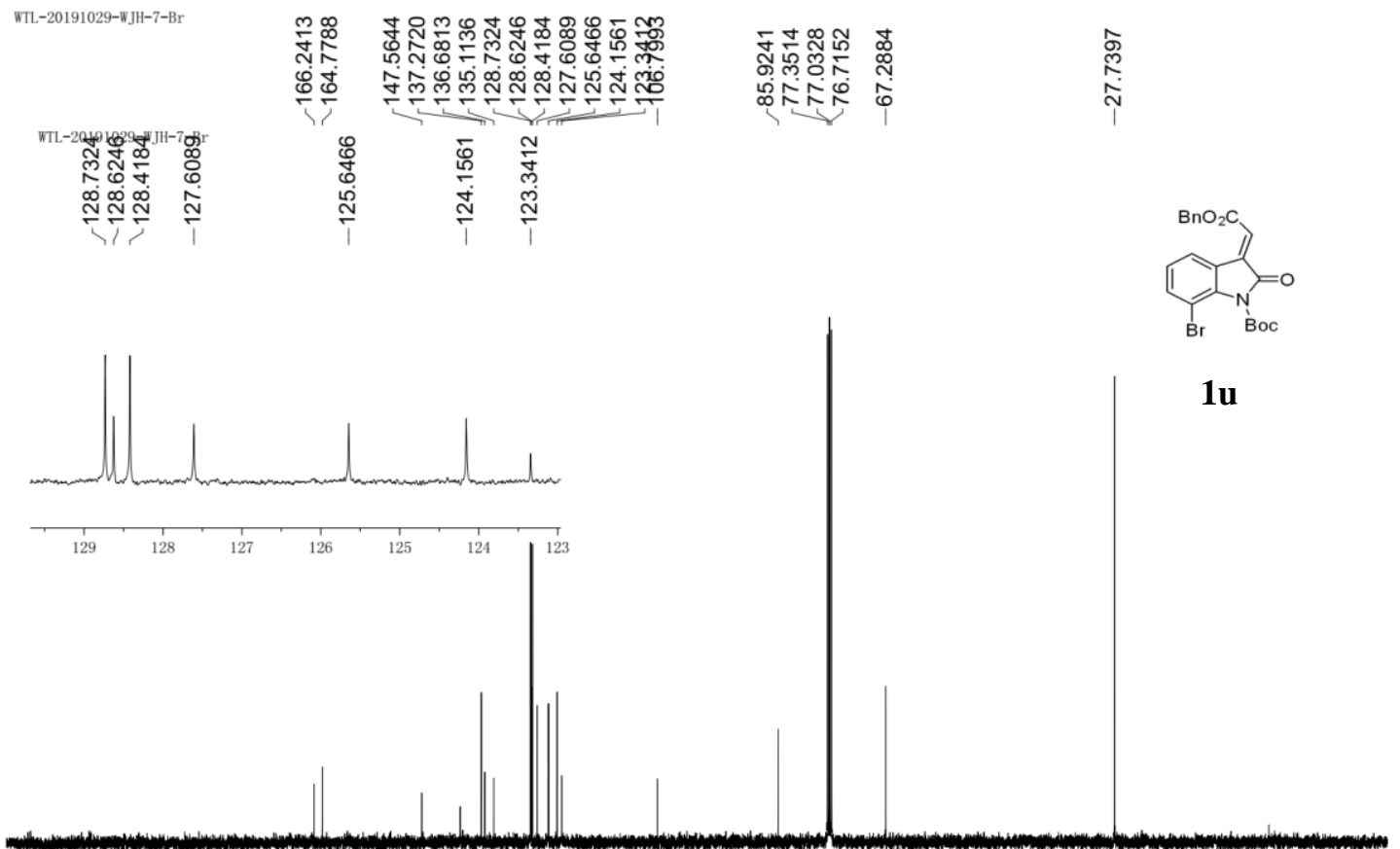

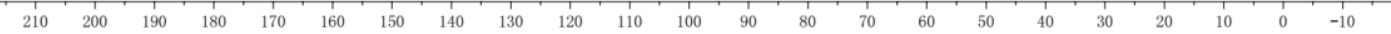

WTL-20191029-WJH-7-Me

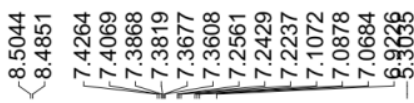

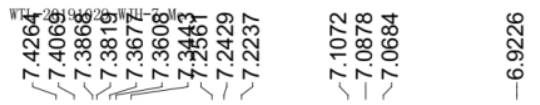
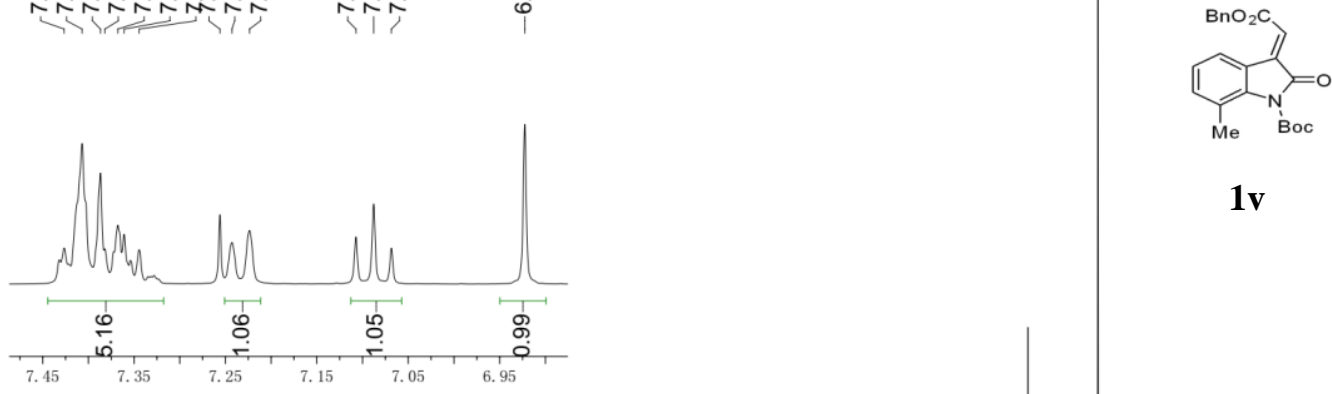

$1 v$

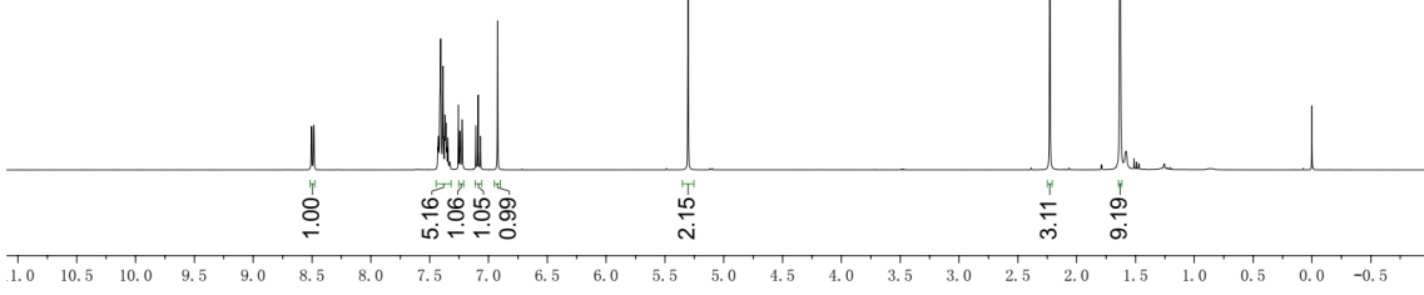



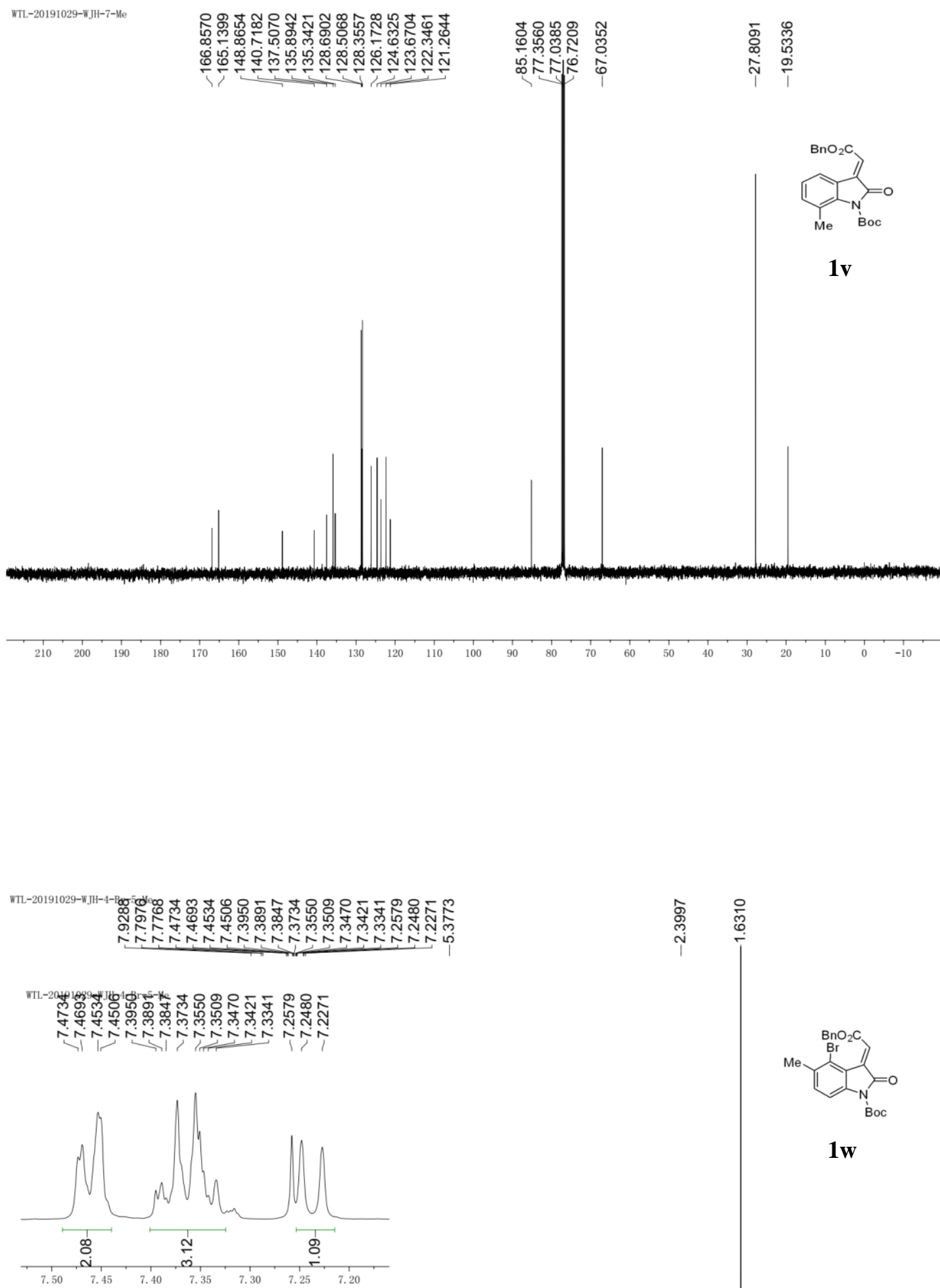

$1 w$

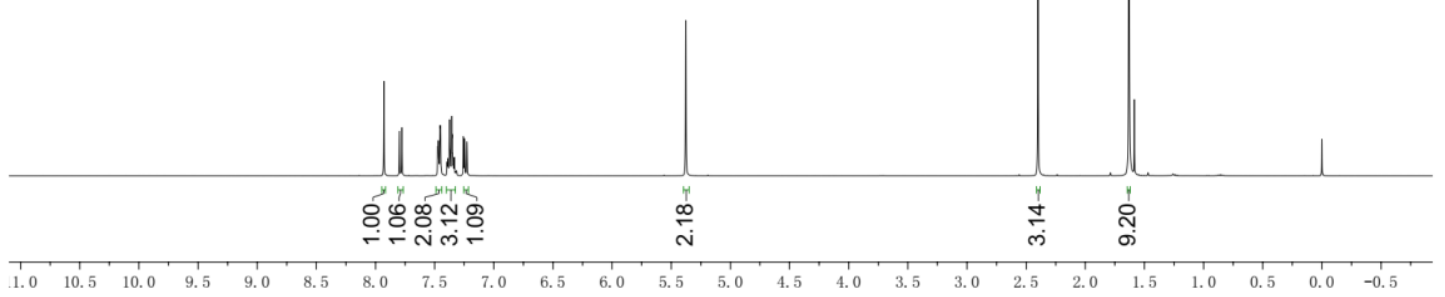




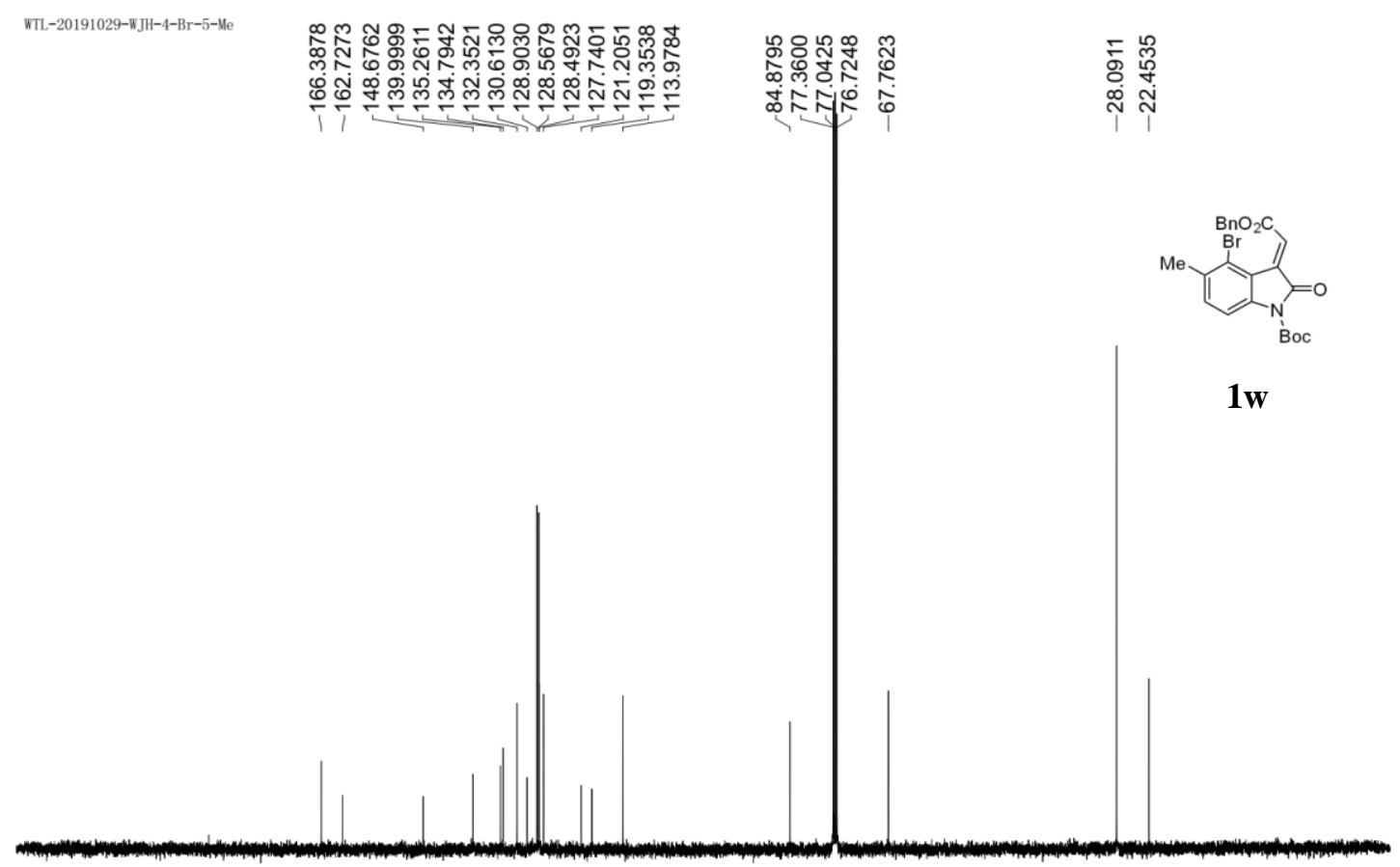

WTL-20191101-WJH-57

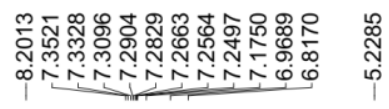

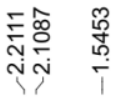

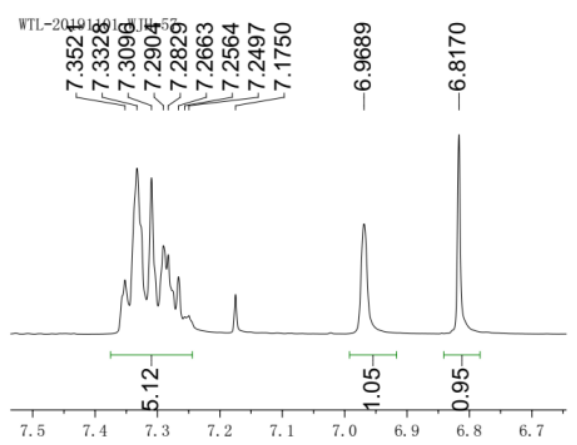

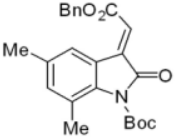

1x

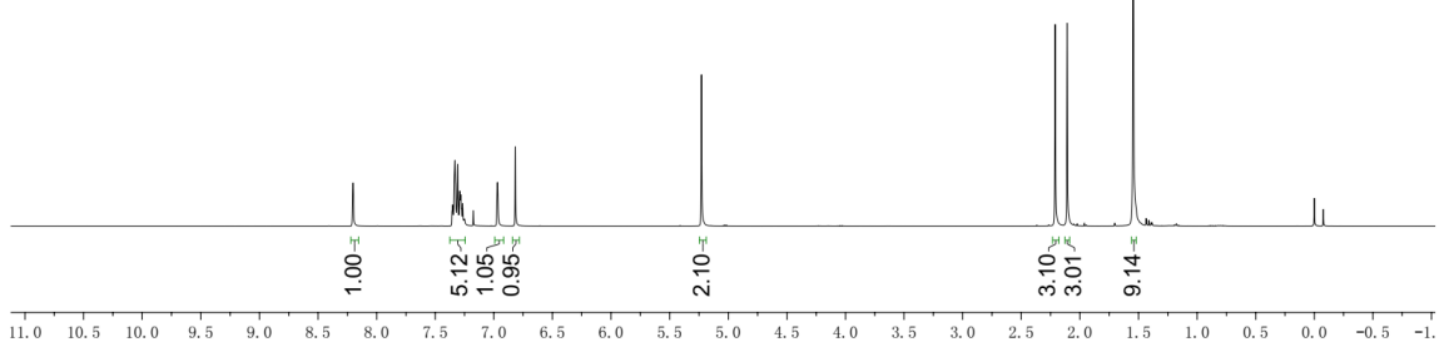



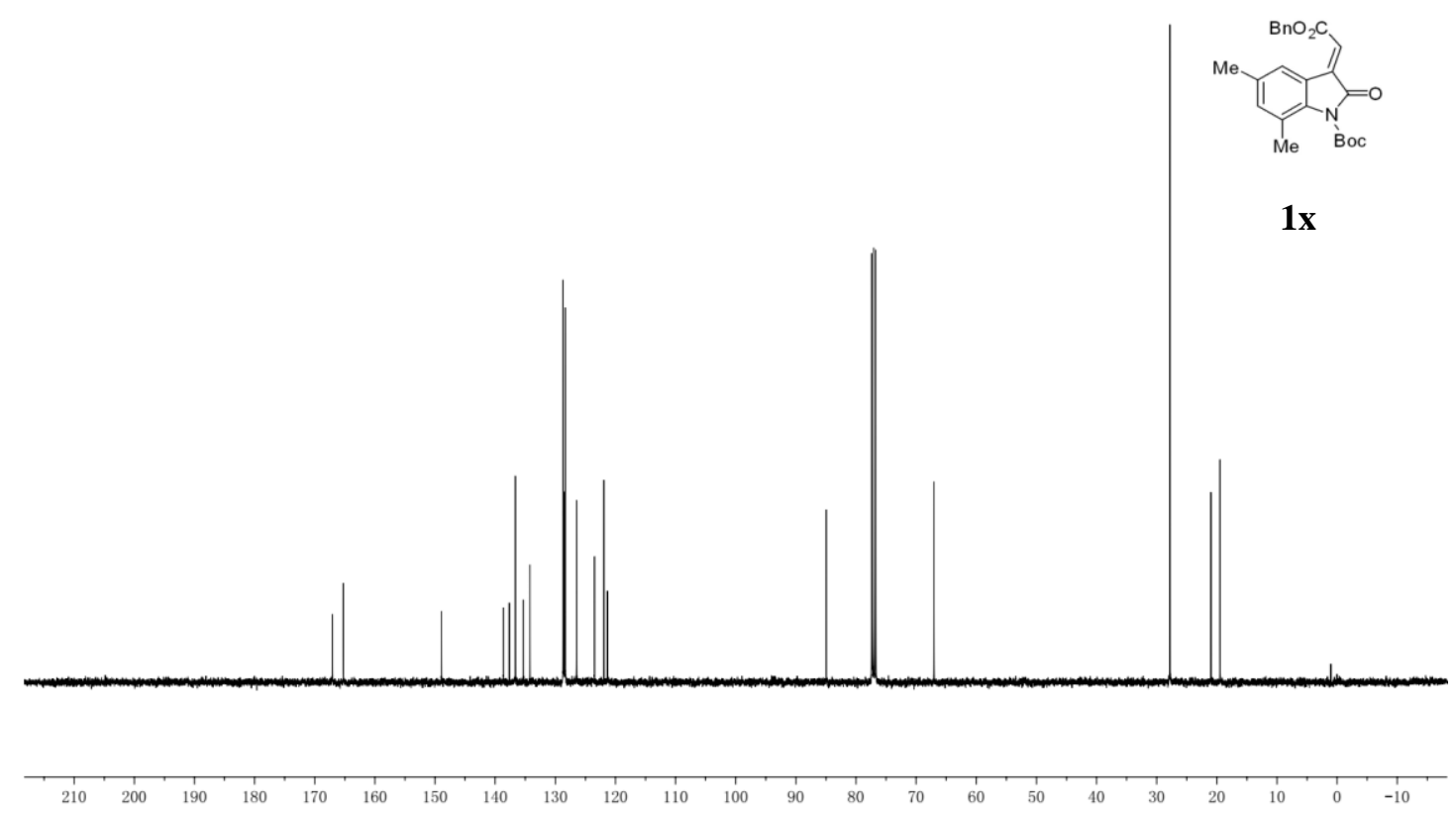

WTL-20191029-WJJH-5-6-F

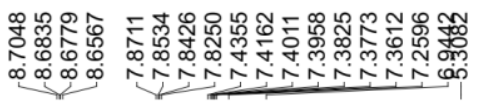

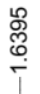
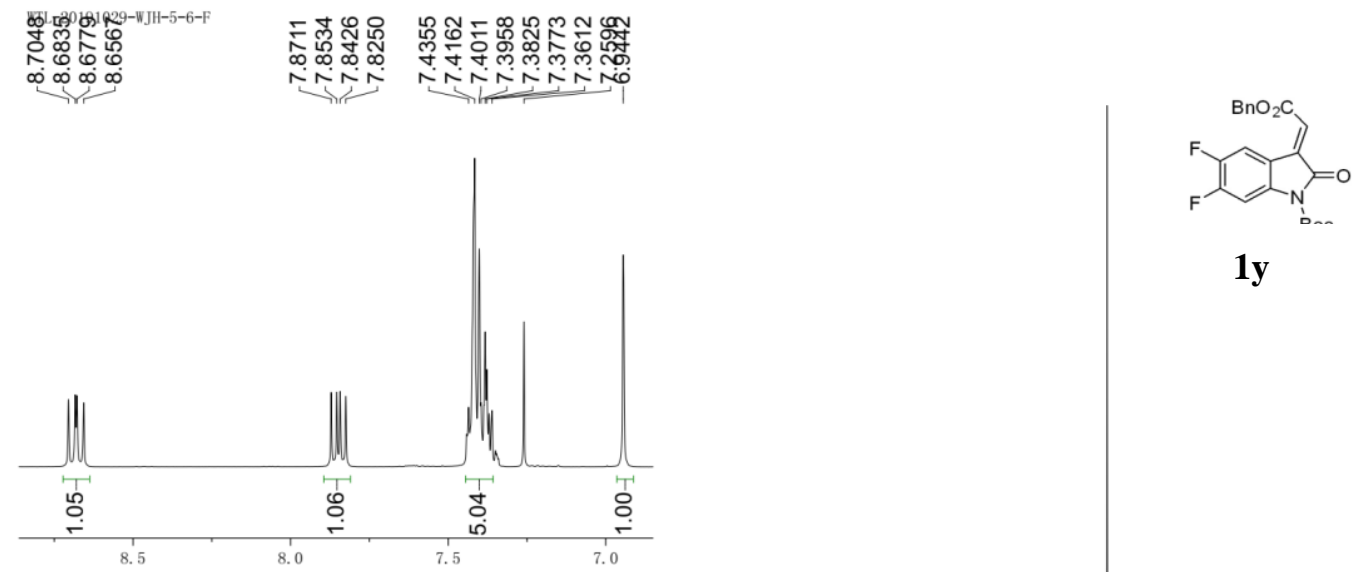

$1 \mathbf{y}$

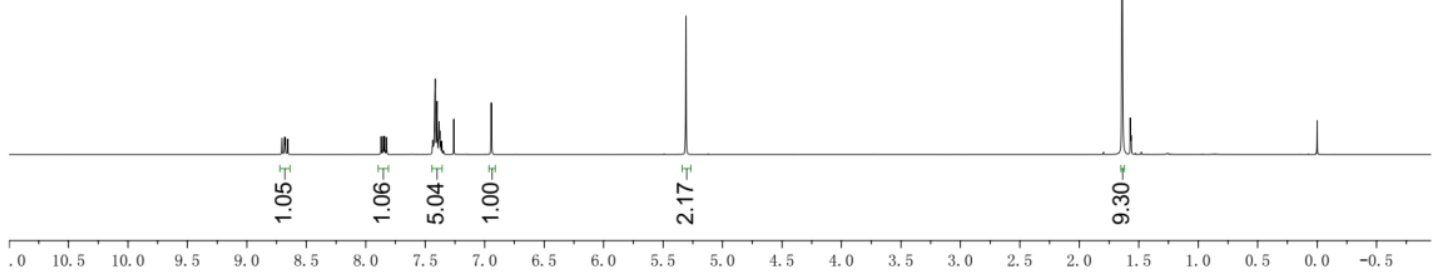




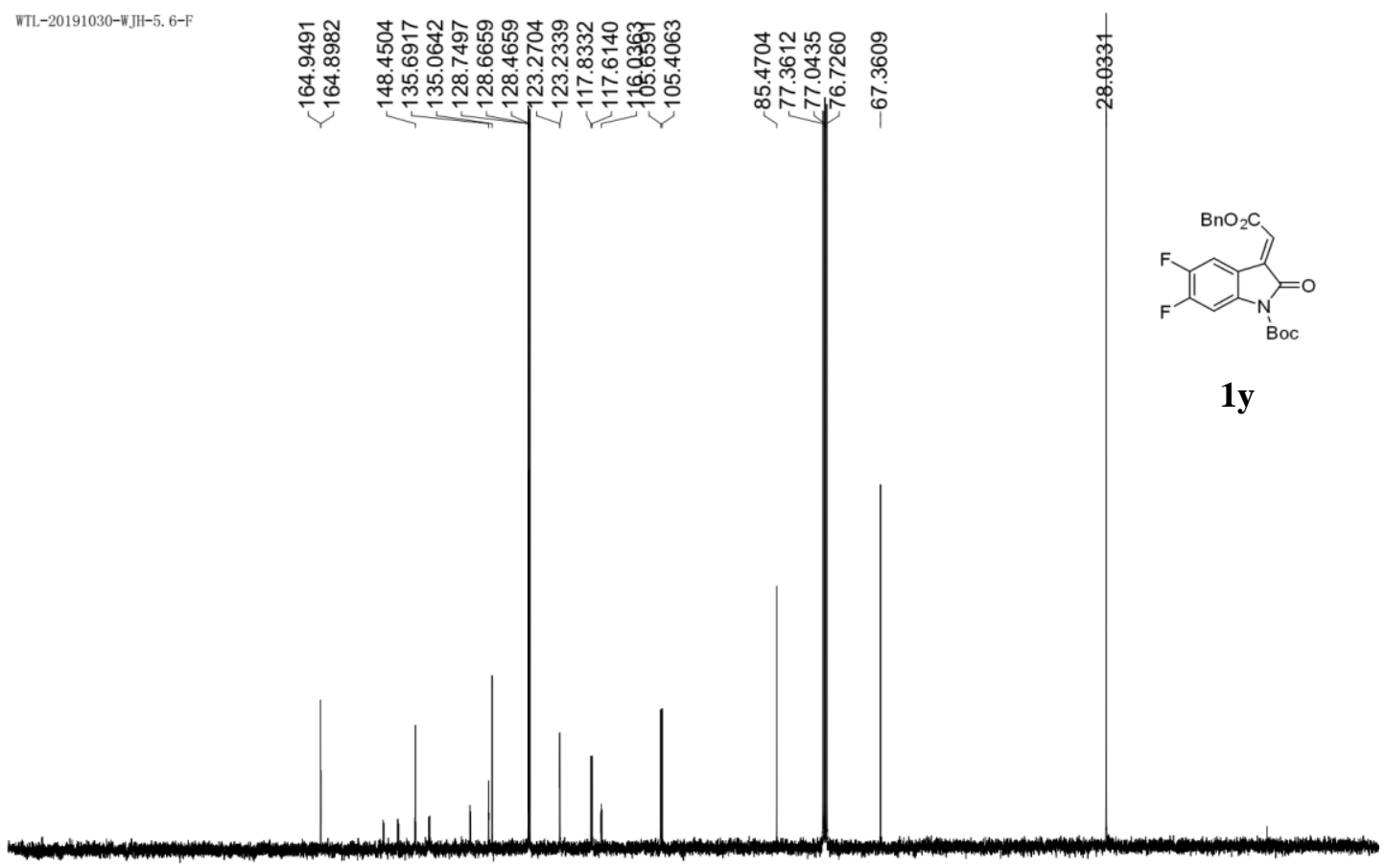

WTL-20191029-WJH-5-6-F
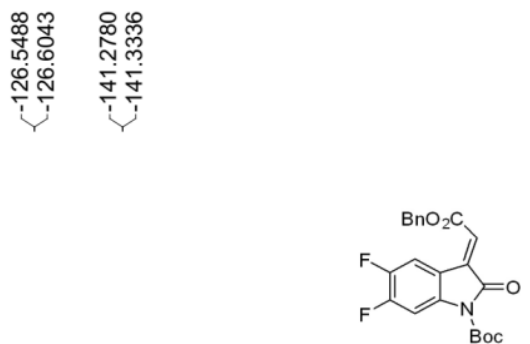

1y

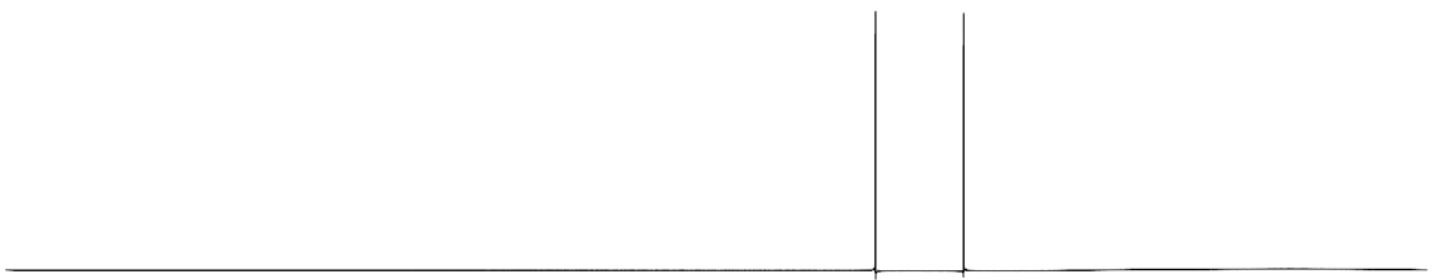

$\begin{array}{lllllllllllllllllllllllll}1 & 10 & 0 & -10 & -20 & -30 & -40 & -50 & -60 & -70 & -80 & -90 & -100 & -110 & -120 & -130 & -140 & -150 & -160 & -170 & -180 & -190 & -200 & -210 & 1\end{array}$ 

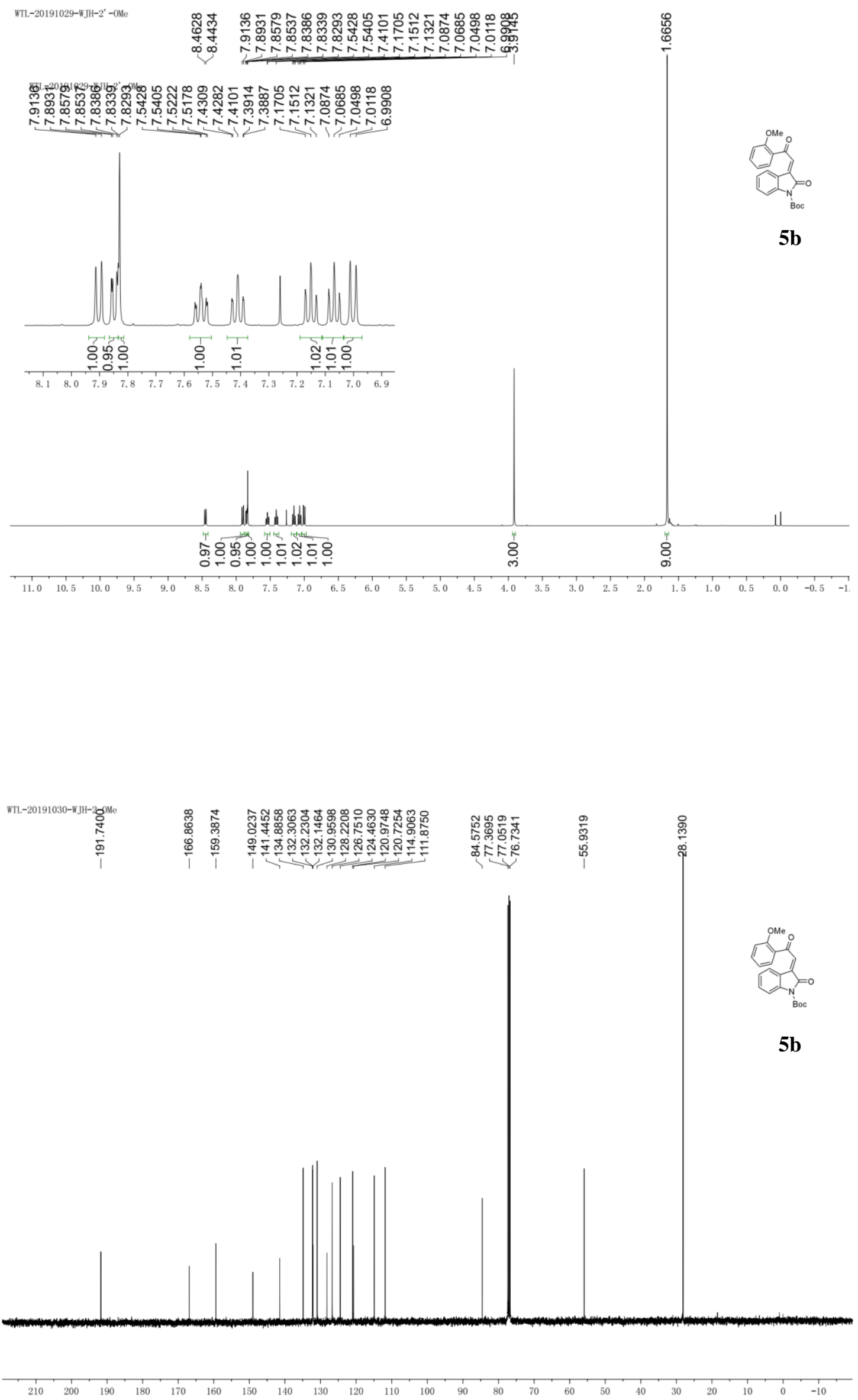


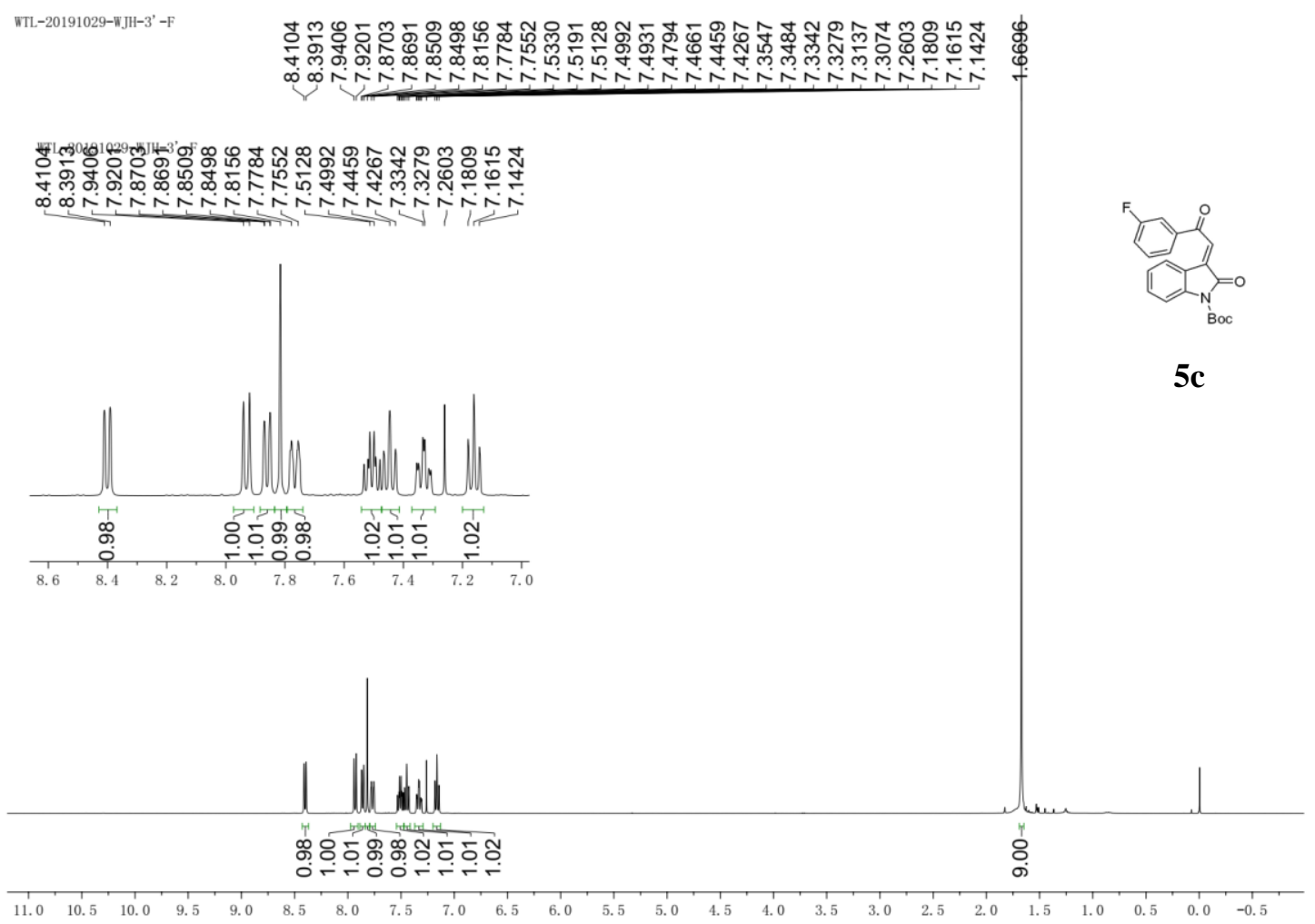

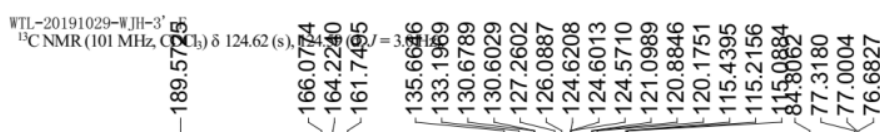

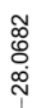

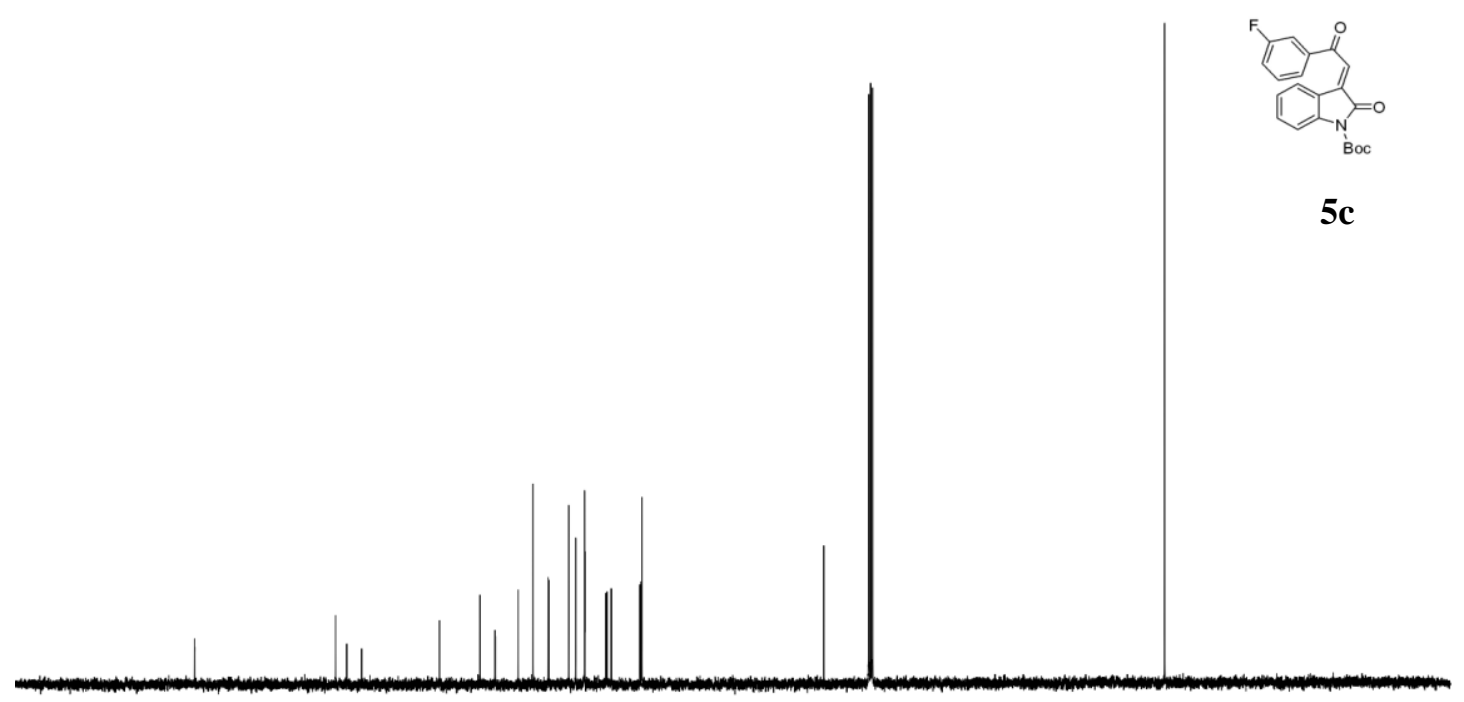

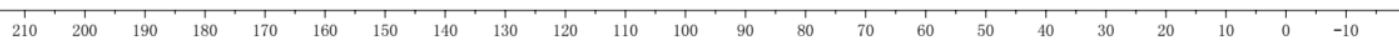


WTL-20191029-WJH-3' -F

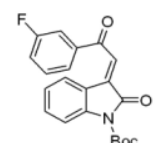

$5 c$

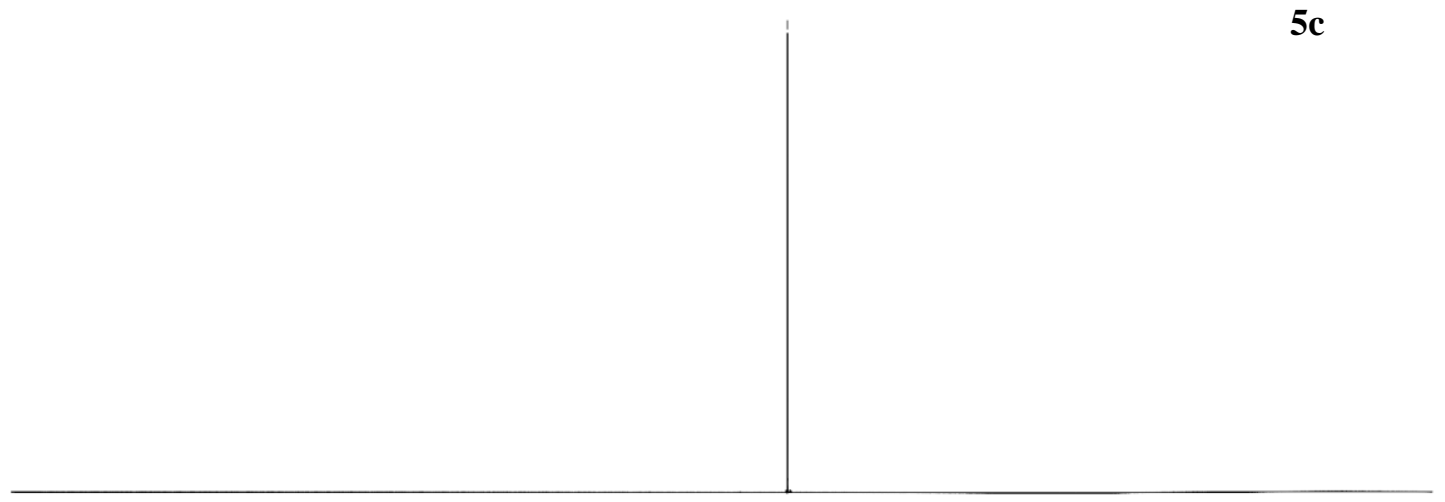

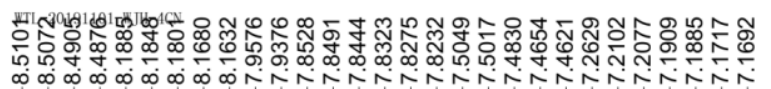

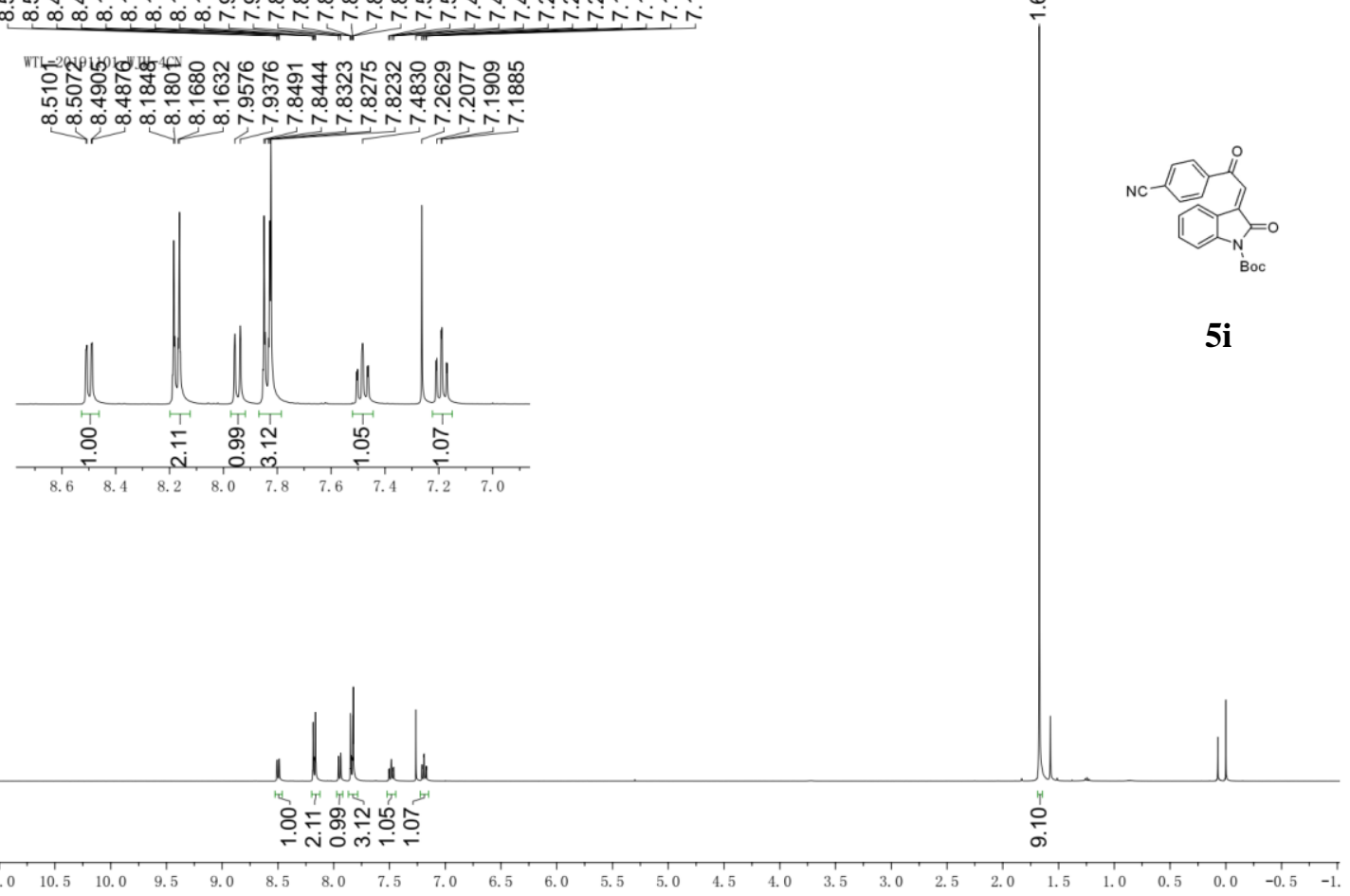



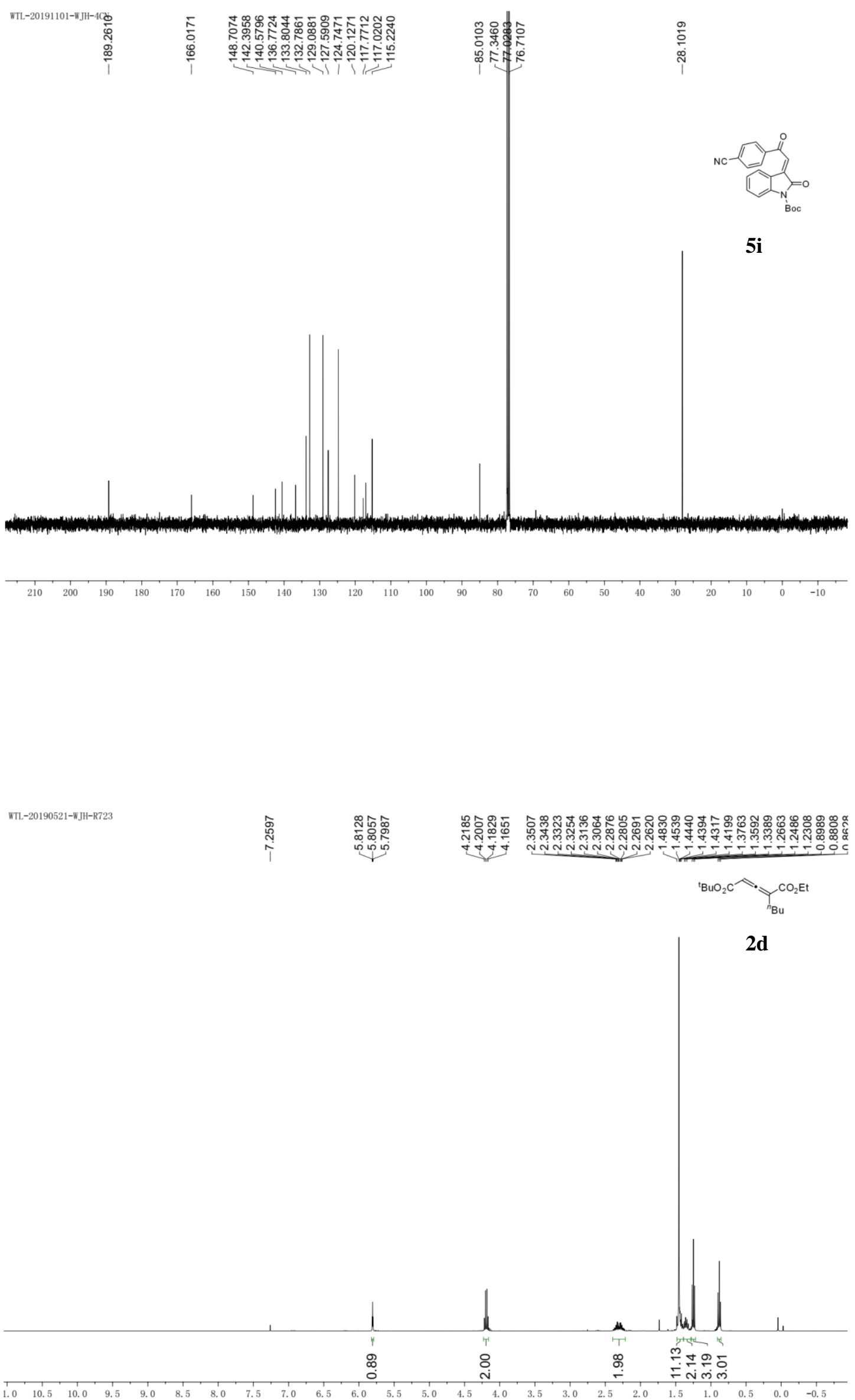

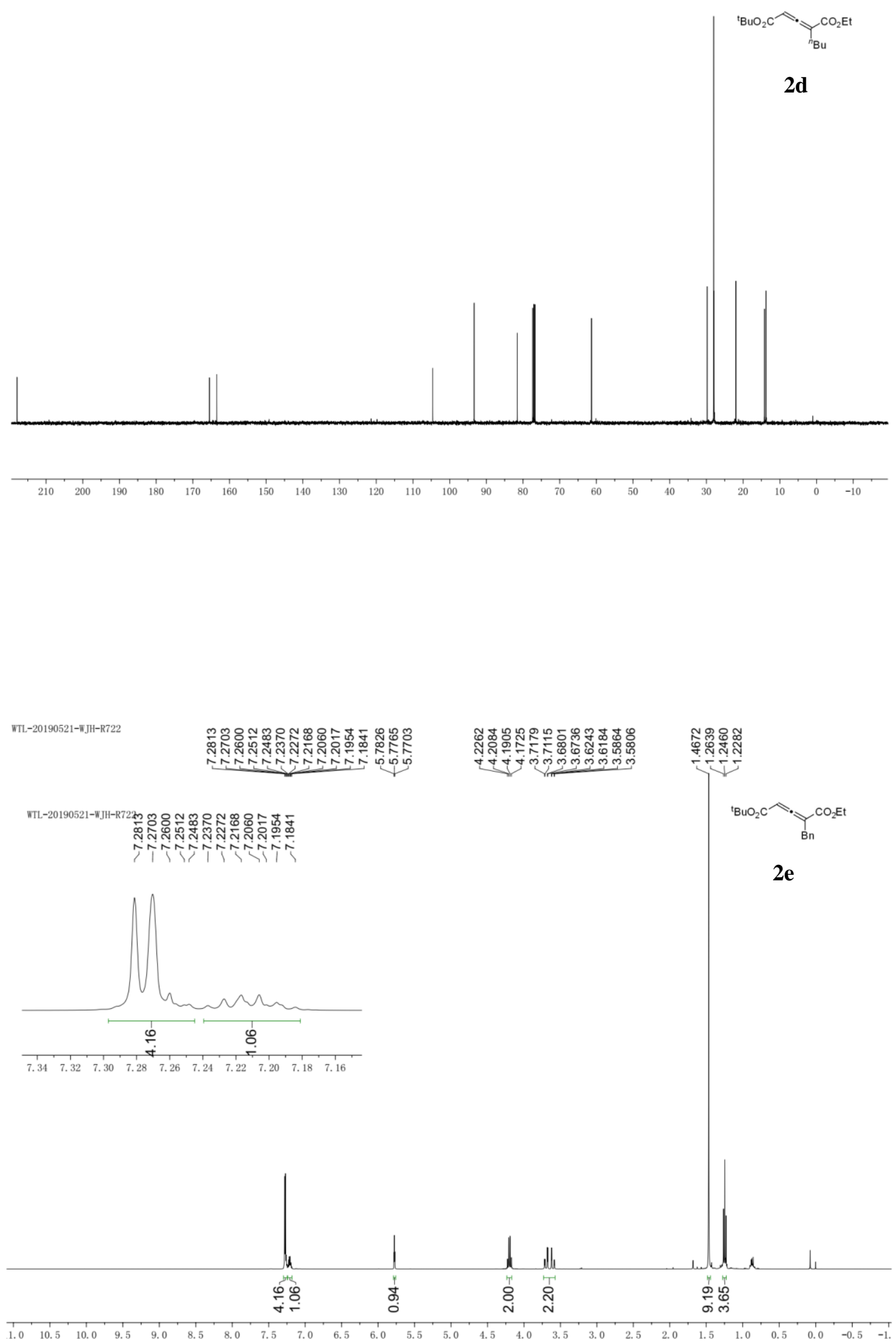

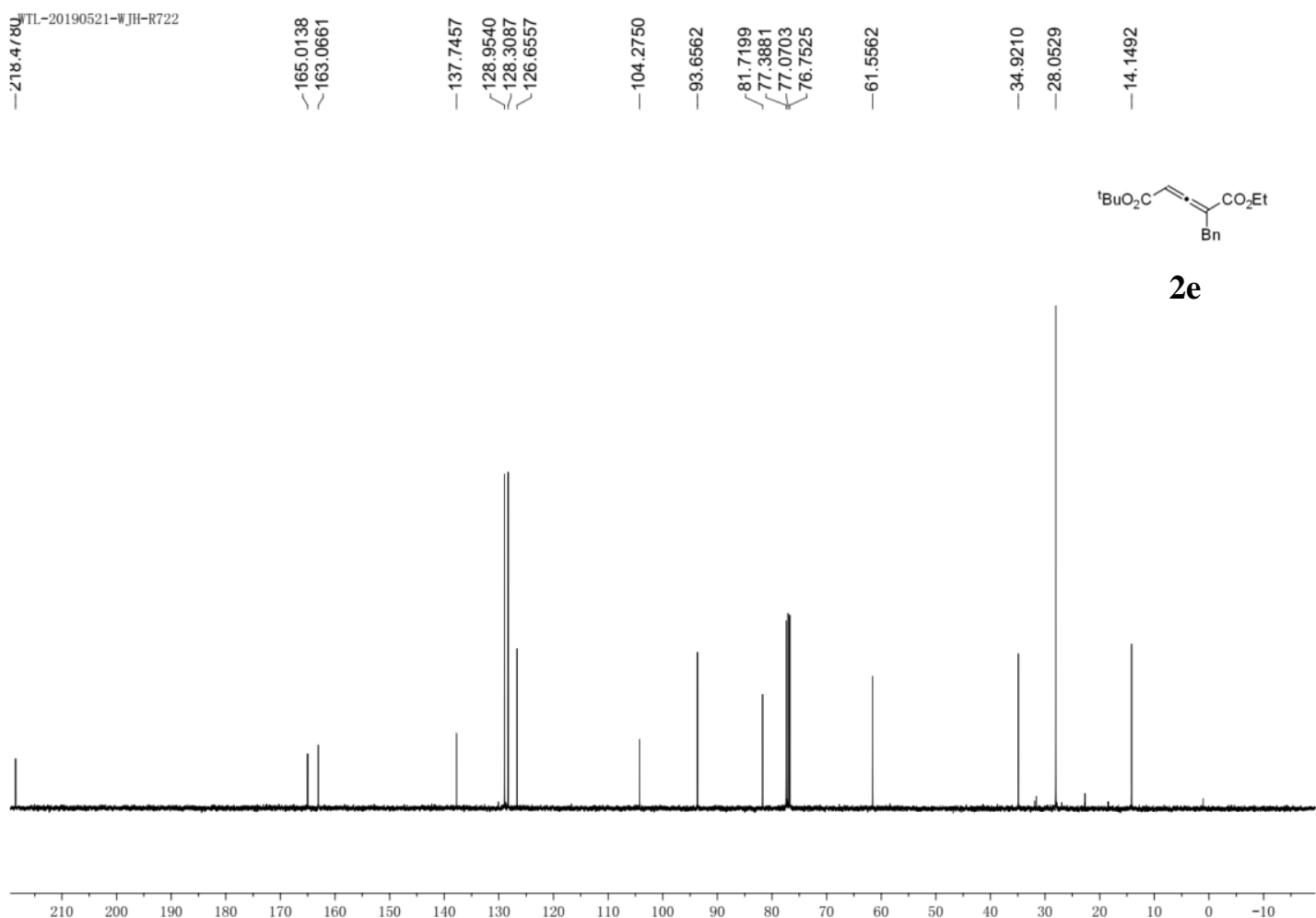

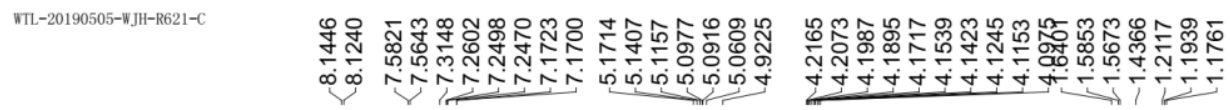

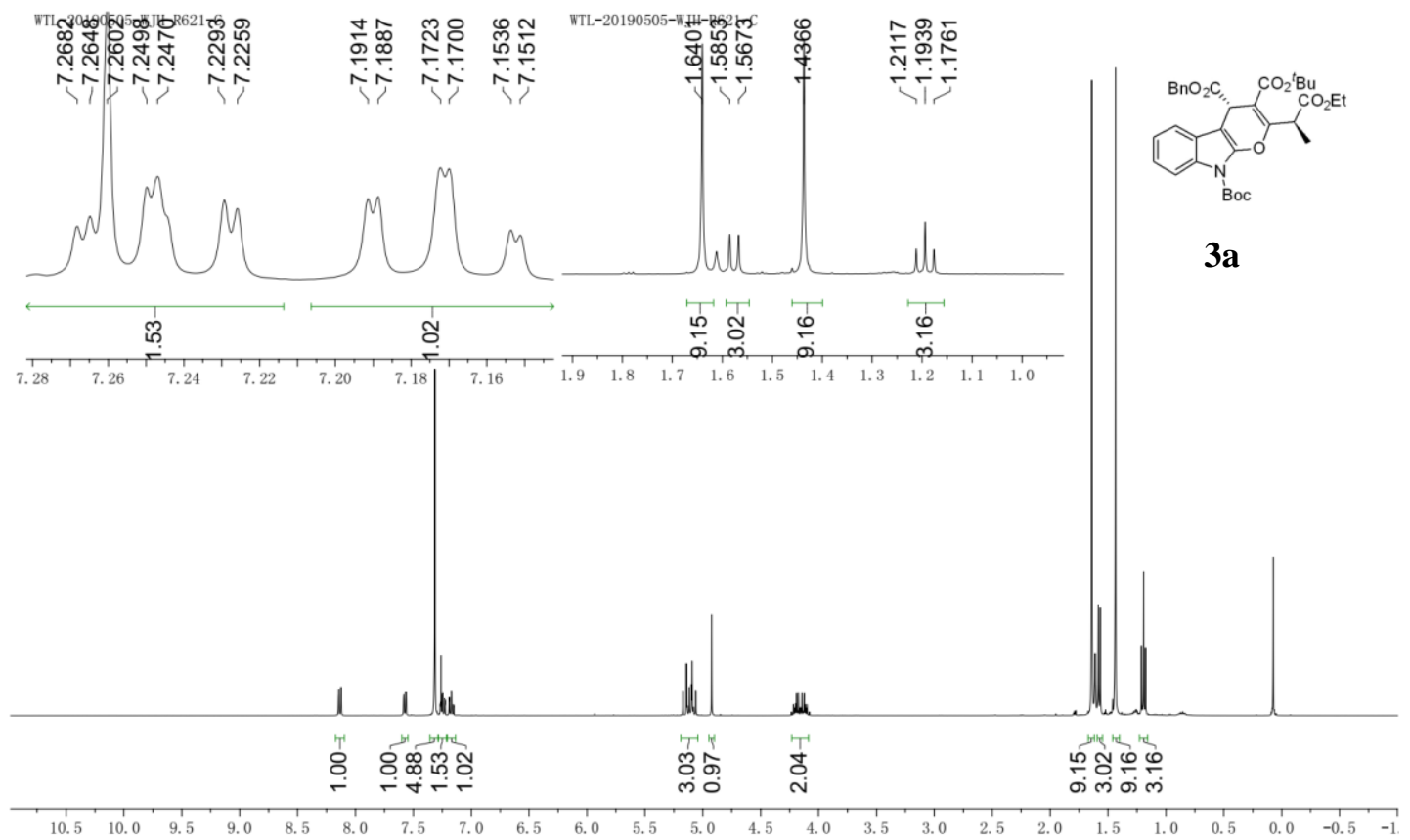



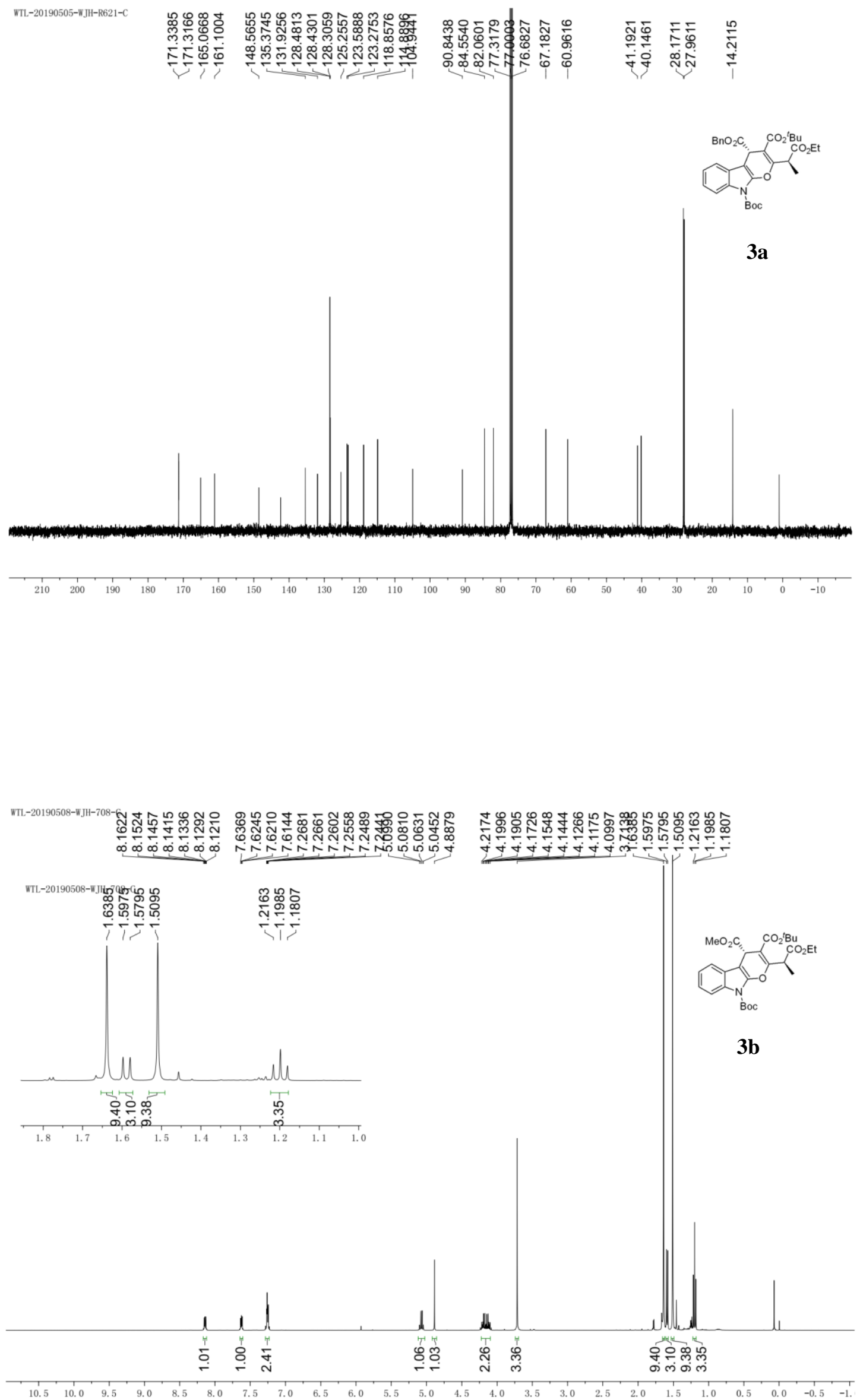

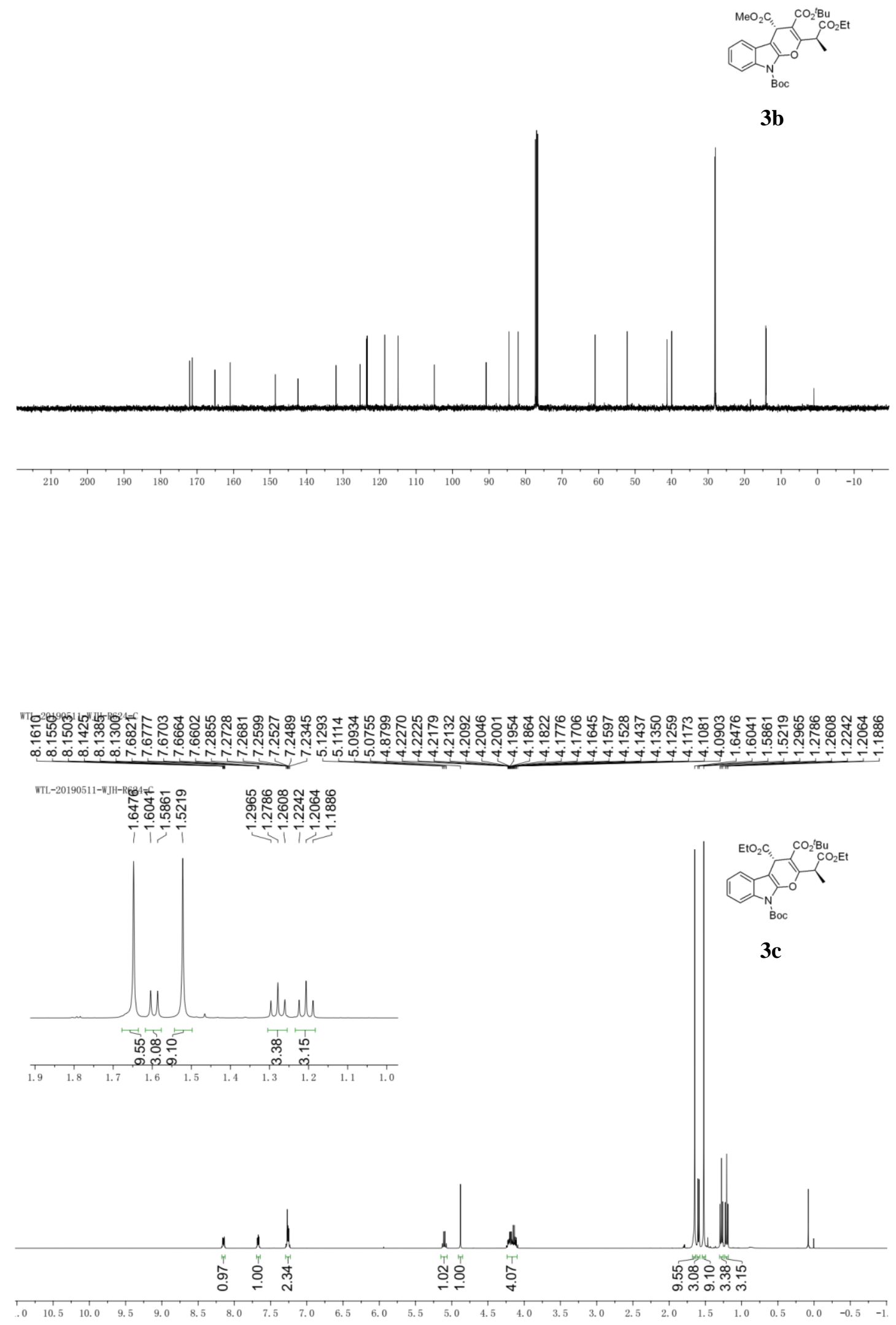

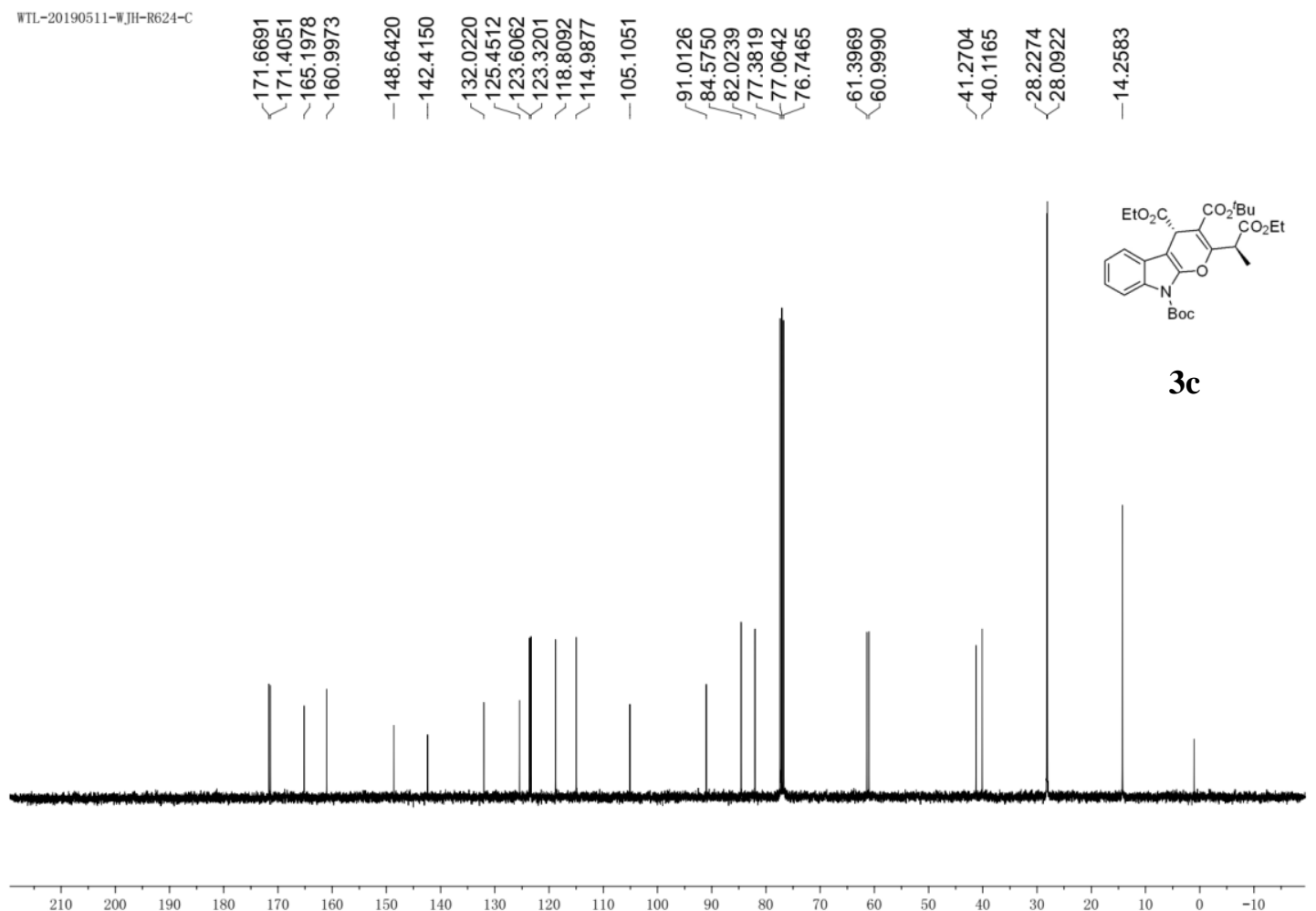

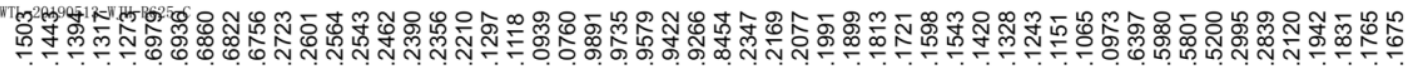 $\infty \infty \infty \infty \infty+i N$}

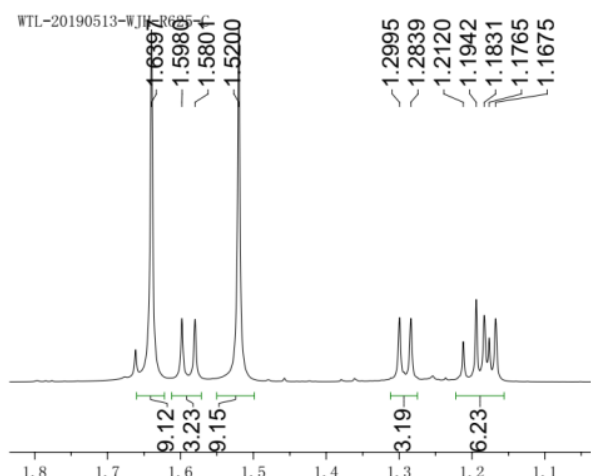

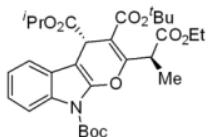

3d

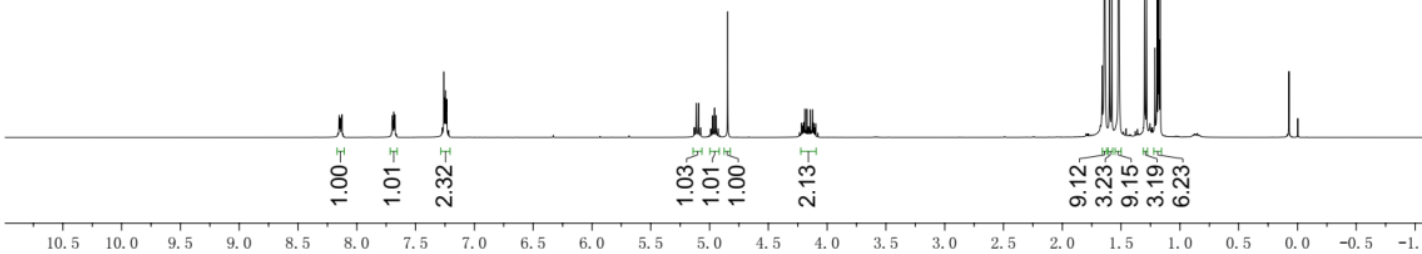



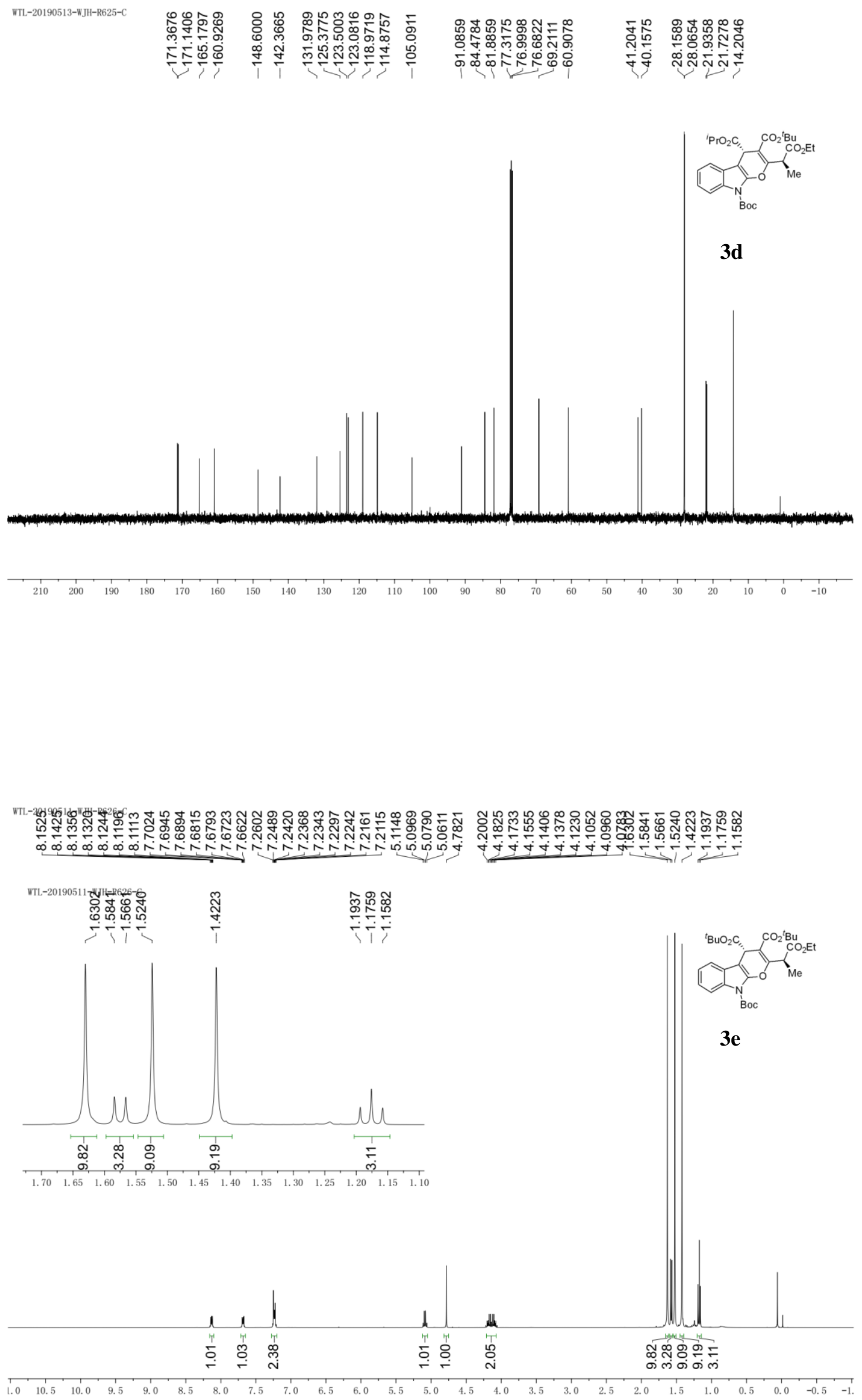

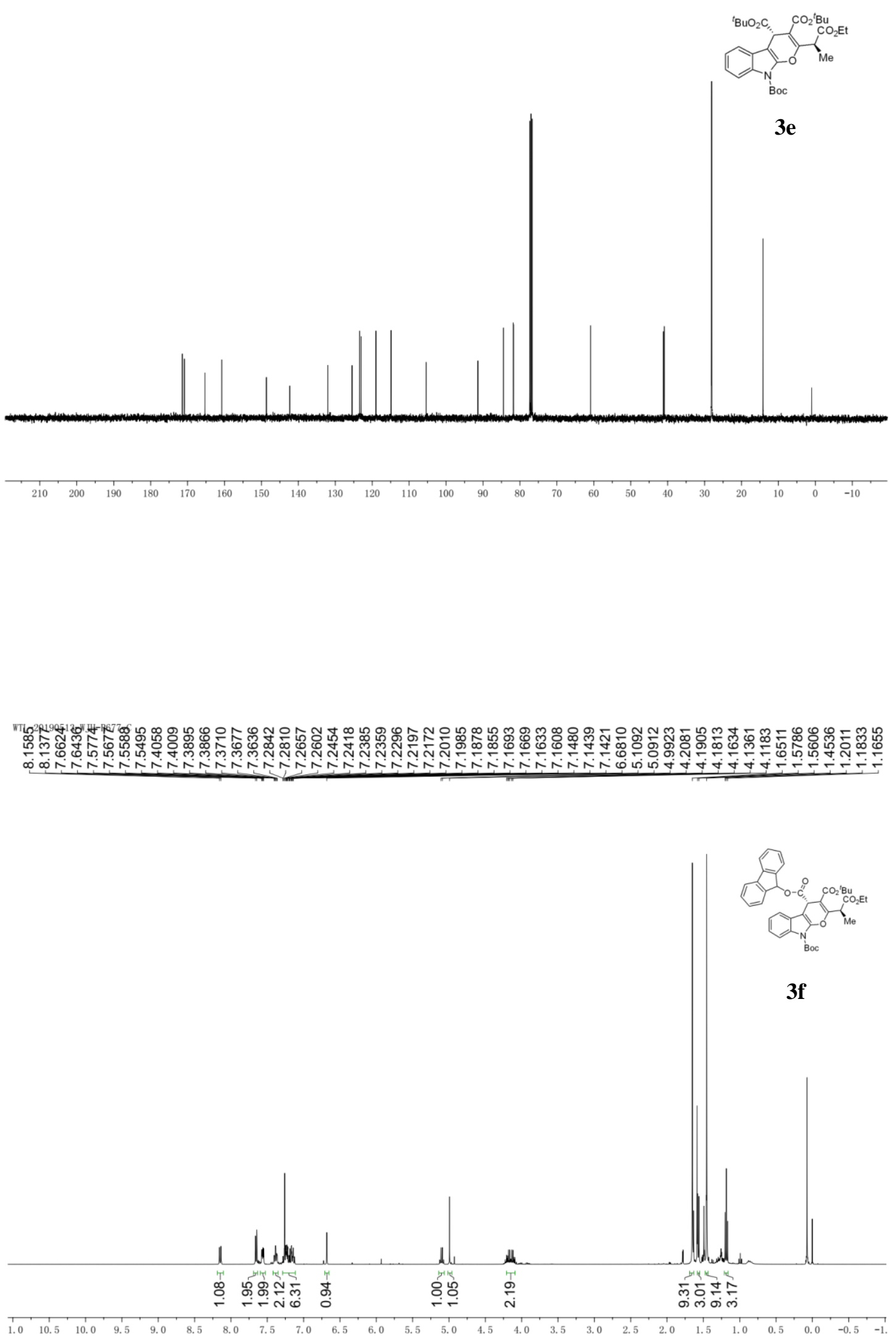

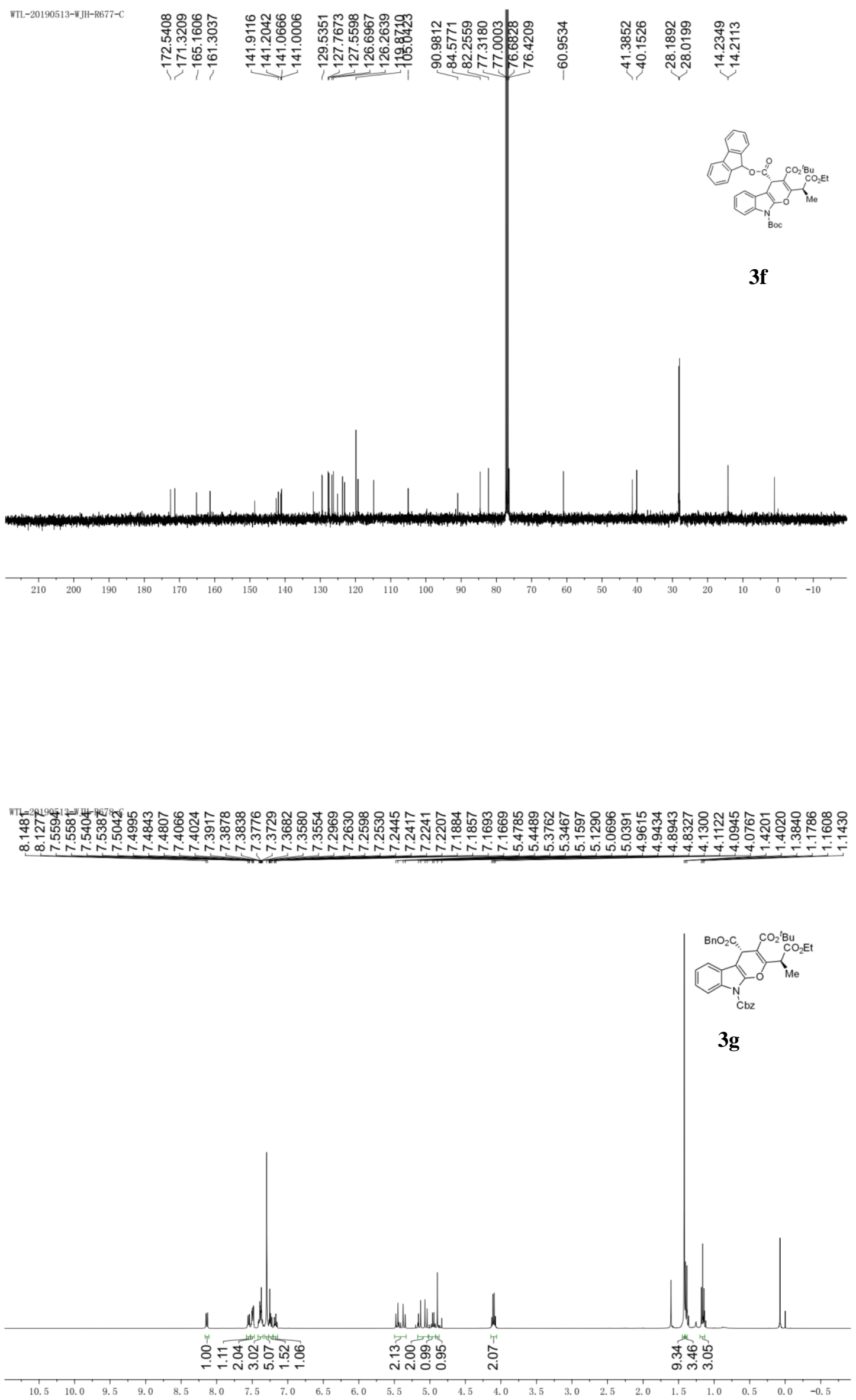

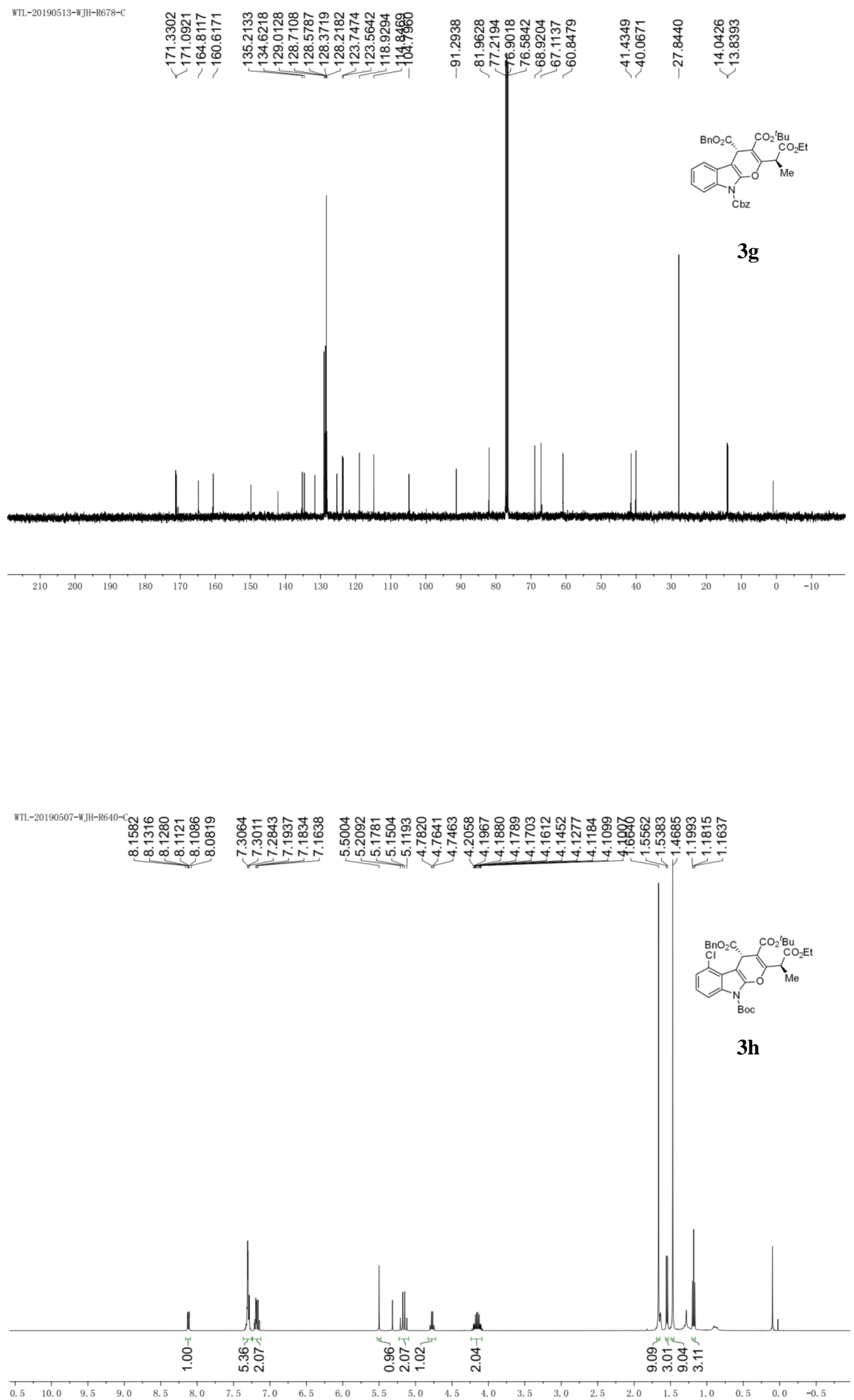


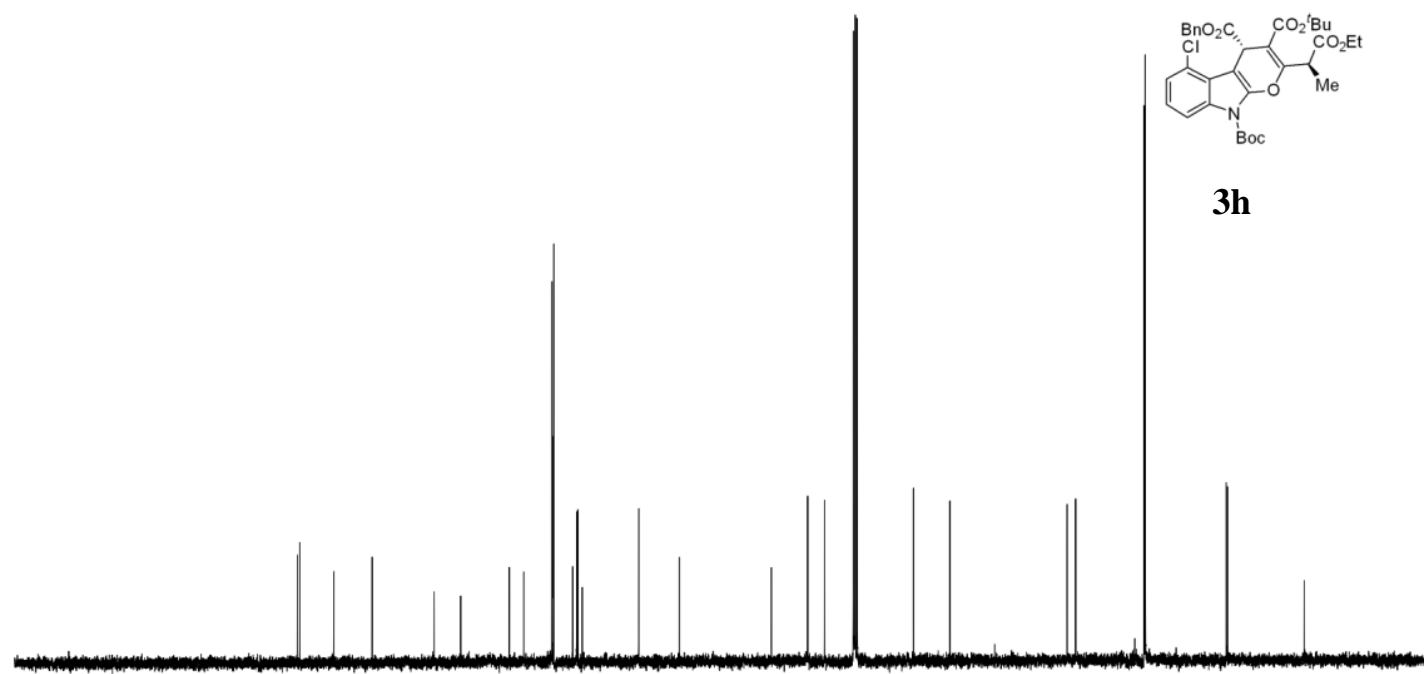

WTL-20190506-WJ.JH-R641-R

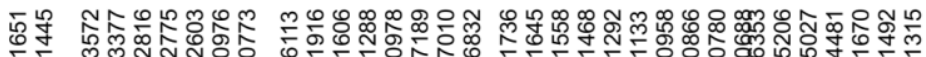

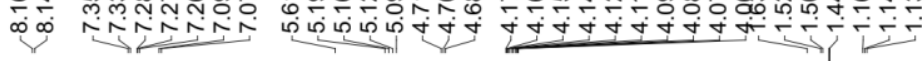

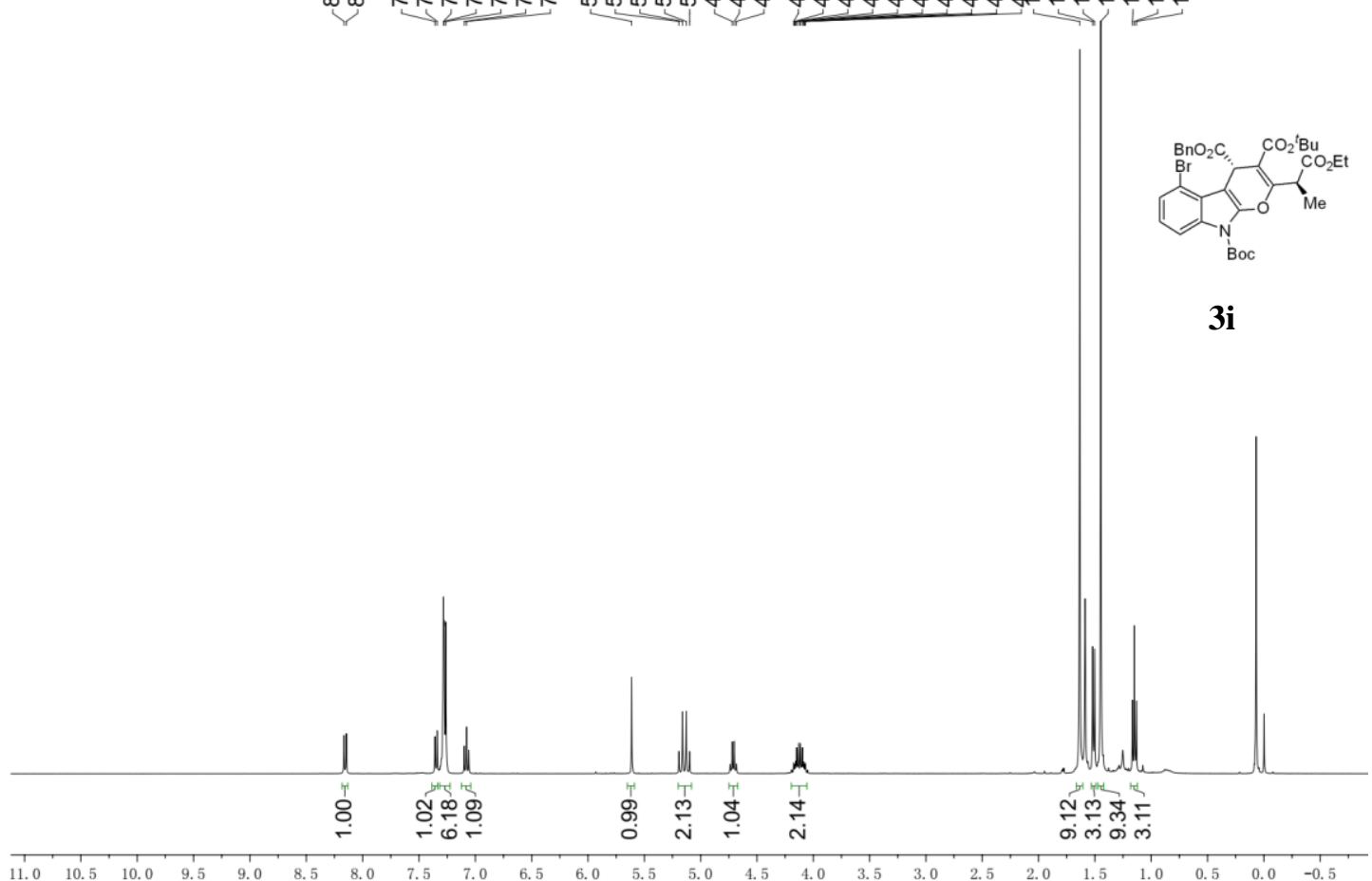



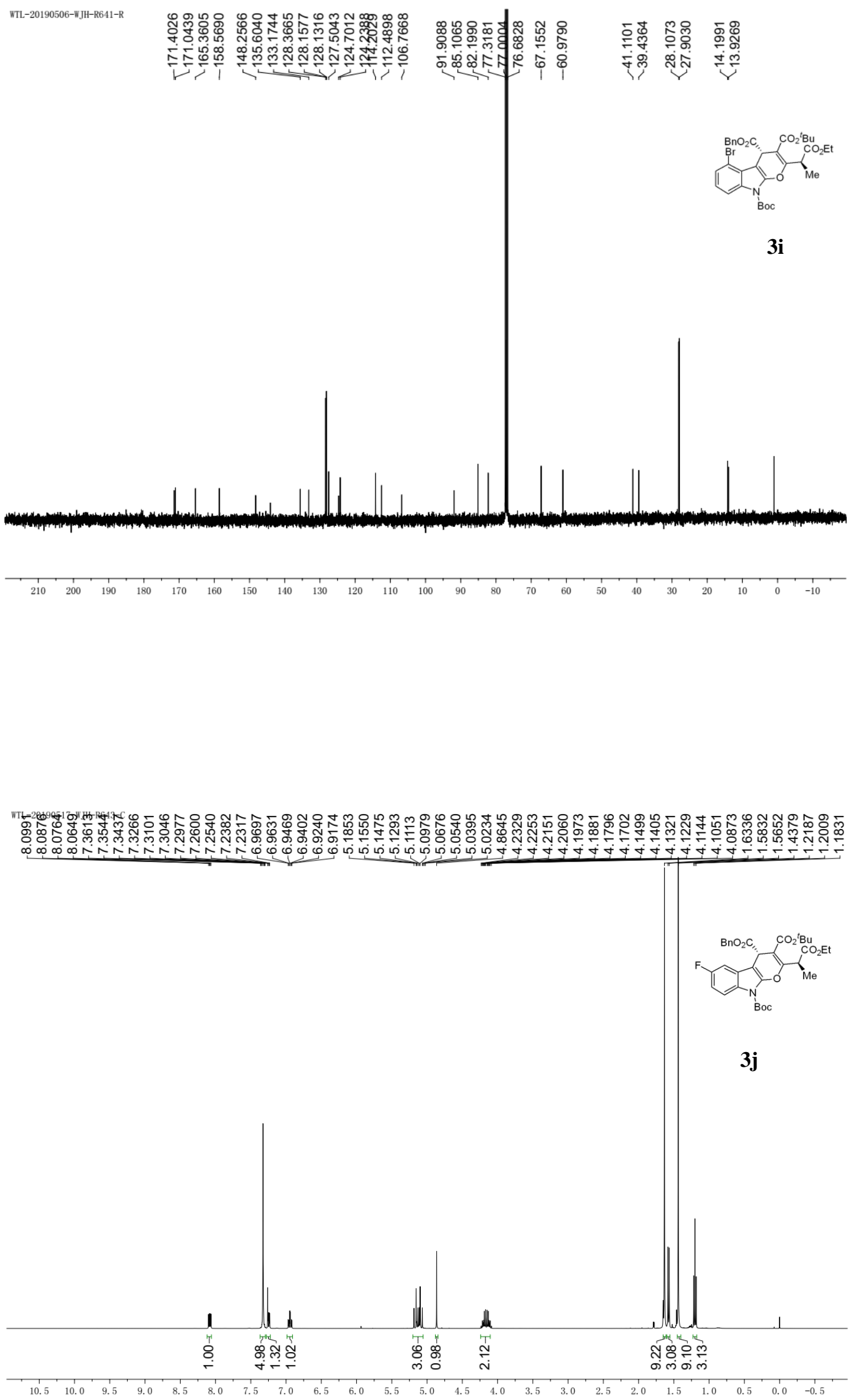

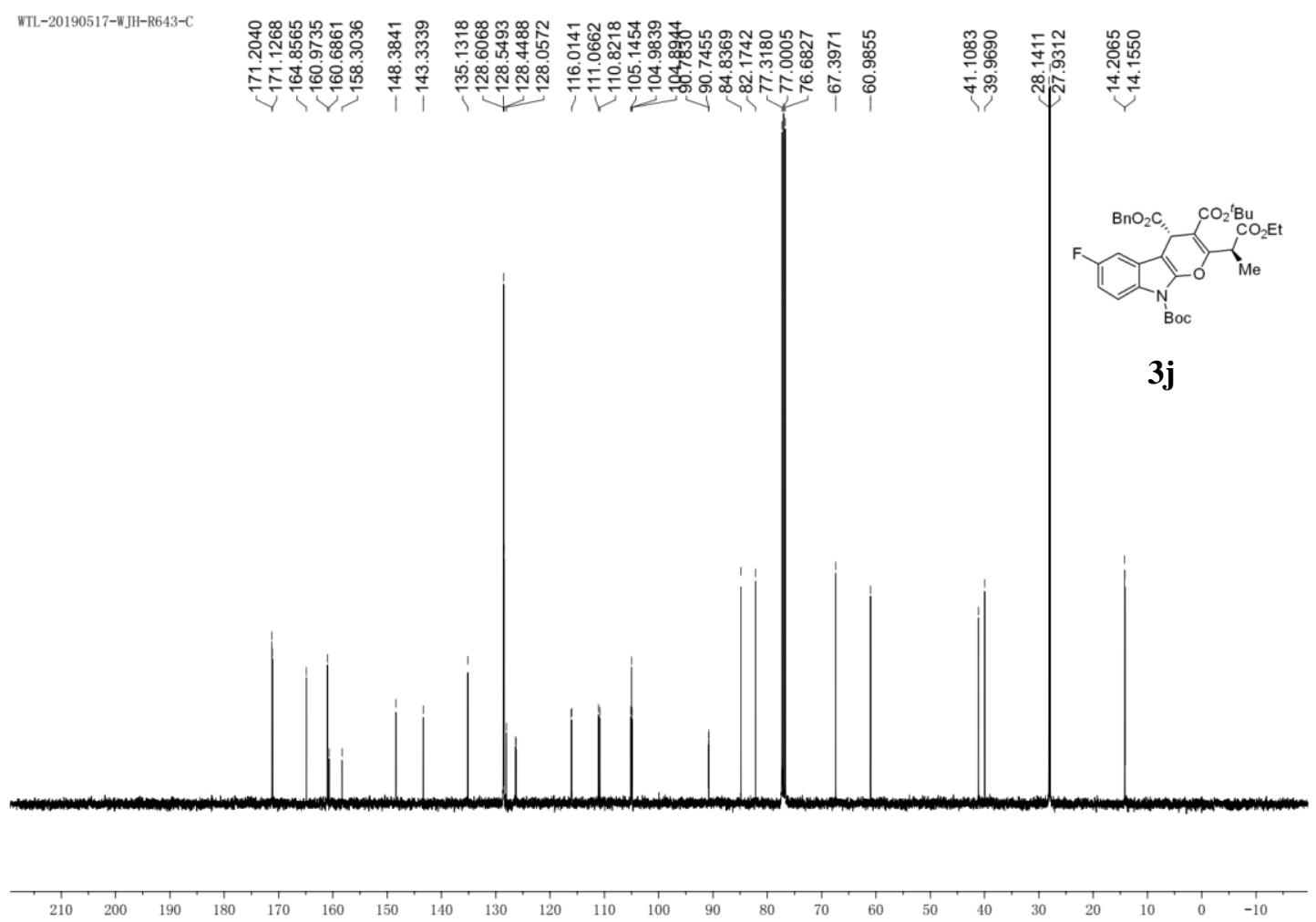

WTL-20190517-WJH-R643-C

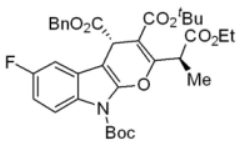

3j 

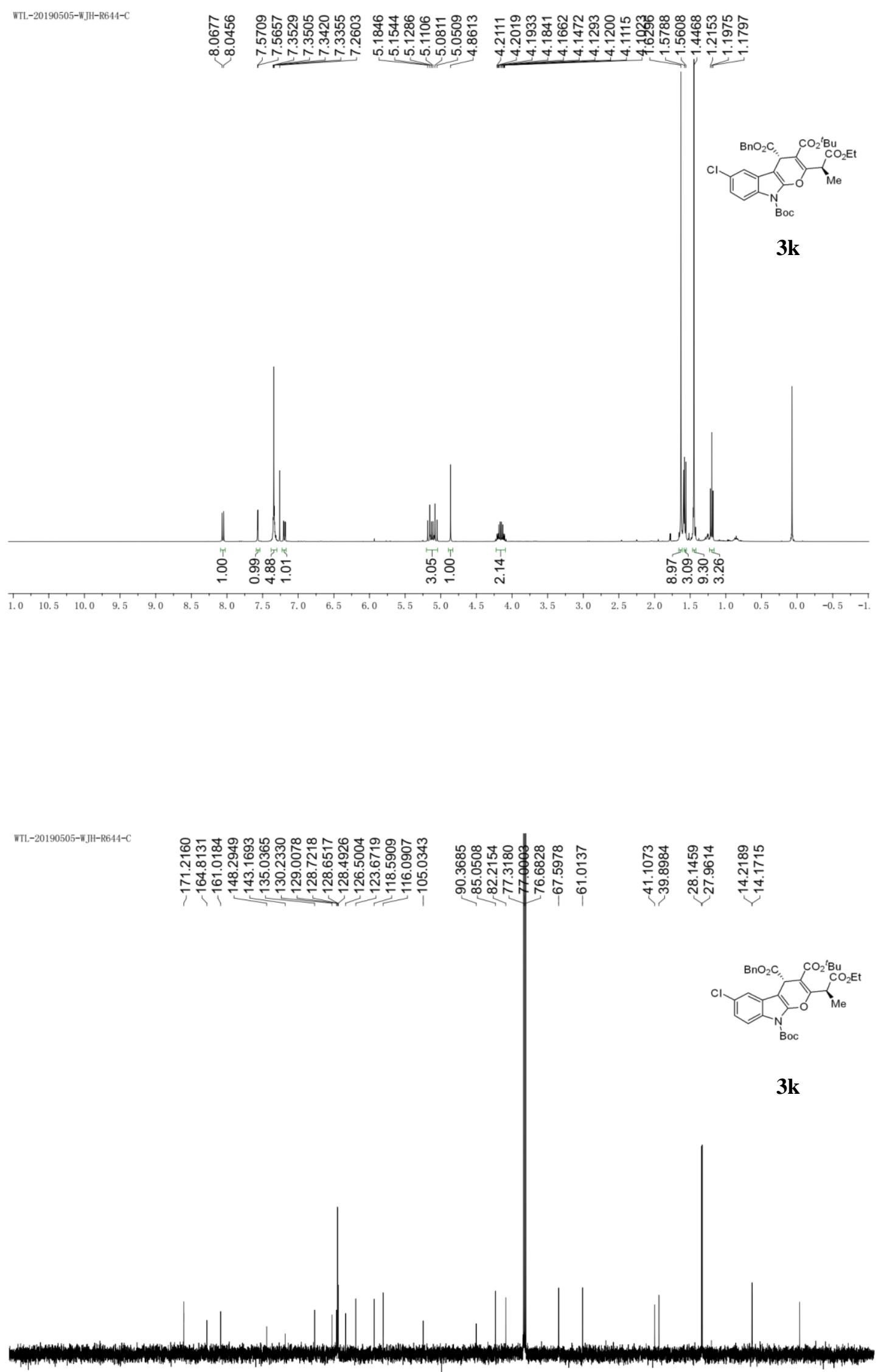

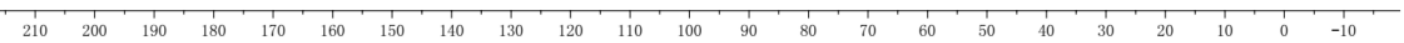



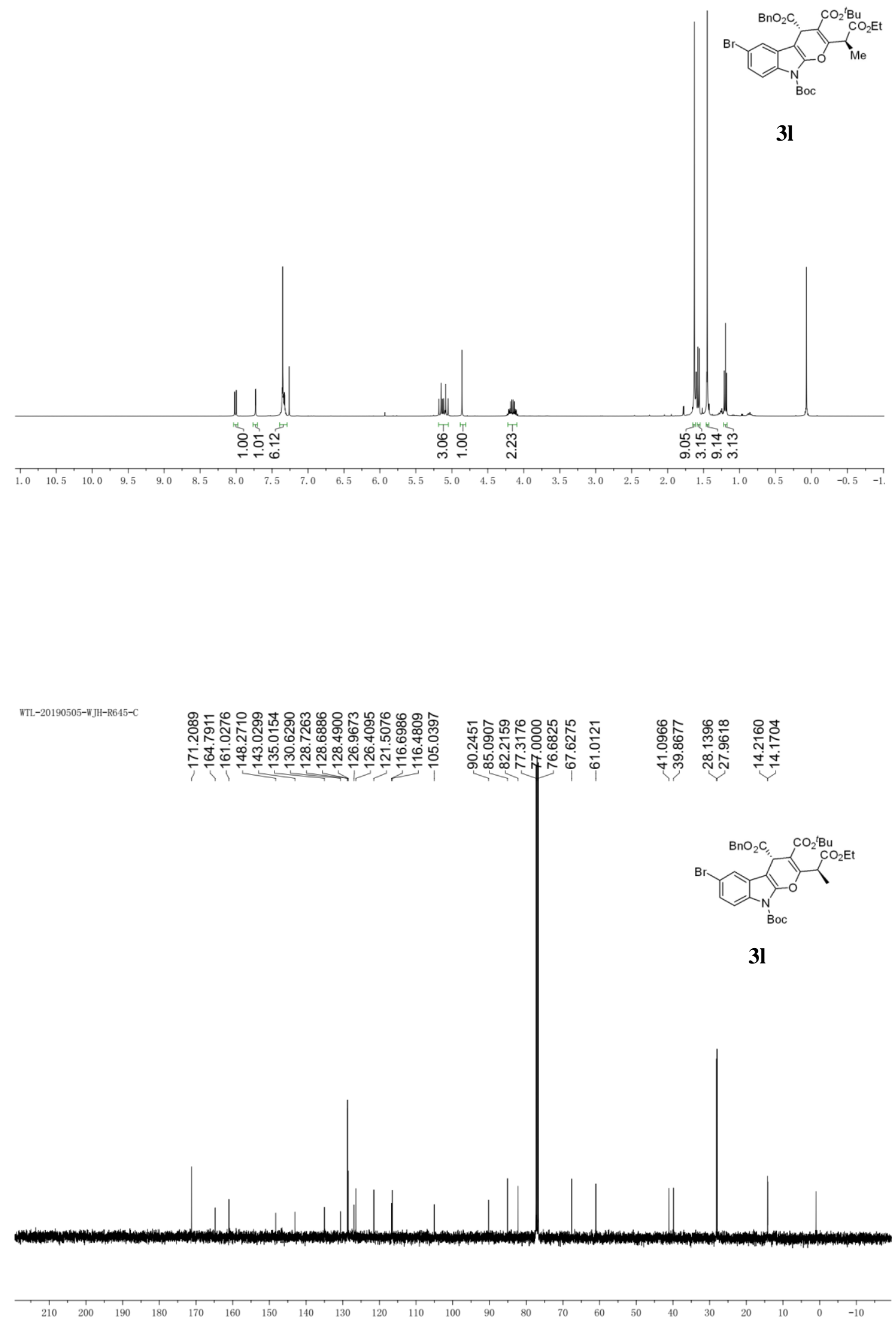

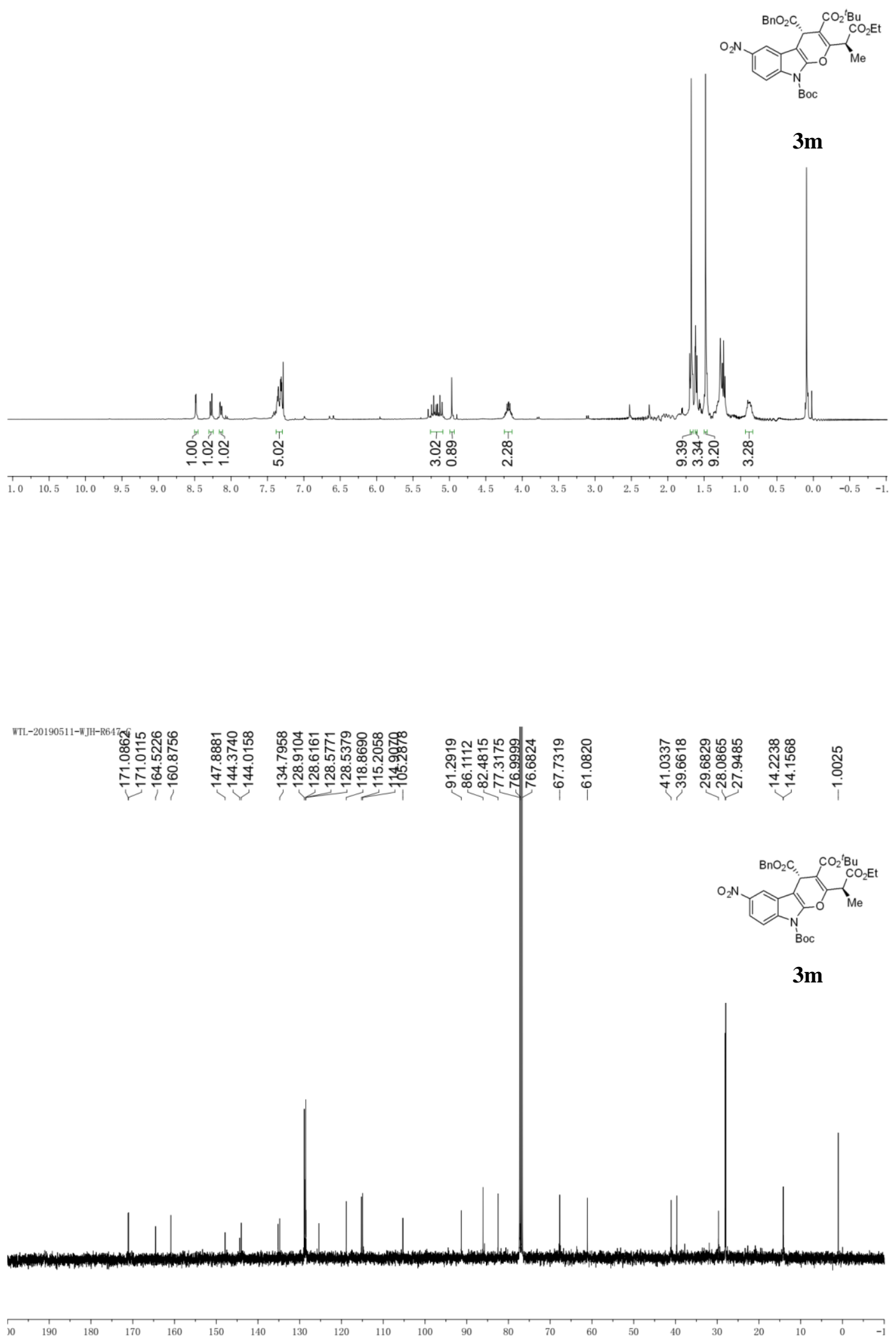

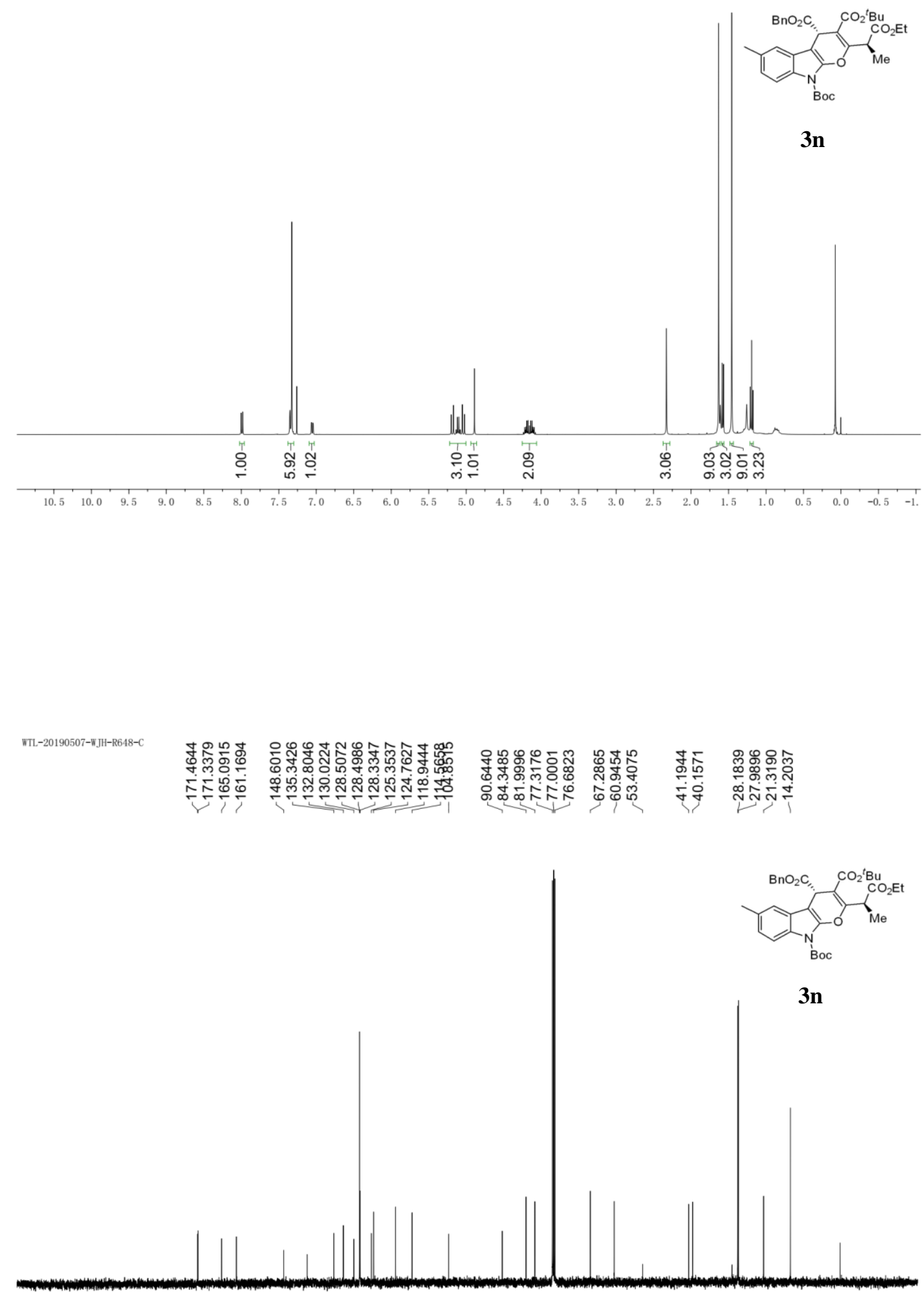

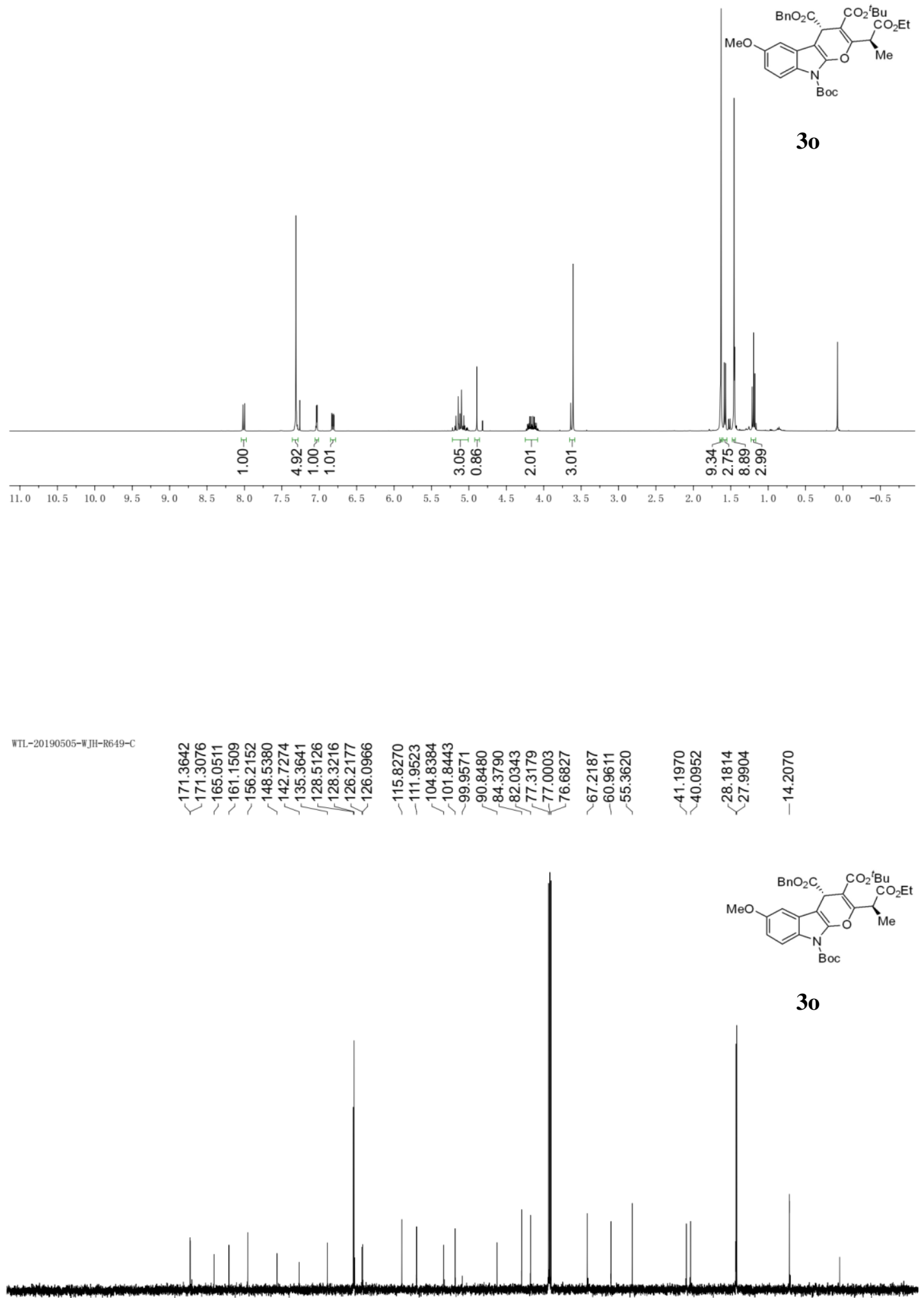


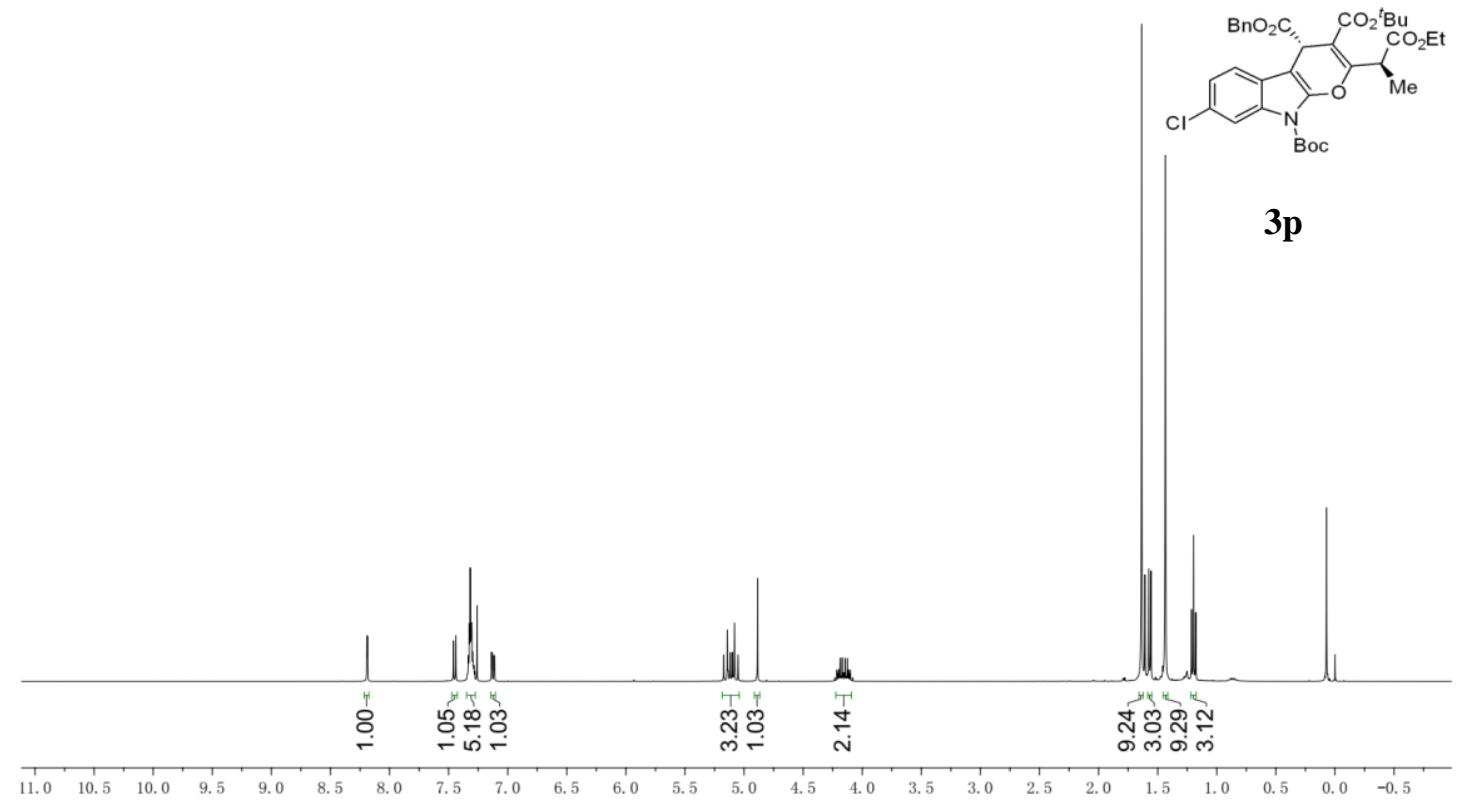

WTL-20190507-WJH-R650-C

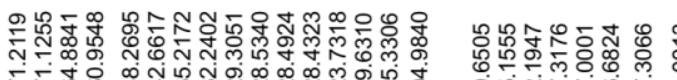

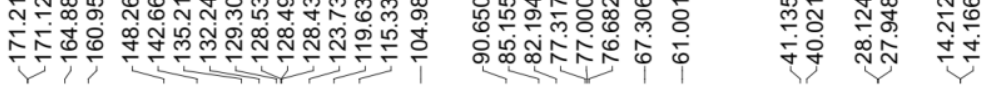

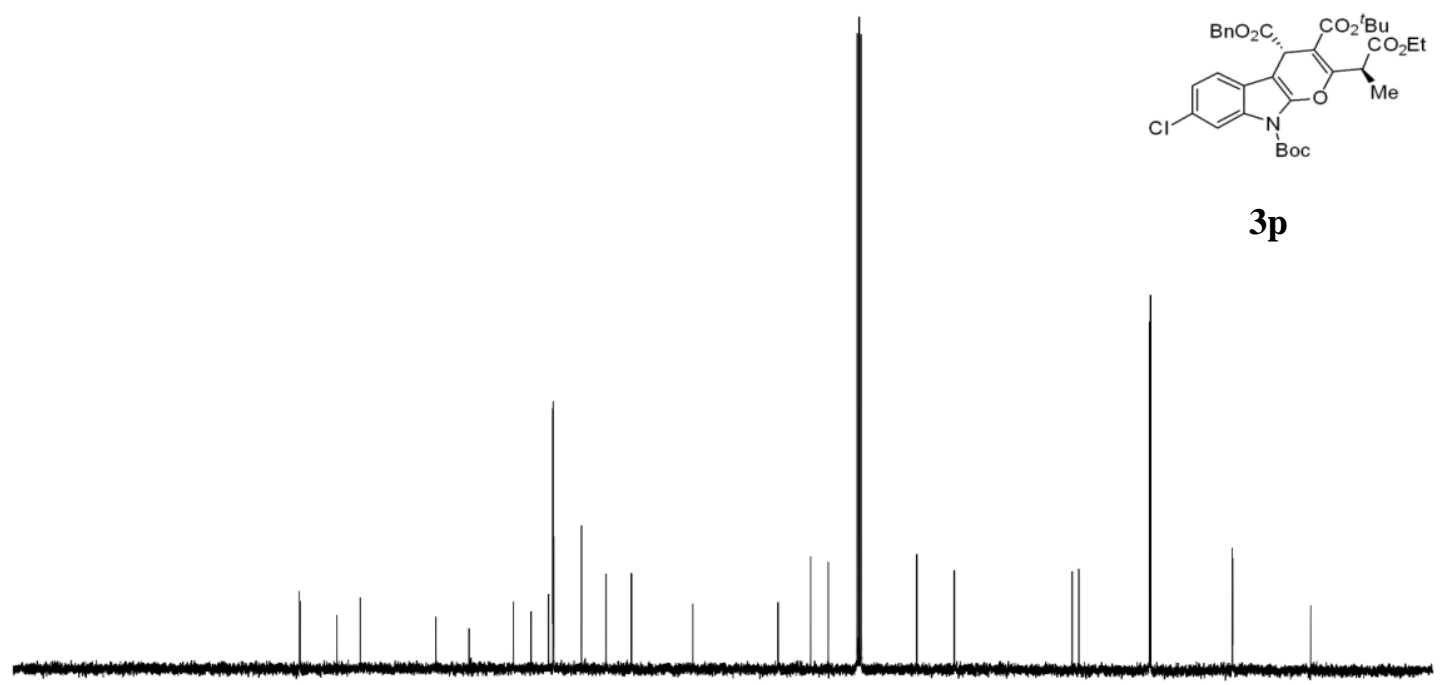

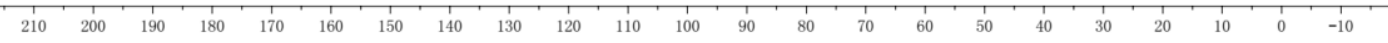



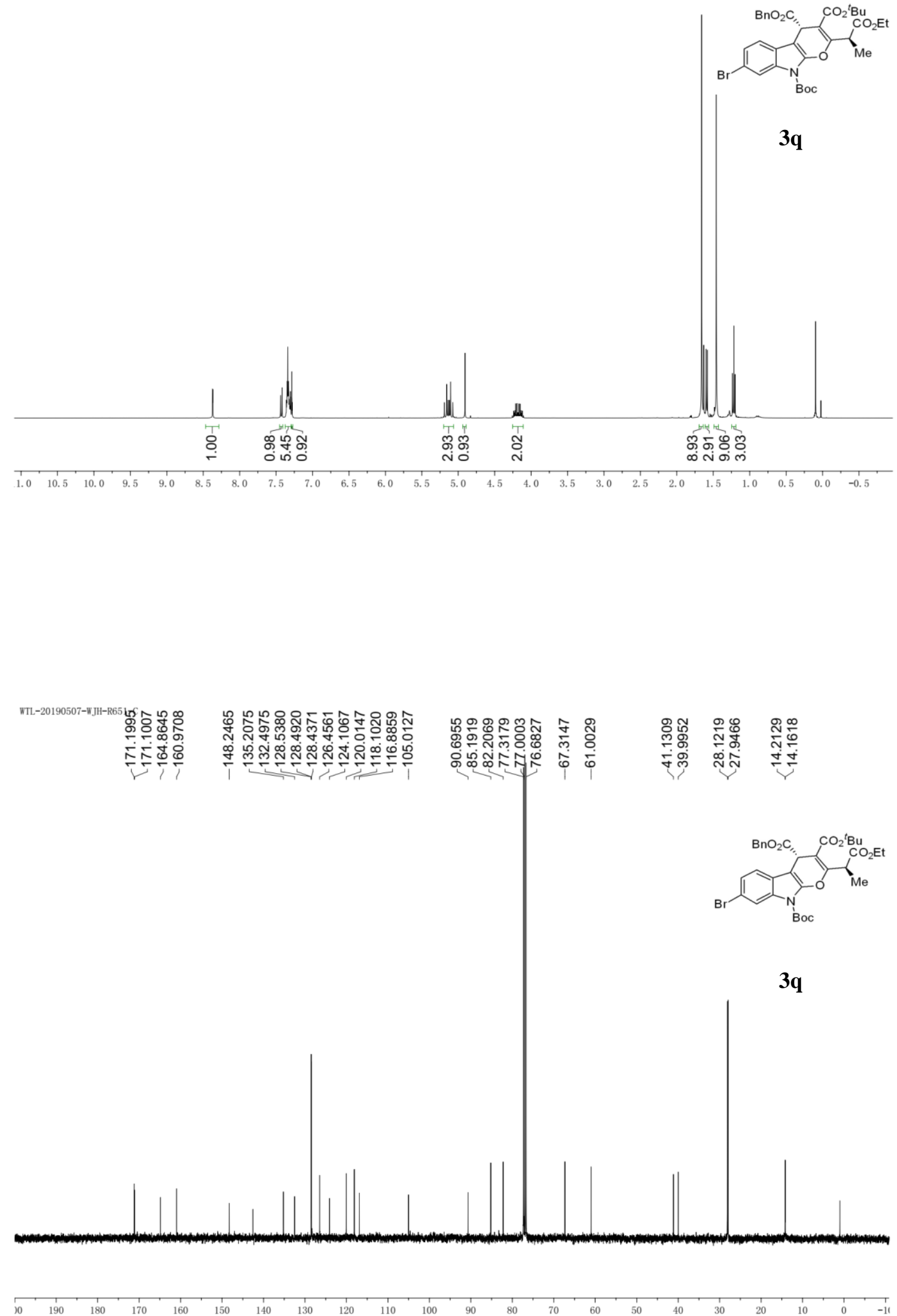


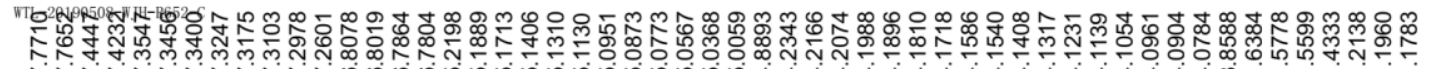

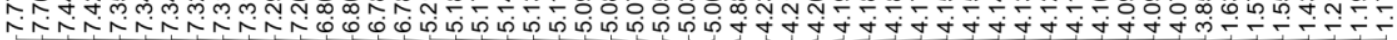

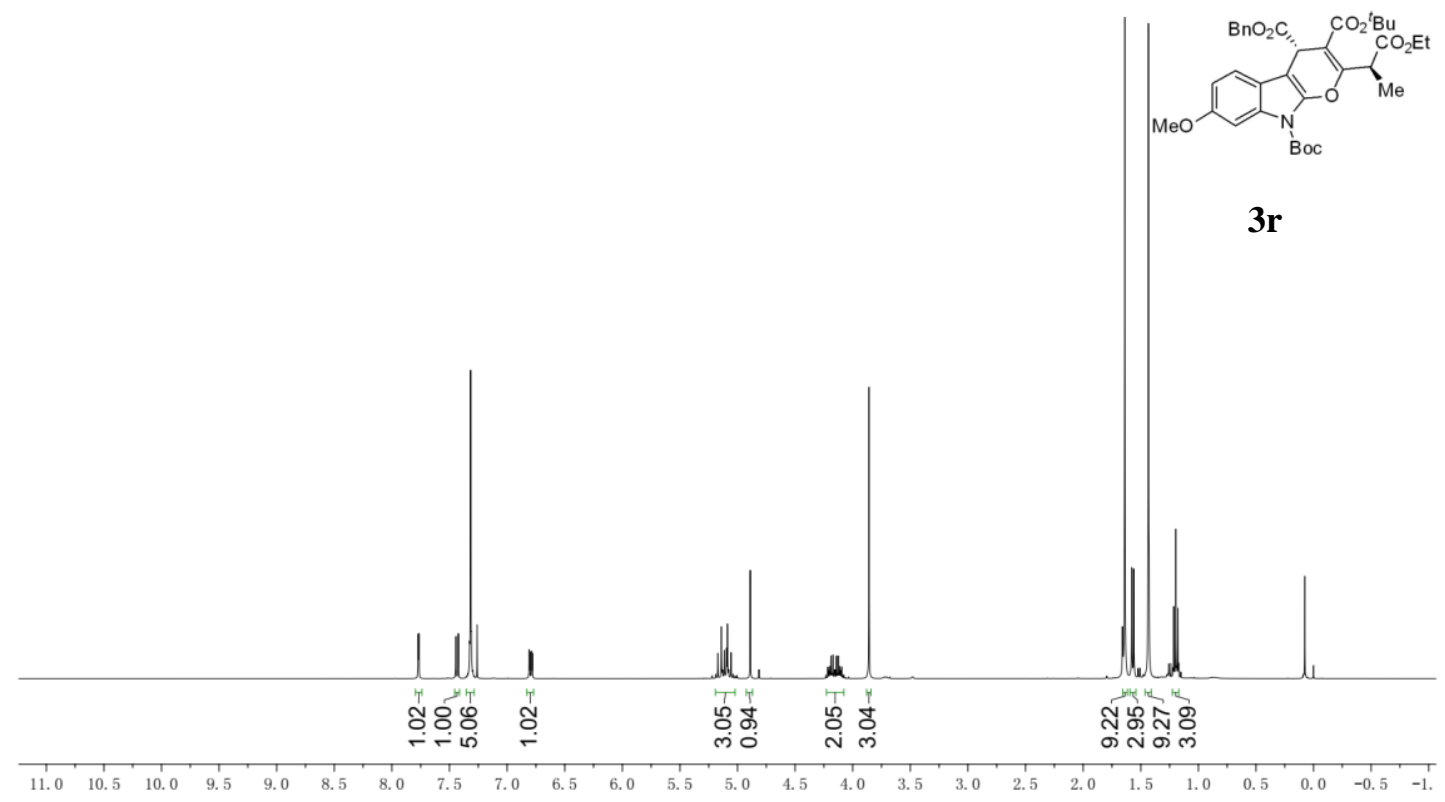

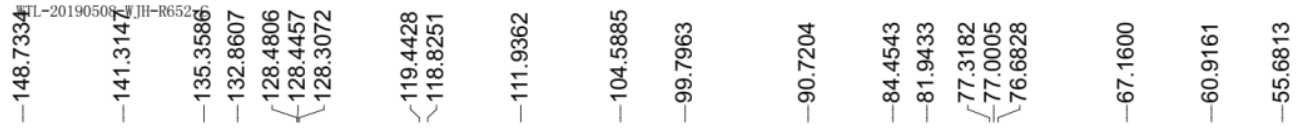
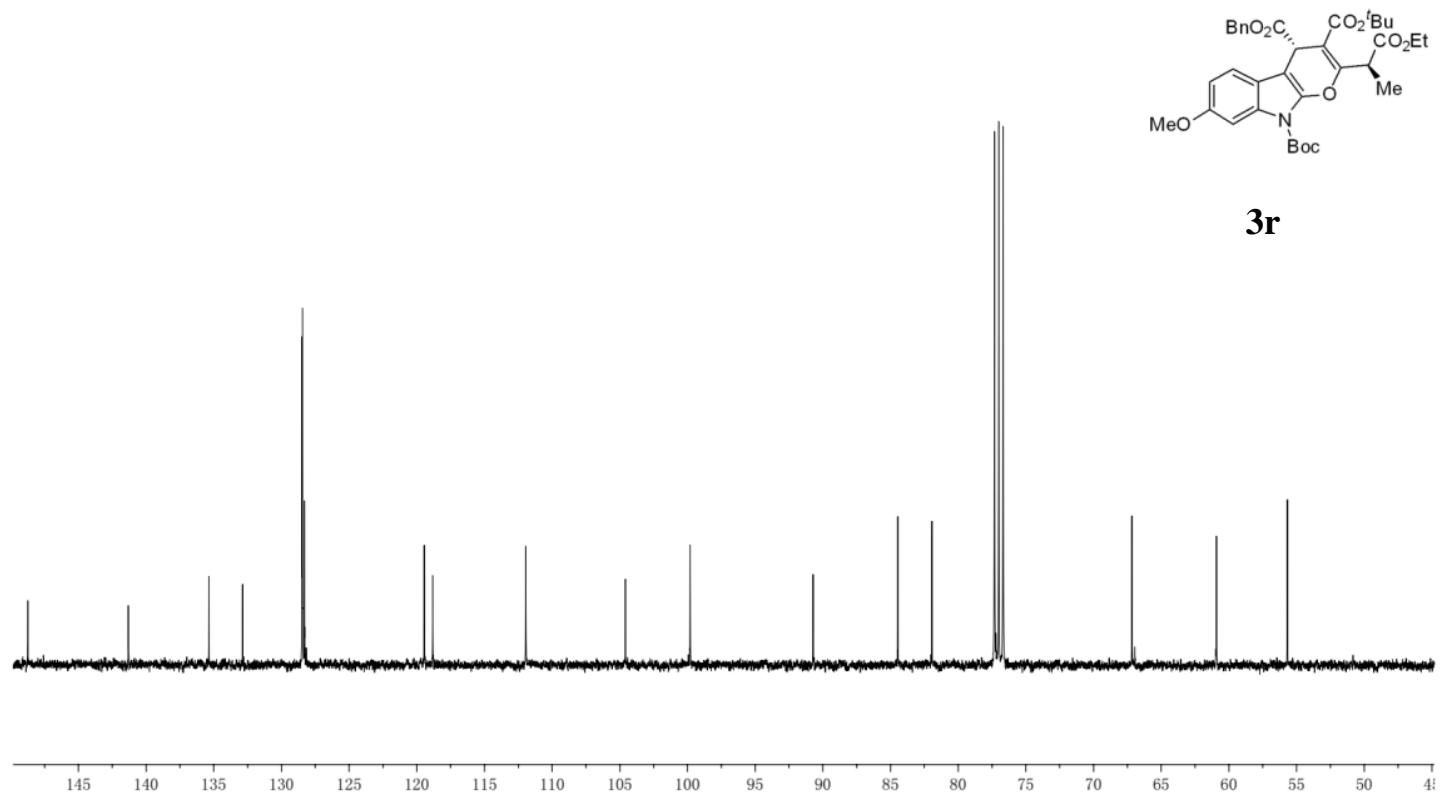


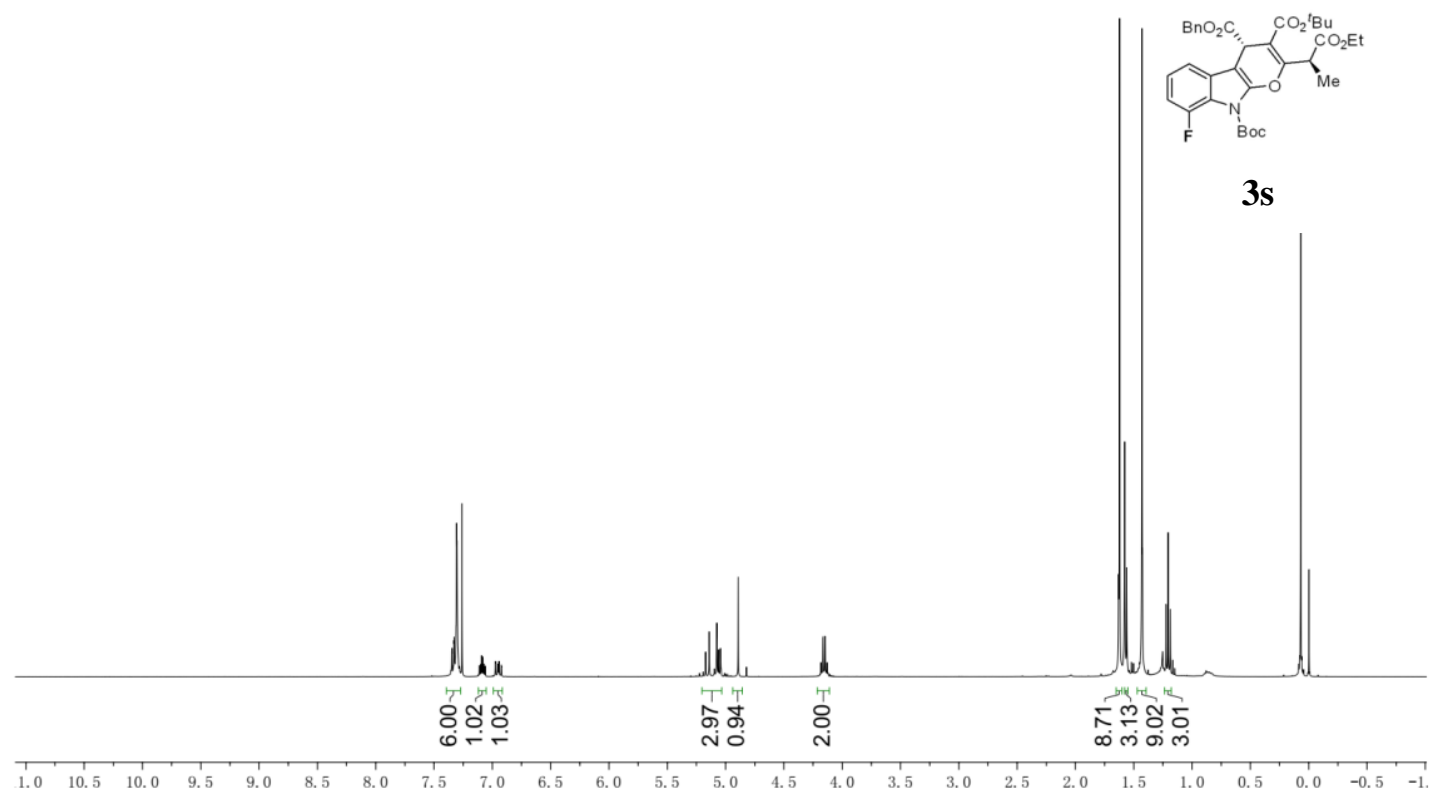

WTL-20190513-WJH-R653-C

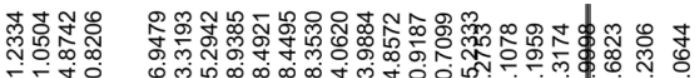

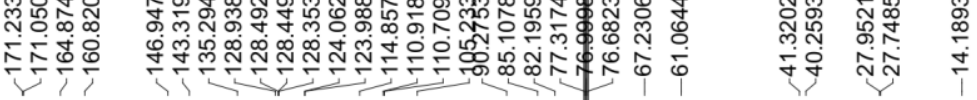

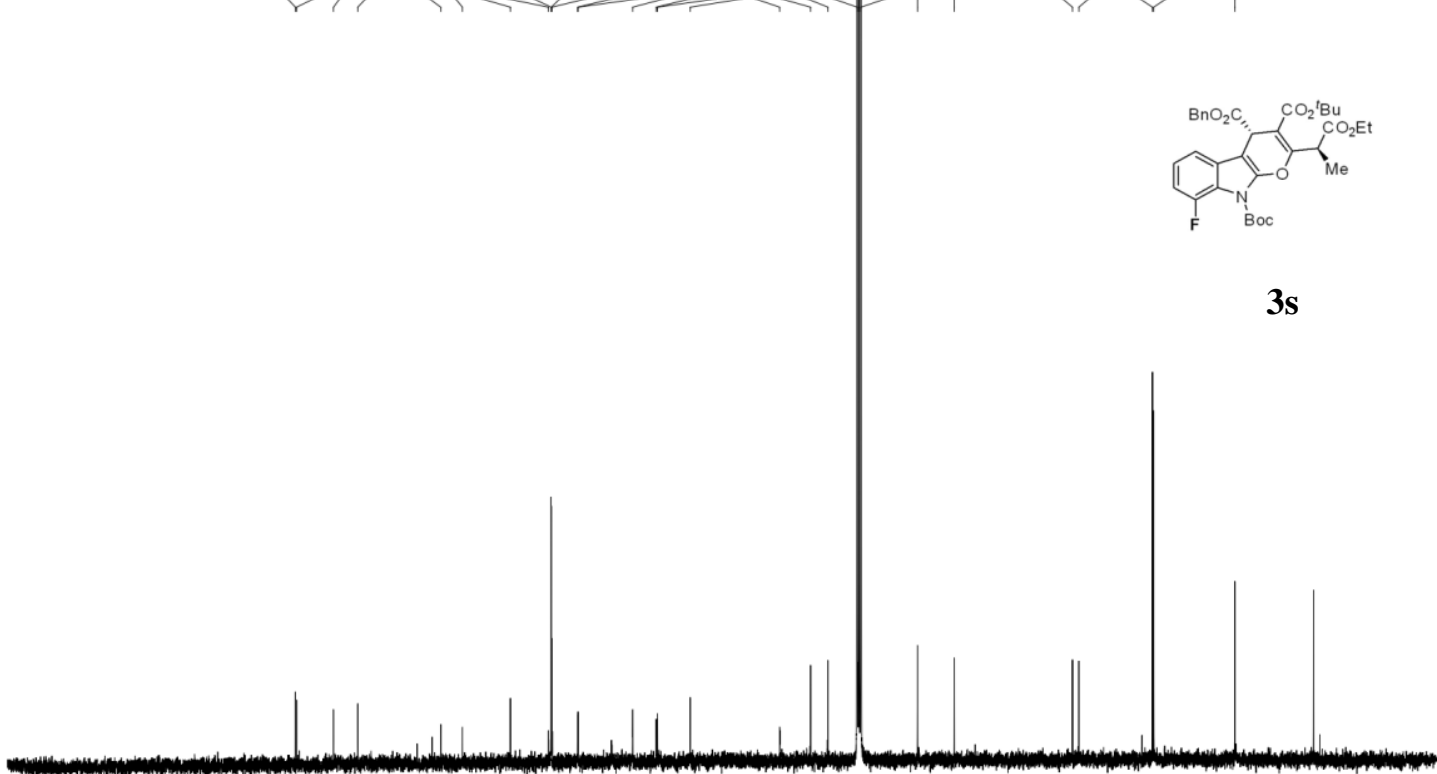

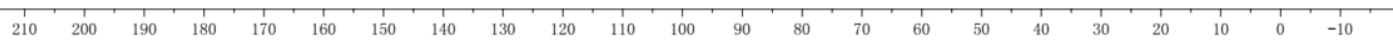




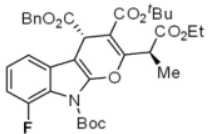

3s

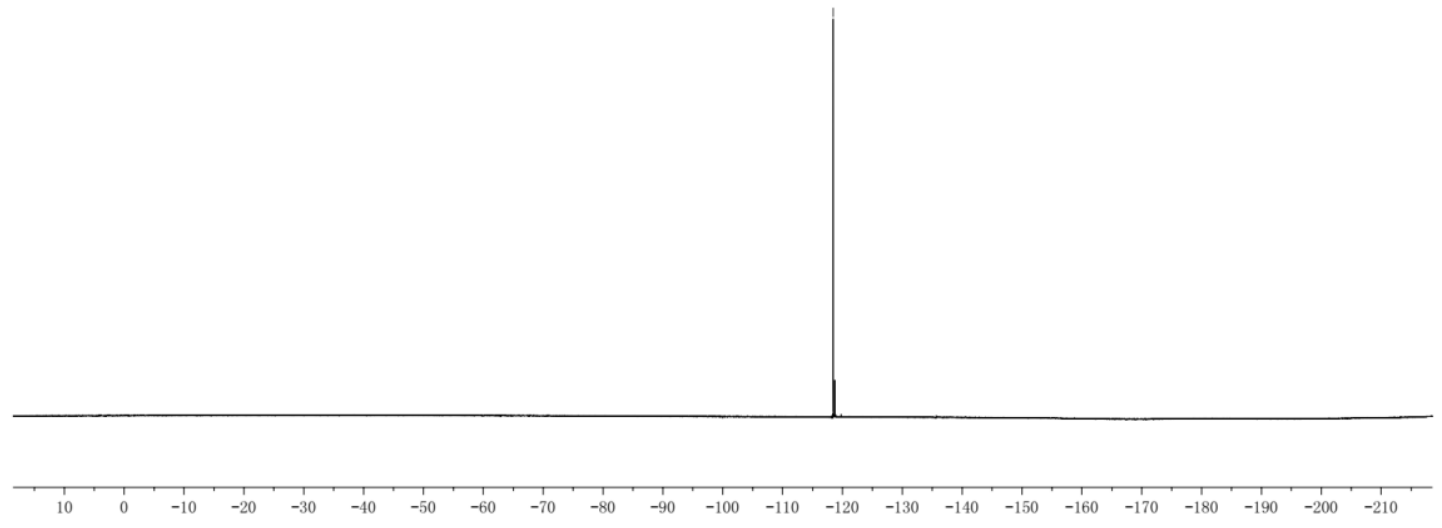

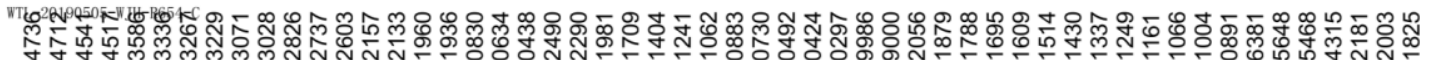

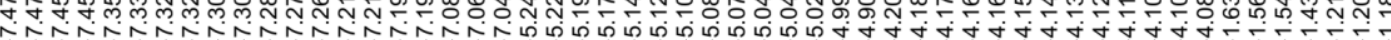

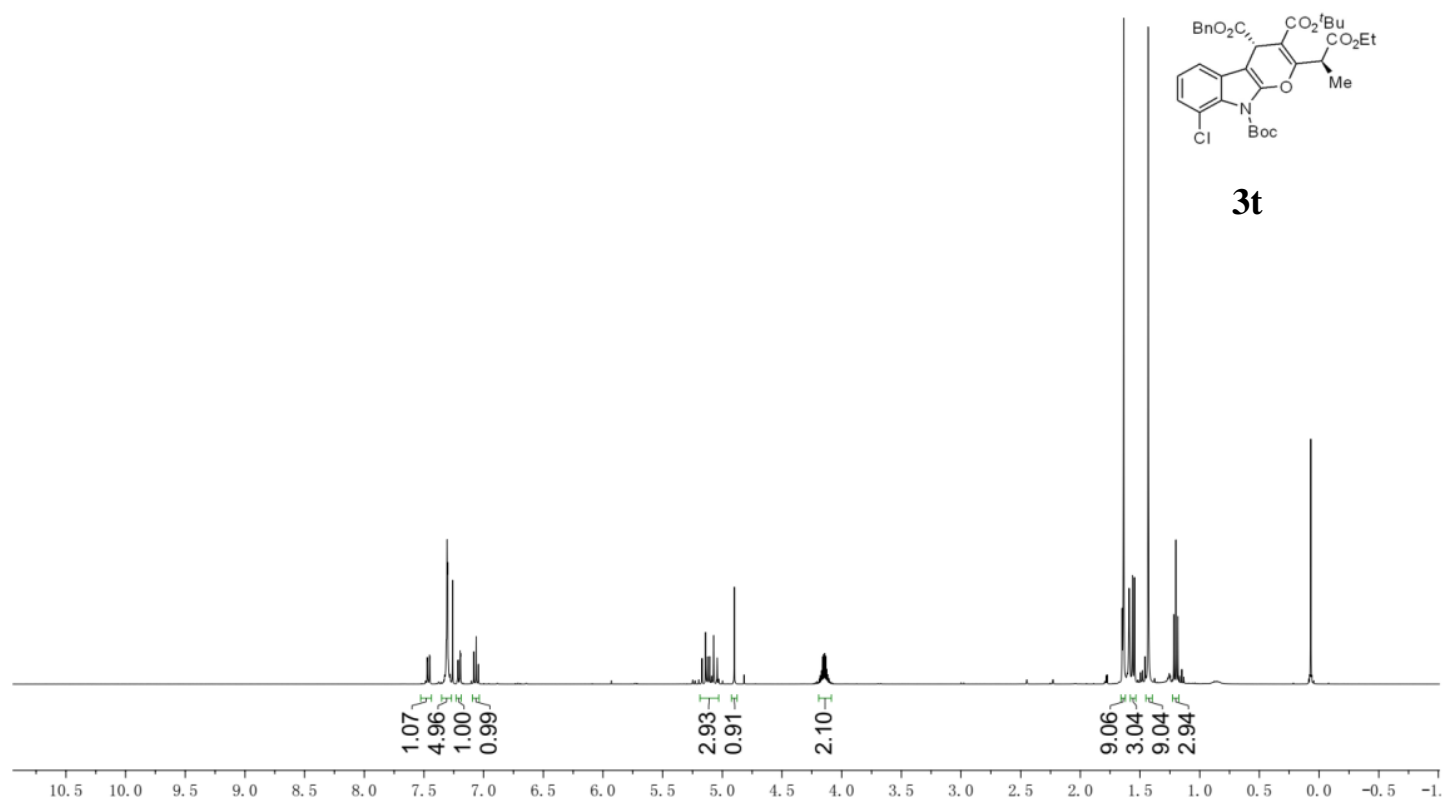



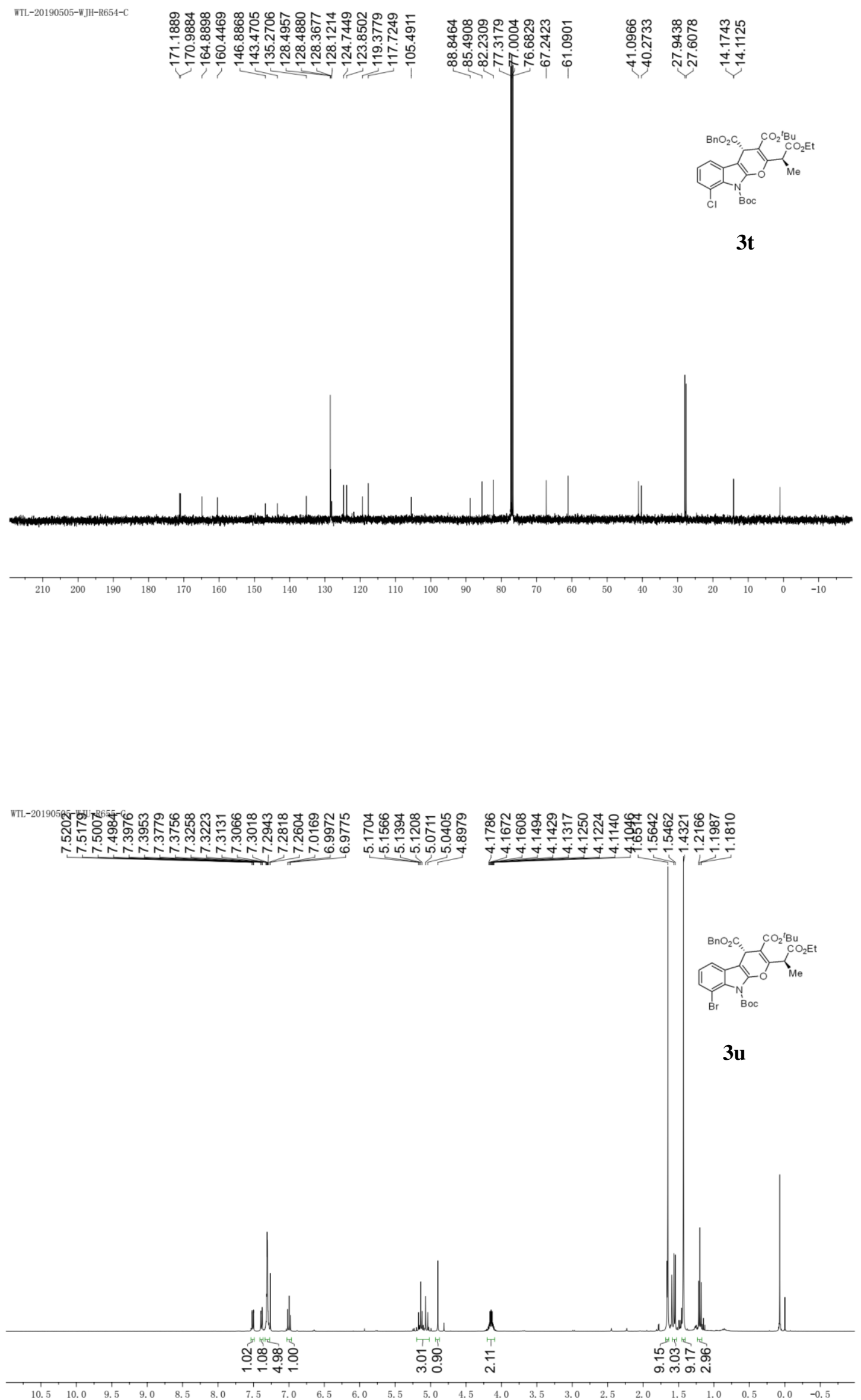

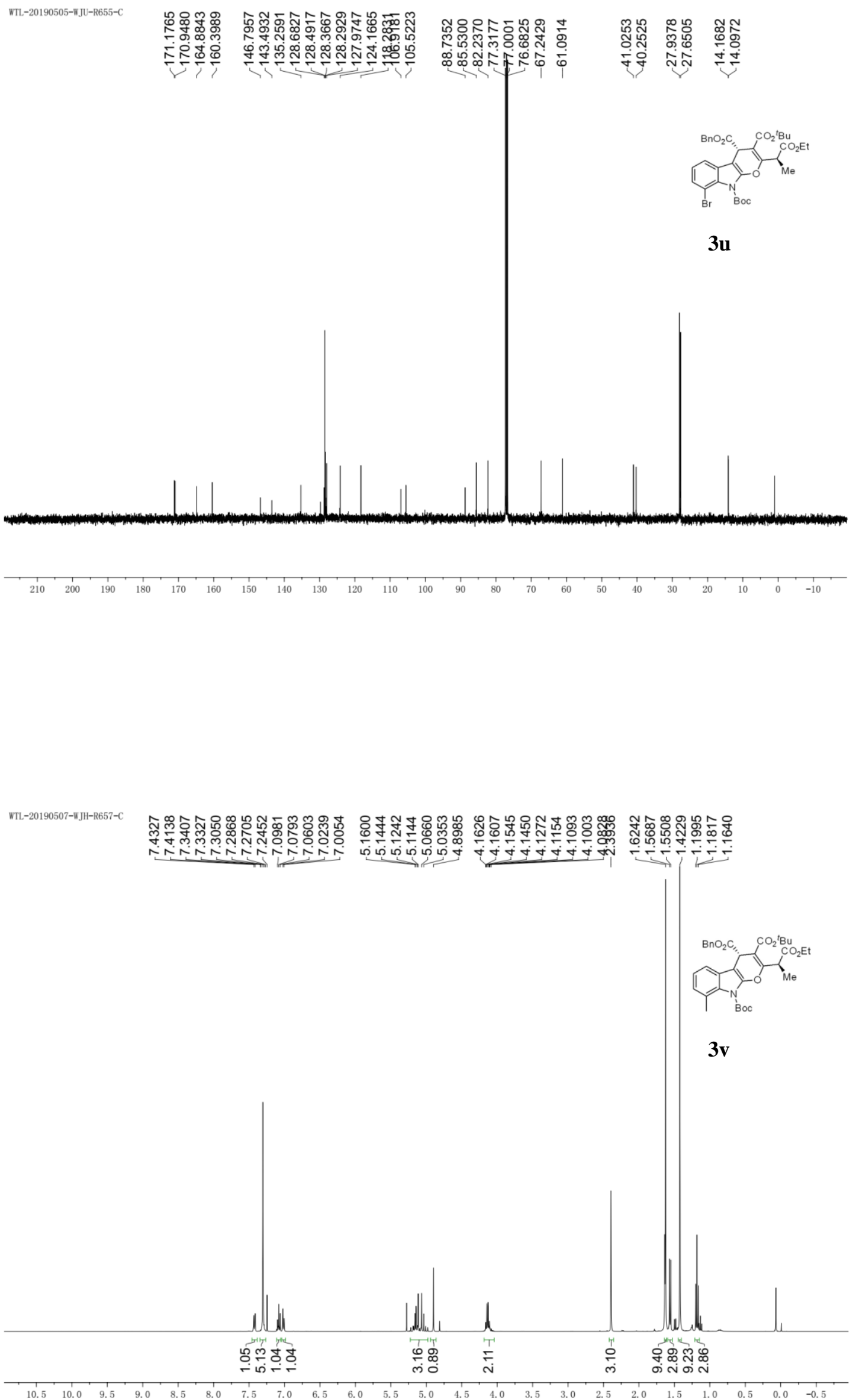
WTL-20190507-WJH-R657-C

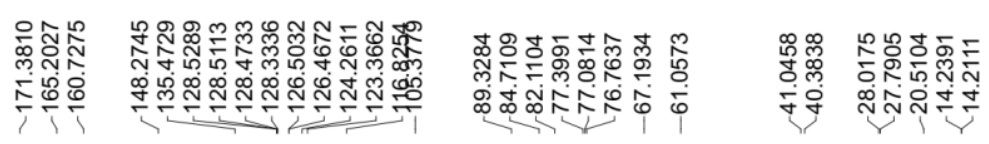

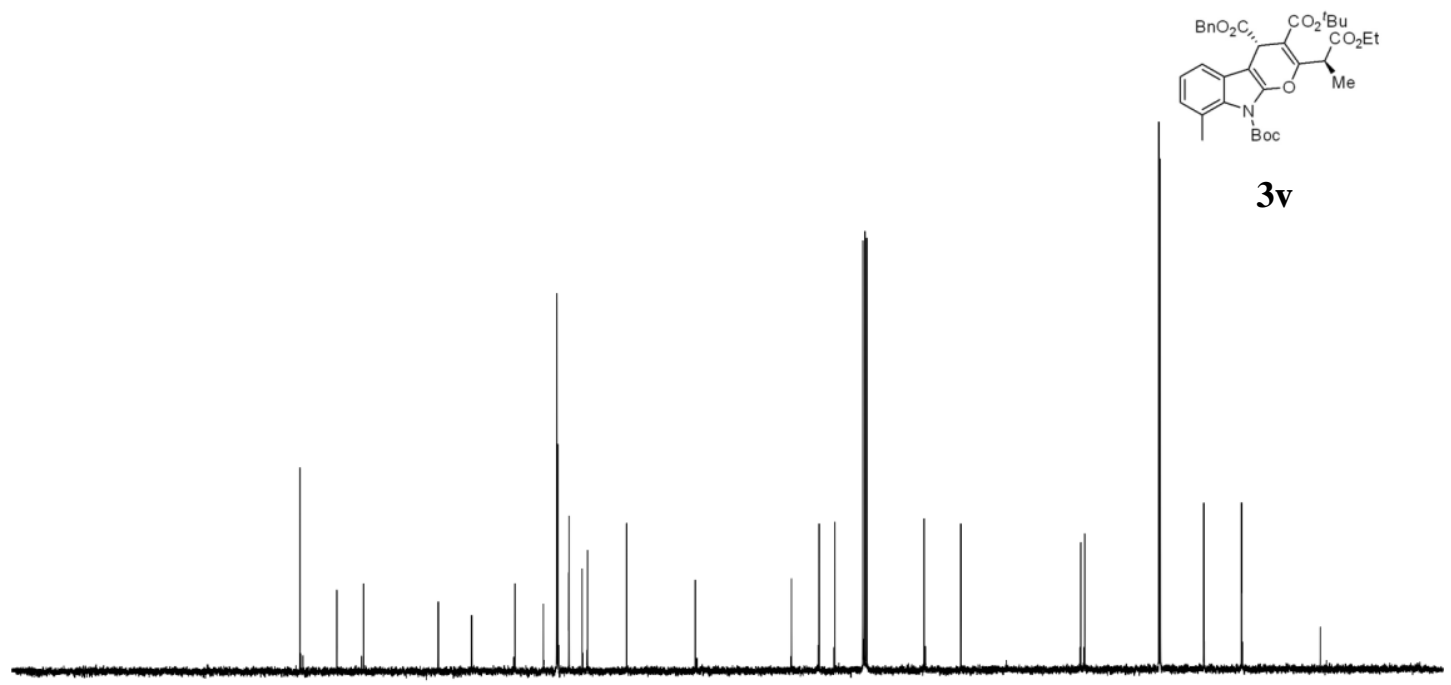

WTL-20190509-WJHT-R680-C

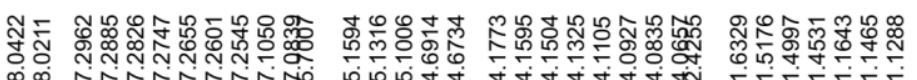

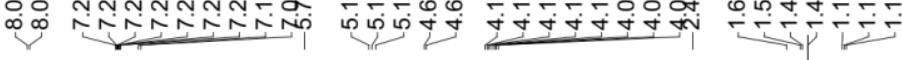

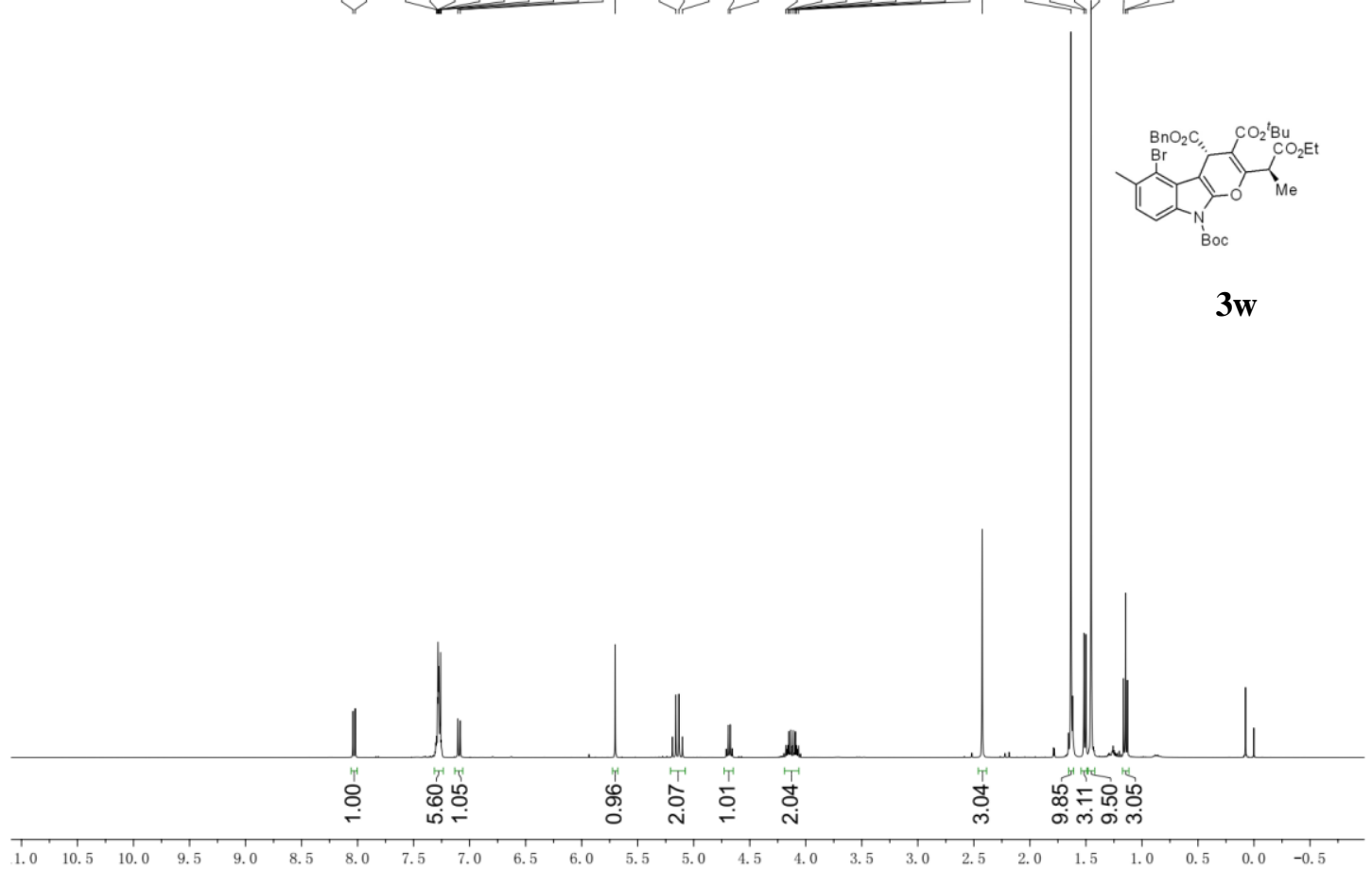




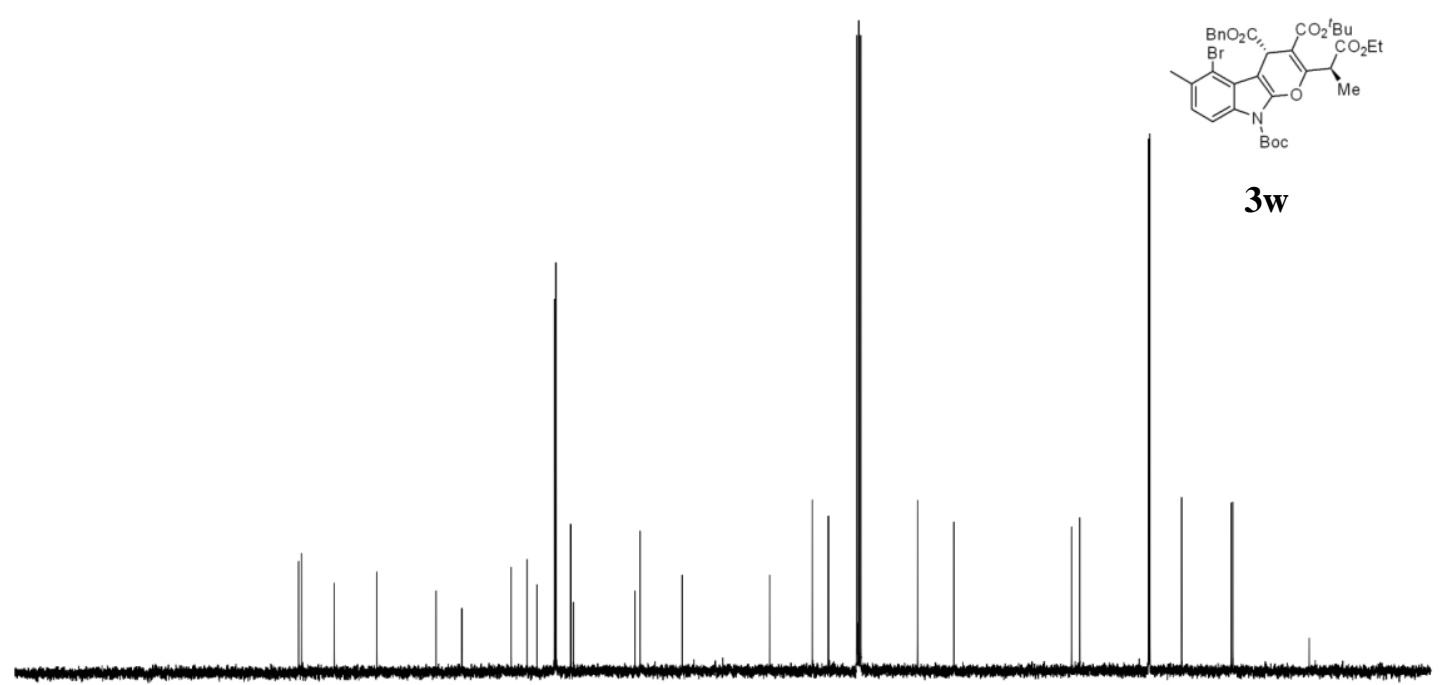

WTL-20190508-WJH-R658-C

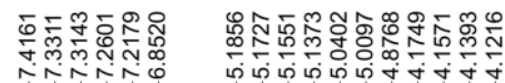

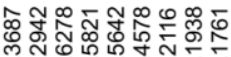

NNi-i:

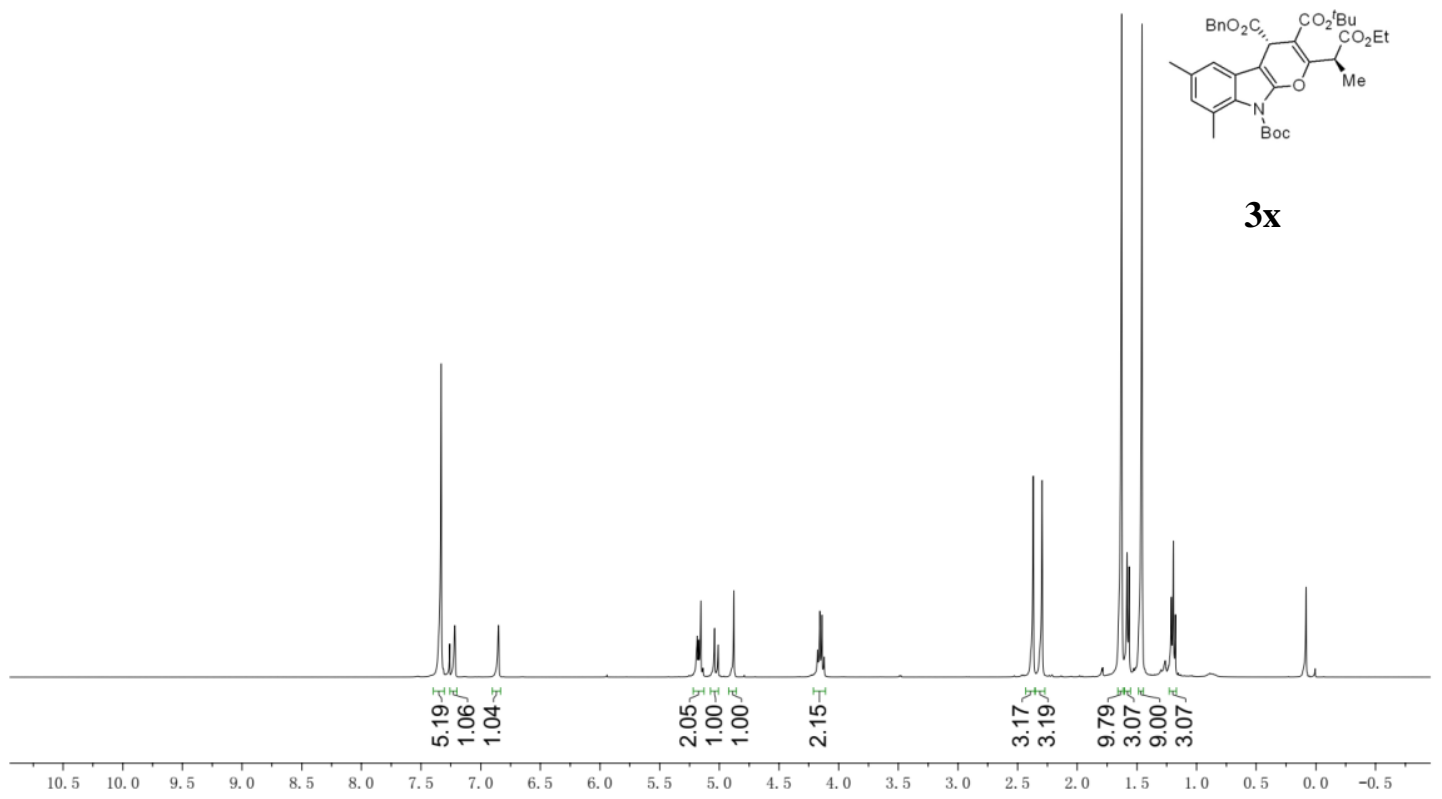



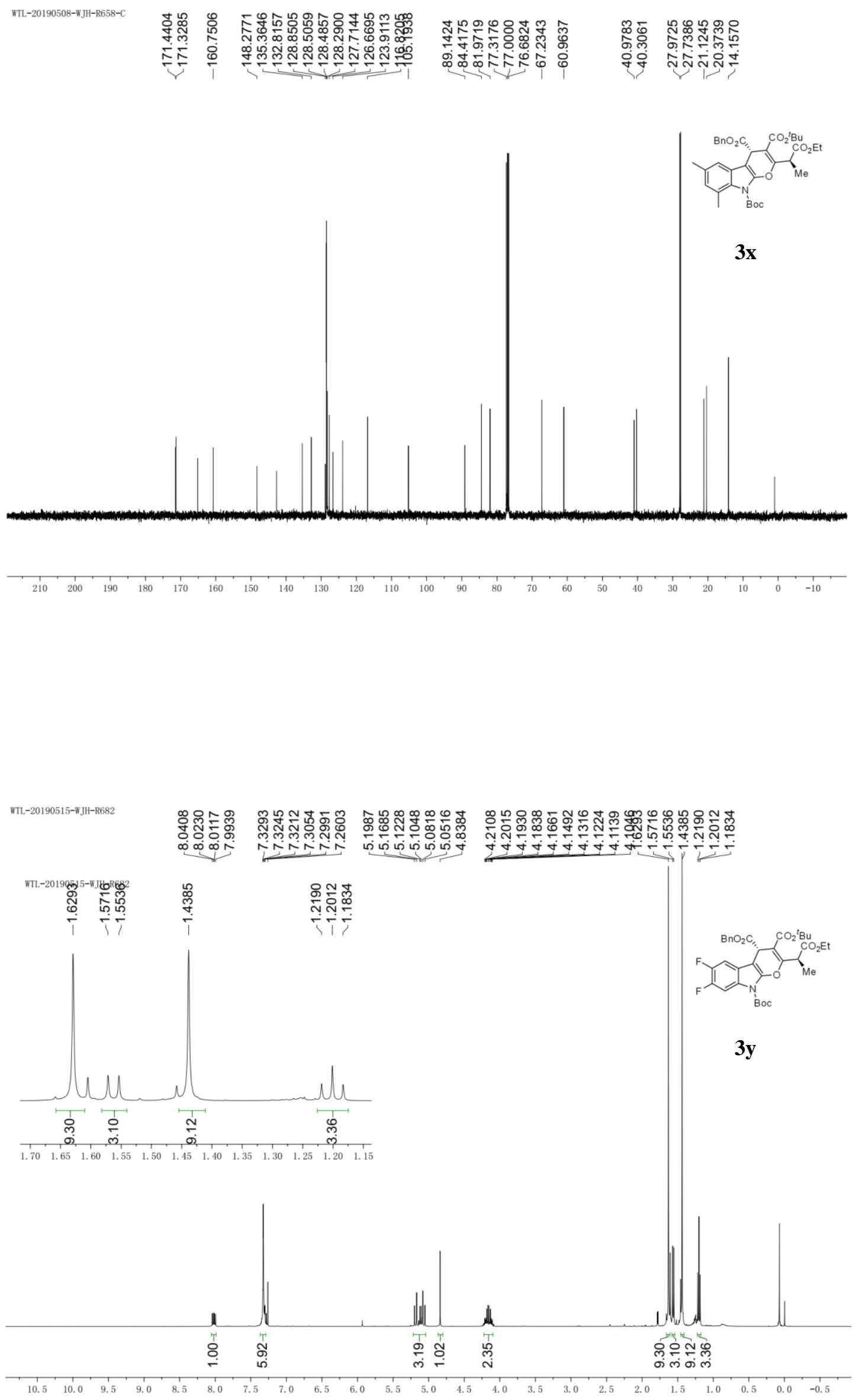


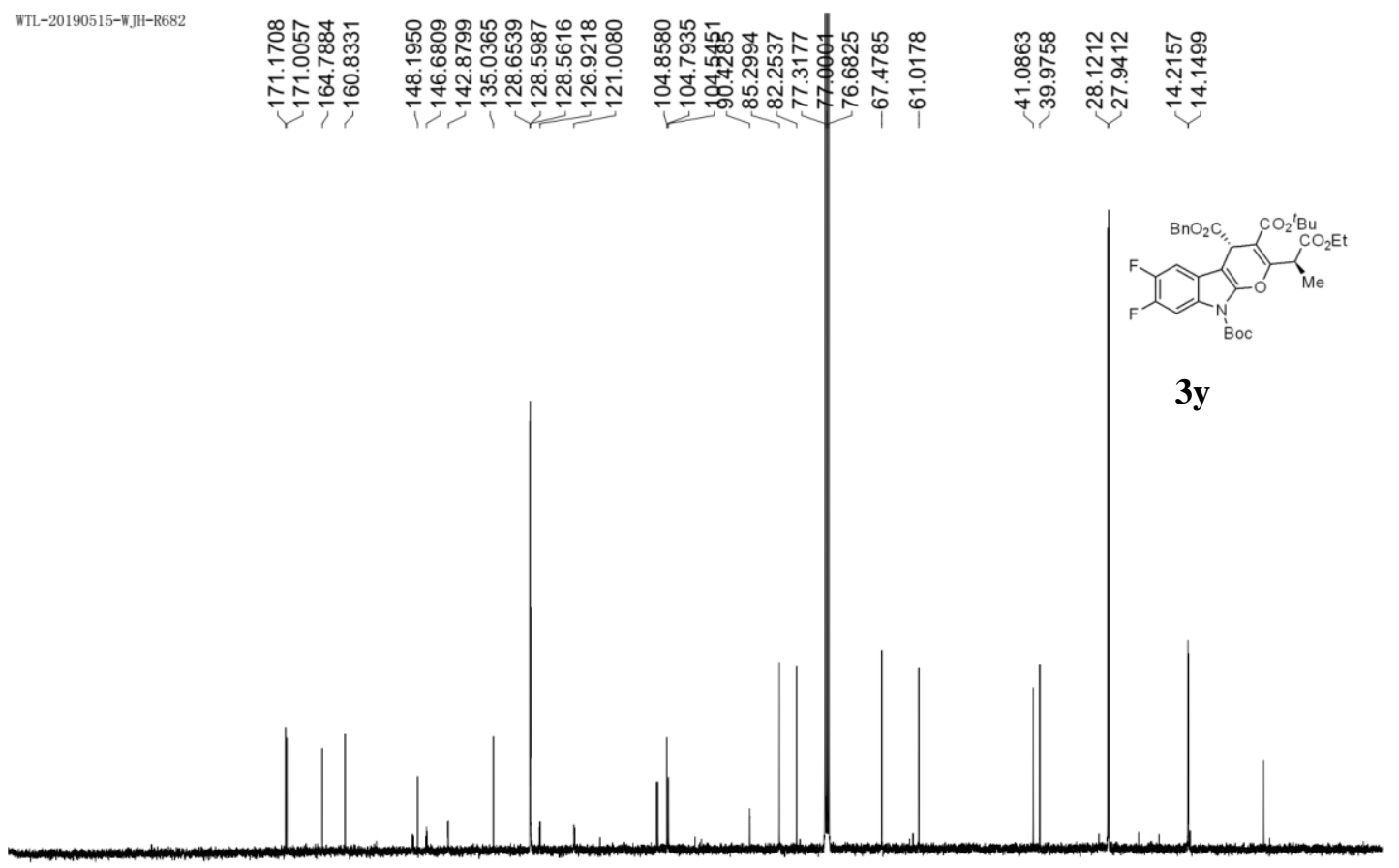

WTL-20190515-WJH-R682

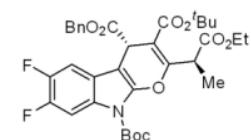

$3 y$

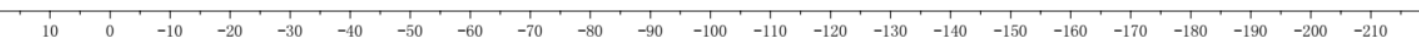




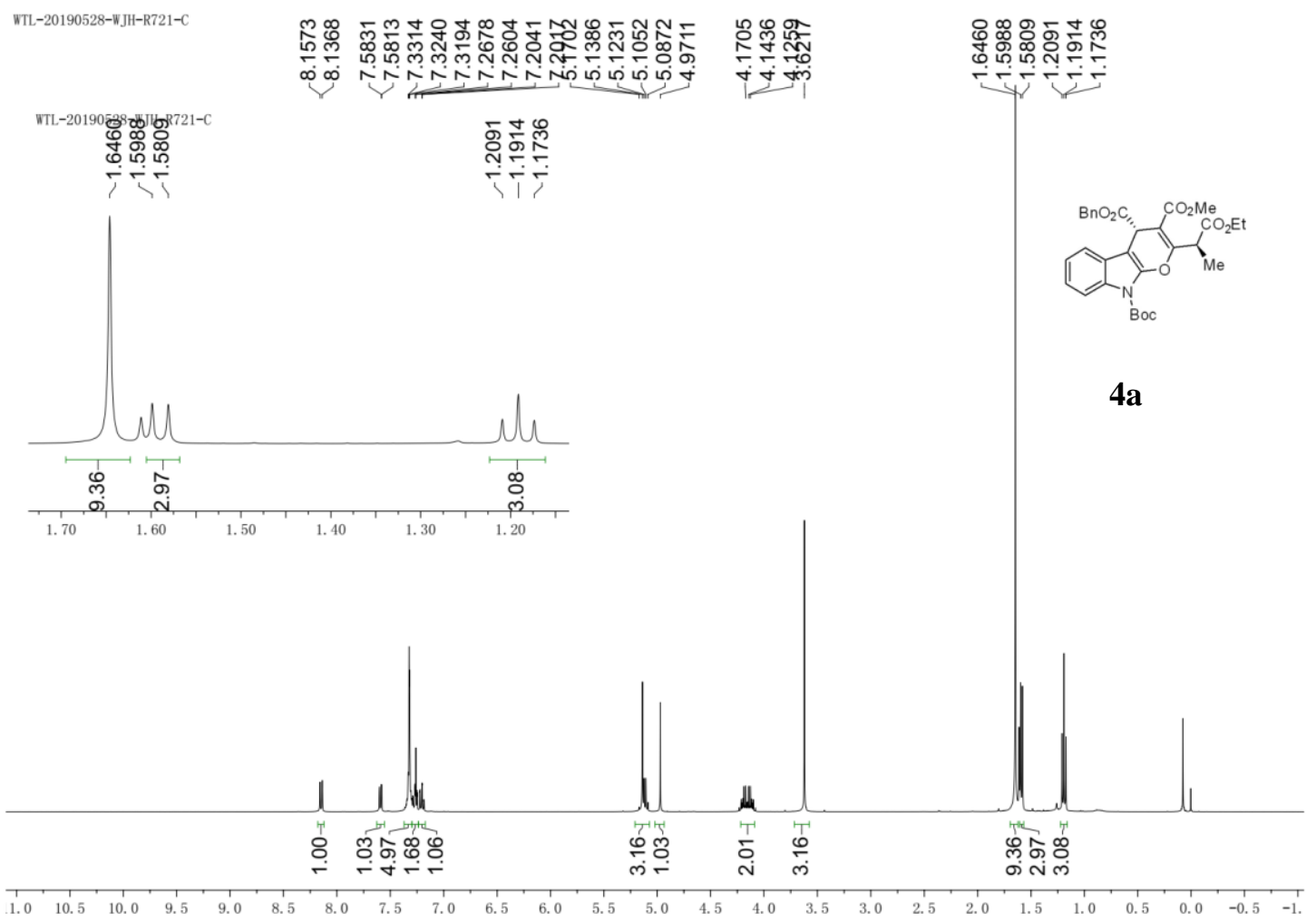

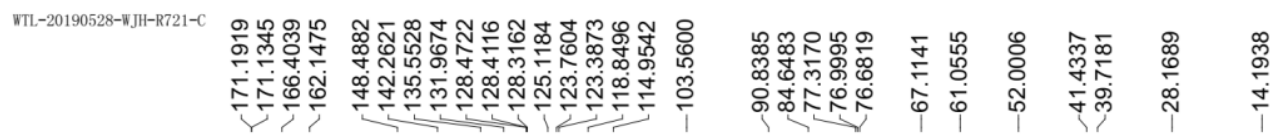
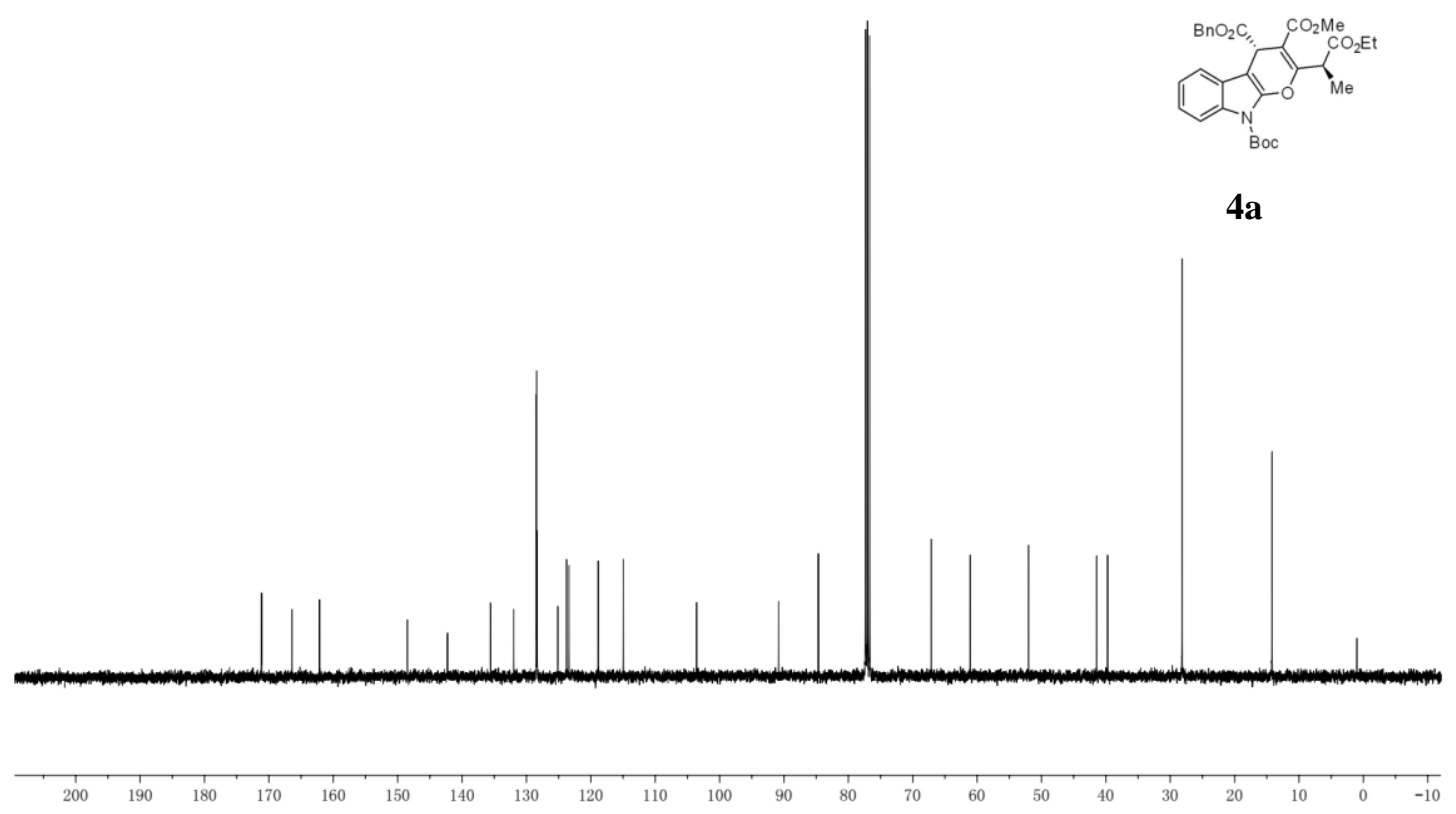

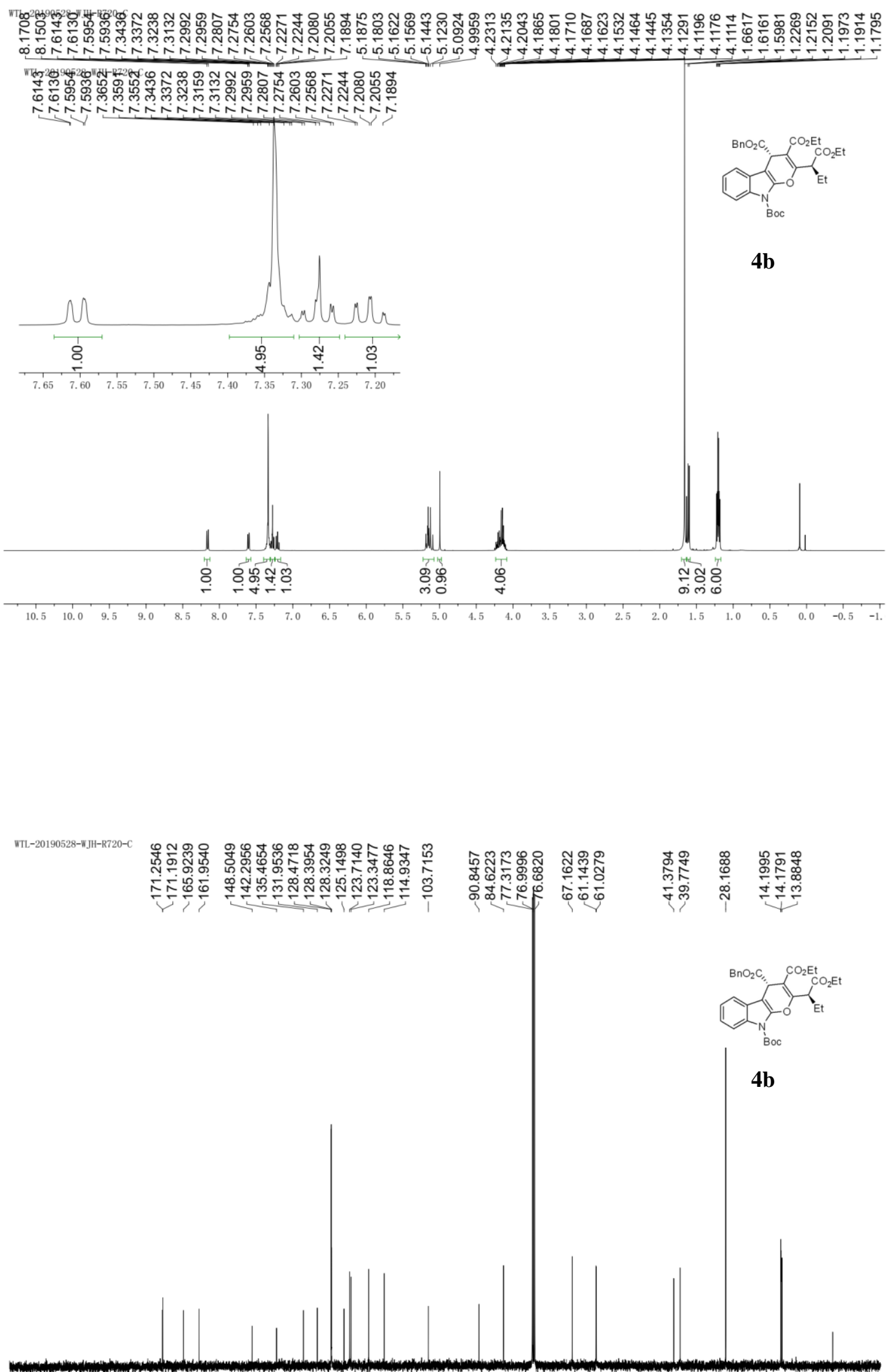

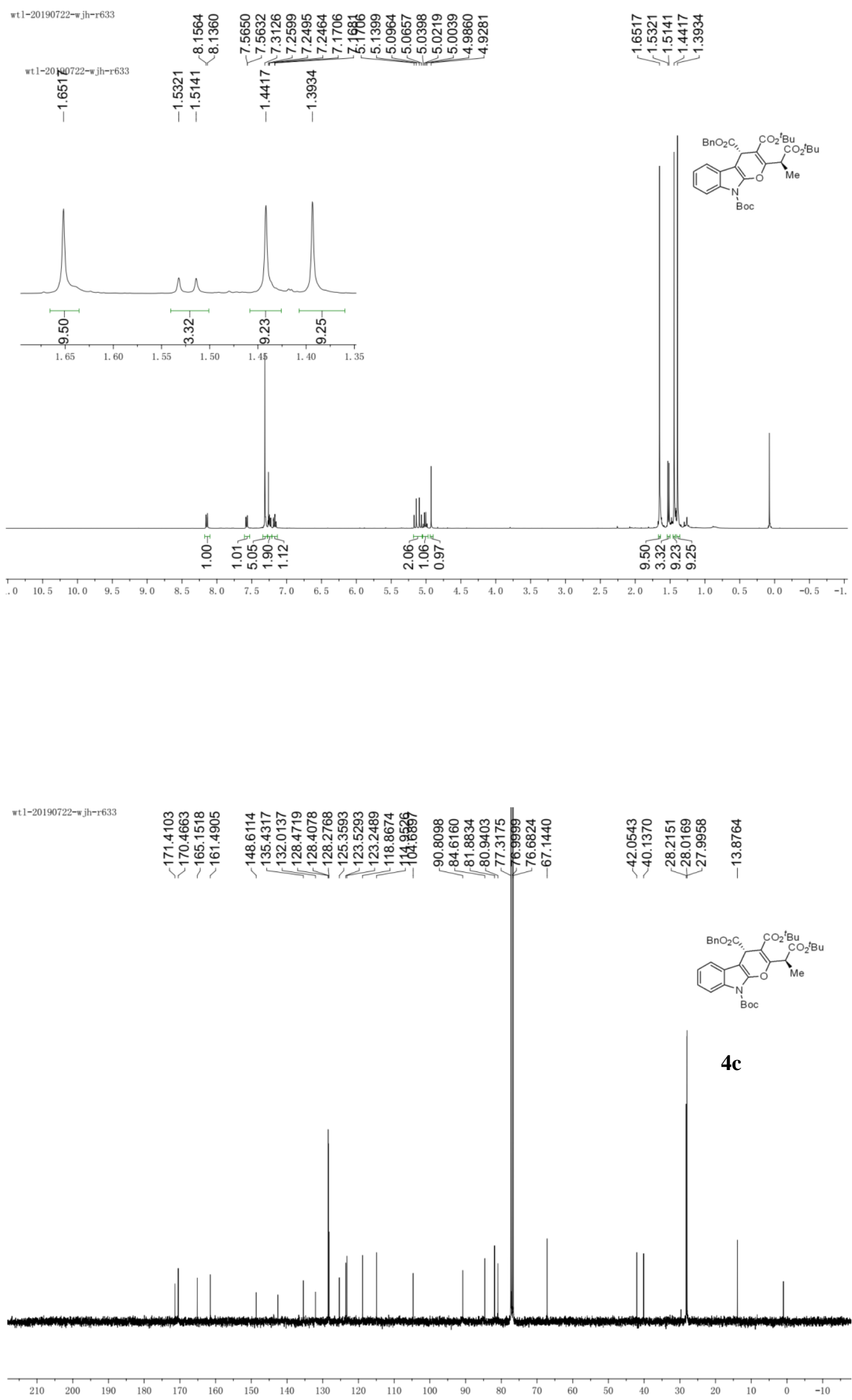


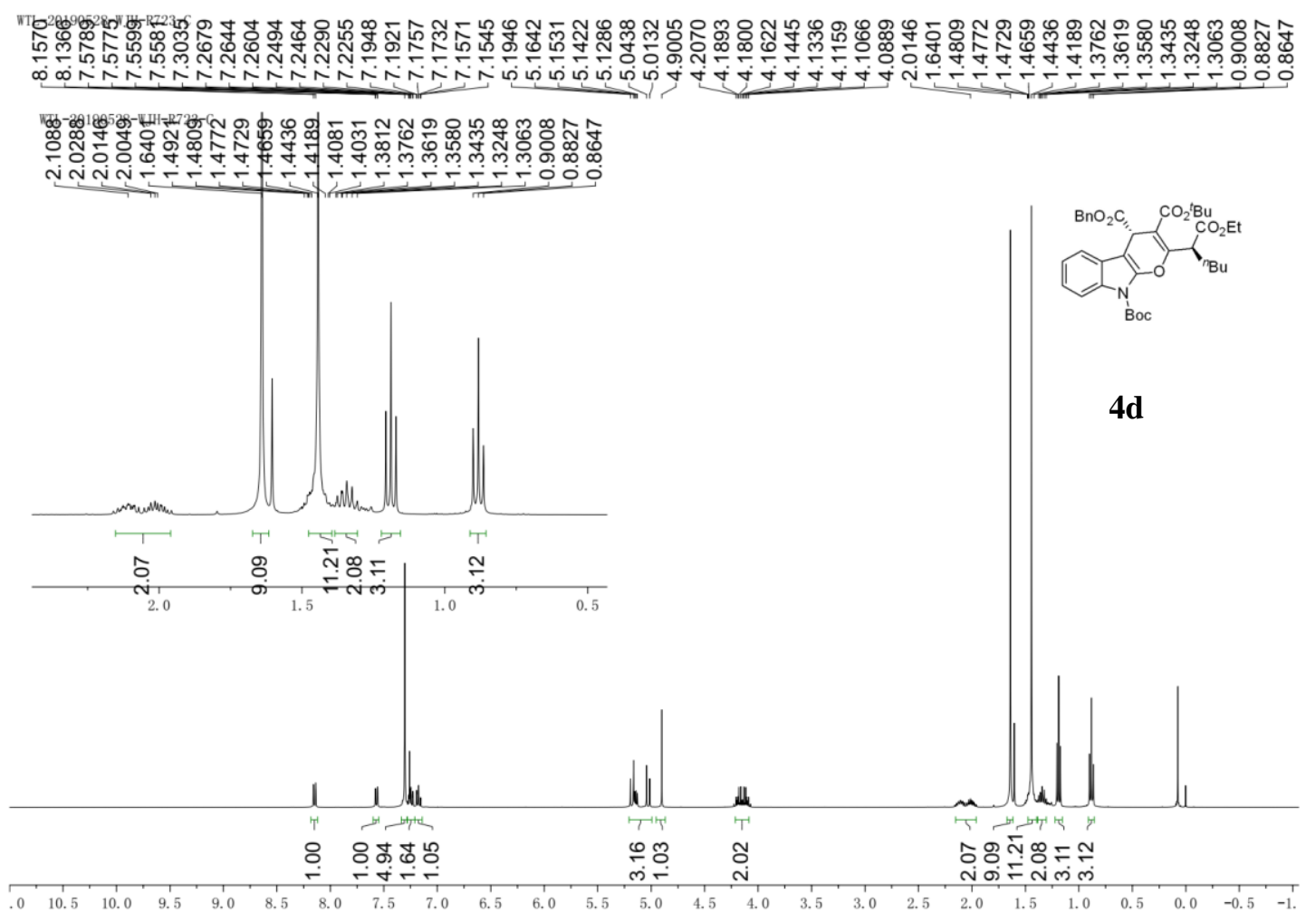

WTL-20190528-WJH-R723-C

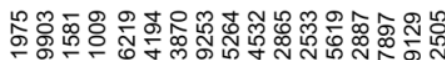

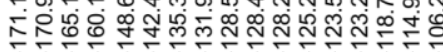

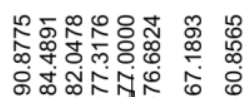

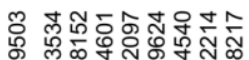

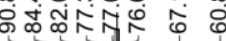

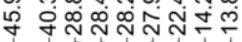

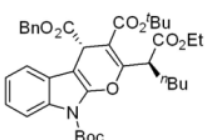

4d

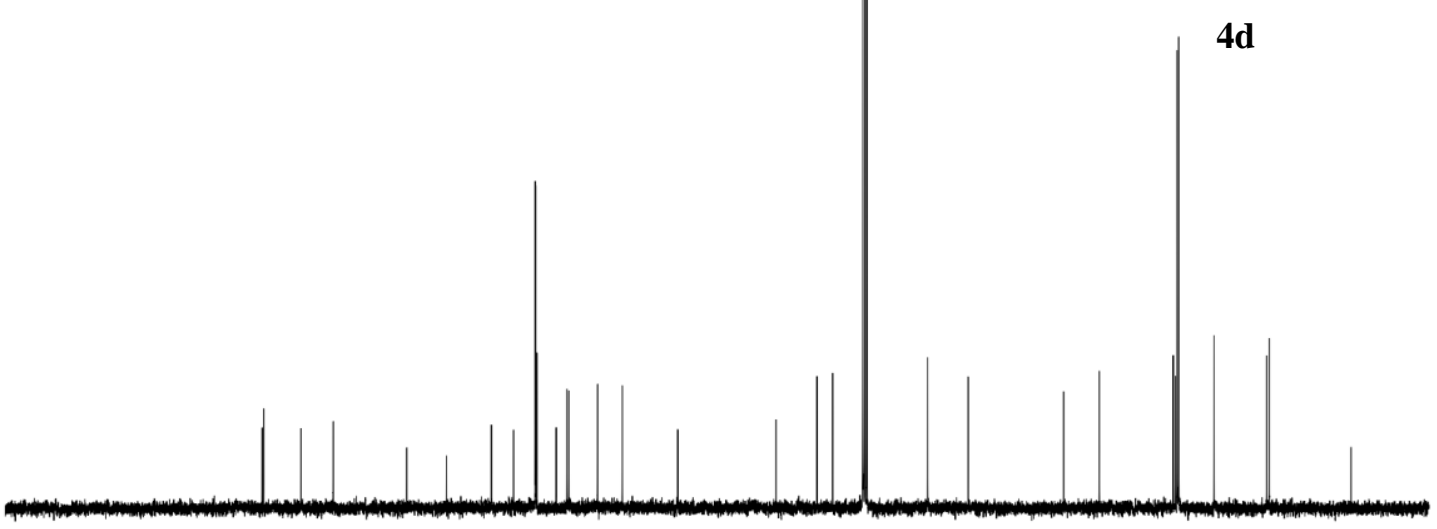

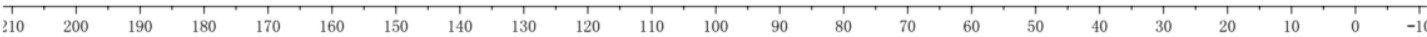




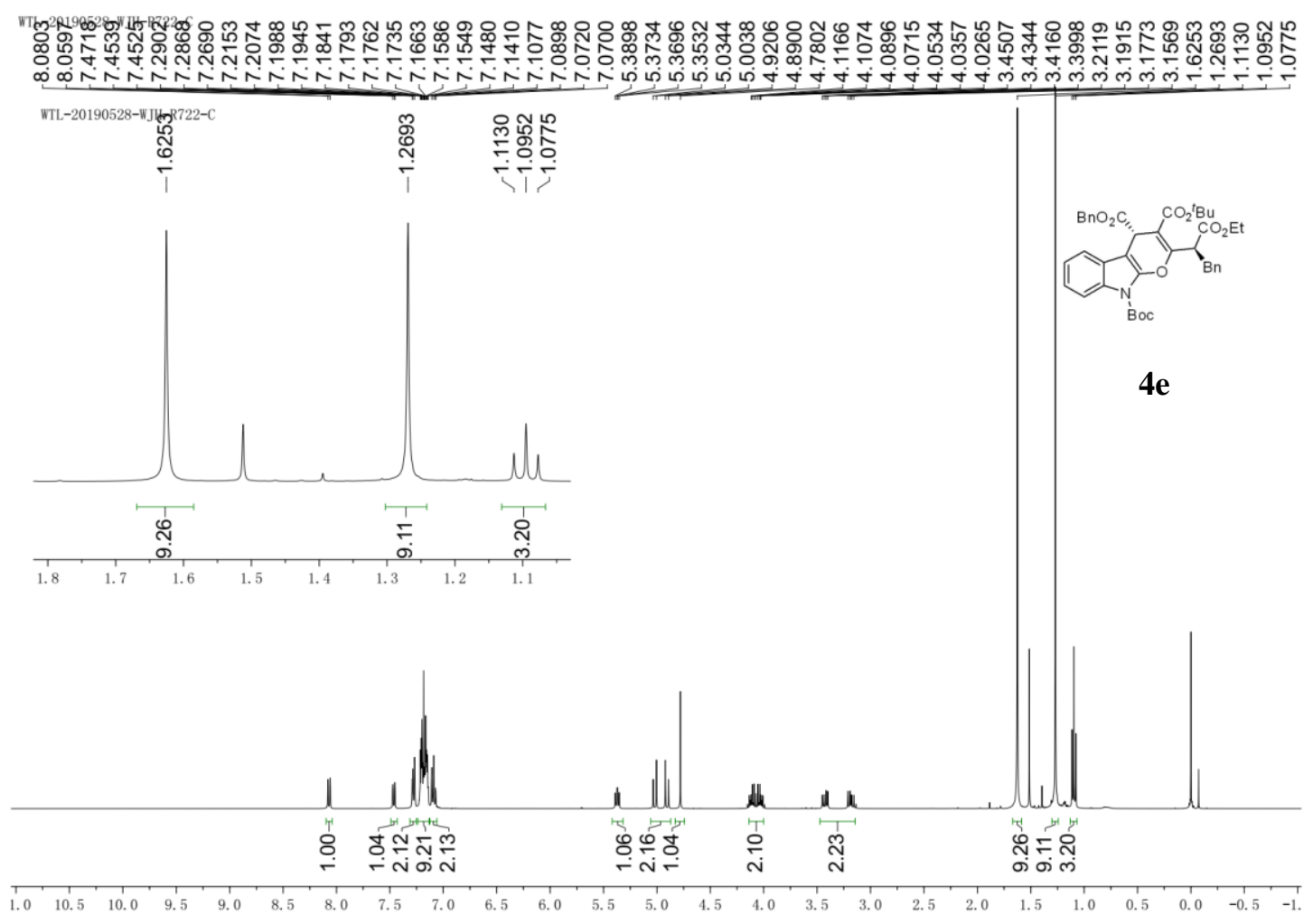

WTL-20190528-WJH-R722-C

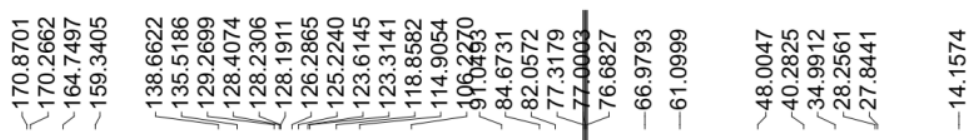

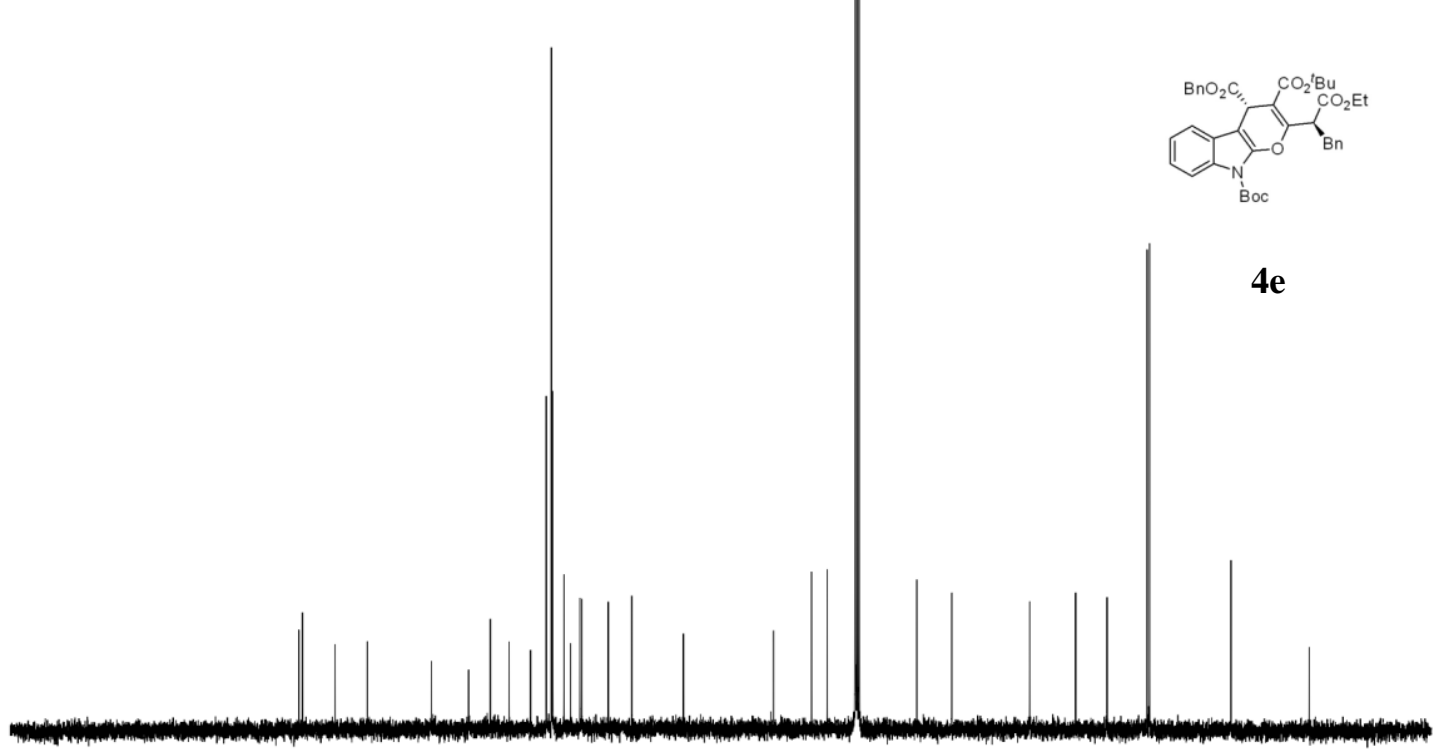

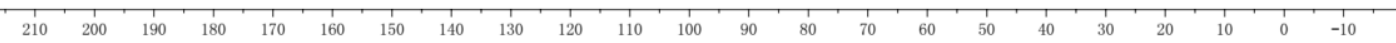



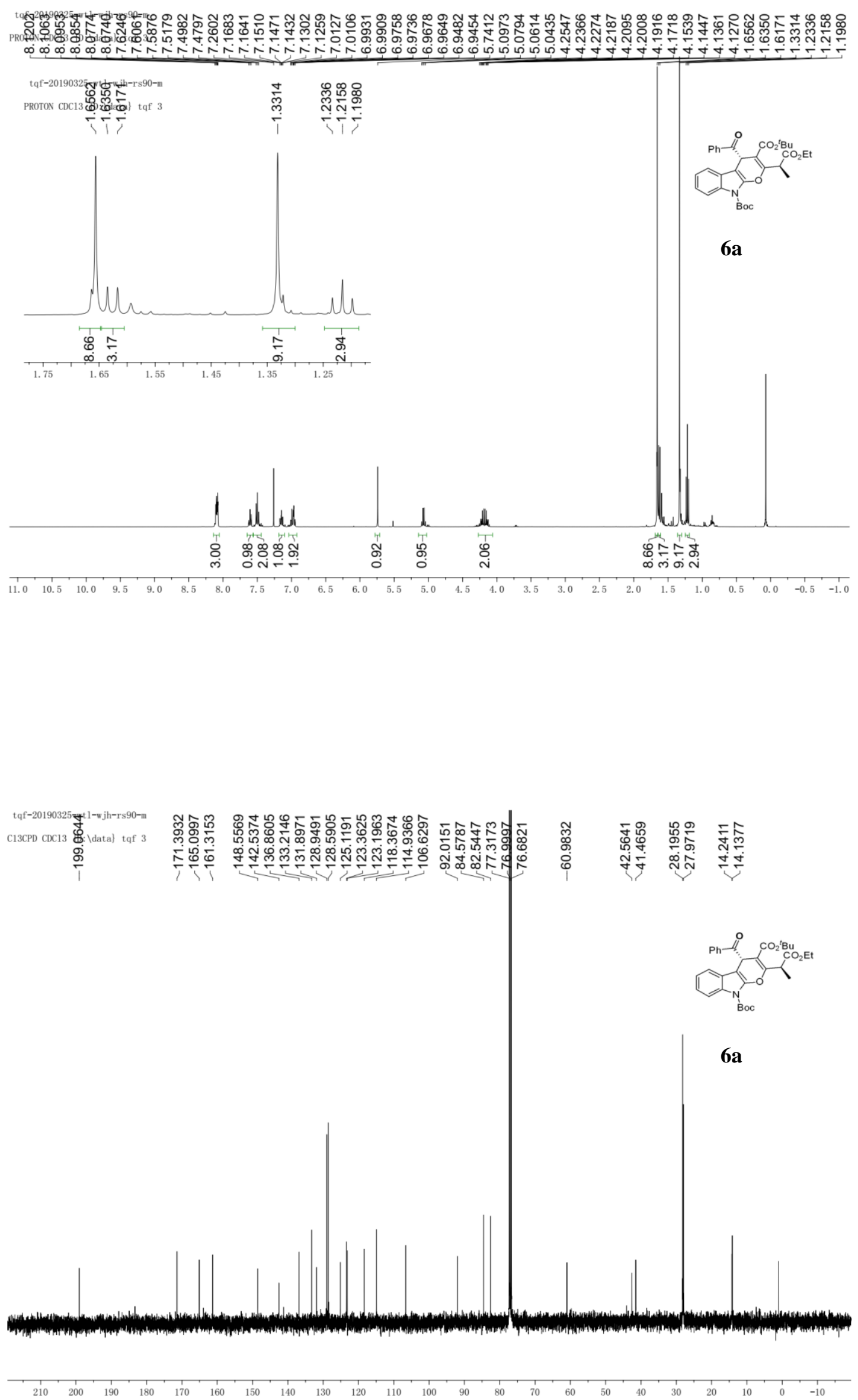


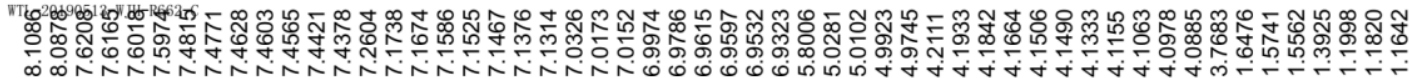
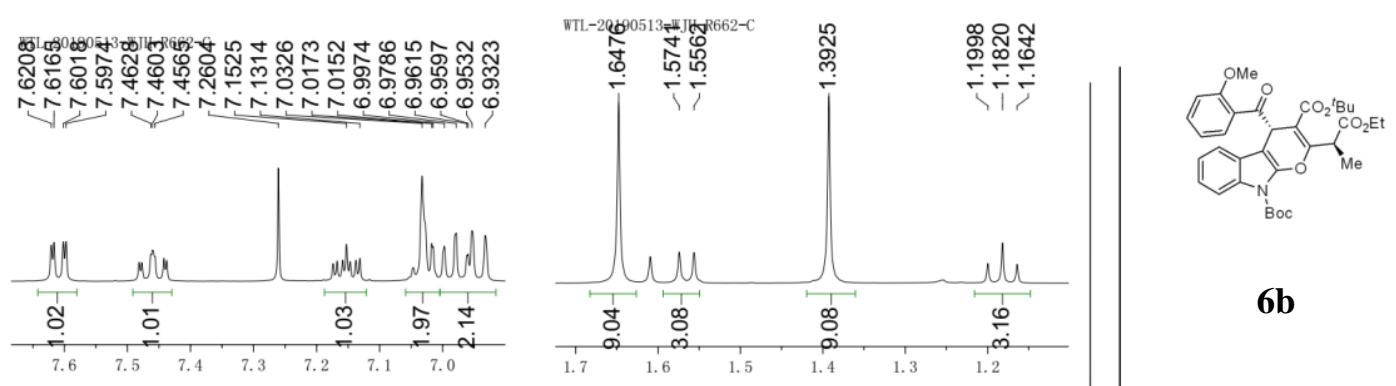

$\mathbf{6 b}$

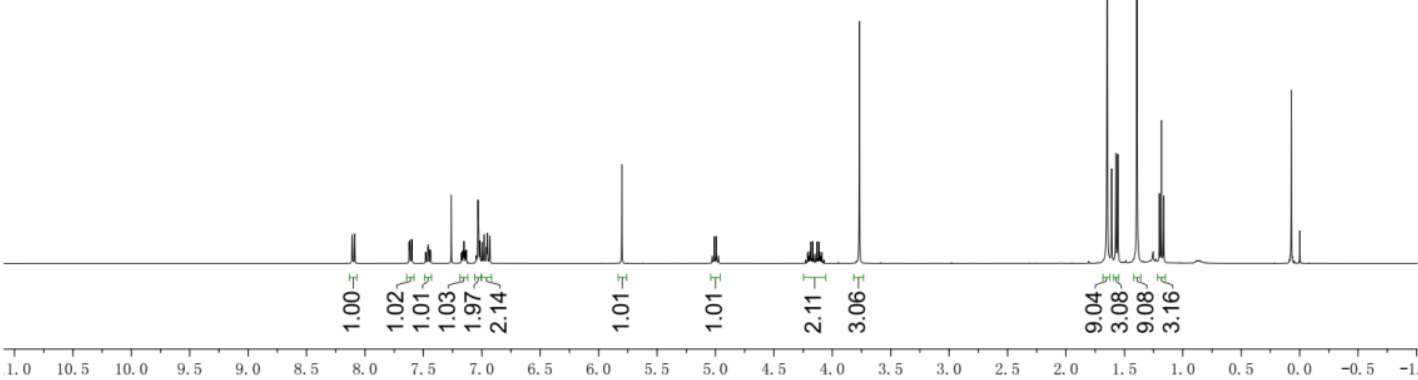

WTL-20190512-UIJH-R662-C

क्ष

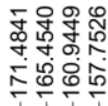

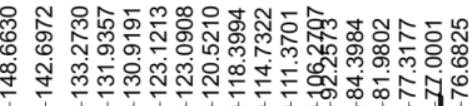

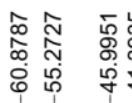

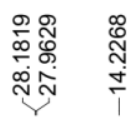

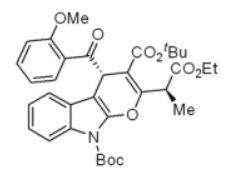

6b

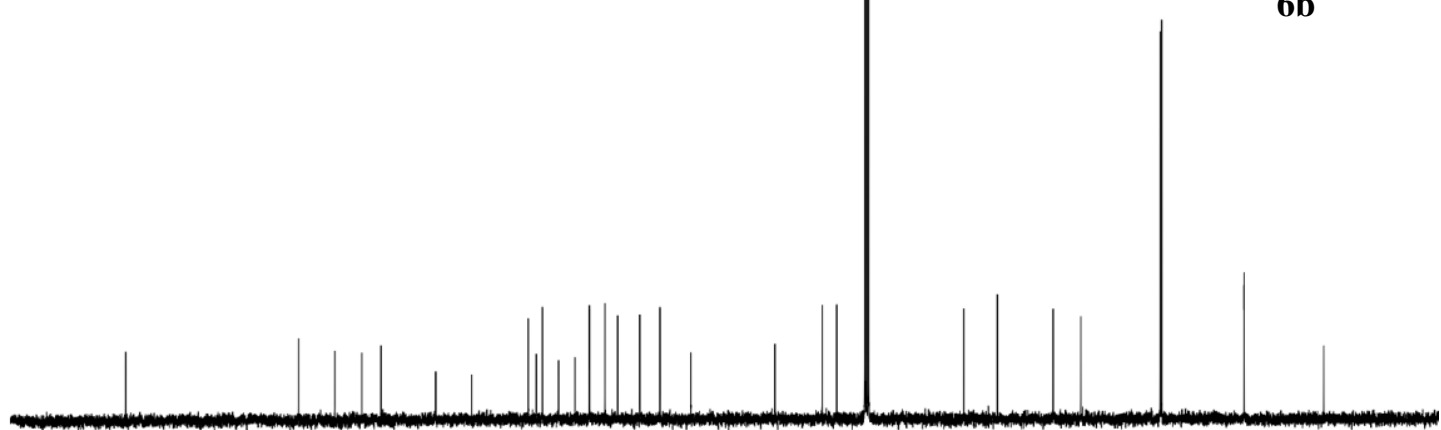

$\begin{array}{llllllllllll}210 & 200 & 190 & 180 & 170 & 160 & 150 & 140 & 130 & 120 & 110 & 100\end{array}$ 


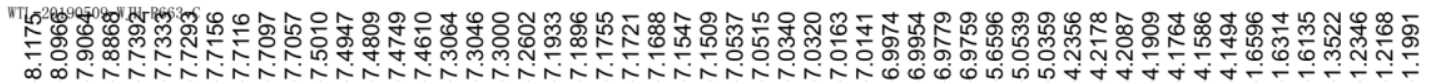

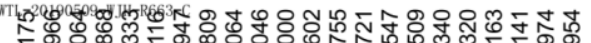

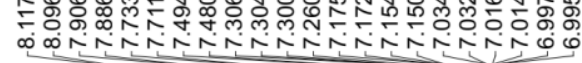
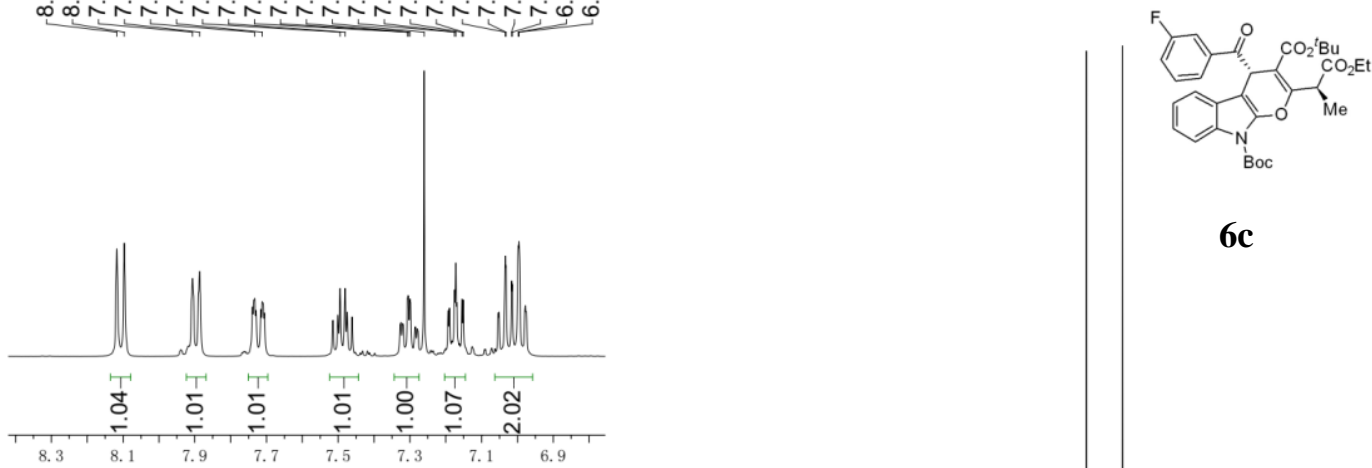

6c

Mendull

(1)

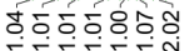

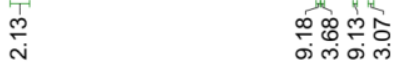

$\begin{array}{llllllllllllllllllllllll}10.5 & 10.0 & 9.5 & 9.0 & 8.5 & 8.0 & 7.5 & 7.0 & 6.5 & 6.0 & 5.5 & 5.0 & 4.5 & 4.0 & 3.5 & 3.0 & 2.5 & 2.0 & 1.5 & 1.0 & 0.5 & 0.0 & -0.5 & -1 .\end{array}$

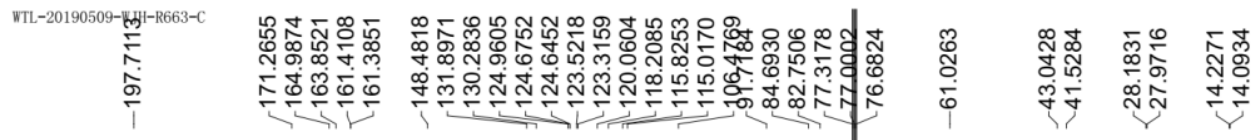

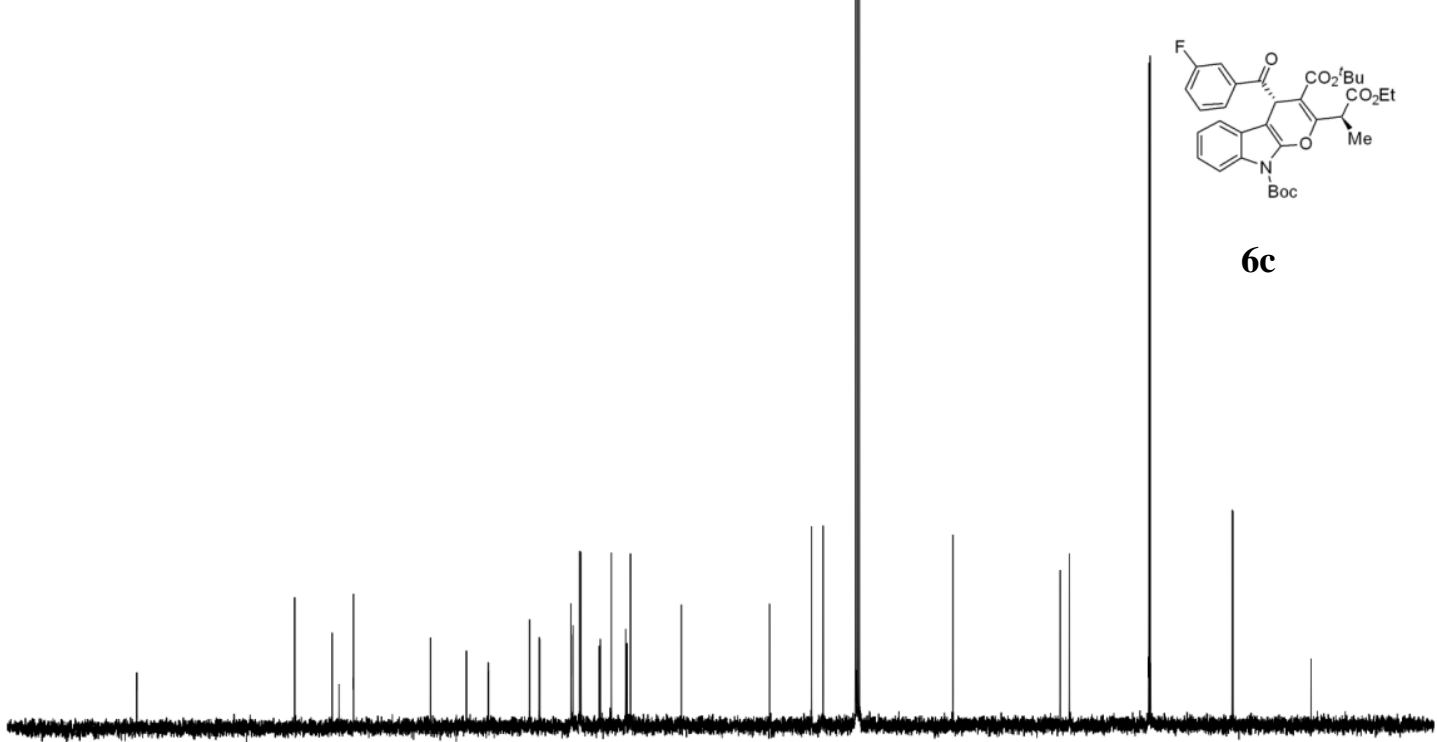

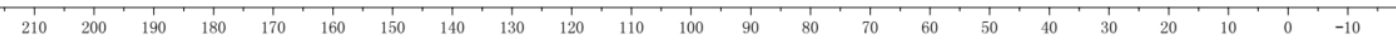


WTL-20190509-WJH-R663-C

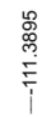

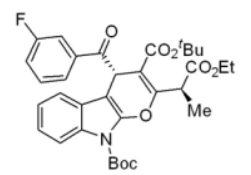

6c

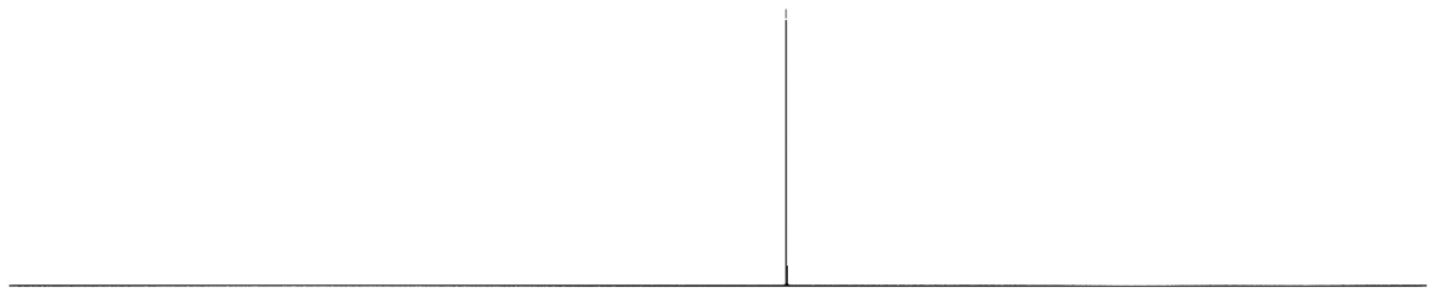

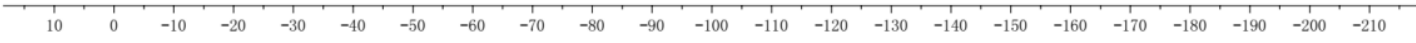

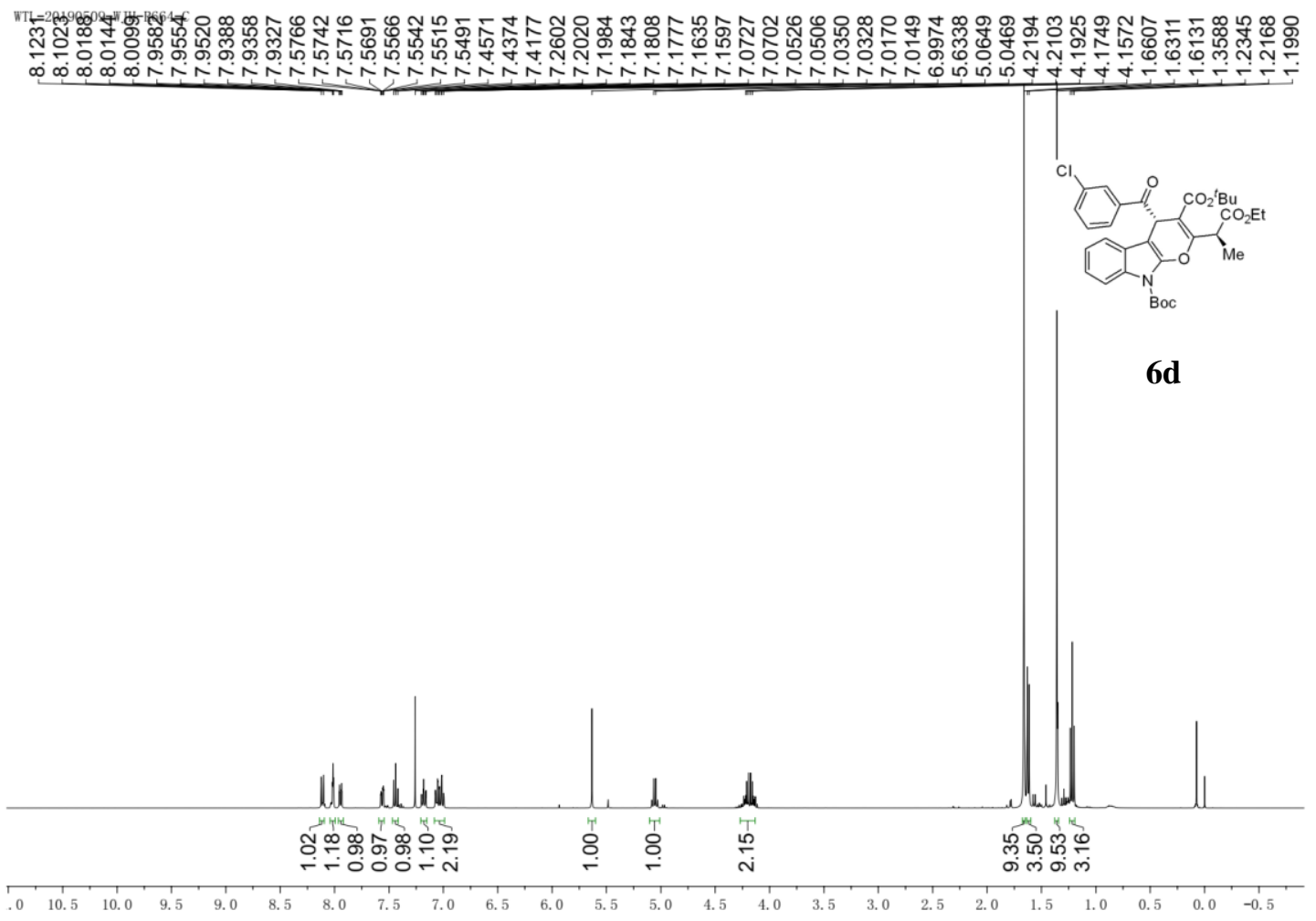




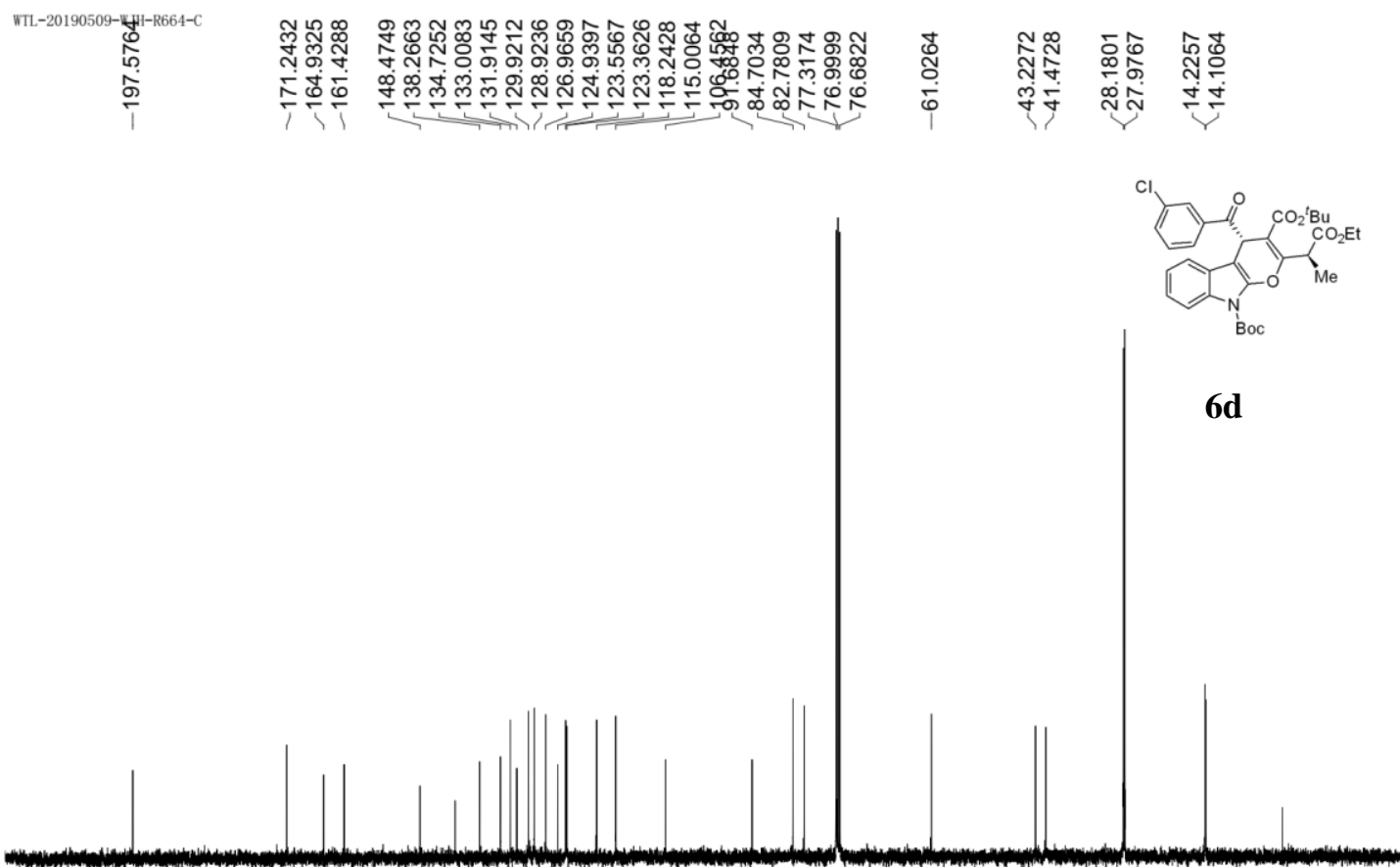

$\begin{array}{llllllllllllllllllllllll}210 & 200 & 190 & 180 & 170 & 160 & 150 & 140 & 130 & 120 & 110 & 100 & 90 & 80 & 70 & 60 & 50 & 40 & 30 & 20 & 10 & 0 & -10\end{array}$

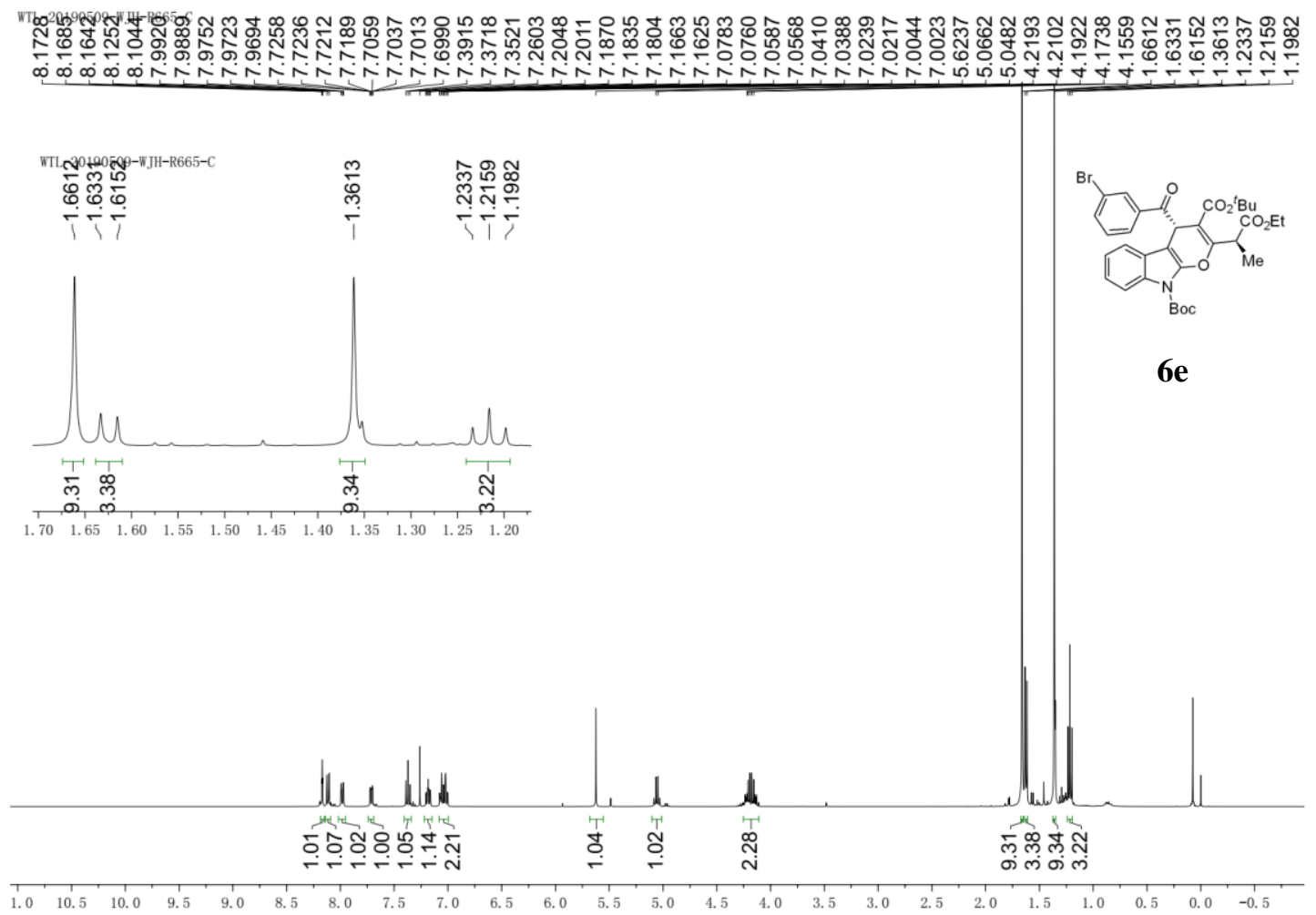




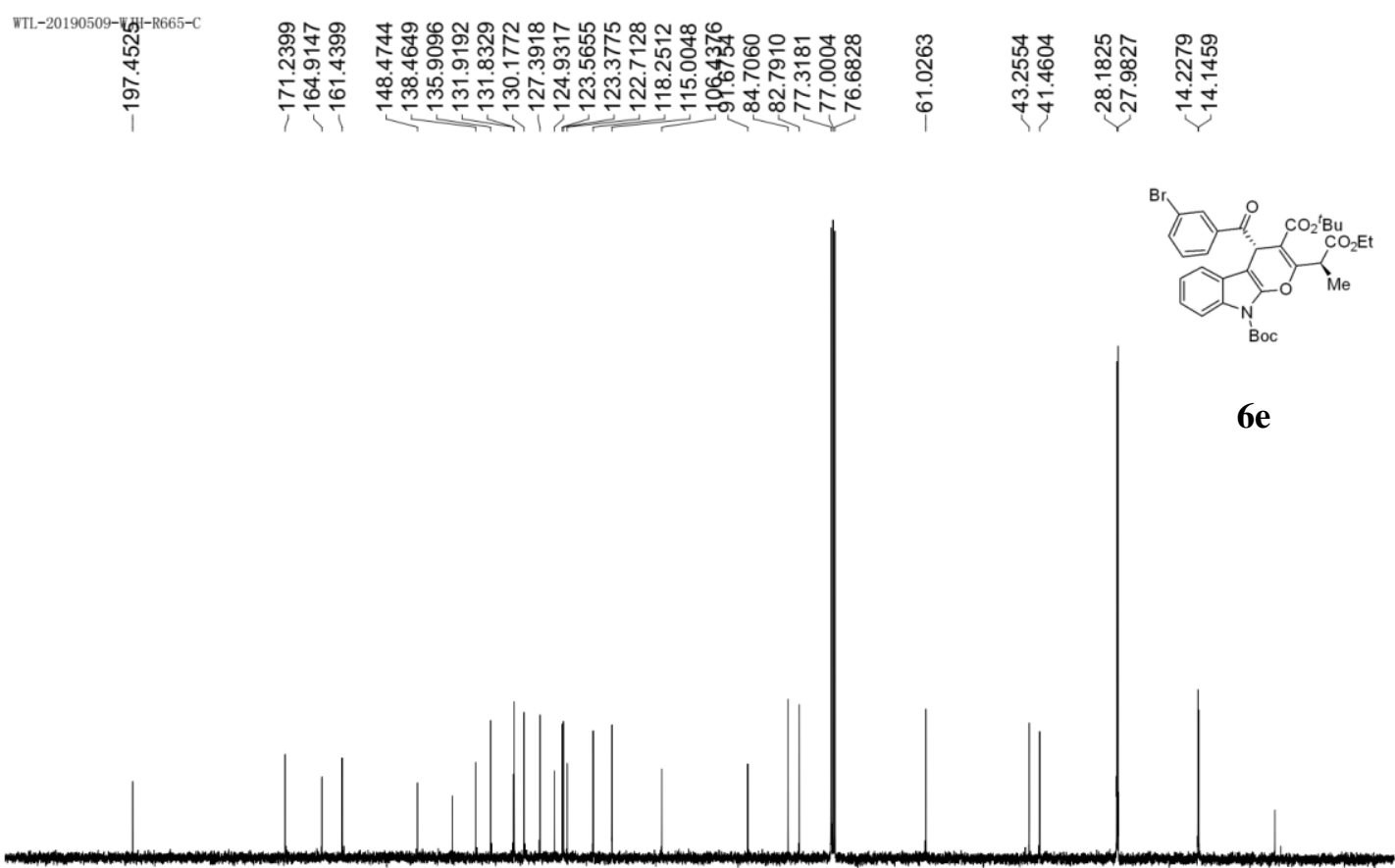

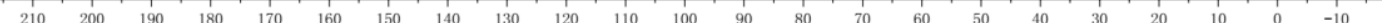

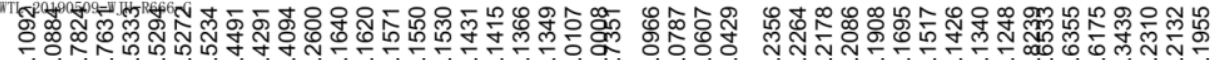

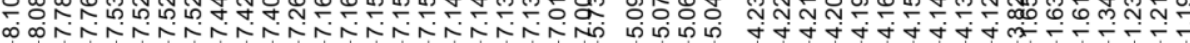

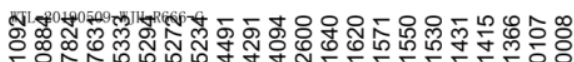

o
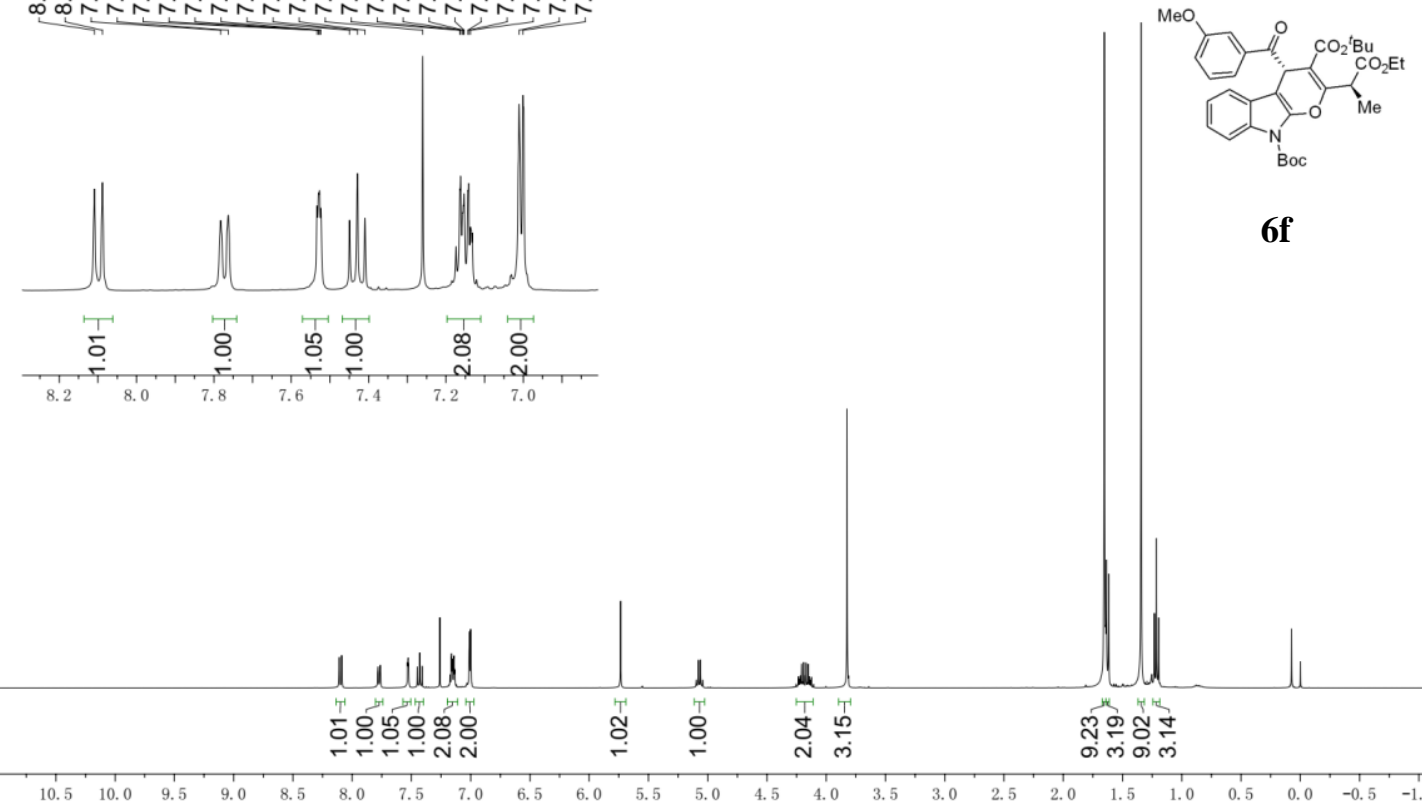


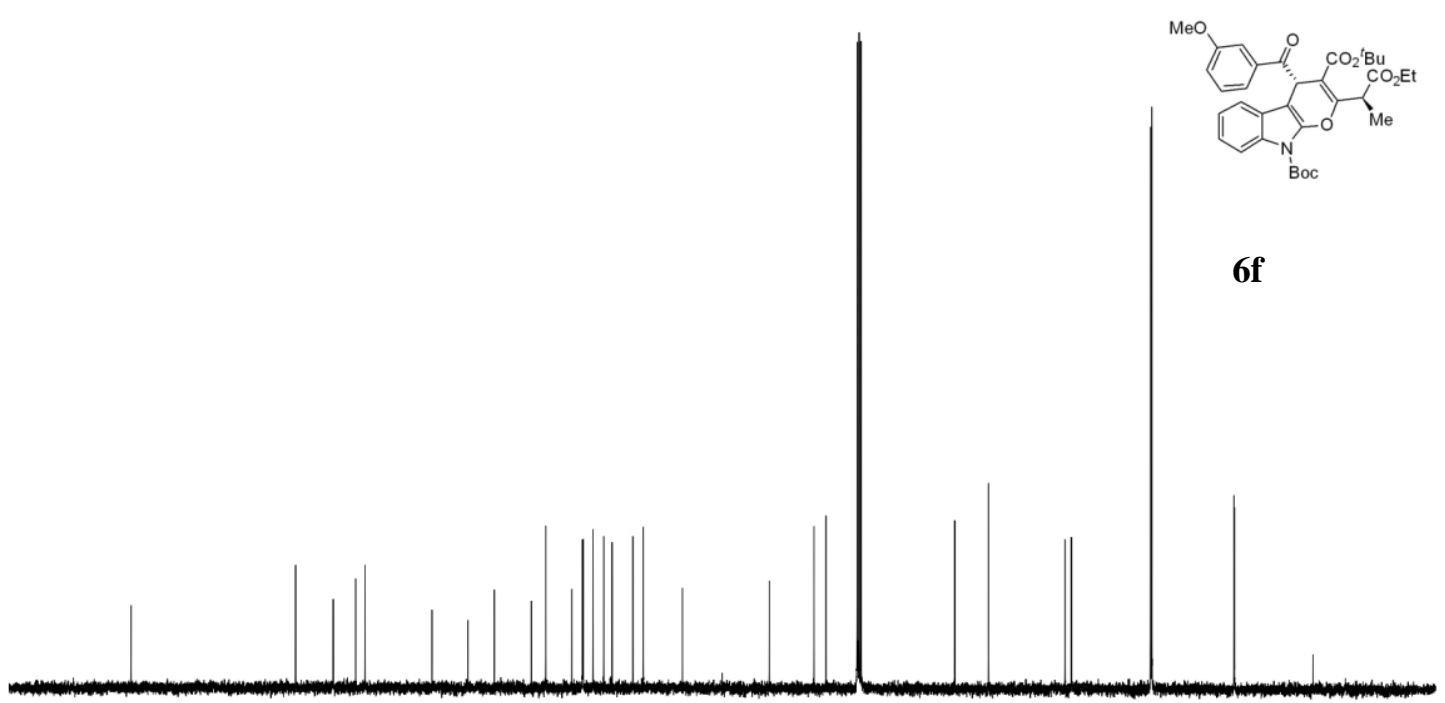

WTL-20190509-WJH-R673-C

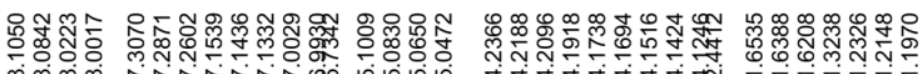

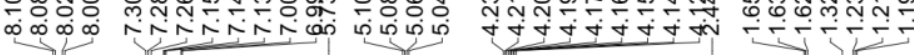

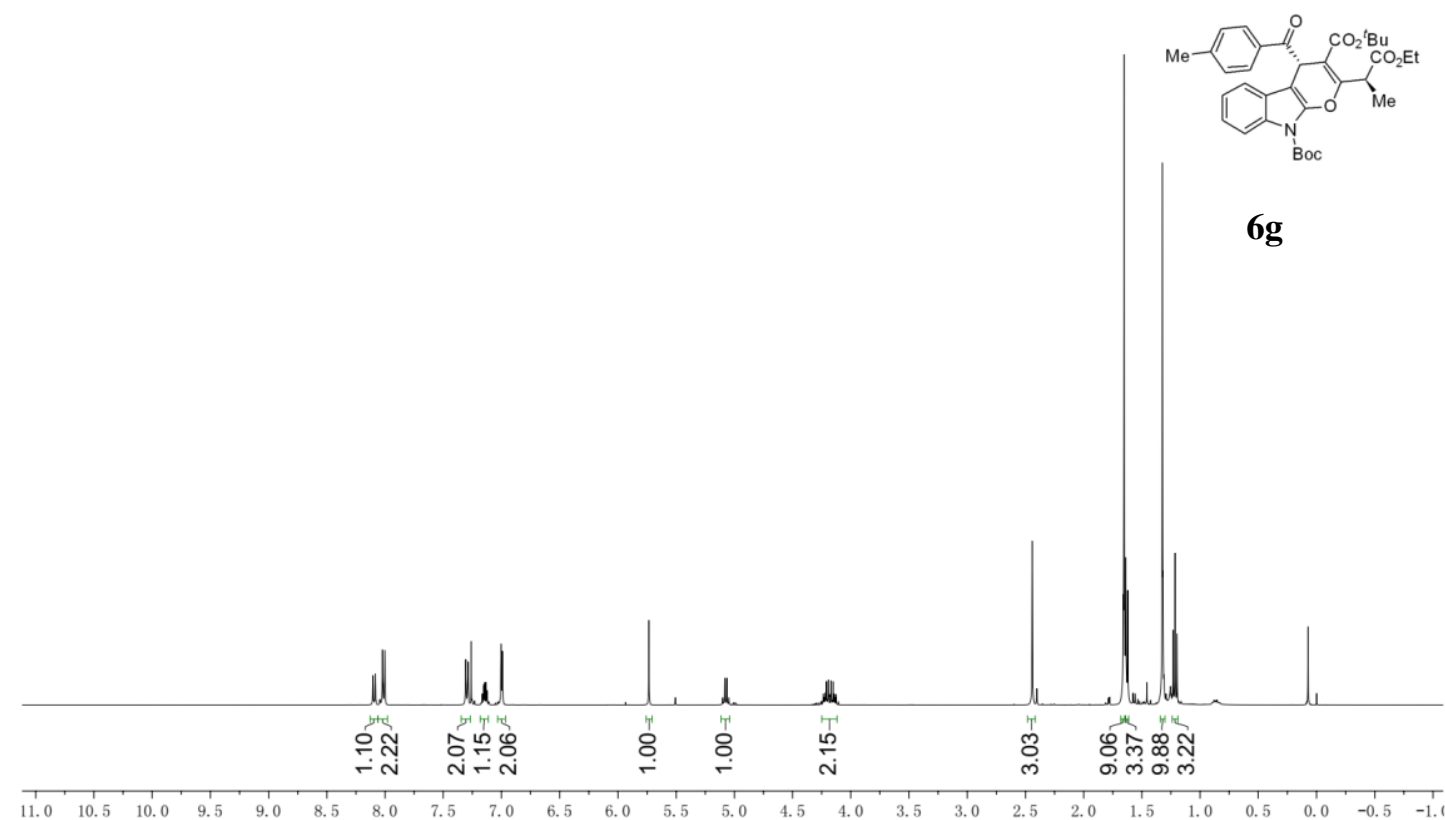



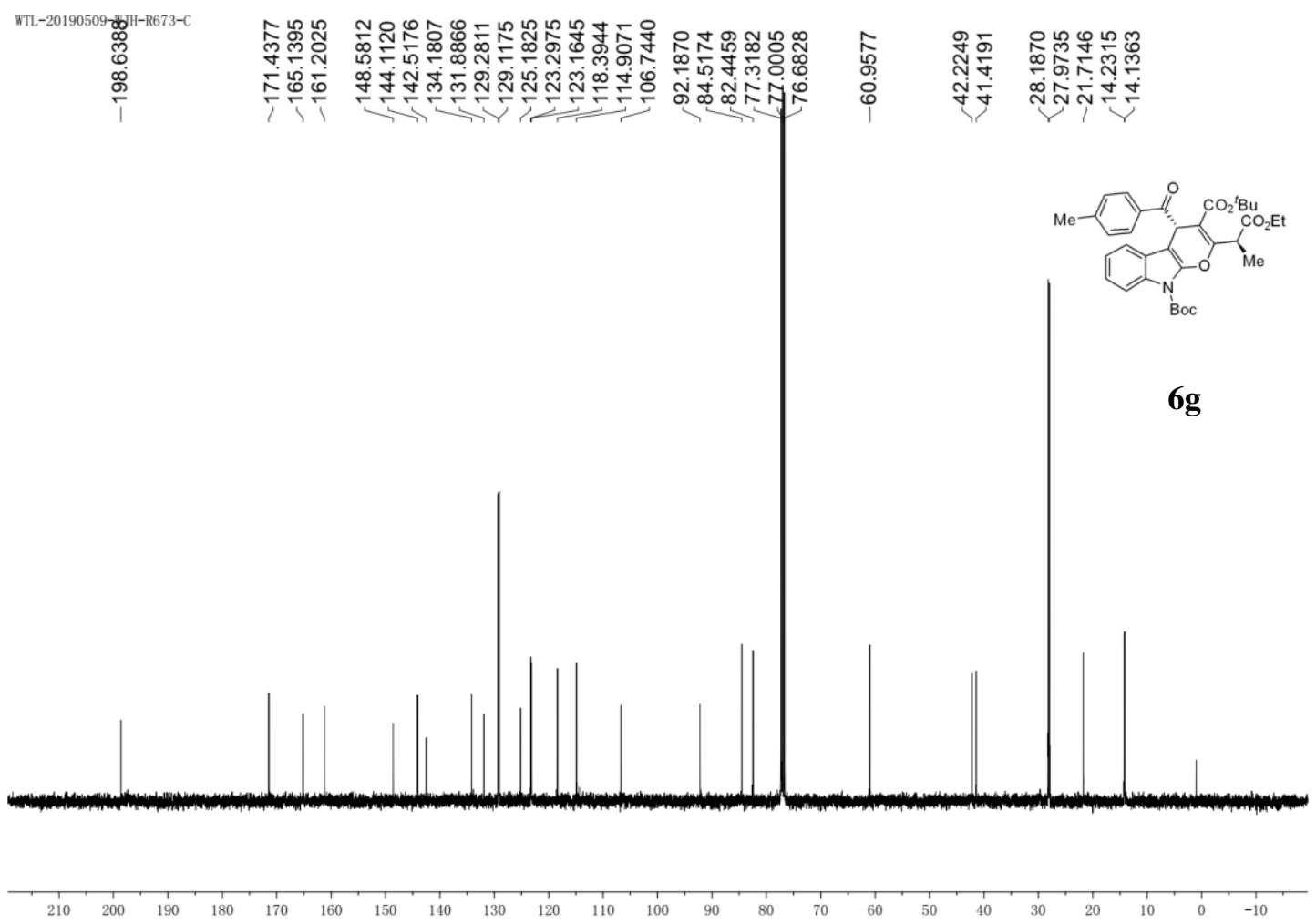

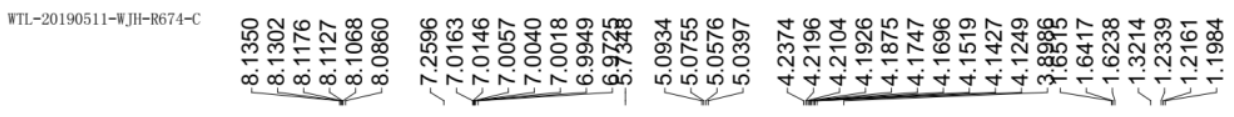
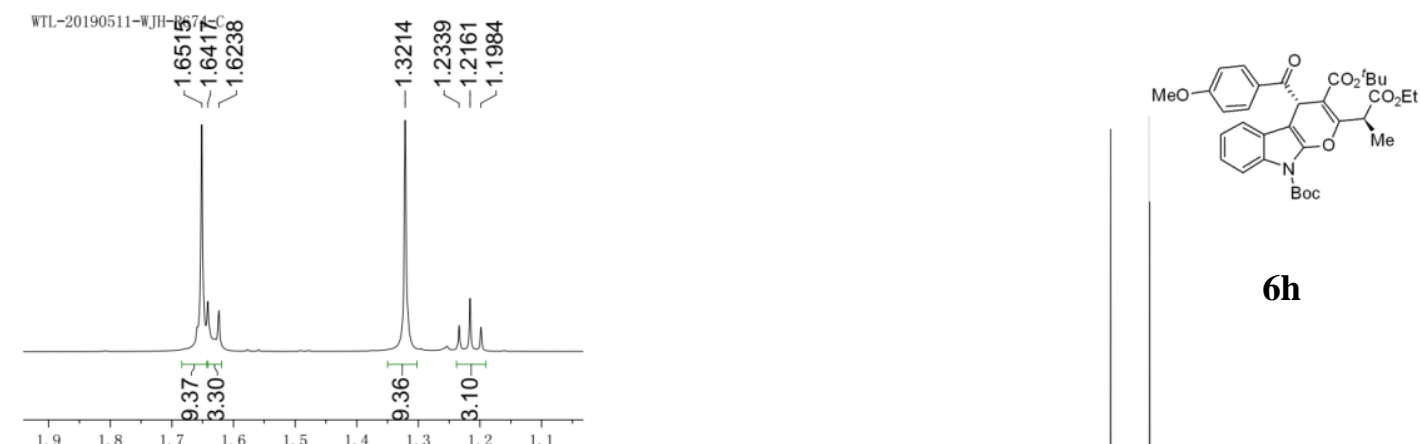

$6 h$

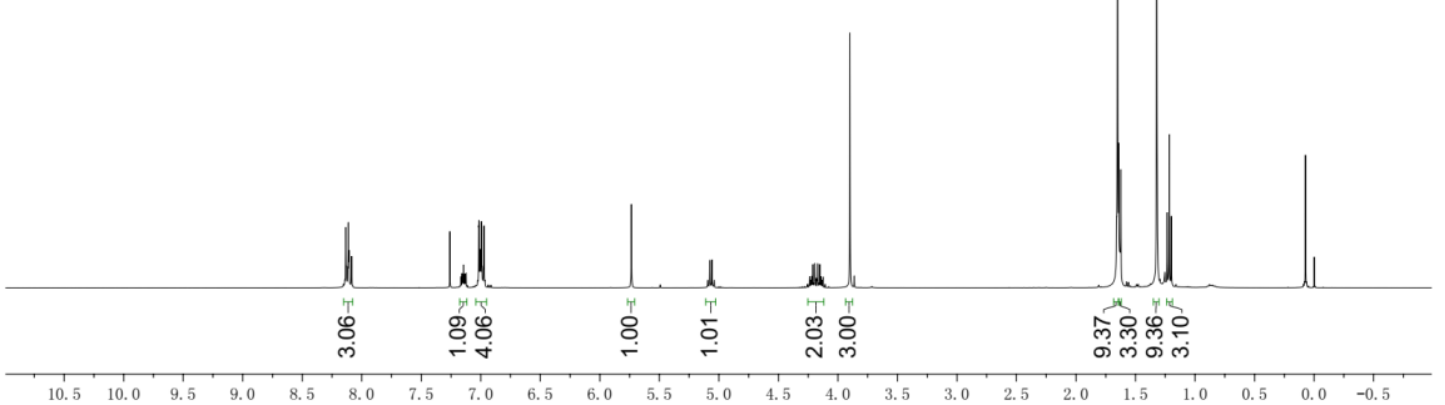




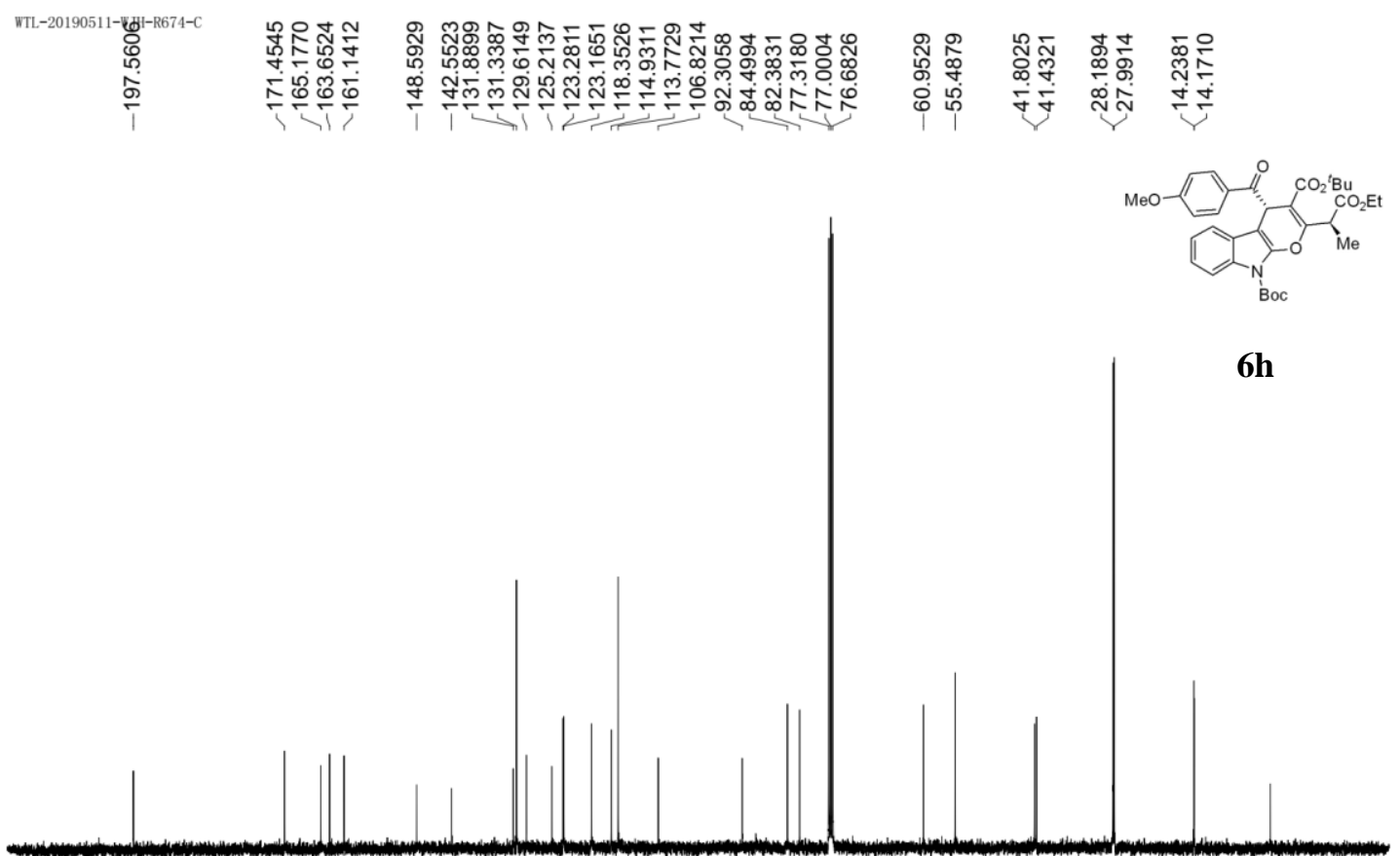

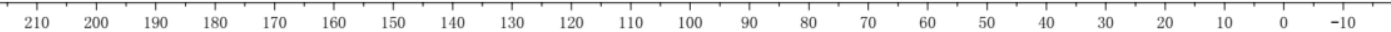

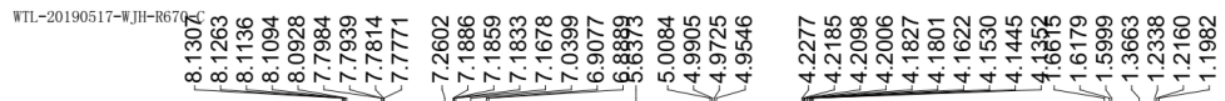

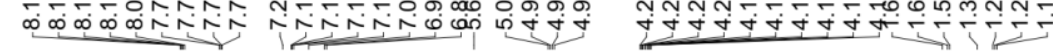

"Tredphd

óm
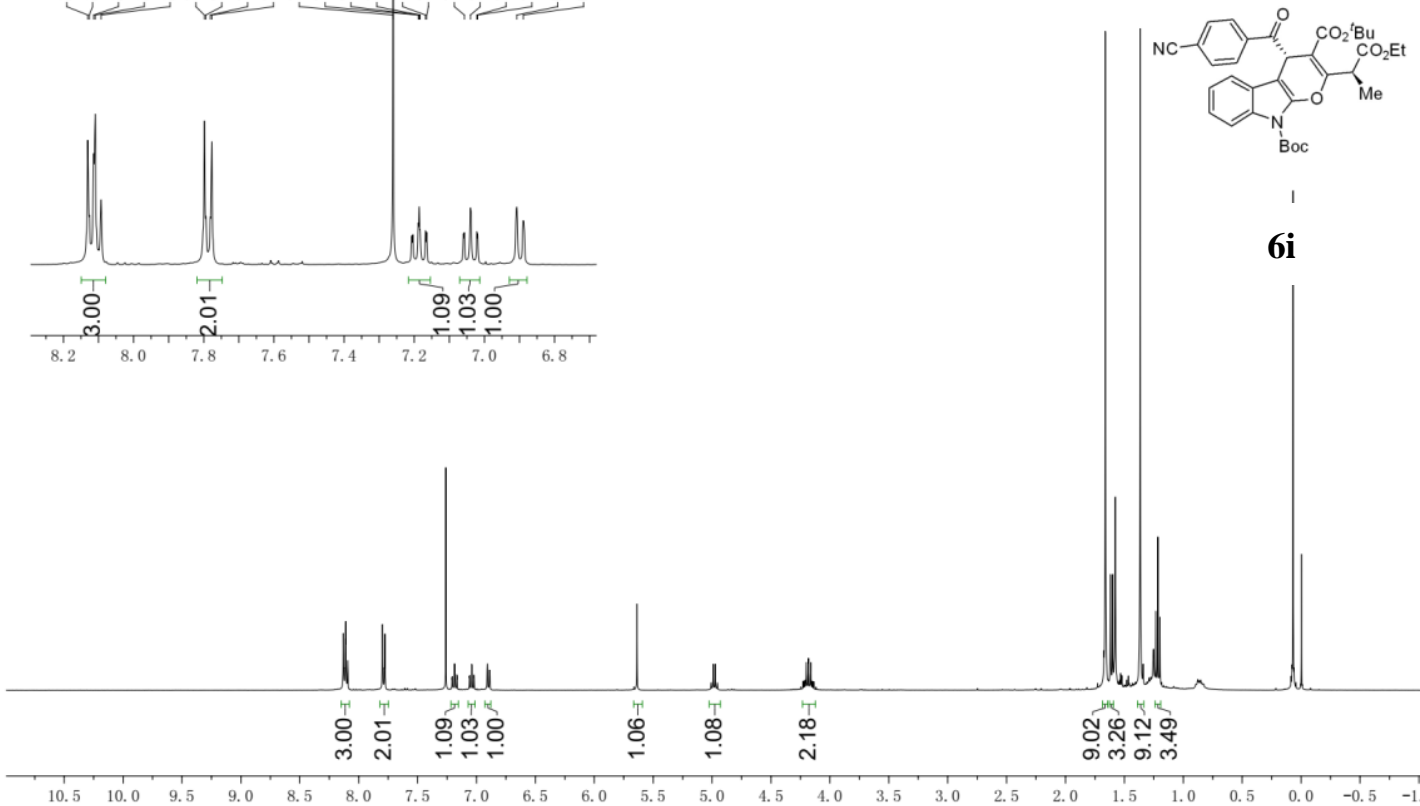

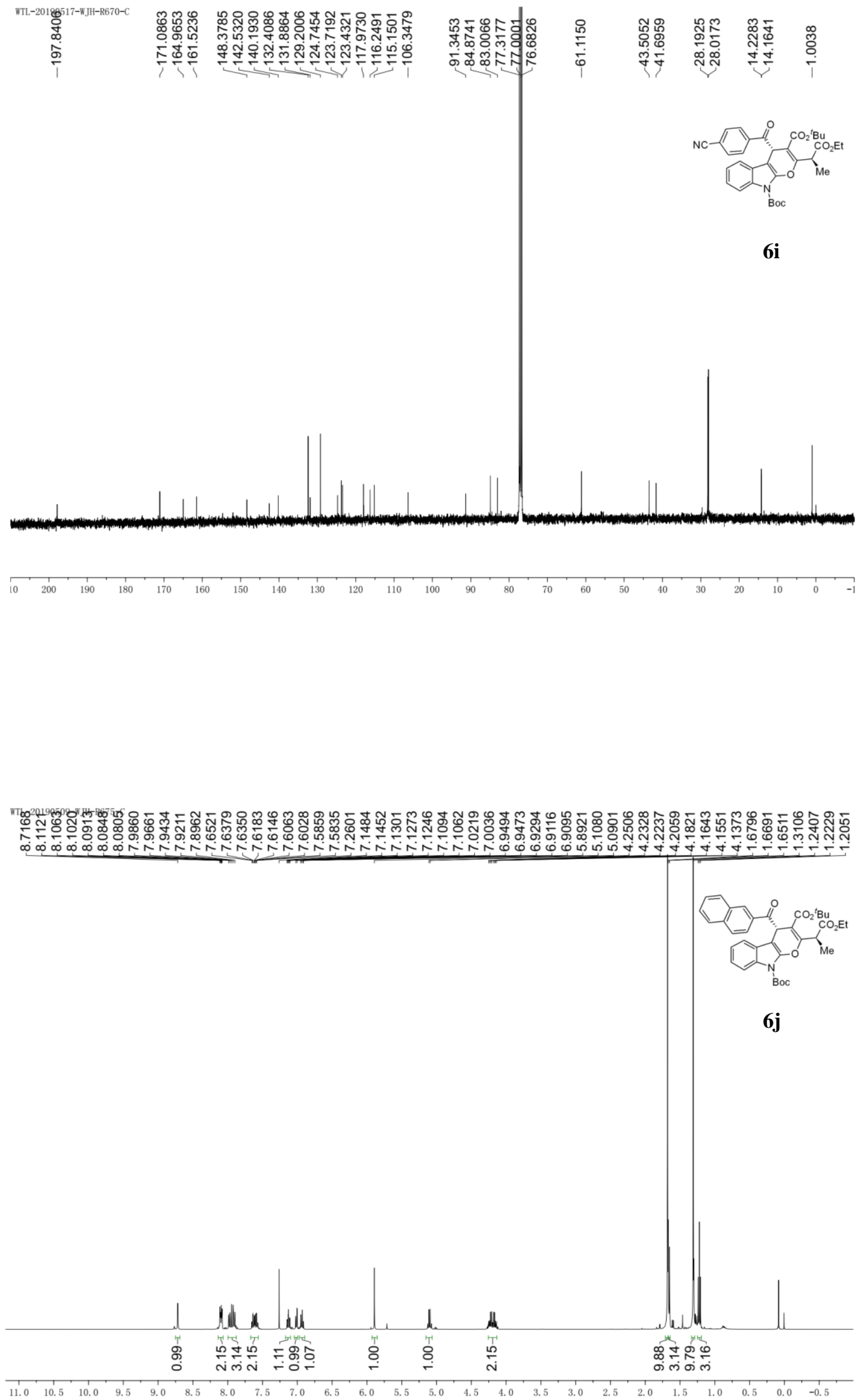


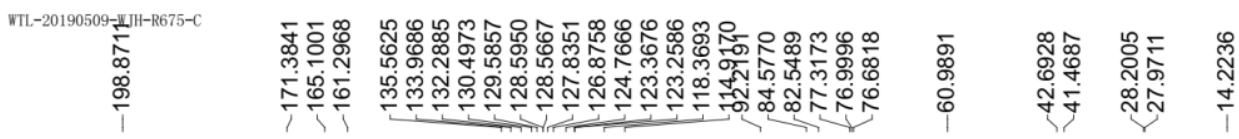
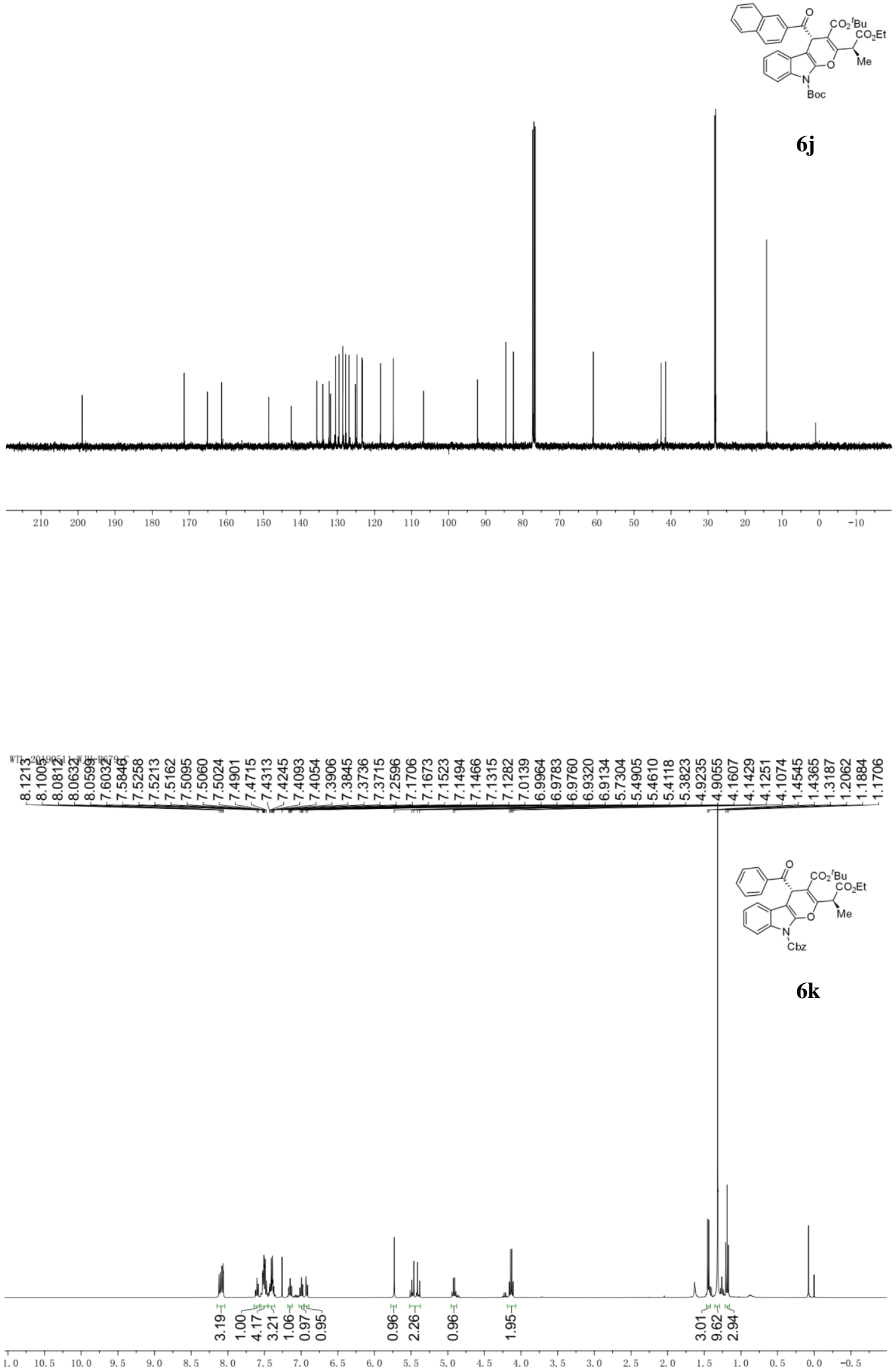

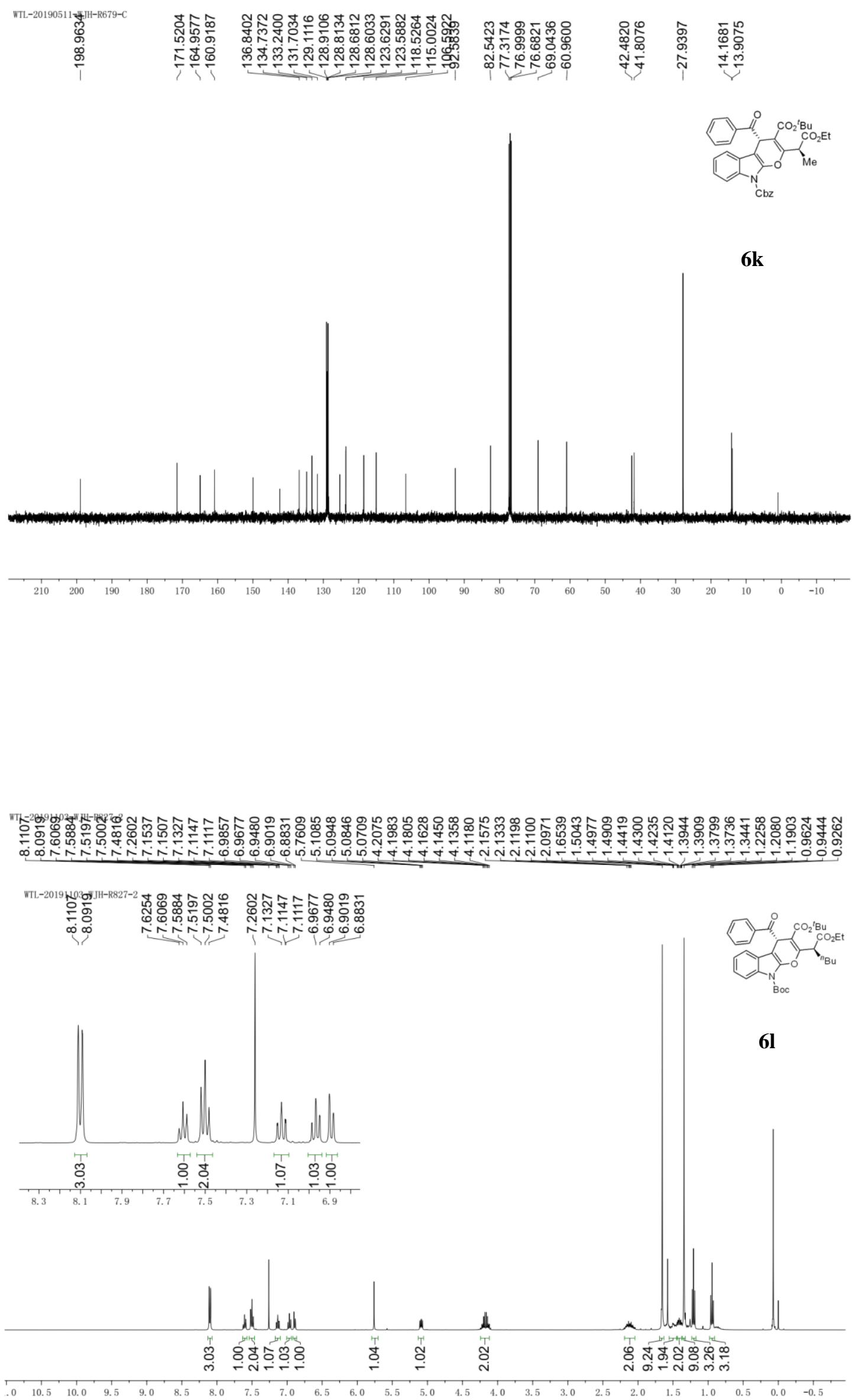


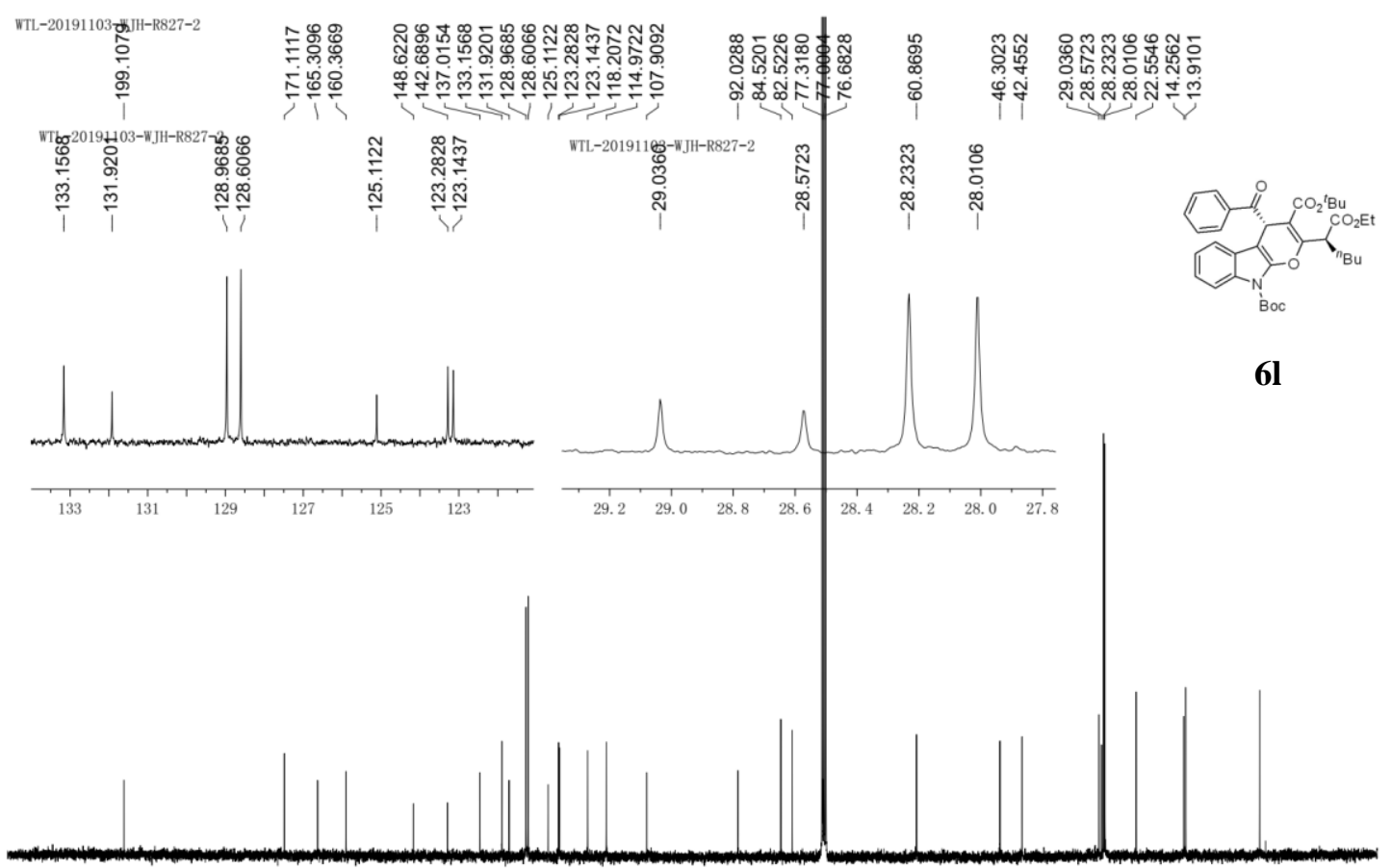

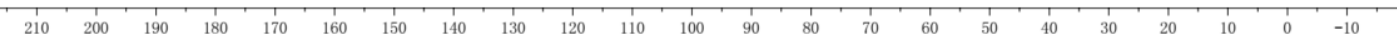

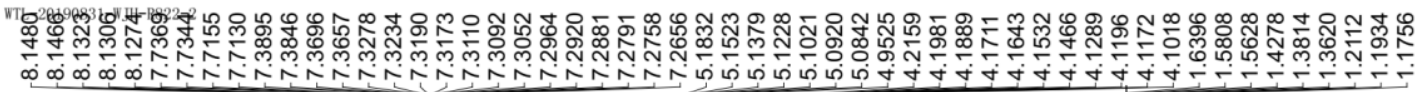

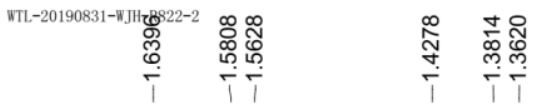
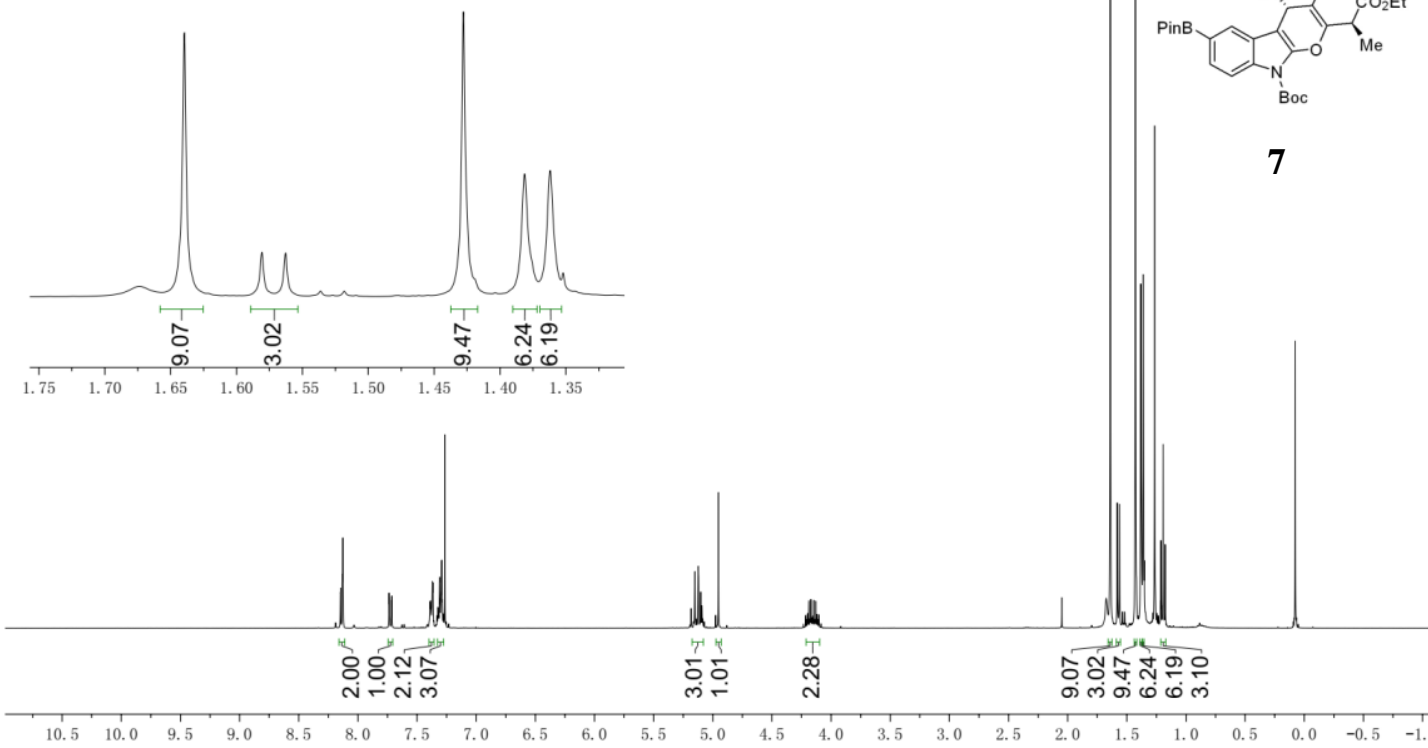

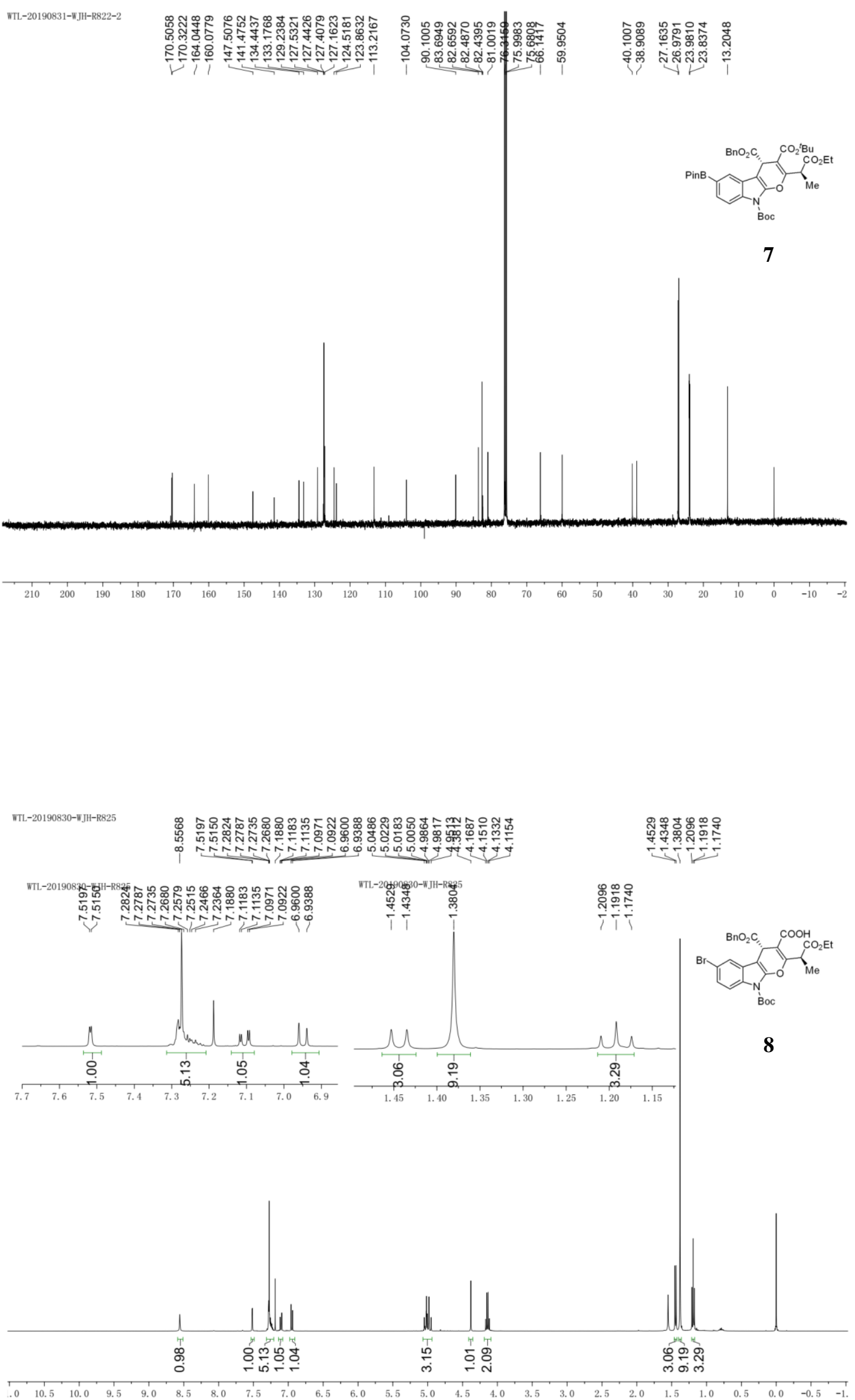

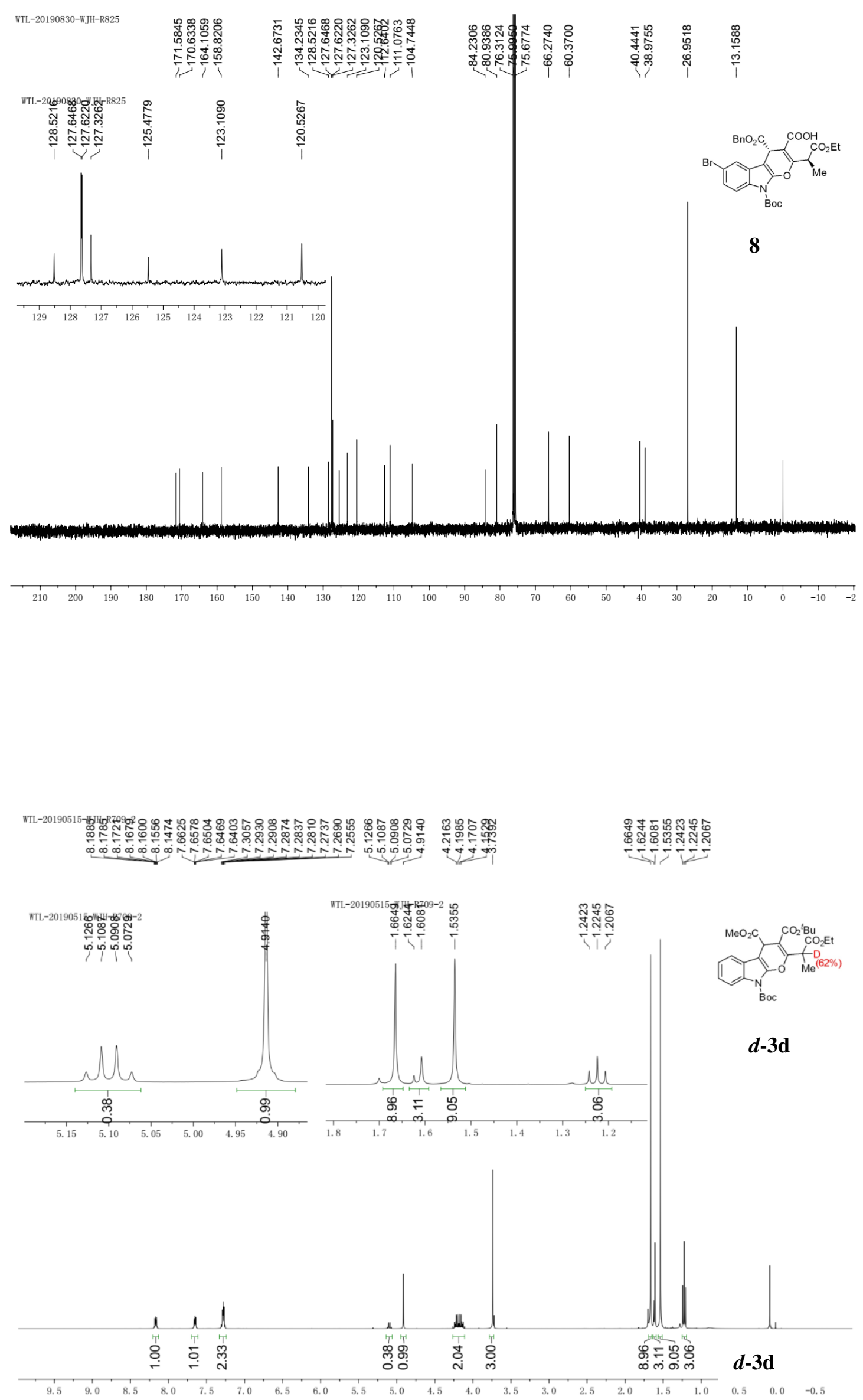


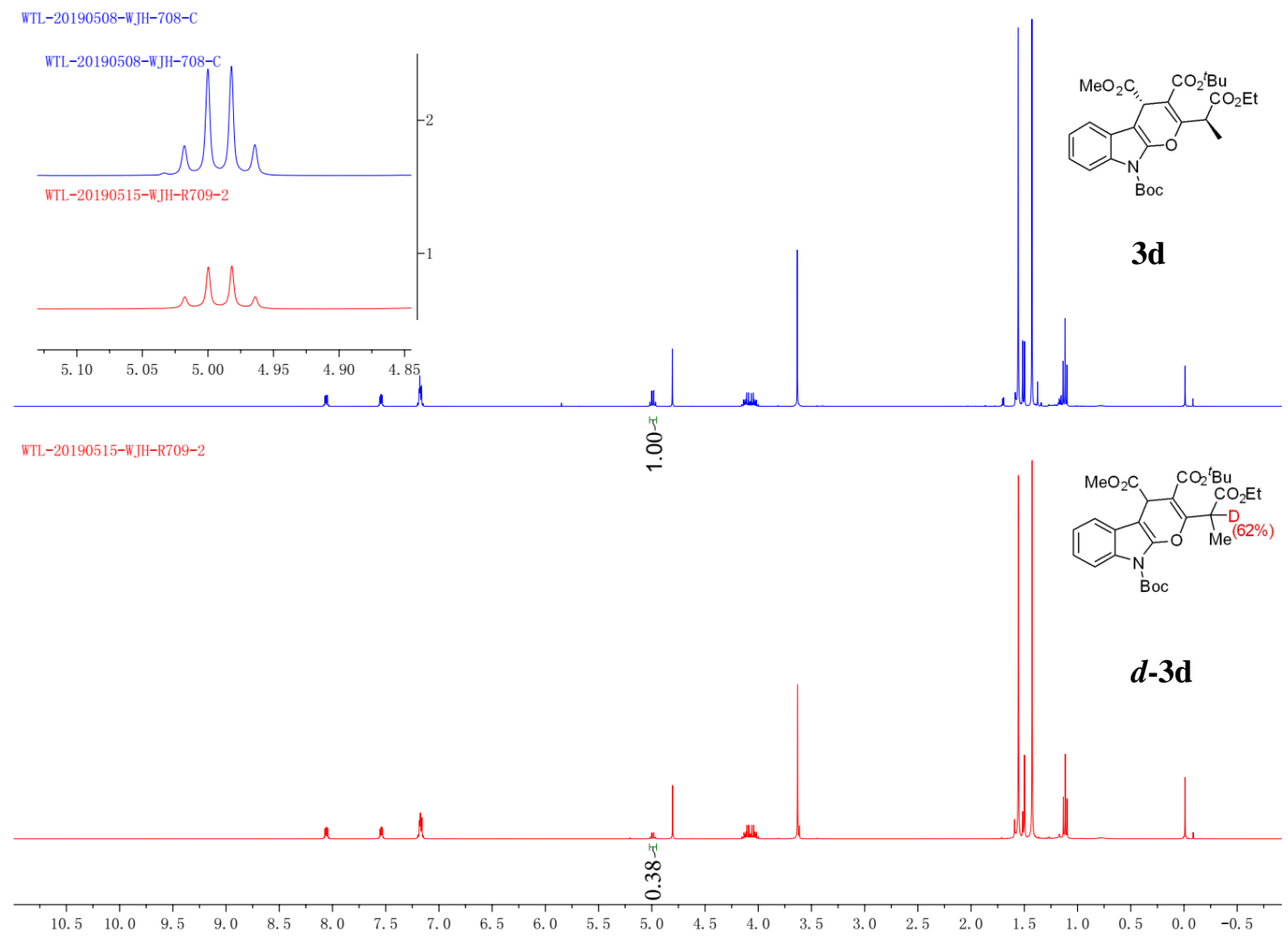

